LBL -15207

DE84 005856
Nuclear Science

Division

\section{Annual Report for the Period \\ July 1, 1981 - September 30, 1982}

\author{
Division Head \\ Joseph Cerny \\ Assistant Division Head \\ Janis Dairikj
}

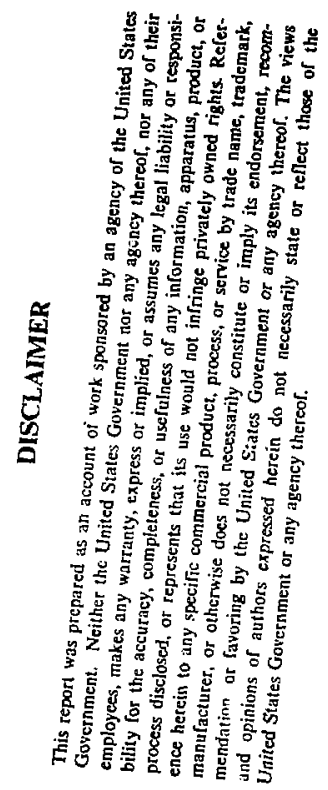

Editor

Jeannette Mahoney

Advisory Committee

William Myers, Chairman, David Clark, J. Michael Nitschke, Arthur Poskanzer

Lawrence Berkeley Laboratory

University of California

Berkeley, California, USA 94720

This work was supported by the Director, Office of Energy Research, Office of High Energy and Nuclear Physics,

Division of Nuclear Physics and by the Office of Basic Energy Sciences, Division of Nuclear Sciences, of the U.S. Department of Energy under Contract No. DE-AC03-76SF00098 
Cover: Microphotograph of stopping tracks of ${ }^{238} \mathrm{U}$ in nuclear track emulsion, detected on the occasion of the first acceleration of $150 \mathrm{AMeV}$ uranium nuclei at the Bevalac, September 25, 1982. Shown in this photograph are the last $0.5 \mathrm{~mm}$ of the stopping, tapering uranium tracks. One of the uranium nuclei collides with a nucleus in the emulsion, resulting in the total breakup of the uranium into light nuclei. 


\section{Introduction}

This report summarizes the scientific research carried out within the Nuclear Science Division between July I, 1981, and September 30. 1982. Heavy-ion investigations continue to dominate the experimental and theoretical research efforts. Complementary programs in light-ion nuclear science, in nuclear data evaluation, and in the development of advanced instrumentation are also carried out.

We began this period looking back upon past achievements as LBL celebrated its 50th anniversary. The real value of these remembrances was to draw our attention to the exciting possibilities of the next 50 years. Of particular significance, the dedication of the (new) third injector at the SuperHILAC was a prelude to the new generation of relativistic heavy-ion physics thal began in early 1982 with the successful acceleration of ${ }^{238} U$ ions at the Bevalac.

Results from Bevalac experiments employing a wide variety of heavy ion beams, along with new or upgraded detector facilities (HISS, the Plastic Ball, and the streamer chamber) are contained in this report. These relativistic experiments have shed important light on the degree of equilibration for central collisions, the time evolution of a nuclear collision, the nuclear density and compressional energy of these collisions, and strange particle production. This work receives continuing guidance from close interactions with the nuclear theory group. The phenomenon of short mean free paths of projectile fragments remains the greatest mystery in Bevalac research. Collaborative "anomalon" emulsion efforts and a new electronic detector being constructed to simulate directly the visual detectors are described in this report.

Reaction mechanism work dominates the heavy-ion research at the 88-Inch Cyclotron and the SuperHILAC. Recent experiments have contributed to our understanding of the nature of light-particle emission in deep-inelastic collisions, of peripheral reactions, incomplete fusion, fission, and evaporation. Nuclear structure investigations at these accelerators continue to be directed toward the understanding of the behavior of nuclei at high angular momentum. Research in the area of exotic nuclei has led to the observation at the 88-Inch Cyclotron of the $\beta$-delayed proton decay of odd-odd $T_{z}=-2$ nuclides; $\beta$-delayed proton emitters in the rare earth region are being investigated at the SuperHILAC.

At all three accelerators the trend is toward more complex detectors measuring more and more parameters per event. To attack the reduction and analysis of stch large quantities of data, the Division has pioneered a unique parallel-processing computing architecture. The successful operation of the Modular Interactive Data Analysis System (MIDAS) prototype during this period demonstrates the principles of this design; subsequent development has provided computational power for data analysis equal to that of a CDC7600.

All three accelerators support strong outside user programs, including both domestic and foreign cooperative efforts. In particular, major collaborations with our German and Japanese colleagues continue to flourish.

Division members published 184 reports and journal articles including $11 \mathrm{Ph}$.D. theses. Affiliated with the NSD during this period were $S$ faculty senior scientists, 18 staff senior scientists, 31 staff scientists, 1 divisional fellow, 20 postdoctoral fellows, 24 graduate students, and 42 technical, administrative, end elerical support staff. Our research continues to thrive from a productive relationship with the UC campus, from the diversity of ideas and talents contributed by our many visiting scientists $(\sim 115)$, and by the cross fertilization frotn other LBL divisions. Honors accorded to Division members include Luciano Moretto's Alexander von Humboldt Award and Michael Perry's Achievement Reward for College Scientists (ARCS) Foundation Award.

The fall meeting of the APS Division of Nuclear Physics at Asilomar (October, 1981) was organized by NSD staff members. Five workshops, focusing on topics from nuclear structure data evaluation and nuclear dynamics to anomalons and cosmic-ray emuision experiments and future planning for heavy-ion experimental needs, were also organized by Division scientists.

The Division is actively engaged in looking to met very high energy heavy-ion physics needs beyond the $1980 \mathrm{~s}$. Over 100 physicists from throughout the world met at LBL in September, 1982 (VENUS-82 Workshop) to review the physias objectives and capabilities of a dedicated heavy-ion accelerator complex providing at least $10 \mathrm{GeV} / \mathrm{N}$ beams over the entire range of the periodic table for fixed target experiments. This Workshop was a prelude to the Tevalac R \& D proposal submitted to DOE in December 1982. Several new detector and accelerator improvements are underway, directed toward investigation of the many exciting avenues yet to be explored. 


\section{PART I: RESEARCH PROGRAMS}

Exotic Nuclei and Nuclear Reactions

Isctopes Project

2

Heavy-Ion Reactions

3

Nuclear Struclure

Polarization Phenomena in Nuclear Physics

Heavy Element Radiochenuistry

Heavy Element Research

Statistical Equilibrium in Deep-Inelastic Heavy lon Reactions

High Energy Nuclear Collisions

HISS

Relativistic Heavy Ion Physics

High Energy Nucleus-Nucleus Collisions

Light Particle Emission in High Energy Nuclear Collisions

Heavy Ion Studies/Pion Studies

Nucleus-Nucleus Collisions

Nuclear Theory 32

Nuclear Electronics 34

Relativistic Heavy Ion Collisions 36

High Energy Heavy Ion Collisions 37

Detector Research and Development 38

Tevalac Project 4 


\section{PART II: ACCELERATORS}

88-Inch Cyclotron Operations and Research

D.J. Clark, D. Eio, L Glasgow, R. Lam, C.M. Lyneis, R.G. Stokstad

Bevalac Research Program

H.G. Pugh

SuperHILAC Research Program

R.M. Diamond

\section{PART III: EXPERIMENTS}

\section{Nuclear Structure}

\section{General}

Decay Properties of Isoscalar Giant Resonances in ${ }^{24} \mathrm{Mg},{ }^{28} \mathrm{Si},{ }^{58} \mathrm{Ni},{ }^{64} \mathrm{Zn}$, and ${ }^{92} \mathrm{Mo}$

P. Bogucki, J.D. Bronson, U. Garg, P. Grabmayr, K.T. Knöpfle, Y.-W. Liu, H. Riedesel, G.J.

Wagner, and D.H. Youngblood

Inelastic Scattering of ${ }^{12} \mathrm{C}$ from ${ }^{208} \mathrm{~Pb}$ to the Second $0^{+}$State of ${ }^{12} \mathrm{C}$

R. Shyam, M.A. Nagarajan, A.C. Shotter, A.N. Bice, and J. Cerny

\section{Exotic Nuclei}

Beta-Delayed Proton Decay of an Odd-Odd $\mathrm{T}_{2}=-2$ Isotope, ${ }^{22} \mathrm{Al}$

M D. Cable, J. Sonkanen, R.F. Parry, H.M. Thierens, J.M. Wouters, Z.Y. Zhou, and Joseph Cerny

Beta-delayed Proton Decay of ine Odd-Odd $\mathrm{T}_{z}=-2$ Isotope ${ }^{26} \mathrm{P}$

M.D. Cable, J. Honkanen, R.F. Parry, S.H. Zhou, Z.Y. Zhou, and Joseph Cerny

Weak Beta-Delayed Proton Groups in the Decay of ${ }^{21} \mathrm{Mg}_{1}{ }^{25} \mathrm{Si}$, and ${ }^{29} \mathrm{~S}$

S.H. Zhou, M.D. Cable, J. Honkanen, R.F. Parry, Z.Y. Zhou, and Joseph Cerny

New Delayed Proton Emitters Measured with OASIS

J.M Nitschke, W.-D. Zeitz, and M.D. Cable

Search for New Ground-State Proton Emitters

J.M. Nitschke, W'-D. Zeitz, and M.D. Cable

Beta Endpoint Measurements of Neutron-Deficient Cesium Isotopes 
Heavy Actinide Products from Reactions of ${ }^{18} \mathrm{O},{ }^{22} \mathrm{Ne}$, and ${ }^{136} \mathrm{Xe}$ with ${ }^{254} \mathrm{Es}$

M. Schädel, R.W. Lougheed, J.H. Landrum, J.F. Wild, R.J. Dougan, A.D. Hoover, E.K. Hulet, G.R

Bethune, A. Ghiorso, M.J. Nurmia, L.P. Somerville, K.J. Moody, and G.T. Seaborg

A New 100-ms Spontaneous Fission Activity Abundantly Produced in the Reaction $99 \mathrm{MeV}{ }^{18} \mathrm{O}+{ }^{254} \mathrm{Es}$ LP. Somerville, M.J. Nurmia, M. Schädel, A. Ghiorso, J.M. Nitschke, RW. Lougheed, J.H. Landrum, and $E . X$. Hulet

An Upper Limit for Spontaneous Fission Branching in ${ }^{254}$ No

L. P. Somerville, A. Ghiorso, M.J. Nurmia, and J.M. Nitschke

Results of Investigations on the New 15- to 24-ms Spontaneous Fission Activities

L.P. Somerville, M.J. Nurmia, A. Ghiorso, and J.M. Nitschke

\section{High Angular Momentum and Gamma De-excitation}

Continuum Gamma-Ray Spectroscopy

R.M. Diamand

High Spin States

F.S. Stephens

The Rotational Inertia of Nuclei

M.A. Deleplanque, H.J. Korner, H. Kluge, A.O. Macchiavelli, N. Bendjaballah, R.M. Diamond, and

F.S. Stephens

Dependence of the Giant Dipole Strength Function on Excitation Energy

J.E. Draper, J.O. Newton, L.G. Sobotka, H. Lindenberger, G.J. Wozniak, L.G. Moretto, F.S. Stepherts, R.M. Diamond, and R.J. McDonald

Isomeric Trapping Following Coulomb Excitation of High Spin States in ${ }^{178} \mathbf{H f}$

J.H. Hamilton, A.V. Ramayva, R.M. Ronningen, R.O. Sayer, H. Yamada, C.F. Maguire, P. Colombani, D. Ward, R.M. Diamond. F.S. Sizphens, I.Y. Lee, P.A. Butler and D. Habs

A Third Discontinuity in the Yrast Levels of ${ }^{158} \mathrm{Er}$

J. Burde, E.L Dines, S. Shih, R.M. Diamond, J.E. Draper, K.H. Lindenterger, C. Schuck, and F.S. Stephens

Lifetimes of Unresolved Transitions from Very High Spins in Nuclei Near $\mathrm{N}=\mathbf{8 2}$

H. Hübel, R.i. Diamond, P. Aguer, C. Ellegaard, D.B. Fossan. H. Kluge, C. Schuck, S. Shih, F.S.

Stephens, a:zd U. Smilanski 


\section{Nuclear Data}

First Conference on Nuclear Structure Data Evaluation

R.B. Firesione, V.S. Shirley, and J.M. Dairiki

$\mathrm{X}$-ray and Auger-Electron Intensities from $\mathrm{K}_{1} \mathrm{~L}_{1}, \mathrm{~L}_{2}$, and $\mathrm{L}_{3}$ Atomic Shell Vacancies Produced by Nuclear Decay

R.B. Firestone

Gamma-Ray Transition Probability Systematics

E. Browne and R.B. Firestone

SPINOZA: A Computer Code for the Scientific Evaluation of Nuclear Data

$R . B$. Firestone

Implementation of the Evaluated Nuclear Structure Data File into DATATRIEVE

R.B. Firestone and E. Browne

\section{Nuclear Reaction Mechanisms}

\section{Polarization Studies}

The Elastic Scattering of Polarized Protons by ${ }^{40} \mathrm{Ar}$

N.T. Okumusoglu, J. Birchall, M.S.A.L. Al-Ghazi, C. Lapointe, J.S.C. McKee, H.E. Conzett, R. M. Larimer and $P$, von Rossen

An Experimental Test of Charge Symmetry in n-p Elastic Scattering

J. Birchall, N.E. Davison, H.P. Gubler, W. P. Lee, J. P. Svenne, W.T.H. van Oers, R. Abegg, C.A. Miller, E.B. Cairns, H. Coombes, C.A. Davis, P. Green, L.G. Greeniaus, W.J. McDonald, G.A. Moss, G. Roy, J. Soukup. G.M. Stinson, H.E. Conzett, and G.R. Platiner

Concerning Tests of Time-Reversal Invariance Via the Polarization-Analyzing Power Equality

Homer $E$. Conzett

Evidence of Time Symmetry Violation in the Interaction of Nuclear Particles

R.J. Slobodrian, C. Rioux, R. Roy, H.E. Conzett, P. von Rossen, and F. Hinterberger

The ${ }^{4} \mathrm{He}(\alpha, \mathrm{p})^{7} \mathrm{Li}^{,}{ }^{7} \mathrm{Li}^{*(0.478)}$ Reaction Near Threshold

$R$ J. Slobodrian and H. E. Conzett

The Radiolysis and Racemizaton of Leucine on Proton Irradiation 


\section{Damped Collisions and Transfer Reactions}

Rigid-rotation and Rolling Limits in Deep-Inelastic Reactions

A.J. Pacheco, G.J. Wozniak, R.J. McDonald, R.M. Diamond, C.C. Hsu, LG. Moretto. D.J. Morrissey,

L.G. Sobotka, and F.S. Stephens

High Energy Gamma Rays Emitted in the Deep-Inelastic Reaction $8.0 \mathrm{MeV} / \mathrm{A}{ }^{139} \mathrm{La}+{ }^{139} \mathrm{La}$

L.G. Sobotka, J.E. Draper, R.M. Diamond, E.L. Dines, R.J. McDonald, LG. Moretto, A.J. Pacheco, F.S. Stephens, and G.J. Wozniak Particle Angular Distributions and Gamma-ray

Particle Angular Distributions and Gamma-ray Multiplicities as Experimental Probes for Angular Momentum Fluctuations

L.G. Moretto

A Study of Charge, Energy, and Angular Momentum Transfer in the Reactions ${ }^{36} \mathrm{Fe}+{ }^{197} \mathrm{Au}$ and ${ }^{56} \mathrm{Fe}+$ ${ }_{107,100} \mathrm{Ag}$ at 7.2 and $8.3 \mathrm{MeV} /$ nucleon

G.J. Wozniak, G.S. Mathews, R.P. Schmitt, R. Regimbart, H. Hübel, R.M. Diamond, and L.G. Moretto

Spin Mistributions of Individual Deep-Inelastic Fragments as Deduced from Continuum $\gamma$-ray Studies

A.J. Pacheco, G.J. Wozniak, R.J. McDonald, R.M. Diamond, C.C. Hsu, LG. Moretto, D.J. Morrissey, L.G. Sobotka, and F.S. Siephens

Sequential Fission Studies of Angular Momentum Misalignment in Very Asymmetric Systems

G. Guarino, D.J. Morrissey, R.J. McDonald, A.J. Pacheco, L. Sobotka, G.J. Wozniak, and L.G. Moretto

Sequential Fission in the Reaction of ${ }^{40} \mathrm{Ar}+{ }^{197} \mathrm{Au}$

D.J. Morrissey. G.J. Wozmiak, L.G. Sobotka, A.J. Pacheco, R.J. McDonald, and L.G. Moretto

Compound Nucleus Decay Via the Emission of Large Fragments

L.G. Sobotka, M.L. Padgett, G.J. Wozniak, G. Guarino, A.J. Pacheco, L.G. Moretto, Y.D. Chan, R.

Stokstad, I. Tserruya, and S. Wald

Alpha-Particle Emission from the Reaction $1354 \mathrm{MeV}{ }^{165} \mathrm{Ho}+{ }^{181} \mathrm{Ta}$

LG. Sobotka, R.J. McDonald, G.J. Wozmiak, D.J. Morrissey, A.J. Pacheco, and L.C. Moretto

Products of $3 \leq Z \leq 9$ from Long-Lived Intermediates in the Reactions $336 \mathrm{MeV}{ }^{40} \mathrm{Ar}+\mathrm{Ag}^{154} \mathrm{Sm}$ and

Au

L.C. Vaz, D. Logan, J.M. Alexander, E. Duek, D. Guerreau, L. Kontalski, M.F. Rivet, and M.S. Zisman

Intrinsic Fragment Spins Generated in the Reactions of ${ }^{20} \mathrm{Ne}$ with ${ }^{197} \mathrm{Au}$ and ${ }^{238} \mathrm{U}$ at $12.6 \mathrm{MeV} / \mathrm{Nucleon}$

D.J. Morrissey, G.J. Wozniak, L.G. Sobotka, A.J. Pacheco, R.J. McDonald, C.C. Hsu, and LG. Moretto

A Study of ${ }^{20} \mathrm{Ne}$ Induced Reactions with ant $\mathrm{Cu}$ and ${ }^{197} \mathrm{Au}$ at 8.6 and $12.6 \mathrm{MeV} /$ nucleon

G.J. Mathews, J.B. Moulton, G.J. Wozniak, B. Cauvin, R.P. Schmitt, S. Sventek, and L.G. Moretto

The Onset of Non-Equilibrium Phenomena in Fusion-Like Processes for ${ }^{16} \mathrm{O}$ Induced Reactions

Y. Chan, M. Murphy, R. G. Stokstad, I. Tserruya, S. Wald, and A. Budzanowski 
Observation of Symmetric Disintegration in the ${ }^{6} \mathrm{Li},{ }^{9} \mathrm{Be},{ }^{12} \mathrm{C}+{ }^{40} \mathrm{Ca}$ Reactions

K. Grotowski, Z. Majka, Y. D. Chan, R. Stokstad, I. Tserruya, S. Wald, G. Guarino, D. J. Morrissey,

L. G. Sobotka, G. J. Wozniak, and L. G. Moretto

${ }^{12} \mathrm{C}^{*}$ and ${ }^{8} \mathrm{Be}$ Production in ${ }^{12} \mathrm{C}+{ }^{208} \mathrm{~Pb}$ Collisions

A.N. Bice, A.C. Shotter, and Joseph Cerny

Transfer and Breakup Reactions in ${ }^{16} \mathrm{O}+\mathrm{CsI}$ at $16.4 \mathrm{MeV} / \mathrm{n}$

M.J. Murphy, B.G. Harvey, D.L. Hendrie, W.W. Pang, K. Van Bibber, and R. Legrain

Kinematics of Heavy Ion Transfer Reactions

B.G. Harvey and M.J. Murphy

Recoil Range Studies of Target-like Products of Multinucleon Transfer from ${ }^{18} \mathrm{O}$ to ${ }^{245} \mathrm{Cm}$ and ${ }^{249} \mathrm{Cf}$

Rose Marie McFarland, Albert Ghicrso, and Glenn T. Seaborg

Excitation Functions for Production of Heavy Actinides from Interactions of ${ }^{18} \mathrm{O}$ with ${ }^{248} \mathrm{Cm}$ and ${ }^{249} \mathrm{Cr}$

D. Lee, K. Moody, M. Nurmia, G.T. Seaborg, H. von Gunten, and D. Hoffman

Actinide Production from the Reaction of ${ }^{129} \mathrm{Xe}$ with ${ }^{248} \mathrm{Cm}$

R.B. Welch. KJ. Moody, D. Lee, K.E. Gregorich, P. Wilmarth, and G.T. Seaborg

Preliminary Results from the Reaction of ${ }^{208} \mathrm{~Pb}$ with ${ }^{197} \mathrm{Au}$

M.D. Perry, K.J. Moody, R.B. Welch, K.E. Gregorich, P. Wilmarth, and G.T. Seaborg

\section{Relativistic Energies: Spectators}

The Breakup of Spectator Residues in Relativistic Nuclear Collisions

A.I. Warnick, H.H. Gutbrod, H.G. Ritter, H. Stelzer, F. Weik, H.H. Wieman, S.B. Kaufman, E.P.

Steinberg, and B.D. Wilkins

Heavy Fragment Yields in the Interaction of $28 \mathrm{GeV}$ Protons with ${ }^{238} \mathrm{U}$

B.V. Jacak, W. Loveland, D.J. Morrissey, P.L. McGaughey, and G.T. Seaborg

Target Fragment Yields in the Interaction of $28 \mathrm{GeV}$ Protons with ${ }^{18]} \mathrm{Ta}$

C. Oertel, L. McGaughey, D.J. Morrissey, W. Loveland, and G.T. Seaborg

Au Target Fragmentation at Intermediate Energies

W. Loveland, K. Aleklett, P.L. McGaughey, and G.T. Seaborg

Ta Target Fragmentation at Intermediate Energies

P. Johnson, W. Loveland, P.L. McGaughey, K. Aleklett, and G.T. Seaborg

Transparency in Heavy Ion-Heavy Nucleus Nuclear Collisions

W. Loveland, K. Aleklett, P.L. McGaughey, and G.T. Seaborg

Target Fragment Angular Distributions in the Reaction of $86 \mathrm{MeV} / \mathrm{u}^{12} \mathrm{C}$ with ${ }^{197} \mathrm{Au}$

R.H. Kraus, Jr., W. Loveland, P.L. McGaughey, K. Aleklett, Y. Morita, and G.T. Seaborg

Target Fragment Angular Distributions in the Interaction of $2.1 \mathrm{GeV} / \mathrm{u}{ }^{12} \mathrm{C}$ with ${ }^{197} \mathrm{Au}$ and ${ }^{238} \mathrm{U}$ 
Anomalous Reaction Mean Free Paths of Nuclear Projectile Fragments From Heavy Ion Collisions at 2

AGeV

E.M. Friedlander, R.W. Gimpel, H.H. Heckman, Y.J. Karant, B. Judek, and E. Ganssauge

Anomalons: Statistical Fluctuation or Physical Fact?

Enwin M. Friedlander

Comment on "Interpretation of Anomalous Mean Free Paths of Projectile Fragments from Relativistic

Heavy-Ion Collisions"

Y.J. Karant and M.H. Mac Gregor

First Observations of ${ }^{238} \mathrm{U}$ Interactions at $1 \mathrm{AGeV}$

Harry H. Heckman, Erwin M. Friedlander, and Yasha J. Karant

Characteristics of the Ionization Tracks and Interactions of Uranium-238 Nuclei in Emulsion

H.H. Heckman. Y.J. Karant, and E.M. Friedlander

Search for Nonintegrally Charged Projectile Fragments in Relativistic Nucleus-Nucleus Collisions

P.B. Price, M.L. Tincknell, G. Tarle, S.P. Ahlen, K.A. Frankel, and S. Perlmutter

Search for Beläayed Gamma Decays of Anomalous Nuclear States

T.M. Liss, S.P. Ahlen, P.B. Price, and G. Tarle

Measurement of the Summed Residual Projectile Mass in Relativistic Heavy-Ion Collisions

J.D. Stevenson, J. Martinis, and P.B. Price

Data Tables of Inclusive Particle Production at Forward Angles from Collisions of Light Relativistic Nuclei

L. Anderson, E. Moeller, S. Nagamiya, S. Nissen-Meyer, L Schroeder, G. Shapiro, and H. Steiner

Invariant Mass Spectra from ${ }^{12} \mathrm{C}$ Interactions

H.J. Crawford

\section{Relativistic Energies: Participants}

The Question of Thermalization in Relativistic Heavy Ion Collisions

A. Baden, H.H. Gutbrod, B. Kolb, H. Löhner, B. Ludewigt, M.R Maier, A.M. Poskanzer, T. Renner,

H. Riedesel, H.G. Ritter, H. Spieler, A. Warwick, F. Weik, and H. Wieman

Global Analysis with Plastic Ball Data

H.H. Gutbrod, H. Löhner, A.M.Poskanzer, T. Renner, H. Riedesel, H.G. Ritter, A. Warwick, F. Weik, H. Wieman

Charged Particle Exclusive Analysis of Central $\mathrm{Ar}+\mathrm{KCl}$ and $\mathrm{Ar}+\mathrm{Pb}$ Reactions at 1.8 and $0.8 \mathrm{GeV} / \mathrm{u}$ 
Compression Effects in Relativistic Nucleus-Nucleus Collisions

R. Stock, R. Bock. R. Brockmann, A. Dacal, J.W. Harris, M. Maier, M.E. Ottiz, H.G. Pugh, R.E. Renfordr. A. Sandoval, L.S. Schroeder, H. Stroebele, and K.L Wolf

High Densities and the Lattst Two-Proton Correlation Results

S. Abachi, J.B. Carrall, K. Ganezer, G.Igo, T.A. Mulera, V. Perez-Mendez, A.L. Sagle, A. Shor, F. Zarbakhsh

A Measurement of pd Quasi Elastic Scattering in $800 \mathrm{MeV}$ pA Collisions and Short-Range Correlations of pn Pair in Nuclei

I. Tanihata, Y. Miake, H. Hamagaki, S. Nagamiya, S. Schnetzer, Y. Shida, and H. Steiner

Cluster Formation Observed with a $4 \pi$ Detector and the Question of Entropy Production in High Energy Nuclear Collisions

H.H. Gutbrod, B. Kolb, H. Löhner, B. Ludewigt, M.R. Maier, A.M. Poskanzer, T. Renner, H.

Riedesel, H.G. Ritter, H. Spieler, A. Warwick, F, Weik, and H. Wiemant

Inclusive Negative Pion Production in ${ }^{40} \mathrm{Ar}+\mathrm{KCl}$ at $1.8 \mathrm{GeV} /$ nucieon

R. Brockmann, J.W. Harris, A. Sandoval, R. Siock, H. Stroebele, K.L. Wolf, H.G. Pugh, L.S. Schroeder, R.E. Renfordt, and M. Maier

Pions Produced Near the Center-of-Mass Velocity in Heavy-Ion Collisions

K.A. Frankel, J.A. Bistirlich, R. Bossingham, H.R. Bowman, K.M. Crowe, C.J. Martoff, D. Murphy, J.O. Rasmussen, J.P. Sullivan, W.A. Zajc, J.P. Miller, O. Hashimoto, M. Koike, J. Peier, W. Benenson, G.M. Crawley, E. Kashy, J.A. Nolen, Jr., and J. Quebert

Strong Coulomb Effects on Pions Produced in Heavy Ion Collisions J.P. Sullivan, J.A. Bistirlich, H.R. Bowman, R. Bossingham, T. Buttke, K.M. Crowe, K.A. Frankel, C.J. Martof, J. Miller, D.L. Murphy, J.O. Rasmussen, W.A. Zajc, O. Hashimoto, M. Koike, J. Peter, W. Benenson, G.M. Crawley, E. Kashy, and J.A. Nolen, Jr.

Funny Hills in Pion Spectra from Heavy-Ion Collisions

John O. Rasmussen

Pions from and about Heavy Ions

John O. Rasmussen

Production of Negative Pions with $183 \mathrm{MeV} /$ nucleon Ne Beams

S. Nagamiya, H. Hamagaki, P. Hecking, S. Kadota, R. Lombard,Y. Miake, E. Moeller, S. Schnetzer, H. Steiner, I. Tonihata, S. Bohrmann, and J. Knoll

Subthreshold $\mathrm{K}^{-}$Production in Relativistic Nuclear Collisions (RNC)

A. Shor, K. Ganezer, S. Abachi, J. Carroll, J. Geaga, G. Igo, P. Lindstrom, T.A. Mulera, V.PerezMendez, A.L. Sagle, F. Zarbakhsh, and D. Woodard 


\section{PART IV:THEORY}

\section{General}

Normal and Pion Condensed States in Neutron Star Matter

N.K. Glendenning, P. Hecking, and V. Ruck

The Hyperon Composition of Neutron Stars

N.K. Glendenning

Quantum Approach to the Nuclear Collision Process

P. Danielewicz

Theory of Direct Nuclear Reactions

N.K. Glendenning

Droplet Model Predictions of Charge Moments

William D. Myers

The Macroscopic Approach to Nuclear Masses and Deformations

W.D. Myers and W.J. Swiatecki

The Nature of Nuclear Dynamics

W.J. Swiatecki

Dynamical Aspects of Nucleus-Nucleus Collisions

S. Bjornholm and W.J. Swiatecki

Nuclear Deformation Energies

J. Blccki and W.J. Swiatecki

W.J. Swiatecki

Nonlinear Mean Field Theory for Nuclear Matter and Surface

\section{Strongly Damped Collisions}

Fragment Spin Correlations in Damped Nuclear Reactions

J. Randrup

Equilibrium Treatment of Spin-Depolarizing Modes in Mass Asymmetric Heavy-Ion Systems 
Energy Equilibration and Anomalous Mass Drift in Heavy Ion Collisions

Luciono G. Moretto

Deep Inelastic Reactions: A Probe of the Collective Properties of Nuclear Matter

LG. Moretto and R.P. Schmitt

Multifragmentation and the Partition of Angular Momentum. A General Statistical Theory

LG. Moretto

Statistics at Work in Heavy Ion Reactions

Luciano G. Moretto

The Effect of Statistical Fluctuations on the Measurement of the Total Energy and the Multiplicity of $\boldsymbol{\gamma}$-Rays Following Deep-Inelastic Reactions

A.J. Pacheco and LG. Moretro

\section{High Energy Collisions}

Calculation of Complete Multi-Fragment Events in a Statistical Model for Nuclear Disassembly

Jorgen Randrup and George Fái

Statistical Simulation of Completc Events in Energetic Nuclear Collisions

George Fai and Jolgen Randrup

Formulation of the Coulomb Effects of Spectator Fragments on Pions from Heavy Ion Collisions

Hafez M.A. Radi, J.O. Rasmussen, J.P. Sullivan, K.A. Frankel, and O. Hashimoto

Calculation of Milon Final Probabilities After Muon-Induced Fission in Four-State Bas
Zhong-yu Ma, Xi-zhen Wu, Jing-shang Zhang, Yi-zhong Zhuo, and J.O. Rasmussen

Deuteron Formation in Nuclear Collisions

M. Gyulassy, K. Frankel, and E.A. Remler

Global Flow Analysis of Nuclear Collisions

M. Gyulassy, K.A. Frankel, and H. Stocker

Spacial Gradients in the Fragmentation Regions at ATeV Energies

M. Gyulassy

Maximum Baryon Densities at $10 \mathrm{GeV} /$ nucleon

M. Guulassy

Energy Densities in $\mathrm{TeV} /$ nucleon Nuclear Collisions

M. Gyulassy

Anomalons as Pineuts Bound to Nuclear Fragments: A Possible Explanation

Wm.C. McHarris and J.O. Rasmussen 
Particle Multiplicities at the $\overline{\mathrm{p}}-\mathrm{p}$ Collider and the $\mathrm{s}^{\mathrm{l} / 4} \mathrm{Law}$

E.M. Friedlander and R.M. Weiner

“Mass Scaling” Phenomena in Heavy Fragment Production in Relativistic Heavy lon Collisions

Fumiyo Uchiyama

Subthreshold K-Production by Coherently Produced $\phi$-Mesons in Fieavy lon Collisions

K.-H. Müller

A Saturating Chiral Field Theory of Nuclear Matter

J. Boguta

\section{PART V: INSTRUMENTATION}

\section{Detectors and Techniques}

Ion Sources for Cyclotrons

David J. Clark

The Plastic Ball Spectrometer: an Electronic 4x Detector with Particle Identification A. Baden, H.H. Gubrod, H. Löhner, M.R Maier, A.M. Poskanzer, T. Renner, H. Riedesel, H.G.

Ritter, H. Spieler, A. Warwick, F. Weik, H. Wieman

A Method for Precise Charge Measurements of Relativistic Nuclei $Z \leq 3$ in Nuclear Emulsion

M.A. Bloomer, H.H. Heckman, and Y.J. Karant

Observation of Large Deviations from the Bethe-Bloch Formula for Relativistic Uranium Ions

S.P. Ahlen and G. Tarle

Calculation of the Stopping Power of Very Low Velocity Magnetic Monopoles -

S.P. Ahlen and K. Kinoshita

Car Grand Unification Monopoles be Detected with Plastic Scintillators

S.P. Ahlen and G. Tarle

The Maximum Likelihood Method Applied to Decay During and After Accumulation of a Sample of Radioactive Nuclei

L.P. Somerville

Time of Flight Detector for HISS

Ryoichi Wada

MUSIC Ionization Chamber

H. Sann

Investigation of the Operation of Wire Chambers in the Self Quenching Streamer Mode 
HISS Phase II Drift Chamber Electronics

Fred Bieser

J. Carroll. J. Geaga, S. Abachi, T. Mulera and V. Perez-Mendez

HISS Cryogenic Control System

C. McParland

Design of a Drift Collection Calorimeter with a Combined Radiator and Field Shaping Structure of Lead Glass Tubing

V. Perez-Mendez, A. Del Guerra, H. Hirayama, T. Mulera and W. Nelson

A Threshold Cerenkov Counter for Isotopic Identification of High-Energy Heavy Ions

J. Stevenson, J. Musser, and P.B. Price

\section{Computers}

Performance of the MIDAS Prototype

Creve Maples, Daniel Weaver, William Rathbun, and Douglas Logan

Dynamic Expression Evaluator for Real Time Applications

Status of Bevalac Software

C. McPorland

\section{PART VI: APPENDICES}

Appendix I

Publications

Appendix II

Thesis Abstracts

Appendix III

Seminars

Appendix IV

Author Index 
PART I: PROGRAMS 


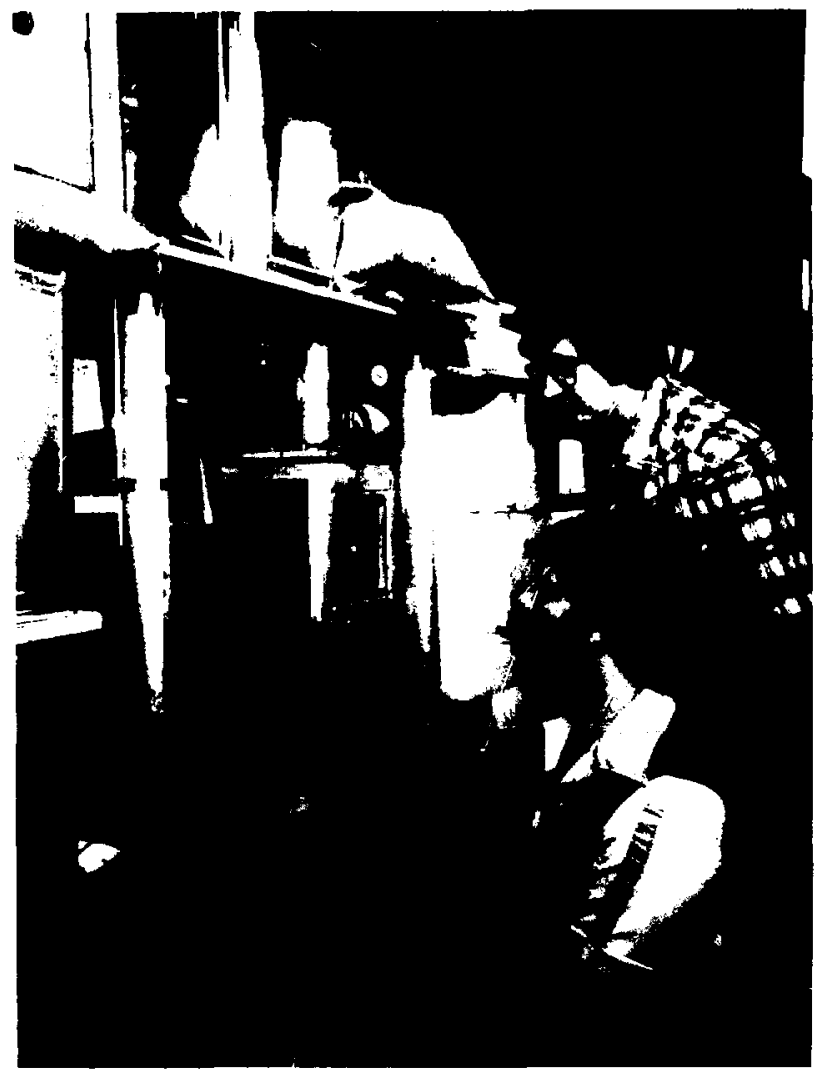

Fig. 1. Roger Parry (seated) and Michael Cable adjusting the helium jet system at the 88-Inch Cyclotron. 


\section{Exotic Nuclei and Nuclear R actions}

Studies of nuclei very far from the valley of stability may reveal new radioactivities; provide tests of theoretical models that predict the existence, masses, aud shapes of exotic nuclei; and develop spectroscopic data on nuclides with abnormal neutron to proton ratios. Several techniques are in use for studying such nuclei produced in low-yield reactions. Current experiments at the 88-Inch Cyclotron are concentrated in two regions of the chart of the nuclides; one in the light-mass region exploring nuclei that are expected to define the proton drip line and the other in the highly neutron-deficient $\mathrm{Cs}$ isotopes.

In the light-mass region, the odd-odd $\mathrm{T}_{\mathrm{z}}=-2$ nuclides ${ }^{22} \mathrm{Al}$ and ${ }^{26} \mathrm{P}$ have been discovered using the $\left({ }^{3} \mathrm{He}, \mathrm{p} 4 \mathrm{n}\right)$ reaction at $110-130 \mathrm{MeV}$ on ${ }^{24} \mathrm{Mg}$ and ${ }^{28} \mathrm{Si}$ targets. The experimental technique employed used a minimal length helium jet to transport these short-lived nuclides $\left(T_{1 / 2}<100 \mathrm{~ms}\right.$ ) from the target region to a collection point in front of a semiconductor counter telescope. Observation of the beta-delayed (jsospin-forbidden) proton decay of these nuclei $(\sim 7-8 \mathrm{MeV}$ protons) established their existence. Both of these nuclides are strong candidntes for beta-delayed einission of two protons with available energies for decay via this mode from the daughter analogue state of $6.1 \mathrm{MeV}\left({ }^{22} \mathrm{Al}\right)$ and $5.3 \mathrm{MeV}\left({ }^{26} \mathrm{P}\right)$. Searches for this new radioactivity are in progress; whether such decays occur by the sequential emission of two protons or by ${ }^{2} \mathrm{He}$ emission as predicted by Goldanskii is of considerabie interest.

The experimental tool used to study the $C_{s}$ isotopes has been the on-line mass separator RAMA (an acronym for recoil atom mass analyzer). This apparatus consists of a helium jet to transport recoil nuclei, which are produced in a target chamber by heavy-ion bombardment, to a hollow cathode ion source. Isotopes are then extracted from this ion source and magnetically analyzed. The mass of interest is collected on the RAMA focal plane by a tape transport system that shuttles the activity to a $\beta-\gamma$-detection station. The decays of ${ }^{119-123} \mathrm{Cs}$ have been observed following their production in $110-140-\mathrm{MeV}{ }^{14} \mathrm{~N}$ bombardment of $\mathrm{CdS}$ targets. Knowledge of the mass excesses of the parent $\mathrm{Cs}$ nuclei and the measurement of the $\mathrm{Q}_{\beta}$ values for their decays have yielded mass excesses for the xenon daughters. These results are then compared with theoretical predictions of the mass surface.

In recent years, this group's research interests and efforts have also included developing techniques to detect unbound resonant states as nuclear reaction products. With these detection capabilities. it has been possible to extract valuable spectroscopic information from reactions such as $\left({ }^{12} \mathrm{C},{ }^{8} \mathrm{Be}\right)$ and $\left({ }^{4} \mathrm{He} .{ }^{2} \mathrm{He}\right)$. Recent studies have used similar techniques to investigate light heavy-ion reaction mechanisms in the range $10-20 \mathrm{MeV} /$ nucleon. In particular, results are reported on the inelastic scattering of ${ }^{12} \mathrm{C}$ (at 132,187 , and $230 \mathrm{MeV}$ ) from ${ }^{208} \mathrm{~Pb}$ to the second $0^{+}$state of ${ }^{12} \mathrm{C}$.

\section{Group Leader}

Joseph Cerny

A. Bice

M. Cable

J. Honkanen

R. Parry

J. Woutars

S.H. Zhou, Institute of Atomic Energy, Beijing, Peoples Republic of China

Z.Y. Zhou, Nanjing Univ. Peoples Republic of China 


\section{Isotopes Project}

\section{Group Leader} J.M. Dairiki

E. Browne

R.B. Firestone

C.M. Lederer

V.S. Shirle!"
The Isotopes Project compiles and evaluates nuclear structure and decay data and develops compilation mettodology. From 1940-1978 the Project had as its main objective the production of the Table of Isotopes. Since publication of the seventh (and last) edition in 1978, the group now coordinates its nuclear data evaluation efforts with those of other data centers via national and international nuclear data networks. The group is currently responsible for the evaluation of mass chains $\mathbf{A}=$ 167-194. All evulurted data are entered into the international Evaluated Nuclear Structure Data Filc (ENSDF) and are published in Nuclear Data Sheets.

During the past 15 months the evaluations of nuclear structure data for all nuclei with masses $A=185,188,189$. and 193 were published in Nuclear Data Sheets. Evaluations of $A=169, A=187$, and $A=190$ have been accepted for publication, and the updated evaluations of three additional mass chains $(A=181, A=$ 174 and $A=192$ ) are in progress.

In addition to the cvaluation effort, the Isotopes Project will produce, on behalf of the U.S. Nuclear Data Network (NDN), a Radioactivity Handbook for applied users. The purpose of the handbook is to provide a compilation of recommended decay data that is detailed enough for use in sophisticated applications, but that is organized clearly so as to be usable in simple routine applications. The handbook will be produced at four-year intervals, beginning in 1984. Recommended decay data will be taken from the current version of ENSDF, with no further updating. Additional calculations and evaluation will be done to provide recommended data on atomic radiations and conversion electrons. Newly-developed computer codes aid in that task.

Most of the additional programming required for production of the Radioactivity Handbook will be in place early in 1983. Final data processing will then commence using DATATRIEVE, a standard DEC database management system. Nonuniformities and holes in the ENSDF data are being corrected as work proceeds. Final output will include data in both tabular and level scheme formats; the existing levelscheme graphics program (used for the Table of Isotopes) has already been modified to handle data in ENSDF formats.

In addition, the group has been asked to present a proposal for production of a complementary volume - the Nuclear Structure Handbook - at the November 1982 meeting of the NAS/NRC Parel on Basic Nuclear Data Compilations.

The Table of Nuclear Moments, a compilation of nuclear magnetic and quadrupole moments published by the lsotopes Project, has been the only such table maintained on a continuing basis. The most recent version was published as an appendix to the Table of lsotopes. User response has led to plans for expanding the amount of data and its presentation in the next edition.

The seventh edition of the Table of Isotopes continues to be the most up-to-date general reference source available for nuclear structure and decay data. Sales through April 1982 total 7317 copies (3535 clothbound and 3782 paperback). Nearly all 7000 copies of the Nuclear Wallet Cards, produced in 1979 by the Isotopes Project on behalf of the U.S. Nuclear Data Network, have been distributed to the user community.

Promoting the science of data evaluation and providing assistance to the user community are both important aspects of the Project's role. Project members organized the very successful First Annual Conference on Nuclear Structure Data Evaluation, held at Asilomar October 27-30, 1981. In addition to answering specific data requests, the Isotopes Project encourages general use of its extensive library, which contains comprehensive data files and major nuclear physics journals. 


\section{Heavy-Ion Reactions}

The behavior of heavy-ion reactions changes rapidly as the bombarding energy is increased significantly above $8 \mathrm{MeV} / \mathrm{n}$. Between this energy and about $100 \mathrm{MeV} / \mathrm{n}$ a transition occurs from the characteristics of low energy phenomena (transfer reactions, complete fusion) to those of relativistic heavy-ion reactions (fragmentation, participant-spectator mechanisms). This group is interested in studying the mechanisms of heavy-ion reactions in this regime and has as its goal the identification and understanding of the competing processes and their dependence on the bombarding energy.

Inclusive measurements are valuable in mapping a region of study, but they do not easily distinguish between the separate contributions of difierent reaction mechanisms. For example, projectile-like masses may arise from reactions in which the projectile is fragmented and from reactions in which the missing charge and mass are captured by the target (transfer reactions). The small streamer chamber at the 88Inch Cyclotron has been used to separate the above mentioned types of reaction mechanism by observing (visually) the presence or absence of charged particles accompanying the projectile--ike fragment. The reaction of $16.4 \mathrm{MeV} / \mathrm{n}^{16} \mathrm{O}$ with $\mathrm{CsI}$ has now been analyzed in detail and has revealed a number of interesting features. At this energy, both transfer and breakup reactions compete. As expected, the closer the projectile-like fragment is to the mass and charge of the projectile, the greater the proportion of transfer events. The number of reactions in which two charged particles accompany the projectile-like fragment turns out to be very small, even for quite large mass transfers. This observation alone places some restrictions on the mechanism of projectile breakup and suggests that sequential decays of excited ejectiles piay a major role.

The streamer chamber, while providing accurate multiplicity information, is hampered by a slow data acquisition rate and a tedious scanning procedure. A $4 \pi$ plastic scintillator array, termed the "plastic box", was designed to remedy these problems and has recently become operational. The device consists of a cube, eight inches on a side, with double walls. The scintillator (NE102) for a single wall is 1 $\mathrm{mm}$ thick and is viewed at one end by a light pipe and phototube. The beam enters and exits along a diagonal line and projectile-like fragments emerge through a slot cut in one side of the cube; the latter particles are detected by a three-element silicon telescope. The entire device is contained in the 60-inch scattering chamber recently acquired from the Jagellonian University in Cracow, Poland.

The principle of operation is simple. Frotons, alpha particles or any other charged particles accompanying the fragment observed in the telescope are registered in the plastic scintillators. A detection efficiency close to $100 \%$ enables the observation of transfer reactions by the absence of a signal in all scintillators. The double walls offer a crude particle identification and aid in the discrimination against gamma rays and neutrons. Although the system is not highly segmented (only six elements), the small number of particles in the final states of the reactions being studied means that useful multiplicity information may be obtained.

The prevailing understanding of heavy-ion reactions classifies reaction mechanisms according to impact parameter. Peripheral collisions lead to few nucleon transfer; central collisions (i.e., complete overlap of projectile and target) to fusion and a compound nucleus. Between these extremes lies a region in which only a part of the projectile may be captured by the target. This process is delected with the plastic box via the presence of the particles not captured by the target. Measurement of the velocity of the heavy, target-like residue also provides information on the reaction mechanism. Complete capture of the projectile by the target implies full momentum transfer and a compound nucleus with a velocity equal to that of the
Group Leaders

B.G. Harvey

R. G. Srokstad

C. Albiston*

Y. D. Chan

M. J. Murphy

S. Wald

I. Tserruya,

Weizmann

Institute,

Israel

K. Van Bibber,

P. J.

Countryman*,

Stanford

University

* graduate

students 
center of mass. Correspondingly, incomplete fusion implies incomplete momentum transfer. In this case (for a light projectile and a heavy target) the velocity of the compound system will be lower. The compound nucleus is highly excited and the ensuing evaporation of protons, neutrons and alpha particles produces a distribution of residue velocities, the average of which indicates the average momentum transfer.

The time of fight system at the 88-Inch Cyclotron was used to measure the momentum transfer for the reactions of ${ }^{16} \mathrm{O}$ with $\mathrm{Al}, \mathrm{Ca}$ and ${ }^{60} \mathrm{Ni}$ at energies up to 20 $\mathrm{MeV} / \mathrm{n}$. At $8 \mathrm{MeV} / \mathrm{n}$ the heavy residues have the velocity of the center of mass, but at higher energies the fraction of the momentum transferred begins to decrease. At $20 \mathrm{MeV}$ it is $80 \%$ of full momentum transfer. This effect appears to be independent of target mass and the missing momentum is thus associated with a loss of mass from the projectile. The onset of incomplete momentum transfer begins at about $5 \mathrm{MeV} / \mathrm{n}$ in excess of the interaction barrier. The fraction of missing momentum increases linearly with the velocity above the threshold valuc. Measuiements of fast light particles in coincidence with the target-like residues are in progress.

In conjunction with the above experimental program, an effort is being made to improve the kinematic models that form the basis for interpreting many of the experimental observables. In particular, Brink's rules have been extended to include recoil effects and combined with a requirement that the transferred nucleons be contained in an overlap region of the target and projectile. These modifications result in an improved agreement with experiment.

This group is also engaged in a study of the decay of compound nuclei formed in the reactions of ${ }^{6} \mathrm{Li},{ }^{9} \mathrm{Be}$ and ${ }^{12} \mathrm{C}$ with ${ }^{40} \mathrm{Ca}$, and of ${ }^{3} \mathrm{He}$ with $\mathrm{Ag}$. In the first case, the objective is to determine whether these light compound systems decay by fission or fission-like processes. The emission of complex fragments such as $\mathrm{Li}, \mathrm{Be}, \mathrm{B}$, etc., is of interest in the study of the ${ }^{3} \mathrm{He}+\mathrm{Ag}$ reaction. Significant yields of these products have been observed and analysis is in progress. Both the above studies are in collaboration with the Moretto group. Andrew Budzanowski, and Kasimir Grotowski of Cracow, Poland have collaborated in the experiment on incomplete fusion, and the fusion-fission of light systems.

Early in the period covered by this report William Rae and John Cole returned to Oxford and Grenoble, respectively, Bernard Harvey left for a year of professional research and teaching at Grenoble, and Carolyn Albiston began a year's leave of absence. Itshak Tserruya spent a sabbatical year with the group. Post doctoral fellows Yuen-dat Chan and Shlomo Wald arrived from Oak Ridge National Laboratory and the Weizmann Institute, respectively. 


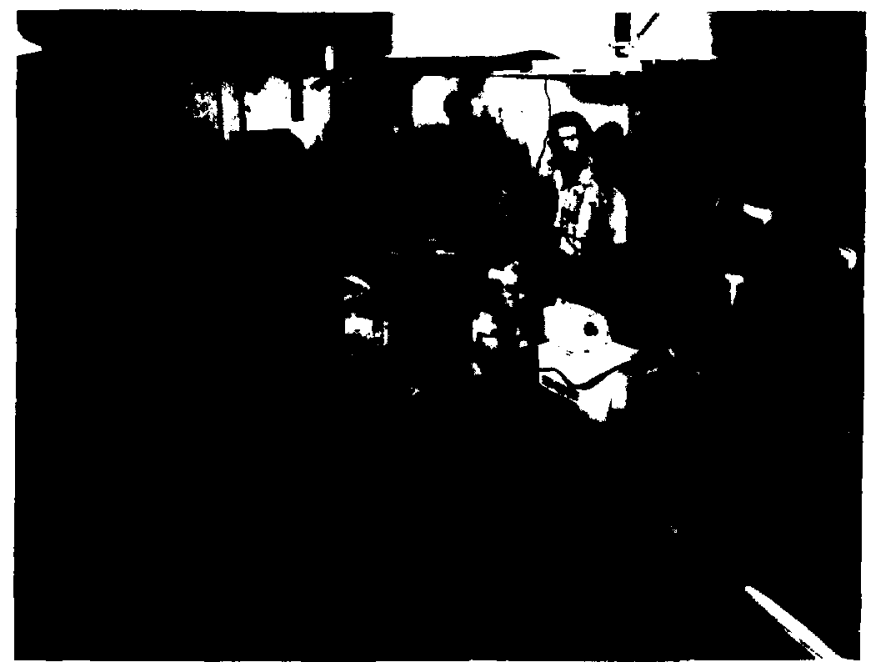

Fig. 1. The "plastic box" shown mounted in the 60-inch diameter scattering chamber. The side walls of the box are visible; the top wall is iemoved. The beam enters the chamber from the lower right. From left to right are P. Countryman, K. Van Bibber, Y.D. Chan, I. Tserruya, R. Burton (mechanical engiieer) R. Stokstad, S. Wald and M. J. Murphy. 


\section{Nuclear Structure}

Group Leaders

R.M. Diomond

F. Stephens

\section{M.A. Deleplanque}

J.E. Draper,
E.L. Dines,"
U.C. Davis

A. Macchiavelli, *

Buenos Aires,

Argentina

* graduate

students
This group is interested in studying and understanding a number of aspects of nuclear structure. For some years the principal effort has been in studying nuclei at high angular momentum. Nuclei arcund the middle of the periodic table can hold up to about $70 \mathrm{~h}$ before the Coriolis and centrifugal forces disrupt them, causing fission. The chance to study nuclei under conditions where these familiar forces become major factors in determining the nuclear structure is attractive, particularly since ${ }^{40} \mathrm{Ar}$ projectiles from the LBL 88-Inch Cyclotron can casily bring the full $70 \mathrm{~h}$ into a compound system. We have learned that nuclei have both collective and singleparticle features at those highest spins. They usually rotate in a rather classical manner, but because the system is finite $(\sim 100$ particles) there are strong irregularities ("backbends") due to just one or two particles realigning their angular momentum. (in such nuclei a single $\mathrm{i}_{13 / 2}$ or $\mathrm{j}_{15 / 2}$ particle can carry angular momentum as high as $10 \%$ of the maximum the system can hold.) The interplay of these two modes of notion is one of the fascinating aspects of studies of high-spin states. However, such studies are complicated by the fact that the $\gamma$-ray spectrum from states above $\sim 30 h$ is up until now unresolvable. The population in this region is spread over too many levels. Several techniques have been developed to study such unresolved spectra: arrays of detectors; large (sum-energy) crystals; and currently the development of $4 \pi$ detector systems.

Information about the collective and single-particle features of high-spin states can be obtained from unesolved $\gamma$-ray spectra by studying average moments of inertia. The properties of rotational bands reffect mainly collective features, and such bands give rise to highly correlated $\gamma$-ray spectra. These correlations can be detected in $\gamma-\gamma$ coincidence spectra without a full resolution of the spectra, and we have been studying such correlations, together with the B. Herskind group in Copenhagen. From them we can obtain moments of inertia appropriate to the bands populated and therefore learn something about the average collective behavior. Recently the group has developed techniques to measure a new moment of inertia, which is sensitive to both collective and single-particle motion, and combination of these two results can indicate both the collective and single-particle contributions in a given rotational frequency interval. Thus, considerable information can be obtained without resolving the $\gamma$-ray spectrum. However, it is becoming increasingly clear that the full elucidation of the physics comes only with with the resolution of the spectrum. This group is now building a system consisting of an approximately $4 \pi$ bismuth germanate inner spherical shell with apertures to about 20 Compton-suppressed intrinsic germanium detectors. This system combines the spin selection of the inner ball with the high resolving power of $\mathrm{Ge}-\mathrm{Ge}$ coincidences, and promises to give detailed information to much higher spins.

The newer study this group has undertaken is the observation of the giant dipole resonance (GDR) in the de-excitation $\gamma$-ray cascades of heavy-ion fusion products. Every state in a nucleus $15 \mathrm{MeV}$ or more above the yrast line is thought to have in it admixtures of the GDR based on states $\sim 15 \mathrm{MeV}$ lower in energy. (This is sometimes called Brink's hypothesis.) When excited, all such states have the possibility of emitting a GDR E1 $\gamma$ ray in competition with the more usual neutron (or particle) emission. In collaboration with the LG. Moretto group we have observed and studied these GDR $\gamma$ rays following deep-inelastic collisions. By gating with different values measured for the total kinetic energy of the fragments, we can select different temperature ranges populated in the nuclei and thus measure the temperature dependence of the GDR. In the first such studies the GDR peak is observed to broaden and drop in energy at the higher temperatures. 
The newest study undertaken by the group is of nucleon transfer between very heavy ions. Two aspects of heavy ions make such studies interesting. First, heavy nuclei are generally more collective, so coherent processes will be enhanced. For example, pairing correlations are stronger, and pair transfer should be enhanced. The second aspect is that angular momeatum transfers are large and orientation dependent. Thus, the populated spin ranges can give information about the initial orientation of the target and projectile nuclei. To see such eriects we have bombarded ${ }^{154} \mathrm{Sm}$ with ${ }^{132} \mathrm{Xe}$ and ${ }^{129} \mathrm{Xe}$ projectiles at the SuperHILAC. Twoneutron pickup by the Xe projectiles is large in these cases (comparable to the largest one-neutron transfers), and there appears to be anomalous spin population in the strongest one-neutron transfer channel. A quantitative analysis of these results is in progress and should tell us how interesting such studies will be.

In addition to these three main efforts, the group studies other aspects of nuclear structure and shapes together with several outside groups: those of Cline, et al., from Rochester; Ward, et al., from Chalk River; and Johnson, et al., from Oak Ridge. These studies are mostly of Coulomb Excitation with very heavy ions ( ${ }^{136} \mathrm{Xe}$ and ${ }^{201} \mathrm{~Pb}$ ) produced by the SuperHILAC.

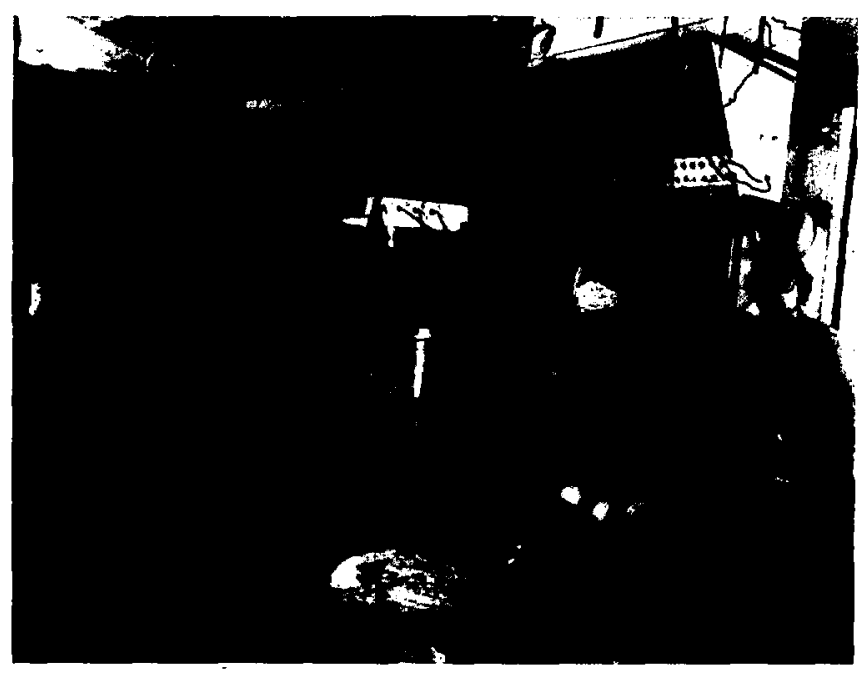

Fig. 1 Marie-Agnes Deleplanque, Gene Dines and Augusto Macchiavelli adjusting Nal detectors around the sum spectrometer. 


\section{Polarization Phenomena in Nuclear Physics}

Group Lender

H. E. Conzerf

D. Eversheim

C. Rioux

R. $M$. Larimer

W. J. Knox,

V. Paolone, *

U.C. Davis

"Graduate

student
This group's research uses the polarized beams from the 88-Inch Cyclotron and is concerned with spin-polarization effects in nuclear scattering and reactions. It particularly addresses such fundamental questions as parity violation by the weak interaction component in pF scattering, time-reversal invariance in reactions, charge symmetry of the nucleon-nucleon interaction, and the three nucleon problem. A most important featurc of polarization effects is that they often display uniquely the operation of a basic symmet: $y$ property of the nuclear interaction.

A major effort during the past year has been devoted to a testing of the principle of time-reversal invariance in nuclear reactions. This principle results in the polarization-analyzing power equality, i.e., the polarization in a reaction $A(a, \vec{b}) B$ is equal to the analyzing power in the inverse reaction with polarized projectile $\mathbf{B}(\vec{b}, \mathbf{a}) \mathbf{A}$ Pievious checks of time-reversal invariance in nuclear reactions have been limited to tests in elastic proton scattering, and this group has shown that all such tests were inadequate, due to either lack of precision or lack of sensitivity to a violation of timereversal invariance. Thus, further experiments are required in order to provide significant tests of this important principle. In collaboration with a group from Laval University, this group has made measurements of the polarizations in several $\left({ }^{3} \mathrm{He}, \vec{p}\right)$ reactions and of the analyzing powers in the corresponding $\left(\vec{p}^{3} \mathrm{He}\right)$ inverse reactions. Substantial differences between the polarization and the analyzing power have been measured. If such differences are confirmed in additional experiments currently underway, it would be firm evidence for the violation of the fundamental postulate of time-reversal invariance of the nuclear interaction.

A collaborative experiment to determine the level of validity of charge symmetry in the nucleon-nucleon interaction is under preparation. The experiment, approved for beam time at TRIUMF, measures the difference $\triangle A$ between the neutron and proton analyzing powers $A_{n}$ and $A_{p}$ in n-p scattering at $500 \mathrm{MeV}$. Designed as a null-measurement requiring no accurately known polarization standards, the experiment determines the difference in angle at which $A_{n}$ and $A_{p}$ cross through zero. It will provide an unambiguous test of a charge-symmetry breaking effect to the level of ${ }^{*}$ $\Delta \mathrm{A} \simeq 0.001$, corresponding to a laboratory angle difference at zero crossing of $\simeq$ $0.05^{\circ}$

Polarization observables are of ten unique probes to provide answers to specific questions concerning nuclear structure or reaction mechanisms. During the past year, several experiments of this nature have been undertaken in collaboration with outside groups as follows:

1. University of Manitoba. Analyzing power measurements have becn made in $\vec{p}$ $+{ }^{40} \mathrm{Ar}$ elastic scattering from 25 to $40 \mathrm{MeV}$ for the determination of the $\mathrm{L}$ dependence of the optical model potential in that energy region.

2. University of Manitoba/Birmingham Measurements were made of analyzing powers in both the ${ }^{117} \mathrm{Sn}(\overrightarrow{\mathrm{p}}, \mathrm{d}){ }^{116} \mathrm{Sn}$ reaction and its inverse, ${ }^{116} \mathrm{Sn}(\overrightarrow{\mathrm{d}}, \mathrm{p})^{117} \mathrm{Sn}$. These data will permit the determination of the separate spin-orbit interactions of the proton and of the deuteron.

3. Ohio State University. Analyzing power measurements in the reactions ${ }^{40} \mathrm{Ca}$, ${ }^{48} \mathrm{Ti}\left(\overrightarrow{\mathrm{d}},{ }^{6} \mathrm{Li}\right){ }^{36} \mathrm{Ar},{ }^{44} \mathrm{Ca}$ at $50 \mathrm{MeV}$ will be used to determine the ${ }^{6} \mathrm{Li}$ spin-orbit strength. Very little is presently known about this interaction.

4. Lawrence Livermore National Laboratory. Analyzing powers were measured in the inelastic proton transition ${ }^{6} \mathrm{Li}\left(\overrightarrow{\mathrm{p}}, \mathrm{p}^{\prime}\right)^{6} \mathrm{Li}^{*}(2.18 \mathrm{MeV})$. Analysis via microscopic distorted-wave calculations will permit the determination of specific components of the effective two-nucleon force in nuclei. 
In future work, this group plans to continue to focus on experiments with polarized particles which examinc the opcration of the basic symmetries - time-reversal invariance, parity conservation, and charge symmelry - in nuclear scattering and reactions. Also, the study of selected polarization observables to answer specific questions concerning nuclear structure and reactions will be pursued. 
Group Leader

G.T. Seaborg

\section{Frank $\boldsymbol{k}^{\dagger}$ \\ K. Gregorich* \\ D. Lee \\ R. McFarland* \\ P. McGaughey* \\ K. Moody" \\ Y. Morita* \\ M. Nurmia \\ M. Perry ${ }^{\dagger}$ \\ L.P. Somerville \\ R. Welch* \\ P. Willmarth* \\ S. Yashita*}

K. Aleklett,

Swedish Research

Council, Sweden

D. Hoffman,

Los Alamos

National

Laboratory

W. Loveland

Oregon State

University

H. von Gunten, University

of Bern,

Switzerland

H. Kudo,

Tokyo

Metropolitan

University,

Tokyo, Japan

W. $L i$

Beijing University, Beijing, Peoples

Republic of China

\author{
*Graduate \\ students \\ tUndergraduate \\ students
}

\section{Heavy Element Radiochemistry}

This group uses all three of the LBL accelerators to identify and characterize new elements and isotopes, to study nuclear reaction mechanisms, and to train students in mociern radiochemical techniques. Currently, research is focused on (1) the synthesis and identification of new isotopes and elements in the actinide and transactinide region, along with attempts to synthesize superheavy elements; (2) the study of low-energy heavy ion reaction mechanisms such as massive transfer, complete fusion and deep inelastic scattering, potentially useful in the synthesis of new elements and isotopes; and (3) the characterization of the mechanisms operating in intermediate energy (10-100 MeV/A) and relativistic ( $\geq 250 \mathrm{MeV} / \mathrm{A}$ ) heavy ion (RHI) reactions through studies of the target fragment yields, energies, angular distributions, etc.

With light $(\mathrm{A}<25)$ heavy ion reaction studies at the 88-Inch Cyclotron, the primary emphasis has been on the use of "transfer" reactions to produce heavy actinides and the study of the mechanism of these reactions. These studies have indicated surprisingly large probabilities for the synthesis of the nuclear species ranging in mass from the target mass to that of the compound nucleus. The group is systematically exploring these reactions by studying the variation of product yields, energies, angular distributions, etc., with projectile and target mass and energy.

Members of the group have also used the 88-Inch Cyclotron and the SC synchrotron at CERN to study intermediate energy heavy ion reaction mechanisms. In particular, they are concerned with studies of the target fragment yields, energies, and angular distributions in light ion-heavy target reactions.

Research at the SuperHILAC has been directeu coward the use of deep inelastic transfer and "cold fusion" processes to produce new isotopes or elements and to obtain an understanding of the mechanisms involved. Eforts are being concentrated on the reactions of ${ }^{48} \mathrm{Ca}$ and the heaviest projectiles $(\mathrm{Kr}, \mathrm{Xe})$ with very heavy targets $\left({ }^{248} \mathrm{Cm},{ }^{254} \mathrm{Es}\right)$. Future work will involve the use of heavier projectiles such as ${ }^{238} \mathrm{U}$.

Research on target fragmentation at the Bevalac has involved single particle inclusive survey measurements of the energy dependence of target fragmentation. No evidence was found for nuclear transparency effects in heavy ion-heavy nucleus collisions. Dramatic changes in fragment angular distributions are observed with increasing projectile energy, with sidewise or backward-peaked distributions observed at the highest projectile energies. Current models of RHI interactions (such as the firestreak, cascade, etc.) do not correctly treat the momentum transfer to the target nucleus in the collision. 


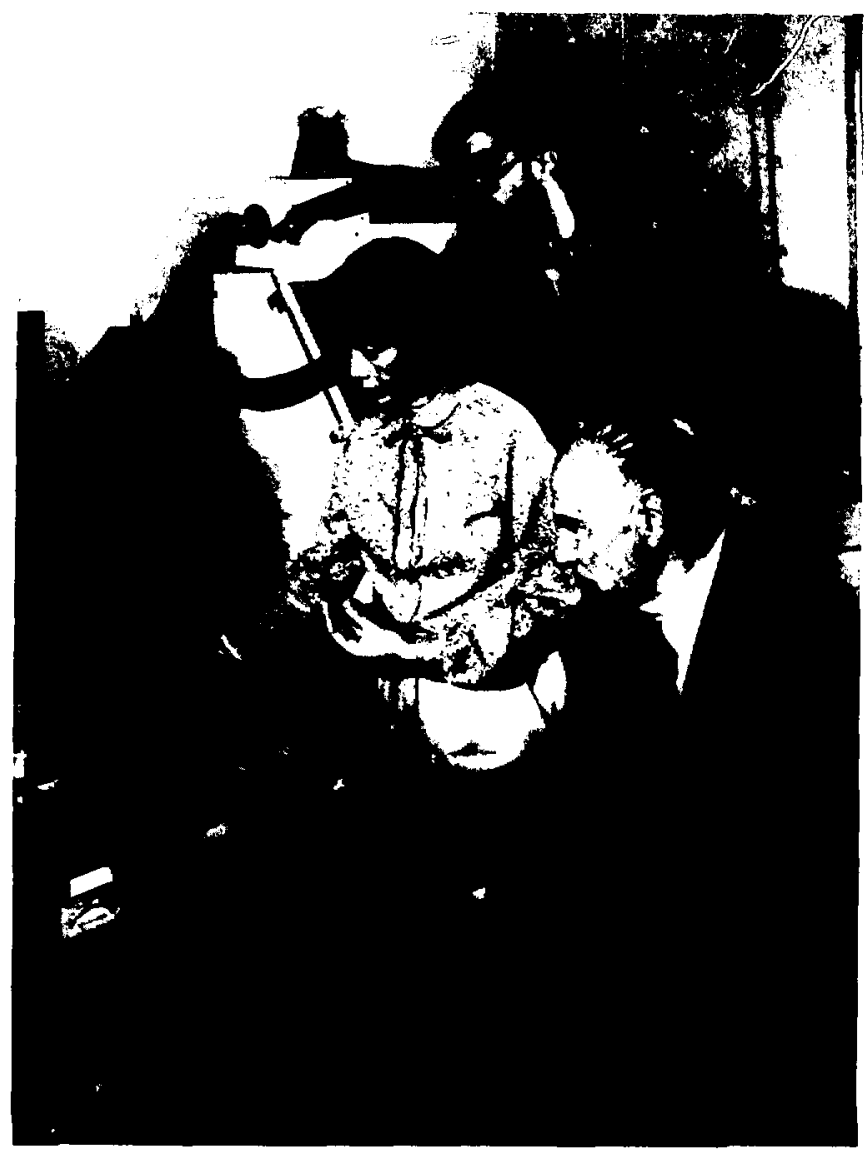

Fig. 1. Diana Lee inserting sample into counting apparatus as Kenton Moody and Glenn Scaborg look on.

XBB 820-9638 
Group Leaders

A. Ghiorso

J. M. Nitschke

L. Frank*

K. E. Gregorich*

D. M. Lee*

K. J. Moody

M. J. Nurmia

L. P. Somerville*

G T. Seaborg

P. A. Wilmarth*

S. Yashita*

\section{Leino, \\ University \\ of Helsinki,}

Finland

\section{J. D. Molitoris, * \\ Stanford \\ University}

W.-D. Zeirz, Hahn-Meitner Institut, Berlin, West Germany

P. Armbruster, S. Hofmann,

P. Lemmertz, G. Münzenberg, GSI, Darmstadt, West Germany

J.-P. Dufour, CEN, Bordeaux, France

\section{Hoffman.}

M. Fowler,

Los Alamos

National

Laboratory

E. $\boldsymbol{K}$. Hulet, R. Lougheed, Lawrence

Livermore

National

Laboratory

* Graduale

students

\section{Heavy Element Research}

The research program of the Heavy Elements Research group is directed toward the synthesis of new nuclides and the determination of the radioactive properties of known nuclides. Graduate students play an important role in this work and thereby are trained in the advanced technology of nuclear physics. Experiments are conducted both at the 88-Inch Cyclotron and at the SuperHILAC, and often there are extensive collaborations with scientists at other laboratories of the UEA and foreign countries.

Research of the group at the 88-Inch Cyclotron deals principally with problems related to the production of neutron-heavy nuclides, and makes use of targets from uranium to einsteinium with particles from protons to argon ions. The workhorse of the group has been the long-tape system for detecting short-lived spontaneous fission (SF) nuclides, and this research has led to the discovery of a number of new SF emitters. The other major system is the MG apparatus which is used to measure coircident $E_{1} / E_{2}$ events from $S F$ emitters; it is presently being modified to have an alpha particle detection capability and thus is now designated MGA. The number of $4 \pi$ detecting stations is being doubled to eight to increase the running efficiency and make maximum use of cyclotron time.

At the SuperHILAC a concentrated effort is underway with SASSY, our gasfilled magnetic spectrometer, to search for superheavy elements (SHE) with half-lives down into the microsecond range. To this end, a strong collaboration was established in 1982 with the Armbruster group at GSI in West Germany. It was agreed to establish teams of scientists that would be exchanged to undertake experiments to search for SHE in bombardments of ${ }^{248} \mathrm{Cm}$ with ${ }^{48} \mathrm{Ca}$, first at LBL using SASSY, and then a little later at GSI using SHIP. In the early part of 1982, studies and calibrations were made with SASSY to understand its capabilities. The joint efforts were quite successful, leading to the production of ${ }^{254} \mathrm{No}$ by ${ }^{48} \mathrm{Ca}$ bombardment of ${ }^{208} \mathrm{~Pb}$, using our old Ortec focal plane detector system. It was then decided to push the development of a new and more efficient silicon detector array, which was quickly incorporated into our system. Unfortunately, a major experimental disaster, which occurred just as the first run with a ${ }^{248} \mathrm{Cm}$ target was to begin, reduced our ${ }^{48} \mathrm{Ca}$ bombardment to only about three days, and as a result, few conclusions can presently be drawn from the experiment.

The second major research tool of the heavy element group is OASIS (an acronym for On-line Apparatus for SuperHILAC Isotope Separation). Recoils from heavy-ion-induced nuclear reactions are stopped in a tantalum catcher, which is kept at a temperature just below its melting point inside an ion source. The recoils diffuse out of the catcher, are ionized and then accelerated to an energy of $50 \mathrm{keV}$. An analyzing magnet selects the desired isotopes and focuses them onto various detectors. The detectors most often used during the present report period were proton telescopes. The isotopically-pure beam can also be guided to a shielded room 4 meters above OASIS where low-background proton and $\beta^{ \pm}, \gamma$ - and x-ray experiments can be carried out. A fast tape system transports the selected isotope within $60 \mathrm{~ms}$ to a detection system that consists of a high purity $G e$ detector for $\beta^{ \pm}$- and $x$-ray spectroscopy, and an n-type $\mathrm{Ge}$ detector for $\gamma$ and $x$-ray measurements. In addition, a single $\Delta E$ detector or a $\Delta E / E$ telescope can be placed in front of one of the Ge detectors to identify different charged particles. All detectors provide coincidence information.

Presently most of the OASIS physics is being done in the focal plane of the separator magnet with emphasis on new isotopes near the proton drip line. Several new delayed proton emitters have been found and efforts are under way to synthesize new isotopes that decay by proton radioactivity. Candidates that are predicted to 
have long enough half-lives and large enough proton branches to be detectable with OASIS are: ${ }^{137} \mathrm{~Tb},{ }^{127} \mathrm{Pm},{ }^{117} \mathrm{La}$, and ${ }^{113} \mathrm{Cs}$. The production of these nuclei is difficult; even with the use of the most neutron-deficient targets and beams from the SuperHILAC, the cross sections are in the microbarn range. Phenomenologically, $\mathrm{p}$ radioactivity is analogous to the very common and familiar $\alpha$-radioactivity. The mathematical interpretation should, however, be simpler since only a single nucleon, rather than a four-nucleon cluster, is involved in the decay.

Two years ago we had already started to use damped collisions to produce isotopes on the neutron-rich side of beta stability. Thus far, the large background inside the "cave" has prevented the exploitation of this technique. With the near completion of the tape station, we are now in the position to study isotopes which previously could not be reached by other production methods. These isotopes have importance for the understanding of cosmological and reactor processes.

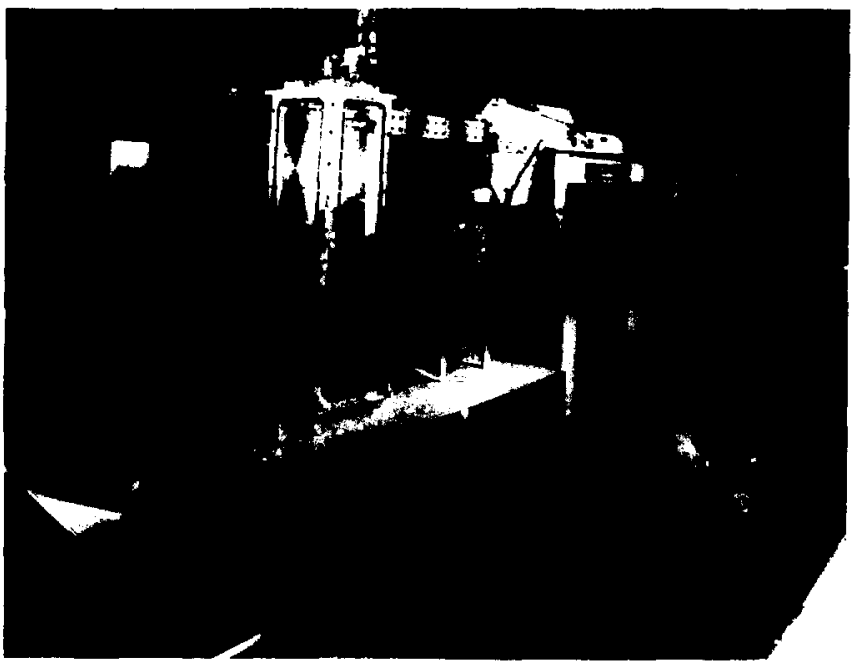

Fig. 1 OASIS Tape Transport Station with Wolf Zeitz, Phil Wilmarth, and Michael Nitschke. 


\section{Statistical Equilibrium in Deep-Inelastic Heavy Ion Reactions}

Group Leader L.G. Moretto

\section{G. Guarino}

J. Hunter A.J. Pacheco*

M.L. Padget**

L.G. Sobotka

G.J. Wozniak

J.H. Gu, Z.Q. Yao, Shanghai, Peoples Republic of China

*graduate students
In deeply inelastic collisions, a certain fraction of the initial orbital angular momentum is converted into spin of the individual fragments. This process can be investigated by studying the decay of the highly excited nuclei emerging from the primary reaction. Measurements of the sequential emission of $\alpha$-particles, fission fragments, and $\gamma$-rays have provided information on different parameters of the exit channel fragments' spin distributions, generated as a consequence of the interaction between the two reaction partners. In principle, these distributions should refiect the underlying mechanism responsible for the angular-momentum transfer. For example, the spin fluctuations (both in magnitude and orientation) not only reflect the diffusive nature of the angular-momentum-transfer process, but they also carry information on the rotational degrees of freedom involved. However, if the rotational modes of the dinuclear complex, reach a state of statistical equilibrium with the intrinsic degrees of freedom, then the distinction among different possible mechanisms would be very difficult, since all of them would lead to essentially the same spin distributions. Thus, it is important to determine whether statistical equilibrium is reached in deepinelastic collisions.

During the last several years, our group has modeled the limit of statistical equilibrium for a very simple configuration of the dinuclear systems. For masssymmetric systems, this model predicts a gaussian distribution for the cartesian components of the spins, with almost equal variances along the three coordinates. The magnitude of the predicted fluctuations compares well with results from the reaction ${ }^{165} \mathrm{Ho}+{ }^{265} \mathrm{Ho}$ at $8.5 \mathrm{MeV} / \mathrm{A}$, studied via $\gamma$-ray multiplicity and anisotropy techniques.

In the last year, this statistical model was extended to mass asymmetric systems. For small to moderate mass asymmetries, this model also predicts that the variances of the spin distributions along the three cartesian coordinates are nearly equal. The magnitude of the predicted fluctuations compares well with the magnitude and alignment of the spins measured for the reactions of $8.5 \mathrm{MeV} / \mathrm{A}{ }^{165} \mathrm{Ho}$ on targets of ${ }^{176} \mathrm{Yb},{ }^{148} \mathrm{Sm}$, and ${ }^{\text {nat }} \mathrm{Ag}$. For very large mass asymmetries, the statistical model predicts that the spin distribution of the heavy fragment will develop a strong inplane asymmetry. A substantial in-plane anisotropy has recently been observed in the measured in-plane angular distributions of sequentially emitted fission fragments from both the reaction of $12.6 \mathrm{MeV} / \mathrm{A}^{20} \mathrm{Ne}$ and the reaction of $8.5 \mathrm{MeV} / \mathrm{A}{ }^{40} \mathrm{Ar}$ with targets of ${ }^{238} \mathrm{U}$ and ${ }^{197} \mathrm{Au}$.

A theoretical investigation of the effect of statistical fluctuations on the total energy and multiplicity of $\gamma$-rays associated with deep-inelastic reactions has been carried out. The transition state theory of fission has been generalized to allow for trajectories that return from saddle to compound nucleus due to high yiscosity at the saddle point. A statistical theory for the distribution of angular momentum between the fragments has been developed for the case of multifragmentation (three or more fragments) produced in heavy ion reactions. The production of three or more major fragments is expected to be a dominant mechanism at bombarding energies greater than $20 \mathrm{MeV} / \mathrm{A}$

The emission of $\alpha$-particles from the deep-inelastic reaction $1354 \mathrm{MeV}{ }^{165} \mathrm{Ho}+$ ${ }^{181} \mathrm{Ta}$ bas been studied. The in-plane $\alpha$-particle data show that the bulk of the $\alpha$ particles in coincidence with the deep-inelastic exit channel can be explained by evaporation from the fully accelerated fragments. The out-of-plane $\alpha$-particle angular distributions together with $\gamma$-ray multiplicities from previous work give a consistent picture of the transfer and partitioning of angular momentum between the two fragments. 
The evaporation of intermediate mass fragments ( $\mathrm{Li}, \mathrm{Be}, \mathrm{B}$, and $\mathrm{C}$ nuclei) from compound nuclei is not well understood. The study of these decay channels is interesting, not only because they are important to the understanding of the deexcitation of highly excited nuclei, but also because they provide a testing ground for attempts to unify evaporation and transition state (fission) theories. We have recently undertaken a study of the emission of complex fragments from the reactions of ${ }^{3} \mathrm{He}+$ ${ }^{n a t} \mathrm{Ag}$ at 130 and $90 \mathrm{MeV}$ bombarding energies. 


\section{High Energy Nuclear Collisions}

LBL:

Group Lerder

A.M. Poskanzer

H.-A. Gustajsson

T. Renner

H. Riedesel

GSI, Darmstadt, West Germany:

Group Leader

H.H. Gutbrod
B. Kolb
H. Löhner
B. Ludewigt
H.-G. Ritler
A. Warwick
F. Weik
H. Wieman

The work of this group is directed to the study of central collisions of relativistic heavy ions with the aim of learning about nuclear matter at high temperature and density. The group is a continuing collaboration between GSI and LBL.

The experiment performed two years ago to study the correlations between slow and fast fragments has been completed and submitted for publication. For the slow fragments an array of 40 detectors, consisting of silicon counters, gas $\Delta \mathrm{E}$ counters, and avalanche counters, was used. For the fast fragments an 80-counter multiplicity array plus the Plastic Wall were used. The analysis focused for central collisions on the violent breakup of the target nucleus as seen by the light fragment muitiplicity and, for peripheral collisions, on the momentum transfer to the target measured by the angular correlation of coincident fission fragments.

With the completion of the Plastic Ball in June 1981 the group moved to exclusive charged particle measurements. The Plastic Ball covers $96 \%$ of $4 \pi$ with 815 modules, each consisting of a $\mathrm{CaF}_{2} \Delta \mathrm{E}$ detector and a plastic scintillator $\mathrm{E}$ detector. The two scintillators are observed by the same photomultiplier tube and the signals are separated electronically by taking advantage of their different decay characteristıcs. In addition, positive pions are identified by their $\pi^{+} \rightarrow \mu^{+} \rightarrow e^{+}$decay, as they were in our previous experiments. The small forward angle region is covered by the Plastic Wall placed $6 \mathrm{~m}$ downstream. The Wall consists of 177 plastic scintillators and identifies particles by their time of flight and energy loss. Data were taken in June 1981 for ${ }^{40} \mathrm{Ca}$ on a Ca target at two energies and in June 1982 for ${ }^{93} \mathrm{Nb}$ on a Nb target, also at two energies. Most of the past year has been spent in developing programs to process the data and in obtaining simulated data from several different theoretical models.

Many types of analysis are now in progress. The degree of thermalization in central collisions is being evaluated and temperatures will be extracted. An attempt is being made to deduce entropy from cluster formation. Two-particle correlations are being studied. Analysis in terms of kinetic energy flow is under way to determine the mechanism and possibly extract density. Jet structure in terms of a minimum spanning tree algorithm is in progress with the possible hope of separating the spectators from the participants. In short, a rich harvest of results is expected from the millions of exclusive charged particle events that have been obtained. 


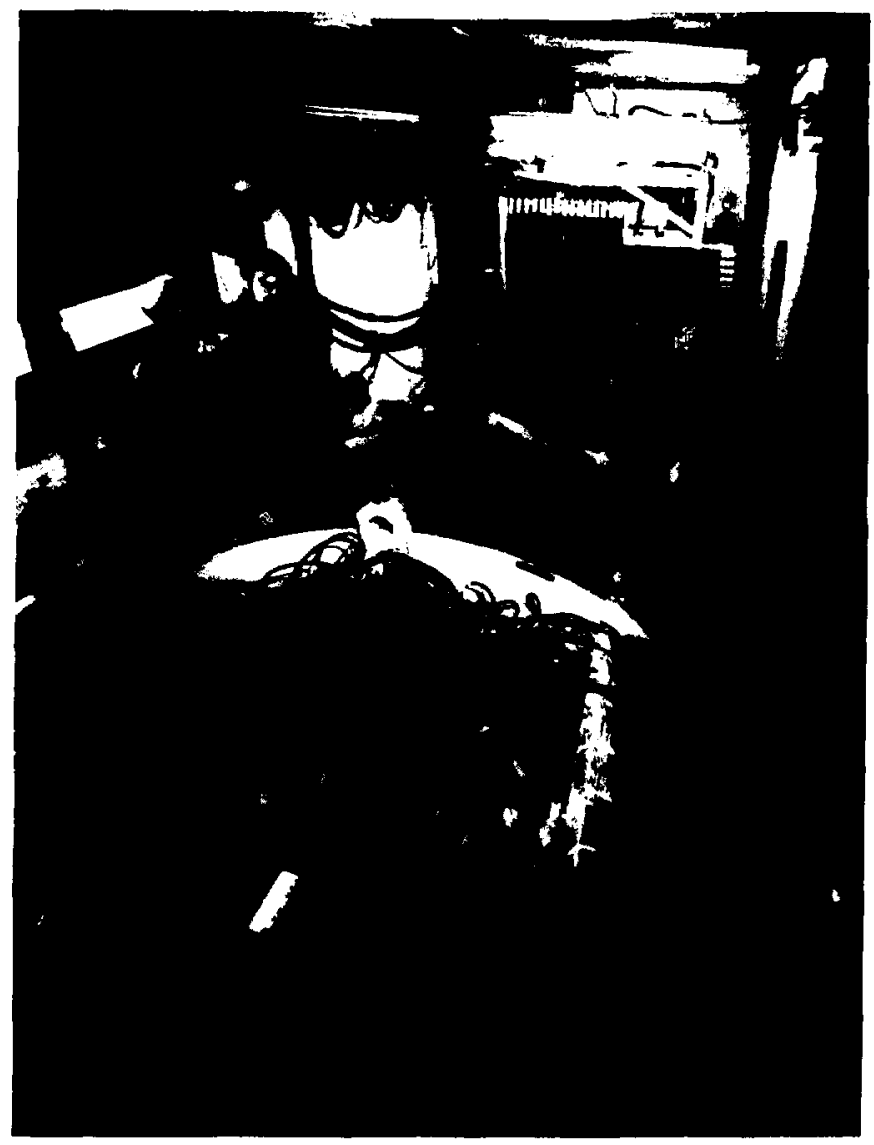

Fig. 1. The Plastic Ball looking downstream to the Plastic Wall. HansGeorg Ritter and Hans Gutbrod are sitting above the Ball and Art Poskanzer is just in front of the Wall.

CBB 817.6464 


\section{HISS}

Group Leader

D. Greiner

\section{E. Beleal \\ F. Bieser \\ M. Bronson \\ P. Lindstrom \\ J. Wolf}

H. Crawford, I. Flores, C. McParland, Space Sciences Laboratory and U.C. Berkeley
The HISS group is a research group which has support responsibility for both the Heavy Ion Superconducting Spectrometer Facility (HISS) and the Bevalac software. The relative ratio of these functions is in a constant state of redefinition with the exception that the Bevalac software support effort is mandated by the administration to be no less than 1 FTE per year. Our research interests are in the area of relativistic heavy ion research. After several years of inclusive measurements as a part of the Heckman group, the HISS group was formed in order to provide a cohesive focus to build and run the facility. As may be expected, our research interests involve using multi-particle measurements. The first experiments we are doing at HISS are directed at using semi-exclusive data to look for unusual states of nuclear matter and to understand the reaction mechanisms in heavy jon reactions.

During FY82 there were many improvements in th: HISS Facility. The refrigeration machinery was moved from the top of the magnet to an enclosure outside the east wall of the cave. The reassembled system, augmented with a "wet engine" from FNAL, has proven to be extremely reliable. Portions of the apparatus are now under computer control and more will be added as time permits. The cave now is finished, has a roof, and is somewhat warmer this winter. The large vacuum tank in the magnet gap is another welcome addition as well as the record-breaking thin window ( $3^{\prime} \times 12^{\prime}$ area tested to over 75 tons of pressure).

The first experiment to be completed using HISS was the proton spectra and correlation experiment by the INS-LBL collaboration $(\exp 512 \mathrm{H})$. The five weekends of running were also shared by the UCLA-LBL collaboration (exp 513H) and the HISS group (exp $512 \mathrm{H}$ ). One further night of running was granted to test the MUSIC detector. Preliminary results from exp 513H were presented at the International Conference on Nucleus-Nucleus Collisions (MSU) and in LBL-15071.

The HISS phase II detector construction effort continued this year. The two large phase II time-of-flight walls were completed, tested, and used in experiments.' The MUSIC detector was completed for testing with nonexotic gas; results were obtained with several beams. The final design for the drift chamber electronics was completed, and 1200 channels were assembled in one ten-day period. Finally the construction began on the $2 \mathrm{~m} \times 5, \mathrm{n}$ phase II drift chamber.

This year the HISS group was given the responsibility for the Bevalac software effort. Headed by Chuck McParland, this work produced a new set of distribution software and also designed, built, and tested a new CAMAC module. This module, called CATS, is a module that tests all the CAMAC functions allowing very rapid system error tracing. 


\section{Relativistic Heavy Ion Physics}

The experimental program of the Relativistic Heavy Ion Physics Group being carried out at the Bevalac focuses on two rather diverse, but forefront, areas of research during FY 1981-82. These are, first, experiments on the properties of relativistic projectile fragments of $\sim 2 \mathrm{AGeV}{ }^{56} \mathrm{Fe}$ nuclei and the evidence for "anomalons" therefrom and, second, the initial uranium beam experiments using emulsion detectors at the Bevalac. These latter experiments were critically important for the verification of the acceleration of the ${ }^{238} \mathrm{U}$ beams at energies $0.150 \mathrm{AGeV}$ (11 May 1982) and 0.950 AGeV (25 September 1982) and gave the first quantitative data on the reaction mean-free-path length of uranium nuclei in emulsion and the energy dependences of the topologies of projectile and target fragments produced in ${ }^{238} \mathrm{U}$ nucleus collisions. An integral part of these programs is the continued development of an Interactive Computer Assisted Measuring System (ICAMS) to improve the rate, accuracy, and scope of data acquisition in nuclear emulsion experiments.

Further investigations of mechanisms of particle production at the high energy densities obtainable from the collider phase of the Tevalac accelerator were carried out in collaboration with R.M. Weiner (University of Marburg, Germany) and G.N. Fowler (University of Exeter, England). The latest data from the CERN-pp-collider were shown to be compatible with the predictions of the hydrodynamical theory and to provide evidence for an increased transparency of hadronic matter, manifested in a decrease of the collision inelasticity. The puzzling features of Centauro-type collisions were shown to be interpretable by an "early" decay of a coherently excited quark-gluon plasma phase through the mechanism of superfluorescence.

Important aspects of the Group's research program in FY 81-82 were the organizing and hosting of two major workshops, i) the Workshop on the "Aromalon Phenomenon", 8-10 February 1982 and ii) the Japanese-American Cooperative (cosmic-ray) Emulsion Experiments (JACEE) Seminar, 19-20 August 1982. Both workshops were attended by researchers from the U.S., Europe, and Japan and were notable for, respectively, i) the establishment of a "world-wide" collaboration on "anomalon" experiments and ii) the in-depth discussion with LBL specialists on new results in ultra high-energy cosmic-ray heavy-ion physics and their implications for the Tevalac. The subject matter of the JACEE Seminar held a feature role in discussions on the possible production of a quark-gluon plasma during the Tevalac Workshop that took place a few weeks later.

The Group's work on the evidence for projectile fragments having an anomalously short reaction mean free path, "anomalons", was selected as one of the "interesting and newsworthy developments in physics during 1981" and was described in the Physics News in 1981, published by the American Institute of Physics, November 1981.

\section{Projectile Fragmentation}

The scanning and measuring of emulsions exposed as part of Bevalac Exp. 559H is in progress. The experiment pertains to the fragmentation reactions of ${ }^{56} \mathrm{Fe}$ and extends our studies on the evidence for anomalons. The experiment also includes irradiations of emulsions to $\sim 2 \mathrm{AGeV}$ beams of ${ }^{3} \mathrm{He},{ }^{6} \mathrm{Li},{ }^{12} \mathrm{C}$, and ${ }^{40} \mathrm{Ar}$ for the purposes of determining reaction mean free paths and charge calibration.

This experiment involves the collaborative eflorts of B. Judek, National Research Council of Canada, E. Ganssauge, University of Marburg, and G. Baroni, University of Rome. We are at present concentrating on the interactions of projectile fragments $Z \geq 3$ as part of a world-wide collaboration that includes, in addition to the above groups, the Lund-Jajpur-Jammu collaboration, SUNY (Buffalo), and
Group Leader H.H. Heckman
M. Bloomer
E. Friedlander
R. Gimpel
S. Janssen
Y. Karant
B. Peterson
J. Sturla
H. Yee
C. Zeitlin
E. Ganssauge,
University of
Marburg,
West Germany

B. Judek, National Research

Council, Otawa, Canada 
the University of Siegen. The immediate objective of this collaboration is to obtain statistically improved data on the short-mean-free-path eifect, e.g., i) the interaction properties of $Z \geq 3$ fragments, ii) lifetime estimates, iii) effects in higher order generations of the extranuclear cascade, iv) the semi-inclusive properties of the collision parameters, and $v$ ) the dependence on projectile nucleus.

\section{Uranium Collisions}

A new generation of relativistic heavy ion physics began with the successful acceleration of ${ }^{238} \mathrm{U}$ at the Bevalac. The nuclear emulsions exposed to the first beams of ${ }^{234} \mathrm{U}$ gave immediate results on the ranges, track structure, and reaction meanfree-path lengths of ${ }^{23 \mathrm{~B}} \mathrm{U}$ in emulsion and characteristic topologies of uranium-nucleus collisions. Based on our preliminary observations, planned uranium experiments will give information on

1. Projectile-related phenomena:

a) charge and multiplicity distributions of projectile fragments

b) angular distribution of projectile fragments; $p_{T}$ of nuclei and nuclear clusters

c) pion (shower particle) production

d) fission processes of $U$, nuclear and electromagnetic

2) Target-related phenomena

a) charged multiplicities and energy spectra of target fragments $E / A \leq 30$ $\mathrm{MeV}$

b) fragments in the mid-rapidity region; search for evidence of the fusion of (light) target nuclei with uranium projectiles.

In support of our future program of research we have the following objectives: i) to extend ICAMS to include additional measuring stations and automatic charge measurements and ii) to develop a 0.3-0.5 MG pulsed magnetic field to permit direct rigidity measurements in nuclear emulsion and other high-spatial-resolution visual detectors. 


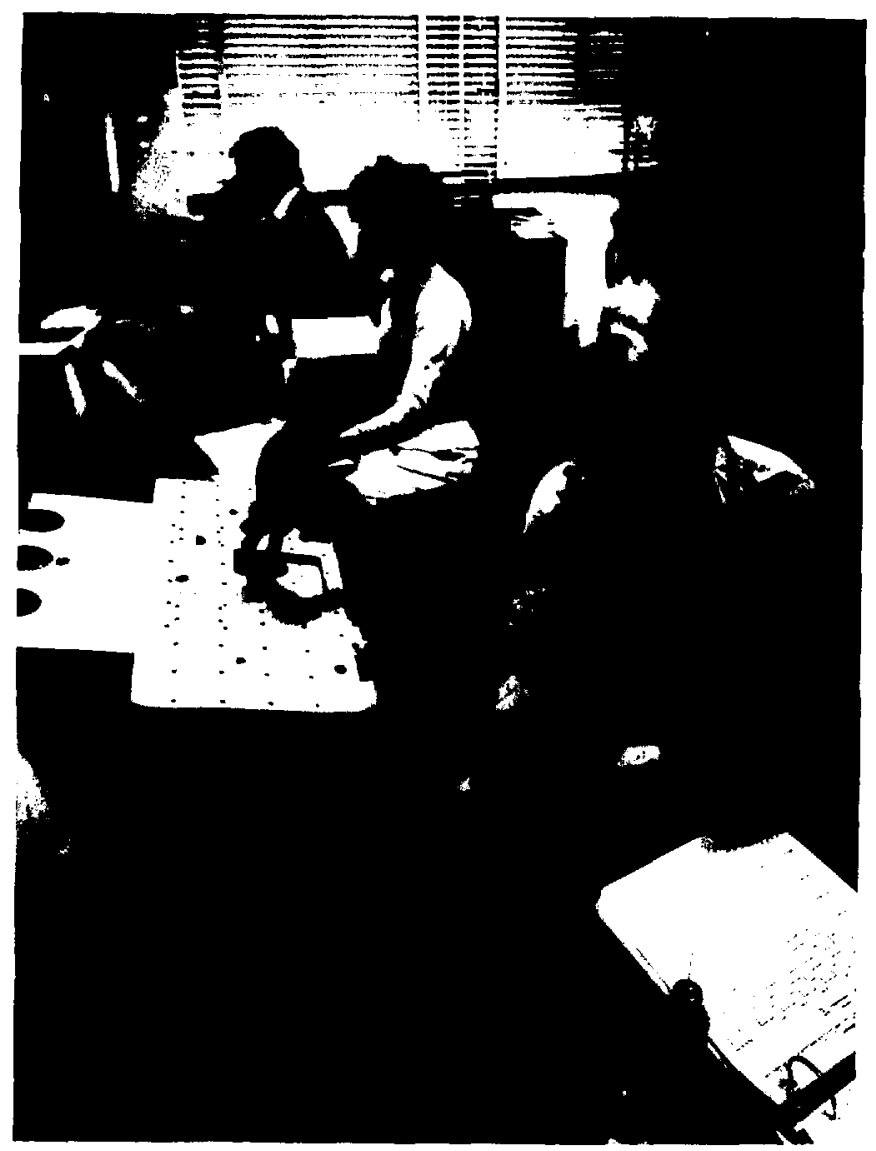

Fig. 1. Scientific data analysts examining nuclear track emulsions.

CBB 819-8239 


\section{High Energy Nucleus-Nucleus Collisions}

Group Leaders

H.G. Pugh

L.S. Schroeder

A. Sandoval.

R. Stock,

GSI, Darmstadt

TASS:

R. Koontz

R. Treuhaft

J.W. Harris, GSI, Darmstadt

P.N. Kirk,

G. Krebs,

J. Gilot,

Louisiana State

University

G. Roche.

J. Vincente,

University of

Clermont-Ferrand
This group has two complementary research components in relativistic heavy ion physics at the Bevalac. One uses electronic detection techniques and operates the TASS two-arm spectrometer system in collaboration with Louisiana State University. This is suitable for a wide range of charged particie inclusive measurements and for two-particle coincidence experiments where a large solid angle is not required. The other operates the STREAMER CHAMBER as a GSI-LBL collaboration to study $4 \pi$ charged perticle exclusive data with this low-data-rate visual-data-storage device. The groups of $\mathbf{K}$. Wolf at Texas A\&M University and A. Dacal at the University of Mexico haye also made major commitments to the streamer chamber research program.

\section{TASS}

The Two-Arm Spectrometer System (TASS) consists of two fully rotatable magrets with accompanying wire chambers and scintillation hodoscopes for particle identification. It was first operated in July 1980 and the first phase of instrumentation is expected to be complete by mid-1983. The main activities on TASS have been:

444H This experiment involved measuring the angular and momentum correlations between high energy particles $\pi^{+}, p$, $d$ emitted in the forward and backward hemispheres. Particle production at backward angles is of considerable interest since it is either strictly forbidden (e.g. nucleon scattering) or severely constrained (e.g., pion production, where the maximum pion momentum is less than $300 \mathrm{MeV} / \mathrm{c}$ in "free" nucleon-nucleon collisions). Thus backward particles may provide a tool to measure exotic or cooperative production mechanisms and the short-range behavior of nucleons in nuclear matter. The observation of forward particles in coincidence should help establish the reaction mechanisms involved. Data taking for the initial phase of studies, primarily of $2.1 \mathrm{GeV}$ proton-carbon interactions was completed in 1982 and comprise the Ph.D. thesis of Robert N. Treuhaft. ${ }^{1}$

599H This experiment is to look into the possible differences of $\boldsymbol{r}^{\circ}$ and $\pi^{+}$ inclusive yields primarily in the central region of rapidity but extending also into the projectile fragmentation domain. The chosen system is ${ }^{40} \mathrm{Ca}+$ ${ }^{40} \mathrm{Ca}$ at $1.05 \mathrm{GeV} / \mathrm{A}$, which, apart from Coulomb effects, should yield a $\pi / \pi^{+}$ratio of unity. A ratio different from unity has previously been reported for the ${ }^{40} \mathrm{Ar}+\mathrm{KCl}$ system at the same energy. For the experiment, a multiplicity detector system was added to TASS at zero degrees to provide a "streamer-chamber-type" tagging of central and peripheral interactions. For pion identification at low momenta it was found essential to add helium bags around the target and to add a Cerenkov detector to veto electrons and positrons. One data acquisition run has been completed. Another will be necessary.

$630 \mathrm{H}$ This cxperiment was to search for anomalous nuclear fragments in the target fragmentation region. The "anomalon" phenomenon has so far been seen only in the projectile fragmentation region but should also occur in the tarcet fragmentation region if target and projectile are interchanged. The difficulty in the target fragmentation region is that the only signature so far observed - shortened mean free path - is more or less unusable because of the rapid variations of mean free path with energy in this regime. In experiment $630 \mathrm{H}$, various targets were bumbarded with $2.1 \mathrm{GeV} / \mathrm{A}$ carbon ions and a search was made for unusual structure in the energy spectra of protons, deuterons, and photons. A thin-walled scatter chamber previously used by the Poskanzer-Gutbrod group was equipped with several $\Delta \mathrm{E}-\mathrm{E}$ 
charged particle telescopes provided by the University of Maryland. Two large photon detectors, a large Nal crystal, and a $\mathrm{Pb}$-glass array were provided and operated by the Johns Hopkins and UCLA groups (486H). The photon speetra up to $400 \mathrm{MeV}$ have been studied to look for sharp peaks that might occur from the decay of an excited anomalon at rest in the target frame; none was observed. The charged particle spectra are smooth and roughly consistent with those previously measured by Sandoval, et al. A final study of the particle-photon correlation data is being made te see if some peak might occir in the effective mass reconstruction that was not visible in the inclusive data. Upper limits are being obtained for the production of anomalons, subject to the constraint that they would have to decay in the relatively simple fashion that we could have observed.

$628 \mathrm{H}, 631 \mathrm{H}$

These two experiments have not been run yet. The first is to search for pionic condensation effects in the inclusive pion spectra from $\mathrm{La}+\mathrm{La}$ at $200 \mathrm{MeV} / \mathrm{A}$. The second, awaiting installation of a liquid hydrogen target for TASS, is to study $\Delta$ production in p-p and p-A collisions by detecting the decay proton and pion in coincidence.

$670 \mathrm{H}$ Following a series of workshops a collaboration has been formed, involving LBL, Johns Hopkins University, Louisiana State University, Northwestern University, UCLA, and the University of Clermont-Ferrand to make a major study of lepton and dilepton production in heavy ion collisions. These particles are believed to be the most reliable prebes of the hot conditions produced in the high density phase of the reaction. The problem in their study is the low yield. TASS is suitable, with the addition of Cerenkov detectors, for measurement of single $l_{b}$, ons, and 300 hours of beam time for such a study have been approved. For the more definitive dilepton measurement a very large acceptance device is required. Tentatively, use is planned of the Lampshade Magnet, which is a large acceptance toroidal field device constructed by CERN. Permission has been received from CERN to borrow this. A detailed plan is being developed for total instrumentation of this new facility. The first experiments using it are planned for 1986.

ISR Experiment

Two of us (LSS and HGP) are involved, with T.J.M. Symons and C.R. Gruhn, in a large collaboration to stuoy $\alpha-\alpha$ collisions at the CERN ISR. This experiment, one of the last to be carried out on the ISR before its final shutdown, has now been scheduled for August 1983. This will provide an opportunity to sample on a small scale the higher energy heavy ion collisions, which we hope will be in the future of LBL. 


\section{Streamer Chamber}

Streamer Chmber: J.P. Brannigan

$P$. Eitner

I.W. Muskovich

G. Odyniec

S. Reinsch

L.M. Tinay

R. Brockmann,

J.W. Harris,

M. Maier.

C. Mott,

R.E. Renfordt,

GSI, Darmstadt

H. Stroebele,

University of

Heidelberg

D. Bangert,

W. Rauch,

University of

Marburg

K.L. Wolf,

Texas A\&M

University

A. Dacal,

M. Ortiz,

University of

Mexico
This group is studying $4 \pi$ charged-particle exclusive data and production of particles such as $\Lambda^{\circ}$ s for which the visual technique is particularly suitable. The LBL streamer chamber is being improved, with a view to eventual ability to measure the most complex events. At the moment, data are being recorded on film, with image intensifiers as appropriate, and measured using a semi-automatic PEPR facility at Heidelberg, as well as with conventional scanning and measuring tables at LBL. Studies are being made of operation in the avalanche mode, of use of CCD cameras, and of other possible long-term improvements. A 400-element hodoscopic array is in process of assembly to add $\mathrm{dE} / \mathrm{dx}$ and end-point measurements for particles in the forward part of the chamber. This is expected to operate in 1983 and to make possible $p, d, t, H e$ and $\pi^{+}$identification even for high multiplicity events.

Recent work has included the following experiments: -

$400 \mathrm{H}$ This experiment used a proton beam to investigate the systematics of backward-going baryons. Complementing a corresponding TASS study, these streamer chamber data provide a look at other fentures of the events, relevant to the backward emission mechanism. The data are now being analyzed and discussed in terms of isobar excitation and absorption or decay.

401H This experiment measured $\mathrm{Ar}+\mathrm{KCl}$ reactions as a function of energy between 0.4 and $1.8 \mathrm{GeV} / \mathrm{A}$, in central and minimum bias confizurations. Several papers have been published on these data. In reference 2 the negative pion excitation function was presented. In reference 3 the data were used to extract a nuclear equation of state at zero temperature for densities up to 3.4 times normal ruclear density. In reference 4 data from completely reconstructed events are used to demonstrate the stopping power of nuclei. It was found that in $\mathrm{Ar}+\mathrm{Pb}$ at $800 \mathrm{MeV} / \mathrm{A}$ complete stopping of the projectile occurs at zero impact parameter, whereas in $\mathrm{Ar}+\mathrm{KCl}$ at 1.8 GeV/A stopping is not complete even at zero impact parameter because the nucleons in the nuclear "corona" do not make enough collisions to produce equilibrium. Such an effect does not occur when a small nucleus strikes a large nucleus, as in $\mathrm{Ar}+\mathrm{Pb}$, In reference $5, \Lambda$ production and polarization data were presented: the large transverse momenta observed for the $\Lambda$ s still have to be explained.

564H This experiment was an improved version of $401 \mathrm{H}$, with $\mathrm{Ca}+\mathrm{Ca}$ and $\mathrm{Ca}$ $+\mathrm{Pb}$. The $\mathrm{Ca}$ beam provides slightly higher energy for production, while the use of a metallic $\mathrm{Ca}$ target and thus identical projectile and target nuclei will help simplify the analysis.

$557 \mathrm{H}$ This experiment is to study reaction mechanisms at the lowest Bevalac energies in $\mathrm{Ar}+\mathrm{KCl}$ and $\mathrm{Ar}+\mathrm{BaI}_{2}$ reactions. Data have been taken from $30 \mathrm{MeV} / \mathrm{A}$ to $90 \mathrm{MeV} / \mathrm{A}$ and analyzed by $\mathrm{K}$. Wolf at Argonne National Laboratory. The measurements have been most useful in indicating directions for charge identification and optimum streamer chamber operation for experiments in this "intermediate energy" range. Aralysis is continuing.

$629 \mathrm{H}$ To study the interactions of heavier projectiles now available at the Bevalac, this experiment will study ${ }^{93} \mathrm{Nb}+{ }^{93} \mathrm{Nb}$ and ${ }^{139} \mathrm{La}+{ }^{139} \mathrm{La}$ reactions. The first objective will be to extend the equation of state study of reference 3 to new conditions. A test run in 1982 used a $\mathrm{Kr}$ beam, the first run with the new Bevatron vacuum liner, and showed that the streamer chamber can function with the increased multiplicity. However, there were problems with laading of the streame- chamber by the higher multiplicity events, and precautions will be taken for a data acquisition run to ensure uniformity of response from event to event. 
SPS experiment

This group is collaborating with the Poskanzer-Gutbrod group and with groups from the University of Marburg and the University of Warsaw to propose an experiment using the CERN SPS. $R$. Stock is spokesman of the collaboration. This experiment, using ${ }^{16} \mathrm{O}$ ions at higher energies than available at the Bevalac (up to $200 \mathrm{GeV} / \mathrm{A}$ would be possible at CERN) would extend Bevalac data into the new regime to be covered by the Tevalac and other possible new facilities. The experiment has been approved for measurements up to $13 \mathrm{GeV} / \mathrm{A}$ LBL and GSI will construct an ${ }^{16} \mathrm{O}$ injector, an MPI Munich streamer chamber will be installed at CERN, and the LBL/GSI Plastic Ball will be moved to CERN for the experiment, which will be carried out in late 1985 or early 1986.

\section{Footnotes and References}

1. R.N. Treuhaft, A (p,2p) Study of High Momentum Components at $2.1 \mathrm{GeV}$, Ph.D. thesis, University of California, LBL-14677 (1982)

2. A. Sandoval, et al., Phys. Rev. Lett. 45 (1980) 874

3. R. Stock, et al., Phys. Rev. Lett. 49 (1982) 1236

4. H. Stroebele, et al., Phys. Rev. 27C (1983) 1349

5. J.W. Harris, et al., Phys. Rev. Lett. 47 (1981) 229 


\section{Light Particle Emission in High Energy Nuclear Collisions}

Group Leaders

O. Chamberlain

H. Steiner

O. Hashimoto

L. Anderson

H. Hamagaki,

K. Omata,

I. Tanihata,

N. Yoshikawa,

INS, Univ. of

Tokyo, Japan

S. Nagamiyn,

Y. Miake,

University of

Tokyo, Japan

K. Kimura

Kyushu Univ., Japan

X. Bai

Institute of

Atomic Energy,

Beijing, China

E. Moeller

Freie Universität,

Berlin, Germany
The main emphasis of our research program is the study of the reaction mechanisms involved in high-energy nuclear collisions. This experimental program includes both inclusive cross-section measurements and particle correlation studies. Most of the measurements involve the detection and identification of light particles ( $\pi^{ \pm}$, $\left.\mathrm{K}^{+}, \mathrm{p}, \mathrm{d}, \alpha \ldots\right)$ over a wide kinematic domain. We are particularly interested in studying processes that are kinematicallly inaccessible in the collisions between two free nucleons. We are also searching for any cooperative phenomena that might result when two massive nuclei interact at high energy, especially in small-impact parameter collisions. Most of these experiments are performed under the INS (University of Tokyo)-LBL collaboration.

Further data analysis of Experiment $299 \mathrm{H}$ has been carried out. A hint of a hydrodynamical bounce-off effect is observed in the two-proton correlation data, although it is not conclusive. A kinematical analysis of inclusive data suggests that there is a third source that can be classified as neither a participant nor a spectator.

As an extension of Experiment $299 \mathrm{H}$ above, pion production with 183 $\mathrm{MeV} /$ nucleon Ne beams has been measured. The mechanism of high-energy pion production at subthreshold beam energies has been studied, and a hint of cooperative accumulation of energy is suggested. Although the immediate goal of this experiment was to search for a possible bump due to pion condensation, no such evidence was observed.

In Experiment $471 \mathrm{H}$, kaon production has been measured with $2.1 \mathrm{GeV} /$ nucleon nuclear beams on various nuclear targets. The inclusive cross section with the $\mathrm{Ne}$ beam shows a much stronger target-mass dependence than that with the $d$ or b beams; this result suggests that collective effects might be important in kaon production. The charged-particle multiplicity associated with kaon production has been measured as well as the multiplicities associated with producing pions and protons. The data indicate that the associated multiplicity is slightly larger when a kaon is detected than in the case of either pions or protons.

The above data combined with the data of $299 \mathrm{H}$ for protons and pions have been anlyzed in terms of mean free paths of product particles. This supports the idea that particles with longer mean free paths probe more sensitively the early, hot, compressed phase of nuclear matter.

The data-taking and analysis of Experiment $472 \mathrm{H}$ have been completed. In this experiment the goal was to measure various two-particle correlation, e.g., p-p, p-d,... Measurements have been made using protons of 400 and $800 \mathrm{MeV}$ and $\mathrm{Ne}$ of 400 and $800 \mathrm{MeV} / \mathrm{A}$. New insight into the production mechanism responsible for backscattered protons in $800 \mathrm{MeV}$ p + A collisions has been obtained by studying forwardbackward correlations. The data suggest that the scattering of incident protons on a two-nucleon cluster is the dominant production mechanism for backward protons. Also, the $\mathrm{p}$-n correlation inside the nucleus has been studied.

In Experiment $512 \mathrm{H}$ large-transverse-momentum particles near kinematical limit and the correlations between two particles emitted at $\theta=90^{\circ}\left(\Delta \theta=180^{\circ}\right)$ have been measured. The HISS magnetic spectrometer was used in conjunction with time-offlight and $\mathrm{dE} / \mathrm{dx}$ measurements. The detection system involves large arrays of scintillation counters, two large drift chambers, and several multiwire proportional chambers. The major data-taking was completed, and the analysis of data is in progress.

In addition, the publication of the data from Experiment E205H has been completed, in which light-particle spectra were measured at forward angles, and such data 
were analyzed in terms of various theories.

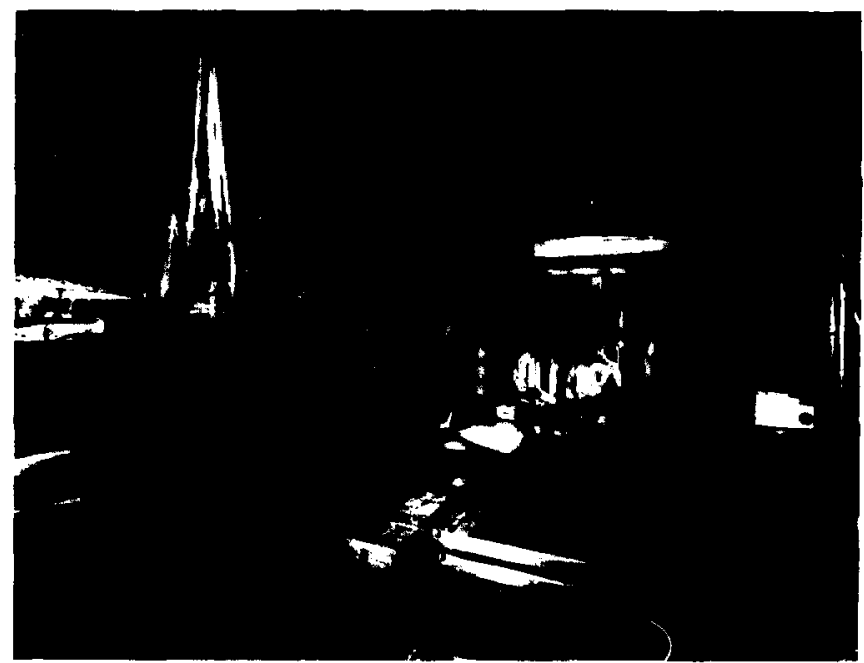

Fig. 1. Some of the group members in the HISS experiniental area.

CBB 826-5362 


\section{Heavy Ion Studies/Pion Studies}

Group Leaders

K. $M$. Crowe

J.O. Rasmussen

\section{J.A. Bistirlich \\ R. Bossingham* \\ H.R. Bowman \\ K.A. Frankel \\ T. Greent \\ J. Kurck* \\ D.L. Murphy* \\ E. YoO}

\section{o. Badowy, Cairo Univ., Egypt}

H.M.A. Rodi, Kuwait Univ., Kuwait

J.P. Sullivan,
Texas A\&M
University,
College Station,
Texas
J.S. Xu,
Fudan Univ.,
Shanghai
W.A. Zajc,
Pennsylvania
State Univ.,
Philadelphia, PA

"Graduate
students
tSummer
student
łUndergraduate
student

The Berkeley research activities of our group center mainly on the study of charged pions or correlated pairs of particles produced in high-energy heavy-ion collisions at the Bevalac. The technical approach involves a large ( $\sim 1.5$ meter long) magnetic spectrometer (JANUS) and combinations of fast scintillators and wire chambers interfaced with computer data systems. One major part of the work is the determination of the cross sections (with an exploration of the angular dependence) for pion production in heavy-ion reactions. The existence of both positively and negatively charged pions facilitates determinations of simple Coulomb effects and therefore the charge density evolution in heavy-ion collisions by observation of the $\pi^{+} / \pi^{-}$ratio as a function of pion energy, bombarding energy, and targetwprojectile charges. In fact, we found that pions produced with low energy in the projectile frame have large $\pi^{-} / \pi^{+}$ratios due to the Coulomb fields of the projectile fragments. Systematic observations of the sharp anomaly were made to compare with various models for production. The effects due to esoteric mechanisms, i.e., condensates, etc., are expected to modify the conventional modes, and these phenomena are being explored.

We are also studying the production cross section for low-energy $\pi^{-}$and $\pi^{+}$at $90^{\circ}$ in the center of mass, where an enhancement in the $\pi^{+}$production has been observed. We want to ascertain that this $90^{\circ}$ peak does disappear at low energy and to attempt to measure the threshold for its appearance. In addition, we are studying production of charged pions below the single nucleon-nucleon threshold to probe the importance of Fermi momentum in the nucleus. By measuring the center-of-mass angular distribution, we want to distinguish between thermal and single particle production modes. We have undertaken thick target studies in order to evaluate the practicality of heavy-ion production of useful $\pi$ beams.

The second major part of our work is an experiment to look at the HanburyBrown-Twiss effect for like-charged pions made by heavy-ion collisions in events where many pions are produced. We look at the production of a pair of closely related pions,++ or,-- at the lab angle $45^{\circ}$. In the past ten years, such exotic phenomena as pion condensation, pionic lasing and quark matter have been predicted as possible for nuelear matter in a heavy-ion collision. Gyulassy, Koonin and others have pointed out that one possible way to see the effect of pion instabilities in heavyion collisions is to look for the correlation of like pions in the situation when the relative momentum is extrapolated to zero.

More quantitatively, one studies the correlation function $\mathrm{C}_{2}\left(\mathrm{k}_{1}, \mathrm{k}_{2}\right)$ for two pions. For the case of two pions, $C_{2}\left(k_{1}, k_{2}\right)=0$ for coherent sources. Highly coherent pions will have a different distribution in relative momenta than that expected for chaotically produced pions, and the range of coherence depends on the size of the interaction region. This technique of pion interferometry has been employed to measure the size and lifetime of the pion source formed in the collision of $1.8 \mathrm{GeV} / \mathrm{A}^{40} \mathrm{Ar}+\mathrm{KCl}$. The values obtained provide useful constraints on models detailing the pion production mechanism. The Bose-Einstein enhancement for low relative momentum was observed.

In addition, we have conducted measurements of neutron-proton pairs produced in the $\mathrm{Ne}+\mathrm{U}$ reaction. Such measurements give information on excited (virtual) state deuteron production and on spin correlations associated with pion condensation. Preliminary results show an enhancement near zero n-p relative momentum. Future work will improve on resolution and statistics for the virtual deuterons and for wideangle n-p correlations measuring quasi-free scattering and collective-flow processes. Cluster production provides the main information on entropy production in relativistic heavy-ion collisions. 
Future work that our group intends to pursue will utilize the experimental techniques and apparatus that we have built. The availability of Bevalac beams of the heavier elements ( $\mathrm{Au}, \mathrm{Pb}$, or $\mathrm{U}$ ) and our upgraded experimental apparatus (addition of a kicker magnet) will call for new pion spectroscopic measurements to search for evidence of highly compressed nuclear matter effects, such as pion condensation, quark matter, etc. The $\pi^{+} / \pi^{-}$ratios will again be exploited for heavier beams to measure the dynamic charge evolution in relativistic heavy-ion collisions.

Now that $\mathrm{Xe}, \mathrm{Pb}, \mathrm{U}$, and other heavy beams at the Bevalac can be accelerated, there are new opportunities in atomic physics. Our earliest Bevalac work involved measuring $\mathrm{K} \times$-ray production cross sections. With Stanfurd collaborators we have measured extensively the $x$-ray spectra from $190 \mathrm{MeV} /$ amu Xe jons. We observed not only target and projectile $x$ rays, but also the radiative electron capture peak.

Various members of the group are involved in collaborative work centered at other laboratories: pion or muon experiments at LAMPF; theoretical studies on muon fission with collaborators in China; theoretical nuclear studies by the Classical Limit S-Matrix Method with collaborators at the Universities of Tennessee, Brazil, and Kuwait; and hybrid plastic and emulsion studies of relativistic heavy ions with collaborators in Nagoya and Cairo. 


\section{Nucleus-Nucleus Collisions}

Group Leader

P.B. Price

S.P. Allen

S. Barwick*

M. Budiansky

T. Coan*

J. Drach

K. A. Frankel

J. Garnett*

K. Kinoshita

T. Liss"

J. Musser*

S. Perlmutter*

M. Salamon*

M. Solarz

J. Stevenson

G. Tarié

M. Tincknell*

\section{B. Grabez, *}

Institute of

Physics,

Belgrad, Yugoslavia

\section{Garwin, *}

University of

Cambridge,

England

Shi-lun Guo,

Institute of

Atomic Energy,

Beijing,

People's Republic

of China

* Graduate

students
All this group's offices and equipment are located in Birge Hall on the UCB campus, which makes it easy to attract new students but restricts to some extent interaction with Nuclear Science Division staff. Current research falls into the following five principal areas.

\section{Relativistic heavy ion research (DOE support)}

a.) Production of heavy ion fragments, including exotic neutron-rich nuclides near the dripline

b.) Production of high-energy gamma rays

c.) Search for Lee-Wick matter

d.) High-order effects in electrodynamics of slowing heavy ions

e.) Response of various detectors to heavy ions

f.) Hadron calorimetry for nucleus-nucleus collisions

g.) Detailed properties of secondary nuclei with anomalously short interaction lengths ("anomalons")

h.) Search for quark-nucleon complexes.

2. Search for highly ionizing particles in $\mathrm{e}^{+} \mathrm{e}^{-}$annihilation (PEP-2 experiment, supported by NSF) and in $\bar{p}$ p annihilation (NSF support)

3. Cosmic-ray astrophysics (NASA support)

a.) Final engineering design and tests of prototype instruments for a $50 \mathrm{~m}^{2}$ detector for a two-year space exposure beginning 1986

b.) Study of highly ionizing particles with anomalously long interaction lengths at mountain altitudes

c.) Design of an experiment to detect anti-iron nuclei in cosmic rays at a level $3 \times 10^{-7}$ of iron

4. New detectors (NASA support)

CR-39 with charge resolution $\sigma_{z} \simeq 0.05 \mathrm{e}$; other plastics with $\sigma_{z}<0.2 \mathrm{e}$ in the vicinity of uranium

5. Search for grand-unification magnetic monopoles (NSF and Calspace support)

a.) Calibration of response of ionization detectors with very low-velocity protons

b.) Sea-level study with huge, thick plastic scintillators

c.) Searches with novel techniques

Doctors Ahlen, Stevenson, and Tarle play a major role in conceiving new experiments and in training students. There is much overlap among the various projects. Detectors devised to solve one problem frequently make possible the solution of all or part of another problem. A result obtained with plastic detectors may stimulate the 
construction of an electronic system to do a second-generation experiment. Expertise gained in resolving isotopes in a balloon-borne cosmic-ray experiment may be used at PEP or the next-generation neutron-rich nuclide experiment. In all these projects, both post-graduate and undergraduate students play an active part.

Among the experiments planned for the next two years at the Bevalac are a high-energy gamma-ray experiment with 100 times higher collecting power; detection of many new nuclides delineating the neutron dripline; hadron calorimetry with large solid angle; search for Lee-Wick matter in U-U collisions; measurement of higher order electrodynamics in $\mathrm{dE} / \mathrm{dx}$ and Cerenkov radiation by ions up to $\mathrm{U}$; detailed studies of anomalons with detectors capable of resolving nonintegral charge; and determination of the response of plastic track detectors to relativistic ions up to $U$ (the latter being of crucial importance for the proof that cosmic rays come from freshly synthesized supernova debris).

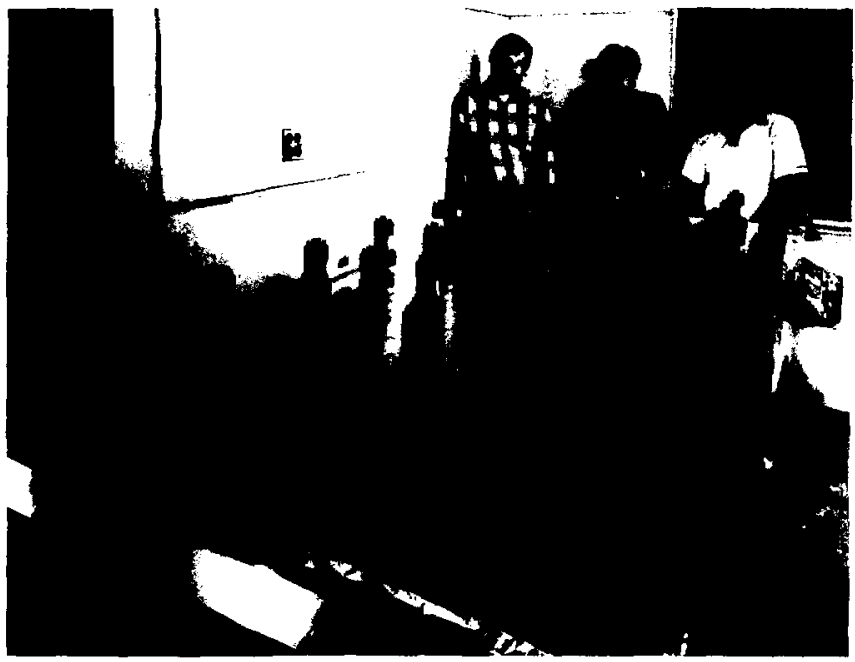

Fig. 1. Shown here is the largest slab of plastic scintillator eyer cast. It will be used to search for super-massive magnetic monopoles moving wih velocities between $5 \times 10^{-1} \mathrm{c}$ and $5 \times 10^{-3} \mathrm{c}$. Such monopoles have been predicted to exist by recently-developed Grand Unification Theories of particle physics.

CBB 820-9777 


\section{Nuclear Theory}

Group Lesders

N.K. Glendenning

M. Gyulassy

W.D. Myers

J. Randrup

W.J. Swiatecki

J. Boguta

P. Danielewicz

G. Fái

M. Redlich

P. Hecking,

Regensburg

A. Klar,

Iowa State

K.H. Müller, THD, Darmstadt

M. Weiss,

Lawrence

Livermore

National

Laboratory
The ruclear theory program encompasses subjects ranging from the general structure of static nuclei (including neutron stars), over nuclear dynamical properties as probed in nuclear collisions at various energies, to the manifestation of the basic quark degrees of freedom.

General nuclear structure is being studied by macroscopic as well as microscopic tools. In particular, a non-linear relativistic mean-field theory has been found to possess remarkable predictive power for a variety of nuclear properties. Because of its simplicity and consistency, this model is a versatile tool for exploring more exotic nuclear properties, e.g., the structure of hypernuclei. Separate studies have focused on generalizing relativistic mean field theory to the study of time-dependent problems. While practically equivalent to time-dependent Hartree-Fock calculations at low energy, the relativistic mean field model provides a natural inclusion of the mesonic degrees of freedom which become significant at higher energies. A relativistic field theory has also been used to study dense neutron matter in the normal and pion condensed phases.

Recently, a program to apply nuclear theory to astrophysics has been initiated. This program includes a study of the pion-condensed phase in stable charge neutral neutron star matter and of the hyperon composition of such matter, star masses, cooling rates, and temperature dependence of the equation of state. All these properties are calculated in the framework of a relativistic theory that is constrained by the bulk properties of nuclear matter, most important of which is the charge symmetry energy.

The large-scale dynamics of nuclear shape changes, such as are encountered in nuclear fission and nucleus-nucleus collisions, continues to be an important area of research. The distinction between the dynamics appropriate for systems with and without symmetries is being clarified. The equations of motion in the latter case (the Chaotic Regime) are being applied to a variety of situations, including the interpretation of cross sections for deep-inelastic, fusion, and fast-fission reactions. Close contact is maintained with experimental attempts to synthesize super-heavy elements, in which the dynamics of fusion may play a decisive role.

In the area of damped nuclear reactions, the angular-momentum-carrying degrees of freedom have been given special attention. The effect of statistical nucleon exchange between the two reacting nuclides on the dynamical evolution of the fragment spins has been studied in detail, leading to a model with considerable predictive power. In particular, it has been shown that the way in which the two-fragment spins correlate is sensitive to the specific reaction mechanism assumed. This suggests that attempts be made to measure the spin-spin correlations between the reaction products, for example, in an experiment in which both products undergo fission later on; calculations show that the angular correlations between such fission fragments are well suited to probe the basic nature of the reaction mechanism.

As the energy is raised in a nucleus-nucleus collision, the binary idealization becomes increasingly inadequate and multi-fragment final states become predominant. The fragment production mechanisms have been studied by various approaches. Some have focused on the production of a specific fragment type, such as nucleon jets appearing as a result of the kinematical boost of transferred nucleons due to the nuclear motion, pions resulting from nuclear collisions at a few bundred $\mathrm{MeV} / \mathrm{n}$, and the production of deuterons within the framework of the intranuclear cascade description. Other studies have attempted a comprehensive description of fragment production where all final states are treated on an equal footing. In relation to current attempts to probe the entropy production in a nuclear collision, it has been found that the eventual de-excitation of unstable ejectiles plays an important role in modifying 
the observed distributions relative to those immediately after the collision process.

The arca of high-encrgy nuclcar collisions is a major part of the theory program and several directions have becn pursued simultaneously, e.g., the development of analytical models for elucidating the constraints of geometry and phase space on the observables, the employment of nuclear hydrodynamics for studying nuclear flow phenomena and their possible detection, and intranuclear cascade studies of the equilibration process. Special topics which have been addressed are $\pi \pi$ and pp correlations, $\pi$ and $\mathrm{K}$ production, multiplicity distributions, composite fragment formatiuu, Coulomb final state interactions, and global event analysis.

A main thrust of this work has been to determine whether we can gain information on the nuclear matter equation of state at high densities. This program necessitates a detailed understanding of the reaction mechanisms at these energies. Very close contact with the Bevalac experimental program is maintained.

Further work has been done on various aspects of the pionic degree of freedom in nuclear matter: the propagation of the pion through matter has been studied within an optical model, the pion condensation of finite temperatures has been given special attention, and a new experimental signature for pion condensation has been proposed and tested. While no positive signatures have been observed, the systems studied are small ( $\mathrm{Ne}+\mathrm{Ne}$ ), and experiments with heavy nuclei are needed.

We have also kept a close watch on the "anomalon" data and have suggested novel explanations of the effect. In particular, topological excitations of nuclei have been considered. This is another area in which application of nonlinear field theoretic models has had interesting consequences.

An important modern frontier is associated with nuclear collisions at ultrarelativistic energies where the fields of nuclear, particle and astrophysics have common ground. For example, the phase transition from hadron to quark matter, which is expected to occur at sufficiently high energy density, is being explored: The onset of the phase transition has been studied in different models and the use of the strange baryon fraction as a signature for a quark phase has been considered. The question of whether high-energy nuclear collisions can generate sufficiently high energy densities to create a quark-gluon plasma is being investigated. Problems with nuclear stopping power and the onset of nuclear transparency are being studied. Cosmic-ray data at $\mathrm{TeV}$ per nucleon energies are being analyzed in a search for signatures that might indicate the creation of a quark-gluon plasma. 


\section{Nuclear Electronics}

\author{
Group Leader \\ F. Goulding \\ D. Landis \\ J. Meng \\ R. Pehl \\ H. Spieler \\ J. Walton
}

The Nuclear Electronics Group develops radiation detectors, signal processing electronics and data acquisition systems for experiments in nuclear science. Considerable emphasis is placed on providing a broad base of expertise in experimental techniques, detector technology and both analog and digital electronics.

Traditionally, the bulk of the detector work centers on semiconductor detectors, both silicon and germanium, and the associated low-noise electronics. At the base of the germanium detector work is the high-purity germanium program. This research has led to unique contributions in development of high-purity germanium detectors. It has also deepened our understanding of purification processes and of the dominant role of defects in germanium.

Another focus of the germanium detector program is on radiation damage and annealing of damage in high-purity detectors. This work is of particular importance in the application of telescopes of thick germanium detectors used for long-range particle spectroscopy. The development of these telescopes and of the logistics for using them (including annealing damage) has been a major endeavor of the group in recent years.

The silicon detector program covers the complete range of detectors used in $x$ ray, low-energy gamma-ray and charged-particle detection. Recent work has focused on position-sensitive detectors for use in large-area or $4 \pi$-detector arrays. The main thrust of this development has been toward microstrip detectors for particle physics experiments. An array of position sensitive detectors using a combination of strip and charge division readout is in use at the SASSY spectrometer at the SuperHILAC to identify new elements and exotic nuclei.

Another aspect of detector development involves microchannel plates, used in time-of-flight detectors for the identification of reaction products. This work corcentrates mainly on simplifying designs without sacrificing performance.

The full potential of a detector cannot be realized without carefully matching the signal processing electronics to the characteristics of the detector. This is especially true in high-resolution spectroscopy with semiconductor detectors at high event rates, where either a time variant or a time invariant pulse shaper may be the optimum choice, depending on the specific circumstances. A set of 21 specially developed gated integrator pulse processors with integrated pile-up rejection circuitry is being built for the high-resolution Ge/BGO Ball under construction by the Diamond/Stephens group. A time invariant pseudo-triangular pulse shaper has been designed for $\mathrm{x}$-ray diagnostics at the Princeton TFTR. This novel design combines simplicity with resolution and throughput characteristics superior to pseado-gaussian shapers in widespread use. A new design of the pile-up rejector channel has increased the throughput of these systems by about 40 percent. Concurrent improvements in preamplifiers have been achieved by using transistor reset circuitry.

An appreciable improvement in noise at nanosecond peaking times results from using low-noise bipolar transistors instead of field-effect transistors (FETs). This is applicable to preamplifiers in fast timing measurements where a four to five-fold reduction in roise over FETs is obtained for subnanosecond rise times. Use of active input terminations which present a $50 \mathrm{ohm}$ input impedance without the associated thermal noise offer great flexibility in practical use, since the preamplifiers can be mounted remotely from the detector, e.g., outside the scattering chamber. Preamplifiers of this type have provided a time resolution of $30 \mathrm{ps}$ FWHM in a silicon detector telescope with ${ }^{28} \mathrm{Si}$ ions of $225 \mathrm{MeV}$. A practical design for silicon detectors has been implemented and preamplifiers are being produced for use at the SuperHILAC and other laboratories throughout the United States. 
Considerable effort has also been directed at improving data acquisition systems and facilities for off-line data analysis. A first step towards improved data acquisition facilities for multi-parameter experiments. at the 88-Inch Cyclotron and the SuperHI$\mathrm{LAC}$ is the development of a CAMAC compatible ADC multiplexer for highresolution data channels. Fast-readout systems for CAMAC will follow.

A considerable increase in computing power for off-line data analysis will be provided by MIDAS (Modular Interactive Data Analysis System). MIDAS reduces computing time by using a large number of parallel processors, which individually can be rather slow and inexpensive.

In the course of the past year the MIDAS prototype, using a complement of four arithmetic processors, a pipeline input processor and an output formatter, was subjected to an intensive series of evaluative tests. Results substantiate earlier claims of a four-fold increase in processing speed over that of a single processor. MIDAS is now in the final phase of being expanded to an eight-processor module from the presently operating four processors. Work is in progress on the interactive display parts, scheduled for completion in 1983.

The group also provides much of the specialized maintanence and design efforts in the Nuclear Science Division. Considerable time is spent in consultation with experimental groups planning new experiments and instruments. Since members of the group participate in projects for other divisions of LBL and laboratories throughout the world, they bring a broad perspective to bear on the problems which arise in nuclear science experiments. 


\section{Relativistic Heavy Ion Collisions}

\section{Group Leader} V. Perez-Mendez

T. Mulera

P. Wiedenbeck

F. Zarbakhsh

G. Igo,

S. Abachi,

J. Carroll,

K. Ganezer,

A. Shor,

UCLA

E. Barasch,

A. Sagle,

U.C. Davis
In addition to the staff listed here, based on a long-standing collaboration between V. Perez-Mendez and G. Igo, shorter lived collaborations have also been formed involving members of the HISS and TASS groups at LBL and the group led bv Ln Madansky at Johns Hopkins University.

The group carries on a diverse program of research in relativistic heavy ion physics using the Bevalac. The unifying theme of this program is the search for evidence for the possibie formation of new states of matter in relativistic heavy ion collisions. This program is currently proceeding on threc fronts.

"Anomalous" production of particles below threshold (for nucleon-nucleon colli$s$ ons at equal energy/nucleon) provides evidence for collective effects that may lead the pioduction of new states of matter. During the 1981-1982 period we published our discovery of a surprisingly high rate of $\mathrm{K}^{-}$production in $2.1 \mathrm{GeV} / \mathrm{A}{ }^{28} \mathrm{Si}+{ }^{28} \mathrm{Si}$ collisions. Preparations for further measurements, including the design of a new beam line/spectrometer were carried out.

Preparations for the continuation of our measurement of proton-proton correlations at small relative momenta were made. These preparations included two peliminary runs using the HISS spectrometer. Use of this facility should allow us to improve greatly on the quality of our previous measurement.

A short run was taken to continue studies of lepton and photon production in heavy ion collisions. These studies have evolved into a large effort (in collaboration with the TASS and JHU groups) first to measure the rate of direct eiectron product.on at the Bevalac and then, after construction of a new large acceptance instrument, $t$ e Dilepton Spectrometer, to study the production of $\mathrm{e}^{+} \mathrm{e}^{-}$pairs. Changes in the eifective mass spectra of such pairs as a function of bombarding energy may provide the clearest signature of the postulated phase transition to the quark-gluon plasma.

A large amount of effort was also devoted to the development of new types of instrumentation for use in the above work. 


\section{High Energy Heavy Ion Collisions}

This group is engaged in a wide variety of experiments using beams accelerated by the Bevalac. In recent years, interests have ranged from nuclear struclure physics to reaction mechanism studies at very high energies. In particular, we have focused our attention on the following problems during the last year.

(1) At the low-energy beam line, we have an active collaboration with a group from Michigan State University. The goal of this program is to study the evolution of the reaction mechanism as a function of bombarding energy between 50 and $150 \mathrm{MeV} /$ nucleon. This is an energy range that remains largely unexplored experimentally and in which a variety of theoretical approaches, including hydrodynamic and thermodynanic modcls, have been applied. Of particular interest is the possibility that abrupt transitions in particle yields might be observed as a function of energy, indicating the presence of a phase transition. Successfui experiments have now been completed using ${ }^{20} \mathrm{Ne}$ and ${ }^{40} \mathrm{Ar}$ beams. So far most of the experimental data have consisted of inclusive particle yields; however, a much more sophisticated multi-element "hit" detector is now being developed at Michigan State University and will be used during the coming year. We also plan to extend the measurements to heavier systems such as Au + Au where thermodynamic concepts should be more applicable.

(2) At the zero degree spectrometer, we are continuing our studies of nuclei far from stability produced in heavy ion fragmentation reactions. We have demonstrated the fcasibility of measuring $\beta$-decay half-lives of very neutron-rich light nuclei by a delayed coincidence technique in which we implant the activities directly into a six-element $\mathrm{Si}(\mathrm{Li})$ detector stack. In our first run with an ${ }^{40} \mathrm{Ar}$ beam we measured two new half-lives and have now begur production runs using an ${ }^{56} \mathrm{Fe}$ beam.

(3) In collaboration with members of the HISS Group, we are starting an experimental program at the HISS facility that will address sevcral problems of current interest. These include the production of very neutron-rich light nuclei at high energies; excitation of giant resonances using the Coulomb field of heavy target nuclei; and, we hope, a searci using an electronic ! haique for the anomalous mean free path effect observed by Friedlander, et ai., in nuclear emulsion.

(4) Away from LBL, we maintain a close interest in the experiments being carried out by the CERN-Heidelberg-Lund collaboration at the CERN Intersecting Storage Rings. This accelerator provides the highest energies available today for nucleus-nucleus collisions and offers an exciting preview of the physics that will be accessible using new re ativistic heavy ion accelerators. 


\section{Detector Research and Development}

\author{
Group Leader \\ C.R. Gruhn \\ W. Pang \\ M. Roach \\ R.A. Loveman, \\ Univ. of \\ Washington \\ *Graduate \\ Student
}

The function of this group is to explore new concepts in detectors and to develop systems that optimize the accuracy of measurement in the specific uses for which they are designed. Some of the work has been done in collaboration with Japanese scientists under the U.S. - Japan Cooperative Science Program. The four main systems under development this year have been i) liquid xenon $\gamma$-ray detectors, ii) liquid argon heavy ion detectors, iii) a Bragg-Curve Spectrometer (BCS) and iv) high density time projection chambers (HDPCs).

\section{Liquid Xenon $\boldsymbol{\gamma}$-Ray Detectors}

Our goal for this detector design is to achieve $25 \mathrm{keV}$ FWHM resolution for 1 $\mathrm{MeV} \gamma$ rays. The best energy resolution achieved both here and in Japan is comparable to that of : $\mathrm{a}(\mathrm{TI})$, but with volumes of less than 0.1 liter. $88 \%$ of the total expected charge $s$ collected in these measurements, although this value is strongly dependent upon our measurement of the energy loss per ion pair in liquid xenon. We now know that the procedure used to measure this loss must be used with extreme caution.

\section{Liquid Argon Hez:y Ion Detectors}

The Bevalac upgrade provides LBL with the ability to accelerate uranium beams to $2 \mathrm{GeV} / \mathrm{n}$ and a Japanese machine under construction will provide similarly energetic heavy ion beams. Beams from these machines, along with planned LBL experiments at CERN and with the proposed Tevalac will result in even higher energy heavy ion collisions, whose event structure involves extremely high multiplicities of particles spanning a broad range in both atomic number and energy. Such experiments require detector systems having the capability of subtending large solid angles along with high pattern recognition. To meet this need, we are developing in collaboration with the Japanese a high-resolution liquid argon calorimeter, in which signals of the free carrier yield and luminescence from the ionization tracks will be viewed simultaneouly.

\section{Bragg-Curve Spectrometer}

The BSC is a charged-particle identifier which has the following advantages:

(1) A signal that measures $Z$ unambiguously;

(2) Combined range and Bragg peak signals which yield a charge resolution higher than that achieved with gas $\Delta \mathrm{E}$-semiconductor $\mathrm{E}$ telescopes;

(3) Large solid angle applications;

(4) Better heavy ion energy resolution than that of semiconductor detectors.

The BCS has been demonstrated to yield energy and charge resolutions of $0.7 \%$ and 1.2-1.4\% FWHM, respectively, for heavy ions lighter than cobalt, and the Bragg peak signal appears to be linear in charge to within $7 \%$ for $\mathrm{Z} \leq 26$. Questions yet to be studied are particle identification for $\mathrm{Z} \geq 40$, particle identification below the $\mathrm{Bragg}$ peak, the geometric mean of the Bragg curve as a particle identification signal, and mass measurements.

\section{High Density Time Projection Chambers}

We have designed and constructed two HDPCs which are to be used for tagging spectator neutrons in colliding light-ion beam experiments at the CERN ISR. These are designed for a high pattern recognition which provides some compensation for a 
non uniform response. Our goal in resolution in the center of the calorimeters is $30 \%$, which permits discrimination between one, two and three spectator neutrons. The calorimeters are currently installed at the split field magnet facility of the CERN ISR, where experiments ill begin shortly. 


\title{
Tevalac Project
}

\section{VENUS 82 Workshop - Prelude to the Tevalac}

\author{
LSS. Schroeder
}

A workshop was held at LBL on Scptember 15-17, 1982, to review the physics objectives and capabilities of a heavy-ion accelerator complex providing nuclear beams over the entire range of the periodic table for fixed target experiments. The complex, injected by the SuperHILAC, would consist of two superconducting rings, one located vertically above the other. The first ring (RI) would be a synchrotron capable of extracting beams for physics research and/or transferring beams to a second ring (R2), which would act as a storer/stretcher ring with the capability of extraction of a nearly DC beam. An initial lower limit of operation of the complex was set at $\sim 50 \mathrm{MeV} /$ nucleon, with an upper limit of $10 \mathrm{GeV} /$ nucleon for uranium beams. The broad aim of this meeting was the development of material for a proposal to be submitted by the end of calendar 1982 to the Department of Energy for initial R\&D funding.

The format for this workshop was different from that of the previous highenergy heavy-ion summer studies in that, while the morning sessions (September 15-17) were devoted to review talks, the afternoon sessions were broken up into parallel working groups on the following subjects:

Working Group

- Theoretical Physics

- Atomic Physics

- Physics at Lower Energies

- Radiochemical Physics

- Exotica and Rare Particle Studies

- Fragmentation Studies

- 4x and Central Collision Physics

- Collider Physics

\section{Conveners}

M. Gyulassy and W. Myers (LBL)

H. Gould (LBL)

B. Harvey (LBL)

W. Loveland (Oregon State)

The charge to the various working groups was to consider the outstanding physics potentials in each of their areas and the optimal parameters in projectile mass and energy for a fixed target facility to carry out such a program. Where appropriate, the types of detectors that would be required were also considered. The working groups met for several hours each afternoon to consider these questions.

On the morning of September 17, reviews from each of the working groups were heard. The major interest focused on questions associated with the formation and 
detection (signatures) of the quark-gluon plasma. In addition, it was evident that uranium beams at energies up to $10 \mathrm{GeV} /$ nucleon also provide an extremcly strong program of atomic, nuclear, astro, and particle physics rescarch, including:

- equation of state of hadronic matter

- new states (pion condensates, anomalons, highly excited nuclear matter $\mathrm{N}^{*}, \Delta$-nuclei)

- new spectroscopy with strangeness quantum number introduced into nucleus (hyper and multi-hyper nuclei)

- nuclear structure, nuclei far from stability, secondary beams of unusual nuclei

- study of liquid to gas transition in nuclei, nuclear hydrodynamics

- 1- and 2-electron heavy-ion atomic physics studies (testing QED)

- cosmic-ray propagation (need to measure fragmentation cross sections) and source abundances

- applications (satellite instrument calibrations and radiobiology)

The physics aspects of the workshop were summarized by $W$. Swiatecki, who indicated that the ultimate focus of such a program would be the question of hadronic deconfinement and the tich new structure of matter that would be possible. H. Grunder reviewed the machine parameters, stating that the experimental community indicated that such an accelerator complex needed to go to at least $10 \mathrm{GeV} /$ nucleon for the heaviest ions, as well as going down to somewhat lower energies (20 $\mathrm{MeV} /$ nucleon) than originally discussed. To undertake such a broad program, a high multiplicity of simultaneous experiments would be required.

For historical purposes, this workshop served as the prelude to the Tevalac R\&D proposal, ${ }^{1}$ which was submitted to the Department of Energy in December 1982.

\section{Reference}

1. The Tevalac - A National Facility for Relativistic Heavy-Ion Research to 10 GeV per Nucleon with Uranium - LBL PUB-5081 (December 1982). 
PART II: ACCELERATORS 


\title{
88-Inch Cyclotron Operations and Research
}

\section{Operations}

\author{
D.J. Clark, D. Elo, L. Glasgow, R. Lam, C.M. Lyneis, R.G. Stokstad
}

The 88-Inch Cyclotron, operated by the Nuclear Science Division, provides a large fraction of the beam time that is used by Division scientists. Variable energy high resolution beams from hydrogen through argon are produced and used for studies of nuclear structure and nuclear reaction mechanisms. The cyclotron is also the Laboratory's major source of medical isotopes and its only source of polarized proton and deuteron beams.

The cyclotron is now operating at an increased value of the energy constant, $K$, of 160 for ions heavier than $A$ $=4$. Ions as heavy as ${ }^{40} \mathrm{Ar}$ can be accelerated to the Coulomb barrier of $5 \mathrm{MeV} / \mathrm{u}$, while lighter heavy ions can reach $20-30 \mathrm{MeV} / \mathrm{u}$. The cyclotron thus operates in the important transition region between low and high energies: $10-30 \mathrm{MeV} / \mathrm{u}$.

As a national accelerator laboratory the 88-Inch Cyclotron is used extensively by outside groups from many institutions in the U.S. and abroad. Table I shows the number of users from LBL and elsewhere. Scheduling of experiments for the cyclotron is done in an open meeting on a weekly basis with a lead time of only eight days. Outside users who must make travel arrangements in advance are accommodated with advance scheduling. A users' organization has been formed and a program advisory committee instituted. The members of the users' executive committee are Karl Van Bibber (Stanford University), Chairman; Frank S. Dietrich (Lawrence Livermore National Laboratory); and Marie-Agnes Deleplanque (LBL). C. Konrad Gelbke of Michigan State University serves as Chairman of the Program Advisory Committee. Charles Goodman (Indiana University) and J $\phi r g e n$ Randrup (LBL) are the other two members.

The 88-Inch Cyclotron plays a significant educational role. At present 13 graduate students from the University of California at Berkeley are employing this facility in their research toward the Ph.D. degree. Three students received their doctorates from UCB in 1982 for research done at the cyclotron. Four graduate students from other universities are also involved in research at the cyclotron.

Table I. Number of users of the Cyclotron during the past year, FY82.

LBL Staff, University of California, Berkeley Campus

Graduate Students

$\begin{array}{cr}\text { U.C. Berkeley } & 14 \\ \text { Other Universities } & 5\end{array}$

Post-doctoral scientists

$\begin{array}{cc}\text { LBL } & 5 \\ \text { Other laboratories } & 3\end{array}$

Outside users and visitors

$\begin{array}{cr}\text { Industry } & \vdots 30 \\ \text { Universities, National Labs. } & 52\end{array}$

TOTAL 
The cyclotron now operates $141 / 2$ eight hour shifts per week with one shift for maintanence at the beginning of the week and one half shift for shutdown on the weekend. It was operated for 20 shifts per week until October 1981 when increased power rates and budget limitations made the reduction in running time necessary. The distribution of cyclotron time is shown in Table II. The list of beams available is given in Table III. The particle distribution of light and heavy ion beams during the past 14 years is shown in Fig. l.

Table Il. Cyclotron operating and maintenance time distribution for the 15 month period $7 / 1 / 81$ to $9 / 30 / 82$ covered by this Annual Report

Operating Time

\begin{tabular}{lr} 
Beam Development & 435 \\
Tuning & 656 \\
Optics & 356 \\
On target & 3942 \\
Waiting for experimenter & 338 \\
\multicolumn{1}{r}{ Subtotal } & \\
&
\end{tabular}

Subtotal

Maintenance Time

$\begin{array}{rr}\text { Weekly maintenance } & 390 \\ \text { Shutdown maintenance } & 591 \\ \text { Ion source preparation } & 487 \\ \text { Unscheduled maintenance } & 314 \\ \text { Other } & 126 \\ & \\ & \text { Subtotal } \\ \text { Total } & 1908 \\ & 7635 \\ \text { Shutdown } & 3045 \\ \text { Holiday } & 288 \\ \text { Total Time } & 10968\end{array}$

Improvement Programs

The staff is involved in programs to improve the ion sources, the machine, extraction, and beam lines. Improvements to ion sources include a new simpler internal PIG (Penning ion gauge) heavy ion source, an upgrade of the polarized ion source, and a proposal to construct an ECR (electron cyclotron resonance) source. Improvements of the cyclotion itself have centered on upgrading the rf system. Improved extraction efficiency is the goal of the magnetic channel project. The installation of a fast slammer valve in beam line 3 provides better protection of the cyclotron from accidents involving radioactive targets.

A new PIG heavy ion source was designed to increase the source lifetime and to simplify parts and maintenance. Design improvements include simplification of the base insulator and elimination of discharges in the connecting line between cathodes. A prototype was designed and fabricated. It is still being debugged but initial use in experimental runs indicates that it will fulfill its design goals. Modifications are now being made to further improve it and more testing will be done but we expect to build another unit soon so that it can go into steady operation.

A new ionizer was installed in the polarized ion source last year. It was tested and put into service early this year. It has provided approximately a factor of 3 increase in the polarized beams. An additional increase in beam intensity is expected when the pumping speed on the ionizer is improved.

Improvements in the cyclotron rf system include a new crowbar unit for the final amplifier plate supply series regulator. It has 4 additional channels and greater speed and reliability. Under construction is a new regulating system using fibre optic cables for signals between ground and $25 \mathrm{kV}$ points. An if filter on the final amplifier plate 
supply is being designed to reduce the number of high voltagc capacitors. This will simplify the systcm and make more spare capacitors available. An all solid-state if driver amplifier has been installed to replace two tube-type distributed amplifiers. The system will be operational after interlock modifications.

A magnetic channel will replace the third section of the electrostatic deficctor. It will provide the stronger deflection strength needed for the higher energy beams made available by the high $K$ operation and by the future ECR source. It consists of a passive iron system about 20 inches long, with no cuils. It reduces the magnetic field in the beam region anc adds a radially focussing gradient. The channel has been constructed and its field has been measured. The measurements indicate that some modifications are needed to improve the field lincarity and to adapt the mechanical support system to the deflector mounting plate.

A fast slammer valve has been installed on the beam line to cave 3 to protect the cyclotron from contamination which could result from a radioactive target accident. The associated electronic controls were also completed.

\section{The ECR Source}

The ECR source has been selected as the best type of advanced ion source for the 88-Inch Cyclotron. The choice was made in May 1982 after the two types of advanced ion source, ECR and EBIS (electron beam ion source), were evaluated. The ECR source was chosen because it will provide an energy increase of 2-4 for heavy ion beams up to krypton at adequate intensities for most experiments $\left(10^{10}-10^{11}\right.$ particles/second external beam) at $100 \%$ duty factor. EBIS, the other source candidate, produces higher charge states, but lower intensity and duty factor. An ECR design workshop was held in August 1982 with participants from Louvain-la-Neuve, Karlsruhe, Oak Ridge and LBL A proposal to build an ECR source was submitted to the Department of Energy in September 1982. Construction will require about 2 years.

The proposed ECR source is a compact 2-stage system using normal conducting coils, a permanent magnet hexapole, and $8 \mathrm{GHz}$ microwave power. The design includes a number of features that contribute to flexibility and a potential for future development. The second stage coils are subdivided so that the mirror ratio may be varied. The hexapole structure is open and allows radial pumping and access to the plasma for diagnostic elements. Particular attention has been given to the design of the extraction system to improve intensity. The beam transport and improvements in the axjal injection system are included in this project. 
Table III. 88-Inch Cyclotron Beam List

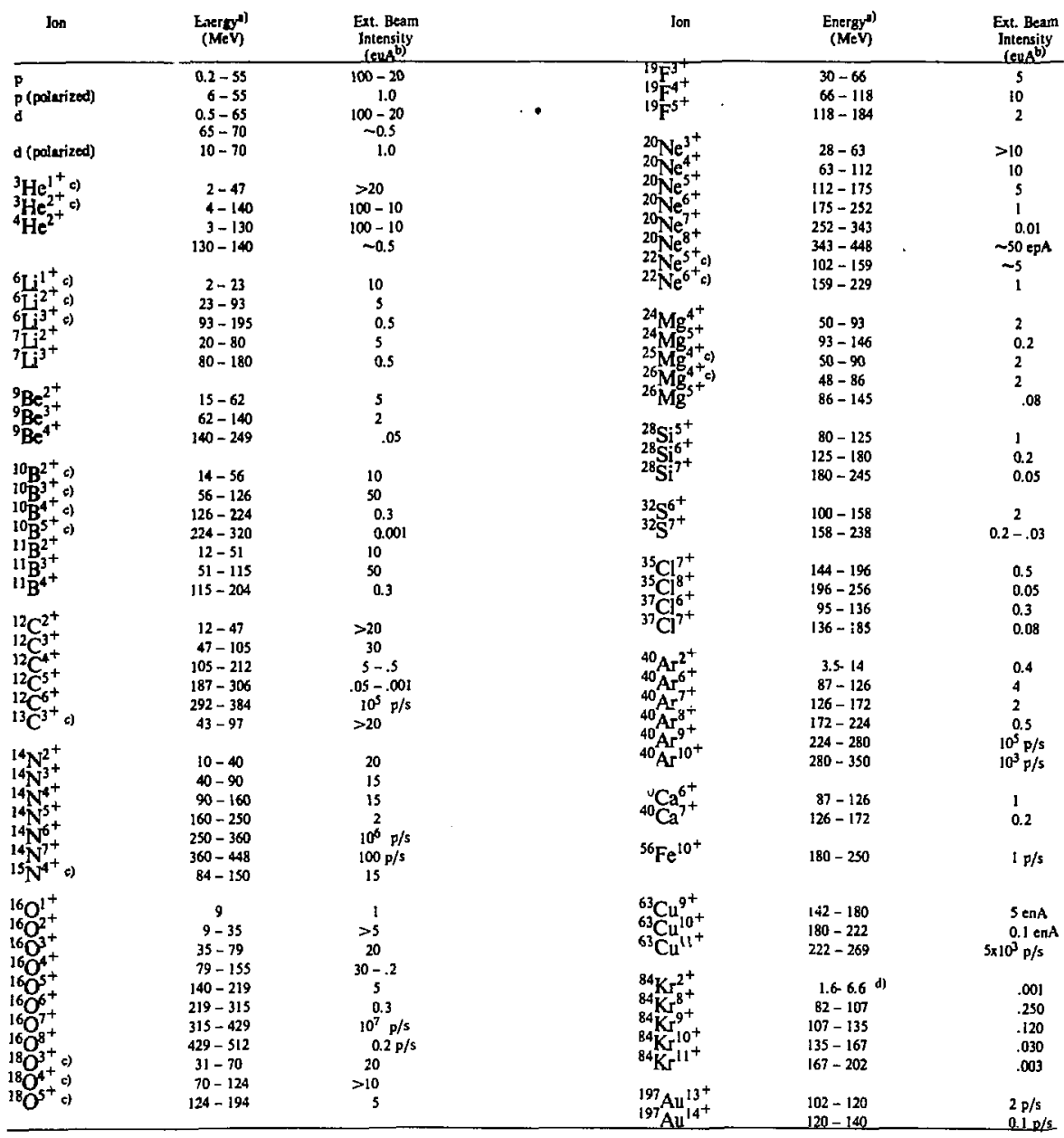

a) Heavy ion range shows nominal maximum energy for a panticular charge state down to energy which can be reached by the next lowest charge state. The maximum energy for several heavy-ion beams has been increased to $\mathrm{E}=160 Q^{2} / \mathrm{A}$ MeV. Besms can also be run at energies below $1 \mathrm{MeV} / \mathrm{nucleon}$.

b) Electrical microamperes exeept as noted, $\Delta E / E \sim 0,3 \%$ Intensity on target will be less, depending on mode of beam transport.

c) Isotopically-enriched source feed.

d)1 Sth harmonic. 


\section{Research}

\section{R.G. Stokstad}

The research programs at the 88-Inch Cyclotron comprise a broad and diversified enterprise that reflects in part the versatility of the accelerator. Basic research in nucleai physics is done with heavy ion and light ion beams. These probes are used to study nuclear structure and nuclear reaction mechanisms. Interspersed in this efi $i t$ is a series of application of nuclear techniques to other disciplines. The following overview categorizes the research in terms of heavy-ion reaction mecharisms, nuclear structure, exotic nuclei, heavy element research, light ion research and applications.

\section{Heavy Ior Reaction Mechanisms}

The study of nuclear reaction mechanisms continues to form one of the main thrusts of the overall research program. Previous developments of beams of beryllium through neon at energies up to $\sim 20 \mathrm{MeV} /$ nucleon have made possible a broad program of investigations into the nature of peripheral reactions, incomplete fusion, fission and evaporation.

The behavior of heavy-ion reactions changes rapidly as the bombarding energy is increased significantly above 8 $\mathrm{MeV} / \mathrm{n}$. Between this energy and about $100 \mathrm{MeV} / \mathrm{n}$ a transition occurs from the characteristics of low energy phenomena (transfer reactions, complete fusion) to those of relativistic heavy-ion reactons (fragmentation, participant-spectator mechanisms). The mechanisms of heavy-ion reactions in this regime are being studied in order to identify and understand the competing processes and their dependence on the bombarding energy. Two main thrusts of this work are in the areas of transfer and fragmentation reactions and of incomplete fusion. Transfer reactions are being studied by a new device, the "plastic box", a $4 \pi$ array of scintillators arranged in the form of a cube which provides the means to distinguish between transfer and fragmentation. The plastic box is being used in a number of experiments where the separation of two-body and three-body reactions is crucial. Incomplete fusion is being investigated with the time-of-flight facility. Systematic measurements for a series of targets and ${ }^{16} \mathrm{O}$ energies reveal an onset of incomplete linear momentum transfer at about $5 \mathrm{MeV} / \mathrm{n}$ above the Coulomb barrier.

The transition between evaporation and fission is also being explored. As the evaporated particle increases in mass beyond helium, the distinction between the two mechanisms blurs and the question of how to describe the production of complex intermediate-mass fragments arises. This is being approached experimentally by observing the yields of helium through neon produced in the reaction of ${ }^{3} \mathrm{He}$ and $\mathrm{Ag}$ and characteristic changes which occur in the shapes of the energy spectra as the mass of the fragment increases (from a Maxwellian shape to a Gaussian shape). These experimental characteristics will be compared with the predictions of theoretical models.

\section{Nuclear Structure}

A major effort is directed toward the understanding of the behavior of nuclei at high angular momentum - up to the limit where they fission. For rare earth nuclei in the upper half of this spin region $(30-60 \hbar)$, the gamma-ray spectrum cannot be resolved and methods have been developed for extracting iniormation from the continuum. In the past few years a new method has been found to measure average effective moments of inertia that include both single-particle and collective contributions. These can be combined with previously measured average "band" moments of inertia (which are mainly collective) in order to separate these two contributions in unresolved spectra for spins in the range of 50-60h. In some light $\mathrm{Er}$ and $\mathrm{Yb}$ nuclei at these spins, evidence has been found for a large single-particle contribution due to high-j pı oton orbi:als. These particular orbitals are from the next higher major shell $(N=5)$ above the valence shell $(N=4)$. In some of the nuclei of this region, the contributions from the two shells are almost completely separated as two broad peaks in the gamma-ray spectrum. Orher regions of nuclei are being examined to see if such large shell effects are common.

\section{Exotic Nuclei}

Research in the area of exotic nuclei consists primarily of decay studies of light to medium mass very neutrondeficient isotopes. Techniques used for these studies involve charged particle and $\beta$ - $\gamma$ spectroscopy of nuclides produced in ${ }^{3} \mathrm{He}$ or heavy-ion bomardments of neutron-deficient targets. These nuclides are transported from the target area via a helium jet system which can be used either to prepare a source directly or to feed the on-line mass separator, RAMA. Recent use of the helium jet system operating alone has permitted discovery of the previously unknown, 
odd-odd. $T_{2}=-2$ isulopes. ${ }^{22} \mathrm{~N}\left(t_{1 / 2} \sim 70 \mathrm{~ms}\right)$ and ${ }^{26} \mathrm{P}\left(t_{1 / 2} \sim 20 \mathrm{~ms}\right)$. Observation of their beta-delayed proton decays has not only provided beta decay and isobaric analog state information but also confirmed a theoretical prediction that both isotopes could decay by a new decay mode, beta-delayed two-proton radioactivity. Subsequent protonproton coincidence experiments have enabled discovery of this new radioactivity (for both nuclides) and provided initial information on the mechanism of two-proton emission.

\section{Heavy Element Research}

Heavy element research is conducted by two NSD groups and a group from Lawrence Livermore National Laboratory. The Long Tape System at the cyclotion was designed to detect a spontaneous fission (SF) activity with $t_{1 / 2} \sim 0.1$ sec against a very high background of longer-lived SF activities. The 0.1-sec activity was never found, down to the sub-nanobarn cross section level, in many experiments utilizing targets of ${ }^{249} \mathrm{Bk}$ and ${ }^{248} \mathrm{Cm}$ with ${ }^{15} \mathrm{~N}$ and ${ }^{16} \mathrm{O}$ projectiles. This is in sharp disagreement with claims put forth by Dubna experimenters; however, in these same experiments a 20-ms SF activity with substantial cross sections was detected which has been tentatively azsigned to ${ }^{260} \mathrm{Rf}$. A 100 -ms SF activity has been found in bombardments of ${ }^{254} \mathrm{Es}$ with $99-\mathrm{Mev}{ }^{18} \mathrm{O}$ and $73-\mathrm{Mev}{ }^{13} \mathrm{C}$ projectiles. In the first case, the cross secton is very large, about 1 microbarn, and in the second, about 0.2 microbarn. Such large cross sections rule out nuclides with $Z$ equal to 104 or more; the best candidate on the basis of comparative yields is ${ }^{261} \mathrm{Lr}$, but ${ }^{260,261} \mathrm{Md}$ are also considered possible.

Reaction mechanisms in the heavy element region, where radiochemical techniques provide unique assignments of nuclear mass and charge to the produced radioactive species, has been concentrated in three areas: (a) the production of actinide nuclei by means of a massve transfer of nucleons from the projectile to the target as a potential means of producing new neutron-rich nuclijes; (b) determination of the preduction of nuclei with a greater nuclear charge than the target and the measurement of the charge dispersion of fission-like products from the reaction of 20 $\mathrm{MeV} / \mathrm{n}{ }^{12} \mathrm{C}$ and ${ }^{16} \mathrm{O}$ beams with targets of gold and rare earths; and (c) measurements of the gamma-ray intensities and population of isomeric states in poorly-known nuclear species produced in heavy-ion reactions. The production and fission of element 104 by the reaction ${ }^{15} \mathrm{~N}+{ }^{249} \mathrm{Bk} \rightarrow{ }^{260} \mathrm{Rf}+4 \mathrm{n}$ is being studied and from about 300 observed events it is seen that element 104 fissions symmetrically. This is in contrast to a preliminary finding based on $\sim 70$ events and indicates that shell effects at the second minimum disappear rapidly with excitation energy. The production of actinide nuclei by heavy-ion induced massive transfer reactions has been studied using an ${ }^{254} \mathrm{Es}$ target and beams from both the 88-Inch Cyclotron and the SuperHILAC. Chemical studies of the electrochemical potential for reduction of divalent nobelium have also been carried out.

\section{Light Ion Research}

Light-ion research using the polarized proton and deuteron beams is generally concerned with spin-polarization phenomena in nuclear reactions and scattering. A most important feature of polarization effects is that they often display uniquely the operation of, hence the possible violation of, a basic symmetry property of the nuclear interaction. Thus, several fundamental experiments have been designed to make significant tests of parity conservation, charge symmetry, and time-reversal invariance in this interaction.

Spin-polarization measurements also provide information concerning nuclear structure and reaction mechanisms that is not otherwise available. Thus, studies are made of analyzing powers and polarization transfer in inelastic nucleon scattering and nucleon-transfer reactions in order to deduce the specific spin-dependent nature of the nuclear interactions. For example, the inelastic nucleon scattering results provide detailed information about the spindependent components of the effective nueleon-nucleon force in nuclei, which is a subject of considerable current interest. Measurements also are made of polarization-transfer in elastic proton-nucleus scattering, since these results will provide a unique means of establishing the existence of a spin-spin component of this nuclear interaction. This research with polarized beams involves collaborations with several groups from different universities and laboratories.

\section{Applications}

Grouped as applied research are the activities of (i) the Division of Biology and Medicine, which include radioisotope production and cellular studies of radiobiological effects with low energy heavy ions; (ii) the investigation of cosmic-ray induced errors in digital semiconductor devices; (iii) detector calibration for relativistic heavy-ion physics and space research; and (iv) a new program, the enhanced adhesion of thin films through heavy-ion bombardment. 

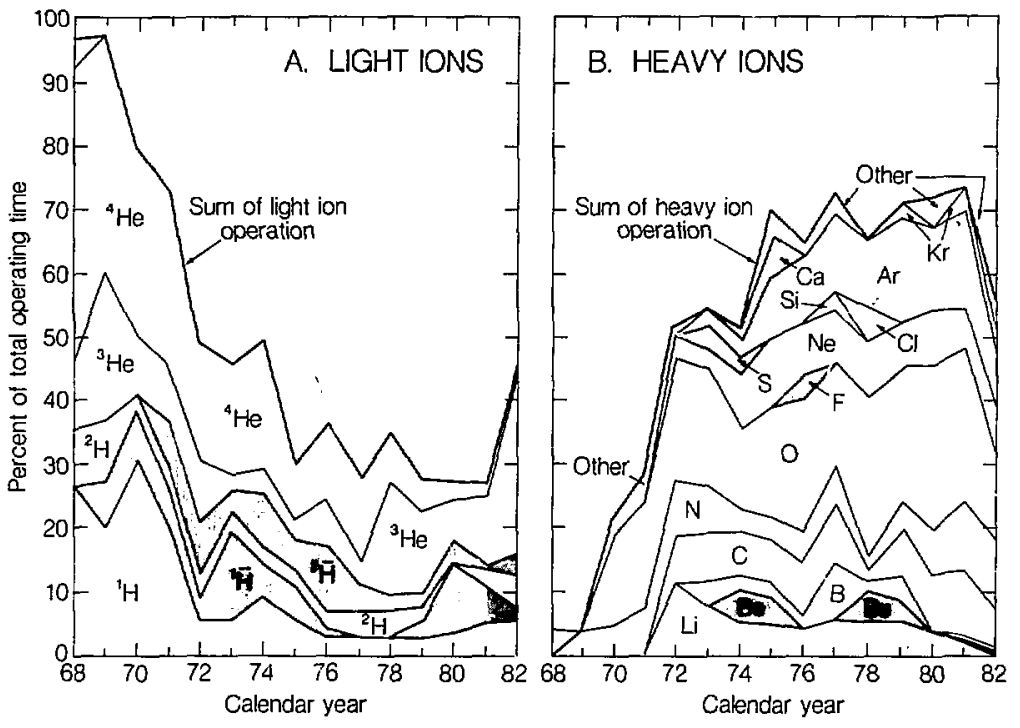

Fig. I Distribution of light and heavy ion beams.

XBL 822-1898 


\title{
Bevalac Research Program
}

\author{
H.G. Pugh
}

The Bevalac is operated by LBL's Accelerator and Fusion Research Division and has the unique capability world-wide to collide heavy and very heavy nuclei against each other at sufficiently high energies inat the internal structure of the composing nucleons is changed. The new ideas of nuclear physics which emerge from relativistic field theories devised to describe such collisions are in many instances strikingly different from our preconceptions of nuclcar behavior. New configurations of nuclear particles are found to be possibly stable and in the extreme limit it is found that the entire system of colliding nuclei may be converted into a new state of matter called the quark-gluon plasma. Such ideas cannot be investigated with conventional probes such as electrons or protons since they involve raising large volumes of nuclear matter to conditions of high density and pressure such as can only be brought about when very large projectiles, i.e., nuclei theinselves, are used. The Bevalac program consists of a systematic study of the behavier of nuclei under these conditions, with hot pursuit of any unusual phenomena that seem to be encountered.

The Bevalac provides beams of ions up to uranium at energies from $50 \mathrm{MeV} /$ nucleon to $2.1 \mathrm{GeV} /$ nucleon $(1.0$ $\mathrm{GeV} /$ nucleon for uranium). To use these beams a large number of user groups converge on LBL from all parts of the United States. An international contingent is led by major groups from GSI, Darmstadt, W. Germany, and INS, Tokyo, Japan, which make a leading contribution to the research program, as well as the many smaller groups engaged, for example, in emulsion experiments.

Experiments are selected by the Bevalac Nuclear Science Program Advisory Committee, which meets twice a year. The present members are Professor Herman Feshbach, MIT, Chairman; Professor Robert Klapisch, CERN, Geneva, Switzerland; Professor David K. Scott, Michigan State University; Professor Erich Vogt, University of British Columbia, Canada; Dr. William A Wenzel, Lawrence Berkeley Laboratory; and Professor Toshi Yamazaki, University of Tokyo, Japan. Within the extremely limited beam time available, collaborations typically receive 100 hours per year. Under these circumstances there is strong emphasis on complex, flexible, and efficient detectors and data acquisition methods, or else on experiments of a decisive quality.

The Bevalac Users' Association meets annually, and its Executive Committee holds telephone conference calls with Laboratory management to discuss current problems. During FY83 the charter of the Association is being revised to give the outside users an enhanced role in the Laboratory's activities. Users' workshops in specialized areas of research serve to coordinate activity. In the past year a JACEE workshop was held in August 1982 to study the state of the art in the study of nucleus-nucleus collisions with cosmic rays, and a Tevalac workshop was held in September 1982 to develop plans for a new accelerator. The Dilepton Workshops have continued on a more informal and more frequent basis and a Dilepton Spectrometer (DLS) project has been developed. In June 1983, the 6th High Energy Heavy Ion Study will be held, in conjunction with the second Anomalon Workshop. This event is expected to attract a large number of visitors from many parts of the world.

The phenomenon of short mean free paths of projectile fragments has continued to be the greatest mystery in Bevalac research. Since the first Anomalon Workshop in February 1982, many additional worler: r-ve entered the field and much improved statistics using visual detectors are anticipated. There have been sevt: ." attempts in the past year to study the short mean free path particles (or "anomalons") by electronic techniques. S.earches have been made for photons emitled downstream from a production target and for sharp photon lines in the spectra emitted from the target fragmentation region. both in the inclusive spectra and in the effective mass spectra of photons in coincidence with deuterons and tritons. Neither of these experiments found an anomalon signal, ruling out certain hypotheses concerning their nature. Several theoretical groups have rearalyzed the emulsion data to seek explanations. It has been suggested that the effect could be explained by molecular nuclei with unusually long lifetimes or that the cross sections observed for anomalons as a function of $Z$ correspond almost exactly to the limit which a nuclear cross section could have, i.e., the suin of the nucleon-nucleon cross sections. A third paper suggests that a relativistic mean field theory consistent with all known facts about nuclear physics would permit nuclei in certain excited states to have extra-long range forces. An important experiment has been conducted using the Plastic Wall to distinguish between the two hypotheses concerning anomalons, i.e., that they correspond to $6 \%$ of nuclear fragments having a 10 times increased cross section or, alternatively, $100 \%$ of nuclear fragments having a $20 \%$ increased cross section coupled with a $10^{-11}$ second lifetime. These can be distinguished by comparison of dense and dilute targets. Preliminary results favor the former hypothesis. A new electronic detector is being constructed to directly simulate the visual detectors. Using the ring-imaging Cerenkov technique, it is hoped that the detector will give direct results 
on the anomalons, and be usable as a trigger for other more sophisticated detectors such as HISS.

Recent work has shed important light on the progress of relativistic heavy ion reactions. These experiments have investigated the degree of equilibration for central collisions, the time evolution of a nuclear collision, the nuclear density and compressional energy of these collisions and strange particle production.

In 1982 jons up to uranium became available and preliminary experiments were carried out with each of the new beams as they were developed.

- The first of the new beams, krypton, was used in early 1982 to test the streamer chamber for high multiplicity events and to indicate several improvements necessary, which are now being implemented.

- Measurements of the range-energy relationship for uranium ions in emulsion showed that the higher-order corrections in the infra-red region of QED cancel each other. The range of $1 \mathrm{GeV} /$ nucleon uranium ions in emulsion is only $4 \mathrm{~cm}$ !

- Characteristic event types for uranium interactions in emulsion have been identified, including binary and ternary fission, grazing and head-on collisions.

- Fission cross sections have been studied as a function of beam energy using solid state detector techniques.

- The equilibrium charge state distribution for uranium ions passing through various materials has been studied. It is found that $90 \%$ of uranium ions at $1 \mathrm{GeV} /$ nucleon can be reduced to the fully stripped state. This is the first time that bare uranium nuclei have ever been produced in the laboratory.

- Atomic physics measurements have been carried out on xenon ions, in particular of X-ray yields when they pass through various materials.

- Inciusive charged particle spectra have been measured for $\mathrm{La}+\mathrm{La}$ collisions at $800 \mathrm{MeV} / \mathrm{nucleon}$.

- Exclusive $4 \pi$ geometry measurements have been made for $800 \mathrm{MeV} /$ nucleon $\mathrm{Nb}+\mathrm{Nb}$ collisions with the Plastic Ball-Wall. Evidence for equilibration was obtained.

- Gold beams were used in experiments to calibrate cosmic-ray detectors.

In experiments to be carried out in the near future, pion multiplicities will be studied for $\mathrm{Nb}+\mathrm{Nb}$ in the streamer chamber. A search will be made for effects due to the pion condensate in La+La, the Plastic Ball experiments will be extended to Au $+\mathrm{Au}$, and measurements will begin towards studying the Iamb shift in hydrogen-like uranium $\left(\mathrm{U}^{\mathrm{Pl+}}\right)$.

Finally, in order to acquire the most coherent and useful set of data, we have chosen to study whenever possible a set of monoisotopic nuclei. Thus $\mathrm{Nb}, \mathrm{La}$ and $\mathrm{Au}$ feature strongly among the early experiments.

\section{New Detectors}

The Plastic Ball-Wall is complete, has taken data, and the first results, on coalescence phenomena, have been submitted for publication. Gas detectors are being added inside the scatter chamber to provide data on very heavy fragments.

The streamer chamber has undergone a fairly extensive upgrade, especially with the addition of a 400-element hodoscope to provide end-point and particle identification measurements in the forward direction. A further improvement is the installation of an ultra-violet laser to produce artificial tracks which are recorded by a CCD camera and used to monitor chamber efficiency and stability. Use of the complete system will begin in summer 1983 and a series of approved experiments will continue through 1984.

HISS has operated successfully with the prototype detectors, but data taking was severuly handicapped by the restriction of the Bevalac to half field during FY82 (with the exception of one weekend, not allocated to HISS). Analysis of data on carbon fragmentation has proved very interesting, with an unexpectedly large proportion of very high nuclear excitation. Confirmation of these results with better statistiss is a high priority in FY83. The Phase II detectors will be completed in FY84. Tests on the MUSIC box indicated that it will reach its design goals of distinguishing one unit of charge at charge 92, but improvemesits still remain to be carried out on the gas handling system and electronics. of value.

The past year has seen major development in our plans for the Dilepton Spectrometer (DLS). A pilot experiment to look for singi: lepton production has been approved by the Program Advisory Committee, and a large collaboration of outside users is preparing the final plan for the dilepton spectrometer. The CERN Lampshade Maynet 
and detectors have been inspected as a basis for the Bevalac experiment and a final choice of procedure will be made by June 1983. This experiment promises to give extremely important information of the internal state of interacting relativistic nuclei. Whether dileptons are observed or not the measurement will be a crucial test of high temperatures reached. If enough dileptons are observed to make studies possible as a function of effective photon mass and of projectile and target masses and beam energy, the spectrometer will surpass other methods of studying hot nuclei.

In accordance with our interest in pursuing studies to their logical conclusion, LBL is continuing involvement in the $\alpha-\alpha$ experiments at the CERN ISR. Since tha: facility will be closed in FY84, continued development requires the CERN PS and SPS. An approved LBL/GSI/Heidelberg/Marburg/Warsaw collaboration will initiate these studies, by providing an ${ }^{16} \mathrm{O}$ ion source using an RFQ pre-injeclor. The initial experiment, to be carried out in FY85-86, will use 6-13 GeV/ nucleon ${ }^{16} \mathrm{O}$ beams from the PS, and will consist of target fragmentation studies with the Plastic Ball, studies of the participant region and particle production with a new streamer chamber to be provided by GSI, and studies of anomalous projectile fragments using emulsions. It is expected that the program will continue in FY86-87 with ${ }^{16} \mathrm{O}$ beams from the SPS at energies up to $200 \mathrm{GeV} /$ nucleon. These experiments will be the first test of nuclear stopping power in this energy region and the overall program will map out the possibilities for creating regions of high density in heavy ion collisions. In accord with our present knowledge, the Tevalac project addresses the beam energy region where high baryon densities can be produced. Our work developing that project and the CERN experimentation will continue hand-in-hand. 


\title{
SuperHILAC Research Program
}

\author{
R.M. Diamond
}

The SuperHILAC is the only accelerator in the United States capable of accelerating very heavy ions far enough beyond the Coulomb barrier to permit studies of compound-nuclear and deep-inelastic reactions. In recent experiments the new injector, ABEL, has reached its design characteristics, producing beams of $\sim 1$ (charge) microampere of $8.5 \mathrm{MeV} /$ nucleon ${ }^{197} \mathrm{Au}$ and ${ }^{238} \mathrm{U}$. Thus the SuperHILAC now furnishes ions with energies from 1.2. $8.5 \mathrm{MeV} /$ nucleon of ${ }^{40} \mathrm{Ar}$ to ${ }^{23 \mathrm{~B}} \mathrm{U}$ with intensities of several (charge) microamperes to one (charge) microampere, respectively. It is a National Facility open to all qualified research groups in the United States and Canada and to collaborating groups in Europe and Asia. The use of two injectors in time-shared mode means that in normal operation the SuperHILAC provides up to three different beams to users: a very heavy-ion beam at 32 pulses/s for the principal user; a different beam at 2 pulses/s for injection into the Bevatron; and either one of these beams, at a different energy if necessary, for a parasite user at the SuperHILAC to test equipment or even do a low duty-cycle experiment. Due to increased power costs during the past year, the total number of hours available to experimenters, 2151 , is slightly reduced over the previous year, and again is about $3 / 4$ of the total scheduled time. Beams used included ${ }^{48} \mathrm{Ca},{ }^{36} \mathrm{Fe}, \mathrm{Kr}, \mathrm{Xe},{ }^{165} \mathrm{Ho},{ }^{199} \mathrm{La},{ }^{197} \mathrm{Au},{ }^{209} \mathrm{~Pb}$, and ${ }^{238} \mathrm{U}$.

A program advisory committec currently composed of Dr. Peter Bond (Brookhaven National Laboratory), Chairman, Dr. C. Lewis Cocke (Kansas State University), Prof. David B. Fossan (State University of New York at Stony Brook), Prof. C. Konrad Gelbke (Michigan State University), and Dr. Jorgen Randrup (LBL) meets twice a year to select the best experiments to be carried out. Ir. 1983, Drs. Cocke and Bond will be replaced by Dr. John Hardy (Chalk River Nuclear Laboratory) and Dr. Shelton Datz (Oak Ridge National Laboratory), and Prof. Fossan will serve as chairman. They work from writter proposals submitted by about two dozen research groups. Last year approximately $52 \%$ of the time went to outside groups and $48 \%$ to groups within LBL. The experimental work performed last year can be divided into four main categories: heavy-ion reaction studies $(\sim 1 / 2)$; nuciear structure studies $(\sim 1 / 6)$; exotic nuclei $(\sim 1 / 6)$; and atomic physics $(\sim 1 / 6)$.

Thus, reaction mechanism work, particularly studies of deep-inelastic reactions, dominates the research program. Major problems involve the transfer and alignment of angular momentum in the product fragments, the energy and angular distributions of light particles emitted and what they tell about the emitting system (whether it is the combired di-nucleus or the resulting fragments after separation, whether evaporative particles or pre-equilibrium ones are involved, and what is the role of composite particles such as $\mathrm{Li}$ and Be nuclei ). For example, from the (low) temperatures deduced from the spectral shapes of the $\alpha$-particles emitted and the kinematics of the fragments, it has been inferred that energy thermalization and particle evaporation are even faster than "fast" fission. Studies of heavy-ion fusion reactions are also being done, for exanole, the variation of the cross section with bombarding energy for heavy projectiles and targets. The experimental results cannot be represented by simple one-dimensional models even allowing for frictional effects. But the inclusion of a neck degree of freedom for the di-nuclear complex gives better agreement; such a model (by Swiatecki) leads to two types of saddle points and requires an extra (energy) push to get the two nuclei to fuse. Tests of this idea are in progress. It should be mentioned that in a number of the reaction mechanism studies performed it was found useful to carry out the inverse reaction, that is, to use the smaller nucleus as the target and bombard it with the larger one, e.8. ${ }^{165} \mathrm{Ho}$ on ${ }^{56} \mathrm{Fe}$, rather than ${ }^{56} \mathrm{Fe}$ on ${ }^{168} \mathrm{Ho}$. The advantages are that the resulting products have larger velocities and so are easier to get through window foils on gas counters and ionization chambers, and the products are focused in a smaller solid angle.

The nuclear structure studies mainly make use of the high-Z projectiles available at the SuperHILAC (Xe and $\mathrm{Pb}$ ) to multiply Coulomb-excite target nuclei, or use inverse reactions to obtain large recoil velocities in order to measure lifetimes by Doppler-shift techniques or to measure $\mathrm{g}$-factors of short-lived states of high spin by means of the enormous transient magnetic fields acting on fast-moving ions in ferromagnetic materials. An example of the last type of problem is furnished by an LBL-Chalk River collaborative work which yiclded the surprising result that the transient field in cooled ferromagnetic gadolinium did not continue to increase with increasing ion velocity as most people had expected, but reached a maximum at a velocity of about $5 \% \mathrm{c}$. Now, having calibrated this field as a function of ion velocity, the technique will be used to measure the g-factors of high-spin states, and hopefully, even the average of regions of continuum states.

The third type of research category is the study of exotic nuclei. A major attempt to produce nuclei of a superheavy element by means of an extended ${ }^{48} \mathrm{Ca}$ bombardment of ${ }^{248} \mathrm{Cm}$ was carried out at the end of 1982 by a collaboration of LBL and GSI scientists. No superheavy element events were observed, and the upper limits on the 
cross sections are currently being refined. The use of a gas-filled magnetic separator SASSY allowed observation into the millisecond region, and the cross section limit for production of superheavy nuclei in this time range is on the I00 picobam level. The heavy element chemistry groups looked for half-lives in the range of hours to a year and have set cross-section limits of the order of some tens of picobarns. The next major effort on superheavy elements is a repeat of the ${ }^{4} \mathrm{Ca}+{ }^{24} \mathrm{Cm}$ bombardment, but done at GSI in March, 1983, again as a collaboration of LBL and GSI nuclear physics and chemistry groups. In the future, the LBL groups may try to make an isotope of element 108 by a ${ }^{48} \mathrm{Ca}$ bombardment of ${ }^{226} \mathrm{Ra}$ in the hope that it might be as long-lived as microseconds. In addition to these studies, the on-line isotope separator OASIS is in operation and the transport system from the separator to the spectroscopy laboratory above it is now finished. OASIS has been used to characterize five new beta-delayed proton emitters and to hunt (so far unsuccessully) for new ground-state proton emitters. This instrument can now separate about $70 \%$ of all the elements, and will be used for isotope studies going out towards both the neutron and proton drip lines.

The final category of research programs is heavy-ion atomic physics. One major class of studies here has to do with the fine structure in the spectra of one-and two-electron ions in order to make rigorous tests of quantum electrodynamics and of relativistic corrections. A number of tests with hydrogen-like ions have been performed, and now new calculations for the two-electron ions will permit even more precise comparisons with experimental studies being done, or planned, with He-like ions of Ar, and possibly up to $\mathrm{Kr}$. To produce these ions at very low velocities so that Doppler shifts are negligibly small, the appropriate gas is bombarded with $U$ ions of 4-5 MeV/nucleon which ionize the gas atoms, but impart recoil velocities corresponding to only a few electron volts. Other studies are measuring the electron-capture cross section at very low velocities. Still another program is attempting to measure correlated electron capture and K-shell excitation by highly stripped, heavy ions (e.g., Li-like ${ }^{40} \mathrm{Ar}$ ) incident on gas targets (this is like an inverse Auger transition). It has been suggested that a similar process is an important energy-loss mechanism in high-temperature fusion plasmas, but such experiments have only recently been performed in the laboratory, in preliminary runs at TUNL, Brookhaven, and LBL. Another program involves the measurement of K-shell ionization probabilities in the collision of high- $Z$ jons as a function of nuclear excitation energy (where the latter reflects the length of the reaction or sticking time). Unfortunately, the initial results with $\mathrm{Xe}$ on $\mathrm{Pb}$ and $\mathrm{Th}$ targets are very much complicated by the large number of internal conversion $\mathbf{K}$ x-rays. But since the internal conversion yield appears to remain approximately constant with an increase in the combined $Z$ of the di-nuclear complex, while the K-vacancy production goes up strongly, the answer is to go to $\mathrm{Pb}-\mathrm{Pb}$ and $\mathrm{U}-\mathrm{U}$ collisions. Successful experiments would provide an atomic clock for these deep-inelastic reaction times. Other programs deal with beam-foil studies of the energies and lifetimes of transitions in highly stripped Ne-like ions of Xe and Ho. Relativistic corrections to the calculated energies are greater than 100\%, and there are much larger effects on the lifetimes of certain types of transitions. The measurements are of direct interest to studies of high-temperature and high-density plasmas, and to schemes for producing $x$-ray lasers in high- $Z$ Ne-like systems. 
PART III: EXPERIMENT

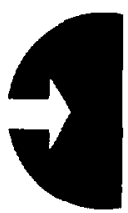




\title{
1. Nuclear Structure
}

\section{General}

\section{Decay Properties of Isoscalar Giant Resonances in ${ }^{24} \mathrm{Mg},{ }^{28} \mathrm{Si},{ }^{58} \mathrm{Ni},{ }^{64} \mathrm{Zn}$, and ${ }^{92} \mathrm{Mo}$}

\author{
P. Bogucki, J.D. Bronson, U. Garg, "† P. Grabmayr, \\ K.T. Knöpfie, ${ }^{\ddagger \text { Y }}$ Y.W. Liu, H. Riedesel, G.J. Wagner. ${ }^{\ddagger}$ \\ and D.H. Youngblood"
}

$\left(\alpha, \alpha^{\prime}, c\right)$ coincidence experiments of the chargedparticle decay c from the isoscalar giant multipole resonances in ${ }^{24} \mathrm{Mg},{ }^{28} \mathrm{Si},{ }^{58} \mathrm{Ni},{ }^{64} \mathrm{Zn}$, and ${ }^{92} \mathrm{Mo}$ excited by $128 \mathrm{MeV}$ inelastic $\alpha$ scattering have been performed at the 88-inch cyclotron of the Texas A\&M University. The inelastically scattered $\alpha$ particles were detected at $0^{\circ}$ in an Enge split-pole spectrograph. This assures axial symmetry of the decay particles $\mathrm{c}$ about the beam axis and, in particular, allows the search for the decays of isoscalar (giant) monopole resonances. Analysis of the data has started recently and is in progress.

\section{Footnotes}

*Permanent address: Cyclotron Institute, Texas A\&M University, College Station, Texas 77843

tPresent address: University of Notre Dame, Notre Dame, Indiana 46556

¥Present address: Max-Planck-Institut für Kernphysik, 6900 Heidelberg, West Germany

§High Energy Physics Lab, Stanford University, Stanford, CA 94305

\section{Inelastic Scattering of ${ }^{12} \mathrm{C}$ from ${ }^{208} \mathrm{~Pb}$ to the Second $0^{+}$State of ${ }^{12} \mathrm{C}^{*}$}

\author{
R. Shyam, ${ }^{\dagger}$ M.A. Nagarajan,,$^{\dagger}$ A.C. Shotter, ${ }^{\ddagger}$ A.N. Bice, ${ }^{\ddagger}$ and J. Cerny
}

\begin{abstract}
A theoretical and experimental investigation of the inelastic scattering of ${ }^{12} \mathrm{C}$ from a ${ }^{208} \mathrm{~Pb}$ target exciting the second $\mathrm{O}^{+}$state of ${ }^{12} \mathrm{C}$ has been performed with the 88-Inch Cyclotron at incident energies of 132, 187, and $230 \mathrm{MeV}$. For those inelastic scattering reactions in which the ${ }^{12} \mathrm{C}$ nucleus is excited to states above 7.37 $\mathrm{MeV}$, but below $15.96 \mathrm{MeV}$, the only particle emission channel open is ${ }^{12} \mathrm{C} * \rightarrow{ }^{8} \mathrm{Be}+\alpha$. These channel fragments are confined to a cone and are detected in coin. cidence by two $\triangle \mathrm{E} \times \mathrm{E}$ counter telescopes in close vertical geometry, with one telescope above and one telescope below the reaction plane.
\end{abstract}

A summed energy spectrum for coincident events in the two telescopes, where at least one telescope records an $\alpha$-particle, is shown in Fig. $1 \mathrm{a}^{\prime}$ for an incident ${ }^{12} \mathrm{C}$ energy of $187 \mathrm{MeV}$ and for the counters placed at $19^{\circ}$ to the beam direction. From kinematic arguments the peak at $178 \mathrm{MeV}$ is determined to arise from the quasielastic breakup of ${ }^{12} \mathrm{C}$ into the $\alpha$ and ${ }^{8} \mathrm{Be}$ channel. Most of the yield of this quasielastic peak is due to the breakup from the $0^{+}, 7.65 \mathrm{MeV}$ state. This may be seen from Fig. $1 b^{1}$ which shows the ${ }^{8}$ Be projected energy spectrum, arising from the quasielastic peak. The different peaks of this spectrum arise from the contribution of different excited states of ${ }^{12} \mathrm{C}$. Thus, the $0^{+}, 7.65 \mathrm{MeV}$ state of ${ }^{12} \mathrm{C}$, which has a $\mathrm{c} . \mathrm{m}$. breakup energy of $0.288 \mathrm{MeV}$, results in a broad peak at $120 \mathrm{MeV}$, while the $9.64 \mathrm{MeV}$ state results in two narrow peaks.

The angular distributions of the quasielastic peak for the three beam energies are shown in Fig. 2. These have been analyzed in a framework of a microscopic folding model and the DWBA. The real parts of the optical potentials in the initial and final channels as 
well as the real part of the transition form factor have been calculated by double folding ${ }^{2}$ the M3Y effective interaction ${ }^{3}$ with the respective densities. The renormalization constants are given in the caption to Fig. 2 . it can be seen that a renormalization constant for the final channel other than 0.6 provides bad fits to the experimental data.

These investigations confirm that for loosely bound projectiles the double folding model overestimates the calculated real pari of the potential and a renormalization of nearly $50 \%$ is necessary to fit the data for inelastic scattering. It is the first time that this effect has been demonstrated by an investigation of inelastic scattering. The M3Y interaction ${ }^{2,3}$ with one nucleon exchange taken into account in the zero order appears to provide a good description of the central part of the nucleon-nucleon effective interaction over a range of projectile energies.

\section{Footnotes and References}

*Condensed from Phys. Lett. 116B (1982) 99

tScience and Engineering Research Council, Daresbury Laboratory. Daresbury, Warrington, WA4 4AD, UK

$\ddagger$ Permanent address: Physics Department, Edinburgh University, Scotland, UK

§Present address: US NRC/ACRS, Washington DC 20535

1. A.C. Shotter, A.N. Bice, D.P. Stahel and J. Cerny, J. Phys. G: Nucl. Phys. 8 (1982) 355

2 G.R. Satchler and W.G. Love, Phys. Rep. 55 (1979) 183

3. G.R. Satchler, Phys. Rev. C22 (1980) 919

Fig. 1.

(a) The yield of coincitent events between the two telescopes. See text.

XBL 807-10713A

(b) The energy of ${ }^{8} \mathrm{Be}$ nuclei in coincidence with an $\alpha$ particle for the transition ${ }^{208} \mathrm{~Pb}\left({ }^{12} \mathrm{C}, \alpha^{8} \mathrm{Be}\right.$ ) ${ }^{208} \mathrm{~Pb}($ g.s. $)$ at $187 \mathrm{Mev}$ bombaraing energy. This projected energy spectrum was taken at $\theta_{\mathrm{LAB}}=19^{\circ}$ with a detectc configuration which has an average vertical angular separation of $\Delta \Phi=5.9^{\circ}$.
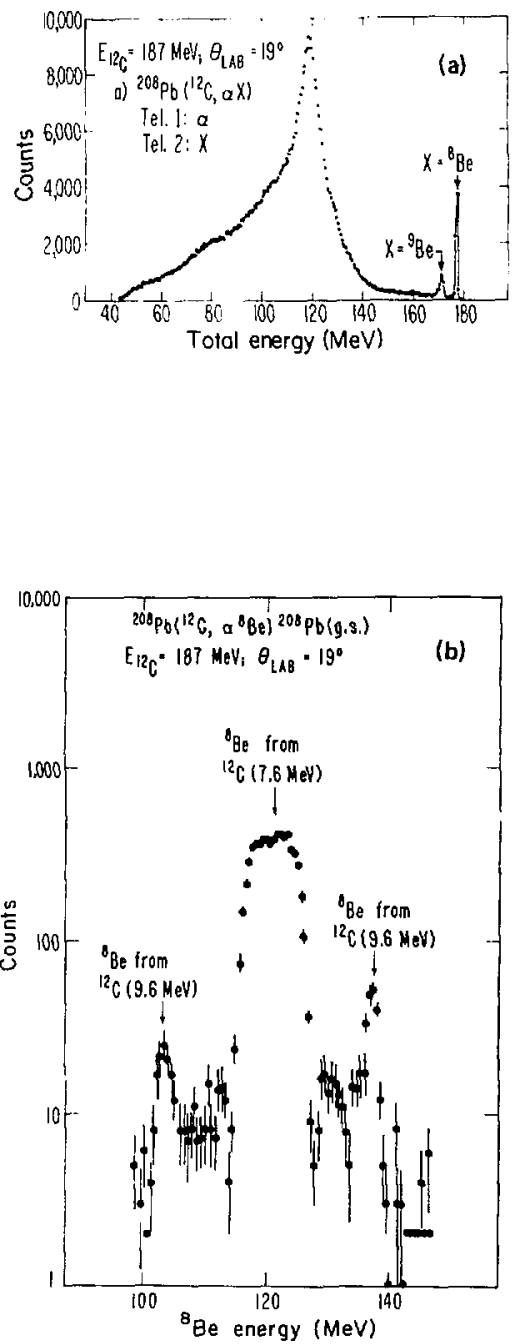

XBL $798-2600$ 


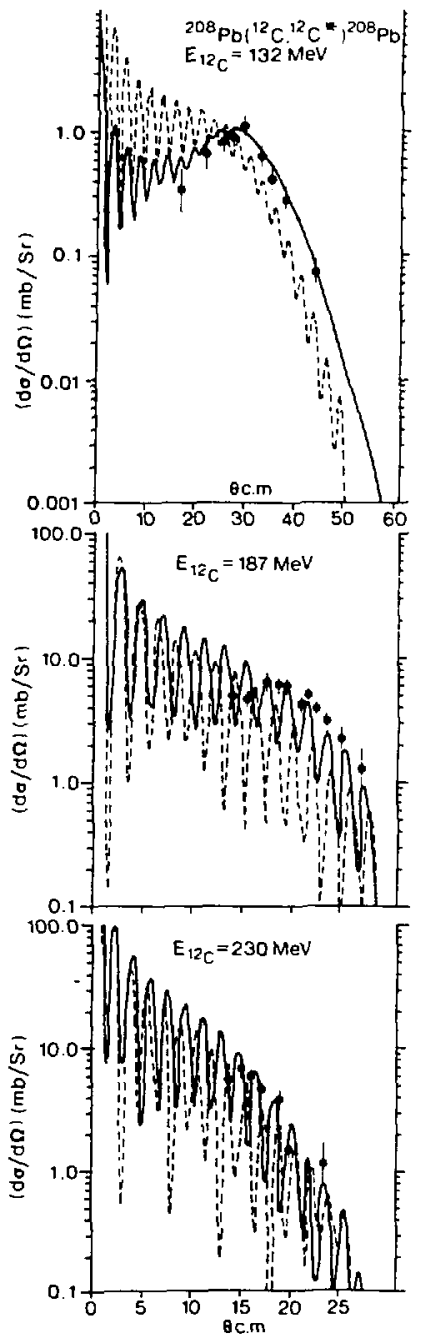

Fig. 2. Inelastic cross section for the excitation of the $\mathrm{O}_{2}^{+}$state in ${ }^{12} \mathrm{C}$ in ${ }^{12} \mathrm{C}+{ }^{208} \mathrm{~Pb}$ collisions. The solid line represents the results of calculations where the renormalization constant of the calculated initial channel real potential was 1.25 and that of the final channel real potential was 0.6. The dashed line represents the results when both the initial and final chanuel real potentials are renormalized by a factor of 1.25 .

XBL $834-9098$ 


\title{
Exotic Nuclei
}

\section{Beta-Delayed Proton Decay of an Odd-Odd $\mathrm{T}_{\mathrm{z}}=-2$ Isotope, ${ }^{22} \mathrm{Al}^{*}$}

\author{
M.D. Cable, J. Honkanen, ${ }^{\prime}$ R.F. Parry, H.M. Thierens, ${ }^{\ddagger}$ \\ J.M. Woute's,' Z.Y. Zhou, ** and Joseph Cerny
}

While the decays of several even-even $T_{2}=-2$ nuclei have been studied, ${ }^{1,2}$ to date no odd-cidd $\mathrm{T}_{2}=-2$ nucleus has been characterized. We report the observation of the $\beta^{+}$.delayed proton decay of the first such nucleus, ${ }^{22} \mathrm{Al}$. The particle stability of ${ }^{22} \mathrm{Al}$ has been a question of interest for many years; most mass models predict ${ }^{22} \mathrm{~A}$ to be at the very limit of particle stability, with some models indicating stability and others not.

${ }^{22} \mathrm{Al}$ was produced via the ${ }^{24} \mathrm{Mg}\left({ }^{3} \mathrm{He}, \mathrm{p} 4 \mathrm{n}\right){ }^{22} \mathrm{~A}$ reaction by bombarding natural magnesium targets with a $110 \mathrm{MeV}{ }^{3} \mathrm{He}$ beam from the 88-Inch Cyclotron. The recoil atoms were transported with ethylene glycol or $\mathrm{NaCl}$-loaded helium through a $1.1-\mathrm{m}$ long, $1.6-\mathrm{mm}$ diameter capillary onto a catcher foil. $\beta^{+}$-delayed protons were detected by a three-element semiconductor telescope consisting of $110 \mu \mathrm{m} \Delta \mathrm{El}, 60 \mu \mathrm{m} \Delta \mathrm{E} 2$, and $1000 \mu \mathrm{m} \mathrm{E}$.

Proton spectra observed at 110 and $60 \mathrm{MeV}{ }^{3} \mathrm{He}$ bombarding energies are shown in Fig. 1. These spectra are dominated by $\beta^{+}$-delayed protons from the decays of ${ }^{25} \mathrm{Si}$ and ${ }^{21} \mathrm{Mg}$, which are produced at relatively high yicld via the $\left({ }^{3} \mathrm{He}, 2 \mathrm{n}\right)$ and $\left({ }^{3} \mathrm{He}, \alpha 2 \mathrm{n}\right)$ reactions. At 110 $\mathrm{MeV}$, two new proton groups are observed at $7.839 \pm$ 0.15 and $8.149 \pm 0.021 \mathrm{MeV}$, very near the predicted absolute proton energies expected. ${ }^{3}$ These groups are not seen in the $60 \mathrm{MeV}$ bombardment, which is below the threshold for producing ${ }^{22} \mathrm{Al}$. The half-life of these groups was measured to be about $70 \mathrm{~ms}$.

As shown in Fig. 2, these two proton groups were assigned to the isospin forbidden proton decay of the lowest $\mathbf{T}=2$ state in ${ }^{22} \mathrm{Mg}$. Using the experimental mass for this state and the calculated ${ }^{3}$ Coulomb energy difference between ${ }^{22} \mathrm{Al}$ and ${ }^{22} \mathrm{Mg}^{*}$, we get a $\mathrm{Q}_{\mathrm{EC}}=$ $18.493 \mathrm{MeV}$ for ${ }^{22} \mathrm{Al}$, which indicates that ${ }^{22} \mathrm{Al}$ is bound against direct proton emission by only $102 \mathrm{keV}$. Comparison of the ${ }^{22} \mathrm{Al}$ yield to that of ${ }^{25} \mathrm{Si}$ and ${ }^{21} \mathrm{Mg}$ indicates an effective cross section for the observed proton groups of about $1.2 \mathrm{nb}$, which corresponds to a total cross section of $40 \mathrm{nb}$.

As is also shown in Fig. 2, the beta-delayed twoproton decay of ${ }^{22} \mathrm{~A}$ is energetically possible. A search for the new decay mode is in progress.

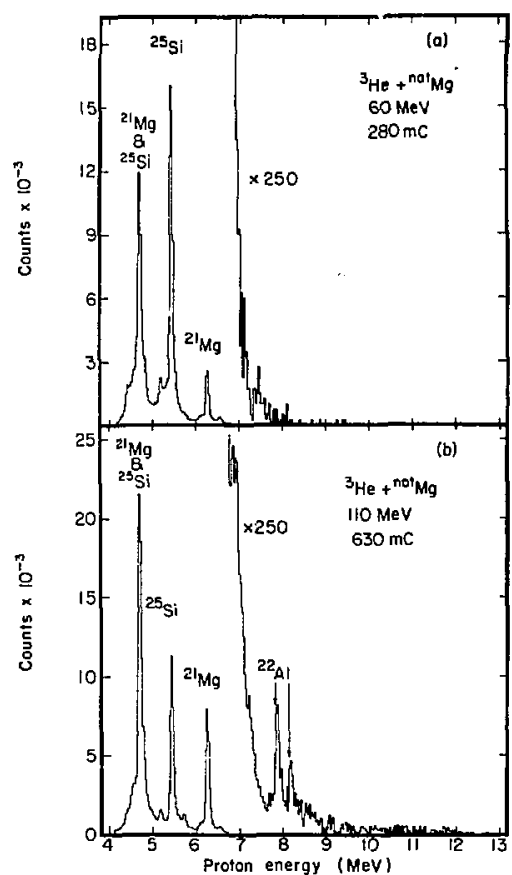

Fig. 1. Beta-delayed proton spectra. The positions of the arrows labeled ${ }^{22} \mathrm{Al}$ are the predicted energies obtained by the method of Hardy, et al. (ref. 3)

XBL. 823-3710

\section{Foornores and References}

"Condensed from LBL-14419, Phys. Rev. C26 (1982) 1778 , with additions 
ton leave from: Department of Physics, University of Jyväskylä, Finland

\$Present address: Department of Physics, University of Ghent. Belgium

Present address: CNC Division. Los Alamos National Laboratory

**On leave from: Department of Physics, Nanking University, China

1. Hagberg, P.G. Hansen, J.C. Hardy, A. Huck, B. Jonson, S. Mattson, H.L Ravn, P. TidemandPetersson, and G. Walter, Phys. Rev. Lett. 39 (1977) 792

2. J. Aystö, M.D. Cable, R.F. Parry, J.M. Wouters, D.M. Moltz, and J. Cerny, Phys. Rev. C23 (1981) 879

3. J.C. Hardy, H. Brunnader, J. Cerny, and J. Janecke, Phys. Rev. 183 (1969) 854

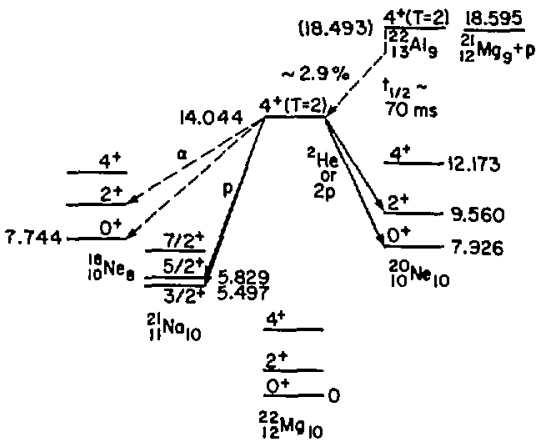

Fig. 2. Partial decay scheme for ${ }^{22} \mathrm{Al}$.

XBL 829-4626

\title{
Beta-delayed Proton Decay of the Odd-Odd $T_{z}=-2$ Isotope ${ }^{26} \mathbf{P}$
}

\author{
M.D. Cable, J. Honkanen, * R.F. Parry.
} S.H. Zhou, ' Z.Y. Zhou, and Joseph Cerny

Recent observation of the $\beta^{+}$-delayed proton decay of ${ }^{22} \mathrm{Al}$ established the existence of the first known member of the odd-odd $A=A n+2, T_{z}=-2$ series. $^{1}$ We wish to report the observation of the second such nucleus, ${ }^{26} \mathrm{P}$. These nuclei are of interest not only for isobaric analog state masses and beta-decay information but are also thought to be at the very limit of particle stability. ${ }^{26 \mathrm{P}}$ is actually predicted to be unbound to direct proton emission by most mass formulae. ${ }^{2}$ As discussed below, this is quite possibly true and not inconsistent with our observation of this isotope. Also of considerable interest is the energetically possible decay mode of $\beta^{+}$-delayed 2-proton emission for both of these nuclei.

${ }^{26} \mathrm{P}$ was produced via the ${ }^{28} \mathrm{Si}\left({ }^{3} \mathrm{He}, \mathrm{p} 4 \mathrm{n}\right){ }^{26} \mathrm{P}$ reaction with $110-130 \mathrm{MeV}{ }^{3} \mathrm{He}$ beams from the 88-Inch Cyclotron. The recoils were transported by a helium jet to a counting chamber and collected on a slowly rotating $\mathrm{Al}$ wheel. A 3-element semiconductor varticle telescope $(110 \mu \mathrm{m} \Delta \mathrm{E} 1,60 \mu \mathrm{m} \Delta \mathrm{E} 2$, and $1000 \mu \mathrm{m} \mathrm{E})$ was used to observe $\beta^{+}$-delayed protons coming from nuclei at the collection site.

Proton spectra observed at 60,110, and $130 \mathrm{MeV}$ ${ }^{3} \mathrm{He}$ energies are shown in Fig. 1. The spectra are dominated by $\beta^{+}$-delayed protons from the decay of ${ }^{21} \mathrm{Mg}$, ${ }^{25} \mathrm{Si}$, and ${ }^{29} \mathrm{~S}$ produced in high yield $\left({ }^{3} \mathrm{He}, x \alpha 2 \pi\right)$ reactions. At $110 \mathrm{MeV}$ and $130 \mathrm{MeV}$, however, two new groups are observed at $7.269 \pm 0.015 \mathrm{MeV}$ and $6.827 \pm$ $0.050 \mathrm{MeV}$ and were observed to decay with a half life of $\sim 20 \mathrm{~ms}$.

As shown in Fig. 2, these two new groups can be assigned to the isospin-forbidden proton decay of the lowest $\mathrm{T}=2$ state in ${ }^{26} \mathrm{Si}$. From the known mass of ${ }^{25} \mathrm{Al}^{2}{ }^{2}$ the ${ }^{26} \mathrm{Si}^{*}$ mass excess can be calculated to be $5.936 \pm 0.015 \mathrm{MeV}$ and a Coulomb displacement estimate of the ${ }^{26} \mathrm{P}_{2}{ }^{26} \mathrm{Si}^{*}$ mass difference gives a calculated ${ }^{26} \mathrm{P}$ mass excess of $18.295 \mathrm{MeV}$. This result indicates that ${ }^{26} \mathrm{P}$ is unbound to direct proton emission by $\sim 34$ $\mathrm{keV}$. Since the proton decay half life even for an $\ell=$ 0 transition is $>1$ second for a decay energy below $\sim 50$ $\mathrm{keV}$ (as calculated by the barrier penetrability code, 


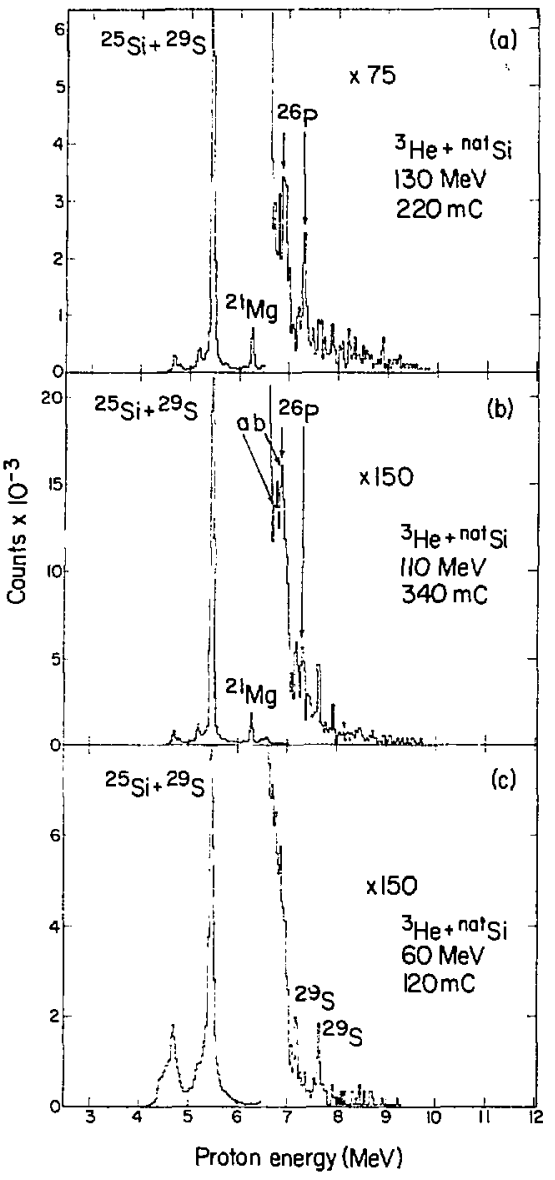

Fig. 1. Beta-delayed proton spectra.

XBL $829-4623$
COCAG), beta decay can be the dominant decay mode, in agreement with our data. The observed half life for ${ }^{26} \mathrm{P}$ beta decay gives a superallowed $\beta^{+}$branch of $\sim 1.9 \%$. As shown in Fig. 2, $\beta^{+}$-delayed $2 p$ emission (an unobserved radioactive decay mode) is energetically possible for this nucleus. Work is progressing to observe this mode of decay.

\section{Foolnotes and References}

"On leave from: Department of Physics, University of Jyväskylä, Finland

ton leave from: Institute of Atomic Energy, Beijing, China

$\ddagger$ On leave from: Department of Physics, Nanking University, China

1. M.D. Cable, J. Honkanen, R.F. Parry, H.M. Thierens, J.M. Wouters, Z.Y. Zhou, and Joseph Cerny, Phys. Rev. C26 (1982) 1778

2. A.H. Wapstra and K. Bos, At. Data and Nucl. Data Tables 19 (1977) 175

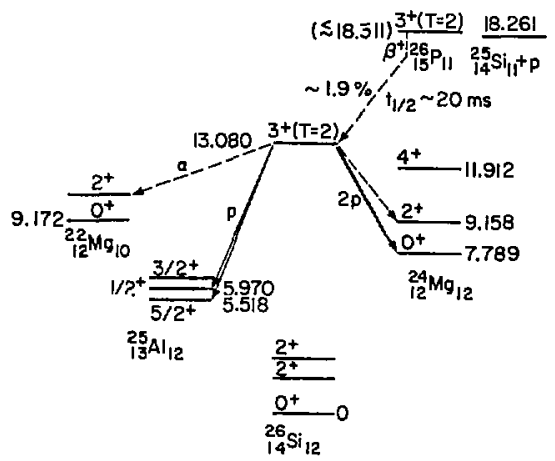

Fig. 2. Partial decay scheme for ${ }^{26} \mathrm{P}$.

XBL 829-4628 


\title{
Weak Beta-Delayed Proton Groups in the Decay of ${ }^{21} \mathrm{Mg},{ }^{25} \mathrm{Si}$, and ${ }^{29} \mathrm{~S}$
}

\author{
S.H. Zhou, " M.D. Cable, J. Honkanen, R.F. Parry, \\ Z.Y. Zhou, and Joseph Cerny
}

During the study ${ }^{1.2}$ of ${ }^{22} \mathrm{~A}$ and ${ }^{26} \mathrm{P}$, several new low-intensity $\beta^{+}$-delayed proton groups have been observed and assigned to ${ }^{21} \mathrm{Mg},{ }^{25} \mathrm{Si}$, or ${ }^{29} \mathrm{~S}$. The results are listed in Table I with intensities given relative to the strongest proton group arising from the decay of each isotope. 3,4 The $\log f t$ values were calculated assuming that the final states of these $\beta^{+}$.delayed proton decays are only the ground states of the corresponding final nuclides, so that the logft values listed in Table I should be considered as the upper limits. The log/t values indicate that all these transitions are allowed. Figure 1 shows the ${ }^{29} \mathrm{~S} \beta^{+}$-delayed $7114 \pm 15 \mathrm{keV}$ and $7581 \pm$ $15 \mathrm{keV}$ proton peaks and ${ }^{25} \mathrm{Si} \beta^{+}$-delayed $6518 \pm 10$ keV proton peak. Those proton peaks are firmly assigned and the others in Table 1 are tentatively assigned.

Tabie I: Proton Energies, Excitation Energies and $\log f t$ Values

\begin{tabular}{|c|c|c|c|c|c|}
\hline ISOTOPE & $\begin{array}{c}\mathrm{E}_{\text {F }} \\
(\mathrm{keh})\end{array}$ & $\begin{array}{c}\mathrm{E}_{\mathrm{cm}} \\
(\mathrm{keV})\end{array}$ & $\begin{array}{c}\text { Relative Intensity } \\
(\%)\end{array}$ & $\begin{array}{c}\mathrm{E}_{\mathrm{z}} \\
(\mathrm{keV})\end{array}$ & $\log / t$ \\
\hline${ }^{25} \mathrm{Si}$ & $6518 \pm 10$ & $6792 \pm 10$ & $0.5 \pm 0.1$ & 9186 & $4.88 \pm 0.01$ \\
${ }^{29} \mathrm{Si}$ & $7114 \pm 15$ & $7370 \pm 16$ & $1.3 \pm 0.4$ & 10348 & $5.35 \pm 0.07$ \\
${ }^{29} \mathrm{~S}$ & $7581 \pm 15$ & $7852 \pm 16$ & $0.5 \pm 0.1$ & 10830 & $5.32 \pm 0.04$ \\
${ }^{21} \mathrm{Mg}$ & $6492 \pm 20^{4)}$ & $6819 \pm 21$ & $0.4 \pm 0.1$ & 9008 & $5.02 \pm 0.03$ \\
${ }^{25} \mathrm{Si}$ & $6719 \pm 50^{2)}$ & $7001 \pm 52$ & $0.05 \pm 0.02$ & 9395 & $5.72 \pm 0.08$ \\
${ }^{25} \mathrm{Si}$ & $6808 \pm 50^{\mathrm{a})}$ & $7094 \pm 52$ & $0.3 \pm 0.1$ & 9488 & $5.86 \pm 0.04$ \\
\hline
\end{tabular}

a) Tentative assignment

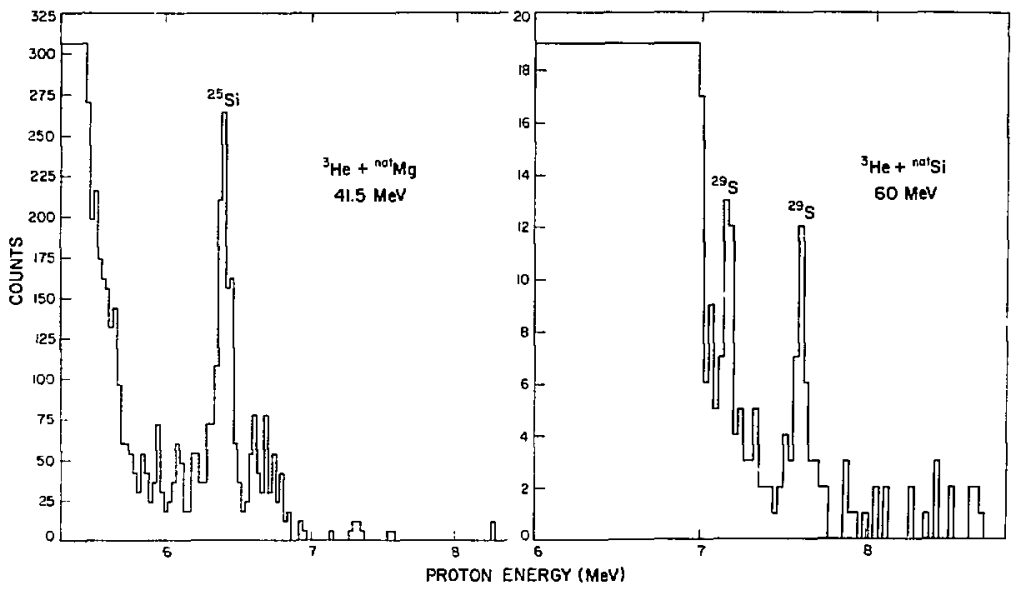

Fig. 1. Proton energy spectra showing $\beta^{+}$-delayed peaks for ${ }^{3} \mathrm{He}$ ions on targets of natural $\mathrm{Mg}$ and $\mathrm{Si}$.

XBL $801 \mathrm{I}-1328$ 


\title{
Footnotes and References
}

"On leave from: Institute of Atomic Energy, Beijing, China

ton leave from; Department of Physics, University of Jyväskylä, Finland

‡On leave from: Department of Physics, Nanking University, China

1. M.D. Cable, J. Honkanen, R.F. Parry, H.M. Thierens, J.M. Wouters, Z.Y. Zhou, and Joseph Cerny, Phys. Rev. C26 (1982) 1778
2. M.D. Cablc, J. Honkanen, R.F. Parry, S.H. Zhou, Z.Y. Zhou, and Joseph Cerny, to be published

3. R.G. Sextro, Ph.D. Thesis, U.C. Berkeley, LBL 2360 (1973)

4. D.J. Vieira, Ph.D. Thesis, U.C. Berkeley, LBL7161 (1978)

\section{New Delayed Proton Emitters Measured with OASIS}

\author{
J.M Nitschke, W.-D. Zeitz, ${ }^{*}$ and M.D. Cable
}

This is a preliminary report on recent results obtained with the SuperHILAC On-line Isotope Separa-

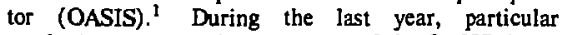
emphasis was put on improvements of the OASIS detector systems. A surface barrier detector array was built which allows the study of charged particle spectra of eight masses simultaneously. As an example of the performance of this detector system, five $\alpha$-spectra of rare earth isotopes are shown in Fig. 1. These were obtained in a bombardment of ${ }^{14} \mathrm{Sm}$ with ${ }^{20} \mathrm{Ne}$ ions. Another detector system that was used extensively consists of two proton telescopes located in the focal plane of the spectrometer magnet, $15 \mathrm{~mm}$ above and below the median plane. These telescopes have solid angles of 32 percent of $2 \pi$ and are composed of two $\Delta E / E$ detectors with thicknesses of $11 / 425 \mu \mathrm{m}$ and $28 / 1035 \mu \mathrm{m}$, respectively. The noise widths of the uncooled detectors are between 15 and $20 \mathrm{keV}$. An electrostatic vertical deflection system at the exit of the magnet switches the direction of the emerging beam within $100 \mu \mathrm{s}$ so that a chosen isotope is first collected on a thin $(20$ $\left.\mu \mathrm{g} / \mathrm{cm}^{2}\right)$ carbon foil in front of the upper and then the lower telescope. The deflection method is used to determine the half-lives of unknown isotopes by observing the growth and decay rates in the two telescopes. The energy calibration was done with ${ }^{241} \mathrm{Am}$ and ${ }^{212} \mathrm{~Pb} \alpha-$ sources as well as low-energy, monochromatic $\alpha$ particles produced from a strong (2.14 $\left.\times 10^{9} \mathrm{dpm}\right)$ ${ }^{241} \mathrm{Am}$ source with suitable absorbers and a spectrometer magnet. We also used the strong ${ }^{241} \mathrm{Am}$ source to produce protons via $\alpha, p$ elastic scattering. The maximum proton energy observed with this method was 2.7 MeV. A third calibration method was based on a ${ }^{239} \mathrm{Pu} /{ }^{9} \mathrm{Be}$ neutron source $\left(6.5 \times 10^{6} \mathrm{n} / \mathrm{s}\right)$ which produces protons of up to about $10.5 \mathrm{MeV}$. All measure-

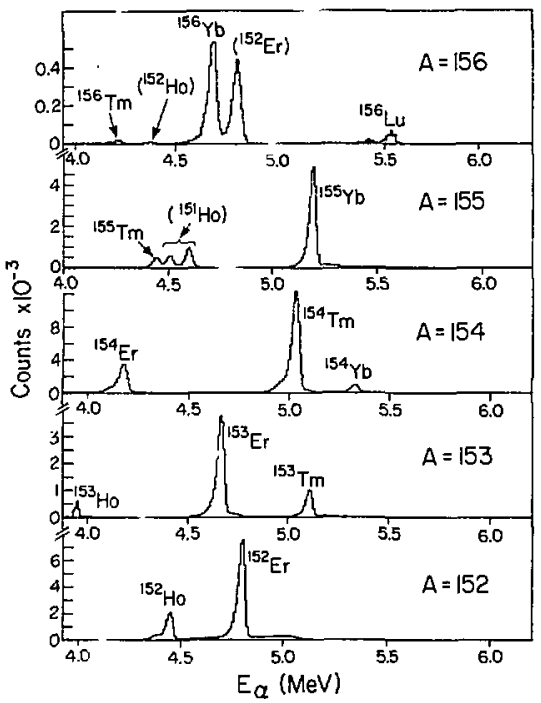

Fig. 1. Alpha spectra of mass-separated rare-earth (RE) isotopes produced in the reaction ${ }^{144} \mathrm{~S}_{\mathrm{r}}\left({ }^{20 \mathrm{Ne}}, \mathrm{xn}, \mathrm{yp}\right) \mathrm{RE}$. Daughter products are sho' in in parentheses.

XBL. $827-972$ 
ments reported below were carricd out with the dual proton telescope.

Targets of ${ }^{90} \mathrm{Zr}$ and ${ }^{\text {nat }} \mathrm{Zr}$ were bombarded with ${ }^{40} \mathrm{Ca}$ beams of 168 to $196 \mathrm{MeV}$ from the SuperHILAC. The ${ }^{40} \mathrm{Ca}$ energy was optimized for the cvaporation of three to six nucleons from the compound nucleus ${ }^{130} \mathrm{Nd}$. The evaporation residue products were stopped in a Ta catcher which was kept at a temperaturc of about $2700^{\circ} \mathrm{C}$. This catcher is part of the OASIS surface ion source. After ionization, acceleration, and mass separation, isotopes of mass 124 through 127 were collected on $20 \mu \mathrm{g} / \mathrm{cm}^{2}$ carbon foils directiy in front of the tclescopes. At the different mass numbers, the following observations were made.

$A=127:$

About 70 protons with energies between 2.2 and $6.15 \mathrm{MeV}$ were observed at this mass number (Fig. 2a). The wide distribution of proton energies is characteristic for delayed proton emission. Due to the poor statistics, only a range of 4 to $40 \mathrm{~s}$ can be given for the half-life of this activity. In Fig. 3, the difference between the $Q$-value for electron capture $Q_{\mathrm{Ec}}$ and the proton separation energy $S_{p}$ is plotted as a function of $Z$ for constant $A$. The mass values were taken from a table by Liran and Zeldes ${ }^{2}$. $\left(Q_{E C}-S_{p}\right)$ represents the highest expected proton energy. Also indicated in Fig. 3 are the energy thresholds for proton emission $\left(\Theta_{p}\right)$ and the range of observed proton energies (schematic spectra, shaded). For $A=127$ it appears that ( $Q_{\text {ec }}$ $S_{p}$ ) is too small for all $Z<60$ to account for the observed maximum proton encrgy of $6.15 \mathrm{MeV}$. This would mean that the new delayed proton precursor is ${ }^{127} \mathrm{Nd}_{60}$ formed in the reaction ${ }^{40} \mathrm{Ca}\left({ }^{90} \mathrm{Zr}, 3 \mathrm{n}\right)$. Calculation, however, shows that ${ }^{127} \mathrm{Pr}$ and ${ }^{127} \mathrm{Cr}$ are formed with cross sections which are a factor $\because \sim 10^{3}$ and $\sim 10^{4}$ higher than the one for ${ }^{127} \mathrm{Nd}$, and one or bath isotopes could therefore be the precursors for the observed proton activity. ${ }^{12} \mathrm{Ce}$ could be the next member in the $T_{z}=9 / 2$ series of the already known delayed proton precursors ${ }^{131} \mathrm{Nd}$ and ${ }^{135} \mathrm{sm}$, Half-life considerations also favor the ${ }^{127} \mathrm{Ce} /{ }^{127} \mathrm{Pr}$ interpretation: for ${ }^{127} \mathrm{Ce}$ a half-life of $32 \pm 4 \mathrm{~s}$ was determined from $\mathrm{x}$ ray decay, ${ }^{3}$, and the prediction for the half-life of ${ }^{127} \mathrm{Pr}$ from the gross theory of beta decay is 4 to $20 \mathrm{~s}$. Both fall within the experimentally-observed half-life range, while the predicted half-life for ${ }^{: 27} \mathrm{Nd}(0.8$ to $4 \mathrm{~s})$ would be too short. If ${ }^{127} \mathrm{Ce}$ or ${ }^{123} \mathrm{Pr}$ are the observed proton precursors, we have a contradiction to the prediction of the Liran-Zeldes mass formula, and the final $Z$ assignment will have to await further experiments.
$A=126:$

The proton spectrum observed at mass 126 is shown in Fig. 2b. From the growth and decay data of the two telescopes, a half-life of $3.2 \pm 0.6 \mathrm{~s}$ was calculated using the maximum likelihood method. The mass predictions plotted in Fig. 3 and charge conservation indicate that $Z$ can only have the values 59 or 60 . The calculated cross section for ${ }^{126} \mathrm{Nd}$ formed in the reaction ${ }^{40} \mathrm{Ca}_{20}\left({ }^{90} \mathrm{Zr}_{40}, 4 \mathrm{n}\right)$ is only $20 \mu \mathrm{b}$, while it is $4 \mathrm{mb}$ for ${ }^{126} \mathrm{Pr}$. This makes the observation of a small proton branch of ${ }^{126} \mathrm{Nd}_{60}$ unlikely and favors the ${ }^{126} \mathrm{Pr}$ interpretation. The observed half-Iife of $3.2 \mathrm{~s}$ is well within the range of predicted values for ${ }^{126} \operatorname{Pr}(1$ to $7 \mathrm{~s}$ ) from the gross theory of beta decay. ${ }^{4}$

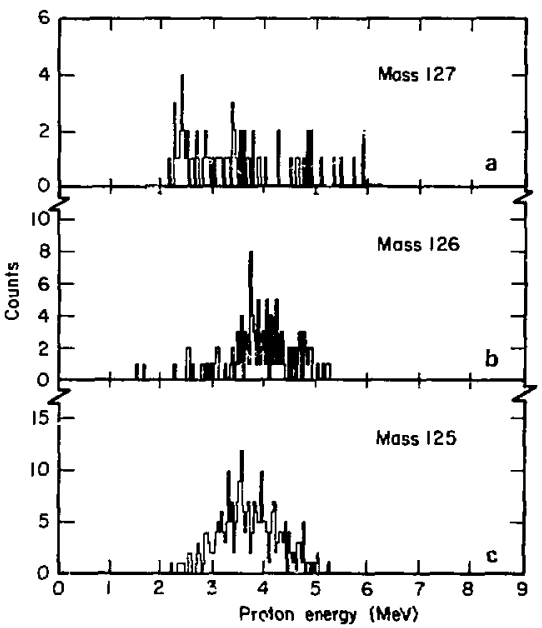

Fig. 2. Delayed proton spectra of mass-separated rareearth isotopes produced in the reaction ${ }^{90} \mathrm{Zr}\left({ }^{40} \mathrm{Ca}, \mathrm{xn}, \mathrm{yp}\right) \mathrm{RE}$. The spectra were recorded with the dual proton telescope described in the text.

XBL $8211-11959$ 


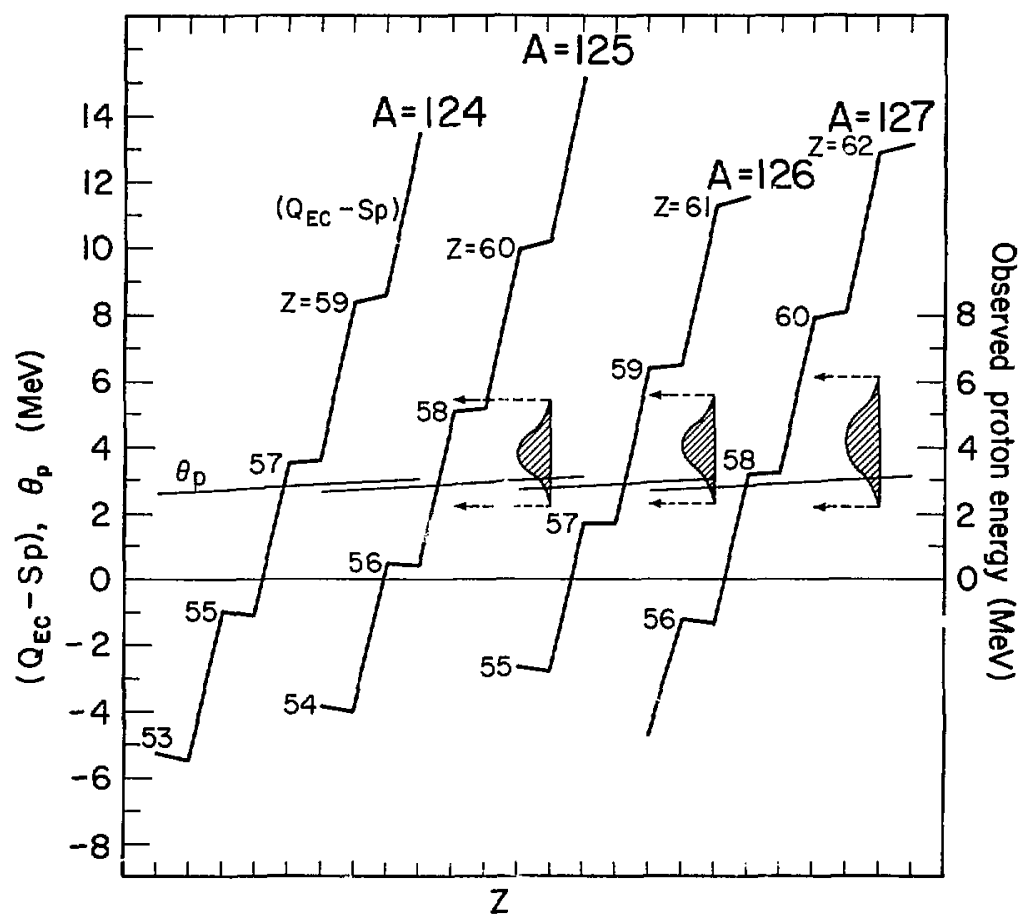

Fig. 3. $\left(Q_{E C}-S_{p}\right)$ and $\theta_{p}$ as functions of $Z$ for constant $A . Q_{E C}=Q$-value for electron capture of the delayedproton precursor $(Z, A), S_{p}=$ proton binding energy of the delayed-proton emitter $(Z-1, A)$, and $\theta_{p}=$ proton emission threshold. The shaded areas indicate schematically the observed proton energy distribution at each mass.

XBL 8211-11558

\section{$A=125:$}

At mass 125 the proton spectrum of Fig. $2 \mathrm{c}$ was observed. From the growth and decay data shown in Fig. 4, a half-life of $8.9 \pm 0.7 \mathrm{~s}$ was calculated for the proton activity. Arguments similar to those given for $A$ $=126$ and 127 lead to a $Z$ assignment of 58 (Ce). ${ }^{125} \mathrm{Ce}$ was first observed via $x$-ray decay by Bogdanov, et al. ${ }^{3}$ and a half-life of $11 \pm 4 \mathrm{~s}$ was found, which agrees well with the observed delayed proton half-life. The gross theory of beta decay predicts 4 to $25 \mathrm{~s}$.

$$
\mathbf{A}=124:
$$

Subsequent to the experiment at mass 125 , the spectrometer was tuned to mass 124 without changing the ${ }^{40} \mathrm{Ca}$ energy. No protons were cbserved. According to Fig. 1, one would expect proton emission from ${ }^{124} \mathrm{Pr}$ or even ${ }^{124} \mathrm{Ce}$ or ${ }^{124} \mathrm{La}$; however, in this brief experiment, the bombarding energy was 25 to $35 \mathrm{MeV}$ too low, which suppressed the cross sections by a factor of 3 to 10 . 


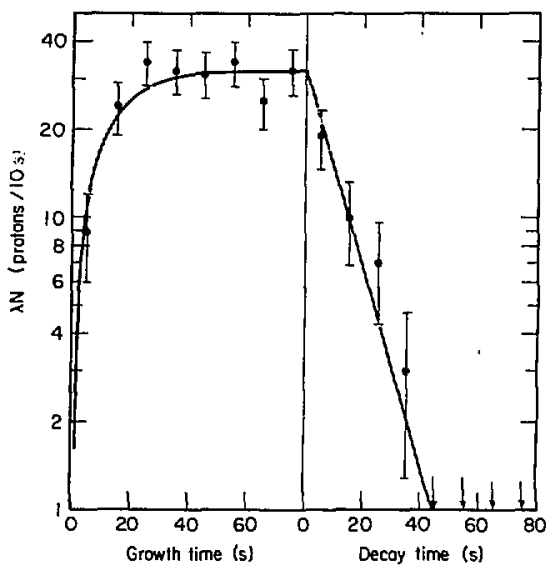

Fig. 4. Growtb and decay curve of the delayed proton activity at mass 125 . The activity was accumulated on each telescope for $80 \mathrm{~s}$ and its decay observed for another $80 \mathrm{~s}$. The data from both telescopes were combined and analyzed with the maximum likelihood method. The best fit for the data is obtained with a half-life of $8.9 \pm 0.7 \mathrm{~s}$ and a production rate of $3.2 \pm 0.2 \mathrm{~s}^{-1}$.
In all experiments $(A=124$ to 127 , the mass calibration of the spectrometer was checked by observm. ing long-lived isotopes of $\mathrm{Ba}$ and $\mathrm{Cs}$ which were created through $\beta^{+}$decay chains.

\title{
Footnotes and References
}

*Hahn-Meitner Institut, Berlin, West Germany.

1. J.M. Nitschke Nucl. Inst. and Meth. 206 (1983) 341 .

2. S. Maripuu, ed., A1. Data Nucl. Data Tables 17 (1976) 476.

3. D. D. Bogdanov, et al., Nucl. Phys. A 307 (1978) 421.

4. K. Takahashi, et al., At. Data Nucl. Data Tables 12 (1973) 101.

XBL 8211-11957

\section{Search for New Ground-State Proton Emitters}

\author{
J.M. Nitschke, W.-D. Zeitz' and M.D. Cable
}

The discovery that ${ }^{151} \mathrm{Lu}$ and ${ }^{147} \mathrm{Tm}$ decay by proton radicactivity from tbe ground state $^{l}$ has been a major advance in the exploration of nuclei far from stability. Using the SuperHILAC on-line isotope separator facility, OASIS, one of the GSI discoveries was confirmed, and an attempt was made to find other proton emitters in the same region.

${ }^{145} \mathrm{Tm}$ was produced by bombarding a $2 \mathrm{mg} / \mathrm{cm}^{2}$ thick ${ }^{92} \mathrm{Mo}$ target with $292-\mathrm{MeV}{ }^{58} \mathrm{Ni}$ ions. The 1.03 $\mathrm{MeV}$ protons from ${ }^{147} \mathrm{Tm}$ were observed with two silicon $(\Delta E / E)$ proton telescopes of $11 / 427 \mu m\left(\Delta E_{1} / E_{1}\right)$ and
$28 / 1035 \mu \mathrm{m}\left(\Delta \mathrm{E}_{2} / \mathrm{E}_{2}\right)$ thickness, respectively. Using standard particle identification algorithms, we were able to reduce the $\beta+$ background sufficiently and prove unambiguously that the charged particles emitted in the ground state decay of ${ }^{167} \mathrm{Tm}$ are protons. This provides an alterutate method of proton identification to the absorber technique used at GSI. An attempt to analyze the second proton emitter $\left({ }^{151} \mathrm{Lu}\right)$ in a similar fashion failed: at mass 151 no protons were found. This is probably due to the short half-life $(85 \mathrm{~ms})$ of the isotope, which decayed for the most part before leaving 
the ion source of the isotope separater.

Both ${ }^{151} \mathrm{Lu}$ and ${ }^{147} \mathrm{Tm}$ have an isospin of $\mathrm{T}_{z}=$ $9 / 2$, and it is therefore compelling to investigate whether ${ }^{143} \mathrm{Ho}$, the next lighter member of the $\mathrm{T}_{2}=$ $9 / 2$ series, is a good candidate for proton radioactivity. Mass tables by different authors ${ }^{2}$ predict proton binding energies for this nucleus between 0.08 and $-0.64 \mathrm{MeV}$. In the reaction ${ }^{92} \mathrm{Mo}\left({ }^{58} \mathrm{Ni}, \alpha \mathrm{p} 2 \mathrm{n}\right){ }^{143} \mathrm{Ho}$, the proton spectrum shown in Fig. 1 was observed. No sharp lowenergy proton lines which would be typical for groundstate proton emission of ${ }^{143} \mathrm{Ho}$ can be distinguished. The spectrum is, however, characteristic of a delayed proton emitter. Figure 2 shows the growth and decay curve for the proton activity from wh! th a half-life of $4.3 \pm 0.3 \mathrm{~s}$ was calculated.

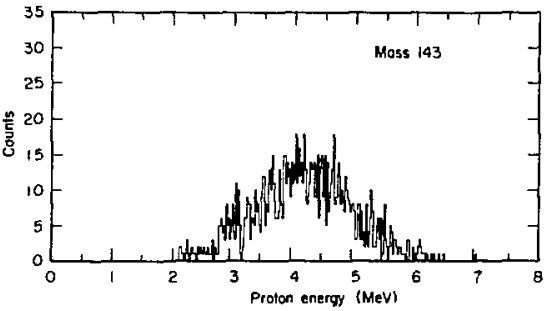

Fig. l. Proton spectrum at mass 143 after hombarding ${ }^{92} \mathrm{Mo}$ with $292-\mathrm{MeV}{ }^{58} \mathrm{Ni}$ ions.

XBL 8211-11953

The atomic number of the delayed proton precursor can be inferxed from Fig. 3 where the difference between the electron-capture Q-value and the proton separation energy $\left(Q_{E C}-S_{p}\right)$ is plotted for mass 143 as a function of $Z$. Proton decay is energetically possible for positive values of this quantity, but the proton width (r) Jecomes comparable to the gamma decay width $\left(\Gamma_{\gamma}\right)$ only for values of $\left(Q_{E C}-S\right)(Z)>\theta_{p}$, where the proton emission threshold $\theta_{p}$ is a function of the orbital angular momentum of the proton and the Coulomb barrier. Comparing the observed proton energy distribution with calculations shown in Fig. 3 , the above inequality is fulfilled for $Z \geq 66$. To narrow down the range of possible Z-values for the new isotope, we made a crossbombardment with ${ }^{56} \mathrm{Fe}+{ }^{92} \mathrm{Mo}$ where an activity with a similar half-life and a proton spectrum similar to Fig. 1 was produced. This justifies the assumption that we synthesized the same isotope for which now only $Z$ values of 66 and 67 are possible. This final uncertainty can be resolved by considering the calculated ratio for the cross sections of the two reactions

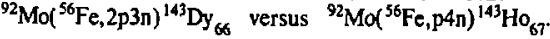

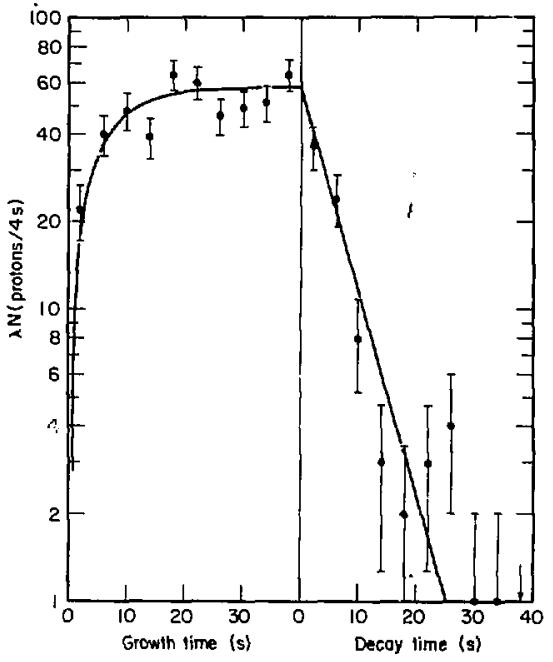

Fig. 2. Growth and decay rates of the proton activity at mass 143 as measured with a dual-proton telescope. The growth and decay periods are $40 \mathrm{~s}$ each. The solid line is a fit to the data obtained with the maximum likelihood method. The calculated half-life is $4.3 \pm 0.3 \mathrm{~s}$.

XBL 8211-11954

This ratio is about 100 , which clearly points to ${ }^{143} \mathrm{Dy}$ as the correct assignment for the new delayed proton activity. The observed half-life of $4.3 \pm 0.3 \mathrm{~s}$ is in good agreement with the prediction of the gross theory of beta decay, ${ }^{2}$ which gives a half-life between 2 and 6 s.

Proton, $\beta-, \gamma$, or $x$-ray coincidence spectra for ${ }^{143} \mathrm{Dy}$ have not been measured so far, which makes it difficult to interpret the proton spectrum in terms of $\beta^{+}$ strength functions.

Figure 4 shows schematically the decay of ${ }^{143} \mathrm{Dy}$ based on mass values from Liran and Zeldes. ${ }^{3}$ If it is assumed that the proton decay from ${ }^{143} \mathrm{~Tb}$ proceeds to the ground state of ${ }^{142} \mathrm{Gd}$ and that the threshold energy for $\beta+$ decay is not higher than a few hundred $k e V$, the expested maximum proton energy is about 7.5 $\mathrm{MeV}$. The expected minimum proton energy for $\mathrm{Tb}_{65}$ is $\theta_{p}=3.30 \mathrm{MeV}$. $\theta_{p}$ has been calculated to be the energy at which a proton with an orbital angular momentum $\ell=0$ has a barrier penetrability of $10^{-4}$, which corresponds roughly to $\Gamma_{p} \sim \Gamma_{\gamma}$. We observe 


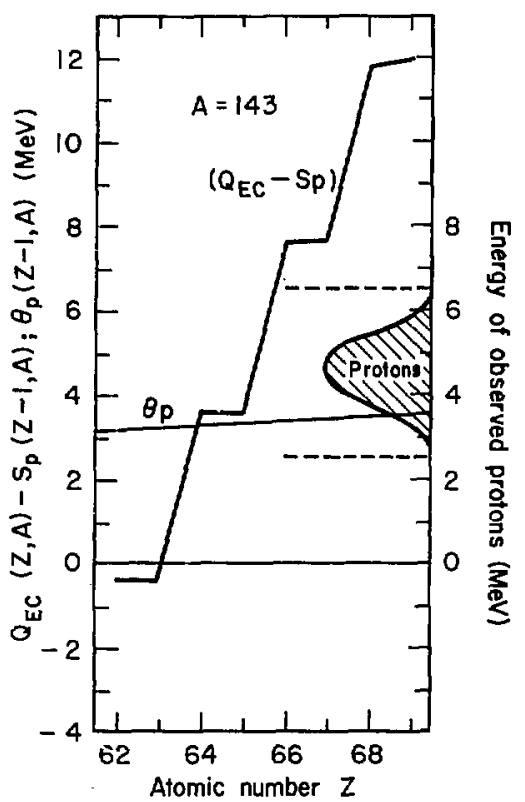

Fig. 3. The difference between the electron capture $Q$ values $\left(Q_{E C}\right)$ and the proton separation energy $\left(S_{p}\right)$, and the proton emission threshold $\left(\theta_{p}\right)$ plotted as a function of the atomic number of the delayed proton precursor (Z,A) for mass 143. Mass values are taken from Liran and Zeldes. ${ }^{3}$ The quantities $\left(Q_{E C}-S_{p}\right)$ and $\theta_{p}$ are relevant for predicting the energy range of beta-delayed protons (see text).

XBL 8211-11955

protons with energies about $1 \mathrm{MeV}$ below $\theta_{p}$, which indicates that the proton branching ratio is probably small. This is to be expected if ${ }^{143} \mathrm{Dy}$ is the first proton precursor in the $A=143$ isobaric chain, and would be consistent with proton branching ratios $\left(b_{b}\right)$ of other isotopes near $\beta$-stability: ${ }^{121} \mathrm{Ba}\left(\mathrm{b}_{\mathrm{b}}=2 \times{ }^{\mathrm{p}} 0^{-4}\right),{ }^{120} \mathrm{Cs}$ $\left(b_{p}=7 \times 10^{-B}\right)$, and ${ }^{11} \times \mathrm{Xe}\left(b_{p}=2.9 \times 10^{-5}\right)$.

A recent paper by W. F. Feix and E. R. Hilf ${ }^{4}$ sheds some light on the question of why the groundstate proton decay of ${ }^{143} \mathrm{Ho}$ was not observed in our experiment. The authors have calculatec $Q_{p}=0.74$ $\mathrm{MeV}$ for ${ }^{143} \mathrm{Ho}$ and obtain a partial proton half-life of
$9.9 \times 10^{4} \mathrm{~s}$ under the assumption that the proton has an orbital angular momentum of $11 / 2$. The $\beta+$ half-life calculated from the gross theory of $\beta$-decay is $2.1 \mathrm{~s}$. This would result in a proton branch of $2 \times 10^{-5}$. Given that the evaporation residue cross section for ${ }^{143} \mathrm{Ho}$ is on the order of $10^{-28} \mathrm{~cm}^{2}$, the effective cross section for producing protons would be about $10^{.33} \mathrm{~cm}^{2}$, which is below the detection limit of the on-line isotope scparator. Furthermore, the detection threshold of our telescope was $0.88 \mathrm{MeV}$ so that protons with an energy of $0.74 \mathrm{MeV}$ as predicted by Feix and Hilf would not have been observed.

\section{Footnotes and References}

tHahn-Meitner Institut, Berlin, West Germany.

*For details of the half-life analysis, see the contribution of $l$. P. Somerville in this report.

**Mass values were taken from the table of Liran and Zeldes. $^{3}$

1. S. Hofmann, et al., Z. Phys. A305 (1982) 111.

2. K. Takahashi, et al., At. Data Nucl. Data Tables 12 (1973) 101.

3. S. Maripuu, ed., At. Data Nucl. Data Tables 17 (1976) 476 .

4. W, F, Feix and E. R. Hilf, Technische Hochschule Darmstadt, Report IKDA 82/12.

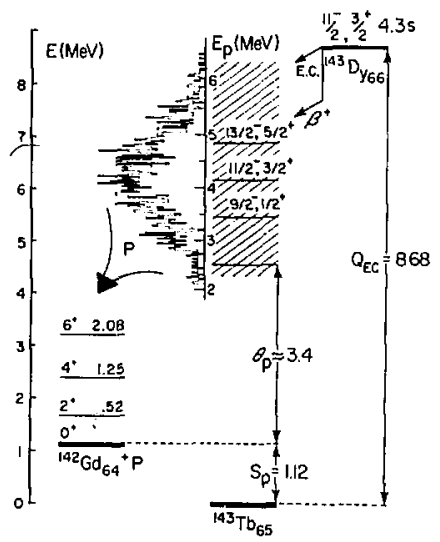

Fig. 4. Schematic representation of the ${ }^{143} \mathrm{Dy}$ decay based on mass values from Liran and Zeldes. ${ }^{3}$

XBL 8211-11956A 


\title{
Beta Endpoint Measurements of Neutron-Deficient Cesium Isotopes
}

\author{
R. Parry, M. Cable. J. Honkanen, * J. Wouters, ${ }^{+}$ \\ S. Zhou, Z.Y. Zhou, ${ }^{\S}$ and Joseph Cerny'
}

The beta decay energies of neutron-deficient ${ }^{119-123} \mathrm{Cs}$ isotopes have been measured. The total decay energies of ${ }^{122 m} \mathrm{Cs}\left(Q_{\mathrm{FC}}=6.95 \pm 0.23 \mathrm{MeV}\right)$ and ${ }^{119} \mathrm{Cs}$ $\left(Q_{E C}=6.26 \pm 0.29 \mathrm{MeV}\right)$ are new measurements based on the $8^{-}$isomeric state in ${ }^{122} \mathrm{Cs}$ decaying to the $2217.3 \mathrm{keV} 8^{+}$level in ${ }^{122} \mathrm{Xe}$ and the $9 / 2^{+}$ground state in ${ }^{119} \mathrm{Cs}$ decaying to the $257.9 \mathrm{keV} 9 / 2^{+}$level in ${ }^{119} \mathrm{Xe}$, respectively. Our measured total decay energies for the cesium isotopes have significantly refined the uncertainty of previous measurements. Comparison of the deduced binding energies of the xenon nuclei from the direct-mass-measured cesium parent isotopes with those of theoretical predictions provides insight into the mass surface in this region of isomer-rich nuclei.

The beta endpoint measurements were accomplished using the 88-Inch Cyclotron on-line mass separator RAMA. Standard beta-gamma coincidence techniques were applied using a $\Delta \mathrm{E}-\mathrm{E}$ beta detector telescope arrangement of NE102 plastic scintillator (15 $\mathrm{MeV}$ positron energy range) and a gamma-ray detector (23\% coaxial Ge crystal) mounted opposite the plastic telescope. Calibration of the beta detector was accomplished using known beta endpoint energies from the strongest decay branches of ${ }^{124} \mathrm{Cs}(4.38 \pm 0.14 \mathrm{MeV})$, ${ }^{66} \mathrm{Ga}(4.153 \pm 0.004 \mathrm{MeV})$, and two branches in the decay of ${ }^{64} \mathrm{Ga}(6.05 \pm 0.03$ and $2.79 \pm 0.08 \mathrm{MeV})$. The cesium isotopes were produced via $140 \mathrm{MeV}{ }^{14} \mathrm{~N}$ bombardment of ${ }^{n !} \mathrm{CdS}$ and the gallium isotnpes by 110 $\mathrm{MeV}{ }^{14} \mathrm{~N}$ on ${ }^{\text {nnt }} \mathrm{Ni}$.

The data were collected using hardwire coincidences between the beta telescope and the gamma detector. Analysis of one component of the beta decay in to the daughter xenon nucleus was accomplished by suitable soltware gating conditions on the desired xenon gamma ray. Endpoint information was established by a "shape fitting" method. Instead of attempting to elucidate a response function correction and using the Fermi-Kurie method, a technique of numerically interpolating between the shapes of calibrated spectra was used, otherwise known as "stretch fitting". 1 A smooth curve is fitted through a standard positron spectrum numerically generating a standard experimental shape. Other intended calibration beta spectra are then normalized in intensity and fit to the standard shape through a nonlinear least squares algorithm, which generates linear stretch factors that are proportional to their endpoint. The result is a linear calibration curve that relates stretch factor to endpoint energy. The un. known nuclei are fit to the standard shape in the same manner. Their stretch factors are then applied to the calibra'ion curve generating endpoint values. This method provides a unique way to avoid both the need for a detector response correction to remove distortion effects and the complicated process of iteration and unfolding common to the Fermi-Kurie technique.

A summary of our measurements, the mass excesses of the xenon nuclei, and comparison with two mass predictions, Liran-Zeldes (L-Z) and GarveyKelson (G-K), is presented in Table $1 .{ }^{2-6}$ The literature values for $Q_{E C}$ of ${ }^{12 i} \mathrm{Cs}$ and ${ }^{123} \mathrm{Cs}$ have unrealistic uncertainties for the decay measurements involved. Or- experimental values of the uncertainties present a smost reasonable estimation of the error. It should be noted that the experimental $Q_{E C}$ for ${ }^{122 m} \mathrm{Cs}$ implies the isomeric level is quite close to the ground state, which is also noted by Epherre, et al. ${ }^{\top}$

An interesting disagreement of about $750 \mathrm{keV}$ between our deduced mass excess of ${ }^{120} \mathrm{Xe}$ and that of the literature values and mass predictions is evident in Table 1. There are several possibilities for this discrepancy. The literature mass excess for this nucleus is in error (the value was determined by measuring the ratio of positron emission to electron capture for the decay of ${ }^{120} \mathrm{Xe}$ to ${ }^{120} \mathrm{I}$ ); the majority of the beta strength is to a higher-lying $2^{+}$state in the Xe daughter; or, finally, the mass excess for the parent, ${ }^{120} \mathrm{Cs}$, has larger uncertainty than reported. The lower. mass cesium isotopes (boginning at ${ }^{120} \mathrm{Cs}$ ) denote an interesing region for experimental study because of the onset of observed beta-delayed alpha and proton emission.

\section{Footnotes and References}

*On leave from: Department of Physics, University of Jyväskylä, Finland

†Present address: CNC Division, Los Alamos National Laboratory, Los Alamos, New Mexico

$\ddagger$ On leave from: lnstitute of Atomic Energy, Beijing, China

SOn leave from: Department of Physics, Nanking University, China

1. C.N. Davids, C.A. Gagliardi, M.J. Murphy, and E.B. Norman, Phys. Rev. C19 (1979) 1463 
2. S. Liran and N. Zeldes, At. Data and Nucl. Data Tables 17 (1976) 431; J. Janecke, ibid., 455

3. A.H. Wapstra and K. Bos, to be published (1983)

4. K. Sofia, B.N. Subba Rao, and J.E. Crawford, Phys. Rev. C24 (1981) 1615

5. J.M. D'Auria, J.W. Gruter, L. Westgaard, G. Nyman, P. Peuser, E. Roeckl, and H. Otto, CERN, Proc. 3rd Int. Conf. Nucl. Far from Sta- bility (1976) 100

6. T. Batsch, M. Nowickj, J. Zylicz, D.D. Bogdanov, V.A. Karnaukhov, L.A. Pietrov, and A. Plochocki, CERN, Proc. 3rd Int. Conf. Nucl. Far from Stability (1976) 106

7. M. Epherre, G. Audi, C. Thibault, R. Klapisch, G. Huber, F. Touchard, and H. Wollnik, Phys. Rev. C19 (1979) 1504

Table I: Measured decay energies of the Cs isotopes and deduced mass excesses of the Xe daughters.

\begin{tabular}{|c|c|c|c|c|c|c|}
\hline \multirow[t]{2}{*}{ Nuclide } & \multicolumn{2}{|c|}{$\begin{array}{c}\text { Decay Energy, } Q_{\mathrm{EC}} \\
(\mathrm{MeV})\end{array}$} & \multicolumn{2}{|c|}{$\begin{array}{c}\text { Xe Mass Excess } \\
(\mathrm{MeV})\end{array}$} & \multicolumn{2}{|c|}{$\begin{array}{c}\Delta=\text { ME (this work) } \\
-M E\left(\text { theory }{ }^{2}\right) \\
(\mathrm{MeV})\end{array}$} \\
\hline & This Work & Literature & This Work & Literature & $\mathrm{L}-\mathrm{Z}$ & $G-K$ \\
\hline $\begin{array}{l}{ }^{123} \mathrm{Cs} \\
{ }^{122} \mathrm{Cs} \\
{ }^{122 m} \mathrm{Cs} \\
{ }^{121} \mathrm{Cs} \\
{ }^{120} \mathrm{Cs} \\
{ }^{119} \mathrm{Cs}\end{array}$ & $\begin{array}{l}4.05 \pm 0.18 \\
7.05 \pm 0.18 \\
6.95 \pm 0.23 \\
5.21 \pm 0.22 \\
7.38 \pm 0.23 \\
6.26 \pm 0.29\end{array}$ & $\begin{aligned} 4.0 & \pm 0.1^{4} \\
7.05 & \pm 0.40^{5} \\
& - \\
5.40 & \pm 0.02^{4} \\
7.3 & \pm 0.5^{6} \\
& -\end{aligned}$ & $\begin{array}{c}-85.19 \pm 0.19 \\
-85.01 \pm 0.20 \\
- \\
-82.27 \pm 0.23 \\
-81.19 \pm 0.26 \\
-78.45 \pm 0.32\end{array}$ & $\begin{array}{c}-85.257 \pm 0.016 \\
-85.344 \pm 0.414 \\
- \\
-82.459 \pm 0.076 \\
-82.028 \pm 0.284 \\
-78.818 \pm 0.156\end{array}$ & $\begin{array}{c}-0.13 \\
0.05 \\
- \\
0.08 \\
0.73 \\
0.27\end{array}$ & $\begin{array}{c}-0.08 \\
0.16 \\
- \\
0.01 \\
0.86 \\
0.47\end{array}$ \\
\hline
\end{tabular}

\title{
Heavy Actinide Products from Reactions of ${ }^{18} \mathrm{O},{ }^{22} \mathrm{Ne}$, and ${ }^{136} \mathrm{Xe}$ with ${ }^{254} \mathrm{Es}$
}

\author{
M. Schädel, * R.W. Lougheed, J.H. Landrum,' J.F. Wild, R.J. Dougan. ${ }^{\dagger}$ \\ A.D. Hoover, ${ }^{\dagger}$ E.K. Hulet, ${ }^{\dagger}$ G.R. Bethune, ${ }^{\ddagger}$ A. Ghiorso, M.J. Nurmia, \\ L.P. Somerville, K.J. Moody, and G.T. Seaborg
}

To find the best target-and-projectile combination that gives the highest yield of product for heavy neutron-rich transplutonium isotopes and to gain knowledge about the production mechanism, we carried out a series of bombardments using ${ }^{254} \mathrm{Es}$ as the target nuclide and ${ }^{18} \mathrm{O},{ }^{22} \mathrm{Ne}$, and ${ }^{136} \mathrm{Xe}$ as the projectile. In our experiments we used chemical techniques to separate element fractions from a catcher foil after the end of the bombardment, and measured these fractions to obtain $\alpha$-energy spectra and to count SF events.

Although the data evalution is still in progress, some interesting observations can be made. Cross sections for the Fm and $\mathrm{Md}$ isotopes and ${ }^{239}$ No from the reactions of $121 \mathrm{MeV}{ }^{22} \mathrm{Ne}, 125 \mathrm{MeV}{ }^{22} \mathrm{Ne}$, and 98 $\mathrm{MeV}{ }^{18} \mathrm{O}$ with ${ }^{254} \mathrm{Es}$ are shown in Fig. 1. Fermium formation cross sections from $97 \mathrm{MeV}{ }^{18} \mathrm{O}+{ }^{248} \mathrm{Cm}$ were taken from ref. 1 and are shown for comparison.

For the ${ }^{22} \mathrm{Ne}+{ }^{254} \mathrm{Es} \mathrm{r}$ uction, we observed only a very slight increase in cross sections as the incident energy increased from $121 \mathrm{MeV}$ to $125 \mathrm{MeV}$. The shape of the isotopic distributions shown do not shift or change significantly. To determine the most probable mass (Ap), we fitted a Gaussian curve to the data and held $\sigma=0.977 u$ (FWHM $=2.3$ ) fixed. For the Fm isotopes, we found $A p=254.5$ and $A p(M d)=256.0$.

More neutron-rich products were observed in the ${ }^{18} \mathrm{O}+{ }^{254} \mathrm{Es}$ reaction at $98 \mathrm{MeV}$ incident energy than were found at $121 \mathrm{MeV}{ }^{22} \mathrm{Ne}$. Ap values of 255.5 for $\mathrm{Fm}$ and 257.1 for Md were determined. This makes the ${ }^{18} \mathrm{O}$ the most favorable projectile for the production of neutron-rich heavy actinides when compared to ${ }^{22} \mathrm{Ne}$. 


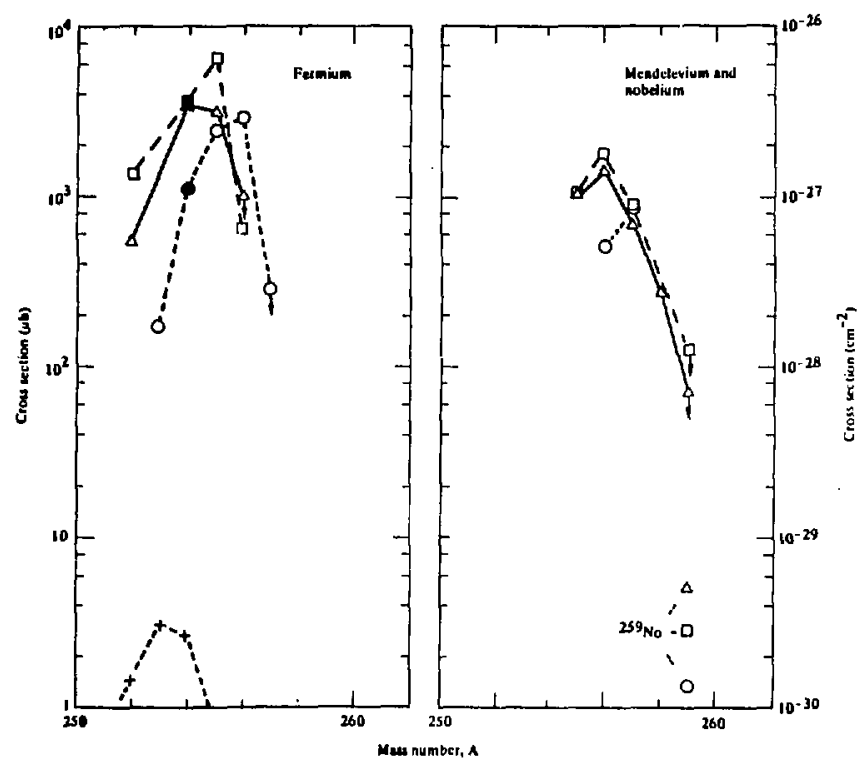

Fig. 1. Cross sections for ${ }^{259} \mathrm{No}$ and $\mathrm{Fm}$ and $\mathrm{Md}$ isotopes in the reaction $12 \mathrm{I} \mathrm{MeV}{ }^{22} \mathrm{Ne}+{ }^{254} \mathrm{Es}$ (symbol $=\Delta$ ), $126 \mathrm{MeV}{ }^{22} \mathrm{Ne}+{ }^{254} \mathrm{Es}$ (symbol $=\square$ ) and $98 \mathrm{MeV}{ }^{18} \mathrm{O}+{ }^{254} \mathrm{Es}$ (symbol $=\mathrm{O}$ ). Cross sections for Fm isotopes in the reaction $97 \mathrm{MeV}{ }^{18} \mathrm{O}+{ }^{248} \mathrm{Cm}($ symbol $=+$ ) from ref. I are shown for comparison. Open symbols are independent; full symbols are cumulative yiejds; arrows indicate that these cross sections are upper limits onl! The curves are drawn to connect the data points.

XBL 8210-3289

The Fm cross section is enhanced by a factor of $10^{3}$ and the $\mathrm{Md}$ cross section by more than $10^{4}$ when the yields from an ${ }^{254} \mathrm{Es}$ target are compared with a ${ }^{248} \mathrm{Cm}$ target. ${ }^{1}$

Assuming an iqual transfer probability for a given number of protons $(\Delta Z)$ and neutrons $(\Delta N)$ in the reactions with ${ }^{18} \mathrm{O}$ as a projectile on ${ }^{248} \mathrm{Cm}$ and ${ }^{254} \mathrm{Es}$ targets, we were able to extrapolate $\left({ }^{254} \mathrm{Es}, \mathrm{x}\right)$ cross sections from the cross sections measured with a ${ }^{248} \mathrm{Cm}$ target. This is possible only in these light ion reactions where the excitation energies are less than the neutron separation energies, whereas there are large differences in cross sections for products with the same number of transferred nucleons $(\Delta Z, \Delta N)$ in reactions with ${ }^{238} U$ projectiles on ${ }^{238} \mathrm{U}$ and ${ }^{248} \mathrm{Cm}$ targets. ${ }^{2}$ This is iecause of higher excitation energies and differences in $\Gamma_{n} / \Gamma_{\text {tol }}$ for the different primary fragments formed. In Fig. 2a, we compare cross sections for a two-proton and $\mathrm{x}$ neutron $(\Delta N)$ transfer in the reaction of ${ }^{18} \mathrm{O}$ with ${ }^{248} \mathrm{Cm}$ and ${ }^{254} \mathrm{Es}$ resulting in $\mathrm{Cf}$ and $\mathrm{Md}$ isotopic distributions, respectively. Positive $\Delta N$ values characterize neutron transfer from the projectile to the target, while negative $\Delta N$ values refer to transfer in the opposite direction. Gaussian curves with $\sigma=0.977 \mathrm{u}$ (FWHM $=2.3$ ) were fitted to the data. Similar results are shown in Fig. $2 b$ for ${ }^{22} \mathrm{Ne}$ as a projectile. Cross sections for the rimeproton transfer are in good agreement with ${ }^{18} \mathrm{O}$. while the small differences in cross section for ${ }^{22} \mathrm{Ne}$ may be due to a slightly higher projectile energy with the ${ }^{254} \mathrm{Es}$ target. We used these extrapolated isotope distributions and a similar method for three- and four- 


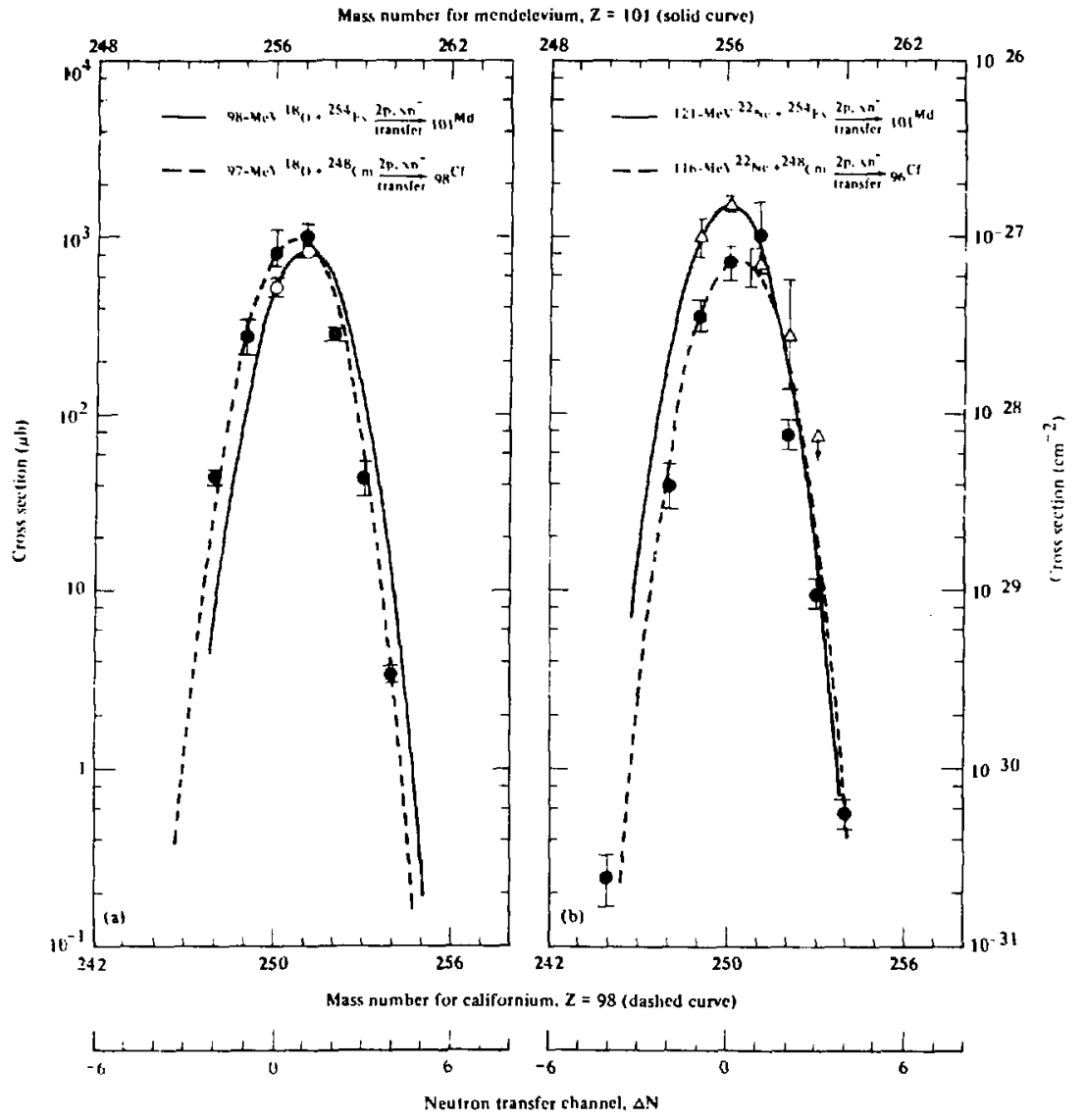

$X B L 8211-3290$

Fig. 2.

(a) Comparison of two-proton, $\mathrm{x}$-neutron transfer $(\Delta \mathrm{N})$ cross sections in the reaction of ${ }^{18} \mathrm{C}$ with ${ }^{248} \mathrm{Cm}$ and ${ }^{254} \mathrm{Es}$ resulting in $\mathrm{Cf}$ (dashed cutve, lower nass number scale) and $\mathrm{Md}$ (solid curve, upper mass number scale) isotopes. Curves are Gaussian with $\sigma=0.977 \mathrm{u}(\mathrm{FWHM}=2.3$ )

(b) Same as $2 \mathrm{a}$ but ${ }^{\mathrm{yith}}{ }^{22} \mathrm{Ne}$ as projectile. 
proton transfer to estimate cross sections for unknown neutron-rich heavy actinides. The results are given in Table 1. It is important to note that these estimates still contain large uncertainties because we are not yet able to predict the exact Ap positi of the isotope distribution. However, it is fascinating to see that exotic nuclei like ${ }^{262} \mathrm{Md}$ or ${ }^{262} \mathrm{Lr}$ should be accessible in reactions of ${ }^{18} \mathrm{O}$ with ${ }^{254} \mathrm{Es}$.

\section{Footnotes and References}

*Gesellschaft für Schwerionenforschung, 6100 Darmstadt, West Germany thawrence Livermore Vationa! Laboratory, Ljuermore, CA 94550

$\ddagger$ Bethure-Cookman College, Daytona Beach, FL 32015

I. D. Lee, H. von Gunten, B. Jacak, M.J. Nurmia, Y.f. Liu, C. Luo, G.T. Seaborg, and D.C. Hoffman, Phys. Lett. C25 (1982) 286

2. M. Schädcl, W. Bruchle, H. Gaggeler, J.V. Kratz, K. Summerer, G. Wirth, G. Herrmann, R. Stakemann, G. Tittel, N. Trautmann, J.M. Nitschke, E.K. Hulet, R.W. Lougheed, R.L. Hahn, and R.L. Ferguson, Phys. Rev. Lett. 48 (1982) 852

Table I: Estimated transfer cross sections in $\mu \mathrm{b}$ for unknown neutson-rich actinides.

\begin{tabular}{|l|cc|cc|cc|c|}
\hline Reaction & \multicolumn{6}{|c|}{ Transfer cross section $(\mu \mathrm{b})$} \\
\cline { 2 - 7 } & ${ }^{260} \mathrm{Md}$ & ${ }^{261} \mathrm{Md}$ & ${ }^{260} \mathrm{No}$ & ${ }^{261} \mathrm{No}$ & ${ }^{261} \mathrm{Lr}$ & ${ }^{262} \mathrm{Lr}$ & $\mathrm{Z}=104$ isotore \\
\hline${ }^{18} \mathrm{O}+{ }^{254} \mathrm{Es}$ & 9. & .3 & 5. & .2 & .7 & .3 & $\leq .05$ \\
${ }^{22} \mathrm{Ne}+{ }^{254} \mathrm{Es}$ & .3 & $\leq .05$ & 2. & .1 & 1. & .2 & $\leq .05$ \\
\hline
\end{tabular}

\title{
A New 100-ms Spontaneous Fission Activity Abundantly Produced in the Reaction $99 \mathrm{MeV}{ }^{18} \mathrm{O}+{ }^{254} \mathrm{Es}$
}

\author{
L.P. Somerville, * M.J. Nurmia, M. Schädel, A. Ghiorso, \\ J.M. Nitschke, R.W. Lougheed, J.H. Landrum, ${ }^{\ddagger}$ and E.K. Hulet ${ }^{\ddagger}$
}

Numerous authors have shown that the use of transfer reactions between neutron-rich actinide targets and neutron-rich projectiles with $A \leq 22$ provides a way to investigate the frontier of very hcavy neutronrich nuclei. ${ }^{1,2}$ The Spontaneous Fission (SF) properties of these heavy nuclei, such as half-life and fission mass distribution, are quite interesting to investigate, particularly since the second barrier to fission is predicted to drop below the ground state for some of them. ${ }^{3}$ Previous transfer reaction studies have made use of targets up to ${ }^{248} \mathrm{Cm}$ (ref. 1). Using an ${ }^{254} \mathrm{Es}$ target one would hope that even heavier, new shurt-lived isotopes could be observed.

The neutron-rich projectile ${ }^{18} \mathrm{O}$ was chosen to bombard a small $3-\mathrm{mm}$-diameter $25-\mu \mathrm{g} / \mathrm{cm}^{2}{ }^{254} \mathrm{Es}$ target for this purpose. The target material was produced by irradiating ${ }^{252} \mathrm{Cf}$ with neutrons at the High Flux Reactor (HIFR) at Oak Ridge, Tennessee. Another article by $M$. Schädel, et al, in this report presents thr cross section measurements for production of longer-lived ${ }_{100} \mathrm{Fm},{ }_{101} \mathrm{Md}$, and ${ }_{102}$ No nuclei in the same reaction ${ }^{18} \mathrm{O}$ ${ }^{100}{ }^{254} E_{S}$ by chemically separating actinide element fractions from recoil catcher foils. To search for short-lived SF emitters with half lives betwecn $10 \mathrm{~ms}$ and 5 seconds, we used the recoil tape-transport system with fixed mica track detectors described in the 1977-1978 issue of the annual report.

The decay curve in Fig. 1 shows that a 100-ms SF activity is clesily observed in the reaction $99 \mathrm{MeV}{ }^{18} \mathrm{O}$ $+{ }^{254}$ Es with a very large production cross section of 1 
$\mu \mathrm{b}$. This cross section is very large compared to the usual nanobarn values measured for other millisecondlived SF activities." The weighted-average half life from two experiments is $105 \pm 7$ ms. From the consistency in hali-life and production cross-section measurements in separate experiments with tape speeds of 0.2 and $0.9 \mathrm{~m} / \mathrm{s}$, tape speed-independent background effects such as neutron-induced fission background can be excluded. The long-lived background that is observed in Fig. 1 is due to production of ${ }^{256} \mathrm{Fm}$ (ref. 2) and ${ }^{256} \mathrm{Md}$, which decays by electron capture to the 2.6-hour SF emitter ${ }^{256} \mathrm{Fm}$.

There are indications that a SF activity with a similar half life may be produced in the reaction 73 $\mathrm{MeV}{ }^{13} \mathrm{C}+{ }^{254} \mathrm{ES}(68-75 \%) /{ }^{250} \mathrm{Cf}(25-32 \%)$ with a much lower cross section of $\sim 170 \mathrm{nb}$, assuming production from the ${ }^{254} \mathrm{Es}$ portion of the target. In the reaction $125 \mathrm{MeV}{ }^{22} \mathrm{Ne}+{ }^{254} \mathrm{Es}(63 \%) /{ }^{250} \mathrm{Cr}(37 \%)$, crosssection upper limits of $80 \mathrm{nb}$ and $130 \mathrm{nb}$ were established for production of a $100-\mathrm{ms}$ SF activity from ${ }^{254} \mathrm{Es}$ and ${ }^{250} \mathrm{Cr}$, respectively.

We do not yet know the identity of this $100-\mathrm{ms}$ SF emitter. But ${ }^{261} \mathrm{Lr}$ and ${ }^{261} \mathrm{Md}$ are two candidates consistent with most of our cross-secti-3n data and the present understanding of the reactions required to produce these isotopes. ${ }^{1,2}$ A 100 -ms SF half life for ${ }^{26} \mathrm{Lr}$, however, creates two interesting problems: (1) SF decay appears to be highly hindered for all other known isotopes of element 103; and (2) this assignment and a possible assignment of ${ }^{262} \mathrm{Rf}$ to a $50-\mathrm{ms} \mathrm{SF}$ activity ${ }^{4}$ would imply an odd-proton hindrance factor of only 2 , compared to at least $10^{5}$ for the similar pairs ${ }^{257} \mathrm{LJ}$ ${ }^{258} \mathrm{Rf},{ }^{255} \mathrm{Lr}-{ }^{256} \mathrm{Rf},{ }^{255} \mathrm{Lr}-{ }^{254} \mathrm{Rf}$, and a tentative $\sim 10^{3}$ odd-neutron hindrance factor for isotopes of element 104. It seems very likely that one of the tentative assignments ${ }^{262} \mathrm{Rf}(50 \mathrm{~ms})$ or ${ }^{261} \mathrm{Lr}(100 \mathrm{~ms})$ is incorrect.

A further intriguing possibility is that the $100-\mathrm{ms}$ SF activity reported here might be the same $100 \pm 50$ ms SF activity produced with a low cross section of 0.5 $\mathrm{nb}$ in the reaction $113 \mathrm{MeV}{ }^{22} \mathrm{Ne}+{ }^{242} \mathrm{Pu}$ by Oganessian, et al. ${ }^{5}$ The Dub.a group has maintained that this 100 -ms SF activity is due to ${ }^{260} 104$ and claimed this SF activity for the discovery of element $104{ }^{6}$ However, we tave not observed an $\sim 100-\mathrm{ms}$ SF activity in other reactions ${ }^{249} \mathrm{Bk}\left({ }^{15} \mathrm{~N}, 4 \mathrm{n}\right){ }^{260} \mathrm{Rf},{ }^{248} \mathrm{Cm}\left({ }^{16} \mathrm{O} .4 \mathrm{n}\right){ }^{260} \mathrm{Rf}$, and ${ }^{249} \mathrm{Cf}\left({ }^{18} \mathrm{O}, \alpha 3 \mathrm{n}\right)^{260} \mathrm{Rf}$ (ref. 4) in which the production cross sections for ${ }^{260} \mathrm{Rf}$ are predicted to be much higher that in the reaction ${ }^{242} \mathrm{Pu}\left({ }^{22} \mathrm{Ne}, 4 n\right){ }^{260} \mathrm{Rf}$. ${ }^{261} \mathrm{Lr}$ or ${ }^{261} \mathrm{Md}$, on the other hand, shoulc have very low production cross sections in the reaction $113 \mathrm{MeV}{ }^{22} \mathrm{Ne}+$ ${ }^{242} \mathrm{Pu}$, which is not in contradiction with the available cross-section data.
A very promising way to test the possible assignment of ${ }^{261} \mathrm{Lr}$ to a $100-\mathrm{ms}$ SF activity would be to search for $100-\mathrm{ms} \mathrm{SF}$ events in the reaction $48.5 \mathrm{MeV}$ ${ }^{10} \mathrm{Be}+{ }^{254} \mathrm{Es}$. Although a ${ }^{10} \mathrm{Be}$ beam would be radioactive and difficult to obtain, the calculated production cross section for ${ }^{261} \mathrm{Lr}$ is nearly a millibarn!

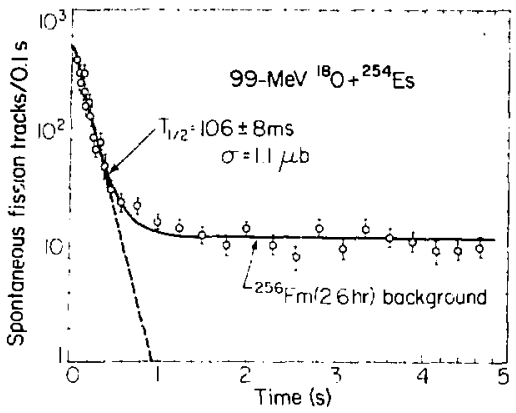

Fig. 1. Decay curve for the bombardment $99 \mathrm{MeV}{ }^{18} \mathrm{O}$ $+{ }^{254} \mathrm{Es}(94.6 \%) /{ }^{250} \mathrm{Cf}(5.4 \%)$ obtained using the recoil tape-transport system described in the 1977-1978 annual report. The tape speed was $0.2 \mathrm{~m} / \mathrm{s}$. See the text for possible assignments for this 100 -ms SF activity.

XBL 835-9513

\section{Footnotes and References}

*Present address: Physics Division, Argonne National Laboratory, Argonne, IL 60439

†Gesellschaft für Schwerionenforschung $\mathrm{mbH}, \mathrm{D}-6100$ Darmstadt, F.R. Germany

\$Lawrence Livermore National Laboratory, Livermore, California 94550

1. D. Lee, et al., Phys. Rev. C25 (1982) 286

2. M. Schädel, et al., Lawrence Livermore National Laboratory Nuciear Chemistry Division Annual Report. Fiscal Y'ear 1982; also this report

3. J. Randrup, et al., Phys. Rev. C13 (1976) 229

4. L.P. Somerville, Ph.D. Thesis, U.C. Berkeley, LBL-14050 (1982)

5. Yu. Ts, Cganessian, et al., Atomnaya Énergiya 28 (1970) 393; Sov. J. Atomic Energy 28 (1970) 502

6. G.N. Flerov, et al., Phys. Lett. 13 (1964) 73 


\title{
An Upper Limit for Spontaneous Fission Branching in ${ }^{254}$ No
}

\author{
L.P. Somerville,* A. Ghiorso, M.J. Nurmia, and J.M. Nitschke
}

The Spontaneous Fission (SF) branching in ${ }^{254}$ No is an important quantity because it is a measure of the strength of the 152-reutron subshell and its effect on the fission probability of nobelium isotopes. A 152 . neutron subshell effect in alpha decay from the ground states of nobelium' and lighter elements ${ }^{2}$ is well known. In many published SF half-life systematics a partial SF half life is plotted for ${ }^{254}$ No. However, no actual measurements have been reported in the literature. An upper limit for SF branching of 0.06 percent can be established from the ratio of the upper-limit cross section for SF and the cross section for production of ${ }^{254}$ No alpha particles in separate experiments by Donets, et al., ${ }^{3}$ and Flerov, et al.; ${ }^{4}$ this is plotted in Fig. 1. Knowing only this upper limit, fig. I shows that compared to the SF half lives of other nobelium isotopes a 152-neutron subshell effect is clearly present.

In a recent experiment we thought we had found a SF brasch from ${ }^{254}$ No. In the bombardment $75 \mathrm{MeV}$ ${ }^{12} \mathrm{C}+{ }^{249} \mathrm{Cf}$ a $47 \pm 13-\mathrm{s}$ SF activity with a production cross section of $\sim 9 \mathrm{nb}$ was observed. The observation time in this experiment was $65 \mathrm{~s}$; it was therefore difficult to determine whether the half life was $47 \mathrm{~s}$ or perhaps longer. A possible SF branch of 0.4 percen. determined from these experiments would be consistent with the 14 SF events that decayed with a half life of $\sim 70$ s observed by Ghiorso, et al., 5 in the reactions 197- and $227-\mathrm{MeV}{ }^{48} \mathrm{Ca}+{ }^{208} \mathrm{~Pb}$. However, in another reaction, $72 \mathrm{MeV}{ }^{13} \mathrm{C}+{ }^{245} \mathrm{Cm}$, an upper limit of 0.05 percent for the fission branch of ${ }^{254}$ No was established, assuming a calculated production cross section of $1 \mu \mathrm{b}$ for ${ }^{254} \mathrm{No}$. This result is in agreement with the upper limit of 0.06 percent established from experiments by Donets, et al., and Flerov, et al.

The origin of the 47-s SF activity is uncertain and will have to be determined in future cross bombardments. The SF branch may still be observable or perhaps an even lower limit may be established from current ${ }^{48} \mathrm{Ca}+{ }^{208} \mathrm{~Pb}$ bembardments. A maximum production cross section of $3.4 \mu \mathrm{b}$ has been measured by Nitschke, et al., ${ }^{\text {for this reaction. }}$

\section{Footnote and References}

*Present address: Physics Division, Argonne National Laboratory, Argonne, Il 60439
1. L.P. Somerville, Ph.D. thesis, Lawrence Berkeley Laboratory Report LBL-14050 (1982)

2. A. Ghiorso, et al., Phys. Rev. 95 (1954) 293

3. E.D. Donets, et al., Atomnaya Energiya 20 (1966) 223; Sov. J. Atomic Energy 22 (1967) 611

4. G.N. Flerov, et al., Atomnaya Energiya 22 (1967) 494

5. A. Ghiorso, unpublished resul's (1976)

6. J. Randrup, et al., Phys. Rev. C13 (1976) 229

7. J.M. Nitschke, et al., Nucl. Phys. A313 (1979) 236

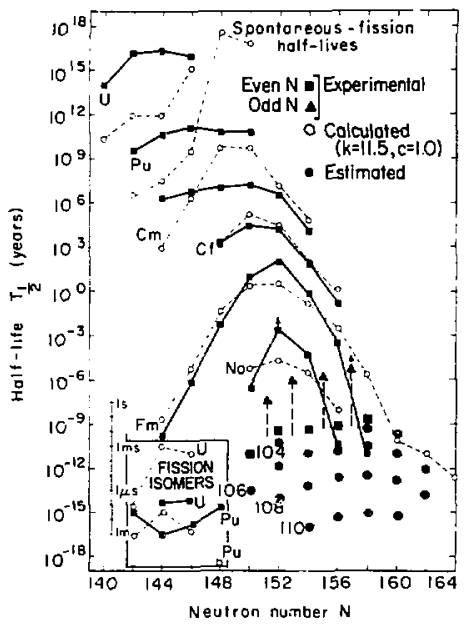

Fig. 1. Spontaneous Fission (SF) half-life systematics for even-even nuclei. ${ }^{1,6}$ The half lives for element y04 nuclei are uncertain. Note the upper limit on the partial SF half life of ${ }^{254}$ No and the extra stability at neutron number 152 associated with a neutron subshell closure.

XBL 756-3150B 


\title{
Results of Investigations on the New 15- to 24-ms Spontaneous Fission Activities
}

\author{
L.P. Somerville, M.J. Nurmia. A. Ghiorso, and J.M. Nitschke
}

In the last annual report ${ }^{1}$ Spontancous Fission (SF) activities with half lives between 15 and $24 \mathrm{~ms}$ were reported in the following bombardments: $80 \mathrm{MeV}$ ${ }^{15} \mathrm{~N}+{ }^{249} \mathrm{Bk}, 92 \mathrm{MeV}{ }^{16} \mathrm{O}+{ }^{248} \mathrm{Cm}, 109 \mathrm{MeV}{ }^{18} \mathrm{O}+$ ${ }^{248} \mathrm{Cm}$, and $96 \mathrm{MeV}{ }^{18} \mathrm{O}+{ }^{249} \mathrm{Cr}$. The production cross sections for the reactions ${ }^{15} \mathrm{~N}+{ }^{229} \mathrm{Bk} .{ }^{16} \mathrm{O}+{ }^{248} \mathrm{Cm}$, and ${ }^{18} \mathrm{O} \div{ }^{249} \mathrm{Cf}$ are consistent with the assignment of an $\sim 20 \mathrm{~ms} S F$ activity to ${ }^{260} \mathrm{Rf}$. Furthermore, an excitation function for the 20-ms SF activity produced in the reactions 78 to $86 \mathrm{MeV}{ }^{15} \mathrm{~N}+{ }^{249} \mathrm{Bk}$ was zonsistent with the shapes of other measured $\left({ }^{13} \mathrm{~N}, 4 \mathrm{n}\right)$ excitation functions. ${ }^{2}$ The measured cross sections for the 15- to $24 \mathrm{~ms} \mathrm{SF}$ activities produced in the reactions 109 to $119 \mathrm{MeV}{ }^{18} \mathrm{O}+{ }^{248} \mathrm{Cm}$ and 88 to $100 \mathrm{MeV}{ }^{15} \mathrm{~N}+$ ${ }^{249} \mathrm{Bk}$, however, now seem much too high for ${ }^{260} \mathrm{Rf}$ or ${ }^{258} \mathrm{Rf}$ ( $13 \mathrm{~ms}$ ) assignments. One hypothesis is that at least two 15- to 24-ms SF activities exist - ${ }^{260} \mathrm{Rf}$ produced in the reactions 78 to $86 \mathrm{MeV}{ }^{15} \mathrm{~N}+{ }^{249} \mathrm{Bk}$ and ${ }^{16} \mathrm{O}+{ }^{248} \mathrm{Cm}$. and at least one other nuclide win $Z<104$ to explain the products of the reactions ${ }^{18} \mathrm{O}+$ ${ }^{248} \mathrm{Cm}$ and 88 to $100 \mathrm{MeV}{ }^{15} \mathrm{~N}+{ }^{249} \mathrm{Bk}$. Within the last year we have performed eleven cross bombardments to try to determine the isctopic assignments that would be consistent with all our data. Table I gives the results of these experiments.

What bave we learned? In the reaction $109 \mathrm{MeV}$ ${ }^{18} \mathrm{O}+{ }^{248} \mathrm{Cm}$ the -20 -ms $\mathrm{SF}$ activity was again observed. Since the measured cross section is $\sim 50$ times larger than that calculated for ${ }^{260} \mathrm{Rf}$ and the measured excitation function is quite broad, this activity is likely to be a transfer product with $Z<104$. Based on the absence of a $2 \mathrm{C}$.ms component when a ${ }^{248} \mathrm{Cm}$ target is bombarded with ${ }^{15} \mathrm{~N}$ or ${ }^{13} \mathrm{C}$ jons, it seems unlikely that this product has mass and atomic numbers near the ${ }^{249} \mathrm{Cm}$ target. In the reaction 86 $\mathrm{MeV}{ }^{15} \mathrm{~N}+{ }^{249} \mathrm{Cf}$ the cross-section upper limil for a possible 20-ms SF activity is only $0.3 \mathrm{nb}$, or $4 \%$ of the calculated maximum ${ }^{249} \mathrm{Cf}\left({ }^{15} \mathrm{~N}, 4 \mathrm{n}\right){ }^{260} \mathrm{Rf}$ cross section. This cross section is much lower than the cross section of $7 \mathrm{nb}$ reported by Demin, et al., ${ }^{3}$ with $93 \mathrm{MeV}{ }^{15} \mathrm{~N}$ ions. ${ }^{260} \mathrm{Rf}$ would be produced in a ${ }^{249} \mathrm{Cf}\left({ }^{15} \mathrm{~N}, \mathrm{p} 3 \mathrm{n}\right){ }^{260} \mathrm{Rf}$ reaction, but the cross section for this reaction is difficult to estimate. We think thai a good estimate could be obtained from the cross section for the quite similar reaction ${ }^{250} \mathrm{Cf}\left({ }^{15} \mathrm{~N}, \mathrm{p} 3 \mathrm{n}\right){ }^{261} \mathrm{Rf}$.
The $\sim 9-\mathrm{ms} S \mathrm{~F}$ activity produced in the reaction $85 \mathrm{MeV}{ }^{12} \mathrm{C}+{ }^{249} \mathrm{Cf}$ is quite likely due to a mixture $\mathrm{Ci}^{\circ}$ ${ }^{258} \mathrm{Rf}(13 \mathrm{~ms})$ and an unidentified 5-ms SF activity, possibly ${ }^{256} \mathrm{R}$ ( ref. 4). The rest of the cross-section upper limits are in most cases too high to draw any conclusions about the 20-ms SF activities, except that they probably are not due to nuclei near the ${ }^{254} \mathrm{Es}$ or ${ }^{250} \mathrm{Cr}$ targets.

Thus, the hypothesis of at least two 20-ms components is still viable. Up to now, in every case that we expected to produce ${ }^{260} \mathrm{Rr}$, a 20 -ms component was observed. The $20-\mathrm{ms}$ component that was observed in the reaction $109 \mathrm{MeV}{ }^{18} \mathrm{O}+{ }^{248} \mathrm{Cm}$ is probably due to a transfer product with $\mathrm{Z}<104$. If, however, in the future it could be shown that a single nuclide could explain all the 15- to 24-ms companents in our data, this activity could not be ${ }^{260} \mathrm{R}$.

\section{Footnote and References}

*Present aúdress: Physics Division, Argonne National Laboratory, Argonne, IL 60439

1. L.P. Somerville, et al., 1980-1981 Lawrence Berkeley Laboratory Annual Report of the Nuclear Science Division, LBL-13366 (1982) p. 48

2. L.P. Somerville, Ph.D. thesis, Lawrence Berkeley Laboratory Report LBL-14050 (1982)

3. A.G. Demin, V.A. Druin, et al., data presented in a talk by V.A. Druin at the Intl. Symp. on Synthesis and Properties of New Elements, Dubna. USSR, (1980); Joint Inst. for Nucl. Research Report D7-80-556, abstract on p. 25 (1980)

4 M.J. Nurmia, et al., unpublished data (1970) 
Table 1

\begin{tabular}{rrr}
\hline Reaction & Half-Life & Cross Section \\
\hline $109 \mathrm{MeV}{ }^{18} \mathrm{O}+{ }^{248} \mathrm{Cm}$ & $22.4 \pm 1.3 \mathrm{~ms}$ & $10 \pm 2 \mathrm{nb}$ \\
$81 \mathrm{MeV}{ }^{13} \mathrm{C}+{ }^{248} \mathrm{Cm}$ & $20 \mathrm{~ms}$ & $\leq 6 \mathrm{nb}$ \\
$97 \mathrm{MeV}{ }^{15} \mathrm{~N}+{ }^{248} \mathrm{Cm}$ & $20 \mathrm{~ms}$ & $\leq 1 \mathrm{nb}$ \\
& $15 \mathrm{~ms}$ & $\leq 2 \mathrm{nb}$ \\
$86 \mathrm{MeV}{ }^{15} \mathrm{~N}+{ }^{249} \mathrm{Cf}$ & $\sim 12 \mathrm{~ms}$ & $\leq 0.3 \pm 0.1 \mathrm{nb}$ \\
& $20 \mathrm{~ms}$ & $\leq 0.3 \mathrm{nb}$ \\
$99 \mathrm{MeV}{ }^{18} \mathrm{O}+{ }^{254} \mathrm{Es}$ & $20 \mathrm{~ms}$ & $\leq 0.5 \pm 0.1 \mu \mathrm{b}$ \\
$99 \mathrm{MeV}{ }^{18} \mathrm{O}+{ }^{250} \mathrm{Cr}$ & $20 \mathrm{~ms}$ & $\leq 1.5 \pm 0.3 \mu \mathrm{b}$ \\
$85 \mathrm{MeV}{ }^{12} \mathrm{C}+{ }^{249} \mathrm{Cf}$ & $\sim 9 \mathrm{~ms}$ & $5 \pm 1 \mathrm{nb}$ \\
$73 \mathrm{MeV}{ }^{13} \mathrm{C}+{ }^{250} \mathrm{Cf}$ & $20 \mathrm{~ms}$ & $\leq 370 \mathrm{nb}$ \\
$73 \mathrm{MeV}{ }^{13} \mathrm{C}+{ }^{254} \mathrm{Es}$ & $20 \mathrm{~ms}$ & $\leq 150 \mathrm{nb}$ \\
$125 \mathrm{MeV}{ }^{22} \mathrm{Ne}+{ }^{254} \mathrm{Es}$ & $20 \mathrm{~ms}$ & $\leq 50 \mathrm{nb}$ \\
$125 \mathrm{MeV}{ }^{22} \mathrm{Ne}+{ }^{250} \mathrm{Cf}$ & $20 \mathrm{~ms}$ & $\leq 80 \mathrm{nb}$ \\
\hline
\end{tabular}




\title{
High Angular Momentum and Gamma De-excitation
}

\author{
Continuum Gamma-Ray Spectroscopy*
}

\author{
R.M. Diamond
}

When angular momentum is added to a nucleus, it is, of course, carried by the individual nucleons, but two limiting types of behavior may be distinguished: 1) a small number of high-j particles align with the rotation axis and 2) the nucleus is deformed and rotates as a whole. At high spin all nuclei seem to show a compromise utilizing both motions. The excited nuclei 'eft as products of $(H I, x n)$ reactions have so many pathways down that none of the $\gamma$-ray transitions have enough intensity to be seen individually until the population gathers near the yrast line. This occurs usually between spin $20-40 h$. All our information on higher states comes from their continuum spectra. Several general types of techniques are being used to study the continuum.

One recent development is the use of $\gamma$-ray multiplicity filters, which involve a number of $\mathrm{NaI}$ detectors to determine the number of coincident $\gamma$ rays per event and thus statistically the $\gamma$-ray multiplicity distribution. A high $\gamma$-ray multiplicity corresponds in general to a large angular momentum. Because of this relationship, a measurement of average $\gamma$-ray multiplicity as a function of $\gamma$-ray transition energy gives direct information on transition energy - spin correlations in the continuum. Examples ${ }^{1}$ for two target-projectile systems at several bombarding energies are shown in Fig. 1. For ${ }^{40} \mathrm{Ar}+{ }^{124} \mathrm{Sn} \rightarrow{ }^{164} \mathrm{Er}^{*}$, there is a pronounced peak in the multiplicity spectrum at all bombarding energies, and it comes at the edge of the 'ump in the intensity spectrum, corresponding to the highest energy and highest spin transition. This peak moves to higher energy with increasing bombarding energy and, hence, higher angular momentum input. This is a nice confirmation of the collective rotation picture for this nuclev: qualitatively agreeing with the rotational expression,

$$
E_{\gamma}=\frac{\hbar^{2}}{2 . g^{(4 I-2)}}
$$

that the $\gamma$-ray energy, $E_{\gamma}$ is proportional to the spin, I.

From the $\gamma$-ray energy of the multiplicity peaks, combined with the maximum spin (obtained from the maximum multiplicity) for a particular reaction, a value for the moment of inertia can be determined by use of eq. (1). The problem is to get this value for the smallest range in spin possible. Other than with crystal balls, the best way is probably to use a total-energy spectrometer, which yields a FWHM of $60.70 \mathrm{~h}$ in spin. The idea is as follows: if one can capture in a large NaI counter most of the $\gamma$-ray energy emitted in the cascades, one can then make cuts at various excitation energies, hence at various spin ranges, and observe the decay spectrum in a coincident external counter viewing the target through a small hole in the sum spectrometer. Examples of such spectra, taken in a $7.6 \times 7.6 \mathrm{~cm}$ $\mathrm{NaI}$ crystal outside a $20 \times 33 \mathrm{~cm}$ sum spe:trometer and in coincidence with consecutive $\sim 4 \mathrm{MeV}$ wide slices of the total energy, are shown in the next figure ${ }^{2}$ for 185 $\mathrm{MeV}{ }^{40} \mathrm{Ar}+{ }^{124} \mathrm{Sn} \rightarrow{ }^{164} \mathrm{Er} *$. The main products are ${ }^{160} \mathrm{Er}$ and ${ }^{159} \mathrm{Er}$, well-deformed prolate nuclei in the ground and $i_{13 / 2}$ bands, respectively, all the way to the highest spins observed. By subtracting one spectrum from the next, we obtain the difference peaks shown at the buttom, which illustrate nicely the increase in $\mathbf{E}_{\text {, }}$ with increase in slice number (excitation energy), and hence spin, as represented by the average multiplicity, $M$, of the two contributing spectra. Converting $M$ to I (essentially by multiplying by two), we can obtain a value for the effective moment of inertia at that average spir from eq. (1).

This moment of inertia is an "effective" one; it is measured along the average of the $\gamma$-cascade pathways and so is an average value for many intersecting (crossing) bands. Another important value is the collective moment of inertia of an individual band. It is smaller and is related to dillerences between $\gamma$-ray energies in the same band and not to the difference between average $\gamma$-ray energies in a pathway (several interesting bands). The angular momentum in these excited band: is carried partly by the rotation of the deformed nucleus as a whole and partly by a few high-j nucleons aligned to the rotation axis, $j_{a}$. The $\gamma$-ray transition energy within the band is then

$$
\mathrm{E}_{\mathrm{\gamma}}=\frac{h^{2}}{2 . g_{\text {oll }}} 4\left(1-\mathrm{j}_{\mathrm{a}}\right)
$$

to be compared with the corresponding form of eq. (1). 


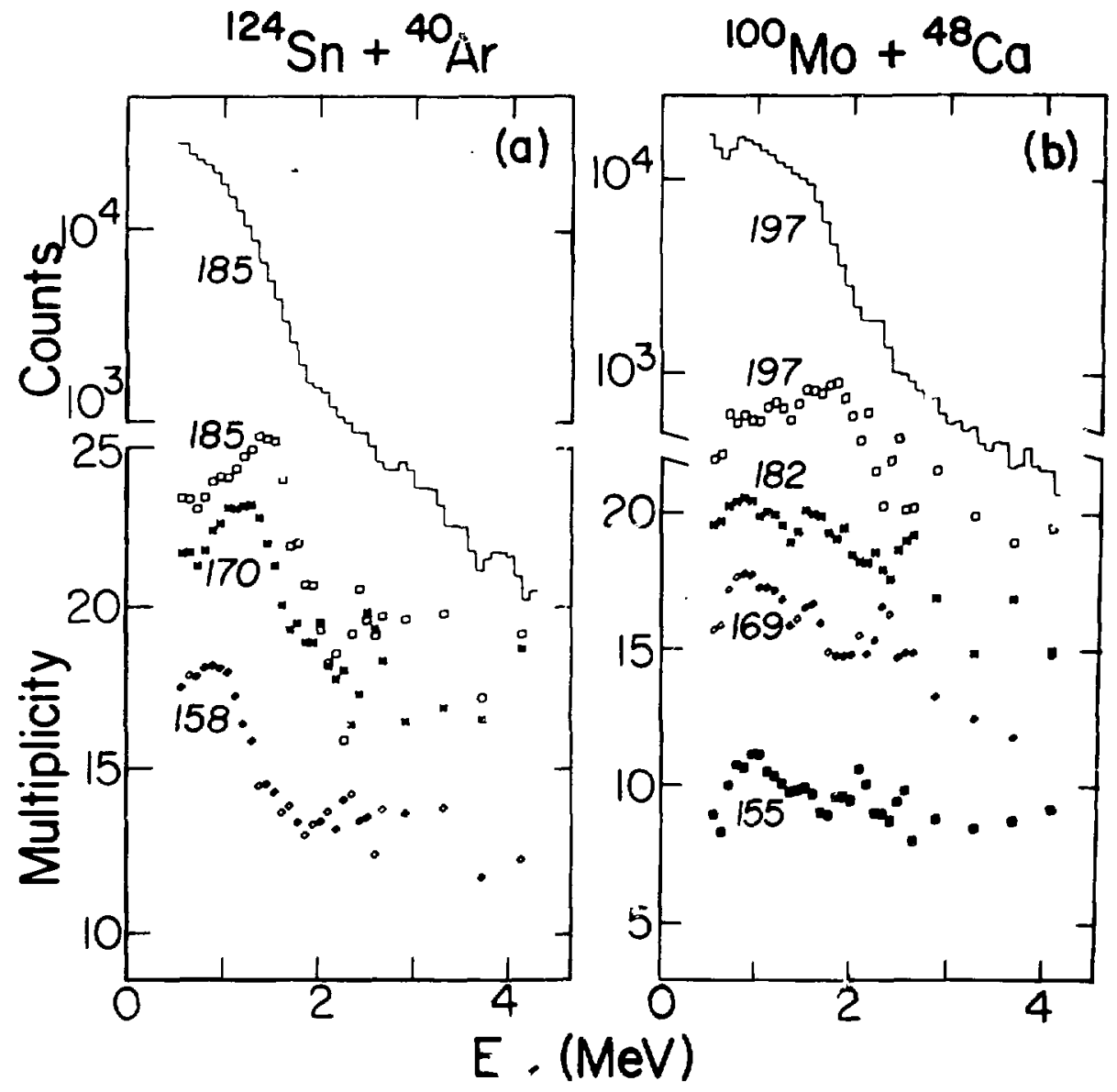

Fig. 1. Plots of multiplicity vs $\gamma$-ray ereergy for the systems (a) ${ }^{124} \mathrm{Sn}+{ }^{40} \mathrm{Ar}$ and (b) ${ }^{100} \mathrm{Mo}+{ }^{48} \mathrm{Ca}$ for the indicated bombarding energies. One $\mathrm{NaI} \gamma$-ray spectrum is also shown for each system (ref. 1 ). 
Can $\Phi_{\text {solt }}$ be deternined experimentally? There is some evidence that this can be done by studies of $\gamma-\gamma$ energy correlations. Consider a nucleus de-exciting through a rotational cascade with a fixed moment of inertia, a true rigid rotor. Then by eq. (1) the $\gamma$-ray spectrum is a set of transitions, evenly spaced at $8 \hbar^{2} / 2$. If this cascade is looked at by two $\gamma$ counters. and the first one is gated on a particular transition, the coincident spectrum in the second counter is the whole spectrum but for the gated line. Thus in a two dimensional $\gamma-\gamma$ correlation plot there will be a valley along the diagonal, with ridges on each side whose distance apart is equal to $16 \hbar^{2} / 2 \boldsymbol{S}_{\text {coll }}$.

To perform such $\gamma-\gamma$ correlation experiments requires good statistics and a method to reduce the number of uncorrelated events. The first successful experiment was made by Andersen, et al. ${ }^{3}$ A background of uncorrelated events was subtracted by a statistical method. However, this procedure actually subtracts too many events, including some correlated ones, so that better, iterative methods have been devised. An additional correction to the two-dimensional array is to divide each point by the corresponding relative detector efficiencies.

Such a two-dimensional array (but without the iterated background subtraction or efficiency correction) for the system ${ }^{124} \mathrm{Sn}+{ }^{40} \mathrm{Ar} \rightarrow{ }^{164} \mathrm{Er} *$ at $185-\mathrm{MeV}$ ${ }^{40}$ Ar energy is shown ${ }^{4}$ in Fig. 3. Four features, believed to be general, can be pointed out. First, there is a distinct valley along the diagonal up to about $1 \mathrm{MeV}$ (spin 40 ) having a measurable width, and there is some possibility this valley also exists in the region above 1.1 $\mathrm{MeV}$. Second, there are a few bridges across this valley beginning as low as $0.55-0.60 \mathrm{MeV}$ and continuing as far up as the valley porsists. Also, there are irreguJarities in the ridges alongside this valley. Third, there is a general filling of the valley above $\sim 1 \mathrm{MeV}$, which is rather complete around $1.1 \mathrm{MeV}$. Finally, on many arrays there are stripes or lines of increased intensity ruuning parallel to the coordinate axes.

The mere existence of the valley strongly indicates the rotational nature of the bands for this range of $\gamma$-ray transition energies, in addition, the half-width of the valley is just the difference between successive transition energies, and so determines $\boldsymbol{F}_{\text {coll }}$. The first results on this point suggest that $\boldsymbol{f}_{\text {coll }}$ is indeed smafler than $f_{\mathrm{ef}}$, with values as low as $\sim 0.6 \mathscr{f}_{\mathrm{eff}}$.

The lowest energy bridges in Fig. 3 are due to known backbends in the nuclei produced. The large one at $0.55 \mathrm{MeV}(\omega \sim 0.27 \mathrm{MeV})$ corresponds to the first backbends in ${ }^{158} \mathrm{Er}$ and ${ }^{160} \mathrm{Er}$ (the major even-even products). There are at least two more higher energy bridges in Fig. 3. It is not clear which orbitals are involved in these higher bridges. However, the general behavior up to about I MeV $\gamma$-ray energy seems reasonably clear - a deep valley reflecting good rotational behavior, and a few large irregularities in both the valley and iidge structure resulting from alignment of specific high-j orbitals. Plausible explanations can also be given for the filling in of the valley above $1 \mathrm{MeV}$ and for the existence of the stripes.

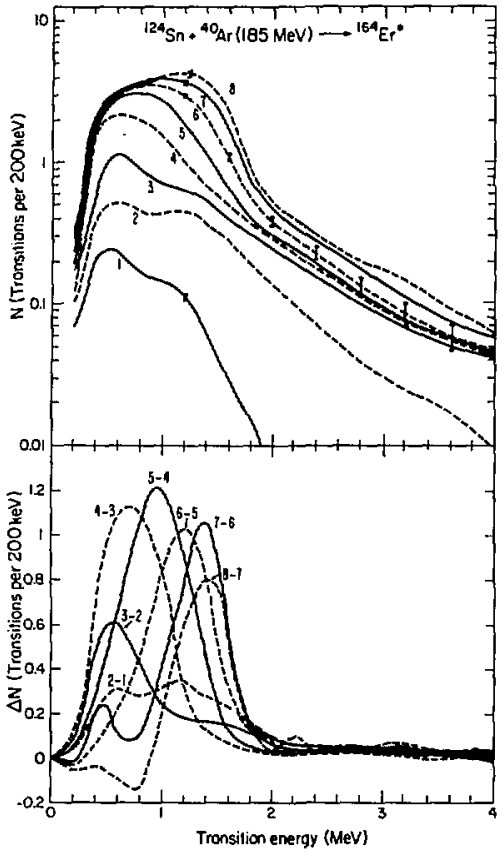

KUL 192-GASA

Fig. 2.

Top: Spectra from a $7.6 \times 7.6 \mathrm{~cm} \mathrm{Nal}$ detector corrected for the detector response function (number of transitions per $200 \mathrm{keV}$ per event) for consecutive $4 \mathrm{MeV}$ wide slices of the coincident total $\gamma$-ray energy spectrum taken with a $33 \times 20 \mathrm{~cm}$ sum crystal for the reaction products (mainly ${ }^{159,160} \mathrm{Er}$ ) of $185 \mathrm{MeV}{ }^{40} \mathrm{Ar}+{ }^{124} \mathrm{Sn} \rightarrow{ }^{164} \mathrm{Er} *$ (ref. 2).

Bottom: the difference in spectra from consecutive slices as indicated in the figure.

XBL 792-645A 
The field of continuum $\gamma$-ray spectroscopy is a rapidly developing and changing one, and the introduction of new experimental techniques such as the use of crystal-ball, $4 \pi$ detector systems can only accelerate the pace. Theoretical developments are also proceeding along similarly expanding lines, so that we may look forward to a wealth of new information on high spin and highly excited states in the next few years and much more detailed knowledge about their structure.

\section{Footnote and References}

*Condensed from LBL-12792

1. M.A. Deleplanque, I.Y. Lee, F.S. Stephens, R.M. Diamond, and M.M. Aleonard, Phys. Rev. Lett. 40, 629 (1978).

2. H.J. Körner, D.L. Hillis, C.P. Roulet, P. Aguer, C. Ellegaard, D.B. Fossan, D. Habs, M. Neiman, F.S. Stephens, and R.M. Diamond, Phys. Rev. Lett. 43, 490 (1979).

3. O. Andersen, J.D. Garrett, G.B. Hagemann, B. Herskind, D.L. Hillis, and L.L. Riedingcr, Phys. Rev. Lett. 43, 687 (1979).

4. M.A. Deleplanque, F.S. Stephens, O. Andersen, J.D. Garrett, B. Herskind, R.M. Diamond, C. Ellegaard, D.B. Fossan, D.L. Hillis, H. Kluge, M. Neiman, F.P. Roulet, S. Shih, and R.S. Simon, Phys. Rev. Lett. 45, 172 (1980).

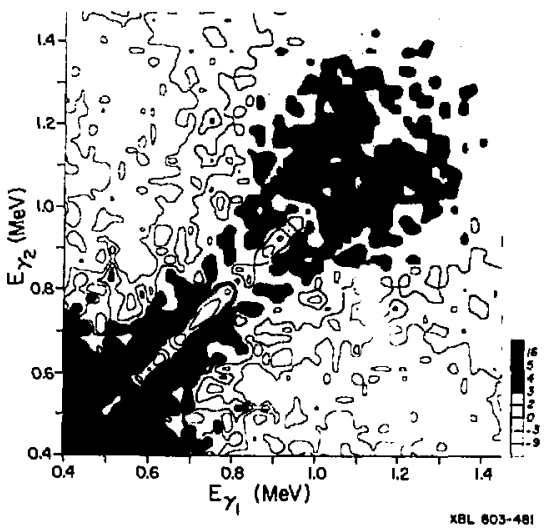

Fig. 3 Energy correlation spectrum from 1: : reaction ${ }^{124} \mathrm{Sn}\left({ }^{40} \mathrm{Ar}, x n\right){ }^{164 \times} \mathrm{Er}$ at $185 \mathrm{MeV}$. T.r data were taken with $\mathrm{Ge}(\mathrm{Li})$ detectors and had ur correlated events subtracted. The plot shows contours of equal numbers of correlated events, where the darker regions have more counts according to the scale at the right edge (ref. 4).

XBL 803-48I

\title{
High Spin States*
}

\author{
F.S. Stephens
}

Nuclei can generate high angular momentum either by alignment along a common axis of the angular momentum of several individual nucleons or by a collective rotation of the nucleus as a whole. Recent developments in this field have been centered on understanding the competition of these two nodes. This can be illustrated in Fig. 1, where level schemes of ${ }^{158} \mathrm{Er}$ and ${ }^{147} \mathrm{Gd}$ are shown ${ }^{1,2}$. The ${ }^{158} \mathrm{Er}$ scheme is quite reguIar and the dominant behavior is collective rotation of a prolate-deformed nucleus as is illustrated at the left of Fig. 1. The ${ }^{147} \mathrm{Gd}$ scheme is quite irregular, with comr plicated decay pathways and isomeric states (dark levels). Its dominant behavior is certainly singleparticle alignment, as is illustrated at the right of Fig. 1. Yet both of these schemes contain elements of the other type of behavior. There are irregularities in the ${ }^{158} \mathrm{Er}$ rotational pattern at spins asound 16 and 26 , which correspond to single particle alignments, and the $49 / 2+$ isomer at $8.6 \mathrm{MeV}$ in ${ }^{147} \mathrm{Gd}$ has a quadrupole moment that suggests that the aligned particles are polarizing the core so a collective oblate shape is developing. I will discuss our present understanding of the interplay of these two types of behavior. 

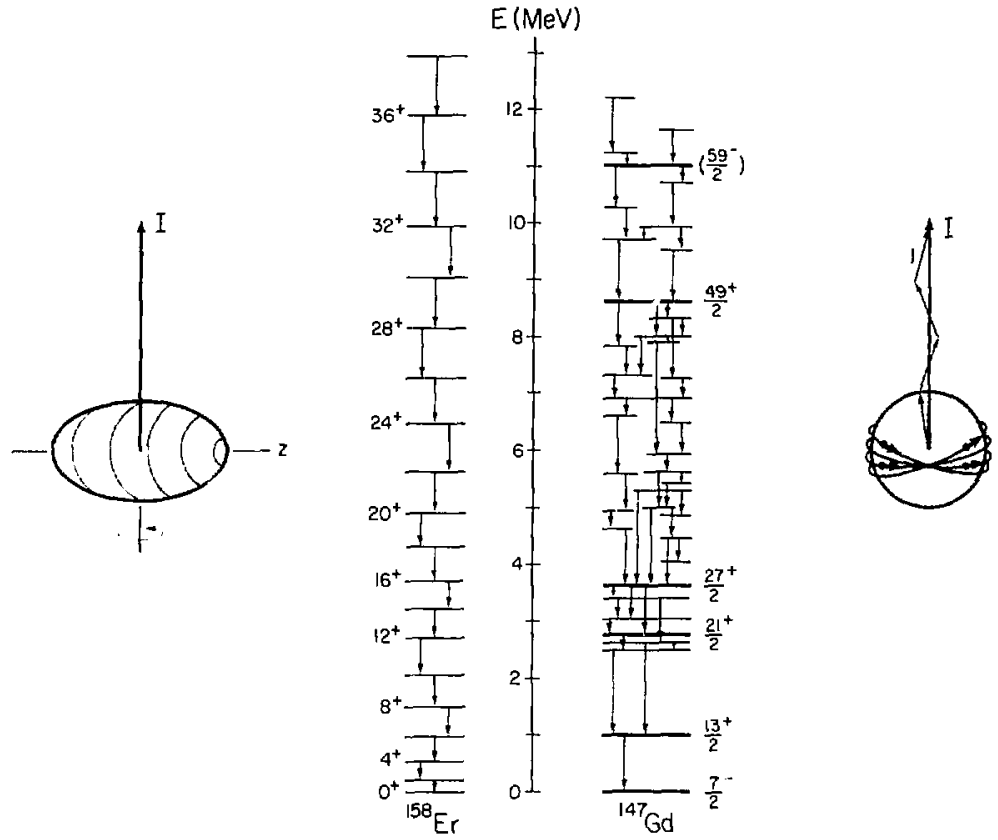

XEL $828-1011$

Fig. 1. Level scheme for ${ }^{158} \mathrm{Er}$ and ${ }^{147} \mathrm{Gd}$, together with illustrations of the dominant source of angular momentum for each case.

$\mathrm{XBL} 828 \cdot 1011$

One of the inost important factors in determining the physics of high-spin states is simply the rotational behavior of rigid classical objects. In Fig. 2 the moment of inertia of such an object is compared with that of a rigid sphere (solid lines) for a variety of shapes and rotational axes. The shape and axis is defined by $\gamma$, which varies from $-120^{\circ}$ to $60^{\circ}$ as the object varies from a prolate shape rotating about its symmetry axis, through oblate and prolate shapes rotating about axes perpendicular to the symmetry axis, to an oblate shape rotating about its symmetry axis. The deformation is given in terms of a quantity $\epsilon$, which is to lowest order just $\Delta R / R$. Values of $\epsilon$ around 0.3 are typical for the familiar deformed rare-earth and actinide nucles. The largest moments of inertia, and therefore the lowest rotational energies, occur for the range of shapes between $\gamma=0^{\circ}$ and $60^{\circ}$. The very largest moment of inertia is for $\gamma=60^{\circ}$, an oblate shape rotating around its symmetry axis, and it is for this reason the earth has such a shape. The full liquiddrop model (LDM) treatment of a rotating nucleus ${ }^{3}$ includes volume, surface, and Coulomb energies, in addition to these classical rotor considerations, and is shown by the dots in Fig. 2. It is apparent that there is no strong shape preference in these additional LDM terms, so that simple classical mechanics determine the 
liquid-drop shapes. This is important, since the liquiddrop model is our best guide to such macroscopic nuclear properties and is even the limit to which some of the microscopic models are normalized.

In order to see how significant these classical shape effects are, one must choose a mass and spin, and for $\dot{A}=160$ and $I=60 \hbar$, an energy scale is given on the right side of Fig. 2. The variation for $\epsilon=0.3$ of about $10 \mathrm{MeV}$ is larger than typical shell effects $(\sim 3$ MeV) so that for this spin the effects considered here should be dominant. The rotational energy varies as $I^{2}$ so that, for $30 \hbar$, shell effects and these classical shape effects should be about equivalent, and below $\sim 20 \hbar$ the shell effects will dominate. The arguments made here would seem to apply only for collective nuclear rotations, and even then only if the nuclear moment of inertia has the rigid body value, neither of which is obviously the case. In fact, however, most people do believe that rotating nuclei at high spins will, on average, have the rigid-body moment of inertia, and this has been shown to be the case for an anisotropic haimonic oscillator potential and independent particle motion ${ }^{4}$. The smaller moments of inertia observed at low spins are due largely to the pairing correlations, which should be quenched above $\sim 30 \hbar$. Furthermore, even in noncollective cases, it has been shown (for a Fermi gas) that the trajectory of lowest levels follows that given by rotating the appropriately shaped rigid body ${ }^{5}$. Thus, these very simple arguments should be valid, and shapes in the $\gamma=0-60^{\circ}$ range should dominate at high spin, i.e., above $-30 \hbar$ in the $A=160$ region. Several types of microscopic calculations have now been carried out for nuclei in this region and all of them agree that the $\gamma=0-60^{\circ}$ range should dominate at high spins, though the models show some differences within the $\gamma=0-60^{\circ}$ range.

A general question arises as to how one can recognize these shapes experimentally. That is, what kind of nuclear structure is expected for shapes in this $\gamma=$ $0-60^{\circ}$ region. It has been recognized since 1952 that a deformed nucleus can rotate collectively around an axis perpendicular to the symmetry axis ${ }^{6}$. The prolate $\gamma=$ $0^{\circ}$ shape is of this type and essentially all the rotational nuclei known have $\gamma$-values equal to, or near, $0^{\circ}$. On the other hand, a nucleus cannot rotate collectively around a symmetry axis; those degrees of freedom are contained in the single-particle motion. Thus the $\gamma=$ $60^{\circ}$ oblate nuclei do not have collective rotation. but build up their angular momentum by aligning along a common axis the contribution of various single particles. These are the two basic behaviors discussef above.

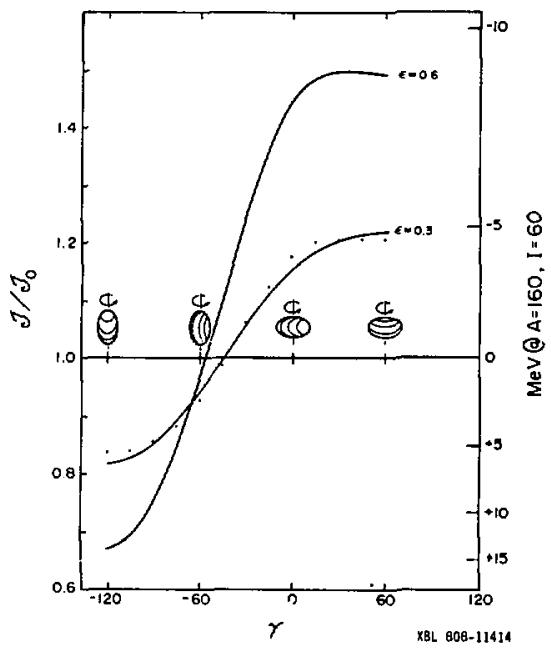

Fig. 2. The ratio of the moment of inertia of a rigid ellipsoid to that of a rigid sphere vs the shape parameter $\gamma$ for two values of the deformation $\epsilon=$ 0.3 and $\epsilon=0.6$. The right-hand scale gives the difference in rotational energy in $\mathrm{MeV}$ for a nucleus with $A=I 60$ and $I=60$. The dots give the ratios for the total liquid-drop energy (rotation + surface + Coulomb) of the nucleus.

XBL 808-11414

The kind of structure associated with the triaxial nuclear shapes between $\gamma=0^{\circ}$ and $60^{\circ}$ has been elucidated recently - since the first clues to this were found in the backbending phenomenon discovered ${ }^{7}$ in 1971. The situation is depicted in Fig. 3. For exactly axially symmetric shapes (upper left) only collective rotation is possible, and different configurations give rise to bands thas cxtend over broad regions of spin. In fact, what is observed to happen is indicated at the upper right, where bands $v$ ith different configuration have different amounts of single-particle angular momentum aligned with the rotation axis and thereby give rise to slightly nonaxial shapes and a pattern of crossing bands. Sequences with spins up to $\sim 40$ h have now been observed with three successive alignments (band crossings) that provide about half the total angular 
momentum of the nucleus. As $\gamma$ nuves closer to $60^{\circ}$ (lower left) the proportion of single-particle to collective angular momentum becumes larger. and the rotational bands become less collective with smaller moments of irertia. This situation probably occurs in nuclei like ${ }^{154} \mathrm{Er}$ in the $\mathrm{I}=30-40 \mathrm{~h}$ region ${ }^{8}$ and in ${ }^{154} \mathrm{Dy}$ near ${ }^{9} \mathrm{I}=40 \mathrm{~h}$, but is certainly the least documented of the regions of Fig. 3. Finally, at the lower right of Fig. 3. the collective motion is very weak or entirely absent. and we now suspect many nuclei in the $\mathrm{N}=82, \mathrm{Z}=$ 64 region are of this type. So far only ${ }^{147} \mathrm{Gd}$ has quadrupole moments measured to indicate a nonspherical shape ${ }^{2}$, but nuclei like ${ }^{152} \mathrm{Dy}$ and ${ }^{150} \mathrm{Gd}$ are very likely of this type. It is not so easy to distinguish these spectra from those of nuclei with spherical shapes, but qua. drupole moments can do this, and there may be additional features of the spectra that can help (the rotation about the perpendicular axes). In any case, the main point here is that there are characteristic spectra associated with the shapes between $\gamma=0^{\circ}$ and $60^{\circ}$, and these are rather easy to distinguish experimentally. Furthermore, the range of spectral types is (or can be) continuous, as it must be since $\gamma$ is (or can be) continuous.

It should be recognized that there is no simple rath through Fig. 3 along which nuclei evolve with increasing spin. It was clear in Fig. 2 that the $y=0$ to $60^{\circ}$ region was quite flat in the LDM limit so that shell effects determine just where in this range a given nucleus wi!l be. Some nuclei like ${ }^{154}$ Dy recently discussed by $\mathrm{Khoo}^{9}$ seem to progress from upper right to lower left and probably on to lower right as the spin goes from 20 to $40 h$. However, its neighbor ${ }^{152} \mathrm{Dy}$ shifts fiom lower-right-type behavior in the $20-40 \mathrm{~h}$ range to some type (as yet not well specified) of collective behavior at higher spins ${ }^{10}$. The shell effects determine such specific i-haviors. However, one generalization we can make is that more single-particle angular momentum generally indicates behavior closer to the $\gamma$ $=60^{\circ}$ limit, and conversely a larger firction of collective angular momentum usually means a shift towards $\gamma$ $=0^{\circ}$.

A wealth of experimental data exists to compare with this picture. Below $\sim 35 \hbar$ there is generally cxcellent agreement between the data and detailed shel] model-type calculations of these effects; above $\sim 35 h$ the data are much less detailed, but seem also to fall into these patterns. Thus, there is a reasonable hope that these simple ideas contain the basic elements of the behavior of nuclei at high spins. There is currently much activity, both experimental and theoretical, to see if that is the case or if there are some important addtional features.

\section{Footnote and References}

* Parts of talks given at : Erice, Sicily; Mt. Fuji, Japan; Fugls $\phi$, Denmark: and Amsterdam, Netherlands.

1. J. Burde, E.L. Dines, S. Shih, R.M. Diamond, J.E. Draper, KH. Lindenberger, C. Schuck, and F.S. Stephens, Phys. Rev. Lett. 48 (1982) 530

2. O. Hausser, H.-E. Mahnke, J.F. Sharpey-Schafer, M.L. Swanson, P. Taras, D. Ward, H.R. Andrews, and T.K. Alexander, Phys. Rev. Lett. 44 (1980) 132; O. Bakander, C. Baktash, J. Borggreen, J.B. Jensen, J. Kownachi. J. Pedersen, G. Sletten, D. Ward, H.R. Andrews, $O$. Hausser, P. Skensved, and P. Taras, preprint (1982)

3. S. Cohen, F. Plasil, and W.J. Swiatecki, Ann. Phys. 82 (1974) 557

4. A. Bohr and B.R. Mottelson, Mat. Fys. Medd. Dan. Vid. Selsk., 30, no. 2 (1955)

5. A. Bohr and B.R. Mottelson, Nuclear Siruciure, vol. 2. (Benjamin, Reading, Mass., 1975) p. 80

6. A. Bohr, Mat. Fys. Medd. Dan. Vid. Selsk., 26, no. 14 (1952)

7. A. Johnson, H. Ryde, and J. Sztarkier, Phys. Lett. 34B (1971) 605

8. C. Baktash, E. der Mateosian, O.C. Kistner, and A.W. Sunyar, Phys. Rev. Lett. 42 (1979) 637

9. A. Pakkanen, Y.H. Chung, P.J. Daly, S.R. Faber, H. Helppi, J. Wilson, P. Chowdhury, T.C. Khoo, I. Ahmad, J. Borggreen, Z.W. Grabowski, and D.C. Radford, Phys. Rev, Lett. 48 (1982) 1530

10. B. Hass, H.R. Andrews, O. Hausser, D. Horn, J.F. Sharpey-Schafer, P. Taras, W. Trautmann, D. Ward. T.L. Khoo, and RK. Smither, Phys. Lett. B84 (1979) 178 


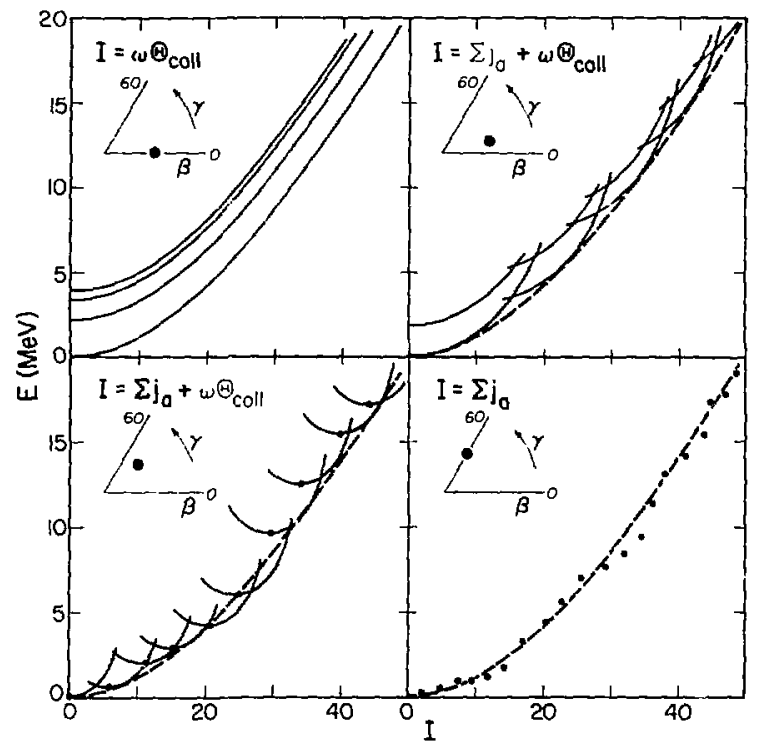

Fig. 3. Schematic excitation energy vs spin plots for various relative amounts of collective angular momentum and single-particle rotation-aligned angular momentum. Bandhead (pure single-particle) energies are shown in the lower two panels. The solid curves correspond to real bands, whereas the dashed curve is the envelope of the real bands.

XBL 804-544

\section{The Rotational Inertia of Nuclei*}

\section{M.A. Deleplanque, H.J. Korner, ${ }^{\dagger}$ H. Kluge, ${ }^{\ddagger}$ A.O. Macchiavelli, N. Bendjaballah," R.M. Diamond, and F.S. Stephens}

Even rotational nuclei do not rotate like a rigid body. Part of the angular momentum is generated by collective motion, but alignments of single-particle angular momentum on the rotation axis also contribute significantly to the total spin. Such behavior can be described through different moments of inertia: the kinematic monent of inertia (j) $=I / \omega$ (where $\omega$ is the angular velocity, also called here rotational frequency) describes the overall motion of the nucleus, whereas the dynamic moment of inertia $S^{(2)}=\mathrm{dl} / \mathrm{d} \omega$, is related to the response of the nucleus to a torque. When there are changes in nuclear structure (such as alignments), $f^{(2)}$ will be different from $\boldsymbol{( 1 )}^{(1)} \boldsymbol{S}^{(2)}$ is a much more sensitive, and therefore more useful, quantity than $\mathcal{A}^{(1)}$.

These two types of moments of inertia can be defined for any sequence of levels; the two most significant ones are $\boldsymbol{F}_{\text {bnd }}$ and $\boldsymbol{F}_{\text {eff }}$ and, in particular, $f_{b a n d}^{(2)}$ and $\mathcal{S}_{\mathrm{ef}}$. The collective motion generates bands within which the rate of change in spin with frequency is the moment of inertia $\Omega^{(2)}=(\mathrm{dI} / \mathrm{d} \omega)_{\text {pand }}$ It is accessible at high spins through $\gamma-\gamma$ correlation experjments. However, a decay path from a highly excited nucleus at high spin down to the ground state consists of a succession of such bands buile on configurations having different alignments, and the rate of change of the total spin with frequency includes both collective motion and alignment effects. It seemed natural to us, 
therefore, to define a new moment of inertia $\boldsymbol{S A f l}_{\mathrm{f}}^{2}=$ $(\mathrm{d} I / \mathrm{d} \omega)_{\text {path }}$. Combined with (2) values at the same frequency, it should give us a measure of changes in alignment with frequency.

We also recently realized that (I) $_{\text {(2) }}$ is accessible experimentally (at least in rotational nuclei) through the height of the $\gamma$-ray spectrum (density of $\gamma$ rays as a function of transition energy $E_{\gamma}$ ). In compound nucleus reactions induced by heavy ions, the continuum $\gamma$-ray spectra are detected in Nel scintillators in coincidence with different slices of total $\gamma$-ray energy (detected in a sum spectrometer). These spectra sample the decay of different (roughly gaussian) direct populations of states. The value of $S_{\text {ef }}$ is related to the height of the $\gamma$-ray spectrum (normalized to the $\gamma$-ray multiplicity). Since, in a rotational nucleus, the transitions are predominantly stretched E2, their number $d \mathrm{~N}$ is $\simeq \mathrm{dI} / 2$, and the spectrum height $h$ is $d N / d E \simeq \mathrm{dl} / 4 \mathrm{dw}$ - $\mathrm{a}_{\mathrm{i}} / 4$. But $\mathrm{h}$ is a measure of $\mathrm{dI} / 4 \mathrm{d \omega}$ only in the frequency region that is fully populated, i.e., up to about $0.35 \mathrm{MeV}$.

To obtain $S_{\mathrm{e}}^{2)}$ at high frequency (up to about 0.7 MeV), we devised a way to correct the $\gamma$-ray spectra for the feeding. Essentially, the difference of two $\gamma$-ray spectra corresponding to slightly different spin feedings (i.e., in coincidence with two consecutive slices of total $\gamma$-ray energy) is proportional to the feeding curve $f(\omega)$. Then

$$
\lambda_{\mathrm{eff}}^{22}\left(\omega_{0}\right) \propto h\left(\omega_{0}\right) \frac{\int_{\omega_{\min }}^{\omega_{\max }} f(\omega) d \omega}{\int_{\omega_{0}} f(\omega) d \omega}
$$

Three different rotational systems have been studied at tie 88-Inch Cyclotron of the Lawrence Berkeley Laboratory, ${ }^{130} \mathrm{Te}+{ }^{40} \mathrm{Ar},{ }^{126} \mathrm{Te}+{ }^{40} \mathrm{Ar},{ }^{124} \mathrm{Sn}+{ }^{40} \mathrm{Ar}$ at $185 \mathrm{MeV}$. These lead essentially to ${ }^{166,165} \mathrm{Yb}$, ${ }^{162,161} \mathrm{Yb}$, and ${ }^{160,159} \mathrm{Er}$, respectively. The total $\gamma$-ray energy was recorded in a sum spectrometer. The $\gamma$-ray spectra were recorded in coincidence with the sum spectrometer in seven $12.7 \times 15.2 \mathrm{~cm} \mathrm{NaI}$ detectors, one at $90^{\circ}$ to the beam, the others as close as possible to $0^{\circ}$ and $180^{\circ}$. For each sum slice, the $\gamma$-ray spectra were normalized to their multiplicity. A statistical spectrum was then subtracted and the remaining yrast part was corrected for the feeding and for angular distribution effects.
Figure 1 shows ${ }_{\mathrm{C}}^{(2)}$ obtained for those three systems, In all three cases, the first backbend shows up as a peak, indicating that alignment generates more angular momentum at that frequency. At high frequency, $\mathrm{Sef}_{\mathrm{fr}}$ increases, starting at $\omega=0.5 \mathrm{MeV}$ in $\mathrm{Yb}$ nuclei and at $0.6 \mathrm{MeV}$ in $\mathrm{Er}$ nuclei. This suggests the alignment of protons, because it is seen at lower frequency in $\mathrm{Yb}$ nuclei where the Fermi level is higher. In fact, both larger triaxial deformation (suggested by low $\boldsymbol{S}_{\text {(2) }}$ values from correlation data, see Fig. 1) and proton alignment could produce such an increase, and recent calculations by $T$. Bengtsson and $I$. Ragnarsson predict that both $h_{9 / 2}, i_{13 / 2}$ proton alignments and triaxial deformation (around $c \sim 0.4$ ) should occur in this frequency range. The crosses in Fig. 1 show predicted Af values deduced from the calculation of spin I as a function of $w$ for the lowest four trajectories of each parity and signature quantum number in ${ }^{166} \mathrm{Yb}$. At high frequency, the lower bump is due to proton alignment and the higher one to the onset of triaxial deformation. Those values agree with the experimental results presented, although the latter are more smeared as they represent an average over many decay paths.

The determination of dynamic effective moments of inertia at high frequencies provides some new insights into nuclear behavior at very high spins. and $S_{\text {bend }}$ are complementary and are at present the only two moments of inertia measurable in the continuum region. However, the determination of $\Phi_{\mathrm{eff}}(2)$ at high frequencies is linked to the feeding correction, which is well understood only in rotational nuclei. It will be an exciting challenge to try to explore less collective nuclei in this way. The nuclear properties change gradually when crossing the region of transitional nuclei from mainly collective on the heavier side to mainly single particle on the lighter side.

\section{Foothutes and References}

"Submitted to Phys. Rev. Lett.

†Permanent address: Physik Department, Technische Universität, Müinchen, West Germany

¥Permanent address: Hahn-Meitner Institute, Berlin, D-1000, Berlin 38, West Germany

\$Permanent address: Comision Nacional De Energía Atomica, Buenos Aires, Argentina

* Permanent address: C.S.T.N., B.P. 1017, Alger, Algeria 


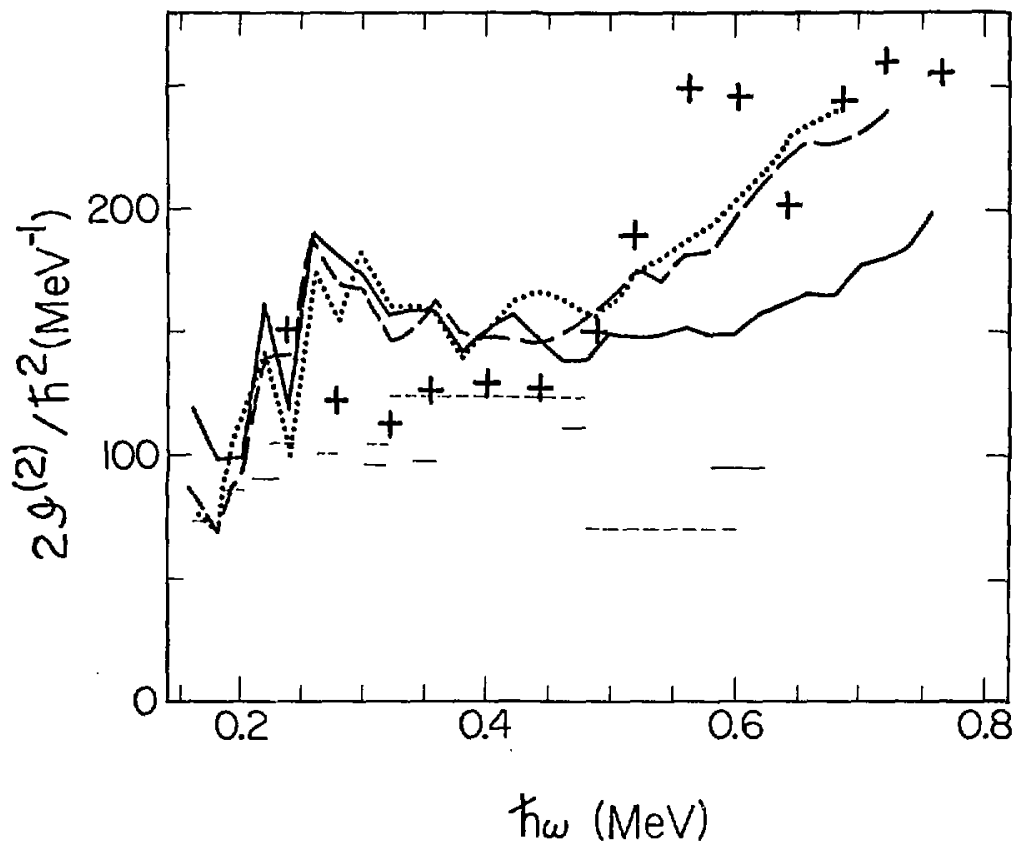

Fig. 1. $\mathcal{S}_{\mathrm{e}}^{2}$ as a function of $\omega$ for the systems ${ }^{124} \mathrm{Sn}+{ }^{70} \mathrm{Ar}$ (thick solid line), ${ }^{126} \mathrm{Te}+{ }^{40} \mathrm{Ar}$ (dotted line), ${ }^{130} \mathrm{Te}+$ ${ }^{40} \mathrm{Ar}$ (thick dashed line). Also shown are some values of $\boldsymbol{S}_{\mathrm{b}}^{(2)}$ for for ${ }^{124} \mathrm{Sn}+{ }^{40} \mathrm{Ar}$ (thin solid lines) and ${ }^{33^{\circ} \mathrm{Te}}+{ }^{40} \mathrm{Ar}$ (thin dashed lines). The crosses are $\mathcal{S}_{\mathrm{e}}^{2}$ values for ${ }^{166} \mathrm{Y} \mathrm{V}$ deduced from $\mathrm{T}$. Bengtsson and $\mathrm{I}$. Ragnarsson calculations.

XBL 823-233A 


\title{
Dependence of the Giant Dipole Strength Function on Excitation Energy*
}

\author{
J.E. Draper, ${ }^{\dagger}$ J.O. Newton, ${ }^{\ddagger}$ L.G. Sobotka, H. Lindenberger. ${ }^{\prime}$ G.J. Wozniak, \\ L.G. Moretto, F.S. Stephent, R.M. Diamond, and R.J. McDonald
}

Spectra of $\gamma$ rays associated with deep-inelastic (DI) products from the $1150 \mathrm{MeV}{ }^{136} \mathrm{Xe}+{ }^{181} \mathrm{Ta}$ reaction have been studied. The yield of $10-20 \mathrm{MeV} \gamma$ rays initially increases rapidly with excitation energy of the products and then more slowly for excitation energies higher than $120 \mathrm{MeV}$.

Several sets of calculations were done to facilitate the interpretation of the measured $\gamma$-ray coincidence spectra. The first set used a constant El strength function (no GDR). Set II employed the ground-state values of the resonance energy (14.6 MeV) and width (6.5 MeV). In Set III the width was increased linearly from 1.0 to 1.5 times the ground-state valuc, as $E^{*}$ increased from 34 to $199 \mathrm{MeV}$. In Set IV the width was increased as in Set III, while the resonance energy of the GDR was decreased linearly from 1.0 to 0.66 times the ground-state value as $E^{*}$ increased. All the calculated spectra were folded with the NaI response function.

In Fig. 1 these calculated $\gamma$-ray spectra are shown for all but the lowest excitation energy bin, omitted due to the large percentage variation of the excitation energy across this bin. At all excitation energies, the calculation with a constant strength function (Set I) substantially underestimates the data, even though this calculation was normalized by assigning radiative widths that are a factor of $\sim 3$ larger than the values found in $(\boldsymbol{n}, \gamma)$ experiments with slow neutrons. For sets Il-IV, the normalization was calculated from the El sum rule. The calculations employing the groundstate values of the GDR (Set II) give a much better representation of the data than does Set $I$, although they overestimate the $15-\mathrm{MeV} \gamma$-ray yield at the highest excitation energies. Better agreement with the $15-\mathrm{MeV}$ $\gamma$-ray region is obtained by increasing the resonance width (Set III), but the best overall agreement is obtained when the peak resonance energy is also decreased (Set IV). Calculations were also made for bin 5 using a resonance energy of $14.6 \mathrm{MeV}$ and widths of 15 and $25 \mathrm{MeV}$; however, these calculations do not reproduce the data as well as the calculation where the peak energy is decreased (Set IV).

The inferred increase in the resonance width might be trivially ascribed to the increasing width of the product mass distribution with increasing $E^{*}$. This explanation does not seem likely because of the weak

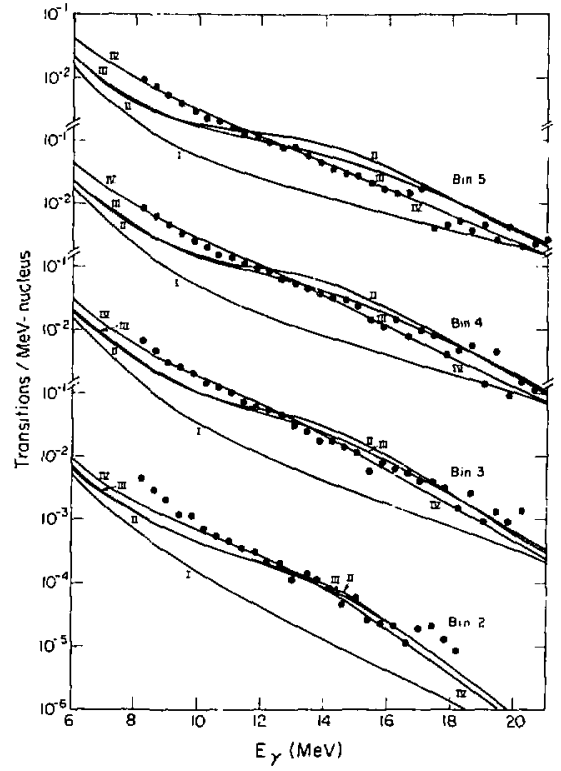

Fig. 1. Experimental (symbols) and calculated (curves) $\gamma$-ray pulse height spectra associated with DI products having mean excitation energies of 80 $\mathrm{MeV}, 119 \mathrm{Mev}, 159 \mathrm{Mev}$, and $199 \mathrm{MeV}$ for bin 2 through bin 5 , respectively. The $\boldsymbol{\gamma}$-ray spectra have been calculated for different widths and resonance energies of the giant dipole strength function (see text) and have been folded with the measured $\mathrm{Nal}$ response function.

XBL $824-488$

$A^{-1 / 3}$ dependence of the ground-state resonance energy. An alternate explanation is that in DI reactions the second moments of the fragment spin distributions can be quite large even at a fixed Q-value. ${ }^{1}$ This large range of angular momenta might lead to a variety of shapes, which would result in different values of the 
resonance energy and thus effectively broaden the resonance. A similar broadening occurs in rare-earth nuclei, where the apparent width is nearly twice that of a spherical nucleus. ${ }^{2}$ An additional possibility is that the resonance width might increase with $\mathrm{E}^{*}$ due to an increase in the rate of dissipation of the collective state into the multitude of n-particle n-hole states available at high excitation energies.

Using simple theory the energy $\hbar \omega$ of the dipole mode can be approximated ${ }^{3}$ as

$$
\omega \simeq\left(\omega_{0}^{2}+\frac{3 V_{1}}{4 M<r^{2}>_{0}}\right)^{1 / 2}
$$

where $V_{1}$ is the symmetry potential, $\left\langle r^{2}\right\rangle$ is the mean squared radius, and $\hbar \omega_{0}$ is approximately $41 \mathrm{~A}^{-1 / 3}$ near the Fermi surface. There are three quantities in the expression for $\omega$ that could depend on the excitation energy $E^{*}$ : (1) For a harmonic oscillator $\hbar_{w_{0}}$ is independent of $E^{*}$, but a more realistic well broadens at the tcp, so the efrective $\hbar \omega_{0}$ might be reduced for large $E^{*}$. (2) The symmetry potential measures the effect of the neutron-proton interaction as a restoring force for the GDR oscillation. Since the participating particles are spread over more shells at high excitation energies, the neutron-proton overlap will decrease $*-1 d V_{1}$ should also decrease. (3) Although onc does not expect a large change in $\left\langle r^{2}\right\rangle$ with $E^{*}$, it should increase due to the particles in higher shells. These effects all decrease the resonance energy of a GDR built on a highly excited state. This agrees with the tentative conclusion from our experimental results.

\section{Footnotes and References}

*Condensed from Phys. Rev. Lett. 49 (1982) 434

†Permanent address: University of California, Devis, California 95616

¥Permanent address: Australian National University, Canberra, Australia

SPermanent address: Hahn-Meitner Institute, Berlin, West Germany

1. P.R. Christensen, et al, Nucl. Phys. A349 (1980) 217

2. F.E. Bertrand, in Proceedings of the International Conference on Nuclear Physics, Berkeley (1980); Nucl. Phys. A354 (1981)

3. A. Bohr and B. Mottelson, Nuclear Structure, Vol. II, W.A. Benjamin, Inc. (1975)

\title{
Isomeric Trapping Following Coulomb Excitation of High Spin States in ${ }^{178} \mathrm{HF}^{*}$
}

\author{
J.H. Hamilton, ${ }_{r}^{\dagger}$ A.V. Ramayya, ${ }^{\dagger}$ R.M. Ronningen, ${ }^{\ddagger}$ R.O. Sayer, \\ H. Yamada, C.F. Maguire, ${ }^{\dagger}$ P. Colombani, ${ }^{* *} D$. Ward, ${ }^{\dagger}$ \\ R.M. Diamond, F.S. Stephens, I.Y. Lee, "P.A. Butler" and D. Habs"
}

In the region of well deformed nuclei between $A$ $=160$ and $180,{ }^{176,178} \mathrm{Hf}$ are unusual in that a number of high spin and/or high $\mathrm{K}$ isomers have been reported' in addition to the well-known $\mathrm{K}^{\mathbf{r}}=8^{-}$and $16^{+}$isomers in ${ }^{178} \mathrm{Hf}$. Furthermore, there are known excited $\mathrm{K}^{\mathbf{2}}=$ $\mathrm{O}^{+}$bands with different moments of inertia that should lead to a crossing with the ground band around spins $14^{+}$to $16^{+}$in ${ }^{176} \mathrm{Hf}$. To investigate possible effects of these different bands on the ground state rotational bands in ${ }^{178,180} \mathrm{Hf}$, we have Coulomb excited these nuclei with $\mathrm{Kr}$ and $\mathrm{Xe}$ ions from the SuperHILAC. Coulomb excitation provides additional and complementary information to (H.I., $\mathrm{xn}$ ) reactions. Because ane starts at the ground state and Coulomb excites up, the levels which are related to the ground-state band (gsb) by collective E2 transitions are the ones most strongly populated, and these may ar may not be yrast levels. Surprisingly, we find for the first time population of the lowest $K^{\pi}=8$ isomer at $1147.4 \mathrm{keV}$ in ${ }^{178} \mathrm{Hf}$ following Coulomb excitation.

Enriched (95\%), thick (35 $\mathrm{mg} / \mathrm{cm}^{2}$ ) targets of ${ }^{178,180} \mathrm{Hf}$ were Coulomb excited with beams of ${ }^{86} \mathrm{Kr}$ and ${ }^{136} \mathrm{Xe}$ with energies in the range of 384 and $594 \mathrm{MeV}$, respectively, at the SuperHILAC. The energies were chosen to keep below the Coulomb barrier. The gamma rays were observed with $\mathrm{Ge}(\mathrm{Li})$ detectors at $0^{\circ}$ and $90^{\circ}$ and in coincidence with back-scattered heavy ions. States up to $14^{+}$in the ground-state band were observed in ${ }^{178} \mathrm{Hf}$ and to $12^{+}$in ${ }^{180} \mathrm{Hf}$. Measurements 
were subsequently made in ${ }^{17} \mathrm{Hf}$ with a thin $\mathbf{0 . 5 0}$ $\mathrm{mg} / \mathrm{cm}^{2}$ ) target with $628,652,682 \mathrm{MeV} \mathrm{Xe}$ tons to look for band crossing at higher spins. Scattered ${ }^{136} \mathrm{Xe}$ particies and Hf recoils were detected by two twodimensional position sensitive avalanche counters in coincidence with each other. States up to spin $16^{+}$and tentatively $18^{+}$in ${ }^{172} \mathrm{Hf}$ were observed. The spins and parities had been established up to the $8^{+}$level. The higher spins and parities were assigned based on the measured lifetimes, and E2 Coulomb excitation yield (see table I), and intensity patterns for different bombarding energies. Lifetimes of the $6^{+}$to $14^{+}$levels were extracted from an analysis of the Dopplerbroadened line shapes and/or from the experimental yields where the semiclassical Winter-de Boer code was used. Up through the $14^{+}$level there is agreement with the rotational model within $10 \%$. When the beam was turned off, the 8-6-4-2.0 cascade in ${ }^{178} \mathrm{Hf}$ was seen with equal $\gamma$-ray intensitites fed by the $4.0 \mathrm{~s} 8^{-}$isomer at $1147 \mathrm{keV}$. Thus for the first time in Coulomb excitation, we have populated a K-isomer trap in ${ }^{178} \mathrm{Hf}$. The isomeric component is $0.9 \%$ of the direct $8^{+}$feed. A similar search was made for the $5.5 \mathrm{hr}, 8^{-}$isomer in ${ }^{180} \mathrm{Hf}$. No evidence was found for this isomer. Correcting for the lack of secular equilibrium, we obtained an upper limit for the isomeric component of $0.3 \%$ of the direct $8^{+}$feed. Direct Coulomb excitation of the isomer ${ }^{178} \mathrm{Hf}$ from the $8^{+}$ground-band member is estimated to be negligible (direct E1 or E3 population from the ground band is $\sim 10^{-12}$ of the $10^{+}$state). It is possible, though unlikely, that the projectile energies are sufficiently close to the Coulomb barrier to populate the isomer by some nuclear interaction, such as charge exchange to ${ }^{178} \mathrm{Ta}$ which populates the isomer. However, $\gamma$ rays from the higher lying states populated by ${ }^{178} \mathrm{Ta}$ were definitely not seen. The $\gamma$ spectrum taken out-ofbeam when the ${ }^{178} \mathrm{Hf}$ isomer was seer had no $\gamma$ rays except those of the isomer above background.

The most likely mechanism is that there is branching from a state which is connected by an E2 transition to the ground-state band but also connected presumably by an El transition to the $8^{-}$band. The higher the spin at which the crossing occurs the larger the branching of that state to the 8 band. Khoo and Lovhoiden using $(\alpha, \pi n)$ reactions observed the $K^{r}=$ $16^{+}$and $14^{-}$isomeric levels to be yrast traps below the $14^{+} \mathrm{gsb}$ member in ${ }^{178} \mathrm{Hf}$, but no crossing of the gsb to the $8^{-}$isomer was reported. Either a weak branch has been missed or branching to the isomer occurs above spin $14^{+}$in ${ }^{178} \mathrm{Hf}$. The latter possibility is supported by our analysis which shows that the level lifetimes up through the $14^{+}$level in ${ }^{178} \mathrm{Hf}$ are consistent with rotational model predictions. In another experiment, the same thick ${ }^{178} \mathrm{Hf}$ target was used in Coulomb excitation studies with ${ }^{16} \mathrm{O}$ ions at the University of Köln tandem. Levels up to the $10^{+}$number of the ground band were seen in that work, but no feeding of the isomer was observed to confirm that the crossing is at higher spins.

\section{Foothotes and Reference}

*Condensed from Phys. Lett. 1128 (1982) 327

†Phys. Department, Vanderbilt University, Nashville, TN

$\ddagger$ Phys. Department, Michigan State University, East Lansing, MI

\$Oak Ridge National Laboratory, Oak Ridge, TN

**Institute de Physique, Orsay, France

††Chalk River Nuclear Laboratory, Chalk River, Ontario, Canada

\#United Kingdom SRC/NATO fellow

"Max Planck Institute, Heidelberg, West Germany

1. T.L. Khoo, F.M. Bernthal, R.G.H. Robertson and R.A. Warner, Phys. Rev. Lett. 37 (1976) 832; T.L. Khoo and G. Lovhoiden, Phys. Rev. Leit. 67B (1977) 271. 
Table I

Lifetimes in ps of states in ${ }^{178} \mathrm{Hf}$ and ${ }^{100} \mathrm{Hf}$.

Average values are compared with rotational nodel predictions.

\begin{tabular}{|c|c|c|c|c|c|c|c|c|}
\hline \multirow[t]{2}{*}{ Transition } & \multicolumn{4}{|c|}{$\tau(\mathrm{DBLS}) \mathrm{ps}$} & \multicolumn{2}{|c|}{ t(Yield) } & \multirow{2}{*}{$\begin{array}{c}\tau \\
\text { (Average) }\end{array}$} & \multirow{2}{*}{$\begin{array}{c}\tau \\
\text { (Rotat. }\end{array}$} \\
\hline & $E_{\mathbf{\gamma}_{\gamma}}$ & $\begin{array}{c}{ }^{6} \mathrm{Kr} \\
\text { Singles }\end{array}$ & $\begin{array}{l}{ }^{86} \mathrm{Kr} \\
\text { Coinc. }\end{array}$ & $\begin{array}{l}{ }^{136} \mathrm{Xe}_{\mathrm{t}} \\
\text { Singles }\end{array}$ & $\begin{array}{l}{ }^{136} \mathrm{Xe}^{\mathrm{a})} \\
\text { Thick }\end{array}$ & $\begin{array}{c}{ }^{136} \mathrm{Xe}^{\mathrm{a})} \\
\text { Thin }\end{array}$ & & \\
\hline $\begin{array}{r}{ }^{178} \text { Hf } 6-4 \\
8-6 \\
10-8 \\
12-10 \\
14-12 \\
16-14 \\
18-16\end{array}$ & $\begin{array}{l}325.6 \\
426.4 \\
512.4 \\
579.7 \\
626.9 \\
658.6 \\
684.4^{\text {b) }}\end{array}$ & $\begin{array}{l}3.96 \prime \prime \\
1.55 s \\
0.82 s\end{array}$ & $\begin{array}{l}4.10 \mathrm{~s} \\
1.3510\end{array}$ & $\begin{array}{l}4.1110 \\
1.621 \\
0.939 \\
0.6514\end{array}$ & $\begin{array}{c}16.1 \\
3.98 \\
1.38 \\
0.75\end{array}$ & $\begin{array}{c}16.4 \\
3.79 \\
1.45 \\
0.75 \\
0.31\end{array}$ & $\begin{array}{l}16.28 \\
3.99 \mathrm{~s} \\
1.48 \mathrm{~s} \\
0.81 \mathrm{~s} \\
0.4810\end{array}$ & $\begin{array}{l}15.0 \\
3.86 \\
1.50 \\
0.78 \\
0.43\end{array}$ \\
\hline 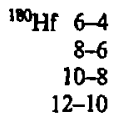 & $\begin{array}{l}322.3 \\
443.2 \\
546.5 \\
642.0\end{array}$ & & $\begin{array}{l}3.0823 \\
1.177 \\
0.489\end{array}$ & $\begin{array}{l}2.8620 \\
1.205 \\
0.556\end{array}$ & $\begin{array}{c}14.4 \\
3.50 \\
1.06 \\
0.55\end{array}$ & & $\begin{array}{l}14.410 \\
3.1515 \\
1.14 \mathrm{~s} \\
0.534\end{array}$ & $\begin{array}{c}13.2 \\
3.07 \\
1.05 \\
0.55\end{array}$ \\
\hline
\end{tabular}

2) Experimental errors are in the range of 5-10\% except for the $14^{+}-12^{+}$transition, where it is $\sim 25 \%$.

b) Tentatively assigned as the $18^{+} \rightarrow 16^{+}$transition.

\title{
A Third Discontinuity in the Yrast Levels of ${ }^{158} \mathbf{E r} *$
}

\author{
J. Burde, E.L. Dines, S. Shih, R.M. Diamond, \\ J.E. Draper, K.H. Lindenberger, C. Schuck, \\ and F.S. Stephens
}

Discrete $\gamma$-rays de-exciting states of compound nuclei formed during heavy-ion fusion reactions can give detailed information about the nuclear structure of such high spin states. Although resolved $\gamma$-rays from noncollective states have been observed for $1 \simeq 38$, for collective states the spins observed are usually below 30.

A study of the high members of the yrast sequence of ${ }^{158} \mathrm{Er}$ was done several years agol when a backbending (attributed to the alignment of an $i_{13 / 2}$ neutron pair) at $I=14$ and an upbend (attributed to the alignment of an $h_{11 / 2}$ proton pair) as high as $I=28$ were observed.

To study still higher members of the yrast sequence in ${ }^{156} \mathrm{Er}$, we used the ${ }^{122} \mathrm{Sn}\left({ }^{40} \mathrm{Ar}, 4 \mathrm{n} \gamma\right)$ reaction with a $170 \mathrm{MeV}{ }^{40} \mathrm{Ar}$ beam at the 88-Inch Cyclotron. Four, self-supporting, thin $\left(0.5 \mathrm{mg} / \mathrm{cm}^{2}\right.$ each) ${ }^{122} \mathrm{Sn}$ targets were used to insure narrow lines without a reduction in the yield.

Four GeLis were placed in a backward direction, $\pm\left(155^{\circ}\right.$ to $\left.160^{\circ}\right)$ with respect to the beam so as to minimize Doppler broadening while a fifth $\mathrm{GeLi}$ was placed alternately at $0^{\circ}$ and $90^{\circ}$ for angular distribiltion measufements.

About $10^{B}$ events (each consisting of a $\gamma-\gamma$ coincidence between any two of the five GeLis gated by one or more detectors of a $5-\mathrm{NaI}$ multiplicity filter surrounding the target) were recorded and analyzed. Figure 1 shows after background subtraction the $\gamma=\gamma$ coincidence spectra from the four backward $\mathrm{GeLis}$ gated by the sum of transitions de-exciting the $18^{+}-32^{+}$levels, as well as spectra gated by the three defined highest lines, $34^{+} \rightarrow 32^{+}, 36^{+} \rightarrow 34^{+}$, and $38^{+} \rightarrow 36^{+}$. From spectra like these, anisotropies (consistent with stretched E2s) and relative intensity determinations, we were able to establish the yrast sequence up to $I=38$. 


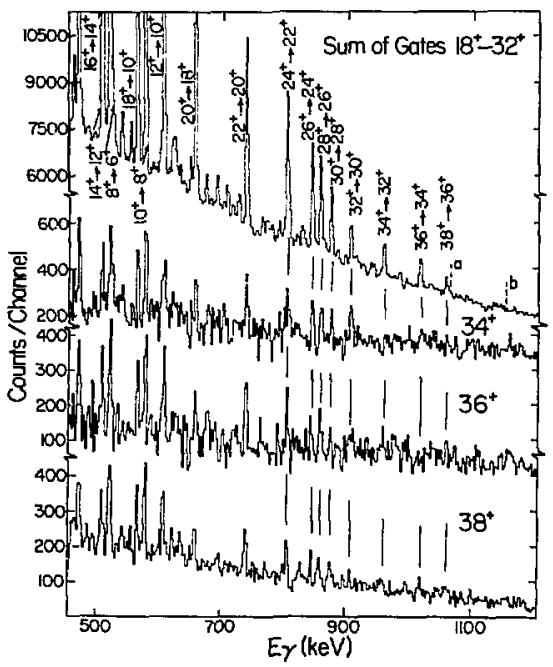

Fig. 1. Coincident $\gamma$-ray spectra from ${ }^{122} \mathrm{Sn}$ $\left({ }^{40} \mathrm{Ar}, 4 \pi\right){ }^{158} \mathrm{Er}$ obtained with indicated gates. XBL 8111-1577

In an attempt to find any higher members, 15 weak lines were taken as possible candidates, and the data were sorted in coincidence with these 15 lines. We then looked at the intensity of each yrast line from these spectra (after background subtraction) divided by the intensity of the same line obtained from the sum of gates on transitions from the $34^{+}, 36^{+}$, and $38^{+}$levels. If a line feeds the whole yrast sequence from above the $38^{+}$level, those ratios must be constant up to $32^{+}$and go above unity for the three higher transitions, because of their suppression in the denominator sum spectrum. Two lines, at $1067 \mathrm{keV}$ and $1155 \mathrm{keV}$ appear to comply with these requirements. We tentatively considered the former to be the most likely $40^{+} \rightarrow 38^{+}$yrast member.

Figure 2 summarizes the high-spin nuclear structure of ${ }^{158} \mathrm{Er}$ best. The moment of inertia, $2 \mathscr{S} / \hbar^{2}$, total spin $I$, and the relative aligned spin, $i$, were platted $^{2}$ vs the rotational frequency $\hat{h} \omega$. The beginning of a third discontinuity at $h \omega \simeq 0.53 \mathrm{MeV}$ is suggested. This would imply an alignment with the rotation axes of an additional high-j nucleon pair, thus continuing the pattern for the nucleus to generate its angular momentum partly from collective rotation, partly from alignment of high-j nucleons.
The two $i_{13 / 2}$ neutrons at the first backbend contribute $10 \mathrm{~h}$ of aligned spin out of a total spin of $16 \mathrm{~h}$ while the two $h_{11 / 2}$ protons responsible for the second discontinuity contribute $6 h$ of aligned spin to a total spin of $28 \hat{h}$. Although the aligned spin is not yet determined, the third discontinuity (at about $I=40 \mathrm{~h}$ ) seems most likely to be caused by either $h_{9 / 2}$ neutrons or $h_{9 / 2}$ protons, of which the former seem the most likely from calculations. ${ }^{3}$

\section{Footnote and References}

*Condensed from Phys. Rev. Lett. 48 (1982) 530

1. I.Y. Lee, et al., Phys. Rev. Lett. 38 (1977) 1454

2 R. Bengtsson and S. Frauendorf, Nucl. Phys. A327f1 (1979) 139

3. S. Frauendorf, Phys. Scripta 24 (1981) 349

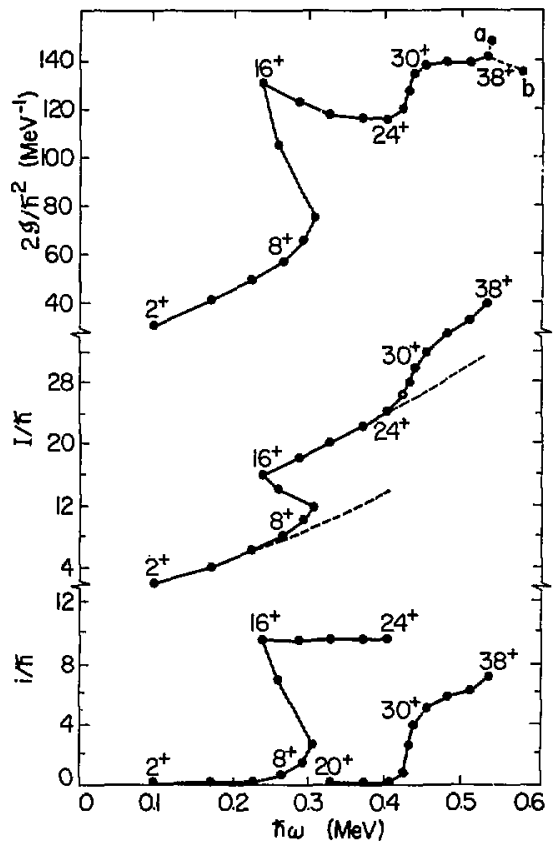

Fig. 2. Plots of moment of inertia (top), yrast spin (midule), and relative spin alignment (bottom) vs the rotational frequency.

XBL 8111-1576 


\title{
Lifetimes of Unresolved Transitions from Very High Spins
}

\author{
in Nuclei Near $\mathbf{N}=82 *$
}

\author{
H. Hübel,,$^{\dagger}$ R.M. Diamond, P. Aguer, ${ }^{\ddagger}$ C. Ellegaard, \\ D.B. Fossan, ${ }^{* *}$ H. Kluge,tt C. Schuck, ${ }^{\ddagger}$ S. Shih, \# \\ F.S. Stephens, ana' U. Smilanskit
}

Properties of nuclei in excited states of very high angular momentum can be studied by investigations of their de-excitation $\gamma$-ray spectra. The unresolved spectra which are connected to the de-excitation of the high spin states populated in heavy-ion compound-nucleus reactions are typically composed of three parts: a statistical component, an E2 bump and a lower-energy dipole part. It has been found that the high-energy edge of this bump moves to higher energies with increasing angular momentum put into the system and that the multiplicity of the transitions in this region is higher than the average multiplicity. Furthermore, angular correlation measurements show that these transitions are predominantly of stretched quadrupole multipolarity.

Recently ${ }^{1}$ the average lifetimes of the transitions in the E2 bump of a good rotor have been measured with the Doppler-shift attenuation method. From these measurements collective E2-enhancement factors were deduced, indicating that the transitions in the E2 bump are, indeed, collective transitions between high-spin states within rotational bands.

In the present paper we use the same method to try to follow the development of collectivity in a nucleus that is spherical at low spin. A comparison is made for two different systems: the ${ }^{157} \mathrm{Er}^{*}$ compound system which leads to evaporation residues around spherical ${ }^{152} \mathrm{Er}$, and the ${ }^{164} \mathrm{Er}^{*}$ compound system which leads to nuclei around rotational ${ }^{159} \mathrm{Er}$.

De-excitation $\gamma$-ray spectra were measured with two $12.7 \mathrm{~cm} \times 15.2 \mathrm{~cm} \mathrm{Nal}(\mathrm{TI})$ detectors located at $0^{\circ}$ and $90^{\circ}$ with respect to the beam direction. They were placed at a distance of $60 \mathrm{~cm}$ from the target to allow for neutron discrimination by time of flight, and were in coincidence with a multiplicity filter. In order to produce the $\mathrm{Er}$ evaporation residues with high recoil velocities, a light-mass target, ${ }^{28} \mathrm{Si}$, was bombarded with heavy projectiles, ${ }^{129} \mathrm{Xe}$ and ${ }^{136} \mathrm{Xe}$, at the LBL SuperHILAC. With projectile energies of $720 \mathrm{MeV}$ and $740 \mathrm{MeV}$, respectively, the recoil velocities were $\mathrm{v} / \mathrm{c} \approx 0.085$. Spectra were measured with a selfsupporting target of $2.3 \mathrm{mg} / \mathrm{cm}^{2}$ thickness and with a target of the same thickness on a $25 \mathrm{mg} / \mathrm{cm}^{2}$ Au backing, alternating cvery few hours. By comparing the Doppler shift for the spectra measured in the $0^{\circ} \mathrm{NaI}$ detector with the unbacked target and with the target on the backing, average lifetimes of continuum r-rays can be deduced if their lifetimes (including feeding time) are of the same order of magnitude as the stopping time of the recoiling Er evaporation residues in the Au foil. The $90^{\circ} \mathrm{NaI}$ detector is used for normalization.

The effect of the different Doppler shifts obtained for the self-supporting and the backed targets can be seen in the ratios of the spectra shown in Fig's 1 and 2. The oscillations in the ratios below $\sim 900 \mathrm{keV}$ result mainly from the Doppler shift of long-lived discrete lines. At higher energies the smooth slope is produced by a larger Doppler shift in the spectra from the unbacked target. Above $\sim 1.6 \mathrm{MeV}$ in the ${ }^{164} \mathrm{Er}^{*}$ system and $\sim 3.5 \mathrm{MeV}$ in the ${ }^{151} \mathrm{Er}^{*}$ system the lifetimes are much shorter than the stopping time of the recoiling $\mathrm{Er}$ atoms in the $\mathrm{Au}$ foil because no difference is observed between the spectra from the backed and unbacked targets.

The data analysis was performed as described in ref. 1. Each level in the continuum is assumed fed either from higher members of the rotational band or by prompt side feeding. For the prompt part, a distribution of initial populations

$$
\sigma_{\mathrm{I}} \sim \frac{\mathrm{I}}{1+\exp \left[\left(\mathrm{I}-\mathrm{I}_{\max }\right) / 2.5\right]}
$$

is used where $I_{\text {max }}$ is the maximum angular momentum 


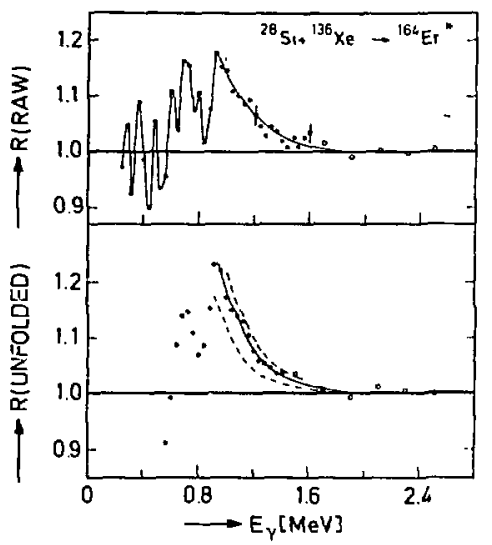

Fig. 1. Upper part: Ratio of the raw (not unfolded) spectra messured at $0^{\circ}$ with the ${ }^{136} \mathrm{Xe}_{\mathrm{C}}$ beam on a self-supporting ${ }^{2} \mathrm{Si}$ target and on a ${ }^{2} \mathrm{Si}$ target on a Au backing. The spectra were normalized using the integrated counts of the $90^{\circ}$ detector. The curve is drawn to guide the eye.

Lower part: Ratio of the unfolded experimental (dots) and calculated spectsa (solid curves). The dashed curves were calculated for the extreme values of $\alpha$ allowed by the errors given in Table I.

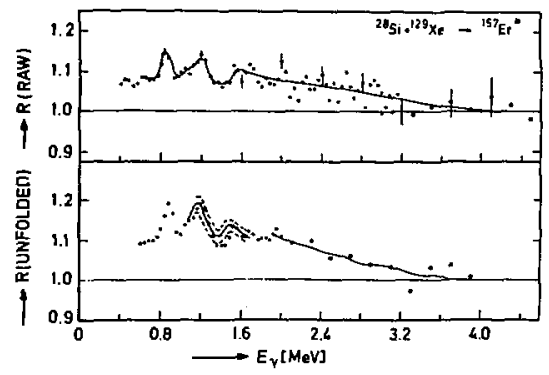

Fig. 2. Same as Fig. 1., but for the ${ }^{129} \mathrm{Xe}$ beam.

brought into the compound nucleus. Within the rotational bands each level decays to the next lower one by an E2 transition and the transition lifetimes are determined according to the rotational model formula

$$
\begin{gathered}
\tau=800 \mathrm{E}_{(\mathrm{kev})}^{-5}\left(<\mathrm{I}_{\mathrm{i}} 2 \mathrm{KO} 0 \mid \mathrm{I}_{\mathrm{f}} \mathrm{K}>^{2} \alpha^{2} \mathrm{Q}_{\mathrm{Q}(\mathrm{b})}^{2}\right)^{-1} \mathrm{~s} \\
\left(\text { or } \tau=\alpha \mathrm{E}^{-5}\right) .
\end{gathered}
$$

The average intrinsic quadrupole moment $Q_{b}$ is obtained by fitting the calculated ratios of spectra for the unbacked and backed targets to the experimental ratios with $Q_{0}$ (or $\alpha$ ) as the only free parameter.

In a first step the procedure described above was applied to both systems, ${ }^{164} \mathrm{Gr}^{*}$ and ${ }^{157} \mathrm{Er}^{*}$. For the rotational ${ }^{16 \mathrm{H}} \mathrm{Er}^{*}$ system we obtain good agreement in both magnitude and slope between the calculated (solid curve in the lower part of Fig. 1) and the experimental ratio of spectra. For the more spherical ${ }^{157} \mathrm{Er}^{*}$ system, however, no satisfactory agreement between the calculated and measured ratio could be obtained. We therefore had to modify the simple model to include the specific features of the ${ }^{157} \mathrm{Er}^{*}$ system.

It is known that the overall amount of stretched E2 transitions is smaller in the light Er nuclei than in the heavier rotational isotopes; approximately 70\% E2 transitions in the E2 bump and an overall E2 contribution of approximately $50 \%$. In the high energy tail above the yrast bump the E2 component is much less than $50 \%$. A reasonable assumption is probably $70 \%$ $100 \%$ for the amount of dipole transitions in the high energy tail of the spectrum.

The inclusion of such a large dipole contribution in the analysis of the Doppler-shift data presents a major problem. There exists no simple relation between transition energy and spin for the dipole transitions (these transitions are probably emitted statistically), and their transition probabilities can vary over a wide range depending on the structure of the states involved in the transition (they are not determined, as for rotational $\mathrm{E2}$ transitions, by a single parameter). Nevertheless, we have attempted to obtain some quantitative results from the data for the ${ }^{157} \mathrm{Er}^{*}$ system with the following assumptions: (i) The quadrupole to dipole ratios deduced from the angular correlation experiment were used. (ii) Feeding time for statistical (dipole) transitions were (a) neglected, and (b) varied in several reasonable ways. Fortunately, it turned out that the feeding times, if reasonably fast, do not have a very large effect on the results and their influence could be taken into account by increasing the uncertainties of the results. (iii) For the transition lifetimes we use the expression

$$
\tau=(1-\mathrm{x}) \alpha_{1} \mathrm{E}^{-\mathrm{t}}+\mathrm{x}_{\alpha_{2}} \mathrm{E}^{-5}
$$

with the fraction $x$ of quadrupole transitions in the 
yrast bump and in the high energy tail regions fixed as discussed above. The two parameters $\alpha_{1}$ and $\alpha_{2}$ (the lifetimes of $1 \mathrm{MeV}$ statistical and E2 transitions respectively) were determined by fits of the calculated yield ratio to the experimental ratio. The results of the fits in the two energy regions are shown by the solid curves in the lower part of Fig. 2. The slope of the data in the high energy region was found to be well reproduced by an $\mathrm{E}^{-3}$-dependence of the first component in (3) and the best fits in this energy region were obtained with $x=$ 0 . In the E2-bump region between 1.0 and $1.6 \mathrm{MeV}$ we find that the best (but still not perfect) fit to the data is obtained with $x=0.7$ and with $\alpha_{1} \approx 10 \alpha_{2}$. The values for the parameters $\alpha_{i}$, the quadrupole moments $\mathrm{Q}_{\text {b }}$, the enhancement factors, and the deformation parameters are summarized in Table I.

Table I.

Results of lifetime measurements of continuum transitions depopulating very high spin states

\begin{tabular}{lcccccc}
$\begin{array}{c}\text { Compound } \\
\text { nucleus }\end{array}$ & $\begin{array}{c}\text { Energy } \\
\text { range (MeV) }\end{array}$ & $\begin{array}{c}\alpha_{1} \\
(\mathrm{ps} \mathrm{MeV})\end{array}$ & $\begin{array}{c}\alpha_{2} \\
(\mathrm{ps} \mathrm{MeV})\end{array}$ & $\begin{array}{c}\mathrm{Q}_{1} \\
(\mathrm{eb})\end{array}$ & $\begin{array}{c}\mathrm{B}(\mathrm{E} 2) \\
\mathrm{B}(\mathrm{E} 2)_{a, p}\end{array}$ & $\beta_{2}$ \\
\hline${ }^{164} \mathrm{Er}^{*}$ & $1.0-1.6$ & - & 0.05520 & 6.11 .6 & 17060 & 0.299 \\
${ }^{157} \mathrm{Er}^{*}$ & $1.0-1.6$ & 0.63 & 0.075 & 5.43 .0 & 150110 & 0.2619. \\
& $2.0-3.5$ & 0.62 & - & - & - & - \\
\hline
\end{tabular}

Footnotes and References

*Condensed from Z. Phys. A - Atoms and Nuclei 304, (1982) 225

fInstitut für Strahlen-und Kernphysik der Universität, Bonn Federal Republic of Germany †Centre de Spectrometric Nucléaire, Orsay, France \$Niels Bohr Institute, Risṕ, Denmark * S.U.N.Y. Stony Brook, New York
††Hahn-Meitner-Institut für Kernforschung, Berlin, West Germany

¥\$Shanghai Institute of Nuclear Research, Shanghai, China

\$8The Weizmann Institute of Science, Rehovot, Israel

1. H. Hübel, U. Smilansky, RM. Diamond, F.S. Stephers, B. Herskind, Pbys. Rev. Lett. (1978) 791 


\title{
Nuclear Data
}

\section{First Conference on Nuclear Structure Data Evaluation}

\author{
R.B. Firestone, V.S. Shirley, and J.M. Dairiki
}

Tt:e 1st Conference on Nuclear Structure Data Evaluation, organized by the Isotopes Project, was held at the Asilomar Conference Grounds, Pacific Grove, $C A$, on October 27 - 30, 1981. Nearly 20 evaluators and interested observers attended the conference. Roundtable discussions were held on the topics of Evaluation as a Science and Evaluation and the Scientific Community. In addition, nine scientific papers on various aspects of evaluation and nuclear structure systematics were presented. The following papers were given by members of the Isotopes Project.

1. Systematic Survey of $\boldsymbol{\gamma}$-ray Multipolarities.

E. Browne and R.B. Firestone

2. Radioactivity Handbook.

J.M. Dairiki
3. Results of the Radioactivity Handbook Survey.

\section{J,M. Dairiki}

4. Are $\log f t$ Values Reliable Guides for Spin and Parity Assignments?

R.B. Firestore

5. Reevaluation of the $\log f t$ Systematics for the Assignment of Spins and Parities.

R.B. Firestone

A complete summary of the roundtable discussions and the text of the submitted papers has been published in the proceedings of the conference (LBL14070).

\section{$X$-ray and Auger-Electron Intensities from $K, L_{1}, L_{2}$, and $L_{3}$ Atomic Shell Vacancies Produced by Nuclear Decay}

\author{
R.B. Firestone
}

$X$-rays and Auger electrons are often the most intense radiations associated with nuclear decay. However, they are seldom accurately measured because of their low energies. The systematics and theory of atomic transitions are now known reliably enough tc calculate $x$-ray and Auger-electron intensities arising from $K, L_{1}, L_{2}$, and $L_{3}$ atomic shell vacancies produced in nuclear decay.

Atomic vacancies are primarily produced in nuclear decay by internal conversion and electron capture decay. I have calculated the internal conversion atomic vacancies using the tables of Rosel, et al., ${ }^{1}$ and the electron capture vacancies using the tables of Bambynek, et al. ${ }^{2}$ To account for fuorescence-, Auger-, and Coster-Kronig processes 1 have used the tables of Krause, et al."

After determining the total intensity of all $x$-ray and Auger lines originating in each atomic shell, it is necessary to divide that intensity into the constituent lines associated with each vacancy. For $x$-rays, I accomplished this with a combination of the systematic data compiled by Salem, et al. ${ }^{4}$ and the theoretical calculations by Scofield. ${ }^{5}$ I used the calculations of Chen, et al., ${ }^{6}$ to distibute the Auger intensity. Usually, values given by theory were as accurate as the experimental data.

Finally, I derived the $x$-ray energies from the atomic binding energies compiled by Bearden ${ }^{7}$ and the Auger-electron energies from calculations by Larkins. ${ }^{8}$ 
The ENSDF nuclear data file (DATATRIEVE version) has been selected as the source of input nuclear decay data, and the final atomic transition energies and intensities have been calculated with my computer code ATOMS. The statistical errors for all data are added in qu. irature and are included with the final intensities. A comparison of experimental and calculated $x$-ray intensities from $A=252$ is shown in Table 1. The complete set of calculated $x$-ray energies and intensities will appear in the Handbook of Radioactivity.

TABLE I. Comparison of Experimental and Calculated X-ray Intensities

\begin{tabular}{|c|c|c|c|}
\hline Decay & Transition & $\begin{array}{l}\text { Intensity } \\
\text { Experiment }\end{array}$ & Calculation \\
\hline \multirow{4}{*}{${ }^{252} \mathrm{Es} \underset{\rightarrow}{\alpha{ }^{241} \mathrm{Bk}}$} & $\mathrm{K}_{\alpha_{1}}$ & 0.00585 & 0.00478 \\
\hline & $K_{\alpha_{2}}$ & 0.00373 & $0.0030 \mathrm{~g}$ \\
\hline & $K_{\beta_{1}}^{\prime \prime}$ & 0.00232 & 0.00234 \\
\hline & $K_{f_{2}}^{\prime \prime}$ & $0.70082 s$ & 0.0006110 \\
\hline \multirow[t]{4}{*}{${ }^{252} \mathrm{Es} \stackrel{\mathrm{EC}}{{ }^{252} \mathrm{Cf}}$} & $K_{\alpha_{i}}$ & 4.74 & 4.38 \\
\hline & $\mathrm{K}_{\mathrm{r}_{2}}$ & $3.1 \mathrm{~J}$ & $2.8 \mathrm{~s}$ \\
\hline & $K_{\theta_{i}}^{\prime 2}$ & 1.92 & 2.14 \\
\hline & $K_{\mathrm{A}_{2}}^{\prime}$ & 0.668 & $0.57 n$ \\
\hline \multirow[t]{9}{*}{${ }^{252} \mathrm{Cf}{ }_{\rightarrow \rightarrow}^{\alpha 248} \mathrm{Cm}$} & $\mathrm{L}_{\mathrm{a}_{1}}$ & 2.83 & 2.54 \\
\hline & $\mathbf{L}_{\mathrm{O}_{2}}$ & 0.313 & 0.28 \\
\hline & $\mathrm{L}_{\beta_{1}}$ & 2.62 & 2.24 \\
\hline & $\mathrm{L}_{\beta_{2}}$ & $0.62 \mathrm{~s}$ & 0.6810 \\
\hline & $\mathrm{L}_{\mathrm{P}_{3}}$ & 0.163 & $0.16 z$ \\
\hline & $\mathrm{L}_{\gamma_{1}}$ & 0.706 & 0.5510 \\
\hline & $\mathrm{L}_{\gamma_{23}}$ & 0.0204 & 0.0161 \\
\hline & $L_{\gamma_{6}}$ & 0.153 & 0.112 \\
\hline & $\mathrm{L}_{\ell}$ & 0.232 & 0.193 \\
\hline
\end{tabular}

\section{References}

1. F. Rosel, et al., Atomic Data and Nucl. Data Tables 21, nos. 2-5 (1978)

2. W. Bambynek, et al., Rev. Mod. Phys. 49 (1977) 77

3. M.O. Krause, J. Phys. Chem. Ref. Data 8 (1979) 307

4. S.I. Salem, et al., Atomic Data and Nucl. Data Tables 14 (1974) 91
5. J.H. Scofield, Atomic Data and Nucl. Data Tables 14 (1974) 121 and Phys. Rev. A9 (1974) 1041

6. M.H. Chen, et al., Atomic Data and Nucl. Data Tables 24 (1979) 13

7. J.A. Bearden, Rev. Mod. Phys. 39 (1967) 78

8. F.B. Larkins, Atomic Data and Nucl. Data Tables 20 (1977) 328 


\title{
Gamma-Ray Transition Probability Systematics
}

\author{
E. Browne and R.B. Firestone
}

The use of $\gamma$-ray transition probabilities for assigning multipolarities has been limited in the past by the fact that only gross lower limits to the half-lives had been suggested. These limits are derived primarily from the compilation of $\gamma$-ray transition strengths by Endt. ${ }^{1-3}$ Recommended upper limits for the transition strengths have been suggested by Endt based on the fastest reliably known transitions of a given multipole. These limits are valid, but they are too restrictive because they fail to account for the effects of nuclear structure. For more detailed systematics, one must look to the older works of Moszkowski ${ }^{4}$ and of Coldhaber and Sunyar."

We have initiated a new, systematic study of $\gamma$ ray transition probabilities based on varied sources such as the Nuclear Structure Database from the Table of

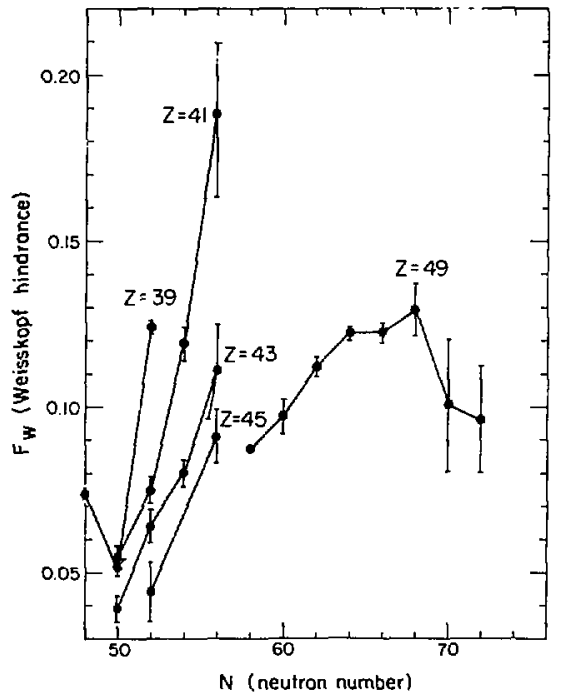

Fig. 1. $g_{/ / 2} \rightarrow p_{1 / 2} M 4$ Weisskopf hindrances.

XBL $8110-7371$
Isotopes, ${ }^{6}$ Endt's compilations, ${ }^{1-3}$ and ENSDF. ${ }^{7}$ For each multipole the transition probabilities are being mapped out in terms of nuclear structure. An example is shown for the $g_{9 / 2} \rightarrow p_{1 / 2} M 4$ transitions near $Z=50$ in Fig. 1. These transition probabilities are observed to vary smoothly.

Tlie available data for the M4 isomeric transition in ${ }^{117} \mathrm{In}$ are summarized in Table 1 . These data are clearly divergent, yet an evaluator is required to select a best value. Previously, most evaluators have chosen the newest data in cases like this. However, with the aid of systematics it is evident that the older data are probably correct. Closer inspection of the newer data indicates that the disagreement may result from an incomplete correction for transient equilibrium to the $\gamma$-ray intensities. This work demonstrates the utility of systematics in selecting the most accurate nuclear data.

Table 1. Hindrance of the $315-\mathrm{keV} \mathrm{M} 4$ iransition in ${ }^{117} \mathrm{In}$

\begin{tabular}{lcc}
$\begin{array}{l}\text { Branching } \\
\text { Ratio (\%) }\end{array}$ & $\begin{array}{c}\text { Hindrance } \\
\text { Factor (FW) }\end{array}$ & Reference \\
\hline $28 \quad(1)$ & $0.12(1)$ & 9 \\
43 & $0.079(5)$ & 10 \\
$26.5(15)$ & $0.129(8)$ & 11 \\
$47.1(15)$ & $0.072(3)$ & 12 \\
\hline
\end{tabular}

References

1. P.M. Endt, At. Nucl. Data Tables 23 (1979) 3

2. P.M. Endt, At. Nucl. Data Tables 23 (1979) 547

3. P.M. Endt, At. Nucl. Data Tables 23 (1981) 47

4. S.A. Moszkowski in "Alpha-, Beta-, and Gammaray Spectroscopy", edited by Kai Siegbahn (North Holland, Amsterdam, 1965), Vol. 2, pp. 863-886 
5. M. Goldhaber and A.W. Sunyar, ibid., pp. 931944

6. R.B. Firestone and E. Browne, "Nuclear Structure Database", LBL-1 1089 (1980)

7. "Evaluated Nuclear Structure Data File", edited and maintained by the National Nuclear Data Ccnter, Brookhaven National Laboratory, on behalf of the International Network for Nuclear Structure Data Evaluation

8. E. Browne and R,B. Firestone in "Proceedings of the Isi Conference on Nuclear Structure Data
Evaluation", edited by R.B. Firestone, V.S. Shirley, and J.M. Dairiki (LBL-14070, 1982)

9. J.H. Wolfe and J.P. Hummel, J. Inorg. Nucl. Chem. 22 (1961) 7

10. O.A. Baedecker, A. Pakkanen, and W.B. Walters, Nucl. Phys, A158 (1970) 607

11. L. Heath, "Gamma-Pay Spectrum Catalogue", ANCR-1000-2 (1974)

12. W. Tang, A Fakkanen, Z.C. Mester, C.D. Coryell, G. Chilos, K. Bos, and A.H. Wapstra, Z. Physik A272 (1975) 301

\title{
SPINOZA: A Computer Code for the Scientific Evaluation of
}

\section{Nuclear Data}

\author{
R.B. Firestone
}

The computer code SPINOZA was designed to assist nuclear data evaluators in testing the consistency and scientific rigor of spin, parity, multipolarity, and other decay level-scheme assignments. This program accepts input data written in the Evaluated Nuclear Structure Data File (ENSDF) format and produces a complete tabular listing of the input data. SPINOZA also indicates to the evaluator scientific errors in the construction of a level scheme.

SPINOZA performs a reliable test of all level spins and parities. Beginning with states of known spin and parity, SPINOZA derives the spins and parities of all levels using the multipolarities of the interconnecting $\gamma$ rays and the $\log f t$ values from $\beta$-decay, All $\gamma$ rays are considered to be stretched multipoles with the exception of those with an E2 multipolarity where an M1 admixture is assumed to be possible. However, the program has an option to consider E2 as a stretched transition. The $\gamma$ rays of unknown multipolarity are presumed to be $\mathrm{M} 1, \mathrm{E} 1$, or E2. Other options allow $\mathrm{M} 2$ or eliminate E2 [e.g., $(\mathrm{n}, \gamma)$ reactions]. Logft values are presumed to follow the selection rules of Raman. ${ }^{1}$
If a transition is found to be inconsistent with all possible spin/parity values (as determined by other transitions), an error message is printed warning the evaluator that the transition cannot be placed. SPINOZA also differentiates between the spin/parity assignments derived from definite and less certain transitions. Finaily, the program generates a listing of the possible spins and parities for each level.

In addition to deducing spins and parities, SPINOZA also verifies the level scheme intensity balances and produces a line-printer level scheme drawing. Future innovations will include a comparison of the transition probabilities with systematics. SPINOZA is expected to be an invaluable aid in the preparation of handbooks from the ENSDF file by maintaining the highest scientific standards.

\section{Reference}

I. S. Raman and N.B. Gove, Phys. Rev. C7 (1973) 1995 


\title{
Implementation of the Evaluated Nuclear Structure Data
}

\section{File into DATATRIEVE}

\author{
R.B. Firestone and E. Browne
}

The most comprehensive information on nuclear structure is contained in the Evaluated Nuclear Structure Data File (ENSDF). This computer file, which consists of about 500,000 records, is edited and maintained by the National Nuclear Data Center, Brookhaven National Laboratory, on behalf of the International Network for Nuclear Structure Data Evaluation. ElvSDF is primarily used for the publication of mass-chain evaluations in the Nuclear Data Sheets, which serve the needs of a broad range of basic and applied users. The file may be used for studies of the systematics of nuclear properties, creation of horizontal evaluations, and the generation of specialized numerical files for complex calculations. At present these applications bave been limited for the following reasons.

First, although the file has a simple structure of 80-character records with well-defined fields, the contents within these fields are not uniform; i.e., characters often appear mixed with numerical quantities. This requires elaborate computer decoding to extract the data and separate the numerical from the non-numerical quantities. Second, the file has about twelve record types with different field definitions that are interconnected only by their sequential order in the file. For example, a " $\gamma$ record", consisting of $\gamma$-ray information, always follows the "level record" containing the level properties corresponding to the de-excited level. This secord organization makes the file inconvenient for use with database management systems that provide fast and efficient access to records. Finally, many connections among records are entirely missing from ENSDF. For instance, the terminal level of a $\gamma$ transition is not in the file.
We have overcome these difficulties by converting the "numbers" in ENSDF into four distinct quantities. Each value is converted into a real number, its experimental uncertainty, an alphanumeric modifier (c.g., AP, LT, or IF), and the number of significant digits required to represent the number. The quantities associated with each record type are then stored in indexed files containing additional special identifier fields to interconnect the different files. For example, the file of $\gamma$-ray records also contains indices connecting each $\gamma$ ray to the original ENSDF dataset identification, radioactive parent, and initial- and final-level records. These files have been stored in the VAX-1l computer at LBL and implemented into DATATRIEVE, a database management system provided by Digital Equipment Corporation.

We are using this database for the selective retrieval of data to present in the Handbook of Radioactivity, for the generation of input data to calculate atomic $X$-ray and Auger electron intensities, and to derive $\beta-, \beta+$, electron capture, conversion electrons, and $\gamma$-ray "binned" average energies per decay. A project to match the nuclear energy levels populated in different reactions and decays with the "adopted" levels reported in ENSDF is in progress. This will resolve a missing link in ENSDF and is indispensable for combining data from different experiments into a new "adopted" file. Although in some instances the matching procedure may require human intervention, we are minimizing this through extensive and reliable programming.

Currently 64 mass-chains $(A=200$ to $A=263$ ) have been implemented into the database, and we expect to install the entire ENSDF file within a year. We expect this numerical database to constitute a powerful research tool for theoreticians, experimentalists, and applied users. 


\title{
2. Nuclear Reaction Mechanisms
}

\section{Polarization Studies}

\section{The Elastic Scattering of Polarized Protons by ${ }^{40} \mathrm{Ar}^{*}$}

\author{
N.T. Okumusoglu, ${ }^{\dagger}$ J. Birchall, ${ }^{\dagger}$ M.S.A.L. Ai-Ghazi, ${ }^{\dagger}$ \\ C. Lapointe, ${ }^{\dagger}$ J.S.C. McKee, ${ }^{\dagger}$ H.E. Conzett, R. M. Larimer \\ and $P$. von Rossen ${ }^{\ddagger}$
}

It has been found that the standard optical model analyses of the elastic scattering of medium energy protons from ${ }^{15} \mathrm{~N},{ }^{18} \mathrm{O},{ }^{24} \mathrm{Mg},{ }^{40} \mathrm{Ca}$ and ${ }^{40} \mathrm{Ar}$ run into difficulties in fitting the cross-section at the minimum around $140^{\circ} \mathrm{c.m}$. and the enhancements at larger angles. Evidence has been found recently for explicit angular momentum dependence of the proton optical model potential (OMP). ${ }^{1-3}$ Furthermore, it has been shown that inclusion of an L-dependent potential may in fact solve the problems in-the optical model analysis of large-angle scattering at low energies.

Examination of the analyzing powers predicted by the L-independent and L-dependent optical model calculations for ${ }^{40} \mathrm{Ar}(\overrightarrow{\mathrm{p}}, \mathrm{p}){ }^{40} \mathrm{Ar}$ elastic scattering at $\mathrm{E}_{\mathrm{p}}=$ $32.5 \mathrm{MeV}$ leads to the conclusion that at large angles the effect of L-dependerce in the OMP is reflected much more in the analyzing power than in the differential cross-section. Similar observations have been made for proton scattering from ${ }^{16} \mathrm{O}$ at $34.1 \mathrm{MeV} .{ }^{1}$ Therefore, analyzing power as well as cross-section data are needed at various energies in order to arrive at more convincing results regarding the L-dependence of the optical model potential.

The purpose of the present work is to provide analyzing power data for ${ }^{40} \mathrm{Ar}(\overrightarrow{\mathrm{p}}, \mathrm{p}){ }^{40} \mathrm{Ar}$ scattering at $25.1,32.5$ and $40.7 \mathrm{MeV}$, complementing existing data at 30 and $50 \mathrm{MeV}$, and to examine a possible $\mathrm{L}$ dependence of the optical model potential.

Protons with a polarization of approximately $82 \%$ were acceleraied to the desired energies by the LBL 88-Inch Cyclotron. The target consisted of argon gas contained in a cell at a pressure of 2 atmospheres. Four sets of detector telescopes were used. They were located symmetrically with respece to the incoming beam so that measurements of analyzing power were made at two angles simultaneously. Each telescape comprised a $0.5 \mathrm{~mm}$ passing detector $(\Delta E)$ and a stopping detector (E) with a total depletion depth of $8 \mathrm{~mm}$. The $\triangle E-E$ systems were used for particle identification. Collimators defined a geometry factor of $3 \times 10^{-6} \mathrm{~cm}$. sr. and provided an angular resolution of $\pm 0.5^{\circ}$ (lab).

The analyzing power measurements at $32.5 \mathrm{MeV}$ are shown in Fig. 1. The curves are optical model fits including $\mathbf{L}$-dependence (full curve), the prediction from the L-dependent fit to the differential crosssections alone (dash-double-dot curve, an L-independent fit (dashed curve) and the fit obtained from the latter with the addition of an L-dependent term with a strength of $\mathrm{V}_{\mathrm{e}}=0.35 \mathrm{MeV}$ (dotted curve). The optical model searches were made with the code SEEK3. The L-dependence included in the calculation is of the form

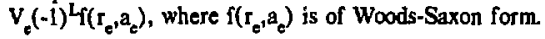

The inclusion of an L-dependent term improves the quality of the fit to the data, as can be seen in Fig. I by comparing the L-independent fit (dashed curve) with the L-dependent result (dotted curve). However, the physical significance of such a term is not yet understood, but may be required to allow for residual effects of the Pauli principle not taken into account by the standard optical potential. The energy dependence of the strength, $V_{e}$, of the L-dependent term used to fit our ${ }^{40} \mathrm{Ar}(\mathrm{p}, \mathrm{p}){ }^{40} \mathrm{Ar}$ elastic scattering differential crosssection and analyzing power data has its largest negative value $(-0.92 \mathrm{MeV})$ near $25 \mathrm{MeV}$, its largest positive value $(+0.33 \mathrm{MeV})$ near $32.5 \mathrm{MeV}$, and becomes negligible at beam energies above $45 \mathrm{MeV}$.

\section{Footnotes and References}

"Condensed from LBL-15186

†Cyclotron Laboratory, department of Physics, University of Manitoba, Winnipeg, Manitoba, Canada R3T $2 \mathrm{~N} 2$.

łlnstitut für Strahlen und Kernphysik, Universität, Born, D5300 Bonn, Germany. 
1. A. M. Kobos and R. S. MacKintosh, J. Phys. G5 (1979) 97

2. R. S. MacKintosh and A. M. Kobos, J. Phys. G4 (1978) L135

3. A. M. Kobos and R.S. MacKintosh, Ann. Phys. 123 (1979) 296

Fig. 1. The analyzing power for ${ }^{40} \mathrm{Ar}(\mathrm{p}, \mathrm{p})^{40} \mathrm{Ar}$ at 32.5 MeV. Full curve - L-dependent fit, dash-double dot curve - L-dependent prediction from best fit to the cross-sections, dashed line - best fit without Ldependent term, dotted line - best fit with L-dependent term added to the latter potential.

XBL 834-9470

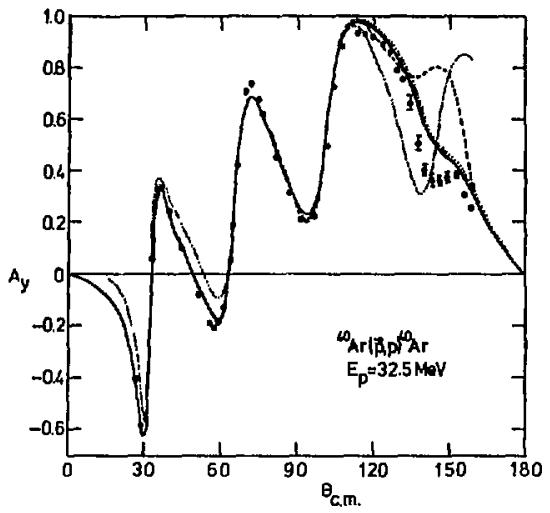

\title{
An Experimental Test of Charge Symmetry in n-p Elastic Scattering*
}

\author{
J. Birchall, ${ }^{\dagger}$ N.E. Davison ${ }^{\dagger}$ H.P. Gubler, W. P. Lee. ${ }^{\dagger}$ \\ J. P. Svenne, ${ }^{\dagger}$ W.T.H. van Oers, ${ }^{\dagger}$ R. Abegg, ${ }^{\ddagger}$ C.A. Miller, ${ }^{\ddagger}$ \\ E.B. Cairns', H. Coombes, C.A. Davis, $P$. Green, \\ L.G. Greeniaus, W.J. McDonald,' G.A. Moss, 'G. Roy, \\ J. Soukup, G.M. Stinson, H.E. Conzett, and G.R. Plattnert'
}

Charge symmetry is a consequence of isotopiz spin invariance, the first "internal symmetry" that was postulated in elementary particle physics. Isotopic spin invariance is broken by the electromagnetic interaction and consequently one expects effects of the order of the fine structure constant $\alpha=1 / 137$. Charge symmetry is a lesser symmetry because it only involves a rotation in isospin space through $\pi$. In the case of nucleons, charge symmetry has as a consequence that observables are unaffected by changing neutrons into protons and protons into neutrons. Thus, the neutron-neutron scattering length and effective range are equal to the proton-proton scattering length and effective range after correcting for electromagnetic effects, e.g., the Coulomb interaction. But also, charge symmetry has as a consequence various equalities among neutron-proton scattering spin-dependent obervables, for instance the polarization of the neutron resulting from the scattering of unpolarized neutrons from unpolarized protons equals the polarization of the recoil proton.

Studies of the low energy nucleon-nucleon scattering parameters have shown that charge-independence breaking interactions exist. The neutron-neutron scattering length is greater than the $T=1^{\prime} S_{0}$ neutronproton scattering length or $\left|a_{n n}\right|<\left|a_{n p}\right|$. Such studies do not allow the unequivocal determination of whether charge-symmetry breaking interactions are also present. The main problem is the complicated influence of the electromagnetic interaction in p-p scattering which forbids an unambiguous determination of the purely hadronic parameters.

Charge symmetry of the $n-p$ interaction leads to the complete separation of the isovector and isoscalar parts of the scattering matrix for $\pi-p$ elastic scattering. In the even (odd) partial waves the isovector part contains spin singlet (triplet) and the isoscalar part spin triplet (singlet) contributions only. The concept of charge symmetry forbids transitions between the two parts of the scattering matrix and thus between the spin triplet and singlet states. the triplet-singlet transition amplitude $f_{T s}$ is thus forced to vanish. This restriction in turn leads to exactly equal polarizations of neutrons and protons in the scattering of initially unpolarized particles. Thus, for a given (neutron) scattering angle $\theta$ in $n-p$ scattering, the concept of charge symmetry implies: 


$$
\Delta P(\theta) \equiv P_{n}(\theta)-P_{p}(\pi-\theta) \equiv 0,
$$

i.c., the equality of scattered and recoil nucleon polarization. Exactly the same considerations hold for the analyzing powers $A_{n}(\theta)$ and $A_{p}(\pi-\theta)$ and their difference $\Delta \mathrm{A}$ in the scattering of polarized nucleons.

If, on the other hand, charge symmetry is given up, isospin mixing via spin triplet-singlet transitions becomes possible and a non-zero difference $\Delta A$ will in general be observed. Such a difference is directly proportional to the amplitude $\mathfrak{l}_{\mathrm{TS}}$ and thus to the existence of isospin-mixing charge-symmetry breaking interactions. From the detection of a non-vanishing analyzing power difference $\Delta A$ the amplitude $f_{T S}$ can be deterrined to the exient that the other interlering amplitude is known, since

$$
\Delta \mathrm{A}=\frac{2}{\sigma_{0}} \operatorname{Re}\left[\mathrm{b}^{*} \mathrm{f}_{\mathrm{TS}}\right] .
$$

Calcuiations of the charge-symmetry breaking effects on the analyzing powers at energies up to 460 $\mathrm{MeV}$ have been made by Cheung, Henley and Miller' and for energies up to $750 \mathrm{MeV}$ by Gersten. ${ }^{2}$ The differences in the analyzing powers were calculated as functions of angle and energy taking into account the direct electromagnetic effect (one photon exchange), $\rho^{0}-\omega$ mixing, and the neutron-proton mass difference effect in charged one pion exchange. Gersten also included the neutron-proton mass difference effect in charged $\rho$-exchange.

The experiment currently in progress at TRIUMF will measure the difference $\triangle A$ between the neutron and proton analyzing powers $A_{n}-A_{p}$ in n-p elastic scattering at $500 \mathrm{MeV}$. Designed as a nufl measurement, requiring no accurately known polarization standards, the experiment will determine the difference in angle at which $A_{n}$ and $A_{p}$ cross through zero $\left(\sim 71^{\circ} \mathrm{cm}\right)$. The two interleaved ${ }^{p}$ phases of the experiment consist of scattering polarized (unpolarized) neutrons from an unpolarized (polarized) proton target of the frozen spin type. The experiment is intended to provide an unambiguous test of charge-symmetry breaking effects to the level of $\Delta A$ $=0.001$, corresponding to a laboratory angle difference at the zero crossing of $\sim 0.05^{\circ}$. Theoretical predictions for this angular difference by Cheung, Henley and Miller $^{1}$ and by Gersten ${ }^{2}$ are factors of 2.5 and 10 , respectively, larger than the experimental accuracy aimed for in the experisnent.
Scattered neutrons and recoil protons originating in the frozen spin target are detected in coincidence in two left-right symmetric detection systems allowing cancellation of many of the systematic errors to first order. Each detection system consists of a neutron array placed at an angle of $32^{\circ}$ and at $5 \mathrm{~m}$ from the frozen spin target and a proton range telescope placed at an angle of $51.5^{\circ}$ on the opposite side of the incident beam. Each proton range telescope contains a time-offlight (t.o.f.) st urt scintillator placed close to the target; three sets of delay line chambers measuring $x$ and $y$ coordinates; and a range counter consisting of a $\Delta E$ scintillator, a wedge-shaped brass absorber at $3 \mathrm{~m}$ from the frozen spin target, an E scintillator, and a veto scintillator. Each neutron array consists of two consecutive planes of 7 scintillator bars, each $1.05 \mathrm{~m}$ wide, $0.15 \mathrm{~m}$ high and $0.15 \mathrm{~m}$ thick with the bars placed one on top of the other. With this detection system one measures the neutron t.o.f. and position and the proton t.o.f., trajectory, and selects a range of proton energies. Meas. urements of the opening angle and coplanarity of the coincident neutron-proton pair will be ttsed iv discriminate against quasi-free n-p scattering from heavier nuclei, e.g., ${ }^{4} \mathrm{He}$ and ${ }^{12} \mathrm{C}$, present in the frozen spin target. Each detection system covers an angular range of $5^{\circ}$ lab on either side of the crossover angle.

\section{Footnotes and References}

*Condensed from paper presented at the Fifth International Symposium on High Energy Spin Physics, Brookhaven National Laboratory, New York, 16-22 Sept. 1982. LBL-15185

tixepartment of Physics, University of Manitoba, WinI... g, Man., Canada R3T 2N2.

†TRUMF, Vancouver, B.C., Canada V6T $2 \mathrm{A3}$

\$Dept. of Physics, University of Alberta, Edmonton, Alta., Canada T6G 2T1.

t†Institut für Physik, Universitât Basel, CH4056 Basel, Switzerland.

1. C.-Y. Cheung, E. M. Henley, and G.A. Miller, Nucl. Phys: A305 342 (1978)

2. A. Gersten, Phys. Rev. C 242174 (1981) 


\title{
Concerning Tests of Time-Reversal Invariance Via the Polarization-Analyzing Power Equality*
}

\author{
Homer $E$. Conzett
}

Among the various tests of time-reversal invariance (TRI), the polarization-analyzing power theorem ${ }^{1}$ has the virtue that it follows directly from TR!. The theorem states that the spin-polarization $(P)$ of a finalstate particle in a (binary) nuclear reaction is equal to the analyzing power (A) for that polarized particle incident in the inverse reaction.

Recently, experimental differences have been reported ${ }^{2}$ between $P$ and $A$ in the two-nucleon transfer reactions:

${ }^{7} \mathrm{Li}\left({ }^{3} \mathrm{He}, \overline{\mathrm{p}}\right){ }^{9} \mathrm{Be}, \quad \mathrm{P}(\theta) ; \quad{ }^{9} \mathrm{Be}\left(\overrightarrow{\mathrm{p}},{ }^{3} \mathrm{He}\right){ }^{7} \mathrm{Li}, \quad \mathrm{A}(\theta)$ and

${ }^{9} \mathrm{Be}\left({ }^{3} \mathrm{He}, \overrightarrow{\mathrm{p}}\right){ }^{11} \mathrm{~B}, \quad \mathrm{P}(\theta) ; \quad{ }^{11} \mathrm{~B}\left(\overrightarrow{\mathrm{p}},{ }^{3} \mathrm{He}\right){ }^{9} \mathrm{Be}, \quad \mathrm{A}(\theta)$.

If these P-A differences are confirmed independently, the clear implication is that time-reversal invariance (TRI) is broken in some component of the nuclear interaction.

In view of these results, it is important to examine the question of why no significant deviations from $P=A$ bave been seen in the previous comparisons. Since elastic scattering is its own inverse process, it has been used in essentialiy all of the tests of TRI that use the polarization-analyzing power equality. I want to show here that all of these previous P-A comparisons fail as adequate tests of TRI either because of a lack of sensitivity to T-symmetry violation or a lack of experimental precision.

The majority of P-A comparisons have been made in pp elastic scattering, the older ones at energies between 142 and $635 \mathrm{MeV}$ before the advent of accelerated polarized beams. ${ }^{3}$ More recently, a comparison was made at $6 \mathrm{GeV} / \mathrm{c}$ from data acquired in measurements of pp scattering in polarized initial and final spin states." Two more accurate comparisons have been made in p-nucleus elastic scattering, $p-{ }^{3} \mathrm{He}$ (ref.5) and $p^{13} \mathrm{C}$ (ref. 6). The previous $\mathrm{P}-\mathrm{A}$ comparisons in nuclear reactions were incidental to the main purpose of the experiments.

The most accurate of these $\mathrm{P}-\mathrm{A}$ comparisions have been made in $\mathrm{p}^{3} \mathrm{He}$ and $\mathrm{p} \cdot{ }^{13} \mathrm{C}$ scattering; it is necessary to scatter from a nonzero spin nucleus, otherwise parity conservation alone ensures that $P=A$. I have found that neither of these comparisons was accurate enough to provide a significant test of TRI, because the equality between $P$ and $A$ depends on the equality of the two possible spin-flip probabilities. And, it is now known from measurements of the depolarization in p-nucleus elastic scattering that the spin-flip probabilities are very small, leading to $\mathrm{P}-\mathrm{A} \simeq 0$ even if the probabilities are not equal as required by TRI. That is, in terms of the spin-dependent cross sections,

$$
\begin{aligned}
& \mathrm{P}=\left(\sigma^{++}+\sigma^{-+}-\sigma^{+-}-\sigma^{-}\right) / 2 \sigma \text { and } \\
& \mathrm{A}=\left(\sigma^{++}+\sigma^{+-}-\sigma^{++}-\sigma^{-}\right) / 2 \sigma,
\end{aligned}
$$

where $\sigma^{-+}$is the cross section for the scattering of a proton from an initial negative spin-state to a final positive spin-state, and

$$
\sigma=\left(\sigma^{++}+\sigma^{+-}+\sigma^{-+}+\sigma^{-}\right) / 2 .
$$

The positive ( + ) direction is along $\vec{k}_{j} \times \vec{k}_{\mathbf{f}}$. Then

$$
\mathbf{P}-\mathbf{A}=(1-\mathrm{D}) \epsilon_{\mathrm{S}}=2 \mathrm{~S} \epsilon_{\mathrm{S}} \text {, }
$$

with the (total) spin-flip probability

$$
S=\left(\sigma^{+-}+\sigma^{-+}\right) / 2 \sigma \text {, }
$$

and the depolarization parameter is given by

$$
\mathrm{D}=1-2 \mathrm{~S}
$$

Thus, defining the spin-flip asymmetry as

$$
\epsilon_{\mathrm{S}} \equiv\left(\sigma^{-+}-\sigma^{+-}\right) /\left(\sigma^{-+}+\sigma^{+-}\right) \text {, }
$$

its absolute limits are $-1 \leq \epsilon \leq i$, but IRI requires that $\epsilon_{\mathrm{s}}=0$. since here the time reversed process of $\sigma^{-+}(\theta)$ is $\sigma^{+-}(\Theta)$. Thus, even though $\epsilon_{\mathrm{S}}$, which is the real measure of time-reversal violation, may be significantly different from zero, a small value of the factor (I-D) would make the P-A comparison quite insensitive to this violation. This $j$, in fact, just the case in these $\mathrm{p}^{3} \mathrm{He}$ and $\mathrm{p}^{13} \mathrm{C}$ experiments.

At higher energies the spin-dependent partial cross sections, measured in pp scattering at $6 \mathrm{GeV} / \mathrm{c}$ with polarized initial and final spin states, were used as 
tests of TRI at values of $P_{\perp}^{2}$ from 0.5 to $1.0(\mathrm{GeV} / \mathrm{c})^{2}$ (ref.4). The experimental quantity

$$
\epsilon_{\mathrm{T}}=[\sigma(++, 0-)-\sigma(--, 0+)] / \sigma
$$

was calculated, where the indices $(\mathrm{jj}, \mathrm{kl})$ denote the spin dir etions of the (beam, target; scattered, recoil) particles, and 0 indicates that the polarization of the scattered proton was not measured. It is readily shown that

$$
\mathrm{E}_{\mathrm{T}}=\left(\sigma^{+-}-\sigma^{-+}\right) / \sigma=\mathrm{A}-\mathrm{P}
$$

so that the concerns expressed in connection with Eo. (1) apply to ihis comparison as well. In fact. these data show values of $1-D=0.15-0.24$, so this cumparision is less sensitive to $\mathrm{T}$-violation than are $\mathrm{P}-\mathrm{A}$ comparisons at lower pp energies where the quantity $1-D$ is considerably larger. From the data of Tables II and III (ref. 4.) one can calculate the normalized T-violating quantity $\epsilon_{\mathrm{S}}$ of Eq. (2). Values of $\epsilon_{\mathrm{S}}$ and 1-D are listed in Table I where it is seen that the errors on $\epsilon_{s}$ vary between 30 and $100 \%$.

It is immediately. obvious from this discussion that tests of TRI in elastic scattering, using the P-A equality, should be made through measurements where the spin-flip probability is expected or known to be large. Even better, more stringent and conclusive tests are provided by $\mathrm{P}-\mathrm{A}$ comperisions in a reaction and its inverse, since the testing of TRI is not then limited to the spin-flip cross sections.

In conclusion, an examination has been made of all of the test of TRI via P-A c mparisions which were made before our report of finding such differences in two $\left({ }^{3} \mathrm{He}, \mathrm{p}\right)$ and $\left(\mathrm{p},{ }^{3} \mathrm{He}\right)$ reactions. ${ }^{2}$ It is seen that these comparisions were considerably less adequate tests of TRI than was believed, either because of lack of experimental precision or lack of sensitivity to $T$ violation. Essentially all of these comparisions were made in elastic $p-p$ and p-nucleus scattering where it is now seen that the sensitivity to T-viclation is directly proportional to the spin-flip probability $S(\theta)$. Consequentiy, kinematical regions in which $S(\theta)$ values are relatively large should be chosen for the P-A comparisons.

Finally, it is seen that more sensitive and exhaustive tests of TRI are likely to be made in P-A comparjsions that involve a nuclear reaction and its inverse. There, the test is not confined to the TRI conditions imposed on the spin-flip eross sections alone, as is the case in elastic scattering. Additionally, large spin-flr probabilities are common to many reactions which are suitable for these tests of TRI.
Table I

Values of 1-D and $\epsilon_{S}$, defined in Eq. 2, in p-p scattering at $6 \mathrm{Gev} / \mathrm{c}$. These are taken from the data listed in Tables 11 and 111 of ref. 4 .

\begin{tabular}{cllll}
\hline \hline $\mathrm{p}_{\perp}^{2}(\mathrm{GeV} / \mathrm{c})^{2}$ & 0.5 & 0.6 & $\mathrm{C.8}$ & 1.0 \\
\hline $\mathrm{I}-\mathrm{D}$ & 0.1410 & 0.139 & 0.206 & 0.258 \\
$\epsilon_{\mathrm{S}}$ & 1.14108 & 0.0899 & 0.1030 & 0.4435 \\
\hline
\end{tabular}

\section{Footnote and References}

* Condensed from invited paper, Proc. First International Conference on Nonpotential Interactiuns and their Lie-Admissible Treatment, Orleans, France, Jan. 5-9, 1982, Hadronic Jour. 5 (1982) 714.

1. R.J. Blin-Stoyle, Proc. Phys. Soc. A65 (1952) 452; G.R. Satchler, Nucl. Phys. 8 (1958) 65: L.C. Biedenharn, Nucl. Phys. 10 (1959) 620.

2. R.J. Slobodrian, C. Rioux, R. Roy, H.E. Conzett, P. von Rossen, and F. Hinterberger, Phys. Rev. Letters 47 (1981) 1803.

3. These data are shown by J. Bystricky, F. Lehar, and P. Winternitz, preprint CRMA-1127, Univ. de Montreal, Oct. 1982, which lists the references to the original work.

4. M. Borghini, W. de Boer, R.C. Fernow, AD. Krisch, H.E Miettinen, T.A Mulera, J.B. Roberts, K.M. Terwilliger, J.R. O'Fallon, and L.R. Ratner, Phys. Rev. D 17 (1978) 24.

5. D.G. McDonald, W. Haeberli and L.W. Morsow, Phys. Rev. 133B (1964) 1178.

6. E.E. Gross, J.J. Malanify, A van der Woude and A. Zucker, Phys. Rev. Letters 21 (1968) 1476. 


\title{
Evidence of Time Symmetry Violation in the Interaction of Nuclear Particles*
}

\author{
R.J. Slobodrian, ${ }^{\dagger}$ C. Rioux, ${ }^{\dagger}$ R Roy, ${ }^{\dagger}$ H.E. Conzett, \\ $P$. von Rossen, ${ }^{\ddagger}$ and $F$. Hinterberger ${ }^{\ddagger}$
}

We report bere on the first test specifically designed to compare the polarization $(P)$ in a nuclear reaction with the analyzing power (A) in the inverse reaction. ${ }^{1}$ We find substantial $P-A$ differences. The clear implication is that time-reversal invariance (TRI) is broken in some component of the nuclear interaction, since the P-A equality follows directly from TRI. ${ }^{2}$

The reactions chosen for the P-A comparison were the two-nucleon transfers ${ }^{7} \mathrm{Li}\left({ }^{3} \mathrm{He}, \mathrm{p}\right){ }^{9} \mathrm{Be}$ and ${ }^{9} \mathrm{Be}\left({ }^{3} \mathrm{He}, \mathrm{p}\right){ }^{11} \mathrm{~B}$ with $14 \mathrm{MeV}$ incident ${ }^{3} \mathrm{He}$ ions, and their inverses studied at the same $\mathrm{CM}$ energies. The Q-values are large, implying considerable mass, energy and momentum rearrangement. The measurements of proton polarizations in $\left({ }^{3} \mathrm{He}, \overrightarrow{\mathrm{p}}\right)$ reactions were performed mostly at the Van de Graaff Laboratory of Universite Laval, using a facility based on $\mathrm{Si}$ polarimeters, and results have been already published. The analyzing powers in $\left(\overrightarrow{\mathrm{p}},{ }^{3} \mathrm{He}\right)$ were measured at the polarized beam facility of the Berkeley 88-Inch Cyclotron. For both the $\mathrm{P}$ and $\mathrm{A}$ measurements, symmetric left-right geometry was used. This symmetry, along with spin reversal, effectively eliminates systematic errors in the A measurements, and it makes the $P$ measurements insensitive to small transverse displacements of the beam on the target.

Because of (a) the substantial P-A differences in our first measurements, and (b) the significance of this result, we repeated and extended the measurements of $A$, and we made completely independent checks on the measurements of $P$. The latter checks were made both at Laval and at Berkeley, with different polarimeters at the two locations. The tests at Laval were twofold. Firstly, some points were remeasured with ${ }^{7} \mathrm{Li}$ and ${ }^{9} \mathrm{Be}$ targets of the same thicknesses as those of the original measurements. The ${ }^{7} \mathrm{Li}$ remeasurements were made with a $500 \mu \mathrm{Si}$ polarization analyzer in place of the usual $1000 \mu$ analyzer. Secondly, measurements were made with significantly thinner targets in order to determine the dependence of the polarization on the energy interval spanned in the target. Finally, an overall experimental check was made routinely in the Laval experiments through a measurement of the proton polarization in ${ }^{2} \mathrm{H}\left({ }^{3} \mathrm{He}, \overrightarrow{\mathrm{p}}\right)^{4} \mathrm{He}$ reaction. The agreement with completely independent meaurements ${ }^{3}$ was always within the errors of the separate results.

Our $P$ and $A$ measurements in the ${ }^{9} \mathrm{Be}\left({ }^{3} \mathrm{He}, \overrightarrow{\mathrm{p}}\right)^{11} \mathrm{~B}$ reaction are shown in Fig. 1 . Since the $\left(E_{C, M}+Q\right)$ energies and the energy widths were not identical for the $P$ and $A$ measurements, an excitation function $A\left(E, \theta_{L}=37^{\circ}\right)$ was measured in the ${ }^{11} B\left(\vec{p},{ }^{3} \mathrm{He}\right)^{9} \mathrm{Be}$ reaction at an angle near the peak of $A(\theta)$. Over an energy span of some $800 \mathrm{keV}$, about $400 \mathrm{keV}$ on either side of the original energy, we find a smooth variation of $A\left(E_{p}\right)$. There are no sharp increases in $A\left(E_{p}\right)$ that could move its value into agreement with $P$ under a small shift in the energy.

At Berkeley, a completely different control experiment was possible with the availability of higher energy protons. That is, the ${ }^{9} \mathrm{Be}\left({ }^{3} \mathrm{He}, \overrightarrow{\mathrm{p}}\right)^{11} \mathrm{~B}$ polarizations at $\theta_{\mathrm{L}}$ $=40^{\circ}$ and $45^{\circ}$ were determined by way of a direct comparison with known ${ }^{12} \mathrm{C}(\mathrm{p}, \overrightarrow{\mathrm{p}})^{12} \mathrm{C}$ polarizations. At each angle, measurements were made of the asymmetries $\epsilon\left({ }^{3} \mathrm{He}, \overrightarrow{\mathrm{p}}\right)$ and $k(\mathrm{p}, \overrightarrow{\mathrm{p}})$ for the polarized protons from the respective reactions. The proton energy in the (p, $\vec{p})$ scattering was selected so that the energy of the protons incident on the polarimeters was the same as those from the $\left({ }^{3} \mathrm{He}, \overrightarrow{\mathrm{p}}\right)$ reantion. The latter polarization was then given simply a:

$$
P\left({ }^{3} \mathrm{He}, \overrightarrow{\mathrm{p}}\right)=\mathrm{P}(\mathrm{p}, \overrightarrow{\mathrm{p}}) \frac{\epsilon\left({ }^{3} \mathrm{He}, \overrightarrow{\mathrm{p}}\right)}{\epsilon(\mathrm{p}, \overrightarrow{\mathrm{p}})}
$$

Since $\mathrm{P}=\mathrm{A}$ in ${ }^{12} \mathrm{C}(\mathrm{p}, \mathrm{p})^{12} \mathrm{C}$ scattering from parity conservation alone, values of $A(\vec{p}, p)$ can be used in Eq.(1). Although literature values of $A(\theta)$ in ${ }^{12} C(\vec{p}, p)^{12} C$ scattering are available near the proton energy used, a separate, high statistics measurement was made of $A(\theta)$ at this energy, $\mathrm{E}_{\mathrm{p}}=24.13 \mathrm{MeV}$. The statistical errors were in the range of $\Delta \mathrm{A}= \pm 0.001$ to 0.003 , with an additional absolute scale uncertainty of $\pm 2.1 \%$ from the beam-monitoring ${ }^{4} \mathrm{He}$ polarimeter. From Eq. (1), then, the $P\left({ }^{3} \mathrm{He}, \overrightarrow{\mathrm{p}}\right)$ values were given directly from the ratio of the measured asymmetries and the measured $A(\vec{p}, p)$ values, and no separate calibration of the polarimeters was required.

Following reports of our preliminary results, ${ }^{4}$ independent determinations of $P$ in the ${ }^{9} \mathrm{Be}\left({ }^{3} \mathrm{He}, \overrightarrow{\mathrm{p}}\right)^{11} \mathrm{~B}$ reaction have have been made by a group at Los Alamos. 5 They report a large discrepancy between their preliminary results and our values, with their measurements of $P$ indicating agreement with $A$ in the inverse reaction, as measured by the authors of this letter. Thus, there is now a clear cxperimental disagreement to be resolved. At the present, however, our lack of detailed knowledge of their experimental procedures precludes an independent evaluation of their results. 
In summery, we have found large differences between $\mathrm{P}$ in the ${ }^{9} \mathrm{Li}\left({ }^{3} \mathrm{He}, \mathrm{p}\right){ }^{9} \mathrm{Be}$ and ${ }^{9} \mathrm{Be}\left({ }^{3} \mathrm{He}, \mathrm{p}\right){ }^{11} \mathrm{~B}$ reactions and $A$ of their inverse processes. From such an inequality between $P$ (in a reaction) and $A$ (in its inverse) it is straightforward to conclude that, prima facie, TRI is violated in these rcactions. Clearly, more experiments are necessary to corroborate these results, and we are pirsuing them.

\section{Footnotes and References}

•Condensed from Phys. Rev. Letters. 471903 (1981).

†Laboratoire de Physique Nucléaire, Université Laval, Québec, Canada GKTP4.

‡Institut für Strahlen und Kernphysik, Universität Bonn, D5300 Bonn, Germany.

1. There have been previous P-A comparisons in reactions, but these have been incidental to the main purpose of the experiments. For example, R. A. Hardekopf, et al., Nucl. Phys A191 (1972) 468, compared their $A(\theta)$ results in ${ }^{3} H(\vec{p}, d)^{2} H$ with the $P(\theta)$ results of others in ${ }^{2} H(d, \vec{p})^{3} \mathrm{H}$. The P-A differences that can be noted at $\mathrm{E}_{\mathrm{d}}=2$ and $3 \mathrm{MeV}$ were, presumably, attributed to experimental errors in the $P(\theta)$ measurements.

2. R. J. Blin-Stoyle, Proc. Phys. Soc. A65 (1952) 452; G. R. Satchler, Nucl. Phys. 8 (1958) 65; L. C. Biedenharn, Nucl. Phys. 10 (1959) 620.

3. R. J. Brown and W. Haeberli, Phys. Rev. 130 1163; W. G. Weitkamp and W. Haeberli, Nucl. Phys. 83 (1966) 46.

4. H. E Conzett, in Poiarization Phenomena in Nuclear Physici -1980, AIP Conf. Proc. No. 69, eds. G. G. Ohlsen, et al., American Institute of Physics, New York (1981) 1422.

5. P. W. Keaton, R. A Hardekopf, P. W. Lisowski, and I. R. Veeser, Bull. Am. Phys. Soc. 26 (1981) 623 .

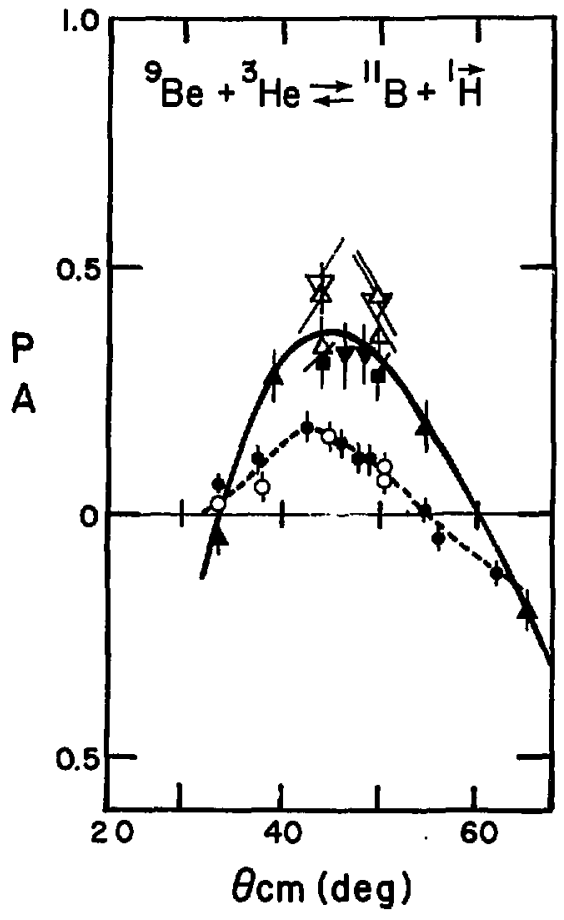

Fig. 1 Comparison of ${ }^{9} \mathrm{Be}\left({ }^{3} \mathrm{He}, \vec{p}\right){ }^{11} \mathrm{~B}$ polarizations with analyzing powers in ${ }^{11} \mathrm{~B}\left(\overrightarrow{\mathrm{p}},{ }^{3} \mathrm{He}\right)^{9} \mathrm{Be}$. The triangles are polarizations measured at Laval, the squares at Berkeley. The dots are the analyzing powers.

XBL 835-909 


\title{
The ${ }^{4} \mathbf{H e}(\alpha, p){ }^{7} \mathbf{L i}^{7}{ }^{7} \mathbf{L i}^{*(0.478)}$ Reaction Near Threshold*
}

\author{
R. J. Slobodriant and H. E. Conzett
}

The present reaction, leading to the ground state of ${ }^{7} \mathrm{Li}$, is the inverse of one widely studied since 1933 and was first investigated by Burcham, at al., ${ }^{1}$ at 38.5 MeV. The transition leading to the first excited state of ${ }^{7} \mathrm{Li}$ at $0.478 \mathrm{MeV}$ can only be studied through the $\alpha-\alpha$ reaction. Recent work has been reported by King, et al., ${ }^{23}$ mainly related to the astrophysical relevance of the ${ }^{7} \mathrm{Li}+\mathrm{p}$ and ${ }^{7} \mathrm{Be}+\mathrm{n}$ channels. The present paper is a detailed account of work performed some time ago which has recently motivated short communications in relation to the ${ }^{7} \mathrm{Lj}$ and ${ }^{7} \mathrm{Be}$ abundance in the universe and its implications for nucleosynthesis. ${ }^{3}$ The analysis of the ${ }^{7} \mathrm{~L}+\mathrm{p}$ channel angular distributions is also relevant to the understanding of the elastic $\alpha-\alpha$ scattering in the neighborhood of the opening of reaction channels, and this aspect of the subject was the motive of the investigation. Our results are in good agreement with those of King, et al., ${ }^{3}$ but provide a better map. ping of the cross sections very close to threshold.

Differential cross-sections were measured at nine energies between 37 and $43 \mathrm{MeV}$ for transitions to the ground and first excited $(0.478 \mathrm{MeV})$ states of ${ }^{7} \mathrm{Li}$ in the reaction ${ }^{4} \mathrm{He}\left(\alpha_{1} p\right)^{7} \mathrm{Li}$. The charged particle spectra were measured with $\mathrm{Si}(\mathrm{Li})$ detectors with a resolution of $100 \mathrm{keV}$ for the protons, permitting a complete separation of the groups from the ground and first excited states. The detector pulses were sent to 400 channel pulse height analyzers and to fast scalers, the latter permitting dead time corrections. Such corrections were kept below $1 \%$.

In general, for a reaction

$$
A+a \rightarrow C \rightarrow B+b \text {, }
$$

where $C$ is assumed to be a compound state of angular momentum $J$, the angular distribution of the reaction products can be expanded in terms of the sum

$$
\frac{\mathrm{d} \sigma}{\mathrm{d} \Omega}=\sum_{k=0}^{L} \mathrm{a}_{k} \cos ^{2 k} \theta \text {. }
$$

If the orbital angular momentum of the initial system is $\ell_{1}$ and that of the final state is $\ell_{2}$ the value of $\mathrm{L}$ which gives the highest power of $\cos ^{2} \theta$ to be found in the angular distribution is given by the minimum value of the set $\ell_{2}, \ell_{2}$ and $\mathrm{J}^{4}$. The original assumption of Inglis ${ }^{5}$ (for the inverse reaction) was that only $\ell=1$ and $\ell=3$ protons were responsible for the reaction, and $\ell=0$ and $\ell=2$ alpha particles were emitted. Thus, a good representation of the reaction should be given by expression (2) with $k=2$ :

$$
\frac{d \sigma}{d \Omega}=A+B \cos ^{2} \theta+C \cos ^{4} \theta \text {. }
$$

However, if $\&=4$ alpha particles are emitted (or feed the ${ }^{7} \mathrm{Li}+\mathrm{p}$ channels in the inverse reaction) it is necessary to consider an additional term $D \cos ^{6} \theta$. The data at $39.94 \mathrm{MeV}$ stow a clear improvement of the fit with $D \cos ^{6} \theta$. The energy region where the $\cos ^{6} \theta$ term is important is sharply defined in energy. This was borne out subsequently and independently by the phase shift analysis of the $\alpha-\alpha$ angular distributions where, due to the evidence provided by the cross sections of the ${ }^{2} \mathrm{Li}+\mathrm{p}$ channels, absorption was included in the $\ell=4$ partial wave. In the notation $\delta_{l}=\alpha_{\ell}+\mathrm{i} \beta_{l}$ where $\delta_{\ell}$ is the scattering phase shift, $\beta_{4}$ is a measure of the absorption of the $\ell=4$ partial wave. It peaks sharply at $19.72 \mathrm{MeV}$ (energy in the center of mass) and is sizeable only over a region of $0.5 \mathrm{MeV}$. The need to include a term in $\cos ^{6} \theta$ has also been established independently by King, et al. ${ }^{3}$ Figure 1 shows the cross sections for ${ }^{7} \mathrm{Li}^{6{ }^{6 S}}+\mathrm{p}$ and ${ }^{7} \mathrm{Li}{ }^{* 0.488}+p$ and their sums compared to results by Burcham, at al., ${ }^{1}$ and King, at al. ${ }^{3}$ Such cross sections were obtained by integrating the angular distributions of protons at each energy.

The main result obtained from the study of the $\alpha+{ }^{4} \mathrm{He} \rightarrow{ }^{7} \mathrm{Li}+p,{ }^{7} \mathrm{Lj}{ }^{* 0.478}+p$ reactions was, historically, independent evidence for the presence of absorptive effects in partial waves with $\ell \geq 4$ in the entrance channel. The present interest is mainly astrophysical. ${ }^{3}$ The complete determination of the problem posed by the interaction of alpha particles above threshold for particle reactions requires the investigation of all open channels, and even closed channels with thresholds near a given energy region. King, at al. ${ }^{3}$ have made measurements at a few points near threshold in the $\alpha+{ }^{4} \mathrm{He} \rightarrow{ }^{7} \mathrm{Be}+\mathrm{n},{ }^{7} \mathrm{Be} * 0.431+n$ channels by capture of the radioactive ${ }^{7} \mathrm{Be}$ recoils and subsequent counting of the decay $\gamma$ rays. A more complete investigation could be pursued either by neutron detection or by the identification of the ${ }^{B e}$ recoils in a $\triangle \mathrm{E}-\mathrm{E}$ detection system. Such systems have been developed and used in heavy ion reaction studies at energies as low as 1 $\mathrm{MeV} / \mathrm{A}$, thus the 16.8 to $16.9 \mathrm{MeV}$ energy of the ${ }^{7} \mathrm{Be}$ recoils at threshold, due to the $\mathrm{CM}$ motion, is clearly sufficient for direct detection. The total reaction cross section as a function of energy, is another observable capable of providing additional constraints for a formal 
description of the reaction and its determination is also desirable. The reactions with a totally symmetric initial state (space, spin and charge) and charge symmetric final states are ideal testing grounds for charge symmetry and its violations. Increased accuracy is certainly possible in these studies, both experimentally and theoretically.

\section{Footnotes and References}

*Condensed from LBL-13892, Z. Phys. A (in press).

†Laboratoire de Physique Nucléaire, Université Laval, Quebes, Canada GIK7P4.

1. W. E. Burcham, G. P. McCauley, D. Bredin, W. M. Gibson, D. J. Prowse, and J, Roblat, Nucl.Phys. 5 (1958) 141.

2. C. H. King, H. H. Rossner, S. M. Austin, W. S. Chien, G. J. Mathews, V. E. Viola, Jr., and R. G. Clark, Phys. Rev. Lett. 35 (1975) 988.

3. C. H. King, $S$. M. Austin, $H$. H. Rossner and $W$. S. Chien, Phys. ReV. C16 (1977) 1712, and refs. therein.

4. J. M. Blatt and L. C. Biedenham, Rev. Mod. Phys. 24 (1952) 258.

5. D. R. Inglis, Phys. Rev. 74 (1948) 21.

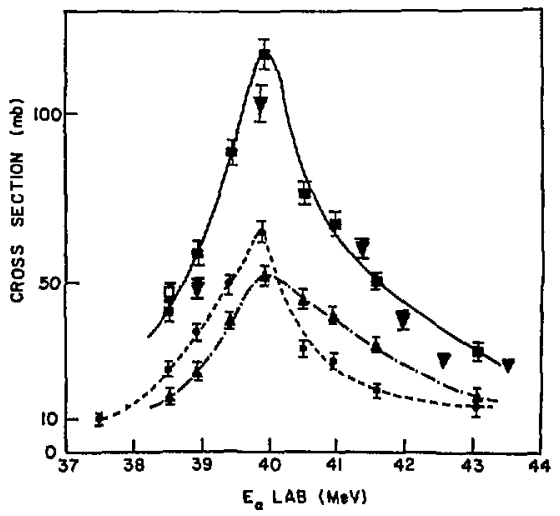

Fig. 1. Cross sections for the proton channels: ${ }^{7} \mathrm{Li}+\mathrm{p}$ is given by dots, ${ }^{7} \mathrm{Li}^{00.478}+\mathrm{p}$ is given by upright triangles. The sum of both channels is shown by solid squares for the work reported here, the open square is taken from ref. I, the inverted triangles from ref. 3. There is good overall agreement. The lines are polynomial fits to the data.

XBL 834-9471

\title{
The Radiolysis and Racemizaton of Leucine on Proton Irradiation*
}

\author{
William A. Bonner, ${ }^{\dagger}$ Richard $M$. Lemmon and Homer E. Conzett
}

Previous experiments have been done by ourselves and others to test the Vester-U]bricht (V-U) hypothesis ${ }^{1}$ for the origin of optically active molecules in nature through the attempted asymmetric radiolysis of racemic amino acids, using natural or artificially produced antiparallel longitudinally polarized beta particles. As an extension of this work, we ${ }^{2}$ have recently subjected DL-leucine to partial radiolysis instead with longitudinally polarized protons of both parallel and anti-parallel spin. Reasons have been given to advance the idea that polarized protons might be more iffective than polarized electrons for engendering such asymmetric effects. Nevertheless, we were unable to detect any enantiomeric inequality in the undeconposed DL-leucine residues - even when such radiolyses were completed to
47-50\% - with protons of either spin polarity, either when they passed completely through the leucine samples or were stopped by them.

After our discovery ${ }^{3}$ that ionizing radiation could cause the racemization of amino acids arcompanying their radiolysis, we suggesied that such radioracemizaton must inevitably diminish the effectiveness of the V$\mathrm{U}$ mechanism for engendering optical activity by asymmetric radiolysis. In particular, it was pointed out that if the rate of radioracemization were greater than the rate of asymmetric radiolysis, it is possible that no enantiomeric inequality might be produced in the undecomposed residue after partial radiolysis. It was later suggested ${ }^{\mathfrak{j}}$ that such effects might be responsible for the lack of asymmetric degradation noted by Hodge, 
et al., ${ }^{6}$ during radiolysis of DL-leucine with longitudinally polarized electrons.

In none of the attempted asymmetric radiolyses mentioned above, using either natural or artificially produced longitudinally polarized electrons, has a systematic attempt been made to assess the actual importance of competing radioracemization or of secondary degradative reactions in a particular experimental system, although it has been noted that negligible radioracemization accompanied the $25-30 \%$ gross radiolysis of solid $\mathrm{D}$ and L-leucine with ${ }^{32} \mathrm{P}$ beta particles at $-196^{\circ}$. Accordingly, further experiments seemed desirable to evaluate the potential importance of these factors in our recent unsuccessful attempts to induce optical activity in DL-leucine by irradiation with polarized protons. ${ }^{2}$

We have conducted four irradiations of $D$ - and $L$. leucine at the 88-Inch Cyclotron under conditions previously employed for irradiations of DL-leucine. ${ }^{2}$ In the present experiments, however, the use of longitudinally polarized protons was unnecessary, and unpolarized protons of the same $0-11 \mathrm{MeV}$ energy range were utilized. The leucine sample disks $(129 \mathrm{mg})$ were irradiated as D- and L-pairs, again alternating each pair between variable and fixed aluminum absorbers in the proton beam, such that the beam passed through the first sample disk and was stopped by the second. Beam intensities were about 14 namps and irradiation times were 144 - 147 minutes, such that the measured integrated beam total was 120 microcoulombs. Since the energy loss in each sample was $5 \mathrm{MeV} / \mathrm{H}^{+}$, each sample received a dose of $\sim 5 \times 10^{8}$ rads. $^{2}$ After irradiation two of the samples were quenched immediately by dissolving in a hot $4.5 \mathrm{M}$ solution of $\mathbf{H C l}$ in 2-propanol, Half of each solution was treated with the appropriate "enantiomeric marker" of the original D- or Lleucine to determine percent degradation, and the resulting mixtures were converted to $\mathrm{N}$-trifluoroacetylleucine isopropyl ester derivatives for gas chromatographic (g.c.) analysis for enantiomeric composition. The g.c. analyses were conducted using columns loaded with the enantiomeric g.c. phases N-docosanoyl-D-valyl- (Dphase) or N-docosanoyl-L-valyl-tert-butylamide (Lphase). The remaining two samples were allowed to stand at room temperature for 21 days, then were dissolved, divided, and similarly converted to N-TFA isopropyl ester derivatives for g.c. analysis. Both Dand L-phase g.c. columns were used for the g.c. analyses of each sample in order to provide a "symmetry check" and to disclose any systematic errors that might be inherent in the experiments or analyses. The results of these experiments are shown in Table I. At the outset Table I shows overall good agreement in both degradation and racemizaton results for each experiment using either the D- or L-phase g.c. columns, indicating no systematic bias in the experiments. We also note that the amount of racemization of the leucine engendered by $5 \times 10^{8}$ rads irradiation with the proton beam is rather low (1.1-1.7\%) compared to that observed during $\gamma$-radiolysis, ${ }^{4}$ and does not vary appreciably if the beam passes through the sample or is ausorbed by $\mathrm{it}$, or if the sample is derivatized immediately after irradiation or after 21 days. The L-leucine pair samples are consistently about $10 \%$, less degraded than the corresponding D-leucine samples (Expts. 1 vs 3 , and 2 ws 4 ), which is probably due to uncontrolled parameters in the proton beam intensity. The degradation caused by the higher energy protons passing through each first leucine sample in the beam, however, is consistantly $5-5.5 \%$ higher than that that caused when the lower energy protons are fully absorbed by the second sample in the beam (Expts. 1 vs 2, and 3 vs 4), suggesting that the average energy deposited in the first sample was about $5 \%$ higher. Finally, at least in the present type of experiment, the data in Table I make it appear improbable that our failure to observe asymmetric radiolysis of DL-leucine at $50 \%$ degradation with longitudinally polarized $0-11 \mathrm{MeV}$ protons ${ }^{2}$ was attributable to competing radioracemization or to secondary (symmetrical) degradation effects.

\section{Footnotes and References}

*Condensed from Origins of Life 12 (1982) 51.

†Department of Chemistry, Stanford University, Stanford, CA 94305

1. T.L.V. Ulbricht, and F. Vester, Tet, 18 (1962) 629-637.

2. R.M. Lemmon, H.E. Conzett, and W.A. Bonner, Origins of Life 11 (1981) 337-341.

3. W.A. Bonner and R.M. Lemmon, J. Mol. Evol. 11 (1978) 95-99.

4. W.A. Bonner and R.M. Lemmon, Bioorg. Chem. 7 (1978) $175-187$.

5. W.A. Bonner, M.R. Yearian and M.A. Van Dort, Nature 280 (1979) 252.

6. L.A. Hodge, F.B. Dunning., G.K. Walters, R.H. White and G.S. Schroepfer, Nature 280 (1979) 250-252. 
Table I

Degradation and Racemization of $\mathrm{R}$ and $\mathrm{L}$-Leucine on Irradiation with 0-11 MeV Protons

\begin{tabular}{|c|c|c|c|c|c|c|c|c|c|c|c|c|c|}
\hline \multirow[t]{2}{*}{ Exp. } & \multirow{2}{*}{$\begin{array}{l}\text { Ler- } \\
\text { cine }\end{array}$} & \multirow[t]{2}{*}{ Beamr) } & \multirow[t]{2}{*}{ Days $\left.^{b}\right)$} & \multicolumn{5}{|c|}{ D-Phase Column } & \multicolumn{5}{|c|}{ LPhase Column } \\
\hline & & & & SD & gL & $( \pm)^{c)}$ & $\begin{array}{c}g \\
\text { Deg }\end{array}$ & $\begin{array}{r}\% \\
\text { Rac } \\
\end{array}$ & sD & gL & $( \pm)^{c}$ & $\begin{array}{c}x \\
\text { Des }\end{array}$ & $\begin{array}{c}\% \\
\text { Rac }\end{array}$ \\
\hline 1 & $\mathrm{~L}$ & PT & 0 & 0.87 & 99.13 & .05 & 44. 1 & 1.7 & 0.85 & 99.15 & .11 & 44.9 & 1.7 \\
\hline 2 & L & A & 21 & 0.82 & 99.18 & .03 & 38.8 & 1.6 & 0.76 & 99.24 & .06 & 39.4 & 1.5 \\
\hline 3 & $D^{d)}$ & PT & 21 & $99.4 T^{e)}$ & $0.53^{e)}$ & .01 & 54.9 & 1.1 & $\left.99.20^{\circ}\right)$ & $\left.0.80^{*}\right)$ & .13 & 54.8 & 1.6 \\
\hline 4 & D) & A & 0 & $99.34^{(t)}$ & $0.66^{\mathrm{e})}$ & .05 & 49.9 & 1.3 & $99.18^{()}$ & $0.82^{4)}$ & .01 & 49.8 & 1.6 \\
\hline
\end{tabular}

a) $\mathbf{A}=$ Bean abuorbed in sumple; PT = Beam passed through sample.

b) 0 = Sample derivatized immodintely after irradiation; 21 - Sample derivatized after 21 days.

c) Standard deviation of 3-4 analyses.

d) Contuined 0,36\% L-Leu.

e) Corrected for L-Leu content of original D-Leu. 


\title{
Damped Collisions and Transfer Reactions
}

\section{Rigid-rotation and Rolling Limits in Deep-Inelastic Reactions*}

\author{
A.J. Pacheco. ${ }^{\dagger}$ G.J. Wozniak, R.J. McDonald, R.M. Diamond, \\ C.C. Hsu. ${ }^{\ddagger}$ LG. Moretto, D.S. Morrissey. L.G. Sobotka, \\ and F.S. Srephens
}

Recently, the magnitude and alignment of the spin transferred to the fragments in the deep-inelistic reactions of $8.5 \mathrm{MeV} /$ nucleon ${ }^{165} \mathrm{Ho}$ on ${ }^{176} \mathrm{Yb},{ }^{148} \mathrm{Sm}$, and ${ }^{\text {nal }} \mathrm{Ag}$ were investigated using continuum $\gamma$-ray multiplicity and anisotropy techniques. The sum of spin magnitudes was obtained from the $\gamma$-ray multiplicity assuming a linear relationship, while the degree of spin alignment was deduced from the anisotropy of the angular distribution with the aid of a statistical model.' From these data, one can conclude that of all the parameters that define the spin distributions, the spin magnitude is the least dependent on model assumptions.

However, in defining the partition of the total internal angular momentum between the two fragments, we have assumed that the intermediate dinuclear complex reaches a state of rigid rotation. To test the validity of this assumption, let us first analyze the sum of the spin magnitudes $I_{1}+I_{2}$. This quantity is expected to be fairly insensitive to any assumption on the rolation regime.

Figure 1 shows $I_{1}+I_{2}$ as a function of Q-value for the three reactions. Two observations can be made. First, the maximum amount of spin cortesponding to the saturation region increases with the total mass of the system. Second, the Q-value where saturation is reached appears to become somewhat more negative for heavier systems. To a large extent, this behavior may be attributed to differences in basic parameters of the reaction, such as the Coulomb barrier, total kinetic energy, mass, or angular momentum. A more significant comparison between the reactions can be made by appropriately transforming both axes in Fig. 1 so as to remove any factor that is not directly connected to the transfer process itself. The choice of new variables is certainly not unique. For the variable related to the excitation energy we have chosen a transformation from $Q$-value to temperature. For the angularmomentum axis we have considered two different scales given by the following equations:

$$
\mathrm{i}_{\mathrm{RR}}=\left(\frac{\mathfrak{I}_{1}}{\mathscr{I}_{1}} \ell_{\mathrm{MAX}}\right)^{-1}\left(\mathrm{I}_{1}+\mathrm{I}_{2)}\right.
$$

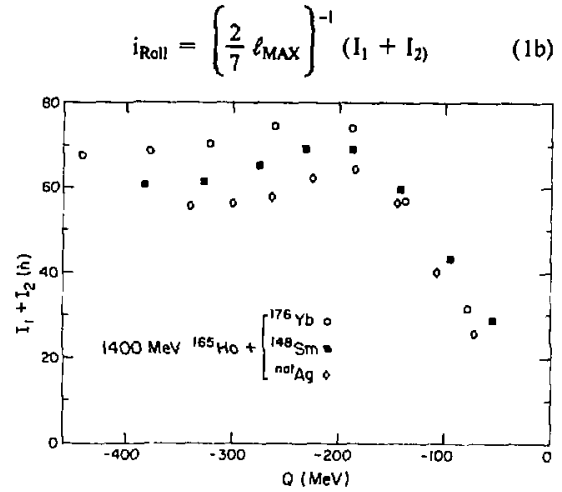

Fig. 1. Sum of the spin magnitudes $\left(I_{1}+I_{2}\right)$ as a function of $Q$-value for the three reaction systems.

XBL 824-327

Here, $\mathscr{I}_{1}$ and $\boldsymbol{F}_{2}$ are the moments of inertia of the individual fragments. $\ell_{\text {MaX }}$ is the maximum incoming angular momentum corresponding to a grazing collision, and we have defined

$$
\begin{aligned}
& \mathscr{H}_{1}=\mathscr{I}_{1}+\mathscr{I}_{2} \\
& \mathscr{I}_{\perp}=\mathscr{I}_{1}+\mu \mathrm{d}^{2}
\end{aligned}
$$

where $\mu$ is the reduced mass and $d$ is the distance between the centers of the nuclei in the dinuclear complex. The transformed variables $i_{R R}$ and $i_{\text {Roll }}$ measure the observed sum of the fragments' spins in units of the maximum value expected from the rigid-rotation and rolling limits, respectively. 
Figure 2 shows plots of the experimental values of $\mathrm{i}_{\mathrm{RR}}$ and $\mathrm{i}_{\mathrm{Roll}}$ as a function of T. A comparison between Figs. 1 and $2 b$ indicates that the transformation from $I_{1}$ $+I_{2}$ and $Q$ to $i_{R R}$ and $T$ succeeds in reducing the experimental points to essentially a single curve. The transformation according to the rolling limit (Fig. 2a) gives a similar curve for the nearly symmetric Ho + $\mathrm{Sm}$ and $\mathrm{Ho}+\mathrm{Yb}$ systems but shows a significant deviation for the more asymmetric $\mathrm{Ho}+\mathrm{Ag}$. These results provide, if not proof, at least a strong support to the assumption of rigid rotation of the intermediate complex.

\section{Footnotes and Reference}

*Condensed from Nucl. Phys. A397 (1983) 313

†Permanent address: Comision Nacional de Energía Atómica, Buenos Aires, Argentina jinstitute of Atomic Energy, Beijing, China §Permanent address: National Superconducting Cyclotron Laboratory, Michigan State University, East Lansing, Michigan 48824

1. L.G. Moretto and R.P. Schmitt, Phys. Rev. C21 (1980) 204

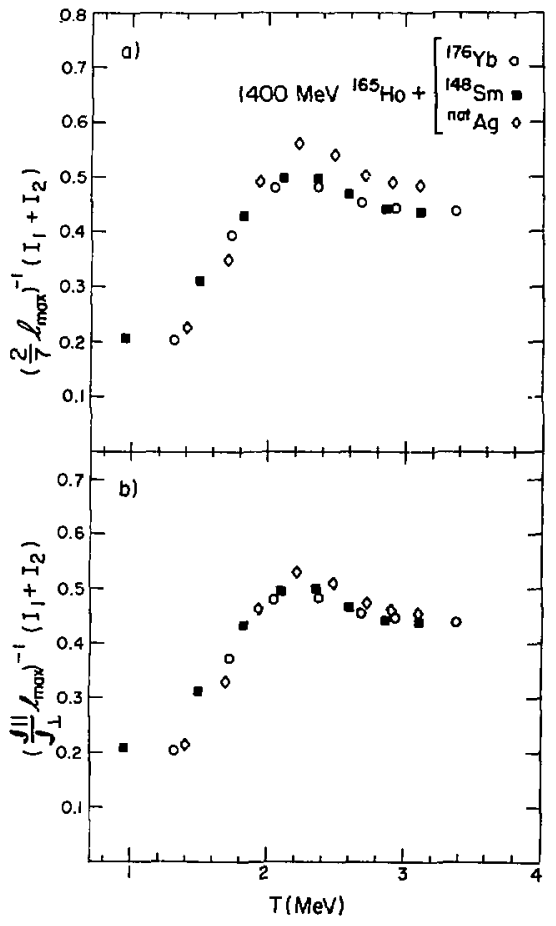

Fig. 2. The sum of spins in reduced units as a function of temper ature. The angular momentum axes have been scaled according to the "rolling" limit for part a) and to rigid-rotation limit for part b).

XBL 824-335 


\title{
High Energy Gamma Rays Emitted in the Deep-Inelastic Reaction 8.0 MeV/A ${ }^{139} \mathrm{La}+{ }^{139} \mathrm{La}$
}

\author{
LG. Sobotka, J.E. Draper, ${ }^{*}$ R.M. Diamond, E.L. Dines, ${ }^{*}$ R.J. McDonald, \\ L.G. Moretto, A.J. Pacheco, F.S. Stephens, and G.J. Wozniak
}

We have recently studied high-energy gamma rays emitted in the reaction $8.0 \mathrm{MeV} / \mathrm{A}{ }^{139} \mathrm{La}+{ }^{139} \mathrm{La}$ to continue our investigation of the Q-value dependence of giant dipole transitions. The experimental apparatus employed was very similar to that described in our previous work. ${ }^{1}$ However, several improvements were made to obtain higher quality data concerning the $Q$. value dependence of the giant dipole resonance energy and width. The first improvement involves the use of a symmetric system. This removes several ambiguities when comparing the experimental $\gamma$-ray spectra to statistical model calculations. Second, the ground state GDR widths for nuclei near $A=140$ are smaller than those for nuclei near $A=160$, which is approximately the average mass in the previous study. Finally, we improved the time of flight separation of neutrons and $\gamma$ rays. This allows us to compare the data to model calculations over a larger $\gamma$-ray energy region, $5 \mathrm{MeV} \leq$ $\mathrm{E}_{\gamma} \leq 20 \mathrm{MeV}$.

The gamma-ray spectra in coincidence with five different Q-value bins are shown in Fig. 1. As one gates on progressively more negative $Q$-values the yield of high-energy gamma rays at first increases but then seems to saturate in the region of the DI peak. This trend, which has been observed in our previous work, is in agreement with simple statistical model predictions.

\section{Footnotes and References}

* Permanent address: Physics Department, University of California, Davis, CA 95616

†Permanent address: Comisión Nacional de Energía Atómica, Buenos Aires, Argentina

1. J.E. Draper, et al., Phys. Rev. Lett. 49 (1982) 434 , and contribution to this report.

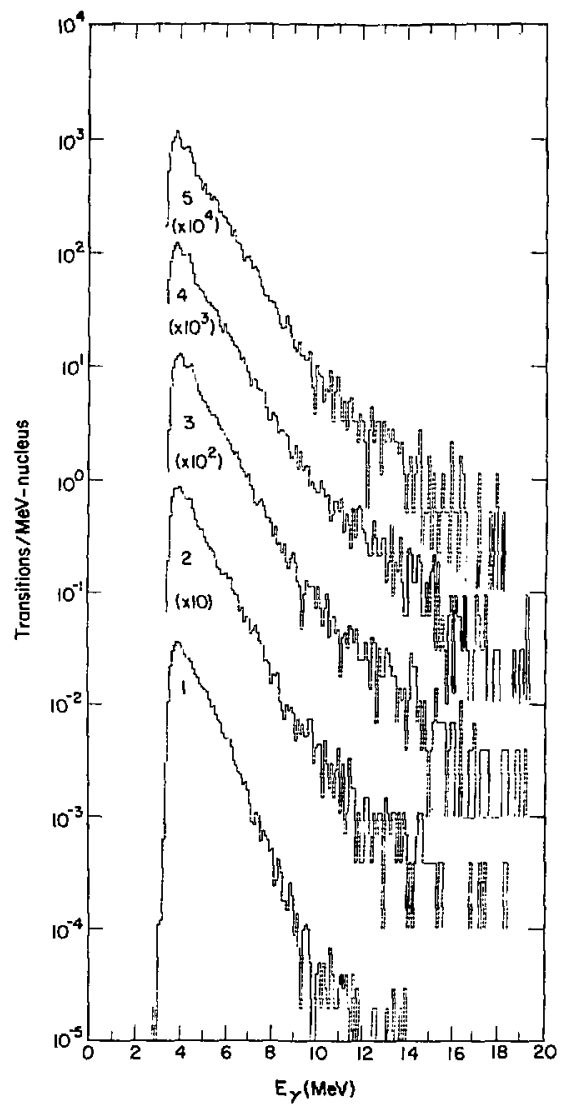

Fig. 1. Coincident gamma-ray spectra for five $Q$ value bins. Bin 1 corresponds to the elastic, quasielastic region and bin 5 to the most deeply inelastic collisions.

XBL $8210-4776$ 


\title{
Particle Angular Distributions and Gamma-ray \\ Multiplicities as Experimental Probes for
}

\author{
Angular Momentum Fluctuations*
}

\author{
L. G. Morctto
}

The sequential emission of " $\mathrm{He}$ is demonstratud in the reaction $\mathrm{Ho}+\mathrm{Ta}$ and the ${ }^{4} \mathrm{He}$ angular distribution is used to test the rigid rotation limit in the reaction $\mathrm{Kr}$ + Ag. A more refined analysis of the dependence of continuum $\gamma$-ray multiplicities on $Q$-value and gammaray energy shows that reasonable agreement with the statistical model is obtained if one assumes the presence of low-energy aligned dipole transitions in the reactions $\mathrm{Ho}, \mathrm{Yb}, \mathrm{Sm}, \mathrm{Ag}_{\mathrm{g}}+\mathrm{Ho}$.
Sequential fission angular distributions from very asymmetric reactions $\mathrm{U}, \mathrm{Au}+\mathrm{Ne}$ provide a severe test for the statistical model.

*Condensed from LBL-14101. Presented at the 20th International Meeting on Nuclear Physics, Bormio, Italy, January 25-29, 1982

\section{A Study of Charge, Energy, and Angular Momentum Transfer in the Reactions}

\author{
${ }^{56} \mathrm{Fe}+{ }^{197} \mathrm{Au}$ and ${ }^{56} \mathrm{Fe}+{ }^{107,109} \mathrm{Ag}$ at 7.2 and $8.3 \mathrm{MeV} /$ nucleon* \\ G.J. Wozniak, G.J. Mathews, ${ }^{\dagger}$ R.P. Schmitt, ${ }^{\ddagger}$ R. Regimbart, ${ }^{\dagger}$ H. Hübel, \\ RM. Diamond, and LG. Moretto
}

Center-of-mass angular distributions for atomic numbers between $13(\Delta Z=-13)$ and $39(\Delta Z=+13)$ are presented in Fig. 1. Because of background from slit scattering, data for the projectile $(Z=26)$ have been omitted. Distributions for the same net charge transfer to and from the projectile (i.e., $\pm \Delta Z$ ) are shown in adjacent columns. The amount of charge transferred increases from 1 to 13 down the columns. For both bombarding energies, one observes the evolution from side peaked to forward peaked angular distributions with increasing charge transfer. (For comparison, a $1 / \sin \theta$ angular distribution is shown at the bottom of the second column.) For the higher bombarding energy the evolution toward forward peaking proceeds faster, presumably because the grazing angle is smaller for the larger bombarding energy. More striking is the marked asymmetry in this evolution for charge transfer to and fiom the projectile, a feature observed at both bombarding energies. For Z-values larger tban $26(\Delta Z>0)$, ine side peak is very weak and quickly disappears for larger charge transfers. For $Z$-values less than $26(\Delta Z<0)$, the side peak is much more intense and persists for much larger charge transfers.
The evolution from side- to forward-peaked distributions has been explained ${ }^{1}$ in terms of a diffusioncontrolled time delay. On the average, small charge transfers require less time than larger ones in a diffusion process. Since small charge transfers populate elements near the projectile, the mean lifetime is short, resulting in side-peaked angular distributions. For larger charge transfers, the longer average lifetimes allow the intermediate complex to rotate more before decaying. This produces forward-peaked angular distributions. The fact that the evolution proceeds more rapidly at the higher bombarding energy is probably due to the larger orbital velocity of the intermediate complex and the smaller angular distance (more forward grazing angle) through which it must rotate to reach $0^{\circ}$.

The large difference in the rate of evolution of the side peak for $\Delta \mathbf{Z}>0$ and $\Delta Z<0$ can be better understood by examining the angular distributions as a function of Q-value. At the $401 \mathrm{MeV}$ bombarding energy (see Fig. 2) the side peak is clearly seen to be associated with the partially damped events, and, in general, the intensity of the side peak decreases with increasing energy dissipation. Since the Z-values above the 
projectile have a smaller fraction of the partially damped events, the side peak for the energy-integrated angular distribution is relatively weak. One should note that for the lowest energy bins both the shape and magnitude of the cross sections are very similar for the same amount of charge transfer to or from the projectile.

\section{Foornotes and References}

*Condensed from Nucl. Phys. A402 (1983) 322
†Permanent address: Lawrence Livermore National Laboratory, University of California, Livermore, CA 94550

¥Permanent address: Department of Chemistry and Cyclotron Institute, Texas A\&M University, College Station, Texas 77843

\$Permanent address: Laboratoire de Physique Corpusculaire, Université de Caen, 1400 Caen, France

* Permanent address: Institut für Strahlen und Kernphysik, University of Bonn, Nussallee 14-16, D-5300 Bonn, Germany

1. P. Russo, R.P. Schmitt, G.J. Wozniak, RC. Jared, P. Glässel, B. Cauvin, J.S. Sventek, and L.G. Moretto, Nucl. Phys. A281 (1977) 509

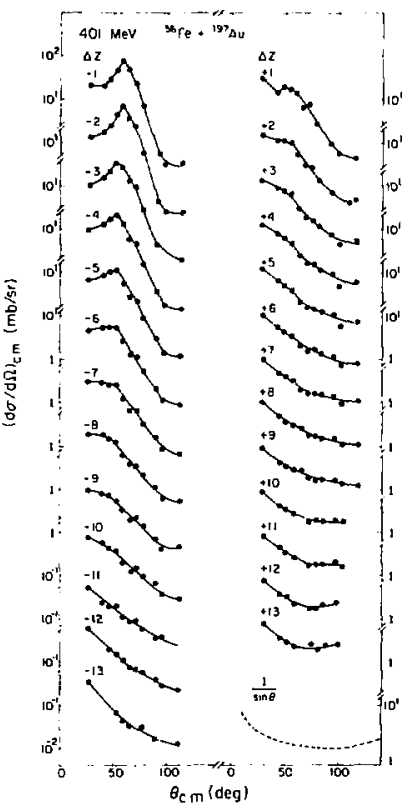

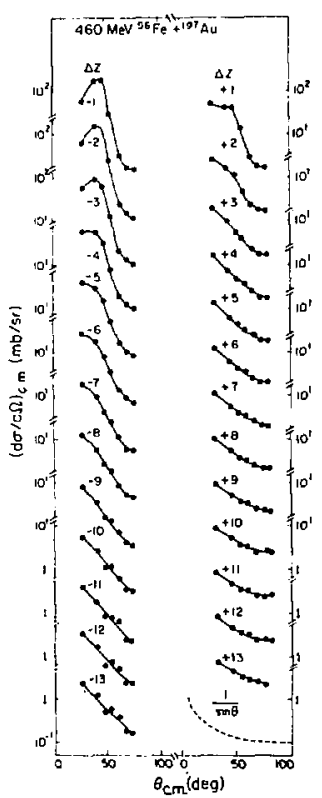

Fig. 1. Experimental c.m. angular distributions for elements detected from the $\left.{ }^{19} \mathrm{Au}+\mathrm{a}\right) 401$ and (b) $460 \mathrm{MeV}$ ${ }^{36} \mathrm{Fe}$ reaction. Distributions for the same net charge transfer to $(\Delta Z>0)$ and from $(\Delta Z<0)$ the projectile are shown in adjacent columns. The curves through the data points serve only to guide the eye.

XBL 795-9723A 


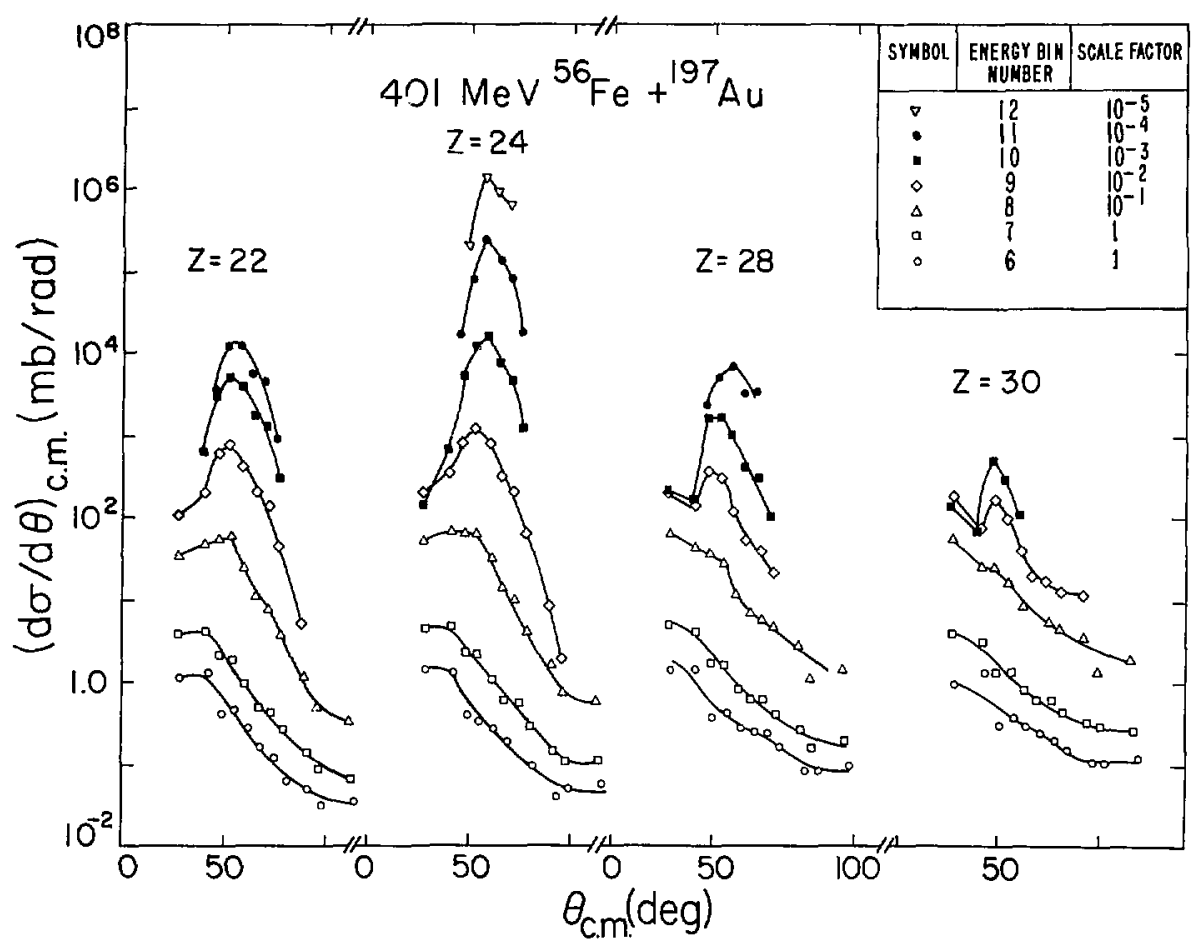

Fig. 2. Experimental c.m. angular distributions for several kinetic energy windows and four selected elements from the ${ }^{197} \mathrm{Au}+401 \mathrm{MeV}{ }^{56} \mathrm{Fe}$ reaction. Bin 7 is approximately centered on the relaxed peak. The highest bin numbers correspond to the largest energies. The curves through the data points serve only to guide the eye.

XBL 795-1413 


\title{
Spin Distributions of Individual Deep-Inelastic Fragments
}

\author{
as Deduced from Continuam $\boldsymbol{\gamma}$-ray Studies*
}

\author{
A.J. Pacheco, ${ }^{\dagger}$ G.J. Wozniak. R.J. McDonald, R.M. Diamond, \\ C.C. Hsu, ${ }^{\ddagger}$ LG. Moretto, D.J. Morrissey, L.G. Sobotka, and F.S. Stephens
}

In this contribution we exidmine some results concerning the spin distribution of each of the two fragments produced in the deep-inelastic reactions of ${ }^{163} \mathrm{Ho}$ on ${ }^{176} \mathrm{Yb},{ }^{148} \mathrm{Sm}$, and ${ }^{\mathrm{mt}} \mathrm{Ag}$ at $8.5 \mathrm{MeV} / \mathrm{nucleon}$. From the measurement of $\gamma$-ray multiplicities, the average sum of the spin magnitudes is extracted. As described in ref. 1 , the average spin magnitudes $\left\langle\mathrm{I}_{1}\right\rangle$ and $\left\langle\mathrm{I}_{2}\right\rangle$ were later assigncd to each nucleus according to the rigid-rotation prescription, and the variances $\sigma_{1}^{2}$ and $\sigma_{2}^{2}$ were calculated with the aid of the statistical model of ref. 2 and the experimental temperatures. Both types of information were finally combined to obtain the individual spin alignments through the average projections $\left\langle\mathrm{I}_{12}\right\rangle$ and $\left\langle\mathrm{I}_{22}\right\rangle$.

Figure 1 shows the evolution of the average spin magnitude $\langle\mathrm{I}\rangle$ (solid curve) and its average aligned component $\left\langle\mathrm{I}_{z}\right\rangle$ (dashed curve) for both reaction partners as a function of Q-value. In all cases the heaviest fragment bears the largest spin according to the rigid-rotation partition. The spin of the projectilelike fragment increases slightly with decreasing total mass because the increase of its moment of inertia (relative to the target-like fragment) prevails over the reduction of the total angular momentum. The Q-value dependence of both $\langle\mathrm{I}\rangle$ and $\left\langle\mathrm{I}_{\mathbf{z}}\right\rangle$ is qualitatively the same as that of the $\gamma$-ray multiplicities. As a function of Q-value, $\langle I\rangle$ shows a rise throughout the elastic and quasielastic region and a saturation at large negative values. At large Q-values, $\left\langle\mathrm{I}_{\mathbf{z}}\right\rangle$ shows a decreasing trend, which leads to a progressive divergence from $<I>$. The magnitude of this effect is a function of the mass of the fragment, and it is related to the alignment of the corresponding spin distribution. The degree of spin alignment is usually measured in terms of the quantity $P_{z z}$ defined as

$$
\mathbf{P}_{\mathrm{zz}}=\frac{3}{2} \frac{\left\langle\mathrm{I}_{\mathrm{z}}^{2}\right\rangle}{\left\langle\mathrm{I}^{2}\right\rangle}-\frac{1}{2}=\frac{1}{\left.1+3 \sigma^{2} /<\mathrm{I}_{\mathrm{z}}\right\rangle^{2}}
$$

With this definition, $P_{2 z}$ varies from $a$ to 1 , those extremes corresponding to a completely misaligned and to a perfectly aiigned system, respectively.

Figure 2 shows the value of $P_{2 z}$ as a function of Q-value for each individual fragment in the three reactions. The same qualitative behavior can be observed in all cases; the alignment increases rapidly with increasing Q-value throughout the quasielastic region, followed by a more or less slow decrease (depending on the mass of the fragment) across the deep-inelastic region. For each system, the heavy reaction partners show a higher degree of alignment.

The differences in :he alignment of each fragment may be understood in terms of the extracted individual spins and the dependence of the calculated spin fluctuations on different parameters. The equilibrium statistical model predicts that the variances of the spin distributions are proportional to the temperature. In addition, it also predicts that the variances decrease with increasing mass asymmetry of the system, although this dependence is rather weak throughout the region investigated in these experiments. In this mass-asymmetry region the variances along the three cartesian coordinates are nearly equal and the average value is larger for the heavy fragment. Finally, for a fixed mass asymmetry the magnitude of the fluctuations in both nuclei vary with the total mass according to the following relation

$$
\sigma^{2} \propto\left(A_{\text {total }}\right)^{s / 3}
$$

Due to the opposite effects derived from the increasing mass asymmetry and the decreasing total mass, the magnitude of the fluctuations induced in the Ho-like fragment is almost constant for the three reactions. Since the spin imparted to this nucleus increases with entrance-channe! mass asymmetry, the net result is that the maximum value of $\mathrm{P}_{2 z}$ (Ho) (Fig. 2) increases with decreasing mass of the target $(0.64,0.70$, and 0.79 for ${ }^{176} \mathrm{Yb},{ }^{148} \mathrm{Sm}$, and ${ }^{\mathrm{nal}} \mathrm{Ag}$ ).

A different behavior is obtained for the extracted alignment of the target-like nuclei. Although the calculated variances become smaller for lighter targets, the trend is not strong enough to compensate for the dramatic decrease in the transferred spins (Fig. 1). In fact, for the lightest nucleus $(\mathrm{Ag})$ at the highest excitation energies $(Q \simeq-300 \mathrm{MeV})$, these randomly oriented components account for alnost the totality of the spin (Fig. 1), thus giving rise to an almost isotropic spin distribution (Fig. 2). 


\section{Footnotes and References}

*Condensed from Nucl. Phys. A397 (1983) 313

†Permar:-nt address: Comisión Nacional de Energía Atómica, Buenos Aires, Argentina \$nstitute of Atomic Energy, Beijing, China

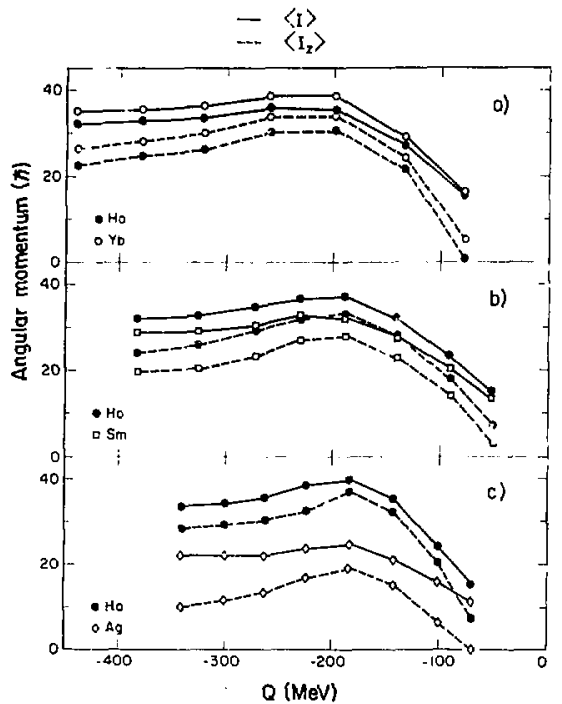

Fig. 1. Average spin màgnitude $\langle\mathrm{I}\rangle$ (solid lines) and average aligned component $\left\langle\mathrm{I}_{\mathrm{z}}\right\rangle$ (dashed lines) as a function of $Q$ value. For each system, the various symbols correspond to the two deep-inelastic fragments.

XBL 824-339
§Permanent address: National Superconducting Cyclotron Laboratory, Michigan State University, East Lar.jing, Michigan 48824

1. A.J. Pacheco, et al., in this Annual Report

2. L.G. Moretto and R.P. Schmitt, Phys. Rev. C21 (1980) 204

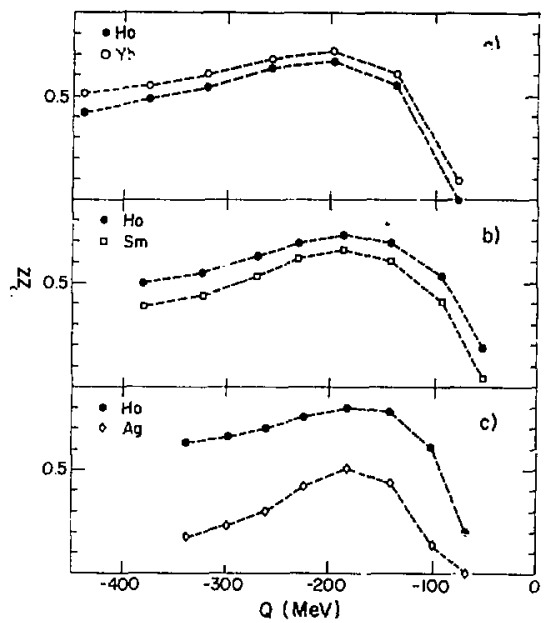

Fig. 2. Alignment parameter $P_{z z}$ as a function of $Q$ value, for each of the two deep-inelastic fragments in the three reactions.

XBL 824-334 


\title{
Sequential Fission Studies of Angular Momentum Misalignment in Very Asymmetric Systems
}

\author{
G. Guarino, D.J. Morrissey. R.J. McDonald, A.J. Pacheco, ${ }^{\dagger}$ \\ L. Sobotka, G.J. Wozniak, and L.G. Moretto
}

In recent years the models of angular momentum transfer in deep-inelastic collisions (DIC) have received a serious test from the analysis of the sequential fission angular distribution. The hypothesis of a rigid rotation limit of the intermediate complex agrees with the presence of a substantial spin alignment along a direction perpendicular to the reaction plane and large fuctuations of the in-plane spin components with zero mean values. The purpose of this experiment was to investigate the degree of misalignmeni of the fragments' spin, in the presence of in-plane angular momentum components, for very asymmetric systems. These systems should severely test the prediction of the equilibrium statistical model.

Projectiles of ${ }^{32} \mathrm{~S}$ ions of $238 \mathrm{MeV}$ from the 88Inch Cyclotron were used to bombard a ${ }^{238} \mathrm{UF}$, target, $0.924 \mathrm{mg} / \mathrm{cm}^{2}$ thick. The projectile-like fragments were detected in a Z-telescope ( $\mathrm{d} \Omega=3 \mathrm{msr}$ ) fixed at $70^{\circ}$. The detection system for the fission fragments (FF) in coincidence consisted of an array of 10 silicon surface barrier detectors $(300 \mu \mathrm{m})$, five in plane and five out of plane, mounted all at the same distance from the target and on the opposite side of the beam.

With two positionsi of this array we have covered the in-plane angular region from $+68^{\circ}$ to $+150^{\circ}$ and the out-of-plane region from $0^{\circ}$ to $75^{\circ}$. The target was rotated both in plane and out of plane to minimize the correction for energy loss for all FF detectors. The coincidence data were transformed in an event-by-zvent mode to the rest frame of the recoiling nucleus with the assumption of $\Phi^{H}=0$ for the direction of the laboratory recoil angle. In the recoil rest frame the FF energy spectra were Gaussian shaped and peaked at energies in agreement with Viola's predictions. ${ }^{1}$ To have an estimate of the bias in the angular distributions introduced from the energy dependence of the fission probability we have calculated $P_{r}\left(E^{*}\right)$ from the ratio of coincidence to singles. Because $\mathrm{P}_{f}$ saturates rapidly to $\sim 1$ we have not introduced this correction in our further analysis.

To increase the statistios the data were divided into only three TKEL bins. The in-plane angular distributions (Fig. 1) show a strong anisotropy. Except for the quasielastic bin, where direct processes are more

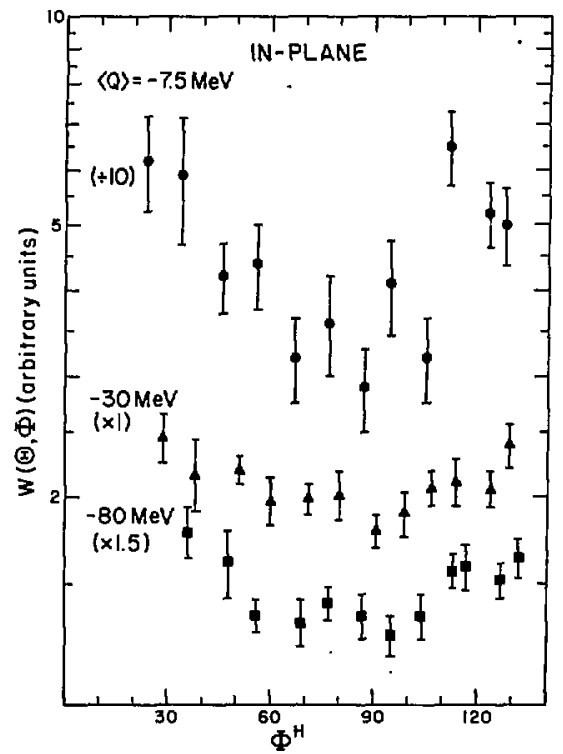

Fig. 1. In-plane angular distributions of sequential fission fragments from the ${ }^{32} \mathrm{~S}+{ }^{238} \mathrm{U}$ reaction are shown for three $Q$-value bins. The distributions are integrated over $Z=9-16$.

XBL $8211-1327$

likely, the anisotropy increases with $Q$ value. This result is well understood in the framework of the statistical model ${ }^{2}$ where for very asymmetric systems the only angular-momentum-bearing mode surviving (tilting) is responsible for the minimum along the direction of the symmetry axis of the fission fragments.

The fit of the data to extract values for the aligned spin component $\langle\mathrm{I}\rangle$ and the thermal widths $\sigma_{x}, \sigma_{y}, \sigma_{z}$ is still in progress. 
Footnotes and References

*Permanent address: National Superconducting Cyclotron Laboratory, Michigan State University, East Lansing, Michigan 48824
†Permanent address: Comisión Nacional de Energia Atómica, Buenos Aires, Argentina

1. V.E. Viola, Jr., Nucl. Data AI (1966) 391.

2. L.G. Moretto and R.P. Schmitt, Phys. Rev. C21 (1980) 204.

\title{
Sequential Fission in the Reaction of ${ }^{40} \mathrm{Ar}+{ }^{197} \mathrm{Au}$
}

\author{
D.J. Morrissey, "G.J. Wozniak, L.G. Sobotka, A.J. Pacheco, ${ }^{\dagger}$ \\ R.J. McDonald, and LG. Moretto
}

The measurement of particle and $\gamma$-ray angular distributions associated with deep-inelastic collisions (DIC) allows one to study the process of angular momentum transfer through the determination of the magnitude and alignment of the fragment spins. Large intrinsic spins can be introduced in the reaction pro ducts through the dissipation of entrance-channel orbital angular momentum. From mechanical considerations and from the rigid rotation limit, the fragments' spins are expected to be aligned perpendicular to the reaction plane. Misalignment of the fragments' spins cccurs when in-plane components of angular momentum are present. These components can be generated either directly by some feature of the reaction mechanism or by nonequilibrium or equilibrium statistical fluctuations in the angular-momentum-bearing modes of the dinuclear system. The angular distributions of sequential fission fragments are quite sensitive to the direction of the spin vector in that it can produce a substantial inplane anisotropy. In-plane measurements of sequential fission angular distributions ${ }^{1,2}$ have given detailed results on the magnitude and direction of the spin vector of one of the fragments from deep inelastic collisions.

A beam of $340 \mathrm{MeV}{ }^{40} \mathrm{Ar}$ was obtained from the SuperHILAC at the Lawrence Berkeley Laboratory. This beam irradiated a metallic gold foil, approximately $1 \mathrm{mg} / \mathrm{cm}^{2}$ thick. The experiment consisted of measurements of coincidences between projectile-like fragments and fission fragments. Projectile-like fragments (PLF) were detected in a telescope that was fixed at $40^{\circ}$ to the beam. The resolution of the Z-telescope was sufficient to separate completely atomic numbers between 6 and 36; however, it was insufficient to resolve the individual atomic numbers of the fission fragments. Fission fragments (FF) were observed on the opposite side of the beam from the PLF in an array of 10 silicon surface- barrier detectors. The array of FF detectors was positioned at various angles in order to cover as large an angular range as possible. After the experiment the data were transformed into the calculated rest frame of the recoiling target nucleus event by event. The energy and angular distributions of the FF were then available as a function of PLF energy and $Z$.

The fission probability of the heavy partner is shown as a function of PLF nuclear charge after integration over PLF kinetic energy in Fig. 1. The sharp minimum at $Z \simeq 18$ indicates the low fissionability of the gold-like nuclei produced with low excitation energies in quasielastic collisions. The expected trend of increasing fissionability with increasing fragment mass (i.e. decreasing PLF charge) is also seen in Fig. 1. The in-plane angular distribution of the FFs in the rest frame of the target nucleus is shown in Fig. 2. The data show a strong in-plane anisotropy (1.5:1) as does the fitted angular distribution function shown by the solid curve.

\section{Footnotes and References}

*Permanent address: National Superconducting Cyclotron Laboratory, Michigan State University, East Lansing, Michigan 48824

†Permanent address: Comisión Nacional de Energia Atómica, Buenos Aires, Argentina

1. D.J. Morrissey, et al., Z. Phys. A305 (1982) 131.

2. R.J. Puigh, et al., Phys. Lett. A336 (1980) 279. 


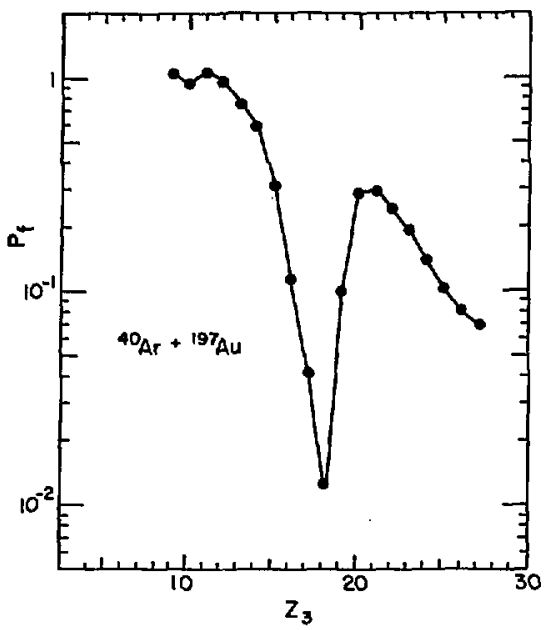

Fig. 1. The fission probability as a function of projectile-like fragment $Z$ integrated over energy is shown.

XBL 833-8959

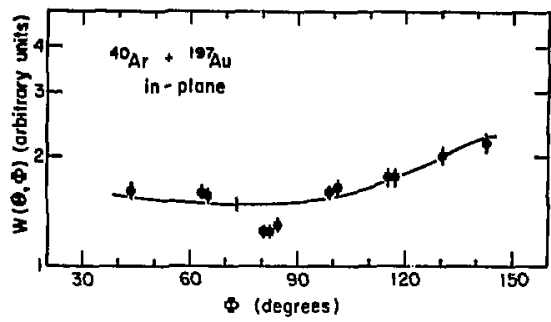

Fig. 2. The in-plane distribution of sequential fission fragments from the reaction of ${ }^{40} \mathrm{Ar}+{ }^{197} \mathrm{Au}$ is shown. This angular distribution shows a significant anisotropy in the rest frame of the recoiling nucleus indicating a preferential direction for the fragment angular momentum.

XBL 8338960

\title{
Compound Nucleus Decay Via the Emission of Large Fragments
}

\author{
L.G. Sobotka, M.L. Padgeti, G.J. Wozniak, G. Guarino, \\ A.J. Pacheco, * L.G. Moretto, Y.D. Chan, R. Stokstad, \\ I. Tserruya, ${ }^{\dagger}$ and $S$. Wald
}

We have studied the emission of $\alpha,{ }^{6} \mathrm{Li},{ }^{7} \mathrm{Li},{ }^{7} \mathrm{Be}$, ${ }^{9} \mathrm{Be}, \mathrm{B}, \mathrm{C}$, and heavier fragments in the reaction 130 $\mathrm{MeV}{ }^{3} \mathrm{He}+{ }^{n t} \mathrm{Ag}$ at the 88-Inch Cyclotron. The intermediate mass fragments were detected using a set of three standard gas-solid state $\Delta \mathrm{E}-\mathrm{E}$ telescopes. Alpha particles were detected in a separate solid-state telescope $(40 \mu \mathrm{m}, 5 \mathrm{~mm})$. Angular distributions were obtained from $20^{\circ}$ to $170^{\circ}$ in the laboratory.

A beam of ${ }^{3} \mathrm{He}$ was chosen because it produces a compound nucleus with high excitation energies and 
because the products of projectile fragments are lighter than the products of interest.

The energy spectra in the center-of-mass system are shown in Fig. 1. The spectra shift to higher energies as the ejectile increases in charge; however, the rate of increase is slower than a simple Coulomb calculation would predict. The spectral shapes also evolve from a Maxwellian for $\alpha$-particles to a shape far more symmetric fo the heavier ejectiles.

From $100^{\circ}$ to $180^{\circ}$ the angular distributions (not shown) range from flat to slightly back ward peaked. This slight backward peaking is expected from angular momentum considerations. At more forward angles the cross sections increase and the spectra become hardes. This might be due to an incomplete subtraction of events caused by target impurities or the presence of some forward focused nonequilibrium process. Nevertheless, at angles $>100^{\circ}$ the spectral shape is independent of angle. This fact, along with the shape of the angular distributions in the backward hemisphere, confirms that these backward data are due to an equilibrium decay process.

These data ase being interpreted in terms of both standard evaporation and fission (transition state) formalisms.

\section{Footrotes}

*Permanent address: Comision Nacional de Energía Atómica, Buenos Aires, Argentina

†Permanent address: Weizmann Institute of Science, Rehovoth, Israel

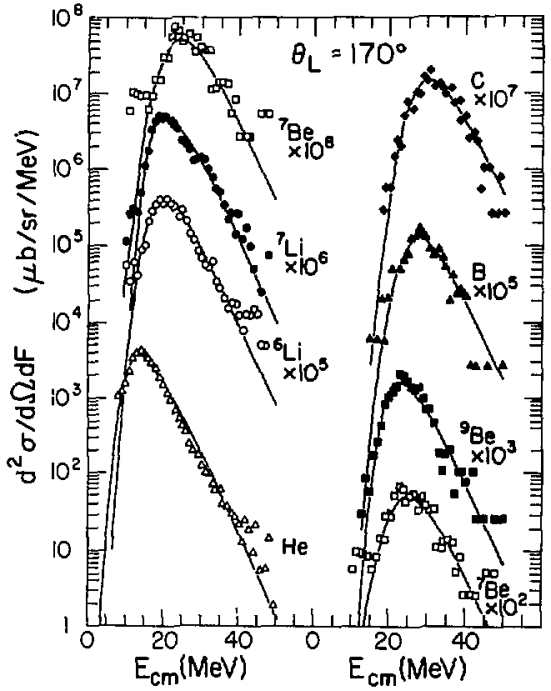

Fig. 1. Center-of-mass kinetic energy spectra for various ejectiles in the reaction $130 \mathrm{MeV}^{3} \mathrm{He}+{ }^{\text {nat }} \mathrm{Ag}$.

XBL 827-874 


\title{
Alpha-Particle Emission from the Reaction $1354 \mathrm{MeV}{ }^{165} \mathrm{Ho}+{ }^{181} \mathrm{Ta}^{*}$
}

\author{
L.G. Soborka, R.J. McDonald, G.J. Wozriak, \\ D.J. Morrissey. 'A.J. Pacheco, ${ }^{\ddagger}$ and L. G. Moretto
}

The emission of alpha particles from the deep inelastic heavy-ion reaction $1354 \mathrm{MeV}{ }^{165} \mathrm{Ho}+{ }^{181} \mathrm{Ta}$ has been studied. Energy spectra and angular distributions of $\alpha$ particles, detected in coincidence with a projectile-like fragment, were acquired both in the reaction plane and out of the reaction plane at a fixed inplane angle. The in-plane data show that the bulk of the alpha particles in coincidence with the deepinelastic exit channel can be explained by evaporation from the fully accelerated fragments. This can be seen in the average velocity vector diagram shown in Fig. Ia, where the root-mean-square velocity $\left(v_{\alpha}^{m}\right)$ of the $\alpha$ particles is plotted along with the velocity vectors for the detected projectile-like fragment (gated on the deepinclastic events), the calculated velocity of the undetected fragment, and the velocity of the system center of mass. As this figure shows, the a-particle velocities are centered around the end of the velocity vector of the target-like fragment. This agrees with the assumption that the $\alpha$ particles are emitted from the fully accelerated target-like fragment. In addition, a low-energy component seen at $30^{\circ}$ can be attributed to emission from the projectile-like fragment. This component is not seen at more backward angles because it drops below the detection threshold, which is shown by the dashec! arc in Fig. 1a.

Furtiner evidence that the fully accelerated DI fragments âre the source of emission can be obtained by determining the Q-value dependence of $v_{a}^{\mathrm{cms}}$. In Fig. 1b the average velocity vector diagram for three different Q-value bins (all in the deep-inelastic region) are plotted along with the corresponding $v_{a}^{\mathrm{rms}}$. A systematic motion for the locus of $v_{a}^{\text {mm }}$ is seen that can only be explained by a source that has a Q-dependent velocity. This trend is explained by the change of the velocity of the target-like fragment with $Q$-value, as shown in Fig. 1 b.

\section{Footnotes}

*Condensed from Phys. Rev. C25 (1982) 1693

†Permanent address: National Superconducting Lab, Michigan State University, East Lansing, Michigan 48824
¥Permanent address: Comision Nacluina! de Energia Atomica, Buenos Aires, Argentina

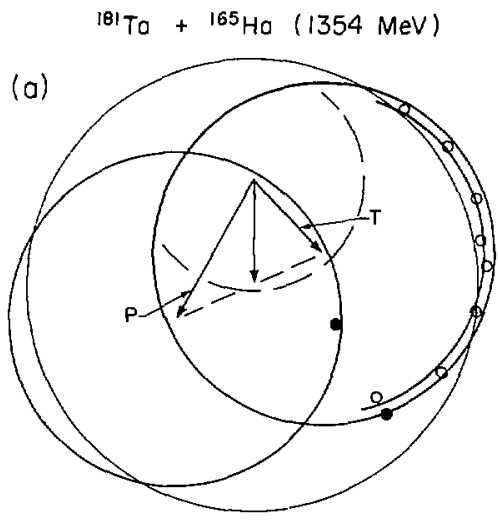

(b)
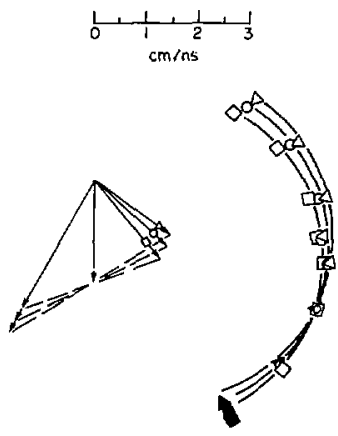

Fig. 1. a) Velocity diagram. The open circles are the rms velocities extracted from the coincident laboratory $\alpha$-particle energy spectra. The solid circles indicate the rms velocities of the two separate peaks that appear at the most forward angle. The full large rings indicate 
the loci of expected $\alpha$-particle velocities for the three different rest frames. For the target-like fragment, the locus of velocities for a $10 \%$ reduction in the expected average emission energy is indicated by a partial ring. The detection threshold is shown as a dashed arc. The letters $P$ and $T$ stand for projectile-like and target-like, respectively. b) The velocity diagrams for three Q-bins (all in the deep-inelastic region). The rms $\alpha$-particle velocities for each bin are indicated. The smallest energy loss bin is indicated by triangles and the largest energy loss data by squares. Threc partial rings are drawn to guide the eye. They have the same radius and are centered on the three different recoil velocities.

XBL 818-2456A

\title{
Products of $3 \leq Z \leq 9$ from Long-Lived Intermediates in the Reactions $336 \mathrm{MeV}^{40} \mathrm{Ar}+\mathrm{Ag},{ }^{154} \mathrm{Sm}$ and $\mathrm{Au}$
}

\author{
L.C. Vaz," D. Logan, J.M Alexander," E. Duek, D. Guerreau, ${ }^{\ddagger}$ L. Kowalski, ${ }^{\ddagger}$ M.F. Rivet, ${ }^{\ddagger}$ and M.S. Zisman
}

In reactions between complex nuclei the products of $3 \leq \mathrm{A} \leq 9$ are produced with much smaller cross sections than either products with smaller $Z$ or heavier products derived from fusion/fission or deeply inelastic scattering. However, they tend to exhibit many of the characteristics of these latter products. Their angular distributions show a fast forward-peaked component while at backward angles a more relaxed component is evident.

In this work we have studied the slow reaction components of products $3 \leq Z \leq 9$ for the reactions of $336 \mathrm{MeV}{ }^{40} \mathrm{Ar}+\mathrm{Ag},{ }^{154} \mathrm{Sm}$ and ${ }^{197} \mathrm{Au}$. Angular distributions and energy spectra were measured using a conventional gas ionization chamber $\Delta \mathrm{E}$, silicon $\mathrm{E}$ telescope. Table I presents for each product a summary of the integrated cross sections for the symmetric component. Shown for comparison are cross sections for evaporative $\mathrm{H}$ and $\mathrm{He}$ as well as those for evaporation residues, fission, total fusion, and total reaction. Several similarities between evaporative $\mathrm{H}$ and $\mathrm{He}$ and products with $3 \leq Z \leq 9$ were noted. The likelihood of emission (measured as the ratio of the product's cross section to the total fusion cross section) is insensitive to the amount of fission of the particular system. Further, the singles energy spectra were much narrower than would be compatible with emission from fully accelerated fission fragments. We therefore investigated the inferences of emission from a composite system via transition state theory, ${ }^{1,2}$ using two extreme assumptions for the nature of the transition state nuclei: 1) an evaporation-like or 3-dimensional state or 2) a fission-like or 1-dimensional state. It is probable that the true state lies somewhere in between.
The first model has many features in common with evaporation theory. It assumes decay lifetimes longer than rotation periods and employs spindependent level densities as the emission driving force. Further assumptions and approximations are that emission occurs perpendicular to the exit channel $\ell$ vector, sharp cutoff transmission coefficients, constant temperature level densities, and first-step emission. In the analysis, the anisotropies of the angular distributions and average energies are used to estimate the rootmean-square spins and barriers to emission for these systems. A comparison of the emission barriers to fusion barriers allows an estimate of the extent of deformation.

The second model is sinilar to the first but with the following qualifications: The angular distribution is assumed to be frozen into one dimension at the saddlepoint configuration, and thus it is the saddle-point level density that is relevant. In addition, the $\ell$ dependence of emission is replaced by a dependence on orientation (or $\mathrm{K}$ value) of the axially symmetric transition state nucleus.

The results of analysis by the first model indicate $J_{\operatorname{mms}}$ in the range of $30-70 h$, i.e., spins that lie in the domain from $\ell_{\mathrm{ER}}$ (the maximum spin for evaporation residual survival) to $\ell_{\text {crit. }}$ Emission barriers were found to be $60-80 \%$ of the fusion barriers. The fission model infers $J_{\text {max }}$ of approximately half the above values and somewhat higher emission barriers. Nevertheless, these two extreme models both lead to the qualitative features of relatively low spins and significant deformation of the emitting systems. 
Table I

Cross sections (mb) for the symmetric component of charged particle emission: $336 \mathrm{MeV}{ }^{40} \mathrm{Ar}+\mathrm{X}$

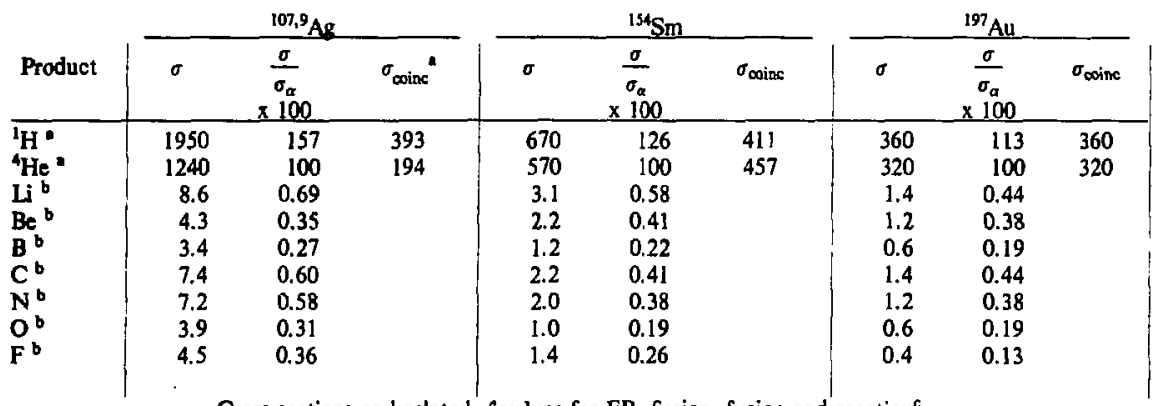

Cross sections and related $\ell$ values for $E R$, fission, fusion and reaction ${ }^{c}$

\begin{tabular}{lrlrlrl} 
ER & $455^{\mathrm{d}}$ & $\left(\ell_{\mathrm{ER}}=71\right)^{\mathrm{d}}$ & 124 & $\left(\ell_{\mathrm{ER}}=39\right)$ & $\sim 0$ & $\left(\ell_{\mathrm{ER}} \sim 0\right)$ \\
Fissione $^{\mathrm{e}}$ & 800 & 1323 & & 1451 & $(\ell=143)$ \\
Fusion $^{\mathrm{e}}$ & 1255 & $\left(\ell_{\text {crit }}=118\right)$ & 1563 & $\left(\ell_{\text {crit }}=137\right)$ & 1451 & $\left(\ell_{\text {crit }}=180\right)$ \\
Reactione $^{\mathrm{R}}$ & 2700 & $\left(\ell_{\max }=172\right)$ & 2565 & $\left(\ell_{\max }=180\right)$ & 2400 & $\left(\ell_{\max }=180\right)$ \\
\hline
\end{tabular}

"Data from ref. 5; uncertainties are $\approx \pm 15 \%$ as given there in detail. Results for the target ${ }^{116} \mathrm{~S} n$ are used for $107.9 \mathrm{Ag}$. The integrated cross section in coincidence with fission is denoted $\sigma$ coinc

bncertainties are $\pm 15 \%, 20 \%$, and $25 \%$ for $\mathrm{Ag},{ }^{154} \mathrm{Sm}$ and $\mathrm{Au}$, respectively.

'For each cross section $\sigma=\pi \hbar^{2}(\ell+1)^{2}$.

dH.C. Britt, et al., Phys. Rev. C13 (1976) 1483

'From refs. 3, 5, and unpublished data.

\section{Footnotes and References}

*State University of New York at Stony Brook, Stony Brook, NY 11794

†Carnegie-Mellon University, Pittsburgh, PA 15213

†ेInstitut de Physique Nucleaire, B.P. No. 1, 91406 Orsay, France

\$Montclair State College, Upper Montclair, NJ 07043

1. T. Ericson and V. Strutinski, Nucl. Phys. (1958) 284; Nucl. Phys. 9 (1959) 689

2. T. Dossing, Ph.D. thesis and unpublished notes, University of Copenhagen, Denmark
3. D. Logan, H. Delagrange, M.F. Rivet, $M$. Rajagopalan, J.M. Alexander, M. Kaplan, M.S. Zisman, and E. Duek, Phys. Rev. C22 (1980) 1080

4. M. Rajagopalan, D. Logan, J.W. Ball, M. Kaplan, H. Delagrange, M.F. Rivet, J.M. Alexander, L.C. Vaz, and M.S. Zisman, Phys. Rev. C25 (1982) 2417; J.M. Miller, D. Logan, G.L. Catchen, M. Rajagopalan, J.M. Alexander, M. Kaplan, J.W. Ball, M.S. Zisman, and L. Kowalski, Phys. Rev. Lett. 40 (1978) 1074

5. M.F. Rivet, D. Logan, J.M. Alexander, D. Guerreau, E. Duek, M.S. Zisman, and M. Kaplan, Phys. Rev. C25 (1982) 2430 


\title{
Intrinsic Fragment Spins Generated in the Reactions of ${ }^{20} \mathrm{Ne}$ with ${ }^{197}$ Au and ${ }^{238} \mathrm{U}$ at $12.6 \mathrm{MeV} /$ Nucleon*
}

\author{
D.J. Morrissey, ${ }^{\dagger}$ G.J. Wozniak, LG. Sobotka, A.J. \\ Pacheco, ${ }^{\ddagger}$ R. M. McDonald, C.C. Hsu, and L.G. Moretto
}

Experimental techniques used to obtain the magnitude and alignment of the transferred spin include $\alpha-$ particle, $\gamma$-ray, and sequential fission fragment angular distribution measurements. The out-of-plane angular distributions of $\boldsymbol{\gamma}$ rays and sequential fission fragments are primarily sensitive to the average random spin component. The angular distributions of $\gamma$ rays and $\alpha-$ particles are rather insensitive to differences in the inplane projections of the random spin component. In contrast, the angular distributions of sequential fission fragments are quite sensitive to such differences in that they can produce a substantial in-plane anisotropy.

Values of the aligned spin $\left\langle\mathrm{I}_{z}\right\rangle$ and the thermal widths were extracted from the sequential fission fragment angular distributions obtained for the reaction of $252 \mathrm{MeV}{ }^{20} \mathrm{Ne}$ with ${ }^{197} \mathrm{Au}$ and ${ }^{238} \mathrm{U}$. The extracted values of $\langle\mathrm{I}\rangle$ are plotted versus Q-value in Fig. 1 for both the ${ }^{20} \mathrm{Ne}^{2}+{ }^{238} \mathrm{U}$ and the ${ }^{20} \mathrm{Ne}+{ }^{197} \mathrm{Au}$ systems. For the formet system, $\left\langle\mathrm{I}_{\mathrm{z}}\right\rangle$ increases steadily with Q-value. A similar increase of $\left\langle I_{2}\right\rangle$ with $Q$-value has been observed in several other reaction systems. Because of the high fission barriers for nuclei near $\mathrm{Au}$, values of $\left\langle\mathrm{I}_{\mathbf{z}}\right\rangle$ were obtained only for the most negative Q-values of the ${ }^{20} \mathrm{Ne}+{ }^{197} \mathrm{Au}$ reaction. A striking difference between the two systems is the much larger values of $\langle\mathrm{I}\rangle$ observed for tbe ${ }^{20} \mathrm{Ne}+{ }^{197}$ All relative to the ${ }^{20} \mathrm{Ne}^{2}+{ }^{238} \mathrm{U}$ system. Since the $\ell_{\text {and }}$ and the rigid rotation partition for the two systems are very similar, the difference most likely refiects the strong bias towards high spin states for the fissioning gold nuclei and the absence of such a bias for the fissioning uranium nuclei. This bias is intoduced by the large fission barrier for gold nucle:.

The values of the aligned spin $\langle\mathrm{I}\rangle$ and the thermal widths extracted from the fitting of the sequential fission fragment angular distributions can be used to determine the two alignment parameters $P_{2 Z}$ and $P_{X Y}$. The results for $P_{Z Z}$ and $P_{X Y}$ obtained from the fitting of the fission fragment angular distributions for the uranium system are sbown in Fig. 2. In contrast to the strong Q-value dependence observed in more symmetric systems (cf., e.g., Refs. 1-3) the extracted values of $\mathrm{P}_{2 z}$ are approximately constant at 0.8 for the ${ }^{20} \mathrm{Ne}+{ }^{238} \mathrm{C}^{Z}$ system. The in-plane alignment parameter shows a stronger dependence on Q-value. It is positive at small $Q$-values and then goes to negative values at larger Q-values.

The predictions of the statistical equilibrium model for two touching spheres (solid curves in Fig. 2) lie substantially below both the $P_{2 Z}$ and $P_{X Y}$ data. Although this discrepancy could be interpreted as indicating that the dinuclear system is not at equilibrium (with respect to the normal modes), a more likely explanation is that the present model of two touching spheres does not allow for either deformation or interfragment separation (neck formation). Indeed, there is extensive evidence for large deformations of the nuclei at their scission configuration following a deep inelastic collision ${ }^{4,5}$. A first order estimate of tbe effect of deformation in the model calculations can be made by allowing the target-like fragment to deform along the line-ofcenter of the dinuclear system. Model calculations of $P_{Z Z}$ and $P_{X Y}$ are shown in Fig. 2 for different ratios of axes $(C / A)$ of the target-like fragment. Both $P_{z z}$ and $P_{X Y}$ are quite sensitive to the deformation of the heavy fragment. In this calculation, $\sigma_{\mathrm{x}}$ and $\sigma_{z}$ increase slowly whereas $\sigma_{y}$ decreases rapidly with increasing $\mathbf{C} / \mathbf{A}$ Thus increasing $\mathrm{C} / \mathrm{A}$ causes $\mathrm{P}_{\mathrm{Zz}}$ to increase because the random component of spin decreases while the aligned component is constant. $P_{X Y}$ approaches zero as $\mathrm{C} / \mathrm{A}$ increases because $\sigma_{y}$ becomes similar to $\sigma_{x}$. In this model, a ratio of axes of 1.6 to 2.0 is needed to reproduce the data in the deep-inelastic Q-value region. Such deformations of the heavy fragment are consistent with values given by the equilibrium configuration of a rotating sphere-spheroid liquid drop model system. ${ }^{4}$

\section{Footmotes and References}

*Condensed from LBL-13055, Nucl. Phys. A389 (1982) 120.

tPermanent address: Department of Chemistry and National Superconducting Cyclotron Laboratory, Michigan State University, East Lansing, Michigan, 48824. \$Permanent address: Comisión Nacional de Energia Atómica, Buenos Aires, Argentina.

\$Permanent address: Institute of Atomic Energy, Beijing, China. 
1. R.J. McDonald, A.J. Pacheco, G.J. Wozniak, H.H. Bolotin, L.G. Moretto, C. Shück, S. Shih, R.M. Diamond, and F.S. Stephens, Nucl. Phys. A373 (1982) 54.

2. D. v. Harrach, P. Glässel, Y. Civelekoglu, R. Männer, and H.J. Specht, Phys, Rev. Lett. 42 (1979) 1728.

3. R.J. Puigh, P. Dyer, R. Vandenbosch, T.D. Thomas, L. Nunnelley, and M.S. Zisman, Phys. Lett. 86B (1979) 24.

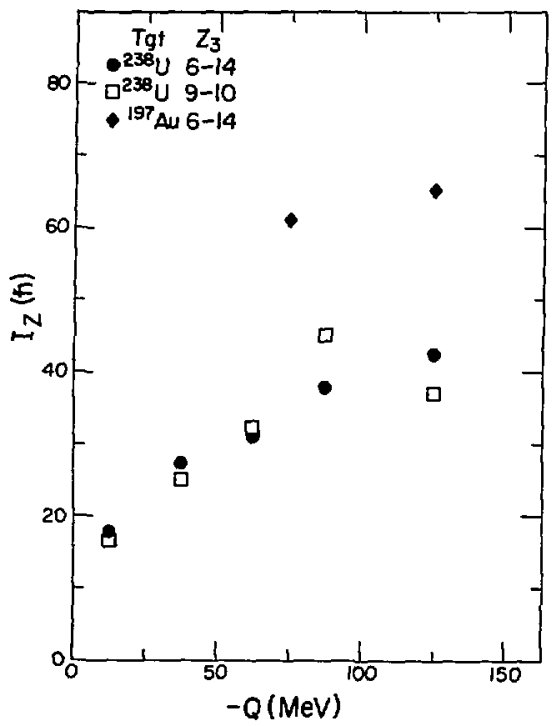

Fig. 1. The measured aligned spin ( $\left.I_{z}\right)$ of the targetlike fragment as a function of Q-value for the $252-\mathrm{MeV}$ ${ }^{20} \mathrm{Ne}+{ }^{197} \mathrm{Au}$ and ${ }^{238} \mathrm{U}$ reactions. Spins were extracted for a broad Z-bin (6-14) for both systems and an additional narrow one $(Z=9-10)$ for the ${ }^{20} \mathrm{Ne}+{ }^{238} \mathrm{U}$ system. The statistical errors are of the same size or smaller than the symbols.
4. L.G. Sobotka, C.C. Hsu, G.J. Wozniak, D.J. Morrissey, and L.G. Moretto, Nucl. Phys. A371 (1981) 510 .

5. K.E. Rehm, H. Essel, P. Sperr, K Hartel, P. Kjenle, H.J. Körner, R.E. Segel, and W. Wagner, Nucl. Phys. A366 (1981) 477.

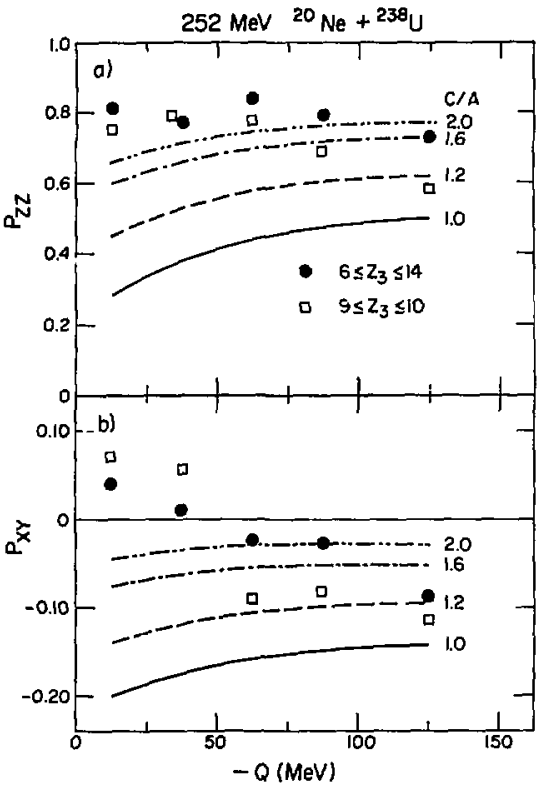

Fig. 2. The measured alignment parameters for the spin distributions obtained for the ${ }^{20} \mathrm{Ne}+{ }^{238} \mathrm{U}$ system are shown for $Z=6$ to 14 (circles) and $Z=9$ and 10 (squares). The solid and dashed curves represent the statistical equilibrium model calculations for different ratios of axes (C/A) of the target-like fragment (see text).

XBL $821-4417$

XBL 822-4472 


\title{
A Study of ${ }^{20} \mathrm{Ne}$ Induced Reactions with ${ }^{n a t} \mathrm{Cu}$ and ${ }^{197} \mathrm{Au}$ at 8.6 and $12.6 \mathrm{MeV} /$ nucleon*
}

\author{
G.J. Mathews, ${ }^{\dagger}$ I.B. Moulton, ${ }^{\ddagger}$ G.J. Wozniak, \\ B. Cauvin,' R.P. Schmitt, "J.S. Sventek, ${ }^{\prime \prime}$ \\ and L.G. Moretto
}

Calculated mean lifetimes for the ${ }^{20} \mathrm{Ne}+{ }^{197} \mathrm{Au}$ reaction are shown in Fig. 1 as a function of incident $\ell$-wave. Results are presented for calculations with and without neck formation. The bifurcation into the neck and no-neck curves occurs as soon as the trajectory for the system reaches the critical distance. Clearly, the inclusion of the neck has a dramatic influence on the lifetime distribution for both systems by displacing the critical $\ell$-wave for trapping of the ion-ion complex to higher incident angular momenta. This increases the complete-fusion cross section substantially. It is interesting that for the ${ }^{20} \mathrm{Ne}+{ }^{197} \mathrm{Au}$ reactions no complete fusion is predicted in the absence of a neck, whereas substantial complete fusion is predicted when a neck is allowed to form. Since the data seem to display a substantial fusion-fission component, this is perhaps an indication of the importance of neck formation. The complete fusion events are treated here according to the method outlined in Ref. 1.

In Fig. 2 experimental and calculated angular distributions are shown for representative fragment $Z$ values both above and below the projectile. It should be noted that these calculated angular distributions have not been normalized. In fact there is only one free parameter in these calculations, which is the ratio of the variance of the reaction lifetime to its mean value, $\sigma^{2} / \tau$. Changing this variable adjusts the slope of the angular distributions for atomic numbers near the projectile. A value of $\sigma^{2} / \tau=0.20$ yielded the best fits for the ${ }^{20} \mathrm{Ne}+{ }^{197} \mathrm{Au}$ reaction. The model calculations have not been corrected for charged-particle evaporation, which may have shifted these data by as much as one to three charge units.

The trends observed in the data are present in the calculations (see Fig. 2). It is particularly satisfying that the side peak in the $175-\mathrm{MeV}{ }^{20} \mathrm{Ne}+{ }^{197} \mathrm{Au}$ data emerges from the model calculations. It is also interesting that the flattening of the distribution at backward angles is less pronounced in the ${ }^{20} \mathrm{Ne}+{ }^{197} \mathrm{Au}$ data. This is predicted by the model simply because for higher $\ell$-waves the fragments do not penetrate deeply enough to form a neck, which would increase the collision lifetime. Nevertheless, for a large range of $\varepsilon$ waves a neck eventually does form (see Fig. 1) leading to a symmetric compound-nucleus fission component in the charge distribution similar to that observed in the data.

\section{Footnotes and References}

"Condensed from LBLI3226, Phys. Rev. C25 (1982) 300

†Lawrence Livermore National Laboratory, University of California, Livermore, CA 94550

†Present address: Department of Nuclear Engineering, Technion-Israel Institute of Technology, Haifa, Israel

§Present address: Dph N/MF CEN, Saclay, France

**Present address: Department of Chemistry and Cyclotron Institute, Texas A\&M University, College Station, Texas 77843

††Present address: Math and Computing Division, Lawrence Berkeley Laboratory, Berkeley, CA 94720

1. G.J. Mathews and L.G. Moretto, Phys. Lett. 87B (1979) 331

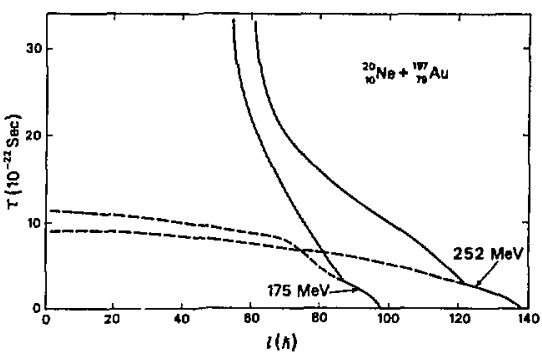

Fig. 1. Calculated average diffusion lifetimes as a function of incident \&-wave for the ${ }^{20} \mathrm{Ne}+{ }^{197} \mathrm{Au}$ reactions.

XBL $8010-2259$ 


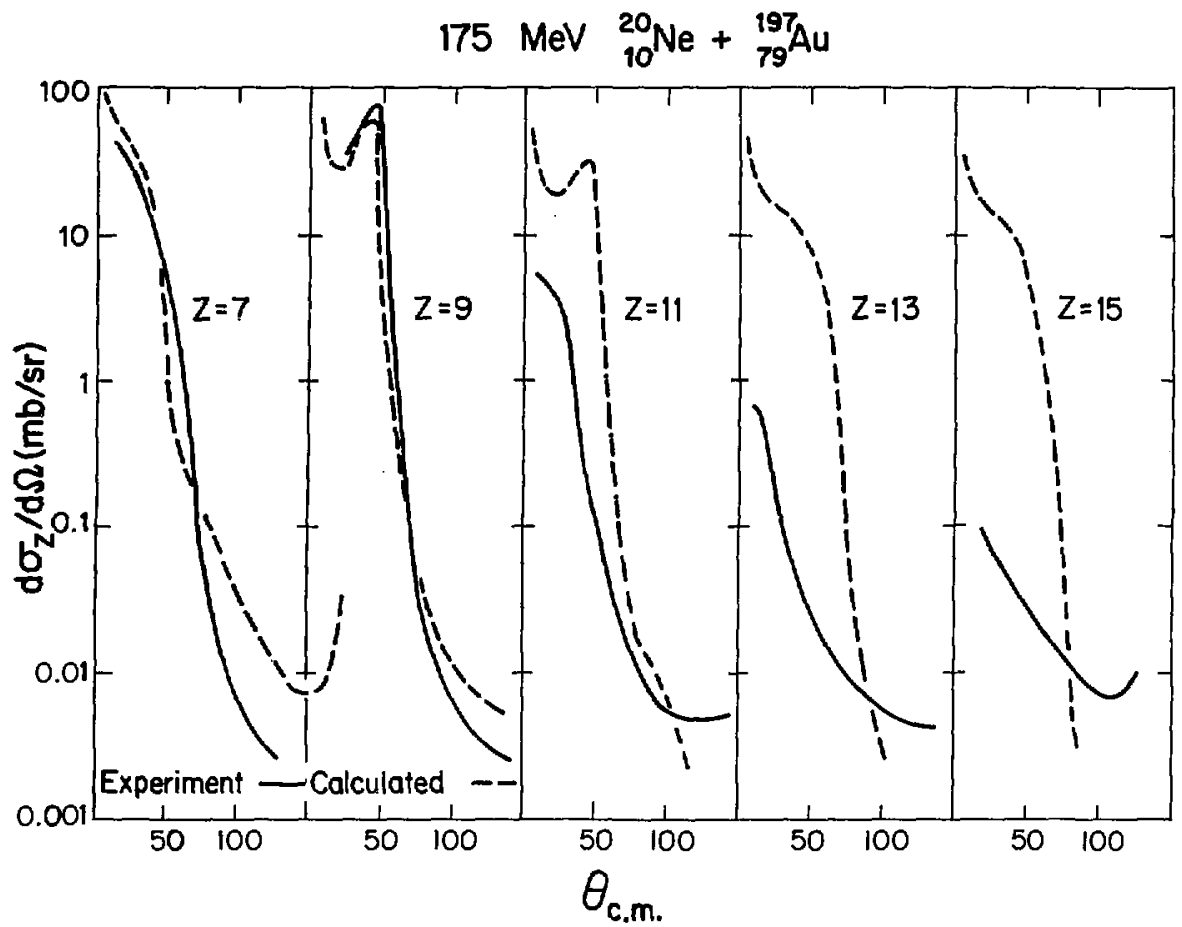

Fig. 2. Calculated and experimental angular distributions for representative products for the ${ }^{20} \mathrm{Ne}+{ }^{197} \mathrm{Au}$ reactions. XBL. 798-2439 


\title{
The Onset of Non-Equilibrium Phenomena in Fusion-Like Processes for ${ }^{16} \mathrm{O}$ Induced Reactions*
}

\author{
Y. Chan, M. Murphy, R, G. Stokstad, I. Tserruyat, \\ S. Wald, and A. Budzanowski ${ }^{\ddagger}$
}

At bombarding energies not too far above the interaction barrier, the complete fusion of heavy ions to form an equilibrated compound nucleus comprises a dominant part of the total reaction cross section. Further increase of the bombarding energy induces reactions which approximate fusion but exhibit nonequilibrium features. In such cases, it is generally believed that only a part of the projectile or target fuse; with the other partner (incomplete fusion), while the remaining part is emitted, presumably at an early stage of the collision and prior to the thermalization of the composite system.

Previous studies of these non-equilibrium features have emphasized the observation of forward-peaked, fast light particles $(n, p, d, \alpha)$ with velocities near to or greater than the beam velocity, sometimes in coincidence with the residual-like fraginents. It follows that the onset of incomplete fusion should also be reflected in a reduced average velocity of the associated heavy residues. (We consider only mass-asymmetric systems with the projectile as the lighter partner.) The present experiments determine the average velocity (or the linear momentum) of the residues, which are then compared with evaporation model predictions. Deviations of the observed velocity centroid and/or spectrum shape may be interpreted in terms of reaction mechanisms other than complete fusion followed by equilibriurs decay.

The experiments were performed at the 88-Inch Cyclotron at ${ }^{16} \mathrm{O}$ bombarding energies of $8.8,13.6$, and $19.6 \mathrm{MeV} / \mathrm{u}$. The energies were chosen to cover the region where one expects to see predominantly complete fusion $(8.8 \mathrm{MeV} / \mathrm{u})$ as well as regions where nonequilibrium phenomena can be important $(13.6,19.6$ $\mathrm{MeV} / \mathrm{u}$ ). Conventional time-of-flight techniques were used to measure the velocity of the residual nuclei. Natural calcium, aluminum, and ${ }^{60} \mathrm{Ni}$ targets with thickness of 235,215 , and $180 \mu \mathrm{g} / \mathrm{cm}^{2}$, respectively, were used. Masses up to about 50 amu could be resolved (Fig. 1a).

For decay processes symmetric about $90^{\circ}$ in the rest frame of the emitter, the velocity dependence of the quantity $F(v) \equiv\left(1 / v^{2}\right) d^{2} \sigma / d v d \Omega$ is expected to be a Gaussian centered at $v_{c m} \cos \theta$, where $v$ is the velocity of the residues in the laboratory, $\theta$ is the laboratory detection angle, and $v_{\mathrm{cm}}$ is the velocity of the center of mass.' Fig. lb shows a spectrum of $N(v) / v^{2}$, which is proportional to $F(v)$, for residues of mass 43 detected at $5^{\circ}$ for $13.6 \mathrm{MeV} / \mathrm{u}{ }^{16} \mathrm{O}+{ }^{+0} \mathrm{Ca}$.

We find that the quantity $\bar{v} / \cos \theta$ depends only weakly on the residual mass or the scattering angle. The deviations of this quantity from $V_{\mathrm{en}}$, averaged over all masses and angles, increase with bombarding energy. The results obtained with ${ }^{27} \mathrm{Al}$ and ${ }^{60} \mathrm{Ni}$ targets exhibit the same qualitative features.

In the following we shall concentrate on the observed angle- and mass-averaged velocity centroids, which we express as a percentage of the velocity corresponding to full momentum transfer followed by equilibrium decay. This quantity is plotted in Fig. 2 vs. the relative velocity of the colliding partners at the interaction barrier $\left(r_{0}=1.5 \mathrm{fm}\right)$. Even though there is some dispersion in the data, ait apparent systematics is seen to emerge; the amount of transferred momentum, expressed as a percentage of the total available linear momentum, is governed essentially by the relative velocity of the projectile and target nuclei at the barrier. Within errors, the deviations of the average centroids from the predictions of full momentum transfer appear to be independent of the target, suggesting that the missing momentum is mainly associated with the ${ }^{16} \mathrm{O}$ projectile.

The data also exhibit an approximately linear behavior in the decrease of the fraction of transferred momentum as a function of velocity as is suggested by the straight line fit to our data points. A similar result has been reported by Viola, et al., ${ }^{2}$ for reactions involving heavier targets. These results suggest a common onset of incomplete momentum transfer in fusion-like reactions at about $5 \mathrm{MeV} / \mathrm{u}$ above the interaction barrier. For comparison, also included in Fig. 2 are the results of ref. 3 obtained from fission fragment correlation measurements using ${ }^{16} \mathrm{O}$ projectiles and a much heavier ${ }^{238} \mathrm{U}$ target. Except for the highest energy point, which lies two standard deviations above the fitted line, these results are consistent with our systematics, and, together, suggest that the relative velocity of the projectile and target at the interaction barrier is a relevant parameter for characterizing incomplete linear momentum transfer in fusion-like processes. The data shown in Fig. 2 are consistent with there being little or no dependence of the missing momentum 
on the mass of the target. These simple results. if validated by additional experiments on a wider range of targets. should prove valuable in the evaluation of theories of pre-equilibrium emission or incomplete fusion.

We appreciate the help of $M$. Blann in various stages of this experiment.

\section{Foulnotes and References:}

*Condensed from LBL-15187; submitted to Phys. Rev. c.

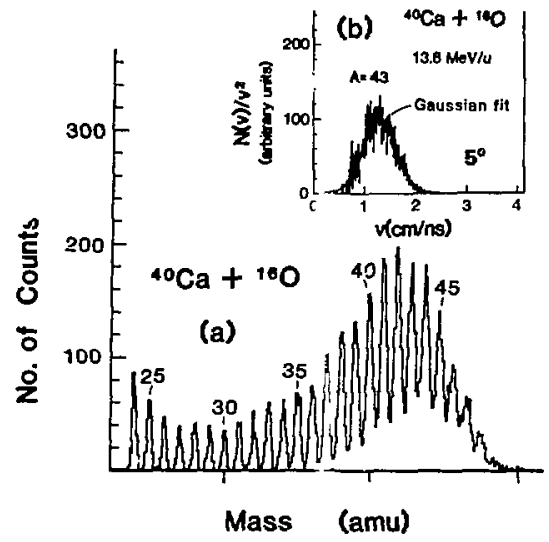

Fig. 1

(a) Partial mass spectrum for ${ }^{16} \mathrm{O}+{ }^{40} \mathrm{Ca}$ at $\mathrm{E}_{\mathrm{L}}=13.8$ $\mathrm{MeV} / \mathrm{u}$ and $\theta=5^{\circ}$ (lab).

(b) $N(v) / v^{2}$ spectrum for $A=43$. $N$ is the number of counts per unit velocity interval. The curve is a Gaussian fit to the spectrum.

XBL 829-11496
†Permanent address: Weizmann Institute of Science, Israel

\$Permanent address: Institute of Nuclear Physics, Cracow, Poland.

1. J. Gomez del Campo, et al., Phys. Rev. C19 2 L70 (1979).

2. V. Viola, et al., Phys. Rev. C26 178 (1982).

3. B. Back, et al., Phys. Rev, C22 1927 (1980).

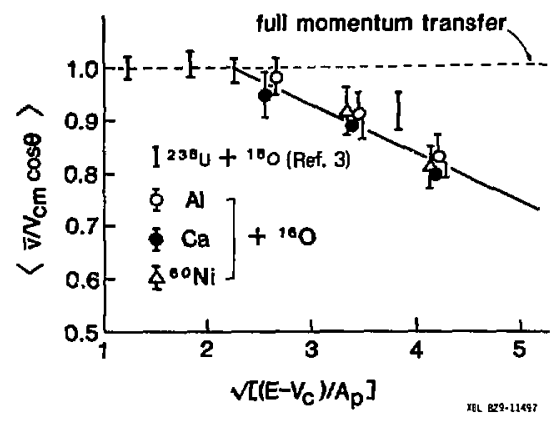

Fig. 2. Systematics of the mean velocities of fusionlike residues, expressed as a percentage of the velocity corresponding to complete momentum transfer. The abscissa is the relative velocity of the projectile and target at the interaction barrier $\left(r_{0}=1.5 \mathrm{fm}\right)$. The energy $E$ and Coulomb barrier $V_{c}$ are evaluated in the laboratory system.

XBL 829-11497 


\title{
Momentum Widths of Heavy-Ion Reaction Products at $27.4 \mathrm{MeV} / \mathrm{n}^{*}$
}

\author{
Martin J. Murphy' and Robert G. Stokstad
}

The inclusive momentum spectra of ejectiles emitted at forward angles in heavy-ion reactions exhibit a characteristic "quasi-elastic" (QE) peak. This peak has a mean velocity approximately equal to the beam velociiy, and is observed for laboratory bombarding energies of 5 to $2000 \mathrm{MeV} / \mathrm{n}$. We report here an inclusive measurement at $27.4 \mathrm{MeV} / \mathrm{n}$, and discuss the status of the currently available body of similar data.

We have measured energy spectra for the reactions, ${ }^{9} \mathrm{Be}+\mathrm{Au} \rightarrow{ }^{6,7} \mathrm{Li}+\mathrm{X}$. A $246 \mathrm{MeV}{ }^{9} \mathrm{Be}$ beam from the 88-Inch Cyclotron was used to bombard a 3.25 $\mathrm{mg} / \mathrm{cm}^{2}$ natural Au target. A $\mathrm{Si}(\mathrm{Li})$ particle telescope with elements $87 \mu \mathrm{m}, 500 \mu \mathrm{m}$, and $5 \mathrm{~mm}$ thick, located at $\theta_{1}=12^{\circ}$, was used to detect and identify reaction products. All of the different ejectile isotopes were resolved and complete energy spectra for ${ }^{6} \mathrm{Li}$ and ${ }^{7} \mathrm{Li}$ were obtained. The energy spectra for ${ }^{6} \mathrm{Li}$ and ${ }^{7} \mathrm{Li}$ are shown in Fig. 1. A Gaussian momentum distribution in the projectile rest frame was transformed to a laboratory energy distribution and used to fit the QE peak. The fitting region encompassed the peak and the high energy side and avoided the low energy tail. The fitted curves are also shown in Fig. 1, along with the resulting centroids and momentum widths. The energy centroids correspond approximately to the beam velocity. We obtained values for the reduced momentum width, $\sigma_{0}$. of $65.4 \pm 2.9 \mathrm{MeV} / \mathrm{c}$ and $60.2 \pm 1.2 \mathrm{MeV} / \mathrm{c}$ for ${ }^{6} \mathrm{Li}$ and ${ }^{7} \mathrm{Li}$, respectively, where ${ }^{1}$

$$
\sigma^{2}=\frac{A_{F}\left(A_{P}-A_{F}\right)}{A_{P}-1} \sigma_{0}^{2}
$$

Fig. 2 is a summary of 5 veral measurements of $\sigma$ and $\sigma_{0}$ at various energies. Ii includes the results of Egelhaaf, et al., ${ }^{2}$ the high energy points at $213 \mathrm{MeV} / \mathrm{n}$ and $2.1 \mathrm{GeV} / \mathrm{n}$, ${ }^{3,4}$ the data of Scott, et al., ${ }^{5}$ reanalyzed by Harvey, ${ }^{6}$ and our new measurement at $27.4 \mathrm{MeV} / \mathrm{n}$. In all cases the widths are for the total momentum distributions at the grazing angle and are obtained from the energy spectra of single isotopes. The fits in each case cover the apparent quasi-elastic part of the energy spectrum. (At energies below $50 \mathrm{MeV} / \mathrm{n}$ this region excludes the obvious low energy tail. At higher energies, where no low energy tail is evident, the fit extends over the full spectrum.) The measurements at 213 $\mathrm{MeV} / \mathrm{n}$ and $2.1 \mathrm{GeV} / \mathrm{n}$ provided widths for a large number of different isotopes. Rather than plot all of the individual points, we have indicated the full ranges of observed widths, as well as the mean values.
The data compiled in Fig. 2 show a similar energy dependence for both $\sigma$ and $\sigma_{0}$. The transition from the low to the high energy limit is not as abrupt as originally suggested, ${ }^{5}$ but it is fully realized over the energy range of $10-200 \mathrm{MeV} / \mathrm{n}$. The factorization according to eq. 1 reduces the mass dependence at most energies, but leaves a residual mass dependence in $\sigma_{0}$ that is more pronounced below $100 \mathrm{MeV} / \mathrm{n}$ than at higher energies. The limited amount of data available at energies below $100 \mathrm{MeV} / \pi$ makes more quantitative statements on the mass dependence of $\sigma_{0}$ difficult.

One must be cautious when using the data for $\sigma_{0}$ (E) in Fig. 2 as evidence for or against the occurance of particular reaction mechanisms (e.g., fragmentation, breakup, transfer, etc.). In particular, the data below $100 \mathrm{MeV} / \mathrm{n}$ suffer from several problems which affect the interpretation of $\sigma$ and $\sigma_{0}$. For instance, all of the results summarized in Fig. 2 have been obtained from inclusive measurements of the reaction spectra. A variety of reaction mechanisms could be represented in these spectra, each contributing its own isotope and energy dependence to the total width. For example, it is known that both transfer and breakup reactions occur at the lower energies. ${ }^{7}$ Secondly, the vailable data below $100 \mathrm{MeV} / \mathrm{n}$ only provide the width of the total momentum distribution. There is evidence ${ }^{8}$ that below $200 \mathrm{MeV} / \mathrm{n}$ only 4 i iongitudinal momentum dispersion has the mass depenuence of eq. 1. As a final point, we note that the fitting procedure to obtain $\sigma$ is itself quite subjective. It requires assumptions about the effect of the low energy tail and qualitative decisions about the part of the spectrum to be included in the fit. Even among the data presented here there are certainly systematic differences due to these necessary judgements.

In conclusion, our measurement of quasi-elastic momentum widths at $27.4 \mathrm{MeV} / \mathrm{n}$ yields results that support a relatively smooth transition in the reduced width $\sigma_{0}$ between 10 and $200 \mathrm{MeV} / \mathrm{n}$. Upon reexamining all of the similar data available at energies from 5 to $2000 \mathrm{MeV} / \mathrm{n}$, we have noted that this transition is just as evident in the "unreduced" width $\sigma$. There are problems with the interpretation of $\sigma_{0}$; it not only conceals a known anisotropy in the longitudinal and transverse momentum dispersions, but also a variety of reaction mechanisms. Therefore, any conclusions about the reaction dynamics at low energies, based upon the presently known values of $\sigma_{0}$, are premature. The reliable deduction of physical information from measurements of $\sigma_{0}$ at low energy must await 
the systematic measurement of each momentum component and separation of the inclusive spectra according to reaction mechanism

We would like to thank Karl Van Bibber and Deter Countryman for discussions and assistance with the measurements.

\section{Footnoies and References}

"Condensed from LBL 15188 - Submitted to Phys. Rev. C

†Present Address: Nuclear Physics Laboratory, L iniversity of Washington, Seattle, WA 98195

1. A.S. Goldhaber, Phys. Letters 53B 306 (1974).

2. Ch. Egelhaaf, et al., Phys. Rev. Lett, 46813 (1981)

3. Y. P. Viyogi, et al., Phys. Rev. Lett. 4233 (1979).

4. D. E Greiner, et al., Phys. Rev. Lett. 35152 (1975).

5. D.K. Scott, et al., Lawrence Berkeley Laboratory Report LbL-7729 (1978), unpublished; C. K Gelbke, et al., Phys. Letts. 70B 415 (1977).

6. D. G. Harvey, Phys. Rev. Lett. (Comments) $\mathbf{4 7}$ 454 (1981).

7. M. J. Murphy, et al., Phys. Letters B (in press).

8. K. Van Bibber, et al., Phys. Rev. Lett $\mathbf{4 3 8 4 0}$ (1979). C. Y. Wong and K Van Bibber, Phys. Rev. C25 2990 (1982).

9. J. B. Natowitz, et al., Phys. Rev. Lett. 471114 (1981).

10. J. Mougey, et al., Phys. Letters 105B 25 (1981).

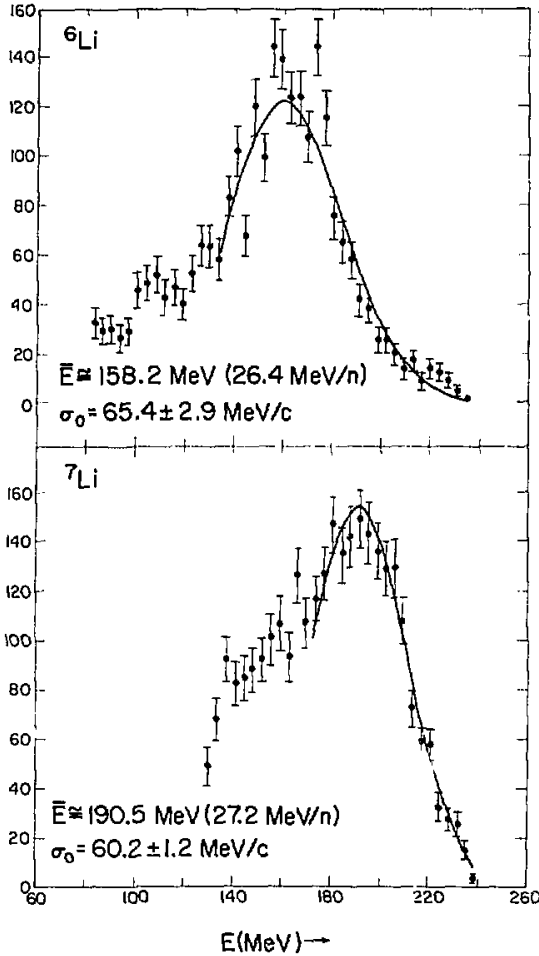

Fig. 1. The inclusive laboratory energy spectra for ${ }^{6} \mathrm{Li}$ and ${ }^{7} \mathrm{Li}$ ejectiles from ${ }^{9} \mathrm{Be}+\mathrm{Au}$ at $27.4 \mathrm{MeV} / \mathrm{n}$. The solid curves are fits to the quasi-elastic parts of the spectra.

XBL 827-1593 

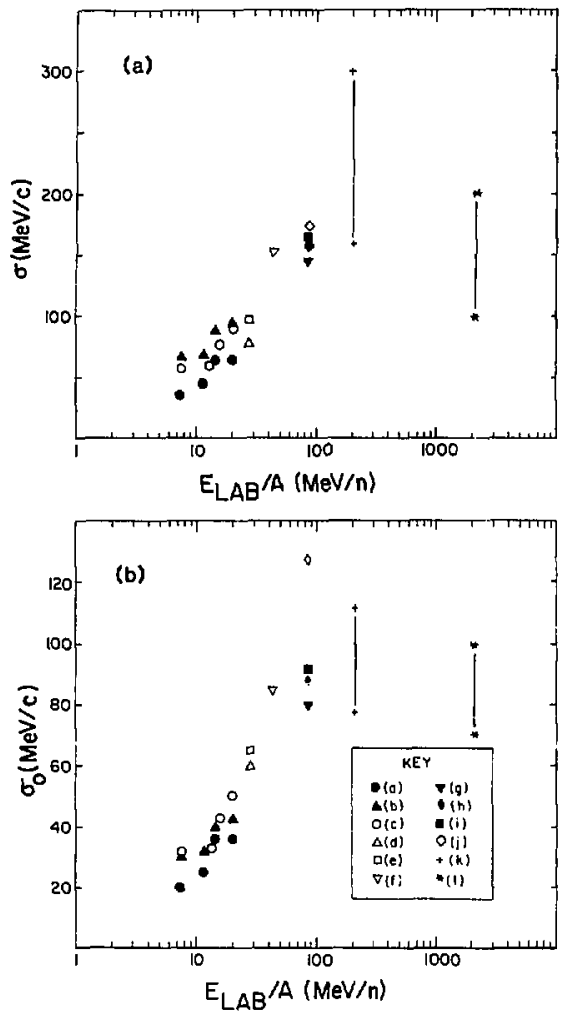

Fig. 2. A compilation of several different measurements of the momentum widths $\sigma$ and the reduced momentum widths $\sigma_{0}$ of ejectiles from heavy ion reactions. The key to the reactions is
a) ${ }^{197} \mathrm{Au}\left({ }^{20} \mathrm{Ne},{ }^{16} \mathrm{O}\right)$, ref. 2
g) ${ }^{12} \mathrm{C}\left({ }^{12} \mathrm{C}^{6} \mathrm{Li}\right)$, ref. 10
b) ${ }^{177} \mathrm{Au}\left({ }^{20} \mathrm{Ne}{ }^{12} \mathrm{C}\right)$, ref. 2
h) ${ }^{12} \mathrm{C}\left({ }^{12} \mathrm{C},{ }^{7} \mathrm{Li}\right)$, ref. 10
c) ${ }^{2 c}{ }^{3} \mathrm{~Pb}\left({ }^{16} \mathrm{O},{ }^{12} \mathrm{C}\right)$, ref. 6
i) ${ }^{12} \mathrm{C}\left({ }^{12} \mathrm{C},{ }^{7} \mathrm{Be}\right)$, ref. 10
d) ${ }^{\mathrm{nat}} \mathrm{Au}\left({ }^{9} \mathrm{Be},{ }^{7} \mathrm{Li}\right)$, This work
e) "nat $\mathrm{Au}\left({ }^{9} \mathrm{Be},{ }^{6} \mathrm{Li}\right)$, This work
f) ${ }^{191} \mathrm{Ta}\left({ }^{20} \mathrm{Ne}, \alpha\right)$, ref. 9
j) $\left.{ }^{12} \mathrm{C}^{12} \mathrm{C},{ }^{10} \mathrm{~B}\right)$, ref. 10
k) ${ }^{232} \mathrm{Th}\left({ }^{40} \mathrm{Ar}, \mathrm{X}\right)$, ref. 3
1) ${ }^{16} \mathrm{O}+\mathrm{X}$ ref. 4

A large number of widths were rieasured at 213 and $2100 \mathrm{MeV} / \mathrm{n}$; the figure indicates the range and mean value of the results.

XBL 829-9684 


\title{
Observation of Symmetric Disintegration in the ${ }^{6} \mathrm{~L},{ }^{9} \mathrm{Be},{ }^{12} \mathrm{C}+{ }^{40} \mathrm{Ca}$ Reactions
}

\author{
K. Grotowshi," Z. Majka, "Y. D. Chan, R. Stokstad. \\ I. Tserruya, S. Wald, G. Guarino, D. J. Morrissey. \\ L. G. Sobotka, G. J. Wozniak, and L. G. Moretto
}

During the last decade fission-like phenomena have been studied for a number of medium-A systems. ${ }^{1}$ This work is intended to test predictions of the liquiddrop and related models in the region $A \sim 50$ where experimental data are scarce. At the 88-Inch Cyclotron coincident fission-like products were observed in reactions induced by ${ }^{6} \mathrm{Lj},{ }^{9} \mathrm{Be}$ and ${ }^{12} \mathrm{C}$ projectiles on a ${ }^{40} \mathrm{Ca}$ target. The energy and charge of the reaction products were measured with $\triangle \mathrm{E}-\mathrm{E}$ telescopes.

Figure I shows coincidence count rates measured at equal telescope angles, $\theta_{1}=\theta_{2}$, for symmetric reaction products with $Z_{1} \simeq Z_{2}$. Keeping one of the detectors at the angle $\theta_{2}$ corresponding to the maximum in Fig. 1, and varying the position of the other detector produces the correlation functions shown in Fig. 2 . These functions refiect the kinematics of secondary fragments remaining after evaporation of nucleons and alpha particles by primary binary fragments. The correlation function found for ${ }^{12} \mathrm{C}+{ }^{40} \mathrm{Ca}$ at $121 \mathrm{MeV}$ has a FWHM about $10 \%$ smaller than the value at $186 \mathrm{MeV}$. An angular correlation function measured out of the reaction plane at $186 \mathrm{MeV}$ has about the same width as the in-plane function.

For the ${ }^{12} \mathrm{C}+{ }^{40} \mathrm{Ca}$ reaction, singles angular distributions were measured with $\triangle \mathrm{E}-\mathrm{E}$ telescopes at six incident energies between $74 \mathrm{MeV}$ and $186 \mathrm{MeV}$. Fig. 3 presents a center-of-mass angular distribution at 186 $\mathrm{MeV}$ for fragments with $\mathrm{Z}=11$. The $1 / \sin \theta$ dependence indicates that a compound system was formed. Assuming a $1 / \sin \theta$ dependence for all angles, the total cross section for the disintegration into two fragments of comparable mass, $\sigma_{\text {fascion, }}$ has been calculated and yields the following values: $18.8 \mathrm{mb}$ for ${ }^{12} \mathrm{C}+{ }^{40} \mathrm{Ca}$ at $186 \mathrm{MeV} ; 4.0 \mathrm{mb}$ for ${ }^{9} \mathrm{Be}+{ }^{40} \mathrm{Ca}$ at $140 \mathrm{MeV}$; and 1.2 mb for ${ }^{6} \mathrm{Lj}+{ }^{40} \mathrm{Ca}$ at $153 \mathrm{MeV}$. For ${ }^{12} \mathrm{C}+{ }^{40} \mathrm{Ca}$ at 186 $\mathrm{MeV}$, the above value of $\sigma_{\text {fission }}$ agrees well with a prediction of the dynamic coalescence and reseparation model of Swiatecki. ${ }^{23}$

\section{Foornore and References}

*Permanent address: Jagellonian University, Cracow, Poland
1. P. David, J. Debrus, H. Fahlbusch and J.Schulze, Nucl. Phys. A319 205 (1979) and references quoted therein; R. Eggers, M. N. Namboodiri, P. Gonthier and J. B. Natowitz, Rep. of the Cyclotron Institute, Texas A\&M University, 1980.

2. J. Blocki, private communication.

3. W. Swiatecki, private communication.

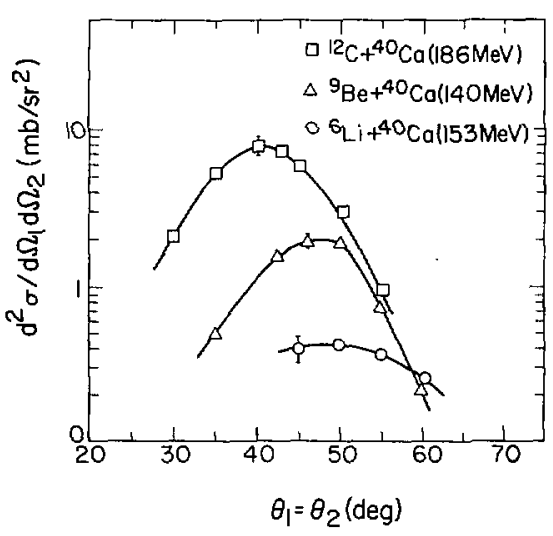

Fig. 1. Coincident count rates measured at equal telescope angles for symmetric reaction products with $Z_{1} \simeq$ $Z_{2}$.

XBL 835-2617 


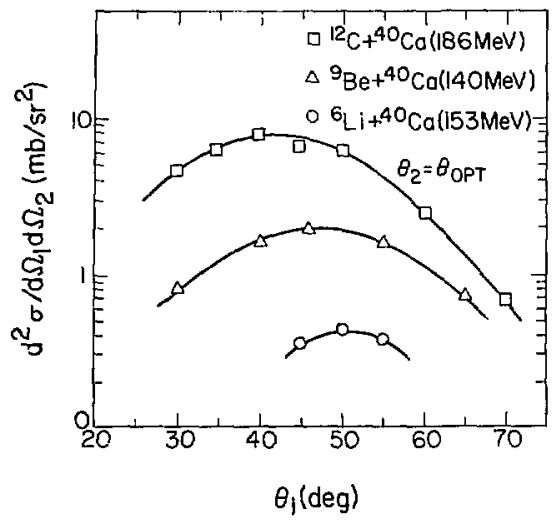

Fig. 2. Angular correlation functions for symmetric seaction products: one telescope is fixed at the angle of the maximum in Fig. 1.

XBL 835-2616

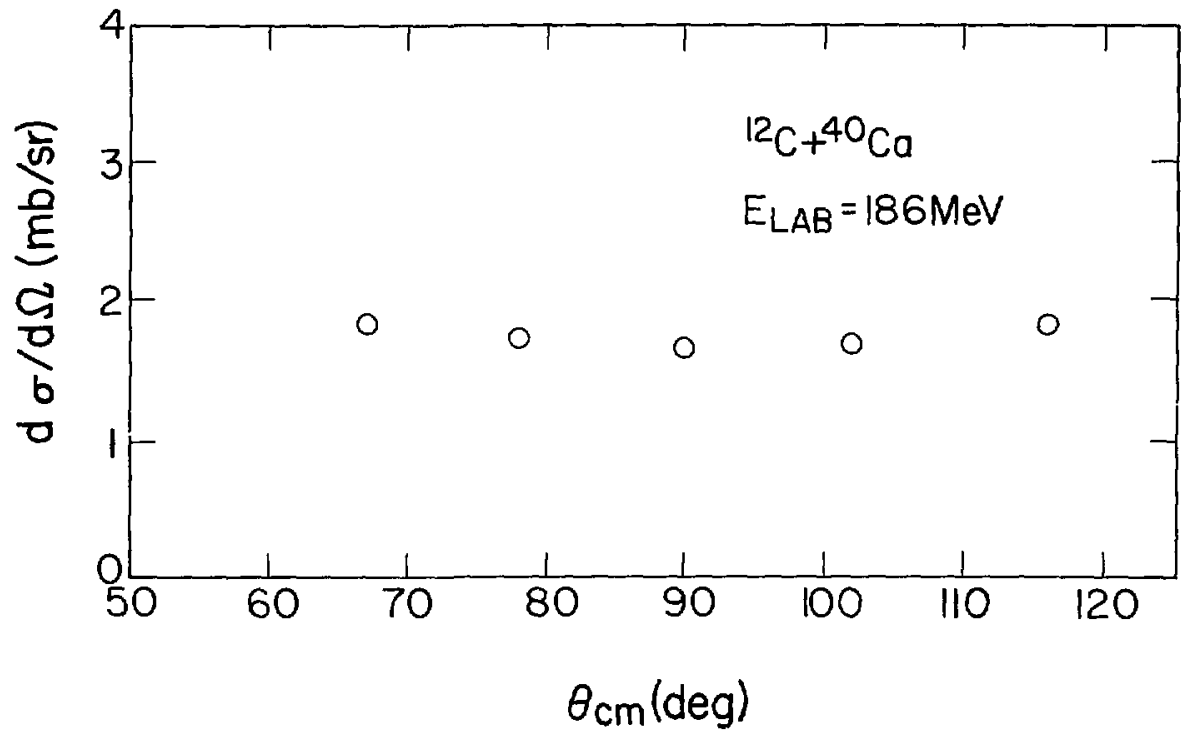

Fig. 3. Singles center of mass angular distribution for fragments with $Z=11$. 


\title{
${ }^{12} \mathrm{C}^{*}$ and ${ }^{8} \mathrm{Be}$ Production in ${ }^{12} \mathrm{C}+{ }^{208} \mathrm{~Pb}$ Collisions*
}

\author{
A.N. Bice, ${ }^{\dagger}$ A.C. Shotter, ${ }^{\ddagger}$ and Joseph Cerny
}

This report presents results of an investigation at the 88-Inch cyclotron into fast $\alpha$-particle production via the production of ${ }^{8} \mathrm{Be}$ (g.s.), ${ }^{8} \mathrm{Be}(2.94 \mathrm{MeV})$, and excited ${ }^{12} \mathrm{C}$ reaction products from the ${ }^{12} \mathrm{C}+{ }^{208} \mathrm{~Pb}$ system at ${ }^{12} \mathrm{C}$ bombarding energies of 132,187 , and 230 MeV. Absolute cross sections have been obtained for the reactions ${ }^{208} \mathrm{~Pb}\left({ }^{12} \mathrm{C}^{12} \mathrm{C}^{1} \rightarrow \alpha+{ }^{8} \mathrm{Be}\right)$, ${ }^{2008} \mathrm{~Pb}\left({ }^{12} \mathrm{C},{ }^{8} \mathrm{Be}(\mathrm{g.s}\right.$ ) $)$ ), and ${ }^{200} \mathrm{~Pb}\left({ }^{12} \mathrm{C},{ }^{8} \mathrm{Be}(2.94 \mathrm{MeV})\right)$ by coincident measurement of three $\alpha$-particles or two $\alpha$ particles for ${ }^{12} \mathrm{C}^{\circ}$ and ${ }^{8} \mathrm{Be}$ detection, respectively. By folding in the probability of detecting correlated particles, the absolute production cross sections were determined, which were then compared with the measured singles $\alpha$-particle cross section at. $187 \mathrm{MeV}{ }^{12} \mathrm{C}$ bombarding energy. From this comparison, further information could be obtained about the reaction channels important in fast $\alpha$-particle production.

A detection system was employed which consisted of three $\Delta E-E$ counter telescopes mounted on a movable platform and arranged in a vertical fashion with respect to the normal scattering plane. A pair of particle telescopes were located symmetrically above and below the scattering plane, i.e., the collimator post between these two telescopes was bisected by the horizontal reaction plane. Further, a third telescope was always located above the reaction plane. Coincidence events between any pair of telescopes were recorded, but simultaneous events in all three were not.

Figure 1 shows a Wilczynski-type diagram for ${ }^{8} \mathrm{Be}$ (g.s.) production at $187 \mathrm{MeV}$. Such a diagram plots contours of the double differential cross section $\mathrm{d}^{2} \sigma / \mathrm{d} \Omega \mathrm{dE}$ for the ${ }^{8} \mathrm{Be}$ (g.s.) reaction products as a function of their kinetic energy and their laboratory scattering angle, highlighting both the energy and the angular distributions. This figure and those at the other bombarding energies show a ridge near beam velocity that extends from the maximum toward backward angles. There is little or no evidence of a ridge extending back from zero degrees as is characteristic of a deep inelastic reaction. The ridge is therefore likely to be associated with an interaction that is peripheral in rature. No other significant features are evident.

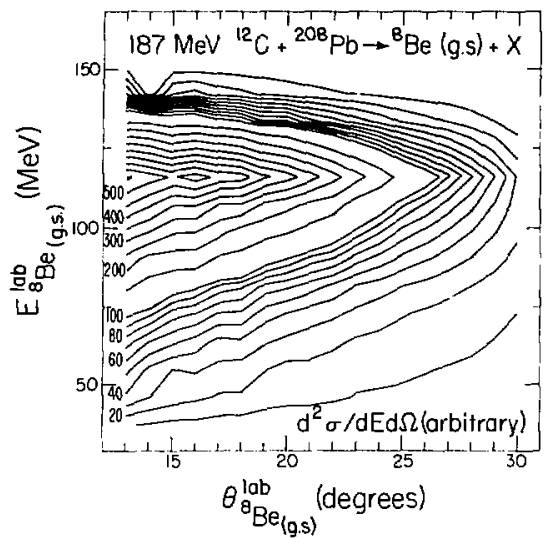

Fig. 1. Wilczynski-type diagram for the production of ${ }^{8} \mathrm{Be}\left(\mathrm{g} . \mathrm{s}\right.$.) nuclei for the system $187 \mathrm{MeV}{ }^{12} \mathrm{C}+{ }^{208} \mathrm{~Pb}$.

XBL $8110-7361$

Figure 2 shows the absolute differential cross section for the production of ${ }^{\mathrm{B}} \mathrm{Be}$ (g.s.) nuclei. These cross sections were obtained by integrating the $\left({ }^{12} \mathrm{C}, \alpha \alpha\right)$ spectra by energy bins with the appropriate detection probability for ${ }^{B} \mathrm{Be}$ (g.s.) nuclei folded in. Increasing cross section with decreasing angles and steeper angular distributions with increasing bombarding energy are evident in this figure. Cross sections to several hundred millibarns are apparent, suggesting that a significant fraction of the inclusive $\alpha$-particles arise from decaying ${ }^{8} \mathrm{Be}$ nuclei.

The observation of a large production cross section for ${ }^{8} \mathrm{Be}($ g.s. $)$ and ${ }^{8} \mathrm{Be}(2.94 \mathrm{MeV})$ nuclei at $\mathrm{E}\left({ }^{12} \mathrm{C}\right)$ $=187 \mathrm{MeV}$ permits the conclusion that projectile fragmentation is largely responsible for the fast $\alpha$-particle production. These measurements, together with those 
of Siwek-Wilczynska, et al., ${ }^{1,2}$ provide an explanation for the origin of over $80 \%$ of the observed $\alpha$-particles at $187 \mathrm{MeV}$ bombarding energy. Although the observed ${ }^{B} \mathrm{Be}$ production cross sections as a function of the bombarding energy are not in disagreement with the simple incomplete fusion model predictions of SiwekWilczynska, et al., it is also clear that projectile spectroscopic and/or final state interactions are important in fragmentation reactions at these bombarding energies. An angular correlation measurement of $\alpha+{ }^{B} \mathrm{Be}$ reaction products would be feasible and very valuable to a further understanding of the breakup mechanism(s) involved.

Footnotes and References

*Condensed from LBL-14100, Nuclear Physics A390 (1982) 161

†Present address: U.S. NRC/ACRS, Washington, D.C. $\ddagger$ Permanent address: Physies Department, University of Edinburgh, Edinburgh, United Kingdom

1. K Siwek-Wilczynska, E.H. du Marchie van Voorthuysen, J. van Popta, R.H. Siemssen, and J. Wilczynsk, Phys. Rev. Lett. 42 (1979) 1599

2. K Siwek-Wilczynska, E.H. du Marchie van Voorthuysen, J. van Popta, R.H. Siemssen, and J. Wilczynski, Nucl. Phys. A330 (1979) 150

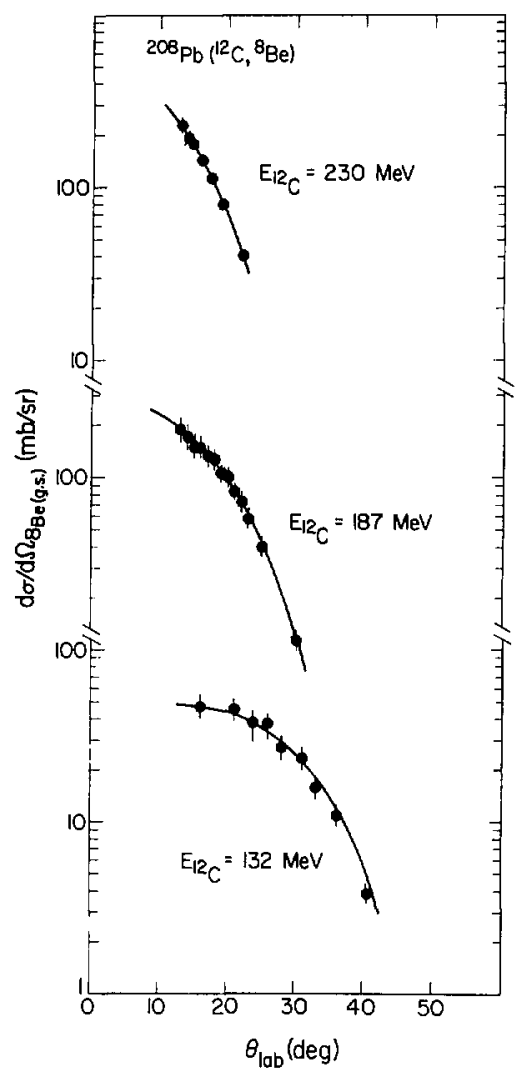

Fig. 2. Angular distributions for the production of ${ }^{8} \mathrm{Be}$ (g.s.) nuclei for the system ${ }^{12} \mathrm{C}+{ }^{208} \mathrm{~Pb}$ at three ${ }^{12} \mathrm{C}$ bombarding energies; 132,187 , and $230 \mathrm{MeV}$.

XBL 818-1161 


\title{
Transfer and Breakup Reactions in ${ }^{16} \mathrm{O}+\mathrm{CSI}$ at $16.4 \mathrm{M}: \mathrm{V} / \mathrm{n}$
}

\author{
M.J. Murphy, B.G. Harvey. D.L. Hendrie, W.W. Pang, K. Van \\ Bibber. ${ }^{\dagger}$ and $R$. Legrain ${ }^{\ddagger}$
}

A streamer chamber triggered by any one of three $\mathrm{Si}(\mathrm{Li})$ particle telescopes at $12.5^{\circ}, 17.5^{\circ}$, and $24.0^{\circ}$ (lab) was used to observe the multiplicity of charged particles in coincidence with the trigger particle in the reaction ${ }^{16} \mathrm{O}+\mathrm{Csl}$ at $16.4 \mathrm{MeV} /$ nucleon. The measurement provided relative probabilities for transfer and projectile breakup as a function of ejectile charge, as well as energy spectra of the heavy ejectiles from transfer and breakup events. The events were photographed by a single camera in the direction normal to the reaction plane defined by the beam and the trigger particle. Simultancously, the signals from the $\Delta \mathrm{E}-\mathrm{E}$ telescope were recorded on magnetic tape along with an event number identifying the photographic frame.

The photographs were scanned for charged particle multiplicity in the forward and backward hemispheres. The positions of the projectile track at the target and of the ejectile track at the chamber exit window were checked for consistency with the target center and the telescope position. Because of the high level of RF noise from the Marx generator, only Z-identification was obtained from the telescope for $Z=3,5-8$. At $Z$ $=4,{ }^{7} \mathrm{Be}$ and ${ }^{9} \mathrm{Be}$ were well resolved.

We assume that tracks in the backward hemisphere arose from charged particle evaporation from the target and that evaporation was isotropic in the laboratory system. The numbers of forward hemisphere events were corrected for the (small) evaporation contribution and summed over telescope angles. The resulting multiplicity distributions are shown in Table $I$.

We interpret multiplicity $(M)=1$ events as transfer reactions anò $M \geq 2$ events as projectile breakup. Table I shows that $Z=7$ ejectiles are produced almost exclusively by the transfer mechanism and that the ratio of breakup to transier increases with decreasing $Z$. Nevertheless, a nine nucleon transfer produces most of the ${ }^{7} \mathrm{Be}$. A striking result is that (except for $\mathrm{Li}$ triggers) multiplicities (M) greater than two are very rare. This observation alone places some restrictions on the mechanism of projectile breakup, suggesting that sequential decays of excited ejectiles play a major role.

As an example, most of the $M=2$ events with $Z$ $=5$ are due to ${ }^{11} \mathrm{~B}$. A prompt ${ }^{16} \mathrm{O}$ fragmentation would produce " $\mathrm{B}+{ }^{5} \mathrm{Li}$. But ${ }^{5} \mathrm{Li}$ is unstable to decay to ${ }^{4} \mathrm{He}$ $+p$, so this mechanism could not give an $M=2$ event. On the other hand, the sequences ${ }^{16} \mathrm{O} \rightarrow{ }^{12} \mathrm{C}^{*} \rightarrow{ }^{11} \mathrm{~B}+$ $\mathrm{p}$ or ${ }^{16} \mathrm{O} \rightarrow{ }^{15} \mathrm{~N}^{*} \rightarrow{ }^{11} \mathrm{~B}+{ }^{4} \mathrm{He}$ would yield ${ }^{11} \mathrm{~B}$ in $\mathrm{M}=$ 2 events. Moreover, the prompt $M=2$ breakup of ${ }^{16} \mathrm{O}$ into ${ }^{9} \mathrm{Be}+{ }^{7} \mathrm{Be}$ would produce equal yields of these nuclides, whereas we observed 4.5 times more ${ }^{9} \mathrm{Be}$ than ${ }^{7}$ Be. A sequence such as ${ }^{16} \mathrm{O} \rightarrow{ }^{13} \mathrm{C}^{*} \rightarrow{ }^{9} \mathrm{Be}+{ }^{4} \mathrm{He}$ could contribute to the extra ${ }^{9} \mathrm{Be}$ yield in $\mathrm{M}=2$ events.

The energy spectra both of transfer and breakup reactions show the familiar quasi-elastic peak at an ejectile velocity close to projectile velocity. However, the peak energies of the breakup spectra, as shown in Table II, are shifted down in energy by $10-20 \mathrm{MeV}$. The energy shifts in $M \geq 2$ spectra are just what would be expected for transfer reactions (or inelastic excitation of ${ }^{16} \mathrm{O}$ ) that leave the primary ejectile at an excitation energy just above the theshold for ${ }^{4} \mathrm{He}$ or $\mathrm{p}$ decay. The small ( $9 \mathrm{Mev}$ ) shift for $Z=6$ triggers is, on this picture, due to the low $(7.2 \mathrm{MeV})$ threshold for ${ }^{16} \mathrm{O}^{*} \rightarrow$ ${ }^{16} \mathrm{C}+{ }^{4} \mathrm{He}$.

Table 1. The associated charged particle multiplicities, summed over angle after correction for target evaporations. Also included is the total number of observed backward-going tracks for each ejectile charge.

\begin{tabular}{|c|rrrrr|c|}
\hline & \multicolumn{5}{|c|}{ MULTIPLICITY } & \\
EJECTILE & 1 & 2 & 3 & 4 & 5 & $N_{\text {BACKWARD }}$ \\
\hline \hline & & & & & & \\
\hline $\mathrm{Li}$ & 22 & 60 & 17 & 5 & 0 & 25 \\
${ }^{7} \mathrm{Be}$ & 11 & 4 & 5 & 0 & 0 & 3 \\
${ }^{9} \mathrm{Be}$ & 40 & 18 & 1 & 1 & 0 & 11 \\
$\mathrm{~B}$ & 66 & 45 & 2 & 2 & 0 & 12 \\
$\mathrm{C}$ & 194 & 85 & 4 & 1 & 0 & 7 \\
$\mathrm{~N}$ & 339 & 16 & 1 & 0 & 0 & 0 \\
\hline
\end{tabular}

\section{Footnotes}

*To be published in Physics Letters

+Department of Physics, Stanford University, Stanford, CA 94305

$\ddagger D P h N-B E, C E N$ Saclay, BP No. 2, 91190 Gif-surYvette, France 
Table II. The measured mean energies of the $M+1$ and $M \geq 1$ specira.

\begin{tabular}{|c|c|c|c|c|}
\hline EJECTILE & $\begin{array}{c}\text { BEAM } \\
\text { VELOCITY } \\
\text { MeV } \\
\end{array}$ & $\begin{array}{c}<E>_{M-1} \\
(T R A N S F E R) \\
M e V\end{array}$ & $\begin{array}{c}<>_{M \geq 2} \\
\text { (FRAGMENTATION) } \\
\text { MeV }\end{array}$ & $<E>_{M-1}-<E>_{m \geq 2}$ \\
\hline${ }^{9} \mathrm{Be}$ & 148 & $142 \pm 9$ & $123 \pm 10$ & $19 \pm 13$ \\
\hline B & 180 & $167 \pm 7$ & $156 \pm 8$ & $11 \pm 10$ \\
\hline C & 197 & $206 \pm 4$ & . $197 \pm 6$ & $9 \pm 7$ \\
\hline $\mathbf{N}$ & 230 & $226 \pm 2$ & $215 \pm 13$ & $11 \pm 13$ \\
\hline
\end{tabular}

\title{
Kinematics of Heavy Ion Transfer Reactions*
}

\author{
B.G. Harvey and M.J. Murphy
}

Any model of the kinematics of heavy ion transfer reactions must give agreement with two experimental quantities. The first of these is the kinetic energy of the ejectiles, which are observed to have broad peaks at energies corresponding to a velocity close to that of the projectile. The second observable is the quantity of angular momentum transferred to the target nucleus. The semiclassical model of Brink ${ }^{1}$ addresses both questions, but it contains approximations that limit its application to transfers of small masses on to the periphery of the target nucleus.

The transfer reaction model of Wilczynski, et al. ${ }^{2}$ implies that the impact parameter becomes smaller the larger the mass transfer, and it accounts quite well for the reaction cross sections. The model of angular momentum transfer proposed by Abul-Magd ${ }^{3}$ explicitly requires that the transferred nucleons be contained in an overlap region of the projectile with the target nucleus. In the present work we have adopted that hypothesis and combined it with the assumption of Brink $^{1}$ that the nucleons are transferred at the velocity with which the projectile arrives in contact with the target nucleus. Unlike Brink, though, we have taken account of recoil so the results are valid for arbitrarily light target nuclei and for large mass transfers. The calculations are nonrelativistic. The geometry of the colliding system at the point where transfer begins is shown in Fig. 1.

The angular momentum transfer $\lambda$ is given by

$$
\lambda=\mu_{i} v_{r e l} R_{i}-\mu_{i} v_{r e l}^{\prime} R_{i}
$$

where the $\mu^{\prime}$ 's are reduced masses, $v_{\text {rel }}$ and $v_{\text {rel }}^{\prime}$ are the relative velocities of projectile-target and ejectile-final nucleus respectively, and

$$
\begin{gathered}
\mathbf{R}_{\mathbf{i}}=\mathbf{R}_{1}+\mathrm{R}_{2}-\mathrm{h} \\
\mathrm{R}_{\mathrm{f}}=2 \mathrm{R}_{1}+\mathrm{R}_{2}-\mathrm{h}-\mathrm{h}^{\prime \prime}
\end{gathered}
$$

The relative velocities take account of the target and final nucleus recoils. Since $\lambda$ is a function of $v_{\text {rel }}^{\prime}$, it depends on the effective $Q$-value $Q_{c}^{l}$ of the transfer.

The angular momentum of the nucleons (of mass m) to be transferred is also given by

$$
\lambda=\frac{\mathrm{m}}{\mathbf{A}_{4}}\left(\mathrm{v}_{\mathrm{ur}}-\mathrm{v}_{\mathrm{s}}\right) \mathbf{R}_{\mathbf{t r}}
$$

where $A_{4}$ is the mass of the final nucleus, $v_{t}$ is the velocity of the transferred nucleons, $v_{5}$ is the velocity of the recoiling target, and $R_{t r}$ is the projected distance between the center of mass of $m$ and the target:

$$
\mathbf{R}_{\mathbf{r r}}=\mathbf{R}_{\mathbf{2}}-\mathrm{h}+\mathrm{h}^{\prime}
$$


For any value of $Q_{5}, v_{5}$ can be obtained by conservation of momentum from:

$$
v_{5}=\frac{A_{4} v_{4}-m v_{t v}}{A_{2}}
$$

where $A_{2}$ is the target mass.

Assuming with Brink that transfer is most probable when $v_{\text {ir }}$ equals the projectile velocity at contact and equating the two expressions above for $\lambda$ yields the value of $Q^{\circ}$, the optimum $Q$ value for which the transfer cross section will reach its peak value. From $Q^{\circ}$ the ejectile kinetic energy at the quasi-elastic peak can be calculated for any angle of observation.

The model gives quasi-elastic ejectile peak energies that correspond to velicities within a few percent of the projectile velocity, even when the target nucleus is lighter than the projectile and recoil effects are large.

Figure 2 shows a comparison of the calculated and experimental ${ }^{4}$ angular momentum transfers in the system ${ }^{20} \mathrm{Ne}+{ }^{181} \mathrm{Ta}$ at $150 \mathrm{MeV}$, as a function of the ejectile $Z$ and of total kinetic energy loss $(-Q)$. The vertical arrows indicate the Q-value corresponding to $Q^{\circ}$. Agreement is excellent for Q Q values near to $Q^{\circ}$, i.e., in the quasi-elastic peak. For much more negative Q-values, the observed ejectiles are at least in part produced by projectile breakup $p^{5}$ with a smaller (or even zero) transfer of angular momentum.

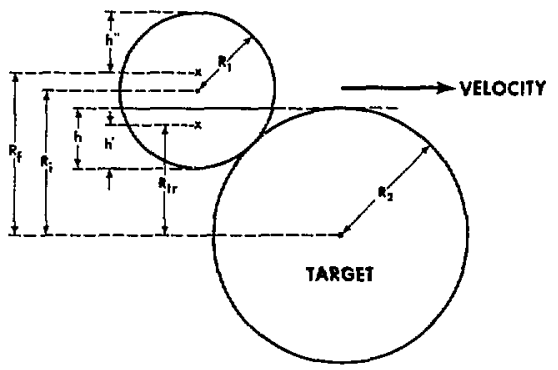

Fig. 1. Geometry of overlap. The nucleons to be transferred are contained in a spherical cap of height $h$ (shaded). $h^{\prime}, h^{\prime \prime}$ are the centers of mass of transferred nucleons and ejectile nucleons. The distances $R_{j}, R_{f}$, and $R_{\mathbf{t r}}$ are defined in the text.

XBL $8212-7368$

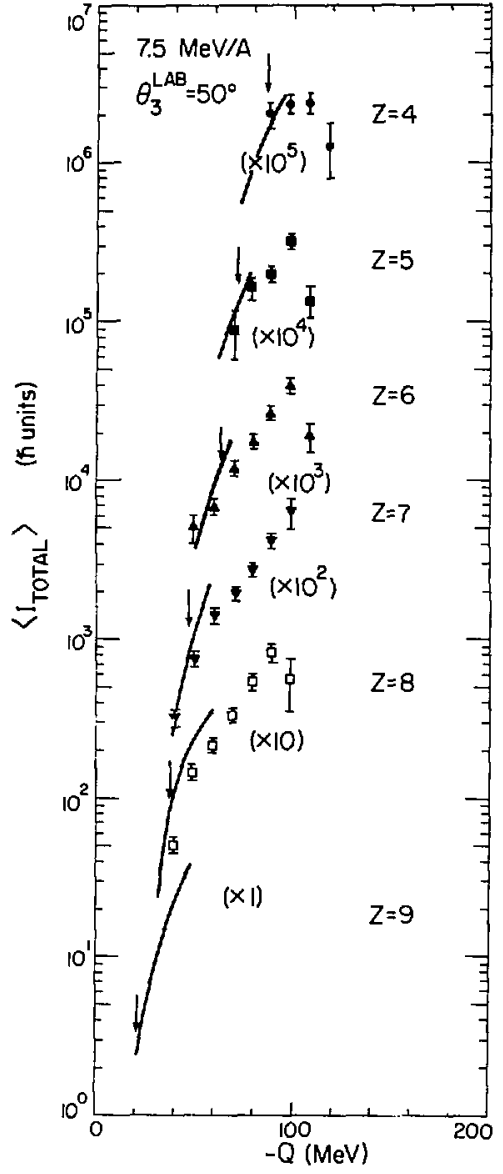

Fig. 2. Comparison of calculated angular momentum transfer (solid lines) with the experimental results of ref. 4.

XBL 80L1-2428A 


\section{Foornote and References}

*to be submitted to Physics Letters

1. D.M. Brink, Phys. Lett. 40B (1972) 37

2. J. Wilczynski et al., Phys. Rev. Lett. 45 (1980) 606
3. A.Y. Abul-Magd, Z. Physik A298 (1980) 143

4. C.C. Hsu, et al., LBL Report 12519 and private communications

5. M.j. Murphy, et al., Transfer and Breakup Reactions in ${ }^{15} \mathrm{O}+\mathrm{CsI}$ at $16.4 \mathrm{MeV} / \mathrm{n}$, preceding contribution to this report

\title{
Recoil Range Studies of Target-like Products of Multinucleon Transfer from ${ }^{18} \mathrm{O}$ to ${ }^{245} \mathrm{Cm}$ and ${ }^{249} \mathrm{Cr}$
}

\author{
Rose Marie McFarland, Albert Ghiorso, and Glenn T. Seaborg
}

Our group is interested in knowing the mechanism, or mechanisms, contributing to the production of ruclides of $Z$ and $A$ between those of the target and compound nucleus in reactions of actinides with light heavy ions (LHI) $(4<\mathrm{A} \leq 40)$ at energies just above the interaction barrier. In this preliminary study, ${ }^{1}$ measurements were made of recoil ranges of alpha and spontaneous fission activities produced in bombardments of ${ }^{245} \mathrm{Cm}$ and ${ }^{249} \mathrm{Cf}$ with ${ }^{18} \mathrm{O}$ projectiles.

All bombardments were done at the 88-Inch cyclotron at Lawrence Berkeley Laboratory. The targets consisted of $0.240 \mathrm{mg}{ }^{245} \mathrm{Cm} / \mathrm{cm}^{2}$ and $0.520 \mathrm{mg}$ ${ }^{249} \mathrm{Cf} / \mathrm{cm}^{2}$ as the actinide trifluoride. Curium-245 was bombarded with $93,99.6$, and $111.6 \mathrm{MeV}{ }^{18} \mathrm{O}$ and ${ }^{249} \mathrm{Cf}$ with $83,89.2,99.8$, and $111.6 \mathrm{MeV}{ }^{18} \mathrm{O}$ projectiles. Recoiling products were caught in a stack of ten 0.1 $\mathrm{mg} / \mathrm{cm}^{2}$ aluminum foils. After a bombardment, each foil was placed over a $\mathrm{Si}(\mathrm{Au})$ surface bartier detector with pulse height analysis, and its decay was followed for up to six months. Nuclides were identified by $E_{\alpha}$ and half-life.

Recoil range is a function of both the energy and angle of separation of the dinuclear complex:

$$
\mathrm{v}_{\mathbf{T}^{\prime}} \cos \Phi_{\mathbf{T}}=\mathrm{v}_{\mathrm{T}} \cos \theta_{\mathrm{T}}+\mathrm{v}_{\mathrm{cm}}
$$

where $v_{T}$ and $\Phi_{T}$ are the lab velocity and recoil angle of the target-like fragment, $V_{T}$ and $\theta_{T}$ are its centerof-mass (c.m.) frame velocity and angle, and $v_{c m}$ is the velocity of the center of mass. The expression on the left is a direct measure of the recoil range.

Complete fusion and massive transfer were eliminated as contributing mechanisms on the basis of the shape of the range distributions. Both should exhibit narrow distributions with a very sharp cutoff at the high-range side. $^{2}$ Figure 1 shows a typical range histo- gram, that of ${ }^{252} \mathrm{Fm}$ formed in the reaction of 111.6 $\mathrm{MeV}{ }^{18} \mathrm{O}$ with ${ }^{249} \mathrm{CF}$. The distribution is broad with a long, bigh-range tail. Therefore, it was assumed that only quasi-elastic and deep inelastic transfer, or QET and DIT, contribute to these reactions.

QET is characterized by an optimal dinuclear separation energy determined by entrance-to-exit channel trajectory matching conditions and a most probable separation angle near the classical Rutherford grazing angle. In DIT, the separation energy is equal to the exit channel Coulomb barrier and, for these very fusile systems, a separation angle near $0^{\circ}$.

Recoil range alone is insufficient to determine both energy and angle of separation. Successive approximations using the characteristics of QET and DIT and the recoil range distributions were made to obtain an angular distribution.

Equation (1) was used to estimate the c.m. angle interval subtended by each foil, assuming separation energy at all angles equal to the exit Coulomb barrier. ${ }^{3}$ A c.m. angular distribution was constructed from these intervals and the recoil range distribution QET and DIT components were graphically resolved, the former as a gaussian centered at a side angle, the latter as an exponential peaked at $0^{\circ}$. The foil-to-angle transformation was recalculated, this time with an angledependent separation energy; $i . e$. at each angle, the energy was taken to bc the weighted average of the optimal and Coulomb energies, according to the relative importance of QET and DIT, respectively, at this angle. The process was repeated until the recoil range distribution predicted came no closer to that measured, and the angle intervals remained the same. Figure 2 is the set of angular distributions derived for $\mathrm{Fm}$ isotopes from $111.6 \mathrm{MeV}{ }^{18} \mathrm{O}+{ }^{249} \mathrm{Cf}$. 
Except for ${ }^{252} \mathrm{Fm}$ from ${ }^{18} \mathrm{O}+{ }^{245} \mathrm{Cm}$, all observed products are from projectile stripping of one or two protons and some neutrons. Projectile-like particle measurements show that DIT is comparable in importance to QET in such reactions. However, there is no forwardpeaked component in any of the derived angular distributions. except for ${ }^{252} \mathrm{Fm}$ from $111.5 \mathrm{MeV}{ }^{18} \mathrm{O}+$ ${ }^{245} \mathrm{Cm}$. This can be interpreted as an effect of looking at target-like residues, which will be severely depleted by fission during de-excitation. DI'T is accompanied by higher excitation than QET, particularly at low bombardment energies, so it is reasonable to expect that QET is the predominant mechanism contributing to the formation of actinide target-like residues.

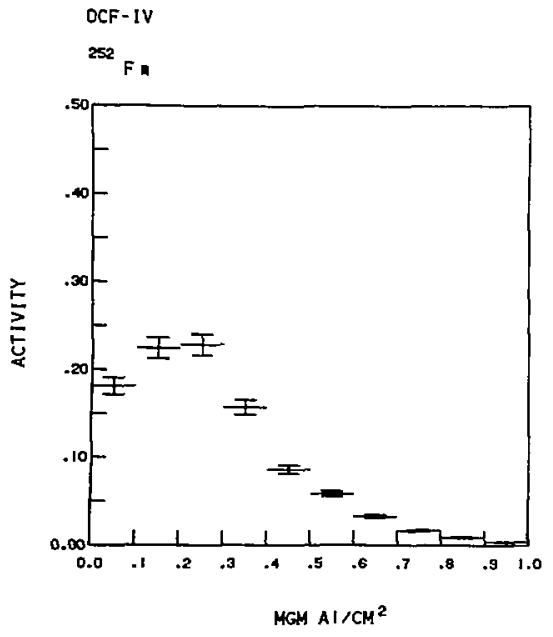

Fig. 1. Recoil range distribution of ${ }^{252} \mathrm{Fm}$ from 111.6 $\mathrm{MeV}{ }^{18} \mathrm{O}+{ }^{249} \mathrm{Cr}$.

XBL 823-9501

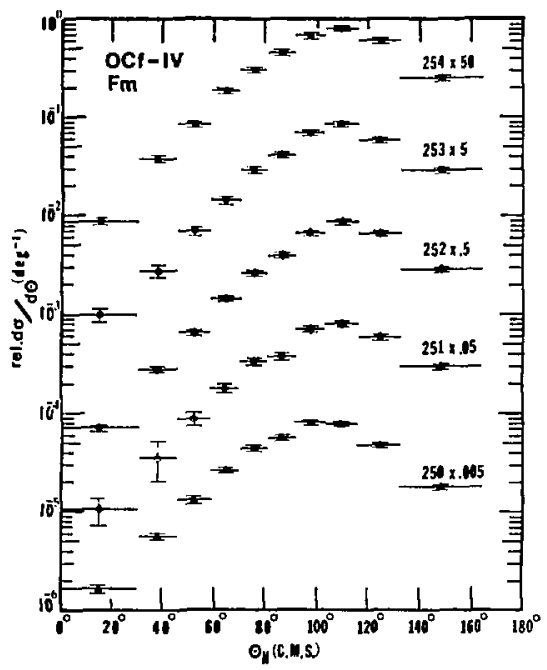

Fig. 2. Center-of-mass angular distributions of $\mathrm{Fm}$ isotopes for $111.6 \mathrm{MeV}{ }^{18} \mathrm{O}+{ }^{249} \mathrm{Cf}$ derived from recoil range distributions.

XBL $826-9542$

\section{References}

1. R.M. McFarland, Ph.D. Thesis, U.C. Berkeley, LBL-15027 (1982)

2. R.L. Hahn, P.F. Dittner, K.S. Toth, and O.L. Keller, Phys. Rev. C10 (1974) 1889

3. R.J. Otto, M.M. Fowler, D. Lee, and G.T. Seaborg, Phys. Rev. lett. 36 (1976) 135 


\title{
Excitation Functions for Production of Heavy Actinides from Interactions of ${ }^{18} \mathrm{O}$ with ${ }^{248} \mathrm{Cm}$ and ${ }^{249} \mathrm{Cr}$
}

\author{
D. Lee, K. Moody, M. Nurmia, G.T. Seaborg, H. von Gunten, ${ }^{*}$ and \\ D. Hoffmant
}

Work on the production of heavy actinides by the interaction of ${ }^{13} \mathrm{O}$ and other light heavy ions on actinide targets ${ }^{1}$ was continued in this period. We measured excitation functions for the production of isotopes of Bk through $\mathrm{Fm}$ in bombardments of ${ }^{248} \mathrm{Cm}$ with 97- to $122-\mathrm{MeV}{ }^{18} \mathrm{O}$ ions and of isotopes of $\mathrm{Bk}$ through $\mathrm{No}$ in bombardments of ${ }^{249} \mathrm{Cf}$ with $9 i_{-}$- to $150-\mathrm{MeV}{ }^{18} \mathrm{O}$ ions supplied by the 88-Inch cyclotron. The isotopes of interest were assayed using radiochemical techniques. An example for excitation functions of $\mathrm{Fm}$ isotopes produced in the reaction of ${ }^{18} \mathrm{O}+{ }^{249} \mathrm{Cf}$ is shown in Fig. 1 . The cross sections are largest for transfer of a few nucleons and decrease rapidly with the number of transferred nucleons. They show appreciable magnitudes even below the calculated Coulomb barrier.

The experimental results were compared with calculations based on a simple model taking into account ground state Q-values for the reactions and Coulomb barriers. These comparisons show that in general about half the kinetic energy of the projectile is transferred to products that survive fission. It was also demonstrated that simple calculations of this type may be helpful in selecting projectile-target systems and optimum energies for the production of as yet unknown heavy isotopes and elements.

The present work allows a comparison of cross sections for production of isotopes of Bk through $\mathrm{Fm}$ from reactions of ${ }^{18} \mathrm{O}$ with ${ }^{248} \mathrm{Cm}$ and ${ }^{249} \mathrm{Cf}$. The shapes of the mass-yield curves for both systems look rather similar. The reactions with ${ }^{248} \mathrm{Cm}$ are generally more favorable for the production of neutron-rich products, whereas those with ${ }^{249} \mathrm{Cf}$ should be used to produce lighter isotopes of an element (see Fig. 2). The cross sections and widths of the mass distributions of the same nuclear transfer, e.g., $(1 p, x n)$ or $(2 p, x n)$ are very similar for ${ }^{18} \mathrm{O}$ reactions with ${ }^{248} \mathrm{Cm}$ and ${ }^{249} \mathrm{Cf}$.

The reactions with lighter heavy ions (e.g., ${ }^{18} \mathrm{C}$ ) compares also very favorably with production cross sections obtained in reactions with very heavy projectiles (e.g, $\left.{ }^{238} \mathrm{U}\right)^{2}$; however, the widths of the mass distributions are about 1 AMU narrower for the lighter projectiles.

\section{Footnotes and References}

*Eidg. Institut für Reaktorforschung, Wurenlingen and Anorganisch-Chemisches Institut, University of Bern, Switzerland

†Isotope and Nuclear Chemistry Division, Los Alamos National Laboratory, Los Alamos, New Mexico 87575

1. D. Lee, et al., Phys. Rev. C25 (1982) 286

2. M. Schadel, et al., Phys. Rev. Lett. 48 (1982) 852

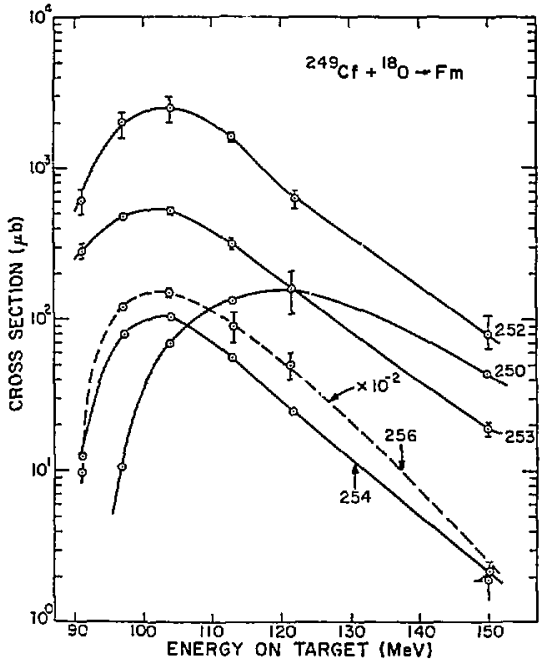

Fig. 1. Excitation functions for Fm isotopes produced in the bombardment of ${ }^{249} \mathrm{Cf}$ with ${ }^{18} \mathrm{O}$. Points are connected only as a visual aid.

XBL 8349099 


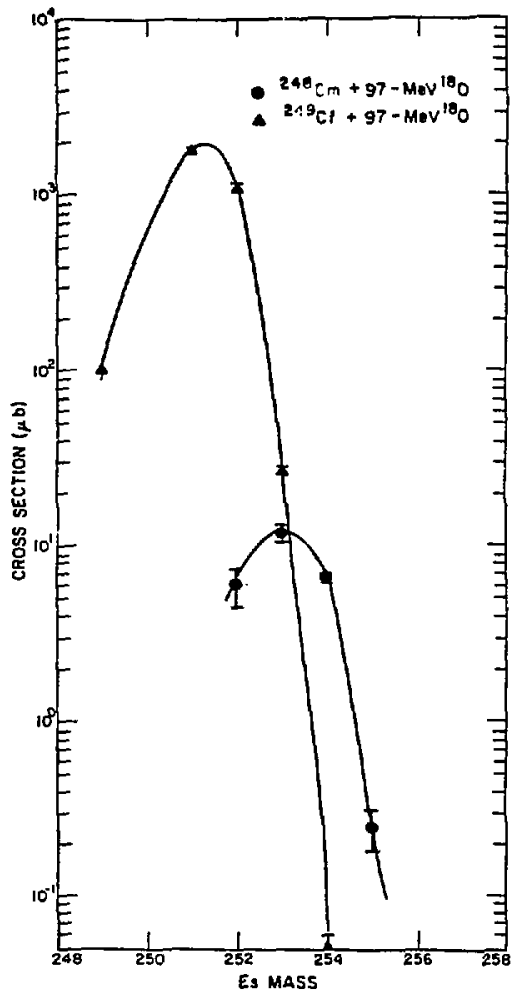

Fig. 2. Comparison of the mass distributions for Es isotopes from the bombardment of ${ }^{248} \mathrm{Cm}$ and ${ }^{249} \mathrm{Cf}$ with $97 \mathrm{MeV}{ }^{18} \mathrm{O}$. (Data for ${ }^{248} \mathrm{Cm}$ from this work and Ref. 1 were averaged.) Points are connected only as a visual aid.

XBL 8349100 


\title{
Actinide Production from the Reaction of ${ }^{129} \mathrm{Xe}$ with ${ }^{248} \mathrm{Cm}$
}

\author{
R.B. Welch, K.J. Mioody, D. Lee, K.E. Gregorich, \\ P. Wilmarth, and G.T. Seaborg
}

We have measured the production cross sections for some actinide nuclides formed in the reaction of ${ }^{129} \mathrm{Xe}$ with ${ }^{248} \mathrm{Cm}$ at a projectile energy between 715 and $785 \mathrm{MeV}$ (1.0 to 1.1 times the Coulomb barrier) in the target, to study the effect of using a more neutrondeficient projectile than those previously used by us in heavy-ion-induced reactions. These data are compared in Fig. 1 with the production cross sections for actinides from the similar réaction of ${ }^{136} \mathrm{Xe}$ with ${ }^{248} \mathrm{Cm}^{1}$ The cross sections for nuclides of a given element above the target $(Z>96)$ appear to be peaked about 2 mass units lighter for ${ }^{129} \mathrm{Xe}$ than for ${ }^{136} \mathrm{Xe}$.

$A^{129} \mathrm{Xe}$ beam was obtained by extraction of ${ }^{129} \mathrm{Xe}$ by the Abel injector of the SuperHILAC from a natural xenon source $\left(26.4 \%{ }^{129} \mathrm{Xe}\right)$ and accelerated to 1089 $\mathrm{MeV}$ by the SuperHILAC into our target system in $\mathrm{S}$ Cave. After passing through a $1.8 \mathrm{mg} / \mathrm{cm}^{2}$ havar window, $0.3 \mathrm{mg} / \mathrm{cm}^{2}$ nitrogen cooling gas, and $2.3 \mathrm{mg} / \mathrm{cm}^{2}$ beryllium target backing, the beam struck a 0.502 $\mathrm{mg} / \mathrm{cm}^{2}{ }^{248} \mathrm{Cm}$ target, present as $\mathrm{Cm}_{2} \mathrm{O}_{3}$. The energy on target, as measured by a $\mathrm{Si}(\mathrm{Au})$ surface barrier detector, was $750 \mathrm{MeV}$, with a FWHM of $70 \mathrm{MeV}$.

The irradiation consisted of nine hours of approximately 250 electrical nancamperes of ${ }^{129} \mathrm{Xe}^{+29}$ through the target. The reaction products were stopped in a gold catcher foil, and chemical procedures were used to create $\mathrm{Cf}$, Es, and $\mathrm{Fm}$ alpha sources for counting. The procedures used are the same as those used by Moody, et al., ${ }^{1}$ for ${ }^{136} \mathrm{Xe}$ on ${ }^{248} \mathrm{Cm}$.

Both of the ${ }^{129} \mathrm{Xe}$ and ${ }^{136} \mathrm{Xe}$ reactions were done at full energy of xenon out of the SuperHILAC and into the same target assembly and are approximately the same energy on target. As expected, the more neutron-deficient projectile $\left({ }^{129} \mathrm{Xe}\right.$ ) makes products that are more neutron deficient, in this case by about 2 mass units for a given $Z$. We intend to do another ${ }^{129} \mathrm{Xe}$ bombardment at the same energy to determine cross sections for neutron-deficient $\mathrm{CF}$, Es, and $\mathrm{Fm}$ isotopes, as well as any Bk and Am isotopes. We also intend to use different energies of ${ }^{129} \mathrm{Xe}$ and compare products with those from analogous ${ }^{136} \mathrm{Xe}$ reactions.

\section{Reference}

1. K.J. Moody, D. Lee, R. Welch, B.V. Jacak, RM. MáFarland, P.L. MeGaughey, M.N. Nurmia, M. Perry, G.T. Seaborg, RW. Lougheed, P.A Buisden, and E.K. Hulet, Nuclear Science Division Annual Report 1979-80, LBL-11588, p. 87.

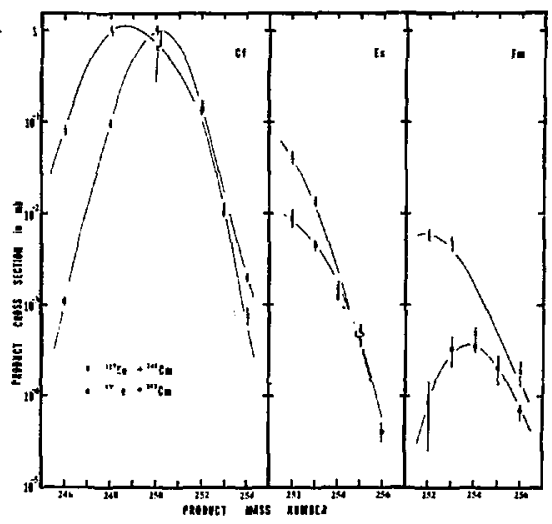

Fig. 1. Comparison of $\mathrm{Cf}, \mathrm{Es}$, and Fm product crcss sections from $750 \mathrm{MeV}{ }^{128} \mathrm{Xe}+{ }^{244} \mathrm{Cm}$ and ${ }^{136} \mathrm{Xe}+$ ${ }^{248} \mathrm{Cm}$.

XBL $8211-3355$ 


\title{
Preliminary Results from the Reaction of ${ }^{208} \mathrm{~Pb}$ with ${ }^{197} \mathrm{Au}$
}

\author{
M.D. Perrv, K.J. Moody, R.B. Welch, K.E. Gregorich, \\ P. Wilmarth, and G.T. Seaborg
}

We have measured the production cross sections for many of the nuclides near both the target and the projectile from the reaction of $8.19 \mathrm{MeV} / \mathrm{A}{ }^{200} \mathrm{~Pb}^{+39}$ ions with a thick target of ${ }^{197} \mathrm{Au}$. The irradiation was performed with as mach as 12 electrical nanoamperes d.c. of beam for approximately three hours. The thickness of the gold target was $49 \mathrm{mg} / \mathrm{cm}^{2}$, which is enough to stop both the beam particles and the deep-inelastic reaction products, but which would allow some fissionlike products to escape. This was desirable to see the lead- and gold-like reaction products better. Since ${ }^{200} \mathrm{~Pb}$ ions will make the reaction products of interest in reactions with eny isolation foil or catcher foil, the ${ }^{197}$ Au material was exposed directly to the beam; therefore any reaction products recoiling backward in the laboratory frame would be lost.

At the end of the irradiation, the whole ${ }^{197} \mathrm{Au}$ target was pulse-height analyzed for gamma rays vith a $\mathrm{Ge}$ (L) detector with a resolution of $1.8 \mathrm{keV}$ FWHM for the ${ }^{60} \mathrm{Co}$ lines. Spectra were taken for approximately one month following the irradiation. Program SAMPO was used to integrate the gamma peak areas, then decay curve analysis was performed to obtain tine cross sections shown in Fig. 1. Corrections for feeding by beta decay are not yet complete. The effective target thickness used in the cross section calculations is the amount of gold necessary to degrade the ${ }^{208} \mathrm{~Pb}$ energy down to the nominal Coulomb barrier, about 14 $\mathrm{mg} / \mathrm{cm}^{2}$.

Many more nuclides were observed than are shown in Fig. 1 because of the lack of known absolute gamma-ray intensities for many of the nuclides in this region. For example, platinum isotopes as neutron deficient as ${ }^{166} \mathrm{Pt}$ were observed for which cross sections could not be determined. There is a similar problem in the light lead and bismuth isotopes.

Both halves of several isomer pairs were observed and their isomeric cross section ratios are listed in Table I. Those products that are formed by simple nucleon transfer seem to populate the ground state in preference to the isomer, while those arising from "deeper" collisions favor the isomeric state.
Table I: Cross section ratios for isomeric states formed in the reaction of ${ }^{208} \mathrm{~Pb}+{ }^{197} \mathrm{Au}$. Included are only those isomer pairs which are shielded or whose parents do not feed them significantly.

\begin{tabular}{lll}
\hline Isomer Pair & Observed Ratio, $\sigma_{\mathrm{v}} / \sigma_{\mathrm{m}}$ \\
${ }^{197} \mathrm{Hg}$ & & $2.5 \pm 1.6$ \\
${ }^{197} \mathrm{Pt}$ & & $0.5 \pm 0.2$ \\
${ }^{196} \mathrm{Au}$ & & $3.5 \pm 1.9$ \\
${ }^{198} \mathrm{Au}$ & & $9.1 \pm 3.0$ \\
${ }^{198} \mathrm{TI}$ & $0.8 \pm 0.3$ \\
\hline
\end{tabular}

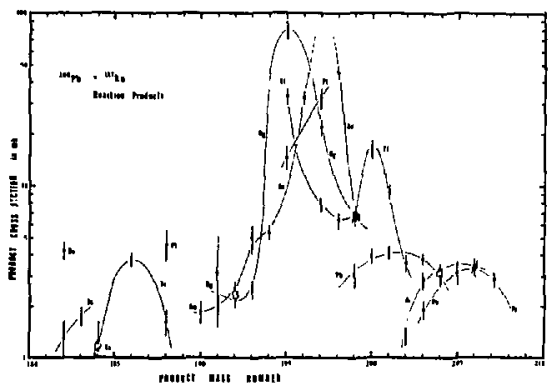

Fig. 1. Reaction product cross sections from ${ }^{208} \mathrm{~Pb}+$ ${ }^{197} \mathrm{Au}$. Not all corrections for feeding during the bombardment have been made. Connecting lines serve merely to guide the eye.

XBL $8211-3356$ 


\title{
Relativistic Energies: Spectators
}

\section{The Breakup of Spectator Residues in Relativistic Nuclear Collisions*}

\author{
A.I. Warwick, H.H. Gutbrod, H.G. Ritter, H. Stelzer, F. Weik,
}

H.H. Wieman, S.B. Kaufman, E.P. Steinberg, and B.D. Wilkinst

Based on the results of a multi-detector experiment, we develop a comprchensive description of the breakup of the excited residue of a Au nucleus after bombardment by relativistic protons, helium, and neon projectiles. We have measured the spectra of the slow fragments $(10<\mathrm{A}<150)$ and the multiplicities of the charged particles emitted from the same event. These multiplicities fall into two categories: the fast, light particles from the primary interaction zone and the slower, heavier charged particles $(2<Z<12)$ from the target residue. We separate the contribution of fission to the yield of intermediate mass fragments fror a more violent spallation mechanism, which produces fragments across the full range of measured masses. ${ }^{1}$

We find, at a fixed projectile energy, that the violence of the initial stage of the collision, as measured by the multiplicity of fast particles emitted, is positively correlated to the excitation of the residue, measured by the spectra of the slow fragments produced.
Slow fragments of a particular mass always exhibit the same energy spectra, essentially independent of the projectile mass and energy. Thus the mass of the fragment produced indicates the excitation of the residue, independently of the fast stage of the collision, which increases dramatically in its violence as the projectile mass or energy is increased. The lighter slow fragments exhibit spectra indicative of more bighly excited residues.

In events producing lighter fragments $(A \simeq 30$ ) at the highest projectile energy ( $42 \mathrm{GeV}$ ) we can account for the entire mass of the event in our measured multiplicities, giving a complete picture of the typical breakup configuration (Table 1). This information enables us to calculate the effect of the late stage Coulomb interaction on the energy spectra of the $A \simeq$ 30 fragments and we find these spectra to be consistent with an initial residue temperature of $20 \mathrm{MeV}$. This value is consistent with that needed to reproduce the measured light fragment mass-yield curve in the statistical calculations of Fai and Randrup. ${ }^{2}$

Table 1

\begin{tabular}{|c|c|c|c|c|}
\hline \multicolumn{5}{|c|}{$\begin{array}{l}\text { Mass balance for } \mathrm{Ne}+\Delta u \text { collisions leading to } \\
\text { the Emission of a Fragment of } \mathrm{A}=20-40\end{array}$} \\
\hline \multirow[b]{2}{*}{ Product } & \multicolumn{2}{|c|}{$5 \mathrm{GeV} \mathrm{Ne}+\mathrm{Au}$} & \multicolumn{2}{|c|}{ 42. GeV Ne + Au } \\
\hline & $\begin{array}{c}\text { Mean } \\
\text { Multiplicity }\end{array}$ & $\begin{array}{c}\text { Approximate } \\
\text { Number of } \\
\text { Nucleons }\end{array}$ & $\begin{array}{c}\text { Mean } \\
\text { Multiplicity }\end{array}$ & $\begin{array}{l}\text { Approximate } \\
\text { Number of } \\
\text { Nucleons }\end{array}$ \\
\hline Trigger fragment & 1 & 30 & 1 & 30 \\
\hline Fast charged particles & 13 & 17 & 42 & 50 \\
\hline Fast neutrons & 29 & 29 & 60 & 60 \\
\hline $\begin{array}{l}\text { Slow charged fragments } \\
(\mathrm{Z}=2-27)\end{array}$ & 6 & 45 & 5.6 & 39 \\
\hline Slow protons & 6 & 6 & 6 & 6 \\
\hline Slow neutrons & 24 & 24 & 24 & 24 \\
\hline Total & & 151 & & 209 \\
\hline
\end{tabular}


tCollaboration of Lawrence Berkeley Laboratory, Argonne National Laboratory, and Gesellschaft für Schwerionenforschung, Darmstadt 1719

2. G. Fai and J. Randrup, Nucl. Phys. A381 (1982) 557

\title{
Heary Fragment Yields in the Interaction of $28 \mathrm{GeV}$ Protons with ${ }^{238} \mathrm{U}$
}

\author{
B.V. Jacak, W. Loveland, D.J. Morrissey, P.L. McGaughey, and G.T. Seaborg
}

During the past year, we have completed the analysis of a study of the production of heavy target fragments in the interaction of $28 \mathrm{GeV}$ protons with ${ }^{23} \mathrm{U}$. The details of the data analysis are summarized elsewhere. ${ }^{1}$ The deduced isobaric yields are shown in Fig. 1 along with the previously published work of Chu, et al. ${ }^{2}$ The measured cumulative yield radionuclide production cross sections for species with $172<A<207$ range, in general, from 1 to $10 \mathrm{mb}$, indicating without a doubt that these fragments are produced in significant yields in the interaction of $28 \mathrm{GeV} p$ with ${ }^{238} \mathrm{U}$. The charge dispersions for the species with $182<A<207$ are moderately well defined and thus the isobaric yields derived from them (Fig. 1) of 5-10 mb for fragments with $180<A<200$ appear to be reasonably well established, although they do exceed significantly the

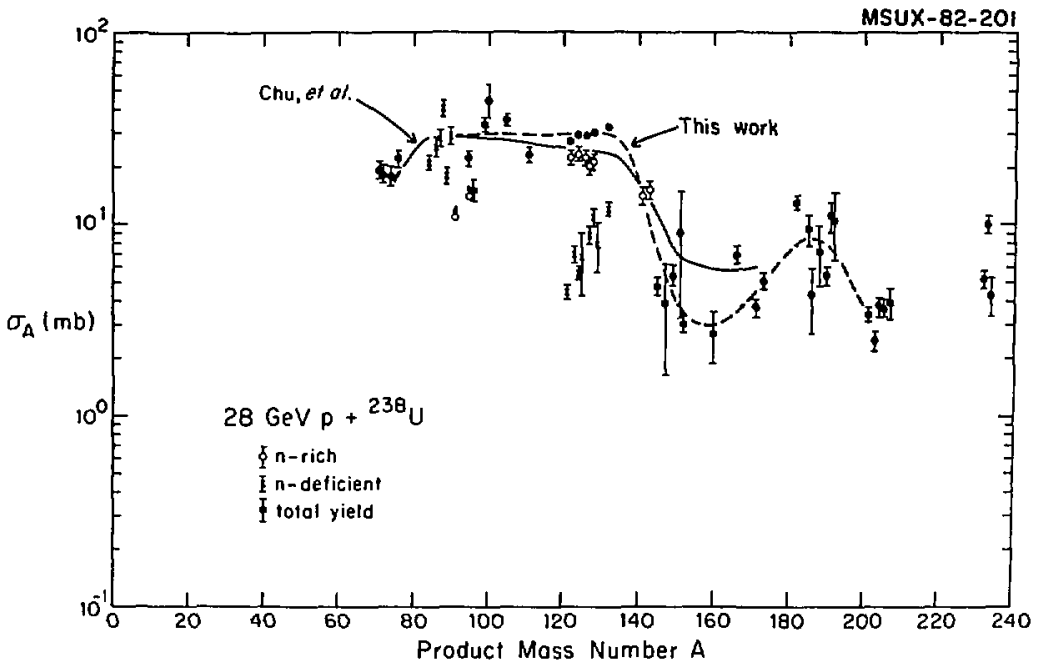

Fig. 1. The target fragment mass distribution, $\sigma(\mathrm{A})$, obtained for this work for interaction of $28 \mathrm{GeV}$ protons with $\mathrm{U}$ is shown along with the previous radiochemical data. Filled circles represent the total isobaric yield while the open circles and crosses represent the isobaric yield of the n-rich and n-deficient species at a given $A$ value. The dashed line is to guide the eye through the data of this work. 
measured radionuclide production cross sections. The production of these heavy fragments is, therefore, a process with a total cross section of $\sim 250 \mathrm{mb}$. In any case, the yields of these fragments are significantly higher than would be expected for fission fragments based on the shapes of the single fragment mass yields.

We have attempted to pursue the question of the origin of the heavy $(160<A<210$ ) fragments in a didactic manner. We have assumed an initial distribution of target fragment mass, charge, and excitation energy as might be appropriate for the first, fast stage of the $\mathrm{p}-\mathrm{U}$ collision and then attempted to compute the final product distribution after de-excitation of these primary residues. In doing so, we can show the types of events that could lead to products in the $160<\mathrm{A}<$ 210 region. Figure 2 shows the shape of the assumed fragment distribution from the $28 \mathrm{GeV} \mathrm{p}+{ }^{238} \mathrm{U}$ reaction after the first, fast step of the reaction.

The de-excitation of the members of this initial fragment distribution was calculated using the EVA3 code of McGaughey and Morrissey. ${ }^{3}$ In Fig. 2 we show those members of the initial fragment population that survived to form fragments with $160<\mathrm{A}<210$. As one can see from examining Fig. 2, these survivors are generally uniformly distributed in mass number from 215 to 235. Because of the steeply falling character of initial distribution with decreasing mass number, this means that the lower the mass number of the initial fragment from the fast, first stage of the reaction, the greater its probability of surviving to become a heavy fragment despite its high excitation energy.

Deexcitation chains that lead to heavy fragments generally start, especially for primary fragments of high $Z$ and $A$, with copious charged particle emission (to escape the region of high fissionability) followed by long chains of successive neutron emission (by nuclei that are very n-rich as the result of charged particle emission). The average length of the de-excitation chain leading from the primary to the final heavy fragment distribution is $53 \mathrm{~A}$ units with $\sim 770 \mathrm{MeV}$ of excitation energy being removed.

\section{Footnotes and References}

"Present address: Department of Chemistry, Michigan State University, East Lansing, Michigan 48824

PPresent address: Radiation Center, Oregon State University, Corvallis, Oregon 97331

1. B.V. Jacak, W. Loveland, D.J. Morrissey, P.L. McGaughey, and G.T. Seaborg, Can. J. Chem. (to be published)
2. Y.Y. Chu, E.M. Franz, G. Friedlander, and P.J. Karol, Phys, Rev. C4 (1971) 2202

3. P.L. McGaughey and D.J. Morrissey (private communication) 1982

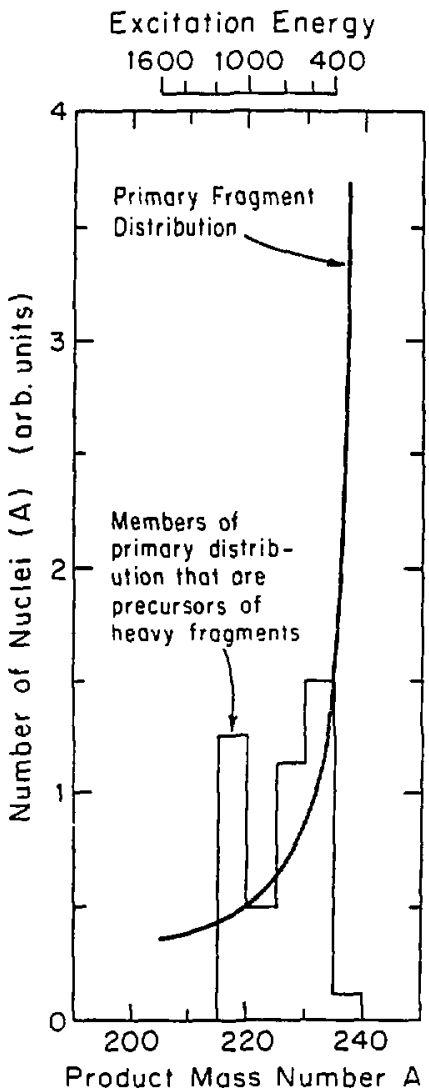

Fig. 2. The shape of the initial primary fragment distribution as a function of product mass number after the fast step of the reaction of $28 \mathrm{GeV} \mathrm{p}+{ }^{238} \mathrm{U}$. Also shown are those members of the initial distribution, which when de-excited produce final fragments with 155 $<\mathrm{A}<210$.

XBL 8349473 


\title{
Target Fragment Yields in the Interaction of $28 \mathrm{GeV}$ Protons with ${ }^{181} \mathrm{Ta}$
}

\author{
C. Oertel. P.L McGaughey. D.J. Morrissey, ${ }^{\dagger} W$. Loveland, ${ }^{\ddagger}$ and G.T. Seaborg
}

Using very similar techniques to those described previously, ${ }^{1}$ we identified and measured the yields of 47 different target fragment radionuclides from the interaction of $28 \mathrm{GeV}$ protons with ${ }^{181} \mathrm{Ta}$. The mass yield curve deduced from these data is shown in Fig. 1.

The comparison of the measured radionuclide yields in this work with that of Chu, et al., ${ }^{2}$ who measured, using a mass-separator, the yields of heavy rare earth nuclides from the reaction of $28 \mathrm{GeV}$ protons with ${ }^{181} \mathrm{Ta}$ is inconclusive. The yields of ${ }^{145} \mathrm{Eu}$ measured in both works are identical; however, the two yield values for the other radionuclide common to both measurements, ${ }^{149} \mathrm{Gd}$, differ by a factor of $\sim 2.4$, while the isobaric yield for $\mathrm{A}=147$ deduced in this work is $\sim 3 / 4$ that deduced by Chu, et al. The isobaric yield for $\mathrm{A}=$ 123 measured in this work is in good agreement with the previous work of Rudstam and Sorensen ${ }^{3}$ who studied the reaction of $18 \mathrm{GeV}$ protons with ${ }^{181} \mathrm{Ta}$. In a similar vein, we should note that the measured light fragment yields $\left({ }^{24} \mathrm{Na}-{ }^{52} \mathrm{Mn}\right)$ determined in this work are in good agreement with the data of Cole and Porile ${ }^{4}$ who studied the reaction of $400 \mathrm{GeV}$ protons with ${ }^{181} \mathrm{Ta}$.

It is interesting to compare the fragment yields measured in this work with the general systematics of light particle induced spallation yields as formulated by Rudstam. ${ }^{5}$ In Fig. 1, we show the isobaric yjeld distribution predicted by the Rudstam CDMD formula with $\sigma=1.967 \sigma_{1}$. One notes that the Rudstam formula predicts a mich steeper decrease of the isobaric yield as the fragment mass decreases than is seen experimentally. Presumably this is due to the fact that the parameters of the Rudstam formula were determined primarily by fitting lower energy data.

Since our primary research effort is directed toward studying nucleus-nucleus collisions, it is of interest to compare the present data for p-Ta collisions with those determined ${ }^{6}$ for heavy ion-Ta collisions. In Fig. 2 we compare the isobaric yield distributions from $\mathrm{p}-\mathrm{Ta}$ and heavy ion-Ta interactions. One is struck by the general similarity in shapes of the isobaric distributions for reactions induced by protons and heavy ions of the same total projectile kinetic energy, a fact previously noted. ${ }^{7-9}$ The total radioactive residue reaction cross section ( $A_{\text {re }}>40$ ) for the heavy-ion-induced reactions exceeds the same quantity for the protoninduced reactions by the ratio of the geometric cross sections, as expected. Enhanced yields of the lightest fragments are seen in the heavy ion reactions, as noted previously. 4,10

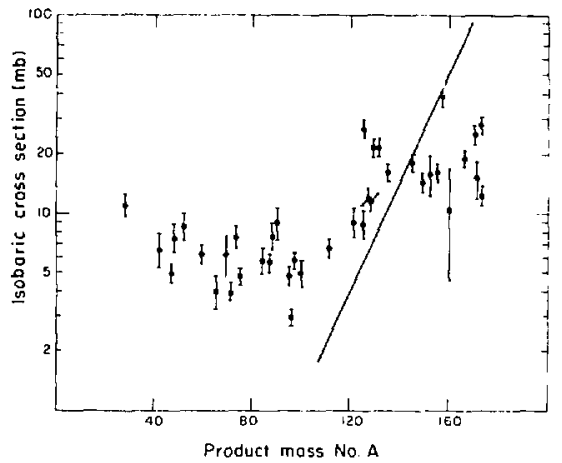

Fig, 1. Target fragment yield distribution from the interaction of $28 \mathrm{GeV}$ protons with ${ }^{181} \mathrm{Ta}$. The solid line shows the predictions of the Rudstam ${ }^{5}$ CDMD formula with $\sigma=1.967 \sigma_{1}$.

XBL 835-907

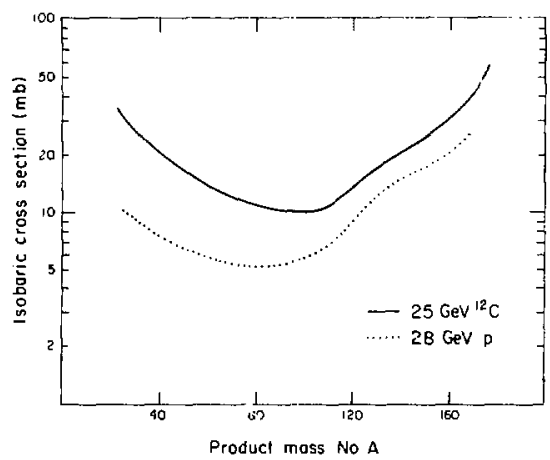

Fig. 2. Comparison of isobaric yield distributions from the fragmentation of ${ }^{181} \mathrm{Ta}$ by $25.2 \mathrm{GeV}{ }^{12} \mathrm{C}$ (ref. 6) and $28 \mathrm{GeV}$ protons.

XBL 835-905 
Footnotes and References

*Present address: Rockwell International, Rocky Flats, Colorado

†Present address: Department of Chemistry, Michigan State University, East Lansing, Michigan 48824

țresent address: Radiation Center, Oregon State University, Corvallis, Oregon 97331

1. D.J. Morrissey, W.D. Loveland, M. de SaintSimon, and G.T. Seaborg, Phys. Rev. C21 (1980) 1783

2. Y.Y, Chu, E.M. Franz, and G. Friedlander, Phys, Rev. ClO (1954) 156

3. G. Rudstam and G. Sorensen, J. Inorg. Nucl. Chem. 28 (1956) 771
4. G.D. Cole and N.T. Porile, Phys. Rev. C24 (1981) 2038

5. G. Rudstam, Z. Naturforsch 212 (1966) 1027

6. C.P. Oertel, M.S. Thesis, Oregon State University (1981) unpublished

7. J.B. Cumming, et al., Phys. Rev, C10 (1974) 739; Phys. Rev. C14 (1976) 1554; Phys. Rev. C17 (1978) 1632

8. S.B. Kaufman, E.P. Steinberg, B.D. Wilkins, and D.J. Henderson, Phys. Rev. C22 (1980) 1897

9. D.J. Morrissey, W. Loveland, and G.T. Seaborg, Z. Phys. A289 (1978) 123

10 N.T. Porile, G.D. Cole, and C.R. Rudy, Phys. Rev. C19 (1979) 2288

\title{
Au Target Fragmentation at Intermediate Energies
}

\author{
W. Loveland," K. Aleklett, ${ }^{\dagger}$ P.L. McGaughey, and G.T. Seaborg
}

We have completed a survey of the energy dependence of Au target fragmentation at projectile energies from 7 to $2100 \mathrm{MeV} / \mathrm{u}$. If we consider those $\mathrm{Au}$ target fragments with $\mathrm{A}=80-100$ as arising from a binary fission process, we can derive a measure of the dependence of the transferred momentum, $p_{r}^{\text {trans }}$, on projectile energy for the events leading to the formation of these fragments. These deduced data are shown in Fig. 1 . The results for the energy region of $18-84 \mathrm{MeV} / \mathrm{u}$ are in good agreement with the data of Galin, et al. ${ }^{1}$ We can also deduce the primary momentum transfer, $\mathrm{pl}_{\mathrm{ins}}^{\text {trans }}$ to the target residue for spallation events by assuming that events with $A>140$ result from a spallation process. These data are also shown in Fig. 1. Examining these data we can see that for a given nucleus the exit channel of the reaction affects the maximum momentum that can be transferred.

Considerable interest has been expressed in understandi.1g the mechanism(s) responsible for the production of light $(A<60)$ fragments in $\mathrm{Au}$ target fragmentation. The Purdue-Fermilab collaboration ${ }^{2}$ has pointed out that the yields of the light fragments in high-energy proton-nucleus interactions follow a power law dependence possibly indicative of a vapor-liquid phase transition. ${ }^{3}$ Warwick, et al. ${ }^{4}$ have shown such 1 power law dependence describes the yields of fragments with $\mathrm{A}<24$ in the interaction of $2.1 \mathrm{GeV} / \mathrm{u}$ heavy ions with $\mathrm{Au}$ (an observation that can be extended to $\mathrm{A}=$
60 using the radiochemical data of Kaufman, et al. ${ }^{5}$ ). In Fig. 2 we show the excitation functions and projectile energy dependence of the fragment kinetic energies for typical $A=40-50$ fragments from Au target fragmentation. The excitation functions are quite broad and the kinetic energies of the fragments vary greatly over the projectile energy region shown consistent with the idea of more than one mechanism contributing to the production of these fragments.

While single particle inclusive data such as that presented here are not sufficient to establish the production mechanism(s) for these light fragments, one can test some of the proposed mechanism(s) for consistency with the single particle inclusive data. For example, it is difficult to reconcile the proposed vapor-liquid phase transition that is said to account for the light fragment yields in high-energy collisions with the single particle inclusive fragment spatial distributions that show the forward to backward ratio $(\mathrm{F} / \mathrm{B})<1$ for these fragments at high energies. If the nucleus were to absorb enough energy to become "gasified" one would expect a significant forward velocity for the system (F/B > 1).

In the same vein, one can note that Lynen, et al. ${ }^{6}$ have shown that the production of target fragments with $10<\mathrm{A}<40$ in the interaction of $86 \mathrm{MeV} / \mathrm{u}^{12} \mathrm{C}$ with

${ }^{197} \mathrm{Au}$ is a binary process involving a target matter source for these fragments. The measured fragment kinetic energy is consistent with an evaporation 
mechanism for the production of these fragments.

\section{Footnotes and References}

-Present adidress: Radiation Center, Oregon State University, Corvallis, Oregon 97331

†Present address: The Studsvik Science Research Laboratory, S-61182, Nykoping, Sweden

I. J. Galin, et al., Phys. Rev. Lett. 48 (1982) 1787

2. J.E. Finn, et al.,Phys. Rev. Lett. 49 (1982) 1321

3. M.E. Fisher, Physics 3 (1967) 255

4. A.I. Warwick, et al, Phys. Rev, C27 (198.3) 1083

5. S.B. Kaufman, et al., Phys. Rev. C22 (1980) 1897

6. U. Lynen, et al., Nucl. Phys. A387 (1982) 129c

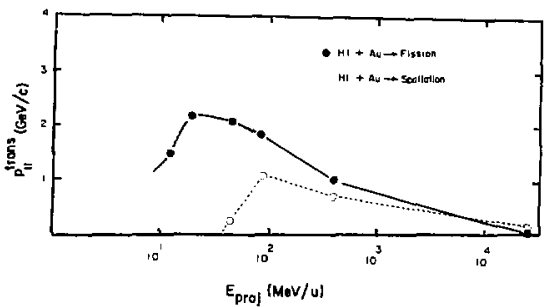

Fig. 1. Variation with projectile energy of the linear momentum transfer to the Au nucleus for two different reaction channels in the reaction of heavy ions with $\mathrm{Au}$.

XBL 834-9092

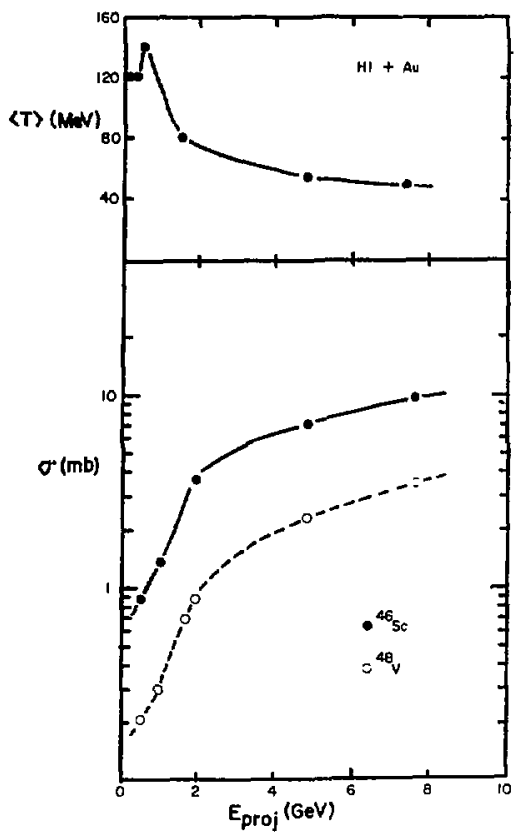

Fig. 2. Dependence on the projectile energy of light fragment a) kinetic energies and b) production cross sections for typical light fragments produced in the interaction of heavy ions with ${ }^{197} \mathrm{Au}$.

XBL 8349093 


\title{
Ta Target Fragmentation at Intermediate Energies
}

\author{
P. Johnson, W. Loveland,* P.L. McGaughey. \\ K. Aleklett' and G.T. Seaborg
}

de Saint-Simon, et al., ${ }^{1}$ have used mass spectrometric techniques to study the yields of alkali metals in the reaction of 13,27 and $77 \mathrm{MeV} / \mathrm{u}{ }^{12} \mathrm{C}$ with ${ }^{183} \mathrm{Ta}$. They find that as the projectile energy increases, the mixima of the alkali metal isotopic distributions shift toward more n-deficient nuclides, which can be interpreted as indicating an increasing excitation energy of the precursors of these products. Surprisingly, they also find that the interaction of $24 \mathrm{GeV}$ protons with ${ }^{181} \mathrm{Ta}$ leads to more $\mathrm{n}$-deficient alkali metal distributions than the interaction of $77 \mathrm{MeV} / \mathrm{u}^{12} \mathrm{C}$ with ${ }^{181} \mathrm{Ta}$, which, if we use the above reasoning, leads to an appar at contradiction of the general trend seen in other heavy elements in which, for projectile energies above $1-3 \mathrm{GeV}$, the excitation energy and momentum imparted to the target nucleus decreases sharply with increasing projectile energy.

We have measured the target fragment recoil properties in the interaction of 75,80 , and $85 \mathrm{MeV} / \mathrm{u}{ }^{12} \mathrm{C}$ and $107 \mathrm{MeV} / \mathrm{u}^{16} \mathrm{O}$ with ${ }^{181} \mathrm{Ta}$ using the thick target catcher recoil technique. Using the formalism described previously, ${ }^{2}$ we have converted these fragment recoil parameters into momenta and energies. In Fig. 1 we compare the values of the fragment longitudinal velocity, $\beta_{1}$, after the first step of the reaction with the kiretic energy $<E>$ of the fragments in the system moving with velocity $\theta_{\text {f }}$ for common fragments from the interaction of $85 \mathrm{MeV} / \mathrm{u}^{12} \mathrm{C}$ and $400 \mathrm{MeV} / \mathrm{u}{ }^{20} \mathrm{Ne}$ with ${ }^{181} \mathrm{Ta}$. In the lower energy reaction, the fragment longitudinal velocities due to the first step of the reaction are $\sim 2 x$ larger while the energies imparted to the fragments during the second step of the reaction are $\sim 3 x$ larger.

Using the simple relationship ${ }^{3}$

$$
\mathrm{E}^{*} \simeq \mathrm{kE}_{\mathrm{CN}}^{*} \frac{\mathrm{p}_{\mathbf{I}}}{\mathrm{PCN}_{\mathrm{N}}}
$$

that has proven useful in analyzing p-nucleus collisions, we can compare the values of $\mathrm{E}^{\prime}$, the fragment excitation energy, for fragments produced in the reaction of $85 \mathrm{MeV} / \mathrm{u}{ }^{12} \mathrm{C}$ and $19 \mathrm{GeV}$ protons ${ }^{4}$ with ${ }^{181} \mathrm{Ta}$. For the $\mathrm{Rb}$ isotopes studied by de Saint-Simon ${ }^{1}$ we calculate that $\mathrm{E}^{*} 86 \mathrm{MeV} / \mathrm{E}^{*} 19 \mathrm{GeV} \simeq 0.7-0.8$, leading to an estimate that the precursor of the $R b$ isotopes has an excitation energy that is some $30 \mathrm{MeV}$ higher in the 19 $\mathrm{GeV}$ reaction. The observed shift in the $\mathrm{Rb}$ isotopic distribution is $\sim 2 \mathrm{~A}$ units when the incident projectile changes from $77 \mathrm{MeV} / \mathrm{u}{ }^{12} \mathrm{C}$ to $19 \mathrm{GeV}$ protons in general agreement with the kinematic data.

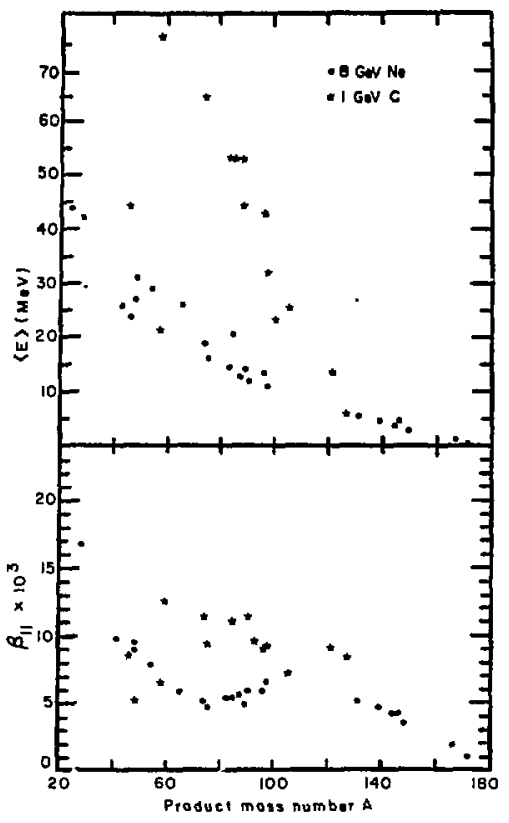

Fig. 1. Comparison of target fragment kinetic properties deduced from recoil data using the two-step yector model for the interaction of $85 \mathrm{MeV} / \mathrm{u}{ }^{12} \mathrm{C}$ and 400 $\mathrm{MeV} / \mathrm{u}^{20 \mathrm{Ne}}$ with ${ }^{181} \mathrm{Ta}$.

XBL 8349094

\section{Footnotes and References}

* Present address: Radiation Center, Oregon State University, Corvallis, Oregon 97331

†Present address: The Studsvik Science Research Laboratory, S-61182, Nykoping, Sweden 
1. M. de Saint-Simon. S. Haan. G. Audil. A. Coc, M. Epherre, P. Guimbal, M. Langevin, A.C. Mueller, C. Thibault, and F. Touchard, Phys. Rev. C26 (1982) 2447

2. W. Loveland, D.J. Morrissey, K. Aleklett, G.T. Seaborg, S.B. Kaufman, E.P. Steinberg, B.D.
Wilkins, J.B. Cumming, P.E. Haustein, and H.C. Hseuh, Phys. Rev. C23 (1981) 253

3. N.T. Porile, Phys. Rev. 120 ( 1960$) 572$

4. U. Trabitzsch and K. Bachmann, Radiochemica Acta 16 (1974) 129

\title{
Transparency in Heavy Ion-Heavy Nucleus Nuclear Collisions
}

\author{
W. Loveland, ' K. Alekiet, ${ }^{\dagger}$ P.L. McGaughey, and G.T. \\ Seaborg
}

We have reported previously the results of our investigation of transparency in heavy ion-heavy nucleus nuclear collisions. Using the total radioactive residue production cross section as a lower limit to the total reaction cross section, we were able to conclude that these lower limit total reaction cross sections (for the ${ }^{20} \mathrm{Ne}+{ }^{181} \mathrm{Ta}$ reaction) remain invariant with projectile energy from 20 to $2100 \mathrm{MeV} / \mathrm{u}$ in agreement with predictions of DeVries and Peng (see Fig. 1 filled circles). In explanation of these data, we noted that for a beavy nucleus such as ${ }^{181} \mathrm{Ta}$, transparency effects associated with nuclear surface phenomena would not be expected to be large.

We were quite surprised, therefore, to read the report of Buenerd, et al., ${ }^{2}$ that in a study of the elastic scattering of $86 \mathrm{MeV} / \mathrm{u}{ }^{12} \mathrm{C}$ from ${ }^{208} \mathrm{~Pb}$, a transparency of $42 \%$ in the total reaction cross section was deduced from an optical model analysis of the data. We therefore used the measured fragment yields for the interaction of 45 and $84 \mathrm{MeV} / \mathrm{u}^{12} \mathrm{C}$ with ${ }^{197} \mathrm{Au}$ to deduce isobaric yields for these reactions, and, using the techniques described previously, ${ }^{1}$ to deduce lower limits for the total reaction cross section for projectiles of these energies interacting with heavy targets. Out results are shown as open circles in Fig. 1. (We have assumed that $\sigma_{R} \propto\left(A_{p}^{1 / 3}+A_{4}^{1 / 3}\right)^{2}$ to scale the results for the ${ }^{12} \mathrm{C}+{ }^{197} \mathrm{Au}$ reaction to the ${ }^{20} \mathrm{Ne}+{ }^{181} \mathrm{Ta}$ reaction.) No indication is seen for an unusual transparency in these lower limit cross sections at the intermediate energies. Since the "lower limit" cross sections are $\sim 80 \%$ of the geometrical cross section, it seems clear that the portion of the total reaction cross section not measured by the radioactive residue cross section (inelastic scattering, multiple fragment breakup, etc.) are not the cause of the discrepancy between the measurements.

\section{Footnotes and References}

*Present acidress: Radiation Center, Oregon State University, Corvallis, Oregon 97331

tPresent address: The Studsvik Science Research Laboratory, S-61182, Nykoping, Sweden

1. W. Loveland, et al., Oregon State University Nuclear Chemistry Annual Report 1980 and 1981

2. M. Buenerd, et al., Phys. Lett. 102B (1981) 242

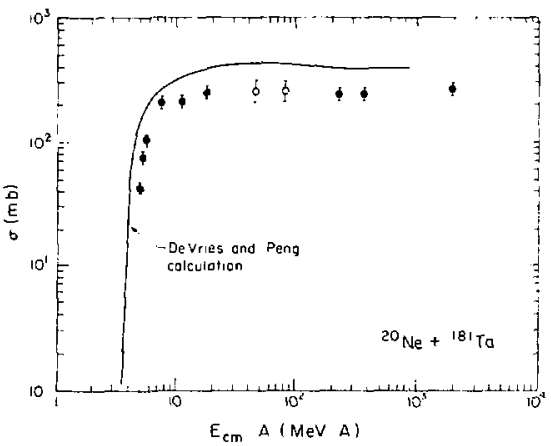

Fig. 1. The dependence of the total reaction fission for the ${ }^{20} \mathrm{Ne}+{ }^{18} \mathrm{Ta}$ reaction on projectile energy. The filled circles represent the data of ref. 1 , the open circles this work.

XBL 834-906 


\title{
Target Fragment Angular Distributions in the Reaction of $86 \mathrm{MeV} / \mathrm{u}$ ${ }^{12} \mathrm{C}$ with ${ }^{197} \mathrm{Au}$
}

\author{
R.H. Kraus, Jr.." W. Loveland, * P.L. McGaughey. K. \\ Aleklett, ${ }^{\dagger}$ Y. Morita, and G.T. Seaborg
}

Using the facilities of the CERN SC synchrocyclotron, we have measured complete fragment angular distributions $\left(0-180^{\circ}\right)$ for 76 different fragments produced in the interaction of $86 \mathrm{MeV} / \mathrm{u}{ }^{12} \mathrm{C}$ with ${ }^{197} \mathrm{Au}$. Angular distributions for eight typical fragments from this reaction are shown in Fig. 1. No corrections have been made to these data for the finite beam spot size, the finite angular resolution of the recoil catchers, or the effect of scattering or stopping of the fragments in the $\sim 225 \mu \mathrm{g} / \mathrm{cm}^{2}$ Au target.

Previously we have measured ${ }^{1}$ the recoil properties of the fragments from the reaction of $84 \mathrm{MeV} / \mathrm{u}$ ${ }^{12} \mathrm{C}$ with ${ }^{197} \mathrm{Au}$ and had analyzed them in terms of the two-step vector model. We can use the angular distribution data to test the validity of this simple model. In Fig. 1, we compare the measured angular distributions

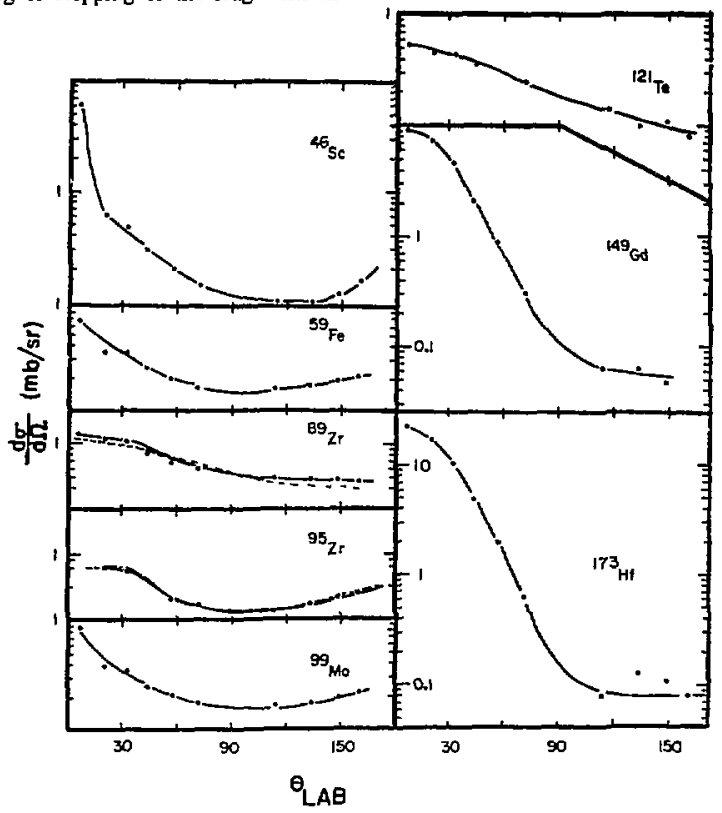

Fig. 1. Typical fragment angular distributions from the interaction of $86 \mathrm{MeV} / \mathrm{u}^{12} \mathrm{C}$ with ${ }^{197} \mathrm{Au}$. The experimental data are shown as filled circles with solid lines to guide the eyc through the data while calculations of the fragment distributions based on the two-step vector model and previously measured fragment recoil data are shown as dashed lines.

XBL 8349095 
for the medium mass fragments ${ }^{89} \mathrm{Zr}$ and ${ }^{99} \mathrm{Zr}$ with calculations of these angular distributions based on the two-step vector model and the previously measured recoil data. The agreement between the data and the calculations seems quite satisfactory. Comparison of the angular distributions of ${ }^{89} \mathrm{Zr}$ and ${ }^{95} \mathrm{Zr}$ shows that the known dectease in $F / B$ as the iragment $N / Z$ increases is due not simply to a lessening in the forward-peaked character of the distribution but to a change in the shape of the distribution. The backward peaks in these distributions can be understood in terms of a significant anisotropy introduced in the second step of the reaction overcoming a weak forward kick given the fragment in the first step of the reaction. The ${ }^{a 9} \mathrm{Zr}$ and ${ }^{95} \mathrm{Zr}$ angular distributions might typify the high and low multiplicity components, respectively, of the mass 80-100 fragments (similar to those observed by Warwick et al. ${ }^{2}$ ).

The heavier fragment $(A>145)$ angular distributions show extremely large anisotropies. However, calculations based on the fragment recoil properties measured previously ${ }^{1}$ would indicate that significant absorption of the fragments moving sidewise and back- ward occurs and that this phenomenon may have contributed to observed anisotropies. To check on this possibility further and to define it better quantitatively, we have repeated our measurement of the fragment angular distributions for the reaction of $86 \mathrm{MeV} / \mathrm{u}{ }^{12} \mathrm{C}$ with ${ }^{197}$ Au using a thinner Au target, i.e., one of thickness $\sim 90 \mu \mathrm{g} / \mathrm{cm}^{2}$. These data are being analyzed. The light fragment $(A<60)$ angular distributions show unusual forward and backward peaking that cannot be accounted for within the simple two-step vector model.

\section{Footnotes and References}

*Present address: Radiation Center, Oregon State University, Corvallis, Oregon 97331

†Present address: The Studsvik Science Research Laboratory, S-61 182, Nykoping, Sweden

1. W. Loveland, K. Aleklett, P.L. McGaughey, K.J. Moody, RM. McFarland, and G.T. Seaborg, Lawrence Berkeley Laboratory Report LBL-14177

2. A. Warwick, et al., Phys. Rev. C27 (1983) 1083

\title{
Target Fragment Angular Distributions in the Interaction of $2.1 \mathrm{GeV} / \mathrm{u}$
}

\section{${ }^{12} \mathrm{C}$ with ${ }^{197} \mathrm{Au}$ and ${ }^{238} \mathrm{U}$}

\author{
Y. Morita, W. Loveland, * and G.T. eaborg
}

Previous attempts ${ }^{2}$ to measure the target fragment angular distributions from the fragmentation of ${ }^{197} \mathrm{Au}$ and ${ }^{238} \mathrm{U}$ by energetic heavy ions have shown that it is possible, albeit marginal, to make such measurements. The observed fragment anisotropes and the variation of this anisotropy with fragment mass number for the interaction of 3 and $12 \mathrm{GeV}{ }^{12} \mathrm{C}$ with ${ }^{197} \mathrm{Au}$ and ${ }^{238} \mathrm{U}$ are in general agreement ${ }^{1}$ with calculations based on a two-step vector model treatment of thick target-thick catcher recoil data. In the interaction of $2.1 \mathrm{GeV} / \mathrm{u}$ ${ }^{12} \mathrm{C}$ with these same heavy targets, it was found ${ }^{2,3}$ that the crude measure of the fragment angular distribution, the F/B ratio, is near unity for many fragments, indicating either isotropy or sidewise-peaked distributions or, in general, symmetry about $90^{\circ}$ in the laboratory system. We thougr it would be useful to try to resolve these ambiguities ' another attempt at measuring fragment angular distributions, i.e., for the interaction of $2.1 \mathrm{GeV} / \mathrm{u}{ }^{12} \mathrm{C}$ with ${ }^{197} \mathrm{Au}$ and ${ }^{238} \mathrm{U}$.
The measured fragment distributions are shown in Figs. 1 and 2. The fragment angular distributions shown in Fig. 1 for the interaction of $2.1 \mathrm{GeV} / \mathrm{u}{ }^{12} \mathrm{C}$ with ${ }^{197} \mathrm{Au}$ are very similar to those observed for the reaction of $1.0 \mathrm{GeV} / \mathrm{u}{ }^{12} \mathrm{C}$ with ${ }^{197} \mathrm{Au}$. A similar situation occurs when comparing the fragment angular distributions from the interaction of $1.0 \mathrm{GeV} / \mathrm{u}$ and 2.1 $\mathrm{GeV} / \mathrm{u}^{12} \mathrm{C}$ with ${ }^{238} \mathrm{U}$ although the ${ }^{97} \mathrm{Zr}$ angular distribution is more forward peaked at the higher projectile energy. This overall similarity is somewhat puzzling, especially for the ${ }^{12} \mathrm{C}+\mathrm{U}$ reaction where recoil data ${ }^{3}$ indicate a decrease in the F/B ratios as the projectile energy increases from 1.0 to $2.1 \mathrm{Gev} / \mathrm{u}$. Furthermore, the recoil properties of the two nuclides ${ }^{97} \mathrm{Zr}$ and ${ }^{99} \mathrm{Mo}$ produced in the interaction of $2.1 \mathrm{GeV} / \mathrm{u}{ }^{12} \mathrm{C}$ with ${ }^{238} \mathrm{U}$ are virtually identical ${ }^{3}$ yet the measured angular distributions greatly differ. Resolution of this matter would seem to involve the measurement of the full $\left(0-180^{\circ}\right)$ 
angular distributions of these fission fragments.

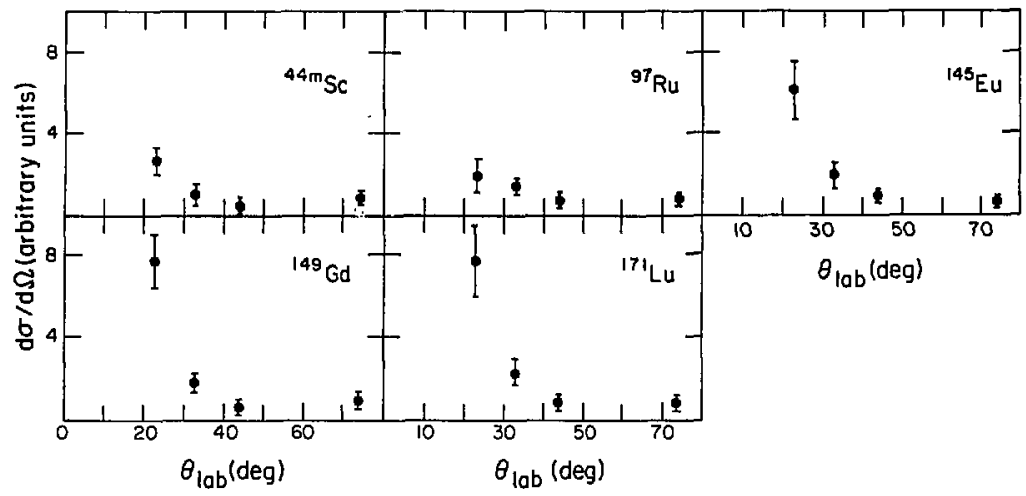

Fig. 1. Target fragment angular distributions from the interaction of $25.0 \mathrm{GeV}{ }^{12} \mathrm{C}$ with ${ }^{197} \mathrm{Au}$.

XBL 835-908

$$
25 \mathrm{GeV}{ }^{12} \mathrm{C}+{ }^{238} \mathrm{U}
$$

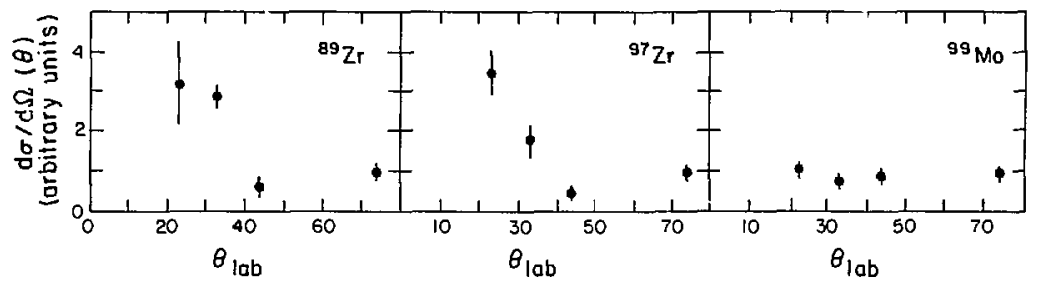

Fig. 2. Target fragment angular distributions from the interaction of $25.0 \mathrm{GeV}{ }^{1} \mathrm{C}$ with ${ }^{238} \mathrm{U}$.

XBL 835-904

\section{Footnotes and References}

*Present address: Radiation Center, Oregon State University, Corvallis, Otegon 97331

1. Y. Morita, W. Loveland, P.L. McGaughey, and G.T. Seaborg, Phys. Rev. C26 (1982) 511

2. S.B. Kaufman, E.P. Steinberg, B.D. Wilkins, and D.J. Henderson, Phys. Rev. C22 (1980) 1897

3. W. Loveland, et al., Oregon State University Nuclear Chemistry Progress Report 1982, DOE/ER/70035-5, p. 57 


\title{
Anomalous Reaction Mean Free Paths of Nuclear Projectile Fragments From Heavy Ion Collisions at $2 \mathrm{AGeV}^{*}$
}

\author{
E.M. Friedlander, R.W. Gimpel, H.H. Heckman, \\ Y.J. Karant, B. Judek, and E. Ganssauge
}

We present (LBL-10573) a detailed description and analysis of two experiments using Bevalac beams of ${ }^{16} \mathrm{O}$ and ${ }^{56} \mathrm{Fe}$. From their results it is concluded that the reaction mean free paths (MFP's) of relativistic projectile fragments (PF's), $3 \leq Z \leq 26$, are shorter for a few centimeters after emission than at large distances where they are compatible with values predicted from experiments on beam nuclei. The probability that this effect is due to a statistical fluctuation is $<10^{-3}$. The effect is enhanced in later generations of fragments, the correlation between successive generations suggesting a kind of "memory" for the anomaly. Various systematic and spurious effects as well as conventional explanations are discussed mainly on the basis of direct experimental observations internal to our data, and found not to explain our results. The data can be interpreted by the relatively rare occurrence of anomalous fragments that interact with an unexpectedly large cross section. The statistical methods used in the analysis of the observations are fully described.

In IBL Report 14562 a brief overview of the status of experiments on the short mean-free-path effect is given. Since the publication of the data presented in LBL-10573, the results of two independent experiments $^{1,2}$ on the MFP's of projectile fragm $i$ ts have been reported and are shown in Fig. 1, superiniposed on the LBL-NRC results. Plotted in this figure are the "charge-independent" MFP parameters $\Lambda^{*} / \Lambda_{\text {beam }}$ for the projectile fragments versus the distance $D$ from their points of emission. The quantity $A^{*}$ is defined in the expression $\Lambda^{*}=\Lambda^{*} Z^{-b}$, where $\Lambda_{\text {beam }}-30 \mathrm{~cm}$ and $b$ $\sim 0.4$ for beam nuclei of charge $Z$. The parameters $\Lambda_{\text {beam }}$ and $b$ are deduced from primary accelerator and/or cosmic-ray beams in each experiment. The results of Jain and Das used Bevalac beams ${ }^{40} \mathrm{Ar}$ and ${ }^{56} \mathrm{Fe}$ at $2 \mathrm{AGeV}$, whereas the experiment of Barber, et al., ${ }^{2}$ involved the re-analysis of MFP data from an extensive series of cosmic-ray balloon flights. All experiments consistently show that the MFP's of projectile fragments are lower than $\Lambda_{\text {bean }}$ for the first few centimeters from their origins, becoming compatible with $\Lambda_{\text {beam }}\left(\Lambda^{*} / \Lambda_{\text {beam }}=1\right)$ for distances $D$ greater than about $5 \mathrm{~cm}$.

\section{Footnotes and References}

*Condensed from Phys. Rev. C 27 (1983) 1489 and LBL-14562 (Yearbook of Science and Technology, McGraw-Hill, Inc., New York, 1982)

†Division of Physics, National Research Councl, Ottawa K1A OR6, Canada

†Fachbereich Physik, Philipps Universität, D-3550 Marburg, Federal Republic of Germany

1. P.I. Jain and G. Das, Phys. Rev. Lett. 48 (1982) 305

2. H.B. Barber, P.S. Freier, and C.J. Waddington, Phys. Rev. Lett. 48 (1982) 856

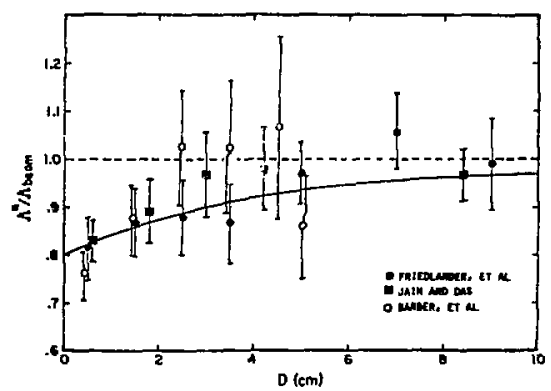

Fig. 1. The MFP parameter $\boldsymbol{\Lambda}^{*}$ versus distance from the origins of PF's. The values of $\Lambda^{*}$ are normalized by the values of $\Lambda_{\text {beam }}$ observed in the three experiments indicated. The dashed !ine at $\Lambda^{*} / \Lambda_{\text {beam }}=1$ is the prediction for normal nuclei; the solid line is the prediction assuming a $6 \%$ admixture of PF's with $\lambda_{a}=$ $2.5 \mathrm{~cm}$.

XBL $8210-3024$ 


\title{
Anomalons: Statistical Fluctuation or Physical Fact?*
}

\author{
Erwin M. Friedlander
}

The Comment discusses:

a) The main statistical arguments used in proving the significance of observations of anomalowsly short mean free paths (MFP's) of projectile fragments (PF's) emitted from relativistic heavy ion collisions and

b) the sample sizes needed to detect similar effects in future experiments assuming a simple model of the phenomenon.

a) The problem of testing the null hypothesis $\mathrm{H}_{0}$. which states (in somewhat simplified physical terms) that PF's have the same MFP as beams of normal nuclei frr $m$ an accelerator, is approached in two ways:

1.) One may assume that the "normal", i.e., "beam" MFP's, $\lambda_{z}$, are known for every charge $Z$ of the PF's (or at least that a reasonable infer(extra)polation is available). Then, taking into account that the exponential distribution law of interaction distances $\mathrm{x}$ is truncated at the value $T$ of the potential path length, one may check whether $P(<x)$, the cumulative distribution function (CDF) of $x$, is uniformly distributed between zero and one. The available data from the LBL/NRC experiment ${ }^{1}$ contradict $\mathrm{H}_{0}$ with a better than 3.7 standard doviation confidence level (Fig. 1).

2.) One may ignore "calibration measurements" on normal beams and test for each PF charge separately the hypothesis that $\lambda_{z}$ is the same irrespective of distance from the PF's origin. Here again the test reduces to checking whether the CDF of the ratio $F=\lambda_{1} / \lambda_{2}$ of MFP's of PF's of the same given charge below and above some cut-off distance is uniformly distributed between zero and one. Fig. 2 shows the distribution of $\mathrm{P}(<\mathrm{F})$ from the data of LBL/NRC and SUNY ${ }^{2}$ along with the mean $P$ of the University of Minnesota group. ${ }^{3}$ The shape of this distribution departs visibly from uniformity, and the mean values of $P$ from the three experimental groups combine to $\sim 5$ standard deviations from the value (P) $=1 / 2$ expected from $\mathrm{H}_{0}$.

b) The simplest alternative hypothesis $\left(\mathrm{H}_{\mathrm{L}}\right)$ used to explain these results is that PF's from relativistic heavy ion collisions include a fraction $\alpha$ of anomalous fragments (nickramed "anomalons") with a MFP, say, g times shorter than the normal MFP. Estimates from

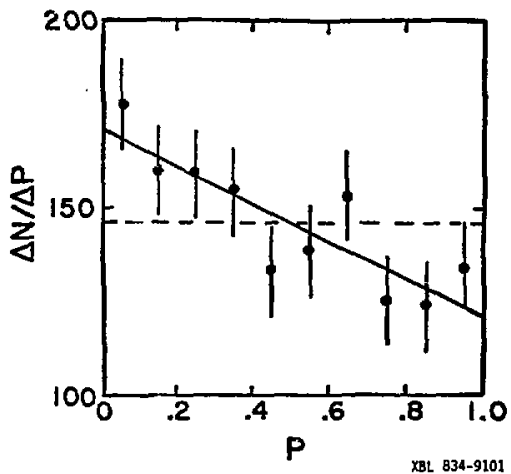

Fig. 1. Distribution of CDF-values $\mathbf{P}$ for the interaction distances from ref. 1.

XBL 8349101

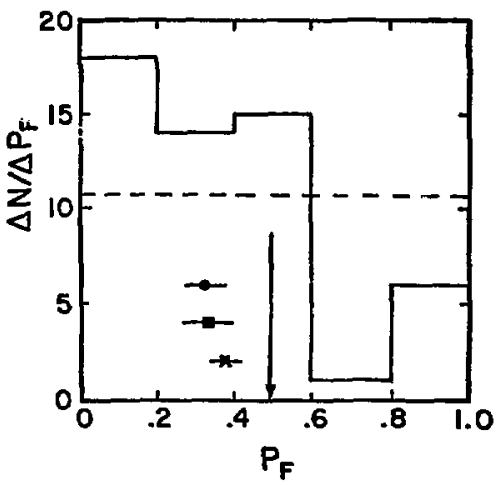

Fig. 2. Distribution of CDF-values $P_{F}$ for the mean free path ratios below and above $2.5 \mathrm{~cm}$ from the PF's origin, for the data of refs. 1 and 2. Mean values of $P_{F}$ are shown by: circle (ref. 1), square (ref. 2), and cross (cosmic-ray data of ref. 3 where only the mean was published). 
experimental data place $\alpha$ and $g$ in vicinity of $\sim 0.06$ and $\sim 4$ respectively. What are then in future experiments, aimed at refining our knowledge of the phenomenon, the sample sizes needed to reach a reasonable level of significance? As an example, it is shown that in the simplest case of a single type of PF + anomalon mixture, observed in a fairly large detector, the minimum number $\mathbf{N}_{\text {min }}$ of events required to show departure from $\mathrm{H}_{0}$ with a confidence of $M$ equivalent standard deviations is given by

$$
N_{\min }=\frac{M^{2}}{3 \alpha^{2}}\left(\frac{g+1}{g+3}\right)^{2} \text {. }
$$

For $M=3$, at least 425 events are needed in this overly simplified and optimistic case. More realistic calculations show $\mathrm{N}_{\text {min }}$ to lie beyond $\sim 2000$ (all this using the values of $\alpha$ and $\mathrm{g}$ mentioned above). Ciearly, detailed Monte Carlo calculations are needed to plan concrete experiments. The arguments presented here show already, however, that considerably higher statistics than those used to detect the effect will be necessary to investigate its details.

\section{Footnote and References}

*Comments in Nuclear and Particle Physics 11 (1983) 127

I. E.M. Friedlander, R.W. Gimpel, H.H. Heckman, Y. Karant, B. Judek, E. Ganssauge, Phys. Rev. Lett. 45 (1980) 1084

2. P.L. Jain and G. Das, Phys. Rev. Lett. 48 (1982) 305

3. H.B. Barber, F.S. Freier, and C.J. Waddington, Abstract HE 3.1.6 in Proc. 17th Int. Conf. on Cosmic Rays, Paris (1981) and Phys. Rev. Lett. 48 (1982) 856

\title{
Comment on "Interpretation of Anomalous Mean Free Paths of Projectile Fragments from Relativistic Heavy-Ion Collisions"*
}

\author{
Y.J. Karant and M.H. Mac Gregort
}

Bayman, Ellis, and Tang (BET) have argued ${ }^{1}$ that the "anomalon effect" occurs only for atomic number $Z$ $\geq 12$ fragments and that this effect can be attributed to the formation of quasimolecular resonances (QMR). However, the BET model appears to be in direct conflict with the experimental data, and the authors have failed to address several important points. Anomalon papers have been published recently by three experimental groups, Berkeley-NRC, ${ }^{2}$ Buffalo, ${ }^{3}$ and Minnesota. ${ }^{4}$ In drawing their conclusions, BET have focused their attention mainly on the Minnesota experimental results. We made $\chi^{2}$ least squares fits ${ }^{5}$ to 17 data points ${ }^{6}$ from the three experimental groups, ${ }^{2-4}$ using both a full $\mathrm{Z}$ range $(Z=3-26)$ anomalon model, ${ }^{25,7}$ and also the BET model (in which only $Z \geq 12$ ) anomalons occur). Appropriate beam mean free paths (mfp's) were used for each data set, including low-Z isotopic effects. The results are shown in Table 1 . As can be seen, a constrained BET fit ( $\alpha=0.25$, as used in Ref. 1) gives a good fit to the Minnesola data, ${ }^{4}$ but poor fits to the other data. ${ }^{2,3}$ An unconstrained BET fit $(\alpha=0.08)$ gives lower $\chi^{2}$ sums but with an unreasonable value for $\lambda$. In the constrained BET solution, the poorest fits are to the low- $Z$ data, but the solution is statistically very marginal over the entire range of $Z$-values.

Table I. $x^{2}$ fits for a full-Z-range anomalon model and for the BET $(Z \geq 12)$ model. ${ }^{7}$ Anomalon fraction $=\alpha$; anomalon $\mathrm{mfp}=\lambda_{0} /(2 Z)^{\beta}$ in $\mathrm{cm}$; average anomalon $\mathrm{mfp}$ over Z-range $=\lambda$. With 17 data points, ${ }^{6}$ we expect $\chi_{\min }^{2} \simeq 15$.

\begin{tabular}{|c|c|c|c|c|c|c|c|}
\hline $\begin{array}{c}x^{2} \\
\text { Sums }\end{array}$ & Total & $\mid \begin{array}{l}\text { Refs. } \\
234\end{array}$ & $3-8$ & $\begin{array}{r}Z \simeq-16 \\
9-15\end{array}$ & $7-26$ & $\alpha$ & $\beta \lambda_{0} \quad \bar{\lambda}$ \\
\hline Full Z-range & 19 & 739 & 8 & 4 & 7 & .06 & $\begin{array}{lll}1 & 22 & 1.1\end{array}$ \\
\hline BET model & 41 & 11237 & 19 & 10 & 12 & $(.25)$ & 11324.0 \\
\hline & 30 & 6159 & 20 & 3 & 7 & .08 & 190.3 \\
\hline
\end{tabular}




\section{Footnotes and References}

\section{•Condensed from UCRI 88198}

†Lawrence Livermore National Laboratory

1. B. Bayman, et al., Phys. Rev, Lett. 49 (1982) 532

2. E. Friedlander, et al., Phys. Rev. Lett. 45 (1980) 1084
3. P. Jain, et al., Phys. Rev. Lett. 48 (1982) 305

4. H. Barber, et al., Phys. Rev. Lett. 48 (1982) 856

5. M. Mac Gregor, UCRL-87368 and UCRO-88010

6. Figure 1 of Ref. $I$ and Tables $I$ of Refs 2 and 3

7. The slope parameter $\beta=1$ was used in Table $\mathrm{I}$; the value $\beta=0$ (used in Ref. 2) gives similar $\chi^{2}$ sums.

\title{
First Observations of ${ }^{238} \mathrm{U}$ Interactions at $1 \mathrm{AGeV}$
}

\author{
Harry H. Hecinilan, Erwin M. Friedlander, and Yasha J. Karant
}

On September 25, 1982, the ultimate objective of the upgraded Bevalac was achieved when ${ }^{238} \mathrm{U}$ ions were successfully accelerated to $0.96 \mathrm{AGeV}$, nearly onequarter trillion volts in kinetic energy! One of the first experiments performed with the extracted beam was the irradiation of nuclear emulsion track detectors to verify the acceleration of tranium and to make initial studies of $1 \mathrm{AGeV}{ }^{238} \mathrm{U}$ interactions in matter.

Within the 3-day period following the 25 September irradiation, we processed and scanned the first set of exposed detectors and were able to have $\mathrm{H}$. Pugh report on our first experimental results on uraniumnucleus collisions to the International Conference on Nucleus-Nucleus Cullisions, Michigan State University,

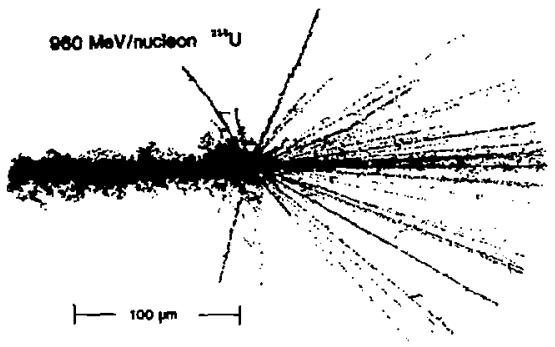

Fig. 1. The catastrophic destruction of both the uranium and target nuclei, the latter being either $\mathrm{Ag}$ or $\mathrm{Br}$ in the emulsion. The forward-going "jet" of tracks are light, bigh-energy nuclear fragments of uranium. The dark tracks at larger angles are low-energy nuclear fragments emitted from the target nucleus.

XBL 829-11834

\section{September 1982 .}

Figures 1-3 show several characteristic interactions of uranium nuclei at about $1 \mathrm{AGeV}$ kinetic energy. In ail the microprojection drawings, the uranium nuclei enter from the left. Because of their high velocity, $\beta=0.87$, and high electric charge, $Z=$ 92e, the uranium ions generate a large number and therefore a halo of $\delta$ rays (energetic knock-on electrons) that produce the broad, "hairy"-like tracks.

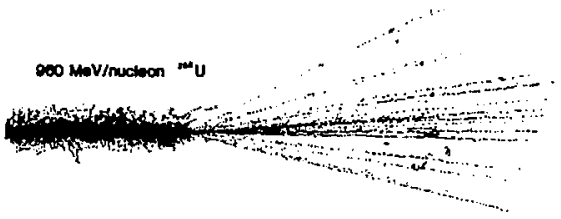

Fig. 2. A nuclear collision where the uranium disintegrates into a shower of nuclear constituents, all confined to a forward cone but with no detectable fragments emitted from the target.

XBL 829-11836

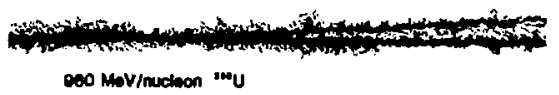

Fig. 3. The uranium is seen to split into two heavy fragments with no associated charged target or lightprojectile fragments. Such an interaction is characteristic of the binary fission of the uranium nucleus.

XBL 829-11833 


\title{
Characteristics of the Ionization Tracks and Interactions \\ of Uranium-238 Nuclei in Emulsion*
}

\author{
H.H. Heckman, Y.J. Karant, and E.M. Friedlander
}

An integral part of the first successful acceleration and extraction of ${ }^{238} \mathrm{U}$ nuclei at the Bevalac on 11 May 1982 was the exposure of nuclear research emulsion detectors to obtain visual confirmation of the acceleration of uranium nuclei. Two exposures of emulsion-detector packets containing 1 by 3 inch, glass-backed 50 - and $200-\mu \mathrm{m}$-thick Ilford $\mathrm{G} .5$ emulsions were made immediately after beam monitors indicated the presence of extracted ${ }^{238} \mathrm{U}$ nuclei. The energy of the ${ }^{238} \mathrm{U}$ ions was, from the machine parameters, estimated to be $147.7 \mathrm{MeV}$ per nucleon.

Withir an hour of their exposure the first emulsions had been developed and the spectacular tracks of ${ }^{238} \mathrm{U}$ were seen, first by the naked eye (Fig. la) and then in detail uncer the microscope. That the tracks were due to uranium was immediately evident from the predominance of collisions involving binary fission of the uranium projectile.

The immediate impression one has when obserying the track of a stopping ${ }^{238} \mathrm{U}$ ion is the immense number of $\delta$ rays (energetic knock-on electrons) it produces and the dramatic narrowing of the ionization track as it loses energy in coming to rest. This is illustrated in Fig. ib, which is a photomicrograph of a uranium ion that entered the top surface of the emulsion at grazing incidence (a few ions did so) and came to rest after traveling a distance of about $1.5 \mathrm{~mm}$. The characteristic tapering of the stopping track (left to right) is attributable to two effects: (i) the decrease in the maximum $f$-ray energies, hence ranges, produced by the ion as its velocity decreases in coming to rest; this tapering is a well-known effect and is observed for all stopping ions of atomic number $Z>3$ in electronsensitive emulsions and (ii) the diminution of the net charge of the ion due to the capture of electrons as its velocity decreases, an effect that compounds the tapering of the uranium track.

The mean range of the noninteracting uranium ions observed in the emulsion detectors was $1.50 \pm$ $0.01 \mathrm{~mm}$, the error being estimated from the dispersion of the ranges obtained in eight difierent emulsion plates. Before entering the emulsions, the uranium beam traversed an Al window, $136 \mathrm{mg} / \mathrm{cm}^{2}$ thick; air, $\sim 62 \mathrm{mg} / \mathrm{cm}^{2}$; and paper, $\sim 10 \mathrm{mg} / \mathrm{cm}^{2}$.

The calculated energy of stopping ${ }^{238} U$ ions for this sequence of materials is $149 \pm \sim 3 \mathrm{MeV} / \mathrm{ariu}$, where the error includes estimates of variations in the thickness of the combination of $A$, air, and paper absorbers and systematic uncertainties in extrapolating the range-energy relation of heavy ions to the high energies and charges of the uranium ions.

Measurements of the mean free path for inelastic uranium-nucleus collisions and the qualitative features of these collisions were also carried out. :

Based on 30 interactions in a total of $93.5 \mathrm{~cm}$ of path length of interacting and noninteracting ${ }^{238} \mathrm{U}$ ions, the interaction mean free path is $\lambda=3.1 \pm 0.6 \mathrm{~cm}$ in the interval $0 \leq \mathrm{E} \leq 115 \mathrm{MeV}$ per nucleon. This value is compatible with that calculated for the mean free path of ${ }^{238} \mathrm{U}$ in emulsion, assuming geometric nuclear sizes, $\lambda_{\text {calc }}=3.6 \mathrm{~cm}$.

Table 1 gives the relative frequencies of several classifications of uranium interactions that were observed and the range interval in which they occurred. To illustrate two of the classifications as defined in Table 1, we show in Fig. lc an example of an event of the $\mathrm{H}+\mathrm{L}$ type, where the uranium fragments to one heavy secondary accompanied by lighter fragments. Figure $1 d$ is an event where the uranium undergoes binary fission, classified as a $2 \mathrm{H}$ event.

Several qualitative features of the uranium interactions in nuclear emulsion at $\mathrm{E} \leq 115 \mathrm{MeV}$ per nucleon are apparent from the data in Table 1 . These are:

(1) About 70 percent of the collisions involve binary fission of the uranium nucleus, with or without L-fragment emission.

(2) One-third of the events exhibit binary fission only. Such events would include both nuclear and Coulomb interactions.

(3) Two-thirds of the events exhibit L-fragment emission, a characteristic signature for a nuclear interaction.

\section{Footnote}

*Condensed from Science, 217, 17 September 1982, also LBL-14629 


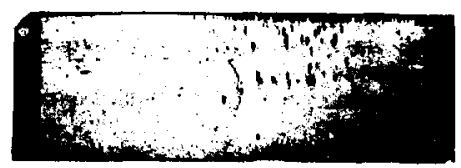

a
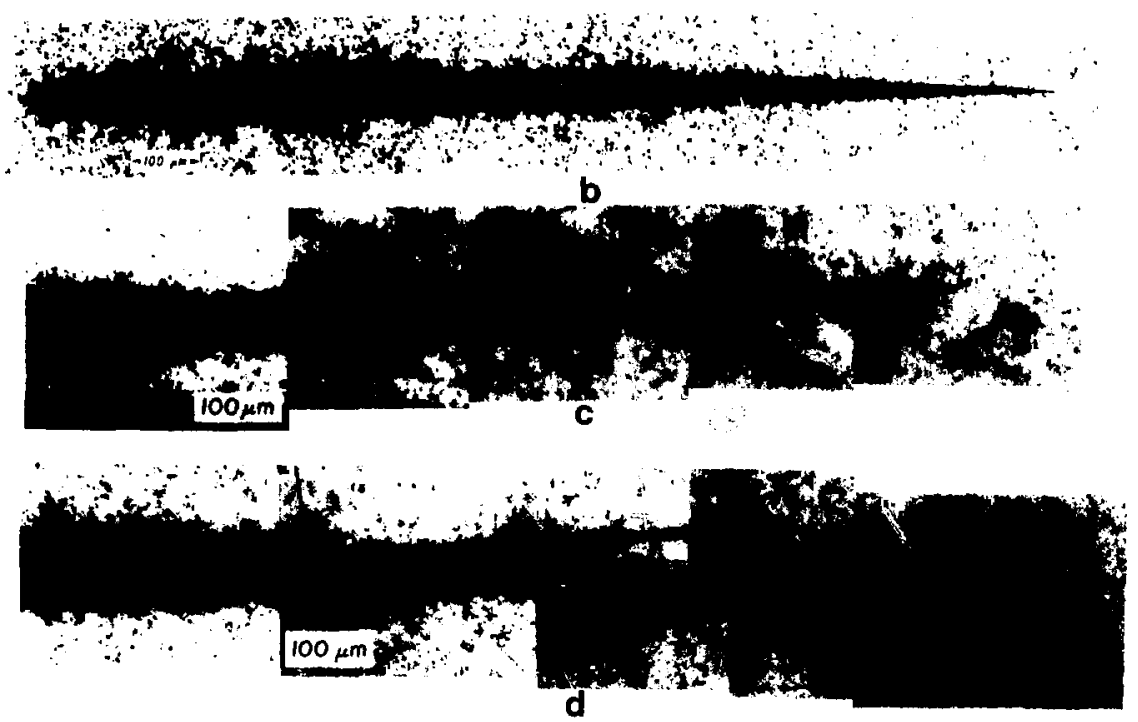

Fig. 1. (a) Contact print of a 1 by 3 inch, $200-\mu \mathrm{m}$ emulsion plate exposed to ${ }^{239} \mathrm{U}$ ions at $115 \mathrm{MeV}$ per nucleon. The tracks of the ions, $1.5 \mathrm{~mm}$ in length, are visible to the naked eye and are seen to enter the upper leading edge of the emulsion and at grazing incidence to the surface of the emulsion.

(b) Photomicrograph of the track of a stopping ${ }^{238} \mathrm{U}$ ion in emulsion. The ion anters from the left and has a range of $1.5 \mathrm{~mm}$ A $100-\mu \mathrm{m}$ scale is indicated.

(c) Interaction leading to the fragmeniation of the uranium nucleus, where both heavy (one) and light projectile fragments are produced.

(d) Example of a coilision leading to binary fission of the uranium projectile. 
Table 1. Characteristics of uranium interactions ( $E \leq$ $115 \mathrm{MeV}$ per nucleon) and number of interactions per $0.5-\mathrm{mm}$ range interyal. In the first column, $\mathrm{nH}$ refers to the number of heavy fragments (fission fragments at . heavier), and $L$ indicates the emission of lighter projectile or target fragments.

\begin{tabular}{|ccccc|}
\hline & & \multicolumn{4}{c|}{ Number in range interval (mm) } \\
\cline { 3 - 5 } Type & $\begin{array}{c}\text { Abundance } \\
\text { (\%) }\end{array}$ & $\begin{array}{l}1.5 \\
\text { to }\end{array}$ & $\begin{array}{l}1.0 \\
\text { to }\end{array}$ & $\begin{array}{c}0.5 \text { to } \\
\text { (ending) }\end{array}$ \\
\hline & & & & \\
L & $10 \pm 5$ & 3 & 0 & 0 \\
$\mathrm{H}+\mathrm{L}$ & $17 \pm 6$ & 1 & 3 & 1 \\
$2 \mathrm{H}+\mathrm{L}$ & $40 \pm 7$ & 5 & 5 & 2 \\
$2 \mathrm{H}$ & $33 \pm 7$ & 2 & 4 & 4 \\
\hline
\end{tabular}

\title{
Search for Nonintegrally Charged Projectile Fragments in Relativistic Nucleus-Nucleus Collisions*
}

\author{
P.B. Price, ${ }^{\dagger}$ M.L. Tincknell, ${ }^{\ddagger}$ G. Tarlé, \\ S.P. Ahlen, K.A. Frankel, and S. Perlmunter ${ }^{\dagger}$
}

Using CR-39(DOP) plastic track detectors with charge resolution $\sigma_{3} \approx 0.06 \mathrm{e}$, we have made the first dynamic search for fractionally charged particles bound to nuclei. Figures 1 and 2 show charge distributions for projectile fragments of $1.85 \mathrm{GeV} / \mathrm{amu}{ }^{40} \mathrm{Ar}$ interactions at mean distances of 3.1 and $1.3 \mathrm{~cm}$ from the

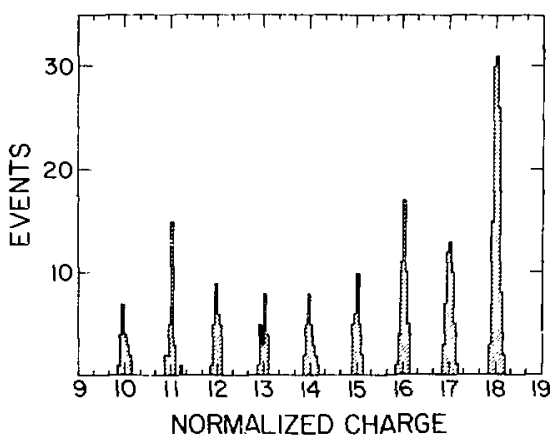

Fig. 1. Charge distribution of projectile fragments measured at a mean distance of $3.1 \mathrm{~cm}$ from their interaction points in a CR-39(DOP) stack. The charge standard deviation, $\sigma_{Z}=0.06 \mathrm{e}$, results from averaging 16 etch pit measurements per event. Abscissa are labeled at intervals of $\mathrm{e} / 3$.

XBI. $834-9035$ points of interactions in CR-39(COP). We find that no more than $3 \times 10^{-3}(95 \% \mathrm{CL})$ of the fragments with 10 $\leq Z<18$ have charges differing from an integer by as much as $0.3 \mathrm{e}$. This rules out models in which anomalons in such charge range have nonintegral charge.

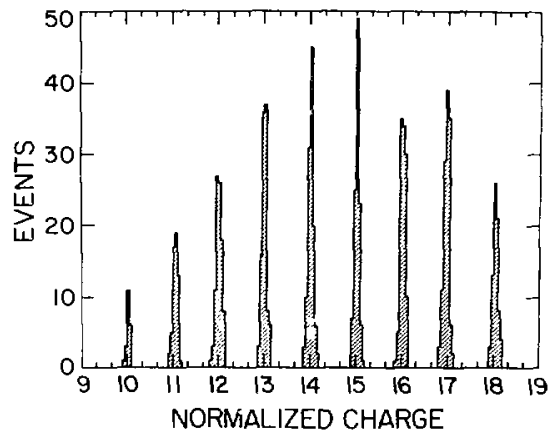

Fig. 2. Charge distribution of projectile fragments measured at a mean distance of $1.3 \mathrm{~cm}$ from their interaction points.

XBL $834-9034$ 
Footnotes

"Condensed from Phys. Rev. Lett. 50 (1983) 566.
†Also at Space Sciences Laboratory

¥Terradex Corporation graduate fellowship

\title{
Search for Delayed Gamma Decays of Anomalous Nuclear States*
}

\author{
T.M. Liss, S.P. Ahlen, P.B. Price, and G. Tarlé
}

It has been shown by other workers ${ }^{1,2}$ that some of the fragments of relativistic heavy-ion collisions have an anomalously short interaction length but may revert to normal nuclei within $\sim 10^{-11}$ sec. Existing data are equally consistent with two interpretations: (1) About 6\% of the projectile fragments are anomalous, having an interaction mean free path (MFP) in nuclear emulsion of $\sim 2.5 \mathrm{~cm}$, an order of magnitude smaller than the MFP for low-mass primary nuclei (ref. 1). (2) Nearly all fragments have an interaction length initially reduced by a factor $\sim 0.65$ and decay by neutral particle emission into normal nuclei with a MFP of $\sim 0.85$ $\mathrm{cm}$ (ref. 2).
The statistical nature of previous experiments is a shortcoming of the evidence for anomalous nuclear states. It would be desirable to identify a feature that could be used to label those products that are in fact anomalnus. One such feature suggested by theoretical models is the emission of one or more high-energy pho'us as the anomalous particle decays into normal rilitter. ${ }^{3}$ A distinguishing characteristic of such a photon would be that its point of emission would be from one to several $\mathrm{cm}$ beyond the target.

By modifying an apparatus used previously to observe direct $\gamma$ rays from nucleus-nucleus collisions, ${ }^{4}$

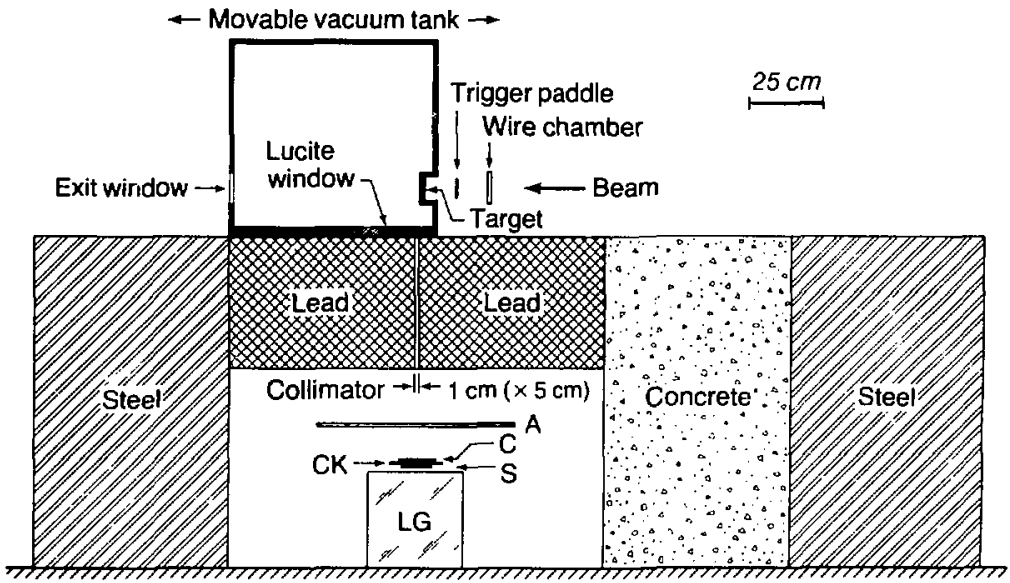

Fig. 1. Schematic of the experimental apparatus 
we have looked for delayed $\gamma$ rays characteristic of the decay of anomalous nuclear states produced as projectile fragments in the collisions of $940 \mathrm{MeV} / \mathrm{amu}{ }^{56} \mathrm{Fe}$ nuclei from the LBL Bevalac with a steel target.

Our experimental configuration is shown in Fig. 1. A 41-cm-thick lead collimator with a hole $1 \times 5 \mathrm{~cm}^{2}$ in cross section permits only $\gamma$ rays emitted at right angles from points 1.4 to $2.4 \mathrm{~cm}$ downstream from the edge of the $0.62-\mathrm{cm}-$ thick steel target to reach the photon detector. The target is placed at the entrance window of a large vacuum tank, which eliminates background from interactions of the beam with air. To be detected, a photon emerging from the collimator must pass through the charged-particle anti-coincidence scintillator, $A$, and produce an $\mathrm{e}^{+} \mathrm{e}^{-}$pair in a 0.18 -cm-thick $\mathrm{Pb}$ converter, $\mathrm{C}$. The pair produce signals in the Lerenkov counter, $C K$, and scintillator, $S$, and deposit the remainder of their energy iat a lead glass calorimeter, LG. The detector is sensitive to photons with lab energies $>15 \mathrm{MeV}$ and $<1000 \mathrm{MeV}$.

During our exposure characterized by $1.63 \times 10^{8}$ interactions only one valid event, at an energy of $70 \pm$ $13 \mathrm{MeV}$, was recorded. Based on this single event we calculate regions in the space of fragment-rest-frame photon decay energy, $E_{0}$, versus proper mean lifetime, $\tau_{0}$, which can be ruled out by our data at a $95 \%$ confidence level assuming single photon decay. This is shown in Fig. 2. The right-hand ordinate is labeled for $\lambda$, the laboratory MFP.

These results cast doubt on models in which anomalous states decay electromagnetically unless the photon energy, $E_{0}$, is $>2000 \mathrm{MeV}$ or $<70 \mathrm{MeV}$.

\section{Footnote and References}

"Condensed from Liss, et al., Phys. Rev Lett. 49 (1982) 775

1. E.M. Friedlander, et al., Phys. Rev. Lett. 45 (1980) 1084

2. H.B. Barber, et al., Phys. Rev. Lett. 48 (1982) 856

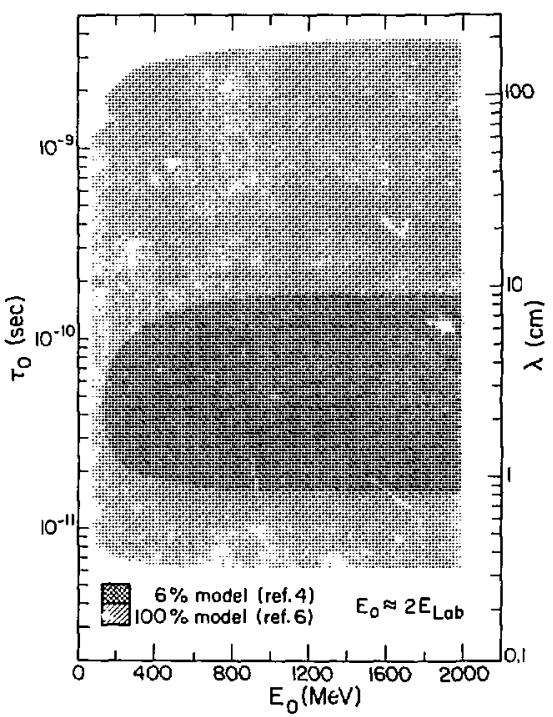

Fig. 2. Regions excluded by this experiment at a $95 \%$ confidence level for single-photon decay of anomalons.

XBL $827-950$

3. Y. Karant, LBL Report No. 9171 (1979) unpublished; also S. Fredriksson, et al., Phys. Rev. Lett. 48 (1982) 14

4. M.P. Budiansky, et al., Nucl. Instr. and Meth. 199 (1982) 453 


\title{
Measurement of the Summed Residual Projectile Mass in Relativistic Heavy-Ion Collisions*
}

\author{
S.D. Stevenson, ${ }^{\dagger}$ J. Martinis, ${ }^{\ddagger}$ and P.B. Price ${ }^{\dagger}$
}

We present the results of a multiparticle measurement that provides the first direct evidence of the validity of the abrasion process. The objective of the measarement was to measure the summed mass of all projectile fragments, $M_{3}$, defined by

$$
M_{h}=\Sigma M_{p l}
$$

The sum is over all projectile fragments produced in a single collision. In the language of the abrasionablation model $M_{2}$ is the mass of the projectile residue before ablation takes place. This measurement allows a direct test of the abrasion assumption independent of the specifics of the ablation process. The method we used to determine $M_{s}$ was to measure the total ki retic energy of al] projectile fragments with a hadron calorimeter. Since projectile fragments are known to have an energy per nucleon within a few percent of that of the beam, a measurement of their total kiustic energy is a measurement of their mass.

The calorimeter was located in the beam line 3.2 $\mathrm{m}$ downstream from the target and subtended angles from $-5^{\circ}$ to $5^{\circ}$ vertically and horizontally when viewcd from the target. The device is triggered by an "inelastic event trigger" scintillator downstream of the target. Figure I shows the summed projectile mass distribution for a $2.1 \mathrm{GeV} /$ nucleon ${ }^{20} \mathrm{Ne}$ beam with carbon and molybdenum targets. The most striking feature of the data is the pronounced difference in the shape of the two spectra. The yield of low-mass projectile residues is a factor of $\sim 20$ higher for the molybdenum than for the carbon target. The depletion of low-mass projectile residues is expected from the abrasion model when the projectile nucleus is larger than the target nucleus, as in the case for the ${ }^{20} \mathrm{Ne}$ and $\mathrm{C}$ data. The solid curve shown in Fig. 1 is based on the abrasion model in which the target and projectile nuclei are taken to be spberes of uniform density with radii $R$ given by $R=$ $1.2 \mathrm{~A}^{1 / 3}$ with $\mathrm{R}$ in fermis. One shortcoming of the abrasion model is that it assumes that the interaction length, $\lambda$, for scattering of a projectile nucleon in the target is small compared with the radius, $R_{T}$, of the target nucleus. The broken curves in Fig. 1 are based on the modified abrasion model with two different values of the interaction length $\lambda$. Values of $\lambda$ between 0 and 1.5 $\mathrm{fm}$ are consistent with both data sets.

\section{Footnotes}

*Condensed from Phys. Rev. Lett. 47 (1981) 990

tAlso at Space Sciences Laboratory $\ddagger$ Also at Department of Physies, University of California, Berkeley

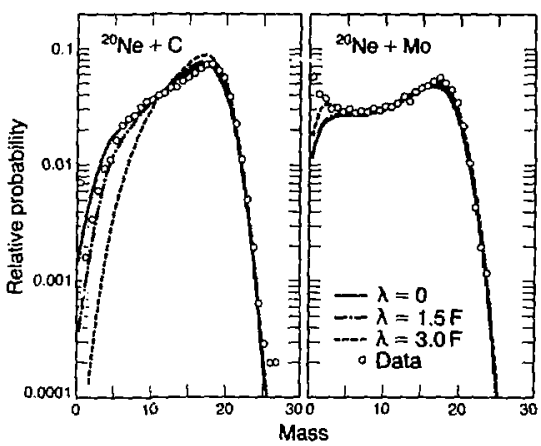

Fig. 1. Plots of the distribution of summed proje_tile masses for $2.1 \mathrm{GeV} /$ nucleon ${ }^{20} \mathrm{Ne}+\mathrm{C}$ and ${ }^{20} \mathrm{Ne}+\mathrm{Mo}$. Masses beyond 20 are due to spreading of instrumental resolution. The curves are based on abrasion calculations described in the text.

XBL 8110-12205 


\title{
Data Tables of Inclusive Particle Production at Forward Angles from Collisions of Light Relativistic Nuclei*
}

\author{
L. Anderson, E. Moeller, ${ }^{\dagger} S$. Nagamiya, S. Nissen-Mever, ${ }^{\ddagger}$ \\ $L$ Schroeder, G. Shapiro, and H. Steiner
}

Data tables of fragmentation cross sections of relativistic light ions have been compiled on eight microfiches. These data were obtained from an experiment whose details are described in ref. 1.

The reaction types and energies are summarized in a TABLE OF CONTENTS together with the identification numbers of the corresponding microfiches. A detailed INDEX for each fiche follows. Cross sections of $Z=1,2$ fragments with the same nominal (lab) rigidity $P / Z(\mathrm{GeV} / \mathrm{c})$ and transverse rigidity $P_{T} / Z(G e V / c)$ are grouped together on a single frame of a fiche. ${ }^{2}$ The INDEX lists the values of $P / Z, P_{T} / Z$ (first two numbers in each entry) and the observed fragment types together with the address of the frame on the fiche where the data are tabulatec. In case of negative pions, cross-section data with the same nominal (lab) momentum $\mathrm{P}(\mathrm{GeV} / \mathrm{c})$ but different transverse momenta $P_{T}(\mathrm{GeV} / \mathrm{c})$ are grouped together on a single frame of a fiche. ${ }^{3}$ Correspondingly, the INDEX lists the values of $P$ (first number in each entry) followed by up to five different $P_{T}$ values together with the address of the frame on the fiche where the data are tabulated.

We have tried to fit some of the data, namely the longitudinal momentum distributions for $\mathrm{p}_{\mathrm{P}}^{\mathrm{P}_{\mathrm{roj}}} \geq 0$ at $\mathrm{p}_{\mathrm{T}}$ $=0$ and the transverse momentum distributions at $\beta_{\text {Fragment }}=\beta_{\text {Beam }}$ in terms of an exponential, which is mos' important for high fragment moinenta, plus either a Gaussian or a second exponential, which dominate the lower momentum behavior. Here, $p_{2}{ }^{\text {Proj }}$ and $p_{T}$ are the longitudinal momentum in the projectile frame and the transverse momentum, respectively. The corresponding fit parameters are summarized on fiche 492 . It should be noted that, although this parameterization gives reasonable fits to the data in most cases, there are some notable exceptions.

\section{Footnotes and References}

*Condensed from LBL-14330

tOn leave from Freie Universität Berlin, West Germany \$Now at Siemens, Munich, West Germany

1. L.M. Anderson, Jr., Ph.D. Thesis, U.C. Berkeley, LBL-6769 (1977) unpublished

2. L. Anderson, W. Bruckner, E. Moeller, S. Nagamiya, S. Nissen-Meyer, L. Schroeder, G. Shapiro, and H. Steirer, LBL-14328 (1982) to be published in Phys. Rev. C

3. E. Moeller, L. Anderson, W. Bruckner, S. Nagamiya, S. Nissen-lMeyer, L. Schroeder, G. Shapiro, and H. Steiner, LBL-14329 (1982) to be published in Phys. Rev. C

\section{Invariant Mass Spectra from ${ }^{12} \mathrm{C}$ Interactions}

\author{
H.J. Crawford*
}

Single particle inclusive measurements in high energy nuclear physics have provided information useful to astrophysics, biology and electronics, as well as providing the foundation for a number of models of interacting nuclear fluids. ${ }^{1.2}$ While such measurements yield information on the endpoints of the evolution of bighly excited nuclear systems, they suffer from the fact that observed particles can be formed in a large number of very different evolutionary paths. In an attempt to learn more about how interactions proceed, we have performed a series of experiments in which all fast nuclear fragments are analyzed for each individual interaction. These experiments were performed at the LBL Bevalac Heavy Ion Spectrometer System (HISS) Facility where we studied the interaction of 1 $\mathrm{GeV} /$ nucleon ${ }^{12} \mathrm{C}$ nuclei with targets of $\mathrm{C}, \mathrm{CH}_{2}, \mathrm{Cu}$, 
and $\mathrm{U}$.

The reaction we have studied can be written schematically as

$$
{ }^{12} \mathrm{C}+\mathrm{T} \rightarrow \text { (channel) }+\mathrm{T}^{\mathrm{V}}
$$

where channel means any final state for the 12 nucleons originally in the $C$ nucleus (e.g., " $B+P$ or three ajphas) and $T$ is the final state of all target nucleons. The "B fragments can be formed only in the "B channel but the "He fragments can be formed in a large number of different channels. In our experiment we attempt to measure complete (i.e., 12 nucleon) channels. After correcting for the acceptance of our system, we are thus able to measure the relative probability of forming each separate channel in the interaction, which gives a picture of the internal nuclear arrangement within the $\mathrm{C}$ nucleus. We then form the invariant mass for each interaction channel and subtract from the mass of the ${ }^{12} \mathrm{C}$ parent to determine the amount of energy transferred to the fragment channel in each interaction. From the relative channel population as a function of this transferred energy we can investigate the nuclear rearrangement in varying energy density environmenis. By analyzing many fragments from a single interaction, we can also take subgroups of fragments and calculate invariant masses for these subgroups to look for preferred intermediate states (e.g., two alphas from a ${ }^{8} \mathrm{Be}$ ).

The experimental layout is shown in Fig. 1 including beam definition devices, large gap superconducting dipole, fragment trajectory drift chambers, and time-of-flight (TOF) scintillator array. The event trigger required a single ${ }^{12} \mathrm{C}$ hit the target and no ${ }^{12} \mathrm{C}$ signature at DS, indicating that the projectile had been scattered or destroyed. Detector performance and resolution are reported elsewhere. The first results from our experiment are shown in Fig. 2, where we have used all channels having $\Sigma Z F=6$ and $\Sigma A F=12$ giving a global excitation energy spectrum. The prominent peak near $30 \mathrm{MeV}$ excitation leads to fragments having $50-200 \mathrm{MeV} / \mathrm{c}$ momentum in the projectile rest frame, fragments primarily sesponsible for observed single particle inclusive momentum distributions. Data out to $400 \mathrm{MeV}$ excitation are from interactions in which large energies are transferred to the ${ }^{12} \mathrm{C}$ system, the energy then being shared among a few multi-nucleon fragments. Energies of $300 \mathrm{MeV}$ shared among three alpha particles have been seen, suggesting a collective mude transfer of energy such as would be expected from a phonon-like exchange mechanism.

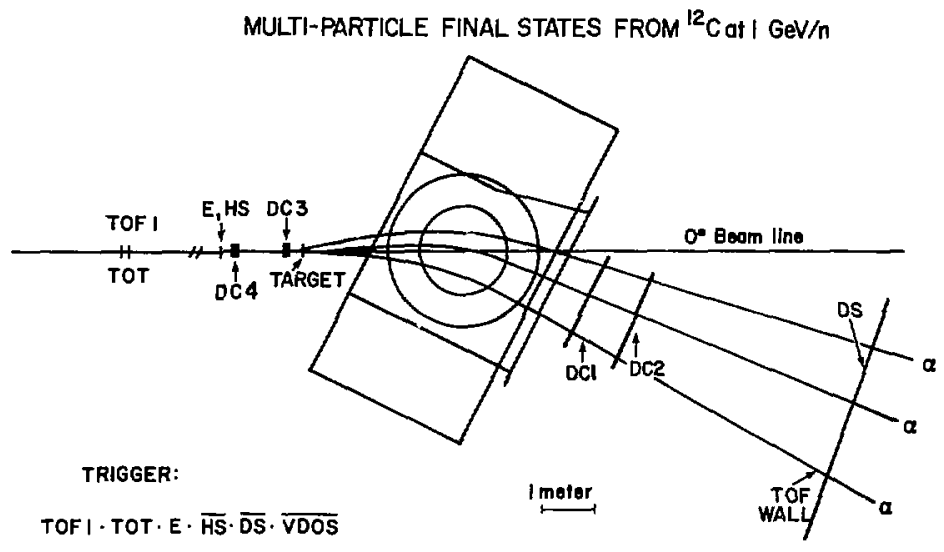

Fig. 1. Experimental setup for ${ }^{12} \mathrm{C}$ at $1 \mathrm{GeV} /$ nucleon showing beam definition scintillators (TOF1, TOT, HS, and $D E$ ), beam definition drift chambers (DC3 and $D C 4$ ), target location (T), large dipole, fragment trajectory drift chambers (DCI and DC 2), trigger veto scintillator (DS), and time-of-fight scintillators (TOF). 


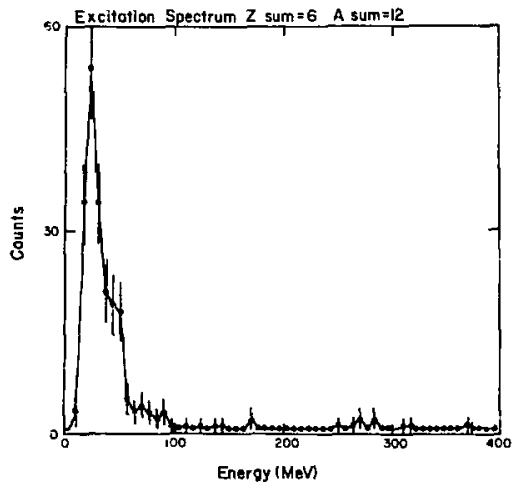

Fig. 2. Preliminary data on invariant mass distribution summed over all channels as labeled.

XBL 835-903
Footnote and References

-Space Sciences Laboratory and U.C. Berkeley

1. D.G. Greiner, et al., Phys. Rev. Lett. 35 (1975) 152

2. A.S. Goldhaber and H.H. Heckman, Ann. Rev. Nucl. Sci. 28 (1978) 161 


\title{
Relativistic Energies: Participants
}

\section{The Question of Thermalization in Relativistic Heavy Ion Collisions}

\author{
A. Baden, H.H. Gutbrod, B. Kolb, H. Löhner, ${ }^{\dagger . t}$ \\ B. Ludewigt.' M.R. Maier, A.M. Poskanzer, T. Renner. \\ H. Riedesel, H.G. Riiter,"H. Spieler,"IA. Warwick," \\ F. Weik," and H. Wieman
}

One of the major questions while studying relativistic heavy ion collisions is whether thermal equilibrium is reached or whether nuclei become transparent in central collisions already at intermediate Bevalac energies. Appropriate quantities to look at for investigation of this subject are the momentum components perpendicular $p_{\perp}$ and parallel $p_{\mid}$to the beam axis in the center-of-mass frame. Figure 1 (upper portion) shows

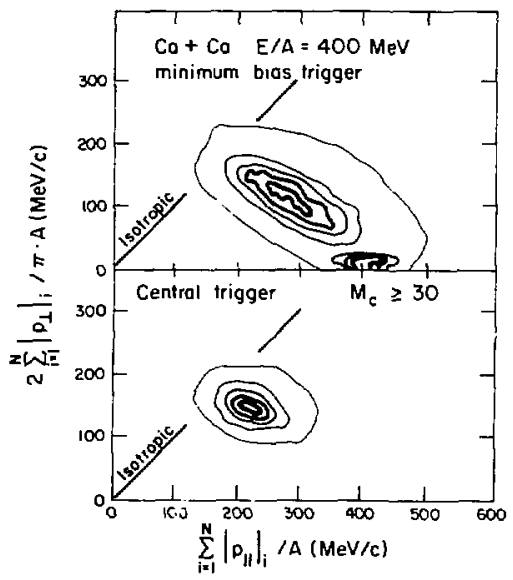

Fig. 1. Contour plots of $\left\langle\mathrm{p}_{1} / \mathrm{A}\right\rangle$ versus $\left\langle\mathrm{p}_{\mathrm{l}} / \mathrm{A}\right\rangle$ for $\mathrm{Ca}$ on $\mathrm{Ca}$ at $400 \mathrm{MeV} / \mathrm{A}$ for a minimum bias trigger, requesting for the projectile some loss of charge. The lower part shows the same quantity for a central trigger (no beam velocity particle within $2^{\circ}$ of the beam) and a charged particle multiplicity cut at 30 . Both graphs contain about 50,000 events each, measured with the Plastic Ball/Plastic Wall spectrometer. The contour lines represent lin mly increasing content.

XBL $8210-1210$ in a contour plot for $\mathrm{Ca}$ or $\mathrm{Ca}$ at $400 \mathrm{MeV} / \mathrm{A}$ in an event-by-event analysis that most of the intensity lies away from the isotropic diagonal. Restricting the data to more central collisions by the trigger and multiplicity sc.siti in (Fig. 1, lower part) the maximum of the distribution moves closer to the isotropic line. In Fig. 2 is shown that the mean of this ratio is increasing as a function of multiplicity but, at least at this energy and target projectile combination, never reaches the value 1 , which would be a necessary (but not sufficient) criterion for thermalization. Cascade calculations with the code of Yariv and Fraenkel, however, show that еver a few spectator fragments mask an isotropic expanding fireball and deform a spherical event in momentum space to a prolate spheroid.

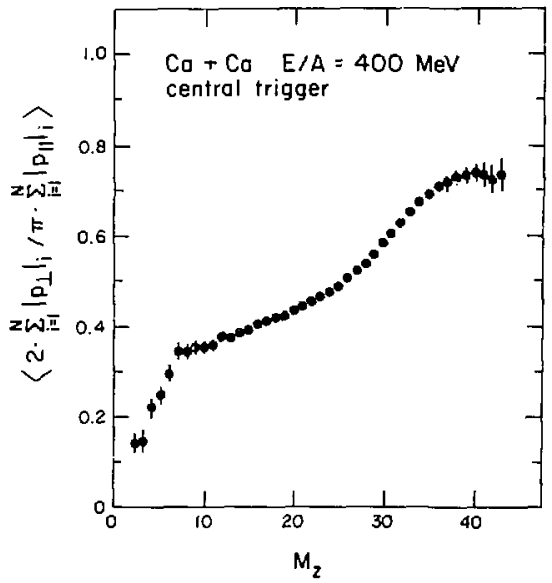

Fig. 2. Ratio of $2 \cdot<p_{\perp}>/ \pi \cdot<p_{1}>$ as function of nucleonic charge multiplicity averaged over 50,000 events. A value of 1 for this ratio would correspond to an average spherical shape of the event in momentum space.

XBL $8210-1209$ 
Footnotes

*Gesellschaft für Schwerionenforschung, Darmstadt, West Germany

+Universität Marburg, Marburg, West Germany
łPresent address: Universität Münster, Münster, West Germany

§Permanent address: Lawrence Berkeley Laboratory, Berkeley, CA 94720

**Present address: Tolcdo Inc., Köln, West Germany

\title{
Global Analysis with Plastic Ball Data*
}

\author{
H.H. Gutbrod, H. Löhner, ${ }^{+3}$ A.M.Poskanzer, T. Renner, \\ H. Riedesel, H.G. Rit:er, ${ }^{\dagger}$ A. Warwick, ${ }^{\dagger}$ F. Weik, ${ }^{\dagger}{ }^{\prime \dagger}$ \\ H. Weimant
}

The global analysis methods (sphericity, thrust) were developed as a tool to detect and distinguish predicted two-jet events at high energy $\mathrm{e}^{+} \mathrm{e}^{*}$ storage rings from events with spherically symmetric emission patterns. ${ }^{1}$ Both methods define a jet axis, the sphericity by minimizing $\sum \mathrm{pi}_{\mathrm{i}}^{2}$ and the thrust by maximizing $\sum p_{i}$ relative to this axis. The sphericity is calculate- analytically by diagonalizing the sphericity tensor. One obtains the orientation of the sphericity axis, e.g., relative to the beam direction, and three eigenvalues which define ar ellipsoid that describes the shape of the event. The thrust analysis yields in addition to the orientation only the magnitude of the thrust, a quantitative measure distinguishing between isotropic and back-to-back emission.

The use of global methods to analyze the more complex events from heavy ion collisions was proposed by several authors. ${ }^{2,3}$ Sphericity $\left(\mathrm{p}^{2}\right)$ overweights leading particles and gives two nucleons a different weight from a deuteron with the same energy per nucleon. Corrections for these shortcomings have been proposed, ${ }^{3}$ e.g., the flow analysis. ${ }^{4}$ Since a global analysis has to be performed for each event, statistical fluetuations due to finite number effects and to limitcd experimental acceptance and efficiency are expected. Experimental oata have to be compared with results from an analysis of theoretically calculated events, which have been filtered for experimental acceptance and efficiency. Most theoretical models have not yet reached the sophistication of the experimental equipment in the sense that they are not able to calculate all the measured quantities. Cascade codes do not include composite particles and hydrodynamical codes do not produce event-to-event fluctuations. This nakes the comparison between experiment and thenry diflicult. Complete events generated with a statistical model calculation by
Randrup and $\mathrm{Fai}^{\mathrm{S}}$ will be extremely usefui to study the effect of finite number fluctuations and experimental biases. As global analysis allows one to determine the shape of the event in pirase space and takes into account all the measured correlations, it should be well suited to distinguish between emission patterns as predicted by the hydirodynamical and the cascade model. Figure I shows the result of a flow analysis of $400 \mathrm{MeV} / \mathrm{u} \mathrm{Ca}$ on $\mathrm{Ca}$ data. The angle of the main axis of the flow ellipsoid is plotted versus the square of the ratio of the largest to the smallest axis. A comparison of those results with theoretical predictions can be done only after the calculated events have been filtered for the experimental acceptance. This procedure is presently being developed for cascade events.

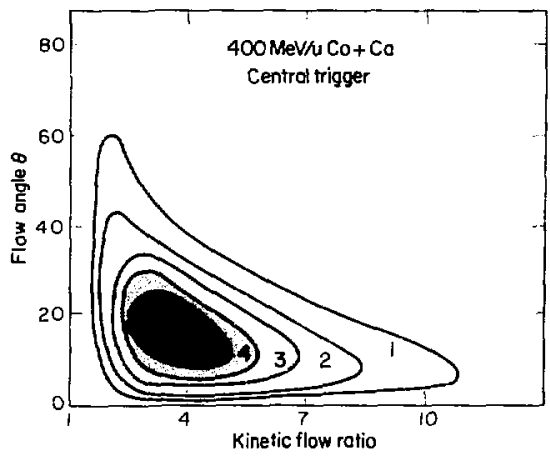

Fig. 1. Flow plot for the reaction $400 \mathrm{MeV} / \mathrm{u} \mathrm{Ca}$ on $\mathrm{Ca}$.

XBL 826-1420 


\section{Focinotes and References}

*Condensed from part of LBL-14980.

†Geselischaft für Schwerionenforschung

† Universität Marburg

Spresent address: Institut für Kernphysik, D-4400

Münster, West Germany

t†present address: Toledo Co., Köln, West Germany
2. J. Kapusta and D. Strottman, Phys. Lett. 100B (1981) 33

3. J. Knoll, Proc. 5th High Energy Heavy Ion Study, LBL-12652 (1981)

4. M. Gyulassy, K.A. Frankel, and H. Stöcker, Phys. Lett. 110B (1982) 185

5. J. Randrup and G. Fäi, Phys. Lett. 115B (1982) 281

1. S. Brandt and H. Dahmen, Z. Physik C1 (1979)

61; S.L Wu and G. Zoberning, Z. Physik

C2(1979) 107

\title{
Charged Particle Exclusive Analysis of Central Ar $+\mathbf{K C l}$ and Ar $+\mathrm{Pb}$ Reactions at 1.8 and $0.8 \mathrm{GeV} / \mathrm{u}$
}

\author{
H. Stroebele, $R$ Brockmann, * J.W. Harris, * F. Riess*.', \\ si. Sandoval, $R$, Srock, *, K.L. Wolf, *\# H.G. Pugh, \\ L.S. Schroeder, R.E. Renfordt ${ }^{\dagger}, K$. Tittel ${ }^{\dagger}$, and M. Maier ${ }^{\dagger}$
}

Our present understanding of the dynamics of relativistic nucleus-nucleus collisions is derived primarily from single particle inclusive measurements. Deeper insight into the interaction may be obtained by a study of multiparticle observables ${ }^{1,2}$ derived from all charged particles produced in these collisions. The analysis of charged particle exclusive data in terms of such multiparticle variables was done for the central collisions of the systems ${ }^{40} \mathrm{Ar}+\mathrm{KCl}$ at $1.808 \mathrm{GeV} / \mathrm{u}$ with a trigger cross section of $180 \mathrm{mb}$ and ${ }^{40} \mathrm{Ar}+$ $\mathrm{Pb}_{3} \mathrm{O}_{4}$ at $772 \mathrm{MeV} / \mathrm{u}$ with a trigger cross section of 990 $\mathrm{mb}$. From the charged particle final stite all $\pi^{-}, 95 \%$ of the hadrens $(p, d, t)$ and $85 \%$ of $\pi^{+}$were identified.

To provide a better determination of impact parameter than given by the Streamer Chamber trigger, we studied the dependence of several observable quantities on the impact parameter using filtered cascade events. ${ }^{3}$ For the reaction $\mathrm{Ar}+\mathrm{KCl}$ the total transverse energy

$$
\begin{aligned}
& E_{i}=\sum_{i}\left(\sqrt{M_{i}^{2}+p_{. L i}^{2}}-M_{i} \delta_{i}\right) \\
& \delta_{i}=0 \text { for pions, } \delta_{i}=1 \text { for protons }
\end{aligned}
$$

was found to exhibit the tightest correlation with impact parameter. This correlation is shown in Fig. 1a where the rms deviations of the total transverse energy distributions are also plotted as a function of impact parameter for intranuclear cascade generated events. For subsequent discussion we use $E_{1}$ as a measure of impact parameter within the range already selected by our trigger condition. Figure lb presents the distribution of $E_{t}$ in the data sample and, for comparison, results from intranuclear cascad́c calculations on which the experimental biases were folded in.

The degree of isotropy of the momenta of the nucleons within one event can be judged by the ratio

$$
\mathrm{R}=\frac{2}{\pi}\left(\frac{\sum\left|\mathrm{p}_{\perp}^{\prime}\right|}{\sum\left|\mathrm{p}_{i}^{\prime}\right|}\right)
$$

which for an isotropic distribution should be 1 . In the $\tau+\mathrm{Pb}$ reaction $\mathbf{R}=0.79$ for all events. Selecting .vents with $E_{t}>5 \mathrm{GeV}$ (the average value) the events show isotropy $(R=0.96)$. In contrast the reaction $\mathrm{Ar}$ $+\mathrm{KCl}$ exhibits an elongation of the momentum distribution in direction of the beam axis ( $R=0.55$ for all events). Even if $R$ is extrapolated to zero impact parameter by means of its dependence on $E_{1}, R=0.6$ ? is obtained; therefore complete degradation of longitudinal momentum is not observed in head-on collisions.

To look for details of the momentum fiux pattern on an event by event basis, a more elaborate approach was adopted, using the normalized momentum flux 
tensor." Events were classified using the cosine of the angle $\theta_{1}$ of the main tensor axis with respect to the beam direction and the aspect ratio $a_{1} / a_{2}$ of the longest

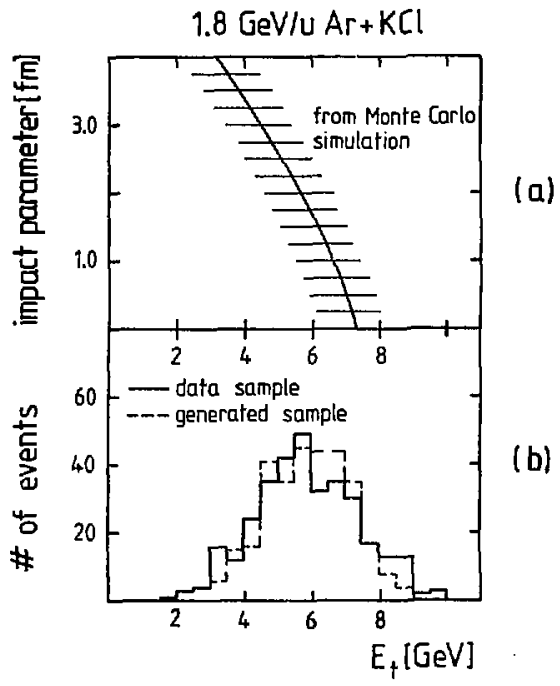

Fig. 1. For the reaction $1.8 \mathrm{GeV} / \mathrm{u} \mathrm{Ar}+\mathrm{KCl}$ :

(a) Correlation between the total transverse energy $\mathrm{E}_{q}$ as defined in the text and the impact parameter $b$ for cascade generated events. The hatched band rorresponds to the $1 \mathrm{rms}$ deviation of the $\mathrm{E}_{1}$ distribution at a given $b$.

(b) $\mathrm{E}_{1}$ spectrum for both the measured data (solid line) and the cascade generated events (dashed line).

XBL 8210-3144

to next longest axis.

Figure 2a shows the $\cos \boldsymbol{\theta}_{1}$ distributions for $\mathrm{Ar}+$ $\mathrm{KCl}$ and $\mathrm{Ar}+\mathrm{Pb}$. The $\mathrm{Ar}+\mathrm{KCl}$ events are aligned along the beam direction with a width at half maximum of $\Delta \theta_{1}=16^{\circ}$. Filtered cascade events reproduce the distribution qualitatively. The experimental $\mathrm{Ar}+\mathrm{Pb}$ events are distributed over a broader range of angles $\left(\Delta \theta_{1}=43^{\circ}\right)$. Cascade events generated for $\mathrm{Ar}+\mathrm{Pb}$ tend to be more forward peaked than the observed distribution $\left(\Delta \theta_{1}=36^{\circ}\right)$. If selection is made on the transverse kinetic energy $E_{1}$ one sees in Fig. $2 b$ that for high $E_{q}$ the distribution is nearly isotropic, contrary to the corresponding cascade prediction. Figure $3 \mathrm{a}$ and $3 \mathrm{~b}$ depicts the mean aspect ratios for both systems studied as a function of $E_{t}$ of the events. Ideally, a completely spherical shape corresponds to $a_{1} / a_{2}=1$. This value is never reached, however, for events consisting of a finite

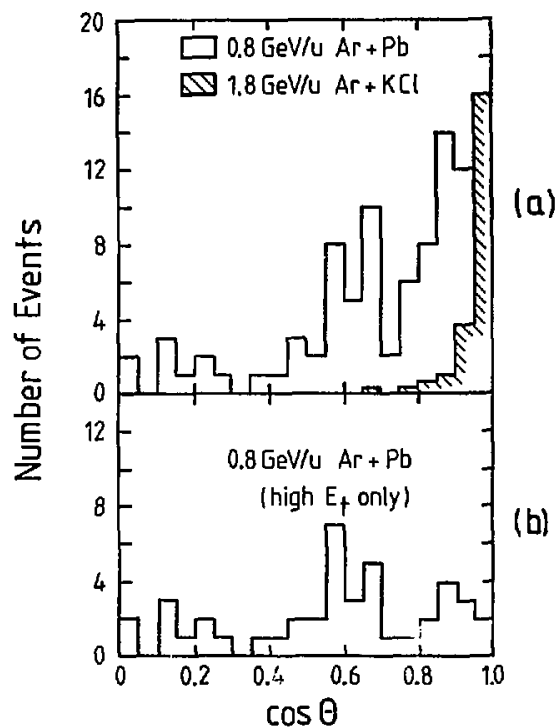

Fig. 2. Spectrum of $\cos \theta$, with $\theta$ being the angle of the main axis of the momentum tensor with respect to the beam direction:

(a) For both systems analyzed. The $\mathrm{Ar}+\mathrm{KCl}$ histogram (hatched) is normalized to the number of entries in the last bin of $\mathrm{Ar}+\mathrm{Pb}$.

(b) Distribution of $\cos \theta$ for events from the $\mathrm{Ar}+\mathrm{Pb}$ final state with $E_{1}$ greater than the average $E_{1}\left(\left\langle E_{1}\right\rangle\right.$ $=5 \mathrm{GeV}$ ).

XBL 8210-3146

number of particles. The mean of the aspect ratio distribution of events with spherical shapes is shown by the solid lines. Thus, $\mathrm{Ar}+\mathrm{Pb}$ events closely approach isotropy at high $\mathrm{E}_{4}$ (Fig. 3a) as expected from the cos $\theta_{1}$ distribution of Fig. 2b. This is not reproduced by the cascade events, which consistently yield higher aspect ratios. The $\mathrm{Ar}+\mathrm{KCl}$ system (Fig. $3 \mathrm{~b}$ ), on the other hand, never becomes completely isotropic even for high $\mathrm{E}_{1}$ events. The cascade-generated events given by the uppermost set of values in Fig. 3c show 
qualitatively the same situation although they are seen to be consistently more elongated.

To understand the nonequilibration of the Ar + $\mathrm{KCl}$ final state a closer look into the cascade events was taken. If only participant nucleons, defined as particles making at least one collision, are considered (represented by crosses in Fig. 3c), the data and cascade sample of events agree in the aspect ratios. This indicates that for central $\mathrm{Ar}+\mathrm{KCl}$ collisions the difference between real and cascade events can be attrihuted to the presence of noninteracting particles in the cascade calculations. In a further step, only particles are considered that have undergone more than two collisions (lowest set of points in Fig. 3c). Under this condition the stape of cascade events approaches the finite particle number limit of $\left\langle a_{1} / a_{2}\right\rangle=1.25$, suggesting that the nonsurface part of the system has become completely equilibrated.

\section{Footnotes and References}

"Gesellschaft für Schwerionenforschung, Darmstadt, West Germany

†Institut für Hochenergiephysik, Universität Heidelberg, West Germany

¥Fachbereich Physik, Universität Marburg, West Germany

§ermanent address: Sektion Physik, Universität Munchen, West Germany

\$Permanent address: Texas A\&M University, College Station, Texas 77843

1. S. Brandt and H.O. Dahmen, Z Phys, Cl (1979) 61; S.L. Wu and G. Zobernig, Z Phys. C2 (1979) 107

2. J. Kapusta and D. Strottmann, Phys. Rev. C23 (1981) 1282; H. Stöcker, et al., Phys. Rev. C25 (1982) 1873; M. Gyulassy, K.A. Frankel, and H. Stöcker, Phys. Lett. 110B (1982) 185

3. J. Cugnon, et al, Nucl. Phys. A379 (1982) 553

4. J. Cugnon, J. Knoll, C. Riedel, and Y. Yariv, Phys. Lett. 109B (1982) 167

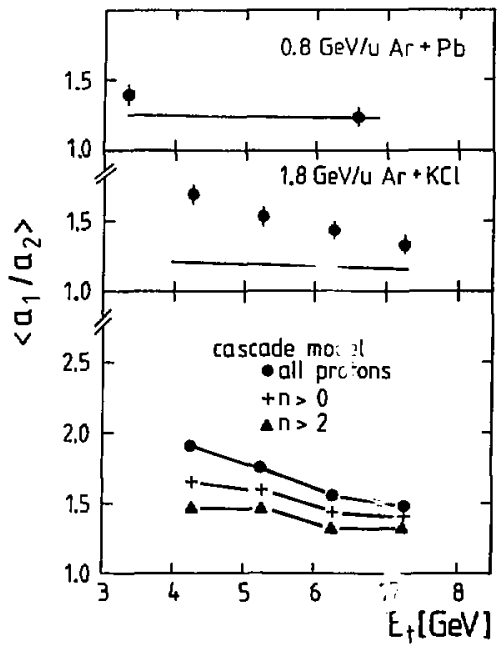

a)

b)

Fig. 3. Aspect ratio (main axis $/ 2^{\text {nd }}$ largest axis) as a function of $\mathrm{E}_{\mathrm{l}}$ for the experimental data from the reaction $\mathrm{Ar}+\mathrm{Pb}$ (a) and $\mathrm{Ar}+\mathrm{KCl}$ (b). The solid lines represent the aspect ratios for isotropic shapes (see text).

(c) Same for cascade generated data with different particle selection: ( $\bullet$ all charged particles; $(+)$ only particles that interacted at least once; $(\Delta)$ only particles that interacted at least three times.

XBL 8210-3145 


\title{
Compression Effects in Relativistic Nucleus-Nucleus Collisions
}

\author{
R. Stock," R. Bock," R. Brockman," A. Dacal, 'J.W. Harris," M. Maier, ' M.E. Ortiz, \\ H.G. Pugh, R.E. Renfordt, A. Sandoval, L.S. Schroeder, H. Stroebele, and K.L. Wolf',
}

Central collisions of nuclei at relativistic energies are predicted by dynamical models ${ }^{1-3}$ to proceed via a compression-expansion cycle of hadronic bulk matter, with a first stage of interpenetration and pile-up of the nucleon densities from target and projectile followed by expansion towards a final freeze-out stage. While these models predict densities several times the nuclear ground state density to be reached at the end of the compression stage, there has been no direct experimental evidence of such densities or of any new physical effects resulting from them. In the present work we propose the total multiplicity of produced pions as an observable linked to the high density stage of the collisions. Data on this variable will be presented for several colliding nuclear systems and discussed in terms of nuclear densities. Values for the bulk compressional energy are extracted and a nuclear equation of state deduced.

The reason total pion multiplicity can be a good measure of the compression stage is an interplay of two considerations: 1) the primary production yield in nucleon-nucleon (nn) collisions rises rapidly over the Bevalac energy range, and 2) thè relative nn kinetic energy in a nucleus-nucleus colitision is continually degraded during compression and is low by the time expansion begins. For these reasions pion production is heavily weighted towards the compression stage.

These statements are best understood in the framework of an intranuclear cascade calculation. The cascade code used, that of Cugne"., et al., ${ }^{3}$ was chosen because it is extensively described in recent publications and has input data in good agreement with pp, тр, and $\mathrm{pn}$ data ${ }^{4}$ in the energy range of the Bevalac. Figure 1 shows the most important results for central collisions (b $b_{\text {max }} \leq 2.4 \mathrm{fm}$ ) of ${ }^{40} \mathrm{Ar}+\mathrm{KCl}$ at 977 $\mathrm{MeV} /$ nucteor. Figure la shows the baryon density in a sphere of $3 \mathrm{fm}$ diameter about the origin of the centerof-mass coordinate system of the participant nueleons, expressed as a ratio to the ground state nuclear density $p_{0}=0.17 \mathrm{im}^{-3}$. It peiks at a time $\mathrm{t}=7 \mathrm{fm} / \mathrm{c}$ (in the laboratory system), corresponding to the end of the compression stage. Figure ib shows that about half the baryon-baryon collisions occur during the compression and expansion stages, respectively. Figure 1c shows that by the end of the compression stage the total number of pions and delia resonances $N_{r+\Delta}$ reaches a plateau where it remains approximately constant through expansion and freeze-out, thus establishing the final pion multiplicity, which is $\mathrm{N}_{\mathrm{r}+\Delta}$. Figure Id shows the maximum density reached as a function of bombarding energy and also the mean density weighted according to the rate of $\pi+\Delta$ production. The latter, which reflects closely the maximum density, will be used in our subsequent analysis.

The LBL Streamer Chamber facility at the Bevalac was used to study the interaction of ${ }^{40} \mathrm{Ar}$ with $\mathrm{KCl}$ at bombarding encrgies from 0.36 to 1.8 $\mathrm{GeV} /$ nucleon. Between 4,000 and 10,000 events were accumulated at each of the energies $360,566,722,977$, $1180,1385,1609$, and $1808 \mathrm{MeV} /$ nucleon in both inelastic and central trigger modes. In addition, data were obtained for ${ }^{4} \mathrm{He}+\mathrm{KCl}$ at $977 \mathrm{MeV} /$ nucleon and ${ }^{40} \mathrm{Ar}$ $+\mathrm{BaI}_{2}$ at $772 \mathrm{MeV} /$ nucleon. Techniques and parts of the data have been presented elsewhere. ${ }^{9}$ For ${ }^{40} \mathrm{Ar}+$ $\mathrm{KCl}$ the central trigger corresponded to a reaction cross section of $180 \pm 20 \mathrm{mb}$, or impact parameters up to $\mathrm{b}_{\mathrm{max}}=2.4 \mathrm{fm}$ in a geometrical model. This value, when used in the cascade code, enables us to predict successfully many experimental quantities such as the proton participant multiplicity distribution.

Figure $2 \mathrm{a}$ shows the negative pion multiplicity $<\mathrm{n}_{-}$(E) $>$observed in ${ }^{* 0} \mathrm{Ar}+\mathrm{KCl}$ collisions as a function of laboratory and $\mathrm{cm}$ energy. The cascade model prediction also shown is systematically too high, with overestimates ranging from a factor of 4 at 360 $\mathrm{MeV} /$ nucleon to 1.35 at $1.8 \mathrm{GeV} /$ nucleon. The overestimate also appears for the other systems studied. For a sequence of data at approximately constant incident energy ${ }^{4} \mathrm{He}+\mathrm{KCl}(977 \mathrm{MeV} /$ nucleon $),{ }^{40} \mathrm{Ar}+\mathrm{KCl}$ (772 MeV/nucleon), and ${ }^{40} \mathrm{Ar}+\mathrm{BaI}_{2} \quad(772$ $\mathrm{MeV} /$ nucleon), the ratios of cascade model predictions to the data are $1.4,2.1$, and 2.4 , respectively. This discrepancy is not due to an inability of the cascade model to deal with pions. Several studies ${ }^{6}$ have found it to work well for pion production in proton-nucleus collisions, for pion-nucleus scattering, and for pion absorption on nuclei. The factor that is present in nucleus-nucleus collisions but not important in pnucleus or $\pi$-nucleus collisions, where the cascade model was successfui, is the feature of density increase or compression. The densities calculated in the cascade for these thrce systems are $\rho / p_{0}=1.9,3.0$, and 3.4 , respectively. The overestimate ratios are proportional to these densities at roughly constant incident energy. 
Now at a given cm energy $E_{i}$ consider the measured multiplicity $\left\langle m_{r}\left(E_{i}\right)\right\rangle$. The same multiplicity is reached in the cascade model at a lower energy $E_{i}^{\prime}<E_{i}$ as indicated in Fig. 2a. As a crude approximation let us interpret the difference $\left(E_{i}-E_{i}^{\prime}\right)$ as that part of the $\mathrm{cm}$ internal energy per nucleon that goes into potential (compressional) degrees of freedom. This energy becomes inactive as far as pion production is concerned. By the time it reappears in kinetic energy of the baryons, overall thermalization has reduced the mean energy of binary collisions to a point where pion production is no longer important. Reading the compressional energy per nucleon $E_{i}^{C}-E_{1}-E_{i}^{\prime}$ from the graph of Fig. 2a at each experimental point and plotting it against the mean compression $p / \rho_{0}$ derived from Fig. Id

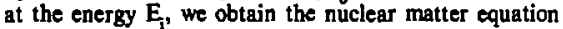
of state graph shown in Fig. 2b. It is noteworthy that above $1.2 \mathrm{GeV} /$ nucleon where the density in $\mathrm{Ar}+\mathrm{KCl}$ becomes constant, so are the values of $\mathrm{E}_{\mathrm{i}}^{\mathrm{C}}$. Furthermore, the reaction ${ }^{10} \mathrm{Ar}+\mathrm{BaI}_{2}$ at $772 \mathrm{MeV} /$ nucleon, for which the density prediction is $\rho=3.4 \rho_{0}$, leads to a value of $\mathrm{E}_{i}^{\mathrm{C}}$ in agreement with that derived from ${ }^{40} \mathrm{Ar}$ $+\mathrm{KCl}$ at $977 \mathrm{MeV} /$ nucleon, for which the density is also $3.4 \rho_{0}$. The values of $\mathrm{E}_{i}^{\mathrm{C}}$ plotted in Fig. $2 \mathrm{~b}$ are offset by $10 \mathrm{MeV}$ to allow for the ground state binding energy of mass 40 nuclej. The dashed curve is a parabola representing an equation of state without phase transitions, corresponding to a compressibility constant $\mathrm{K}=250 \mathrm{MeV}$, i.e., a "hard" equation of state. The value of $200 \mathrm{MeV}$ extracted from excited nuclear energy levels yields the partial curve shown for comparison. The best fit to the data is fornd at $K=240$ MeV. The horizontal and vertical error bars shown are statistical only. No estimate of the systematic errors implicit in our treatment has been made, as this should be the subject of more penetrating future theoretical studies, transcending the present simplistic approach.

\section{Footnotes and References}

-Gesellschaft für Schwerionenforschung, D-6100 Darmstadt, West Germany

IInstituto de Física, UNAM, Mexico City 21 D.F., Mexico

‡Universität Marburg, D-355 Marburg, West Germany

§ermanent address: Texas A\&M University, College Station, Texas, 77843

I. H. Stöcker, et al., Phys. Rev. Lett. 44 (1980) 725; J.R. Nix and D. Strottman, Phys. Rev. C23 (1981) 2548
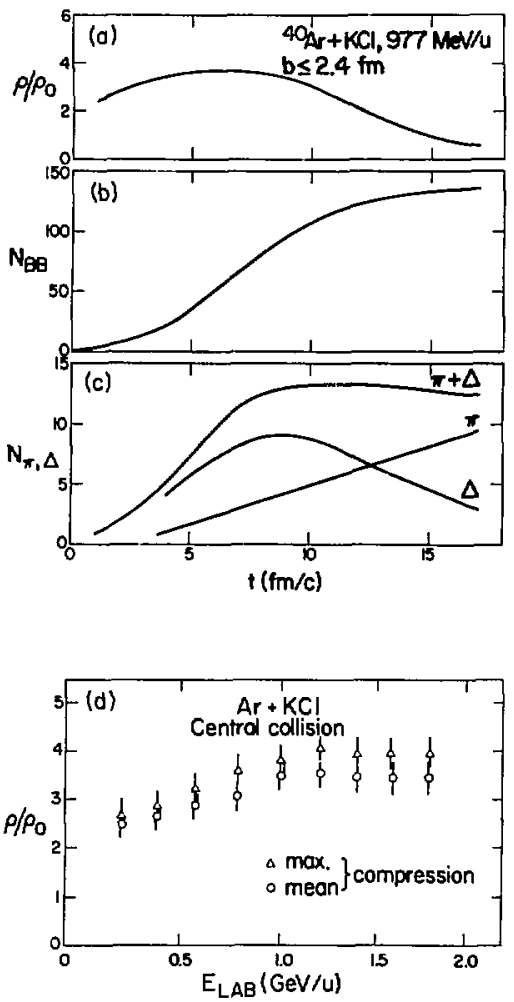

Fig. 1. Results of a cascade calculation for near-central collisions (b $\leq 2.4 \mathrm{fm}$ ) of ${ }^{40} \mathrm{Ar}+\mathrm{KCl}$ at a laboratory bombarding energy of $977 \mathrm{MeV} /$ nucleon. The time dependence of the reactions is shown for (a) the baryon density, relative to the ground state value, (b) the integrated number of baryon-baryon collisions, and (c) the instantaneous number of pions and $\Delta$-resonances. Part (d) shows the maximum baryon density attained as a function of bombarding energy and the mean density weighted by the rate of $\pi+\Delta$ production.

(a-c) XBL 826-1434, (d) XBL, 826-815 

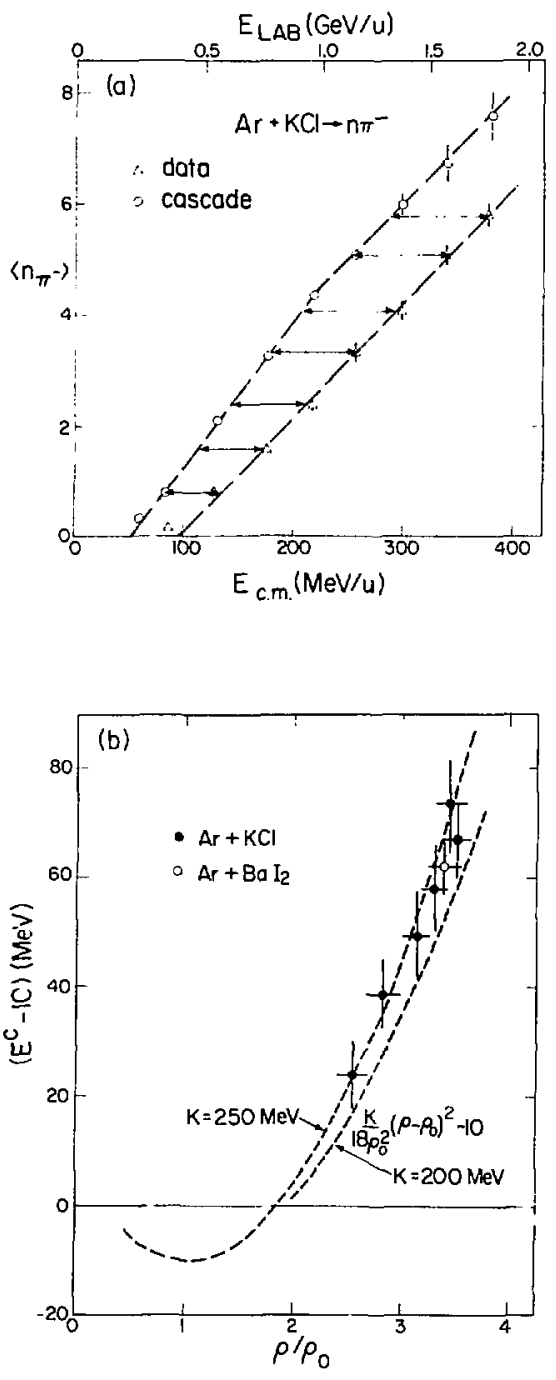

2. Y. Yariv and Z. Fraenkel, Phys. Rev. C20 (1979) 2227 and $C 24$ (1981) 488

3. J. Cugnon, et al., Nucl. Phys. A352 (1981) 505 and A379 (1982) 553

4. F. Shimizu, et al., University of Tokyo preprint (1982); G. Alexander, et al., Nucl. Phys. B52 (1973) 221; V. Flaminio, et al., CERN-HERA 79-03 (1979)

5. S.Y. Fung, et al., Phys. Rev, Lett. 40 (1978) 292; A. Sandoval, et al., Phys. Rev. Lett. 45 (1980) 874; J.W. Harris, et al., Phys, Rev. Lett. 47 (1981) 229

6. M. Sternheim and $\mathbf{R}$ Silbar, Phys. Rev. D6 (1972) 3117; Y. Yariv and Z. Fraenkel, to be published. In calculations with the Cugnon code for pion absorption, quantitative agreement is obtained with the data of K. Nakai, et al., Phys. Rev. Lett. 44 (1980) 1446
Fig. 2. (a) The mean $\pi$ multiplicity as a function of bormbarding energy for near-central collisions of ${ }^{40} \mathrm{Ar}+$ $\mathrm{KCl}$. The triangles show the data. Open circles show the results of cascade calculations, where vertical lines at $E_{\mathrm{LAB}} \geq 1.4 \mathrm{GeV} /$ nucleon estimate the uncertainty due to multiple pion production from single NN collisions, not included in the calculation. Horizontal arrows are the values of $\mathrm{E}_{i}^{\mathrm{C}}$, the compressionai energy per nucleon determined at each experimental point.

(b) The values of $\mathrm{E}_{i}^{\mathrm{C}}$ as a function of the calculated mean baryon density. Points determined at 1.6 and 1.8 $\mathrm{GeV} /$ nucleon are not shown since values of $E_{\mathrm{C}}$ and $\rho / \rho_{0}$ are nearly identical to the results at $1.2 \mathrm{GeV} /$ nucleon. The dashed lines represent equations of state with an incompressibility constant $\mathrm{K}$ of 250 and $200 \mathrm{MeV}$, respectively.

(a) XBL 826-1436, (b) XBL 826-1435 


\title{
High Densities and the Latest Two-Proton Correlation Results
}

\author{
S. Abachi, J.B. Carroll, * K. Ganezer, " G.Igo,* T.A. Mulera, \\ V. Perez-Mendez, A.L. Sagle, A. Shor, F. Zarbakhsh
}

Relativistic nuclear collisions offer the possibility of studying nuclear matter at high densities and temperatures. At sufinciently high energy densities, it may be possible to study quark deconfinement effects. The maximum temperatures achieved at the Bevalac are i00-150 MeV. Recent estimates suggest that at these temperatures a baryon density of $4-5 \rho_{0}$ ( $\rho_{0}$ is normal nuclear density) would be needed to see a transition to quark matter. $^{1}$

The Monte Carlo cascade calculations of Cugnon, et al., ${ }^{2}$ suggest that densities as high as $4 p_{0}$ cah be achieved in $\mathrm{Ca}+\mathrm{Ca}$ collisions at $2 \mathrm{GeV} / \mathrm{n}$. In these calculations, the collision has a highly compressed phase followed by a rapid expansion. There are, however, many approximations in these calculations. Since the effects of these approximations are difficult to estimate, it is important to determine experimentally if compression is occuring.

We are attempting to study the collision process by measuring the size of the proton emitting source. This size may not be a direct measure of the dense nuclear matter because the protons could be emitted during a Jatter expansion phase. Nevertheless, we wish to see if the size we measure is consistent with the occurence of compression.

The spatial extent of the proton emitting source is obtained from measurements of the correlation between two protons emitted at small relative momenta. Results for the correlation function $R$ were first reported ${ }^{3}$ for $\mathrm{Ar}+\mathrm{KCl} \rightarrow \mathrm{p}+\mathrm{p}+\mathrm{x}$ at $1.8 \mathrm{GeV} / \mathrm{n}$. Measurements were made for protons traveling at the beam rapidity $Y_{B}$ and at $Y_{B} / 2$. The multiplicity of fast charged particles was also sampled with a set of sixteen counters.

A reanalysis of this data is currently underway using an improved algorithm for generating the uncorrelated background. The most recent results are shown in Fig. 1. The peaks in $\mathbf{R}$ are smaller and the shapes of the data agree much better with the predictions of Koonin's' model. Only the data in the first bin is significently different from the model calculations and this may be due to experimental resolution and efficiency.

When no multiplicity, $M$, selection is made, the peaks in $\mathrm{R}$ are roughly at the same height for protons at $Y_{B}$ and $Y_{B} / 2$. Both of these sets of data are described fairly well with curves for $r_{0}=3 \mathrm{fm}$. The corresponding sharp sphere radius of $r_{1}=4.7 \mathrm{fm}$ is slightly larger

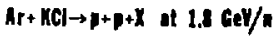
PMELIMIKLAY RESULTS of MenKaLYsis
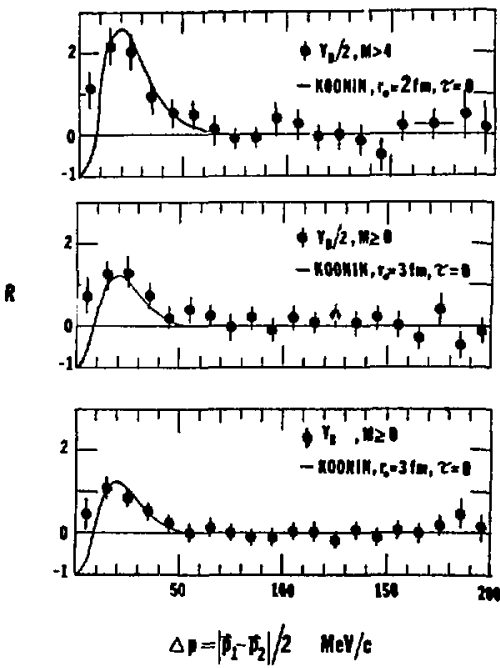

Fig. 1 The correlation function at central rapidity and at the beam rapidity. The data at central rapidity are shown for two different multiplicity requirements.

XBL 8349194

than the size of an Argon nucleus. These data are not associated with central collisions $(M \gtrsim 0)$. From a cross-calibration between streamer chamber data and the multiplicity counters, the average total charged multiplicity, $n$, is estimated to be 12 for data at $Y_{B}$ and IS for data at $Y_{B} / 2$, corresponding to maximum impact parameters of 6 and $5.5 \mathrm{fm}$, respectively.

The data at $Y_{B} / 2$ for $M \gtrsim 4$ is associated with more central collisions. The estimated $n$ is 25 and the maximum impact parameler is only $3 \mathrm{fm}$. We are surprised to find that the peak in $R$ for $Y_{B} / 2$ protons gets larger when this multiplicity requirement is 
imposed. The data seem well described by a curve for $\mathrm{r}_{0}=2 \mathrm{fm}$.

These results seem inconsistent with participant/ spectator models where one would expect the size of the fireball to increase as the impact parameter decreases. We find just the opposite effect.

The smaller $t_{0}$ for high multiplicity events may be an indication that compression has occured during small impact parameter collisions. While this result is sug " gestive of high density, a firm conclusion cannot be drawn from the present data because the multiplicity counters were not optimum in selecting central collisions. It will be important to see if a small $r_{0}$ is also measured when a more suitable central collisicn trigger is user.
Foothotes and References

*University of California, Los Angeles

tUniversity of California, Davis

1. Summary Report of Biclefeld Workshop (May 1982), edited by T. Ericson, M. Jacob, H. Satz, and $W$, Willis.

2. J. Cugnon, et al., Nucl. Phys. A352 (1982) 565.

3. F. Zarbakhsh, et al., Phys. Rev. Lett. $\$ 6$ (1981) 1269.

4. S. Koonin, Phys. Lett. B70 (1977) 43.

\title{
A Measurement of pd Quasi Elastic Scattering in $800 \mathrm{MeV}$ pA Collisions and Short-Range Correlations of pn Pair in Nuclei*
}

\author{
1. Tanihata, Y. Make, ${ }^{\dagger}$ H. Hamagaki, S. Nagamiya, ${ }^{\dagger}$ \\ S. Schnetzer, ${ }^{\ddagger}$ Y. Shida, and H. Steiner
}

The momentum spectrum of pd quasi-elastic scattering (QES) in the large momentum transfer region has been used to study the strength of shortrange correlations of pn pairs. To study the pd QES at large momentum transfer we have measured protondeuteron coincidence spectra in collisions of $800 \mathrm{MeV}$ protons on nuclear targets. Protons were accelerated at the Bevatron with a beam intensity of about $3 \times 10^{8}$ pps. Deuterons were detected by a magnetic spectrometer at a seattering angle $\theta$ of $15^{\circ}$. Backscattered protons were detected by a set of $\Delta \mathrm{E}-\mathrm{E}$ counters consisting of three layers of plastic scintillators whose thicknesses are $0.5,1.5$, and $20 \mathrm{~cm}$, respectively. The $\Delta \mathrm{E}-\mathrm{E}$ counter covered scattering angles from $103^{\circ}$ to $133^{\circ}$ and was located in the same reaction plane as the magnetic spectrometer at the opposite side of the target. The spectrometer covered the momentum range from $500 \mathrm{MeV} / \mathrm{c}$ to $2 \mathrm{GeV} / \mathrm{c}$ with a solid angle of about 12 $\mathrm{mSr}$, and the $\triangle \mathrm{E}-\mathrm{E}$ covered from $350 \mathrm{MeV} / \mathrm{c}$ to 750 $\mathrm{MeV} / \mathrm{c}$ with $75 \mathrm{mSr}$.

Targets used were $\mathrm{C}, \mathrm{NaF}, \mathrm{KCl}, \mathrm{Ag}$, and $\mathrm{Pb}$ of about $0.5 \mathrm{~g} / \mathrm{cm}^{2}$ thickness. This combination of detection angles corresponds to a scattering angle of $145^{\circ}$ in pd center of mass. In the following discussion the event that leaves an excitation energy in the residual nucleus of less than $100 \mathrm{MeV}$ is defined as QES. Figure 1 shows momentum spectra of the proton and the deuteron in the QES region. Calculations based on piane-wave-impulse approximation (PWLA) have been made to fit the shape of the proton spectrum. In the calculations, the Gaussian distribution. of $\left\langle\mathrm{P}_{\mathrm{m}}\right\rangle$ ari the free pd-elastic-scattering cross sections here assumed. The fit was made only to the proton spectrum because a momentum resolution of the forward spectrometer was too poor to see the natural width of the deuteron. The best fit is drawn in Fig. 1a. The parameters used are $\sigma_{\mathrm{pm}}=(85 \pm 15) \mathrm{MeV} / \mathrm{c}$ and $\mathrm{BE}=60$ $\mathrm{MeV}$. The calculated deuteron spectrum is also shown in Figure $1 \mathrm{~b}$ for reference. The value of fitting parameters does not differ for the $\mathrm{p}+\mathrm{C}$ reaction, suggesting an independence of $\sigma_{\mathrm{pn}}$ on the target.

The rms momentum and $\sigma$ are related as $\left\langle\mathrm{p}^{2}\right\rangle^{1 / 2}=\sqrt{3} \sigma$, hence $\left\langle\mathrm{p}_{\mathrm{pm}}^{2}\right\rangle^{1 / 2}=150 \mathrm{MeV} / \mathrm{c}$. This value is considerably smaller than the value expected from the uncorrelated pn pairs, showing the large correlation of negative sign $\sqrt{2}<\mathrm{p}_{\mathrm{p}} \mathrm{p}_{\mathrm{n}}>^{1 / 2}=(-230+30)$ $\mathrm{MeV} / \mathrm{c}$. The comparison of pd, QES and (p,d) cross sections suggests that the bigh-momentum nucleon is associated with another high-momentum nucleon moving in the opposite direction.

Finally, the target mass dependence of the ratio (R) of momentum-integrated pd QES cross sections to that of pp QES supplies information on the deuteron mean free path.

The mean free path of the high-energy (1.7 $\mathrm{GeV} / \mathrm{c}$ ) deuteron has been estimated as $1.7 \pm 0.3 \mathrm{fm}$ through the mass dependence of the ratio of pd and pp 
quasi-elastic-scattering cross sections.

Footnotes

condensed from a paper in preparation.

†Present address: Department of Physics, Faculty of Science, University of Tokyo, Tokyo, Japan

†Present address: KEK National Laboratory for High Energy Physics, Ibaraki, Japan

Fig. 1. Momentum spectra of backward proton (a) and forward deuteron (b) from pd QES events. The dashed curves show the results of a PWIA calculation.

XBL $831-7580$
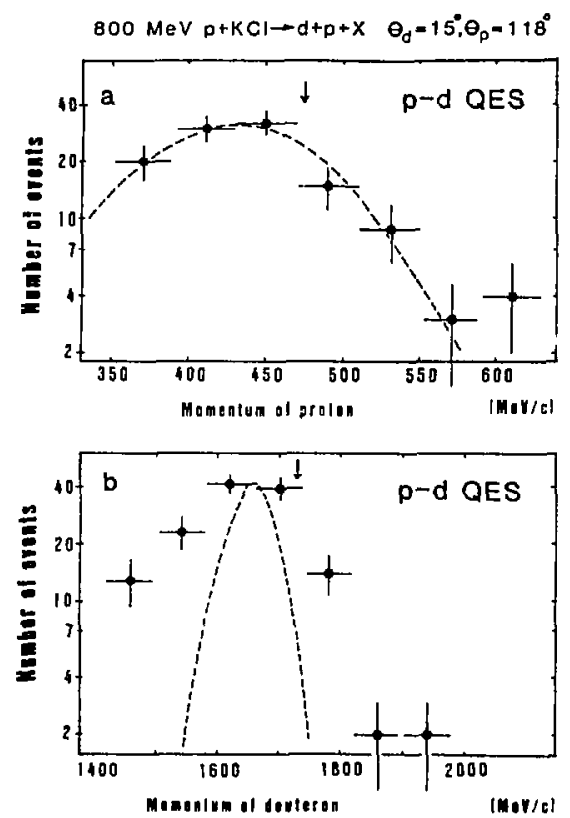

\title{
- Custer Formation Observed with a $4 \pi$ Detector and the Question of Entropy Production in High Energy Nuclear Collisions*
}

\author{
H.H. Gutbrod, ${ }^{\dagger}$ B. Kolb, ${ }^{\dagger}$ H. Löhner, ${ }_{1}^{\ddagger 1}$ B. Ludewigt, ${ }^{\ddagger}$ M.R Maier, ${ }^{\ddagger}$ \\ A.M. Poskanzer, T. Renner, H. Riedesel, ,t H.G. Ritter, ${ }^{t}$, H. Spieler, ${ }^{1 * * *}$ \\ A. Warwick, F. Weik, ${ }^{\dagger} \#$ and $H$. Wiemant
}

With the assumption that relativistic nuclear coll:sions allow one to study the equation of state of nuclear matter, the hydrodynamical model predicts an increase in entropy for a phase transition at high nuciear density. Recently it was pointed out ${ }^{\prime}$ that entropy can be related to cluster production, in particular to the ratio of deuteron yield to proton yield. This has stimulated a dispute among theorists over whether the information about the early state inherent in this ratio gets lost during the expansion phase or is disturbed only via second order effects; whether the deuteron and proton yields are the proper observables; or whether one should use the quantities deuteron-like and proton-like defined ${ }^{2}$ as $\mathrm{d}$-like $=\mathrm{d}+1.5 \cdot\left({ }^{3} \mathrm{He}+{ }^{3} \mathrm{H}\right)+3 \cdot{ }^{4} \mathrm{He}$ and p-like $=$ $\mathrm{p}+\mathrm{d}+\mathrm{t}+2 \cdot\left({ }^{3} \mathrm{He}+{ }^{4} \mathrm{He}\right)$. Until now these studies have been limited to single particle inclusive data, which mix 
together reactions of all impact parameters. With the Plastic Ball/Wall spectrometer ${ }^{3}$ for the first time $4 \pi$ data are available to investigate this subject. Figure 1 shows the ratios averaged over many events as a function of charged particle multiplicity $(a, b)$ and as a contour plot on an event-by-event basis (c,d). The inclusion of heavier clusters obviously decreases the dispersion in the ratio and supports the choice of these variables in the analysis. The increase in deuteron production with increasing multiplicity (Fig. 1a,b) can be described by a model introduced by Sato and Yazaki, which takes into account both the size of the deuteron and the volume of the participant region. The coalescence radius $p_{0}$ in momentum space is related to the deuteron radius $r_{d}$ and that of the participant volume $r_{p}$ via

$$
\frac{d-l i k e}{p-l i k e^{2}}=\frac{4 \pi}{3} p_{0}^{3} \simeq \frac{1}{\left(r_{d}^{2}+r_{p}^{2}\right)^{3 / 2}}
$$

while $I_{p}$ can be related to the observed charges by $r_{p}=r_{0}(2 p-\text { like })^{1 / 3}$ with $r_{0}$ as a free parameter. Figure 2 shows a fit to the data with $r_{d} / r_{0}=2.6$.

From the comparison of the data with different projectile-target combinations and different energies taken with the Plastic Ball/Wall spectrometer, we might be able to obtain information about the compression occurring during the collision.
Footnotes and References

"Condensed from LBJ - 14888

†Gesellschaft îür Schwerionenforschung, Darmstadt, West Germany

‡Universität Marburg, Marburg, West Germany

\$Present address: Universität Münster, Münster, West Germany

**Permanent address: Lawrence Berkeley Laboratory, Berkeley, Ca 94720

†+Present address: Max-Planck-Institu für Kernphysik, Heidelberg, West Germany

¥¥Present address: Toledo Corp., Köln, West Germany

1. P. Siemens and 3. Kapusta, Phys. Rev. Lett. 43 (1979) 1486

2. G. Bertsch and J. Cugnon, Phys. Rev. C24 (1981) 2514

3. A. Baden, H.H. Gutbrod, H. Löhner, M.R. Maier, A.M. Poskanzer, T. Renner, H. Riedesel, H.G. Ritter, H. Spieler, A. Warwick, F. Weik, and H. Wieman, Nucl. Instr. Meth. 203 (1982) 189

4. H. Sato and K. Yazaki, Phys. Lett. 98B (1981) 153 


\section{$\mathrm{Ca}+\mathrm{Ca} \mathrm{EA}=400 \mathrm{MeV}$ CENTRAL TRIGGER}

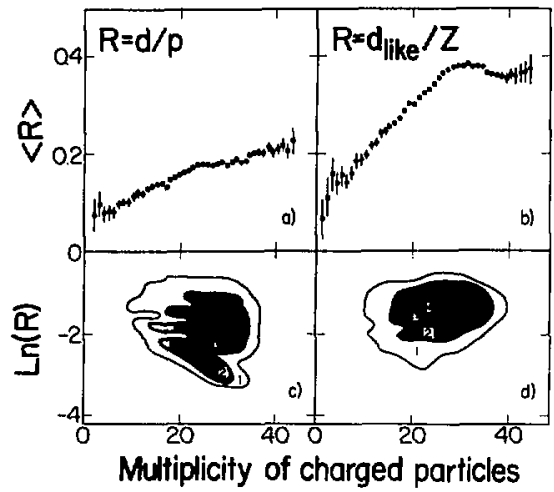

Fig. 1. a,b: Ratio of deuteron to protion production (a) and ratio of deuteron-like to proton-like particles (b) as a function of the total observed charged particie multiplicity for the reaction $\mathrm{Ca}$ on $\mathrm{Ca}$ at $400 \mathrm{MeV} / \mathrm{u}$. c,d: Corresponding event-by-event contour plots of the logarithm of the ratios versus the charged particle multiplicity. Relative intensities are indicated by the contour lines.

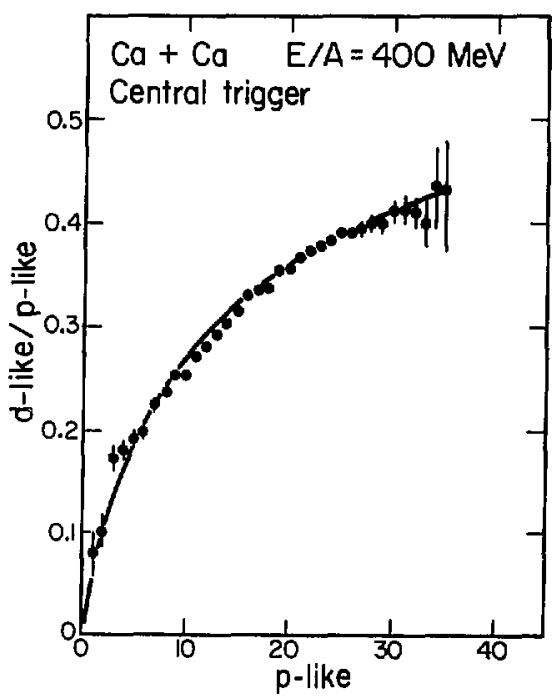

Fig. 2. Ratio of the number of deuteron-like to protonlike particles as a function of the number of proton-like particles. The solid curve represents a fit to the data.

XBL-829-1162

XBL 826-1433

\title{
Inclusive Negative Pion Production in ${ }^{40} \mathrm{Ar}+\mathrm{KCl}$ at $1.8 \mathrm{GeV} /$ nucleon
}

\author{
R. Brackmann, J.W. Harris, A Sandoval,* R. Stock, H. Stroebele, K.L. Wolf,*s \\ H.G. Pugh, L.S. Schroeder, RE. Renfordt, , and M. Maier, ${ }^{\ddagger}$
}

Inclusive negative pion spectra obtained in a Streamer Chamber experiment for central collisions in the reaction $\mathrm{Ar}+\mathrm{KCl}$ at a bombarding energy of 1.8 $\mathrm{GeV} /$ nucleon have been investigated and compared with the result of an intranuclear cascade calculation by Cugnon. 'The cascade code has been modified so the angular dependence of the $\Delta$-production in $\mathrm{NN} \rightarrow \Delta \mathrm{N}$ and its decay $\Delta \rightarrow N_{\pi}$ reproduced the characteristic NN $\rightarrow \mathrm{NN}_{\pi}$ forward-backward angular distribution observec at these energies.
The mean negative pion multiplicity is overpredicted in the cascade calculation by $20 \%$ as discussed in ref. 2. Other inclusive observables, e.g., mean total energy or the slope parameters in the energy spectra, agree within $10 \%$ or better with the data: In analyzing more differential quantities, we observe deviations in the emission pattern for high energy pions.

The angular distribution of the pions in the NNc.m. system has been fitted to 


$$
\frac{d \sigma}{d(\cos \theta)}=\left(1+a \cos ^{2} \theta\right)
$$

for different total pion c.m. energy bins. The fit parameter $a$ is plotted as a function of the pion c.m. energy in Fig. 1b. About $70 \%$ of the pions are emitted at low energies (Fig. 1a) with an isotropic angular distribution (Fig. 1b). Pions in the energy range $290<-\mathrm{E}<550$ $\mathrm{MeV}$ are emitted preferentially in the forward-backward direction and for $E>550 \mathrm{MeV}$ the distribution is again more isotropic. The cascade model, in contrast, predicts an increasingly forward-backward emission for energies $\mathrm{E}>240 \mathrm{MeV}$ (Fig. 1b).

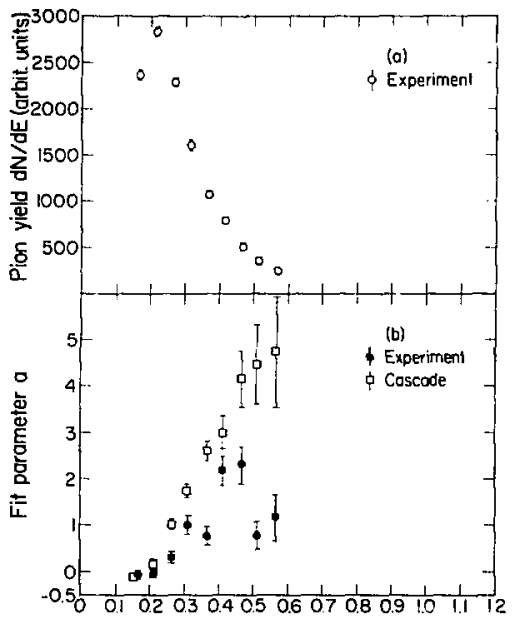

Fig. 1.

Neg. pion totol $E_{c . m .}(\mathrm{GeV})$

a) Experimental yield of negative pions as a function of the total pion c.m. energy.

b) From the fit of the angular distribution to $\frac{d \sigma}{d(\cos \theta)} \sim\left(1+a \cos ^{2} \theta\right)$, the fit parameter $a$ is plotted as a function of the total pion c.m. energy.

XBL 828-1088

\section{Footnotes and References}

*Gesellschaft für Schwerionenforschung, Darmstadt, West Germany

†Institut für Hochenergiephysik, Universität Heidelberg, West Germany
The transyerse momentum distribution as function of $p_{i}$ also shows differences between the data and the cascade result (Fig. 2). While the data approach more an isotropic distribution, solid line, the cascade shows an almost constant $\left\langle\mathrm{p}_{\perp}\right\rangle$ as a function of $\mathrm{p}_{\mathrm{I}}$ corresponding to a prolate shape of the momentum emission.

The origin of the deviations is not fully under3tood. One reason may be that the isotropically emitted pions from multiple production processes are omitted in the cascade calculation, although their contribution to the total cross section amounts to $15 \%$. The effect of this process has to be studied in more detail.

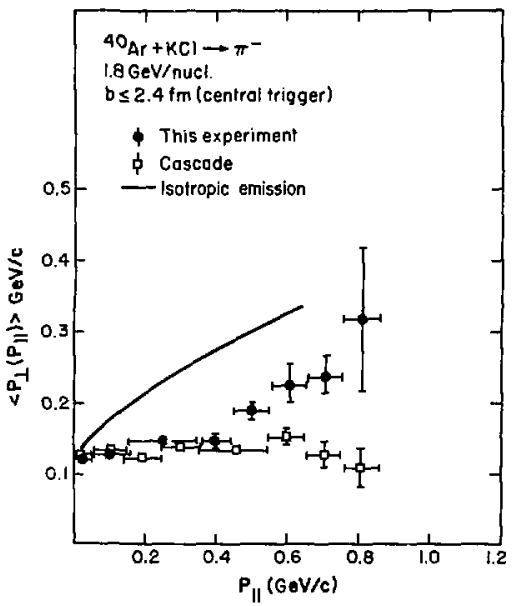

Fig. 2. Mean transverse momentum of the negative pions as a function of the paraliel momentum component.

XBL 828-1087

†Fachbereich Physik, Universität Marburg, West Germany

§Permanent address: Texas A\&M University, Coliege Station, Texas 77843

1. J. Cugnon, D. Kir.zt, and J. Vandermeulen, Nucl. Phys. A379 (1982) 553

2. R. Stock, et al., Phys. Rev. Lett. 49 (1982) 1236 


\title{
Pions Produced Near the Center-of-Mass Velocity in Heavy-Ion Collisions*
}

\author{
K.A. Frankel, J.A. Bistirlich, R Bossingham, H.R. Bowman, K.M. Crowe, C.J. Martoff, \\ D. Murphy, J.O. Rasmussen, J.P. Sullivan, ${ }^{\ddagger}$ W.A. Zajc, J.P. Miller, ${ }^{\dagger}$ O. Mashimoto, \# \\ M. Koike, " J. Peter," W. Benenson," G.M. Crawley," E. Kashy," J.A. Nolen, Jr.," and J. Quebert"t
}

The cross section for producing $\pi^{+}$and $\pi^{-}$at velocities close $: 0$ that of the center of mass was studied for wes ${ }^{40} \mathrm{Ar}+{ }^{40} \mathrm{Ca}$ reaction at $\mathrm{E} / \mathrm{A}=1.05 \mathrm{GeV}$. The $\pi^{+}$ and $\pi^{-}$data show a flat plateau around $y_{\text {f.m. }}=0$. The $\pi^{-} / \pi^{+}$ratio of $1.5 \pm 0.2$ is much lower than published theoretical predictions. Data were also obtainst for carbon and usanium targets.

The $\pi^{-} / \pi^{+}$for $\mathrm{Ar}+\mathrm{Ca}$ is $1.5 \pm 0.2$ for data taken at the center of mass. Calculations by Cugnon and Koonin ${ }^{1}$ predict the ratio $\mathbf{R}$ to be about 5.5 for $\mathrm{Ar}$ $+\mathrm{Ca}$ at this point. The structure predicted in their calculation is also inconsistent with our essentially flat spectrum.

We have also taken data with carbon and uranium targets. The data on carbon are essentially flat, as are the data on uranium for $\pi^{+}$. The uranium $\pi^{-}$data have a slight falloff going forward from mid-rapidity. The invariant cross sections near nucleon-nucleon center-ofmass rapidity are as follows: $\mathrm{MeV}^{* 2}$

For $\mathrm{C}, \sigma_{+} \sim 2.5 \mu \mathrm{b} \mathrm{sr}{ }^{-1} \mathrm{MeV}^{-2}, \sigma_{-} \approx 4.2$;

for $\mathrm{KCl}, \sigma_{+} \sim 8 \mu \mathrm{b} \mathrm{sr} \mathrm{MeV}^{-2}, \sigma_{-} \sim 12 \mu \mathrm{b} \mathrm{sr}^{-1}$

for $U, \sigma_{+} \approx 24, \sigma_{-} \approx 85$.

For the few data points at which our $\pi^{+}$data directly overlap those of Wolf, et al., ${ }^{2}$ there is remarkable agreement. We disagree only on their lowest and third lowest energy data points at $30^{\circ}$ (lab) reflected about the center of mass. Thus, our data indicate a flatter cross section near rest in the c.m. system.

\section{Footnotes and References}

"Condensed from Physical Review C25 (1982) 1102

†Physik Institute der Universität Zürich, Zürich, Switzerland

$\ddagger$ Cyclotron Institute, Texas A\&M University, College Station, Texas

§University of Pennsylvania, Philadelphia, Pennsylvania †+Department of Physics, Boston University, Boston, Massachuset1s 02215

\#Institute for Nuclear Study, Tokyo, Japan

§§Institut de Physique Nucleaire, Orsay, France

* Michigan State University, East Lansing, Michigan 48824

††Universite de Bordeaux, Le Haut-Vigneau, 33170 Gradignan, France

1. J. Cugron and S.E. Koonin, Nucl. Phys. A355 (1981) 477

2. K.L. Wolf, H.H. Gutbrod, W.G. Meyer, A.M. Poskanzer, A. Sandoval, R. Stock, J. Gosset, C.H. King, Nguyen Van Sen, and G.D. WestfalI, Phys. Rev. Lett. 42 (1979) 1448

\section{Strong Coulomb Effects on Pions Produced in Heavy Ion Collisions*}

\author{
J.P. Sullivan, J.A. Bistirlich, H.R. Bowman, R. Bossingham, T. Butke, K.M. Growe, \\ K.A. Frankel, C.J. Martoff ${ }^{\ddagger} J$. Miller, D.L. Murphy, J.O. Rasmussen. W.A. Zajc,

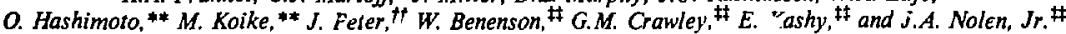

Doubly differential cross sections for the production of $\pi^{+}$and $\pi^{-}$near the velocity of the incident beam for pion laboratory angles from 0 to 20 degrees are presented. Beams of $20 \mathrm{Ne}$ with $\mathrm{E} / \mathrm{A}=280,380$, and $480 \mathrm{MeV}$ and ${ }^{40} \mathrm{Ar}$ with $\mathrm{E} / \mathrm{A}=535 \mathrm{MeV}$ incident on $C, \mathrm{NaF}, \mathrm{KCl}, \mathrm{Cu}$, and $\mathrm{U}$ targets were used. A sharp peak in the $\pi^{-}$spectrum and a depression in the $\pi^{+}$ spectrum is observed at $0^{\circ}$ near the incident projectile velocity. The effect is explained in terms of Coulomb interactions between pions and fragments of the incident beam. Least squares fits to the data using ine Coulomb correction formulas of Gyulassy and 
Kaufimann ${ }^{1}$ and an effective projectile fragment charge are made. The relationship between these data and previously measured projectile fragmentation data is discussed and a simple parameterization of projectile-mass, target-mass, and beam-energy dependence of the differential cross sections is given.

Figures $1-4$ show cuts through the peak in the $\pi^{-}$ spectra (hole in the $\pi^{+}$spectra) as a function of laboratory angle and laboratory momentum for each of the nearly-equal-mass projectile-target combinations studied. The vertical error bars are statistical. The horizontal error bars on the graphs of cross section versus angle show the angular resolution and, on the graphs of cross section versus momentum, the momentum resolution. The solid curves are least squares fits of functions based on Gyulassy and Kauffmann's theoretical expressions. These solid curves have had the resolution of the spectrometer folded into them. The dashed curves are the same functions before folding with the resolution and the dotted curves are the fitted pion spectra in the absence of Coulomb effects.

In the case of the $\pi^{+}$data at $\mathrm{E} / \mathrm{A}=380 \mathrm{MeV}$, we have an overlap with Nakai, et al. ${ }^{2}$ There is a $30 \%$ disagreement in normalization. We therefore apply a normalization factor of 0.75 to their data and combine it with our data to produce the Lorentz-invariant cross section contour plot of Fig. 5. Here the approximate symmetry about the center of mass has been used to reflect the data sets of both studies about the center of mass.

For all the projectile-target combinations that were studied, a peak in the $\pi^{-}$differential cross section (and in the $\pi^{-} / \pi^{+}$ratio) was observed slightly lower than the velocity of the incident beam. The shifts of the peaks from the momentum associated with the beam velocity are, within our experimental uncertainties, independent of beam energy over the range reported here. For the neon beam this pion momentum shift is $3.2 \pm 1.6 \mathrm{MeV} / \mathrm{c}$ in the rest frame of the incident beam. For ${ }^{40} \mathrm{Ar}$ the corresponding shift is $2.0 \pm 1.7$ $\mathrm{MeV} / \mathrm{c}$. The depression in the $\pi^{+}$cross section near beam velocity is broader than the corresponding $\pi^{-}$peak but has approximately the same downshift as does the $\pi^{-}$peak.

\section{Footnotes and References}

*Condensed from Physical Review C25 (1982) 1499

†Cyclotron Institute, Texas A\&M University, College Station, Texas

†Physik Institute der Universität Zürich, Zürich, Switzerland
\$University of Pennsylvania, Philadelphia, Pennsylvania **Institute for Nuclear Study, University of Tokyo, Tanashi, Tokyo 188, Japan

††Institut de Physique Nucleaire, B.P. No. 1, 91406 Orsay Cedex, France

¥¥Michigan State University, East Lansing, Michigan 48824

1. M. Gyulassy and S.K. Kaufimann, Nucl. Phys. A362 (1981) 503

2. K. Nakai, J. Chiba, I. Tanthata, M. Sasao, H, Bowman, S. Nagamiya, and J.O. Rasmussen, Phys. Rey. C20 (1979) 2210

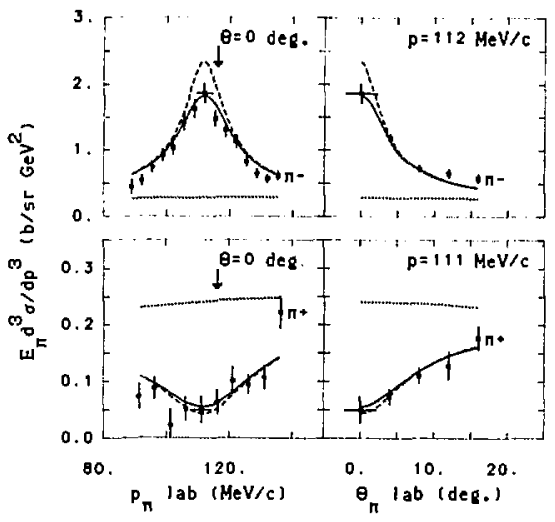

Fig. 1. Lorentz invariant cross section cuts for $\mathrm{Ne}+$ $\mathrm{NaF} \rightarrow \pi^{ \pm}$at $\mathrm{E} / \mathrm{A}=281 \mathrm{MeV}$. The left side of the graph shows the cross section versus momentum at 0 degrees in the laboratory for $\pi^{-}$(top) and $\pi^{+}$(bottom). The right side shows the cross section yersus laboratory angle at a fixed laboratory momentum near the peak in the $\pi^{-}$spectrum. The solid line is from a least squares fit of a function based on the Coulomb correction equations of Gyulassy and Kaufimann. ${ }^{1}$ This solid line has the experimental resolution folded into it. The dashed line is the same function before folding with the resolution. The dotted line shows the cross section predicted by the uncharged pion source function to which the Coulomb corrections were applied. The arrows on the left-hand graphs mark the velocity of the incident beam.

XBL 816-10182 


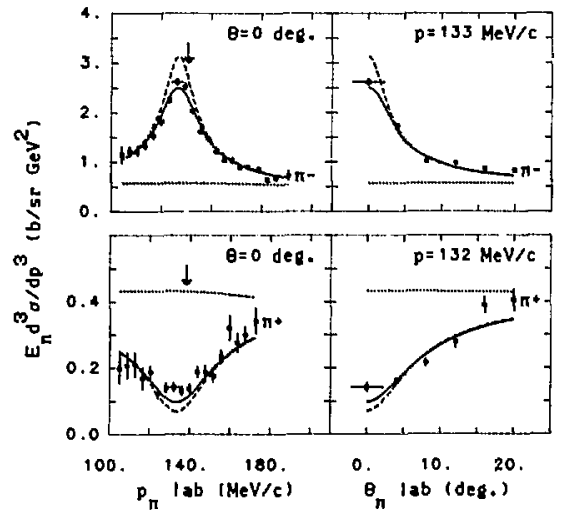

Fig. 2. Lorentz invariant cross section cuts for $\mathrm{Ne}$ $+\mathrm{NaF} \rightarrow \pi^{ \pm}$at $\mathrm{E} / \mathrm{A}=380 \mathrm{MeV}$. See also the caption for Fig. 1.

XBL 816-10183

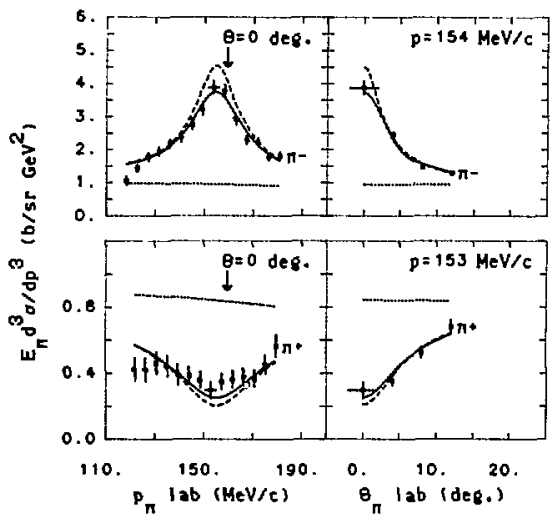

Fig. 3. Lorentz invariant cross section cuts for $\mathrm{Ne}+$ $\mathrm{NaF} \rightarrow \pi^{ \pm}$et $\mathrm{E} / \mathrm{A}=483 \mathrm{MeV}$. See also the caption for Fig. 1 .

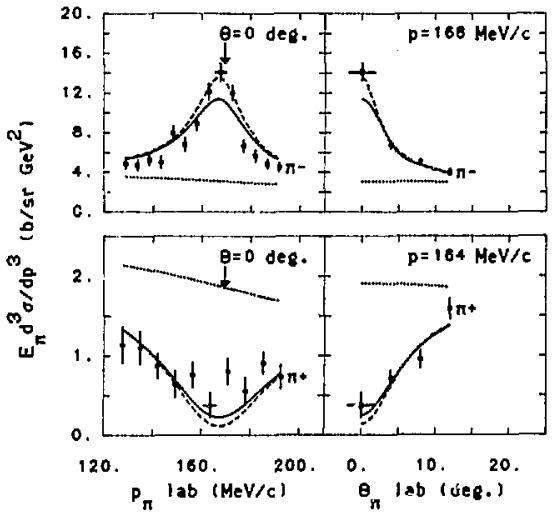

Fig. 4. Lorentz invariant cross section cuts for Ne + $\mathrm{NaF} \rightarrow \pi^{ \pm}$at $\mathrm{E} / \mathrm{A}=534 \mathrm{MeV}$. See also the caption for Fig. 1 .

XBL 816-10186

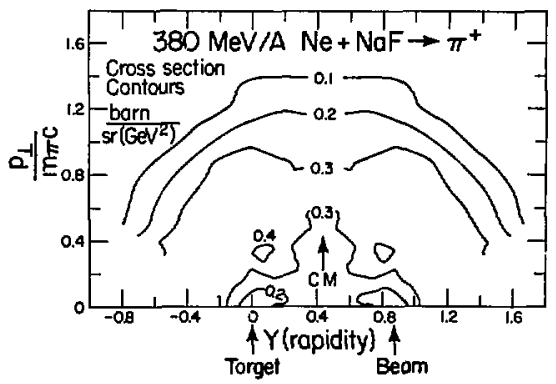

Fig. 5. Contours of Lorentz invariant cross section $\left(E_{1} d^{3} \sigma / d p_{r}^{3}\right.$ on a rapidity $\left[y=\tanh ^{-1}\left(p_{l} / E\right)\right]$ us $p_{\perp}$ plot for Ne $+\mathrm{NaF}$ at E/A $380 \mathrm{MeV}$. The present results have been combined with the data of Nakai, et al., ${ }^{2}$ at larger angles to produce this plot. The cross sections from Nakai, et al., have been multiplied by a factor of $3 / 4$ to correct for the difference between our normalization and theirs.

XBL 815-2270 


\title{
Funny Hills in Pion Spectra from Heavy-Ion Collisions*
}

\author{
John O. Rasmussen
}

I shall restrict my discussion for the most part to the hills and valleys in heavy ion pion spectra that show up at the lower pion energies.

\section{Three Kinds of Funny Hills}

We first examine the main $\pi^{+}$hills observed by our TOSABE (TOkyo-OSaka-BErkeley) collaboration using a scintillation range telescope on the ${ }^{20 \mathrm{Ne}}+\mathrm{NaF}$ system At 800 AMeV there is a main peak of $\pi^{+}$in the backward direction at a lab rapidity of -0.4 . Of course, by symmetry there must be a corresponding forward peak. These peaks are explained in the isobar model as the decay of aligned $\Delta(1232)$ resonances of spin $3 / 2$. For $\mathrm{Ne}+\mathrm{NaF}$ at $400 \mathrm{AMeV}$ the peak has pulled in to near target rapidity but we may still attribute the peak to decay of a $\Delta(1232)$, now virtual. We called attention to a second kind of funny hill in the $800 \mathrm{AMeV}$ data involving much slower pions (c.m.) and situated near $90^{\circ}$ (c.m.) at $\sim 0.5 \mathrm{~m}$ c momentum. This funny hill of the second kind did not appear in 400 AMeV data.

We next consider the sharpest and most dramatic feature, the $\pi^{-}$peaks near beam velocity. To follow the chronological orde: of their first observation I shall call them for this talk funny hills of the third kind. Their thorough investigation is the subject of a separate article. ${ }^{1}$ I shall just show one example from yet more recent work ${ }^{2}$ In Fig. 1 we see isometric and contour plots of the Lorentz-invariant cross section for $\pi^{-}$in Ne $+\mathrm{NaF}$ at $138 \mathrm{AMeV}$. The $\pi^{+}$spectra always show a depression near beam velocity. We believe the $\pi^{-}$peak and $\pi^{+}$hole are consequences of Coulomb focusing by projectile fragments. Quantitative fits on this basis are made by Radi, et al., ${ }^{3}$ in which the primary fragment distribution and momentum dispersions of compound nuclei before nucleon evaporation play the central role.

$$
\pi^{-} / \pi^{+} \text {Ratios near Center of Mass }
$$

In subsequent work Cugnon and Koonin ${ }^{4}$ restudied the pion Coulomb problem by Monte Carlo methods with relativistic trajectories. Their plot of $\pi^{-} / \pi^{+}$ratios at $0^{\circ}$ for the $\mathrm{Ar}^{40}$ on $\mathrm{Ca}^{40}$ collision at 1.05 AGeV shows the familiar beam velocity peak ("third kind") but in addition a secondary peak at rest in the center of mass. Their $\pi^{-} / \pi^{+}$ratio is about 5.5 at the center of mass.

Our group ${ }^{\dagger}$ then measured both $\pi^{-}$and $\pi^{+}$spectra at and near rest in the c.m. frame for $1.05 \mathrm{~A} \mathrm{GeV}{ }^{40} \mathrm{Ar}$ on natural $\mathrm{C}, \mathrm{Ca}$, and $\mathrm{U}$ targets. The spectrum for ${ }^{40} \mathrm{Ca}$ is quite flat over the whole region of our spectrometer acceptance including the nucleon-nucleon center of mass, in contrast to the theoretical prediction.

In Table $I$ are summarized the measured $\pi^{+} / \pi^{-}$ ratios at the nucleon-nucleon center of mass.

Table I

$\pi^{-} / \pi^{+}$ratios at the Nucleon-Nucleon c.m. for Heavy Ion Collisions

\begin{tabular}{cccc}
$\begin{array}{c}\text { Lab Energy } \\
\text { per Nucleon } \\
(\mathrm{MeV})\end{array}$ & Projectile & Target & $\begin{array}{c}\pi^{-} / \pi^{+} \text {Ratio } \\
\text { at c.m. } \\
( \pm 10 \%)\end{array}$ \\
\hline \multirow{2}{*}{1050} & ${ }^{40} \mathrm{Ar}$ & $\mathrm{C}$ & 1.6 \\
& & $\mathrm{Ca}$ & 1.5 \\
655 & ${ }^{20} \mathrm{Ne}$ & $\mathrm{U}$ & 3.2 \\
& & $\mathrm{NaF}$ & 1.76 \\
\hline
\end{tabular}

Faced with the large discrepancy between theory and experiment on the $\pi^{-} / \pi^{+}$ratios, we undertook extensive new Monte Carlo trajectory studies. The most important difference from Cugnon and Koonin was probably our introduction of absorption for any trajectories going through nuclear matter. Table II summarizes our results. 
Table II

Zero Energy (c.m.) $\pi^{-} / \pi^{+}$Ratio Factors

\begin{tabular}{lll} 
Spectator Coulomb factor & 1.40 & $1.36^{\star}$ \\
Neutron-excess factor & 1.083 & 1.17 \\
Participant Coulomb factor & 1.11 & 1.10 \\
\hline & & \\
Final Product (theory, ref. 5) & 1.68 & 1.75 \\
Experiment & $1.76 \pm 0.1$ & $1.5 \pm 0.2$
\end{tabular}

-Calculated by scaling from Ne Monte Carlo Results

Possible Pion Orbiting about Fireball(s)

In Fig. 2 you see $0^{\circ}$ and $90^{\circ}$ (c.m.) cuts of the $\pi^{+}$and $\pi^{-}$invariant cross sections for $\mathrm{Ne}$ plus $\mathrm{NaF}$ at $655 \mathrm{AMeV}$. The $\pi^{+}$funny hill of the second kind has its counterpart in $\pi$ ! It is thus highly unlikely that there is a simple Coulomb explanation for the $90^{\circ} \mathrm{c.m}$. hills of the second kind. We did not have sufficient beam time to measure far enough out along the $90^{\circ}$ c.m. line to cover the $\pi^{+}$bump region, but the data are consistent with such a bump for $\pi^{+}$as well as $\pi^{-}$. For $\pi^{-}$we show in the solid line a comparison with work of Nagamiya, et al. ${ }^{6}$ This line represents an interpolation in beam energy between $400 \mathrm{AMeV}$ and $800 \mathrm{AMeV}$, and it represents an extrapolation down in pion energy. Their lowest measured pion point would be on the right margin of Fig. 2, and the extrapolation follows a Boltzmann form, Gaussian in momentum, exponential in energy.

At the high-momentum edge, where the two data sets nearly overlap, our data appear to be approaching theirs. At the ofigin the extrapolation from the Nagamiya work matches our data, and the nature of the hill of the second kind is delineated. The excess cross section above the extrapolated line forms a broad hill from $0.4-0.6 m_{x} c$ dropping to zero above and below this momentum region.

What could be the significance of this midrapidity hill? It appears that simple application of the uncertainty principle to this ubiquitous momentum of $\sim 0.5 \mathrm{~m}_{\mathrm{c}} \mathrm{g}$ gives a distance of $\sim 2$ pion Compton wavelengths, or $\sim 3 \mathrm{fm}$. This distance is around the size expected for the hot fireball source region.

We postulate that this mid-rapidity funny hill may indicate pion orbiting in or about the fireball. (Cf. discussion in report "Pions from and about Heavy
Ions" in this report.)

\section{Footnotes and References}

*Condensed from invited paper presented at the Relativistic Heavy Ion Winter Sehool, Banf, Alberta, Canada, February 22 - 26, 1982 (LBL-14174)

†Principal collaborators on our JANUS magnetic pion spectrometer measurements at mid-rapidity have been the following: James Bistirlich, Harry Bowman, Roy Bossingham, Kenneth Crowe, Kenrieth Frankel, Jeff Martoff, James Miller, Don Murphy, John Rasmussen, John Sullivan, William Zajc, and Eunice Yoo, U.C. Lawrence Berkeley Laboratory; Osamu Hashimoto and Masahiro Koike, Institute for Nuclear Studies, University of Tokyo; Jean Quebert, University of Bordeadx, Walter Benenson, Gary Crawley, Edwin Kashy, and Jerry Nolen, Michigan State University; and Jean Peter, Laboratory for Nuclear Research, Orsay

1. J.P. Sullivan, J.A. Bistirlich, H.R. Bowman, R. Bossingham, T. Buttke, K.M Crowe, K.A. Frankel, C.J. Martoff, J. Miller, D.L. Murphy, J.O. Rasmussen, W.A. Zajc, O. Hashimoto, M. Koike, J. Peter, W. Benenson, G.M. Crawley, E. Kashy, and J.A. Nolen, Phys. Rev. C25 (1982) 1499. Also J.P. Sullivan, Ph.D. Thesis, U. C. Berkeley, LBL-12546 (1981) unpublished.

2. D. Murphy, J. Bistirlich, H. Bowman, R. Bossingham, $\mathrm{K}$ Crowe, $\mathrm{O}$. Hashimoto, $\mathrm{K}$ Frankel, J. Rasmussen, J. Sullivan, W. Zajc, J. Peter, W. Benenson, G. Crawley, E. Kashy, and J. Nolen, Bull. Am. Phys. Soc. 26 (1981) 1148 
3. H.M.A. Radi, J.O. Rasmussen, J.P. Sullivan, K.f. Frankel, and O. Hashimoto, Phys. Rev. C25 (1982) 1518

4. J. Cugnon and S.E. Koorin, Nucl. Phys. A355 (1981) 477

$E / A=138 \mathrm{MEV} \quad \mathrm{NE}+\mathrm{NAF} \rightarrow \mathrm{X}+\pi-$
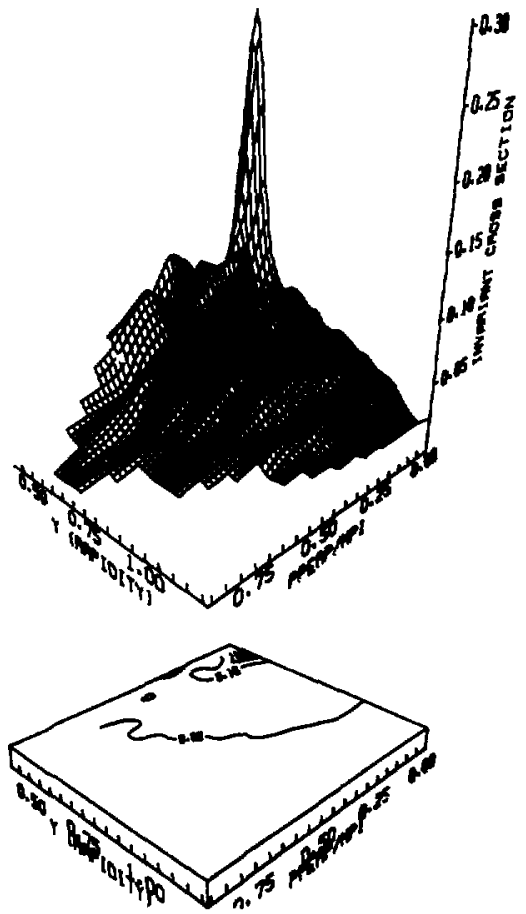

Fig. 1. Isometric and contour plots of $\pi^{-}$data ${ }^{2}$ for ${ }^{20 \mathrm{Ne}}+\mathrm{NaF}$ at $138 \mathrm{AMeV}$. Cross section is in units of b sr ${ }^{-1} \mathrm{GeV}^{-2}$. Peak is near beam velocity.

XBL 823-8269
5. H.M.A Radi, J.O. Rasmussen, KA Frankel, 3.P. Sullivan, and H.C. Song, Phys. Rev. C27 (1983) 606

6. S. Nagamiya, M.-C. Lemaire, E. Moeller, S. Schnetzer, G. Shapiro, H. Steiner, and I. Tanihata, Phys. Rev. C24 (1981) 971

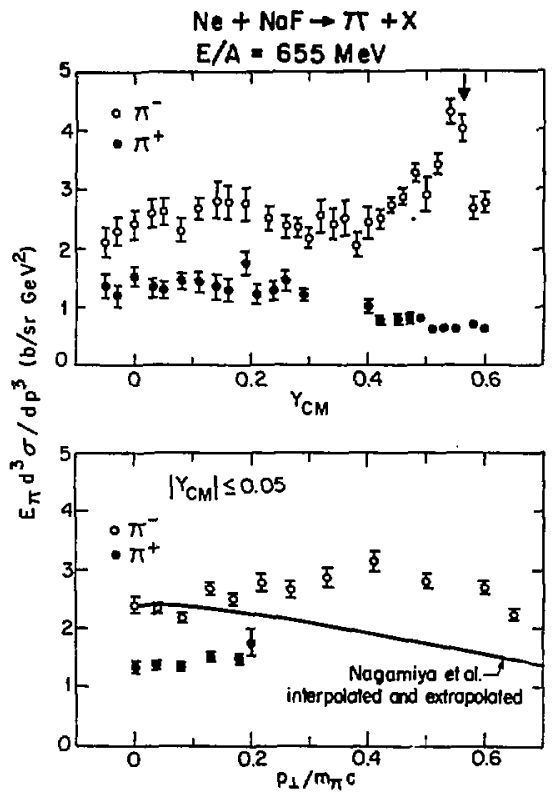

Fig. 2. Cuts of $\pi^{-}$and $\pi^{+}$production cross sections for ${ }^{20} \mathrm{Ne}$ on $\mathrm{NaF}$ at $655 \mathrm{AMeV}$. Upper half shows cut at $0^{\circ}$. The lower half shows the cut at $90^{\circ}$ (c.m.) with comparison to data extrapolated from Nagamiya, et al. ${ }^{6}$ Lorentz invariant cross sections are in units of $\mathrm{b} \mathrm{sr}^{-1} \mathrm{GeV}^{-2}$.

XBL $8112-12880$ 


\title{
Pions from and about Heavy Ions*
}

\author{
John O. Rasmussen
}

\section{Pion Thermometry}

The various particles from high energy heavy ion collisions exhibit spectra that asymptotically approach an exponential fall-off with energy suggesting a thermal distribution. There are two main problems: one, the slopes depend on angle, and two, the apparent temperatures indicated by the different particles are not the same. We restrict ourselves here to the case of symmetric systems, where target and projectile are nearly the same charge and mass. The slopes determined at the more forward angles are not reliable indicators of any thermal equilibrium situation. In the case of protons there is a persistence of initial momentum through several collisions, since elastic scattering is forwardpeaked at energies greater than $300 \mathrm{AMeV}$. In the case of pions there is a natural forward-backward peaking from the initial formation in a nucleon-nucleon collision, such distribution arising from the role of the $\Delta$ (1232) intermediate. Thus, we believe that the $90^{\circ}$ c.m. spectra of various particles are the least admixed with particles from direct processes and may provide the best thermometry of the hot reaction region.

Fig. 1 is taken from Nagamiya, et al., ${ }^{1}$ and it shows proton and pion slope parameter $E_{0}$, which is not the temperature, but is closely related to a temperature. To get a temperature from a Boltzmann distribution, one should plot just the differential cross section without multiplying by the relativistic total energy to make it Lorentz-invariant. The effective temperatures will be somewhat lower than Nagamiya's $E_{0}$ values.

The correction to $E_{0}$ to get a temperature vaiue is rather easy to make.

$$
\sigma_{\mathrm{INV}}=\mathrm{E} \frac{\mathrm{d}^{3} \sigma}{\mathrm{dp}^{3}},
$$

where $E$ is the relativistic total energy of the pion. Taking the natural logarithm of both sides and differentiating with respect to $\mathbf{E}$ gives us

$$
-\frac{1}{E_{0}}=\frac{1}{E_{x}}-\frac{1}{T} \text {, }
$$

where $E_{x}$ is the total energy at which the slopes are to be determined. If we choose to evaluate the effective temperature at a kinetic energy around twice the $\mathbf{E}_{\mathbf{0}}$ value (total $\mathbf{E}$ three times greater), then the above equations tell us that $T$, the effective temperature, is 0.75 times $\mathrm{E}_{0}$. With this in mind we can determine temperatures from Fig. 1.

The pion probe indicates temperatures smaller than those given by the simple fireball model, in which all the available kinetic energy of the geometrically overlapping matter (participants) is assumed to be therinalized. For example, at $\mathrm{E}_{\mathrm{pb}}=0.18 \mathrm{AGeV}$ there is 45 $\mathrm{MeV}$ kinetic energy per nucleon available for a fireball model temperature of $2 / 3$ of this, or $30 \mathrm{MeV}$. This is to be compared with a pion temperature of 0.75 times $25 \mathrm{Mev}$, or $18 \mathrm{MeV}$. Note also that the temperature indicated by the pions for a given system is always less than that indicated by the protons.

In an aitempt to resolve the discrepancy between proton and pion temperatures Phil Siemens and I some years ago proposed ${ }^{2}$ a blast-wave model. We derived expressions for the spectra from a system in which the available energy was divided between thermal and ordered motion of a spherically expanding blast wave. If the energy were about equally distributed between these forms, the difference in apparent temperatures was resolved. Today I would not want to defend the literal blast wave but rather point out that any ordered motion of the thermal sources in the perpendicular direction can give systematic differences in the limiting slopes of the various mass particles boiling off. Arguments have been given that a "bounce-off" effect occurs $^{3}$, and this effect provides an ordered sidewise component in the thermal sources. Furthermore, cascade-code calculations of Gyulassy and Frankel ${ }^{4}$ show a fuctuation in sideways momentum, even of head-on central collisions. Nagamiya in ref. 5 presents a kaon temperature which does not fit into my general theme that it is ordered perpendicular motion that causes the difference in slopes. The temperature inferred from the kaon $\left(\mathrm{K}^{+}\right)$production is even higher than that of the proton, rather than lying between protoin and pion temperatures. I would merely point out that the kaons are not likcly to be very good thermal probes, since their mean free paths in nuclear matter are longer than the other particles. Since they require the greatest energy for production, they may also probe just the most violent initial parts of the collision, before any thermal equilibration has set in.

\section{Pionic Orbits of Nuclear Size?}

Heavy ion pion spectra show hills or ridges in the cross sections at very low energy in the c.m., around 15 $\mathrm{MeV}$ or momentum of $0.4-0.5 \mathrm{~m}_{x} \mathrm{c}$. What is striking about the mid-rapidity bumps ${ }^{x}$ is that a simple 
application of the uncertainty relation gives a distance about equal to the nuclear radius. That is, $h$ over the bump momentum of $0.5 \mathrm{~m}, \mathrm{e}$ gives a size parameter of 2 pion Compton wave lengths, or about $2.8 \mathrm{fm}$. Of course, the bump may be shifted somewhat by Coulomb effects, so we will need in the future to accumulate data for both $\pi^{+}$and $\pi^{-}$for systems of widely differing mass to see if the mid-rapidity bumps move inward with increasing mass, as the uncertainty principle argument would predict. Ideally, we should have data somewhat sorted between peripheral and central collisions to extrack the most information. We could then take Fourier transforms of the momenturn spectrum to infer the spatial wave functions of the pions.

It is worthwhile to trace some of the roots of these notions about possible pion orbiting. Kitazo and Sano ${ }^{6}$ solved equations for thermal and chemical equilibrium among particles in the hot dense matter of heavy ion collisions, and they drew attention to a "zero-energy" component of the pion spectra. Likewise, Zimanyi, Fai, and Jakobsson considered such a component, which they gave a finite width according to the nuclear size and uncertainty principle. ${ }^{7}$

Pion Confinement in the Fireball

To get some orientation on pion orbiting conditions I have developed a computer code to solve for eigenvalues of pions in a nuclear optical potential. The methods of Ericson and Myhrer ${ }^{8}$ have been employed except that we obtain eigenvalues first in the real part of the potential, using the WKB approximation and applying Bohr-Sommerfeld quantization conditions, with modifications of ref. 9 where there is a singularity in the effective mass.

With the nuclear potential turned of I have verified that the code gives about the right Bohr value for the pionic binding energy in lowest s- and p- states. Then the first calculations with the double density of a fireball made clear that the central part of the rucleus will have a negative effective pion mass for both $\pi^{+}$and $\pi$ under a broad range of neutron-torproton ratios. The mass-singularity near the surface th'ss provides always a confining boundary for inner solutions.

\section{Footnotes and References}

*Condensed from LBL Report 14852, to appear in the Proceedings of the Summer School on Nuclear Dynamics, Pearson College, Victoria, B.C.

1. S. Nagamiya, et al., Phys. Rev. C24, 971 (1981)
2. P.J. Siemens and J.O. Rasmussen, Phys. Rev. Lett. 42, 844 (1979)

3. H. Stocker, J.A. Maruhn, and W. Greiner, Phys. Rev. Lett. 44, 725 (1980)

4. M. Gyulassy, K.A. Frankel, and H. Stöcker, Phys. Lett 110B, 185 (1982).

5. S. Nagamiya, Proc. Fifth High Energy Heavy Ion Study, Berkeley (May, 1981). LBL Report No. 12652, p. 141

6. H. Kitazoe and M. Sano, Lett. Nuovo Cim. 14, 400 (1975)

7. J. Zimanyi, G. Fäi, and B. Jakobsson, Phys. Rev. Lett. 43, 1705 (1979)

8. T.E.O. Ericson and F. Myhrer, Phys. Lett. 74B, 163 (1978)

9. V.B. Mandelzweig, A. Gal and E. Friedman, Ann. Phys. (N.Y.) 124, 124 (1980)

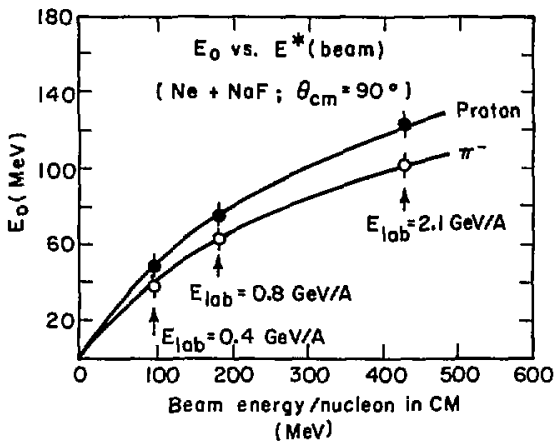

Fig. 1. Summary of slope parameters $E_{0}$ for various beam energies. (from Nagamiya, et al.)

XBL 788-1495B 


\title{
Production of Negative Pions with $183 \mathrm{MeV} /$ nucleon Ne Beams*
}

\author{
S. Nagamiya, ${ }^{\dagger}$ H. Hamagaki, ${ }^{\ddagger}$ P. Hecking, S. Kadota, ${ }^{\ddagger}$ R. Lombard, ${ }^{\uparrow}$ Y. Miake, ** \\ E. Moeller," ${ }^{\prime}$ S. Schnetzer, $\#$ H. Steiner, "II. Tanihata, ${ }^{\ddagger}$ S. Bohrmann, "** and J. Knolltt
}

Cross sections for negative pion production in 183 $\mathrm{MeV} /$ nucieon $\mathrm{Ne}+\mathrm{NaF}$ and $\mathrm{Ne}+\mathrm{Pb}$ collisions have been measured at laboratory angles from $20^{\circ}$ to $90^{\circ}$. The first goal of this experiment yas to study the mechanism of subthreshold pion production for largeangle, high-energy pions.

The second goal of the experiment was to test a recent theoretical prediction of a pionic instability effect related to pion condensation. According to Gyulassy, ${ }^{l}$ if this effect exists, then it should reveal itself in the inclusive pion spectra. Namely, the pionic instability induces an excess pion production at c.m. $90^{\circ}$ and at a c.m. momentum around $2-3 \mathrm{~m}_{x} \mathrm{c}$.

The experimental procedures are almost the same as those reported in Ref. 2 .

In Fig. 1 the energy spectra of $\pi^{-}$at c.m. $90^{\circ}$ observed for $\mathrm{Ne}+\mathrm{NaF}$ collisions are displayed together with the previously published data for the same projectile and target combination but at different beam energies. As shown, we have observed highenergy pions at subthreshold beam energies in nuclear collisions down to cross section of $\sim 1 \quad(\mu \mathrm{b}$ $\mathrm{GeV}) /\left(\mathrm{sr}(\mathrm{GeV} / \mathrm{c})^{3}\right)$. We have found no evidence for the pionic instability effect predicted by Gyulassy. The production mechanism of high-energy pions has been studied using both hard-collision and phase-space models. A naive hard-collision model without rescattering effects does not explain the data when conventional internal momentum distributions are assumed. How. ever, if both pion absorption and rescattering are included, then the model agrees to within a factor of about 2 with the data. For the production of highenergy pions it turns out that the $\pi \mathrm{N}$ rescattering is especially important. The phasi-space model also fits the present data reasonably well. From the qualitative agreement of the data with both these models, it seems likely that processes inyolving more than two nucleons play an important role for producing energetic pions at subthreshold beam energies. The relationship between these two models is still not clear, but it may be that the microscopic rescattering and absorption effects can be effectively replaced by the statistical behavior of a rather large number of nucleons.

\section{Foothotes and References}

*Condensed from LBL-14033 and Phys. Rev. Lett. 48 (1982) 1780

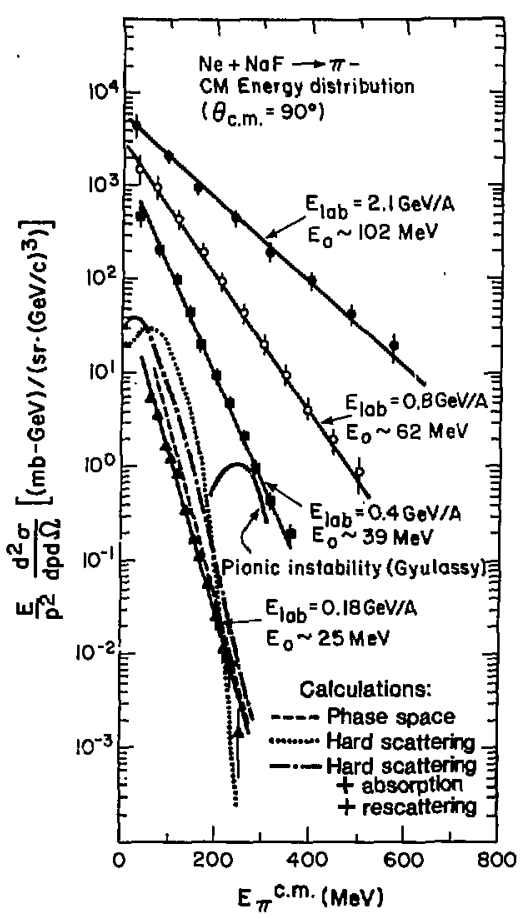

Fig. 1. Energy spectra of $\pi^{-}$at $\theta^{\mathrm{cm}}=90^{\circ}$ in $\mathrm{Ne}+$ $\mathrm{NaF}$ collisions at four beam energies, $0.18,0.4,0.8$, and $2.1 \mathrm{GeV} /$ nucleon. $\mathrm{E}_{0}$ is the inverse slope factor when the cross sections are parametrized by exp($\left.E_{.}^{c . m} / E_{0}\right)$. The solid curve is the predicted cross section for pionic instability as calculated by Gyulassy. ${ }^{1}$ The dashed line is the result of a phase-space-model calculation for $E_{\text {bean }} L a b / A=200 \mathrm{MeV}$. The dotted line is the calculation of a simple hard-collision model and the dot-dashed line is the result from the hard-collision model which includes both pion absorption and rescattering.

XBL 8112-13235 
†Also Department of Physics, Faculty of Science, University of Tokyo, Hongo, Bunkyo-ku, Tokyo, Japan

$\ddagger$ On leave from Institute for Nuclear Study, University of Tokyo, Tanashi-shi, Tokyo, Japan

§Present address: DPhN/ME, CEN de Saclay, 91191 Gif-sur-Yvette, France

**On leave from Department of Physics, Osaka University, Osaka, Japan

tton leave from Institut für Theoretische Physik, Freie Universität, Berlin, 1000 Berlin 33, Germany

$¥$ \#resent address: National Laboratory for High-
Energy Physics (KEK), Oho-machi, Tsukuba-gun, Ibaraki-ken, Japan

§\&Also Department of Physics, University of California, Berkeley

***Max Planck Institut für Kernforschung, D-6900 Heidelberg, Germany

††Gesellschaft für Schwerionenforschung (GSI), D6100 Darmstadt, Germany

1. M. Gyulassy, Nucl. Phys. A354 (1981) 375

2. S. Nagamiya, et al., Phys. Rev. C24 (1981) 971

\title{
Subthreshold $\mathrm{K}^{-}$Production in Relativistic Nuclear Collisions (RNC)*
}

\author{
A. Shor, ${ }^{\dagger}$ K. Ganezer, ${ }^{\ddagger}$ S. Abachi, ${ }^{\ddagger}$ J. Carroll, ${ }^{\ddagger} J$. Geaga, ${ }^{\ddagger}$ G. Igo, ${ }^{\ddagger}$ P. Lindstrom, $\cdot$
} T.A. Mulera, V.Perez-Mendez, A.L. Sagle, F. Zarbakhsh, and D. Woodards

We have recently observed copious production of $\mathrm{K}^{-}$in the reaction ${ }^{28} \mathrm{Si}+{ }^{28} \mathrm{Si}$ at $2.1 \mathrm{GeV} /$ nucleon. At this bombarding energy, $\mathrm{K}^{-}$production is subthreshold in nature, i.e., a minimum of $2.5 \mathrm{GeV}$ in projectile energy is required for $\mathbf{K}^{-}$production in nucleon-nucleon collisions. The $\mathrm{K}^{-}$yicld we measured is more than 20 times larger than phase space calculations which include the internal nuclear (Fermi) momentum of the colliding nucleons. Calculations which employ spectra of the relative energies between $p, \Delta$ and $\pi$ 's generated by a cascade code ${ }^{1}$ also underpredict the observed $\mathrm{K}^{-}$ yield by over an order of magnitude.

The enhanced $\mathrm{K}^{-}$production is an indication of multi-nucleon processes occurring in RNC. Collective effects such as $\mathbf{K K}$-condensation ${ }^{2}$ or $\mathbf{\phi}$-Bremsstrahlung ${ }^{3}$ may account for the large $\mathrm{K}^{-}$yield. Enhanced production of strange particles would also result if a quarkgluon plasma (or fluctuations leading to such a plasma) were to be produced in RNC. Even if such processes seldom occur, the effects on $\mathrm{K}^{-}$production may be discernable since the conventional backgrounds for the production of $\mathrm{K}^{-}$are small.

The experimental technique involves magnetically bending negative secondaries away from the positively charged fragments and transporting them along a magnetic beam line. Detector stations following each successive bend allow for high background rejection and very sensitive particle measurements. Figure 1 presents a data sample of 4 million events and shows the relative time of flight between the first two detector stations after cuts were made on Cerenkov pulse heights. The peak with the $K^{-}$TOF represents a differential cross section $D^{2} \sigma / \mathrm{dpd} \Omega$ of $1.65 \pm .35 \mathrm{mb} / \mathrm{sr}-\mathrm{GeV} / \mathrm{c}$. The upper limit for anti-proton production is $\mathrm{d}^{2} \sigma / \mathrm{dpd} \Omega<3$ $\mu \mathrm{b} / \mathrm{sr}-\mathrm{GeV} / \mathrm{c}$.

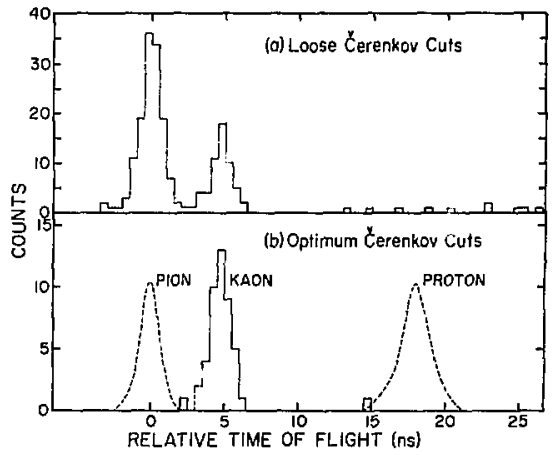

Fig. 1. Time of flight spectra for the first two detector stations. The effect of cutting on Cerenkov pulse height can be seen in the difference between the two spectra.

XBL 824-9006 
Recent reports of observation of fractional charges ${ }^{4}$ has led to the theoretical speculation that fractional charge may occasionally be liberated in RNC where color screening may be in effect ${ }^{5}$. The experimental techniques we employ for the $\mathrm{K}^{-}$measurements are well suited for direct measurements of fractional charges. For example, with our existing measurement with ${ }^{28} \mathrm{Si}$, we obtain an upper limit of $\mathrm{d}^{2} \sigma / \mathrm{dpd} \Omega<3$ $\mu b / s r-G e V / c$ for $Z=-2 / 3$ particles with a mass range of $M_{r}<m<M_{p}$ at $P / Z=1 \mathrm{GeV} / \mathrm{c}$ (assuming absorption in the thick target and counters is comparable to that for an anti-proton).

\section{Footnotes and References}

•Condensed from Phys. Rev. Lett. 48 (1982) 1597.
†LBL and UCLA, Los Angeles CA 90024 †UCLA Los Angeles, CA 90024

\$California State University, Hayward, Hayward, CA 94542

1. J. Harris, Priqate Communication

2. V.P. Berzovoii, et al., Sov. J. Nucl. Phys. 30 (1979) 581.

3. K.H. Müller, LBL-14715 (1982).

4. G.S. LaRue, et al., Phys. Rev. Lett. 46 (1981) 967.

5. R. Slansky, et al., Phys. Rev. Lett. 47 (1981) 887. 


\section{PART IV: THEORY}




\title{
1. General
}

\section{Normal and Pion Condensed States in Neutron Star Matter*}

\author{
N.K. Glendenning, P. Hecking, and V. Ruck
}

In a companion paper' a relativistic field theory of charge neutral and stable dense matter was formulated. The theory is solved in the mean field approximation. The Lagrangian is regarded therefore as an effective one, whose coupling constants are constrained by the known bulk properties of nuclear matter, the saturation density, binding, compressibility, and charge symmetry energy.

In the present work, numerical solutions to the coupled nonlinear equations defining the fields are found for the normal and pion condensed phases. The known properties of nuclear matter are insufficient to define completely the coupling constants. This freedom is exploited to investigate two extreme situations that we refer to as "stiff" or "soft" equations of state, referring to their high density behavior.

Numerous properties of the system are calculated as a function of baryon density. One such is the pion condensate energy shown in Fig. 1 for the "stiff" equation of state. Neutron-rich matter very much favors pion condensation as compared to symmetric nuclear matter. However, for the "stiff" equation of state the maximum stai mass is hardly affected by the presence of the condensate, as seen in Fig. 2. However, the "soft" equation of state can be ruled out on the basis of the condensed state, since it will not support a star mass as large as several observed masses $(\sim 1.4 \mathrm{M})$.

Cooling times of neutron stars are strongly influenced by the presence of a pion condensed state. ${ }^{2}$ Therefore, cooling times and present temperatures for a star like the Crab pulsar are calculated. However, the present accuracy of the measurements does not permit a discrimination among our results, which are tabulated in the original report.

\section{Footnote and References}

*Condensed from LBL-14505

1. N.K. Glendenning, B. Banerjee, and M. Gyulassy, LBL-12704 (accepted by Arnals of Physics)

2. O.V. Maxwell, Astroph. J. 231 (1979) 201

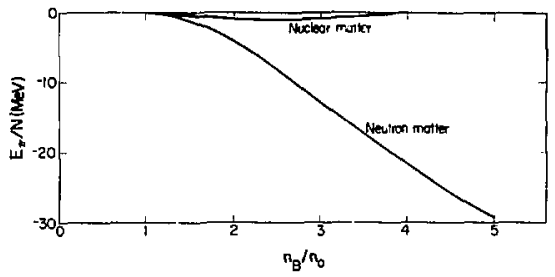

Fig. 1. Pion condensate energy in neutron star matter and nuclear matter as a function of baryon density.

XBL 829-4546

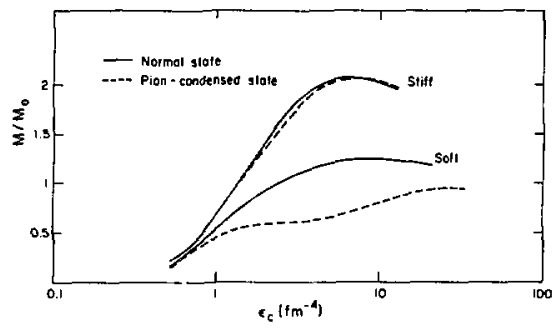

Fig. 2. Neutron star mass in units of solar mass as a function of central energy density for four states of charge neutral matter, the normal and pion condensed phases for "stif" "and "soft" equations of state.

XBL 829-1155 


\title{
The Hyperon Composition of Neutron Stars*
}

\author{
N.K. Glendenning
}

A description of neutron star matter is developed here in the framework of a relativistic ield theory of interacting nucleons, hyperons, and mesons. The theory is solved in the mean field approximation. Relativistic covariance of the theory is retained throughout.

The jinportant bulk properties of nuclear matter are imposed as constraints. These are the saturation deisity, binding energy, compressibility, and isospin symmetry energy. Subject to these constraints the equation of state is calculated for cold dense chargeneutral matter that is in chemical equilibrium.

As the density of matter rises, the Fermi energy of the neutrons will eventually exceed the threshold for decay, first to protons and then to the hyperons, most especially the $\Lambda, \Sigma, \Xi$, and $\Delta$, with the emission of electrons, muons, pions, kaons, etc. Any photons or neutrinos produced can leak out of the star, and its energy is lowered. The kaon decays are, of course, not forbidden on a time scale that is large compared to $10^{-10} \mathrm{sec}$. However, the hyperons become Pauli blocked and a net hyperon population is built up. The stable ground state of the star, achieved as a result of the various elementary processes, is sought.

At some sufficiently high density, the nature of the ground state may undergo a phase transition to the pion condensed state. We do not allow for a structural change in the ground state here, but we do allow a condensate of free pions to develop when the chemical equilibrium would require it. Likewise, of course, the electrons and muons are in chemical equilibrium with the hadrons but may be treated as free particles because the interaction is electro-weak. Our complete Lagrangian is $(h=c=1)$,

$$
\begin{gathered}
\mathscr{L}=\sum_{\beta} \bar{\psi}_{\beta}\left(\mathrm{i} \partial-\mathrm{m}_{\beta}+\mathrm{g}_{\sigma \beta^{\sigma}}{ }^{\left.-\mathrm{g}_{\omega \beta} \omega_{\mu} \gamma^{\mu}\right) \psi_{\beta}}\right. \\
-g_{\rho} \rho_{\mu 3} \mathrm{~J}_{3}^{\mu}+\mathscr{L}_{\sigma}+\mathscr{L}_{\omega}+\mathscr{L}_{\beta}+\mathscr{L}_{\lambda}-U(\sigma) \\
+\sum_{\lambda=e^{-}, \mu^{-}} \bar{\psi}_{\lambda}\left(\mathrm{j} \partial-\mathrm{m}_{\lambda}\right) \psi_{\lambda}
\end{gathered}
$$

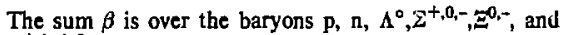
$\Delta^{++}+, 0,-$ and $\beta$ stands for their quantum numbers, the spin, isospin, 3rd component of isospin, and hypercharge. These are Yukawa-coupled to the scalar and vector mesons $\sigma$ and $\omega_{\mu}{ }^{t}$ The rho meson and nucleons are coupled through the isospin four-current of the baryons, $J_{y}$. The free Lagrangians for the mesons are denoted by $\mathscr{L}_{0} \ldots \mathscr{L}_{n}$ the lepton Lagrangians by $\mathscr{L}_{\lambda}$, and finally the $\sigma$-field self-interactions by $U(\sigma) .^{2}$ The field equations derived from $\mathscr{L}$ are solved in the mean field approximation. The Dirac equations for the baryons have the spin degenerate eigenvalue spectrum for particles given by

$$
\begin{gathered}
E_{\beta}(p)=g_{\alpha \beta \alpha \beta}+g_{\rho \beta} \rho_{03} I_{3 \beta}+\left[\left(p-g_{\alpha \beta} w\right.\right. \\
\left.\left.-g_{\alpha \beta} I_{3 \beta \rho}\right)^{2}+\left(m_{\beta}-g_{\alpha \beta} \sigma\right)^{2}\right]^{1 / 2}
\end{gathered}
$$

The meson field equations reduce to

$$
\begin{gathered}
\mathrm{m}_{\sigma}^{2} \sigma=-\frac{\mathrm{dU}}{\mathrm{d} \sigma}+\sum_{\beta} \mathrm{B}_{\sigma \beta}<\bar{\psi}_{\beta} \psi_{\beta}> \\
\mathrm{m}^{2} \omega_{0}=\sum_{\beta} \mathrm{g}_{\alpha \beta}\left\langle\bar{\psi}_{\beta} \gamma_{0} \psi_{\beta}>\quad \mathrm{n}_{\mathrm{B}}=\sum_{\beta} \mathrm{h}_{\beta}\right. \\
\omega=0=\rho_{3} \\
\mathrm{~m}_{\rho}^{2} \rho_{03}=\sum_{\beta} \mathrm{g}_{\rho \beta} \frac{1}{2}\left\langle\bar{\psi}_{\beta} \gamma_{0} \tau_{3} \psi_{\beta}\right\rangle
\end{gathered}
$$

The baryon currents that couple these equations are explicit functions of the fields and the fermi momenta. Charge neutrality and chemical equilibrium impose relations among the Fermi momenta $k_{\beta}$. The total charge is

$$
\begin{aligned}
& Q=\sum_{\beta} q_{\beta}\left(2 J_{\beta}+1\right) \frac{k_{\beta}^{3}}{6 \pi^{2}} \\
& -\sum_{\lambda} \frac{k_{\lambda}^{3}}{3 \pi^{2}}-\rho_{r} \theta\left(\mu_{\pi}-m_{r}\right)
\end{aligned}
$$

where the charge on species $\beta$ is $\mathrm{q}_{\beta}=I_{3 \beta}+\mathrm{Y}_{\beta} / 2$.

The three terms are the electric charge densities of baryons, leptons, and negative pions.

Chemical equilibrium with respect to the various transmutations of the particles can be expressed in 
ierms of two independent chemical potentials $\mu_{\mathrm{N}}$ and $\mu_{\mathrm{e}}$ (corresponding to baryon and electric charge conservation) by

$$
\mu_{\beta}=\mu_{n}-\mathrm{q}_{\beta} \mu_{c}, \quad \mu_{\pi}=\mu_{\mu}=\mu_{c}
$$

where $\mu_{\beta}$ is the chemical potential for baryon type $\beta$. The strangeness quantum number is not conserved on the time scale of the star so that the lowest energy state is achieved with no constraint on this quantum number. The Fermi momenta, $\mathrm{k}_{p}$, of the baryons are solutions of $\mu_{\beta}=E_{p}\left(k_{p}\right)$, wher these equations have real solutions; otherwise $\mathbf{k}_{p}$ vanishes. Similarly,

$$
\begin{gathered}
\mu_{e}=\sqrt{k_{e}^{2}+m_{e}^{2}}=\sqrt{k_{\mu}^{2}+m_{\mu}^{2}} \\
=\sqrt{k_{r}^{2}+m_{\tau}^{2}} .
\end{gathered}
$$

Since the pions are bosons, they can condense in the zero momentum state when $\mu_{e} \geq \mathrm{m}_{\mathrm{r}}$.

Equations (3-9) comprise a set of coupled transcendental equations for the $(8+N)$ quantities

$$
\sigma, \omega_{b}, \rho_{03}, \mu_{n}, \mu_{e}, k_{e}, k_{n}, k_{n} k_{p}, k_{n} \cdots k_{g}
$$

which deternuine the properties of the system at the density (4) and charge (7), when $\mu_{e}<m_{r}$. The lowest energy state has $\mu_{e} \leq m_{r}$ When the equality holds, then $\rho_{x}$ replaces $\mu_{e}$ as an unknown, and its value is prescribed by (7).

The energy density $\rho$ and pressure $P$ can be found in terms of the above field configurations being the time-like and the (equal) space-like diagonal components. We find

$$
\begin{aligned}
& \rho=\mathrm{U}(\sigma)+\frac{1}{2} \mathrm{~m}_{\sigma}^{-\rho^{2}}+\frac{1}{2} \mathrm{~m}_{\omega}^{2} \omega_{0}^{2}+\frac{1}{2} \mathrm{~m}_{\rho}^{2} \rho_{03}^{3} \\
& +\rho_{\Omega} m_{\pi}+\sum_{\beta} \frac{2 J_{\beta}+1}{2 \pi^{2}} \\
& \int_{0}^{k_{\beta}} \sqrt{p^{2}+\left(m_{\beta}-B_{\sigma \beta} \sigma\right)^{2}} p^{2} d p \\
& \mathrm{P}=-\mathrm{U}(\sigma)-\frac{1}{2} \mathrm{~m}_{0}^{2} \sigma^{2}+\frac{1}{2} \mathrm{~m}_{\omega}^{2} \omega_{0}^{2}+\frac{1}{2} \mathrm{~m}_{\rho}^{2} \rho_{03}^{2} \\
& +\frac{1}{3} \sum_{\beta} \frac{2 J_{\beta}+1}{2 \pi^{2}} \int_{0}^{k_{\beta}} \frac{p^{4} d p}{\sqrt{p^{2}+\left(m_{\beta}-g_{\beta} \sigma\right)^{2}}} .
\end{aligned}
$$

star. The matter then arranges itself according to the solution of the Oppenheimer-Volkoff equations, which follow from the general theory of relativity for a static spherically symmetric geometry.

Although the four bulk nuclear properties mentioned in the introduction are the most important constraints on the theory that we can think of that would influence the structure of the star, they are not sufficient to determine all the parameters. The singleparticle properties of finite ni zlei serve to further narrow the choice. ${ }^{3}$ Following Moszkowski's ${ }^{4}$ application of SU(3) symmetry and based on quark counting arguments the nucleons and $\Delta s$ are coupled with the same constants to the meson fields, but the couplings of strange baryons relative to nucleons are reduced by a factor $\left(g_{s} / B_{N}\right)^{2}=2 / 3$.

Fig. 1 shows un populations of dense matter as a function of the baryon number density. While neutrons are the dominant population of stable charge neutral matter at densities in the vicinity of normal nuclear density, the populations of mrotons, pions, and hyperons rise steeply as their thresholds are reached so that at a few times nuclear density the neutron becomes a iraction of the total baryon population. This contrasts with scenarios based only on neutrons, protons and leptons, in which case the matter remains relatively pure in neutrons. $^{s}$

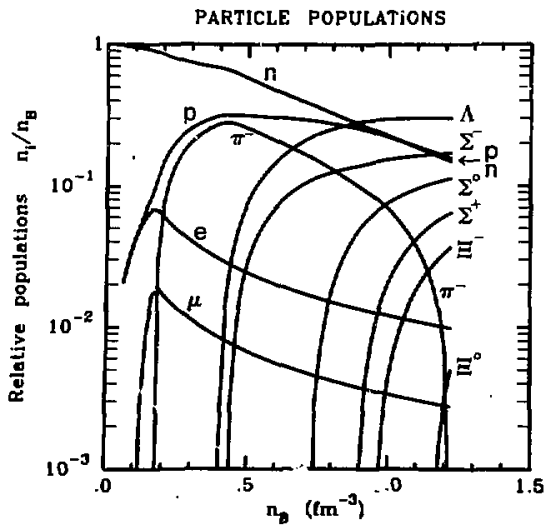

Fig. 1. The particle populations in dense neutral matter plotted as a ratio to the total baryon number density are shown as a function of baryon density.

XBL $823-8693$

These are required to find the structure of a neutron 
Through the dependence on the density, the particle populations will vary as a function of radius in a star. For a star of 1.82 solar masses (the maximum star mass for this theory) these populations are displayed in Fig. 2. The total baryon number density is represented by the top curve, and it is broken down into its com: ponents by the other curves above the axis. Below the axis (for convenience) the pion population is shown. One sees that the strange baryons represent about $2 / 3$ of the baryon population in the deep interior and that charge neutrality is maintained not by a paucity of charged particles (as in models that consider only neutrons, protons and leptons) but by an abundance of charged particles of both signs. The electron and meson populations are both extremely small, not exceeding 0.012 and $0.003 \mathrm{fm}^{-3}$ anywhere. The pion condensate is very influential in quenching the lepton populations (see Fig. 1) at intermediate density.

For stable configurations of collapsed matter, this, theory predicts a maximum mass of 1.82 solar masses and maximum moment of inertia of $2.1 \times 10^{45} \mathrm{~g}-\mathrm{cm}^{2}$, which exceed the observational lower limits of 1.6 and $1.5 \times 10^{44} \mathrm{~g}-\mathrm{cm}^{2}$, respectively. The fractional gravitational redshift of a spectral line emitted at the surface of this star and observed at infinity is $\Delta \mathrm{X} \lambda=0.42$, which for a stable star is large.

The composition and strangeness of a neutron star will never be directly measured. However, it is not excluded that observable properties may be influenced by the hadronic composition. Several things come to mind in addition to the much studied problem of neutron star cooling. There can be vibrational modes associated with the isospin and strangeness composition. Moreover, since the charge is carried mainly by massive baryons, rather than half of it on electrons and muons, as is usually assumed, the electrical conductivity will surely be influenced. The active lifetime of pulsars depends sensitively on the electrical conductivity because it plays a decisive role in the decay rate of the magnetic field, which is thought to be responsible for their beamed radiation.

\section{Footnote and References}

*Condensed from Phys. Lett. 114B (1982) 392

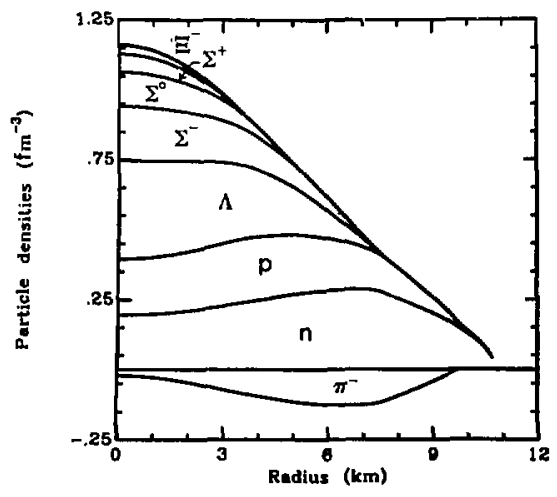

Fig. 2. The proper number densities (as measured in a locally inertial reference frame) of the various particles are plotted as a function of the star radius. On this scale the electron and meson populatin- are not much larger than the thickness of the linis. The vertical . distance between lines represents the population. densities.

XBL 823-8692

1. J.D. Walecka, Ann. of Phys. 83 (1974) 491

2. J. Boguta and A.R. Bodmer, Nucl. Phys. A292 (1977) 413

3. F.E. Serr and J.D. Walecka, Phys. Lett. 79B (1978) 10

B.D. Serot and J.D. Walecka, Phys. Lett. 87B (1980) 172

J. Boguta, Nucl. Phys. A372 (1981) 386

J. Boguta, Phys. Lett. 106B (1981) 245

J. Boguta, Phys. Lett. 106B (1981) 250

4. S.A. Moszkowski, Phys. Rev. D9 (1974) 1613

5. G. Baym in Les Houches, Session XXX, ed. by $\mathbf{R}$. Balian, M. Rho and G. Ripka (North-Holland, Amsterdam, 1978) p. 747.

J. Boguta, Phys. Lett. 106B (1981) 255. 


\section{Quantum Approach to the Nuclear Collision Process}

\section{P. Danielewicz}

Contrary to common belief, nuclear collisions cannot be described classically at hundreds of $\mathrm{MeV} /$ nucleon beam energies. At the initial stage of a 200-800 MeV/nucleon nuclear collision, the mean time $\tau$ between successive nucleon-nucleon collisions is of the order of $1 /\left(n_{0} \sigma v\right) \approx(1.7-3.5) \mathrm{fm} / \mathrm{c}$. The value of $\tau$ implies uncertainties of energies in subsequent collisions $h / \tau \approx(55-115) \mathrm{MeV}$. The uncertainties are comparable with values of nucleon energies in the midrapidity frame.

We have examined the role of quantum dynamics in high-energy nuclear collisions. We have carried out numerical calculations of collisions in an interpenetrating nuclear-matter model. The quantum dynamics has been compared to a classical Markovian dynamies given by the Boltzmann equation. In quantum calculations, methods of nonequilibrium Green's functions have been employed. These methods, which bave been initiated by Kadanoff and Baym, ${ }^{1}$ haye been already proposed ${ }^{2}$ to extend the TDHF method for low-energy nuclear collisions. In the calculations we have solved the Kadanoff.. Baym equations of motion for the single-particle Green's functions, with the self-energies in the Born approximation. We have solved the equations both for a two Fermi-spheres Hartree-Fock initial state and a correlated initial state of nuclear matter.

Results of the calculations for $E_{1 \mathrm{bb}}=400$ $\mathrm{MeV} /$ nucleon are presented in Figs. 1 and 2 . In the

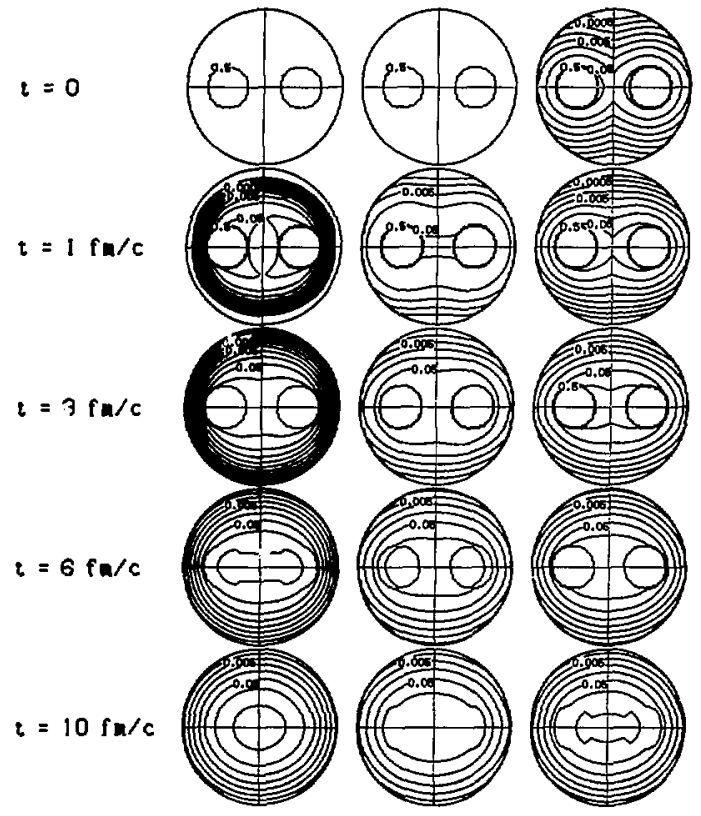

Fig. 1. Evolution of the nucleon momentum distribution $f\left(p^{\perp}, p^{2}, t\right)$. Leftmost figures - Boltzmann equation evolution; central figures - Green's functions equations of motion evolution for a two Fermi spheres Hartree-Fock initial state; rightmost figures - evolution for a correlated initial state. Horizontal axes are the collision axes. The momentum space is restricted to $900 \mathrm{MeV} / \mathrm{c}$ as shown by the outer circles.

XBL 8211-3430 
distributions resulting from the Boltzmann equation, the effects of energy conservation in binary collisions can be seen. The particles initially fill a hollow shell in momentum space, determined by the two Fermi spheres, and they cannot substantially populate the high momenta region. In the quantum dynamics, both the energy conservation in binary interactions and the single-particle energies are ill defined. In quantum calculations the particles move directly into the central region between the Fermi spheres, and they also spread over the whole momentum space. In contrast to what one would expect from the above, the approach to the thermodynamic equilibrium is slower by about $50 \%$ in the quantum case. This is due to an effective reduction of the binary collision time and also to a development of a gap between particle and hole frequencies. The gap is of the order of $1 /\left(m \lambda^{2}\right)$, with $\lambda=1 /(n \lambda)$ a mean free path, and the effect seems to be related to energy spacing of levels in a finite spatial region. In Fig. 2 we depict the evolution of the momentum distribution anisotropy. In the correlated initial-state quantum calculation, a value of $1 / 2$ for the anisotropy is achieved only after $10 \mathrm{fm} / \mathrm{c}$ of the evolution. The value of the time seems too large to justify the hydrodynamic description for the heavy-ion collisions at the considered beam energy.

\section{References}

1. L.P. Kadanoff and G. Baym, Quantum Statistical Mechanics, Frontiers in Physics Series (Benjamin, New York, 1962)

2. C.Y. Wong and H.H.K. Tang, Phys. Rev. Lett. 16 (1978) 1010; Phys. Rev. C20 (1979) 1419; H. Orland and R. Schaeffer, Saclay Report DPhT/78/41; C.M. Shakin and M.S. Weiss, Report UCRL-80500

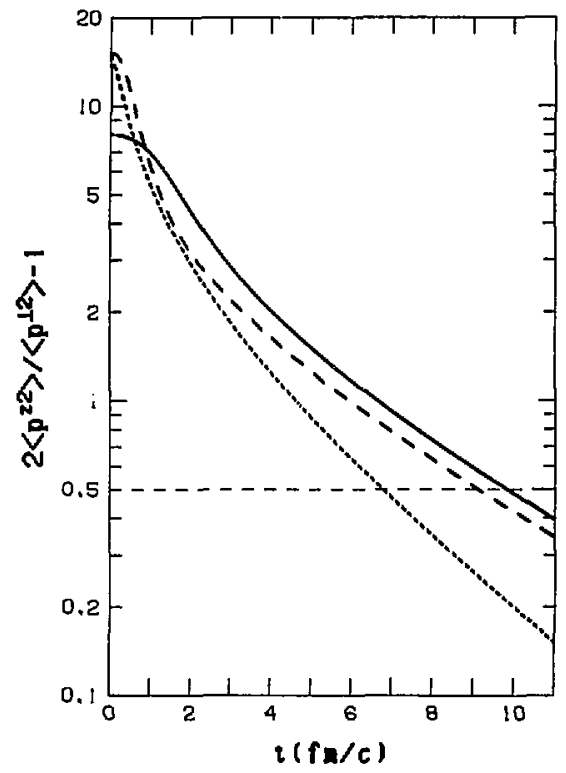

Fig. 2. Evolution of the momentum distribution anisotropy. Short-dashed line corresponds to the Boltzmann equation, long-dashed line to the Green's function equation of motion and the Hartree-Fock initial state. The dashed horizontal line at 0.5 is a guide to the eye representing $\left\langle\mathrm{p}^{22}\right\rangle=1.5\left(<\mathrm{p}^{\perp 2}>/ 2\right)$.

XBL 829-11826 


\title{
Theory of Direct Nuclear Reactions*
}

\author{
N.K. Glendenning
}

The spontaneous disintegration of long-lived, naturally occurring isotopes provides one source of information on nuclei. However, only a limited number of nuclei are accessible for study by this natural process and only under a narrow range of tircumstances. On the other hand, nuclear reactions can be induced in the myriad of pairwise combinations provided by stable or long-lived nuclei and over the wide range of energies provided by the accelerators in the physics laboratories of the world. Reactions, therefore, provide the greatest volume and widest range of nuclear data. The energy loss of a beam particie can be directly interpreted as an excitation energy in the target nucleus. Usually, however, the data acquire meaning for the structure of nuclei only after they have been interpreted through a reaction theory. The synthesis of such accumulated information inta a coherent theory of the nucleus is the main subject matter of nuclear physics. There are a number of volumes on nuclear structures that review this ongoing process.

This book is about the theory of direct nuclear reactions. It emphasizes the microscopic aspects of the reactions and their description in terms of the changes induced in the motions of the individual nucleons by the reaction. However, since collective motion can be accurately described by a few collective parameters, I sometimes depart from a strictly microscopic description; any account of direct reactions would otherwise be incomplete.

The book begins essentially at the beginning, assuming only a modest knowledge of quantum mechanics and some acquaintance with angular momentum algebra, and ends by describing some of the most recent topies. The principal results of the theory are described, with emphasis on the approximations involved so as to provide a guide as to how well the theory can be expected to hold under specific experimental conditions, and also to suggest areas in which improvements can be made. Applications to the analysis of experiments are also emphasized, not only because reactions are interesting in thernselves but because they can be used to measure nuclear properties. Indeed, most of our detailed knowiedge of nuclear properties has been discovered by means of reactions.

The goal of the book is thus to provide the novice with the means to become competent to do research in direct nuclear reactions and to provide the experienced researcher with a detailed discussion of the advanced topics.

Topics covered include coupled equations and the distorted-wave Born approximation; form factors and their nuclear structure content; the basis of the optical potential as an effective interaction; reactions such as inelastic single and two-nucleon transfer reactions; the effect of muclear correlations; the role of multiple-step reactions; the theory of inelastic scattering and the relationship of the effective interaction to the free one; reactions between heavy ions; the polarizability of nuclear wave functions during a reaction; and the calculation of components of the optical potential arising from specific collective or transfer reactions.

\section{Footnote}

-Pseface to a book to be published by Academic Press.

\section{Droplet Model Predictions of Charge Moments*}

\author{
William D. Myers
}

The Droplet Model was originally developed to provide a macroscopic description of the binding energy and spatial distribution of a saturating, two-component, leptodermous system, and the model coefficients were later chosen to correspond to the values they would have in atomic nuclei. ' Like the liquid drop model that preceded it, the Droplet Model nuclear binding energy predictions agree with the measured values to within a percent or less for the heavier nuclei (10 MeV out of $1000 \mathrm{MeV}$ ). Similar accuracy (or even better) seems to 
be obtainable in the description of the density distribution as well.

The most frequently measured quantity, the mean square radius $\left\langle\mathrm{r}^{2}\right\rangle$, can be calculated from the expression

$$
\left\langle\mathrm{r}^{2}\right\rangle=\left\langle\mathrm{r}^{2}\right\rangle_{\mathrm{u}}+\left\langle\mathrm{r}^{2}\right\rangle_{\mathrm{r}}+\left\langle\mathrm{r}^{2}\right\rangle_{\mathrm{d}},
$$

where

$$
<r^{2}>_{u}=\frac{3}{5} R^{2}\left(1+\alpha_{2}^{2}+\frac{5}{9} \alpha_{4}^{2}+\ldots\right)
$$

is the contribution from the size of the uniform distribution and its shape. The next term,

$$
\left\langle\mathrm{r}^{2}\right\rangle_{\mathrm{r}}=\frac{12}{175} \mathrm{C}^{2} \mathrm{R}^{2}\left(1+\frac{14}{5} \alpha_{2}^{2}+\frac{595}{108} \alpha_{4}^{2}+\ldots\right)
$$

is the contribution from the redistribution, and its shape dependence. The last term,

$$
<r^{2}>_{d}=3 \sigma^{2} \text { or } 3 b^{2}
$$

is the contribution from the diffuseness. It is interest ing to note that the diffuseness correction has no shape dependence and that it is the same for all nuclei (so long as we assume that the diffuseness itself is a constant).

In order to assess the relative importance of the different terms we can consider the example of ${ }^{238} \mathrm{U}$. The spherical Droplet Model value of $R_{2}$ is $7.030 \mathrm{fm}$, and this becomes $7.027 \mathrm{fm}$ when the effect of deformation (using the values $\alpha_{2}=0.139$ and $\alpha_{4}=0.065$ provided by $\mathrm{Moller}^{2}$ on the size of the distribution is included. The mean square radius of such a distribution is $29.627(1+0.0218) \mathrm{fm}^{2}$, where the first part comes from the size and the second from the shape. The redistribution contribution is $0.879 \mathrm{fm}^{2}$, and the diffuseness contribution is $3(0.95)^{2}=2.707 \mathrm{fm}^{2}$, for a total of $33.860 \mathrm{fm}^{2}$. The predicted RMS radius is 5.819 $\mathrm{fm}$, which is to be compared with the experimental value of $5.843 \pm 0.012 \mathrm{fm}$.

\section{Foomote and References}

*Condensed from LBL-14310

1. W.D. Myers, Droplet Model of Alomic Nuclei (IFI/Plenum Data Co., New York, 1977)

2. P. Möller, private communication, March 1982

\title{
The Macroscopic Approach to Nuclear Masses and Deformations*
}

\author{
W.D. Myers and W.J. Swiatecki
}

After sixty years of atomic mass measurements, the binding energies are known today experimentally for $\sim 1900$ nuclei in their ground-state equilibrium configurations, as well as for dozens of nuclei in deformed, fission-barrier saddle point shapes, and for hundreds of interaction-barrier shapes corresponding to pairs of nuclei in contact. These binding-energy (or mass) measurements are often made with a precision corresponding to a small fraction of an $\mathrm{MeV}$ and, together; they represent an immense amount of information of practical relevance for many branches of nuclear physics, nuclear engineering, and astrophysics. They also represent an exacting challenge to theoretical efforts at understanding the basic properties of the unique many-body problem presented to us by an atomic nucleus.
The major part of the binding energy of nuclei may be accounted for by a simple "Liquid Drop" formula, consisting of a volume entergy [assumed to depend quadratically on the relative neutron excess I, defined as (N-Z)/A], a surface energy proportional to the surface area, and the electrostatic energy of a uniform distribution of electric charge inside the nucleus. Originally conceived more than 45 years ago for the purpose of cilculating only such ground-state masses, the model began: to assume a wider range of applicability when it was recognized that the gross properties of nuclear fission could be understood in terms of the shape dependence of the surface and electrostatic energies of the nuclear drop. ${ }^{1}$ Unfortunately, however, there was a historically understandable tendency to associate the Liquid Drop Model (even in its static 
aspects) with a system of strongly interacting particles characterized by short mean free paths (and treated according to classical mechanies). Because of this misconception and the failure of idealized versions of the Liquid Drop Model to account for nuclear dynamics (c.g., excited nuclear states), the soundness of the Liquid Drop Model, even in its description of the gross, static aspects of nuclear binding energies, began to be questioned when the nuclear Shell Model was found to be a good approximation to nuclear structure.

Major advances in treating the shell effects took place about fifteen years ago. (Before this time the shell effect deviations had usually been treated in an ad hoc way, by use of tabulated empirical correction functions.) Later it was recognized that the main features of the shell effect deviations could be understood in terms of the bunching of the quantized nucleon levels into bands, the bunching being governed by the symmetries of the nuclear shape in question and thus disappearing when the symmetries were destroyed by a deformation. A semi-empirical algebraic treatment of shell effects, made possible by these insights, ${ }^{2}$ was soon followed by quantitative calculations (requiring, however, numerical solutions of the Schrödinger equation in an appropriate potential well). ${ }^{3}$ The result of these calculations was not only a dramatic reduction of the discrepancies between theoretical and experimental masses (from around $10 \mathrm{MeV}$ to around $1 \mathrm{MeV}$ ) but also the explanation of the long-standing puzzle of the mass asymmetry of nuclear fission in terms of shell effects at the fission barrier, and potentially most significant, these shelleffect calculations predicted the possible existence of an island of relatively stable nuclei beyond the known limits of the periodic table of elements.

Concurrently with this conquest of the nuclear shell effects, there followed a substantial further improvement in the treatment of the Liquid Drop Model (the so-called Droplet Model) and the development of successful "Proximity" and "Folding" techniques for calculating the nuclear interaction between approaching nuclei, essential for a description of the energies of interaction-barrier configurations. Taking together these three contributions, Liquid-Drop, Proximity, and Shell Effects, one can today estimate theoretically the binding and deformation energies of known or hypothetical nuclei with an accuracy often approaching or even exceeding $1 \mathrm{MeV}$.

Footnote and References

* Condensed from Ann. Rev. Nuc. Part. Sci. 1982

1. N. Bohr and J.A Wheeler, Phys. Rev. 56 (1939) 426

2. W.D. Myers and W.J. Swiatecki, Nucl. Phys. 81 (1966) 1

3. V.M. Strutinskii, Nucl. Phys. A95 (1967) 420

\title{
The Nature of Nuclear Dynamics*
}

\author{
W.J. Swiatecki
}

It is becoming more and more apparent that the nature of nuclear macroscopic shape dynamics is profoundly affected by the symmetries of the configurations in question. On the one hand, if the nuclear configuration is free of symmetries (the "Chaotic Regime") the static potential energy, considered as a function of shape, is now known to be described quite accurately by a leptodermous (liquiddrop) type of expression and, although this is by no means established, the dynamics of shape changes is expected to follow a simple equation of motion, based on the one-body dissipation concept. The resulting shape dynamics is predicted to be severely overdamped: the nucleus should behave like a viscous fluid, with a novel type of viscosity, described by the Wall or Walland-Window Formula. On the other hand, when the nuclear system is dominated by symmetries, the single- particle level structure of the nucleons acquires special features (bunchings and crossings or near-crossings of levels) and the nucleus becomes more like a viscoelastic solid, in some cases actually dominated by elasticity. This symmetry-dominated regime of nuclear dynamics has been vividly illustrated by the prediction, discovery, and further elucidation of the giant quadrupole resonances. Adding to this the striking pair correJation effects associated with time-reversal symmetry (responsible for superconductivity and superfluidity in macroscopic bodies), it is clear that nuclear dynamics is a fascinatingly rich and complex field for study of which, at the moment, we only possess an approximate understanding of a few especially simple limiting cases.

Fig. 1. shows insights into the nature of nuclear statics and nuclear dynamics in which the presence or absence of symmetries plays a dominant role. 


\section{HOW DOES IT ALL FIT TOGETHER?}

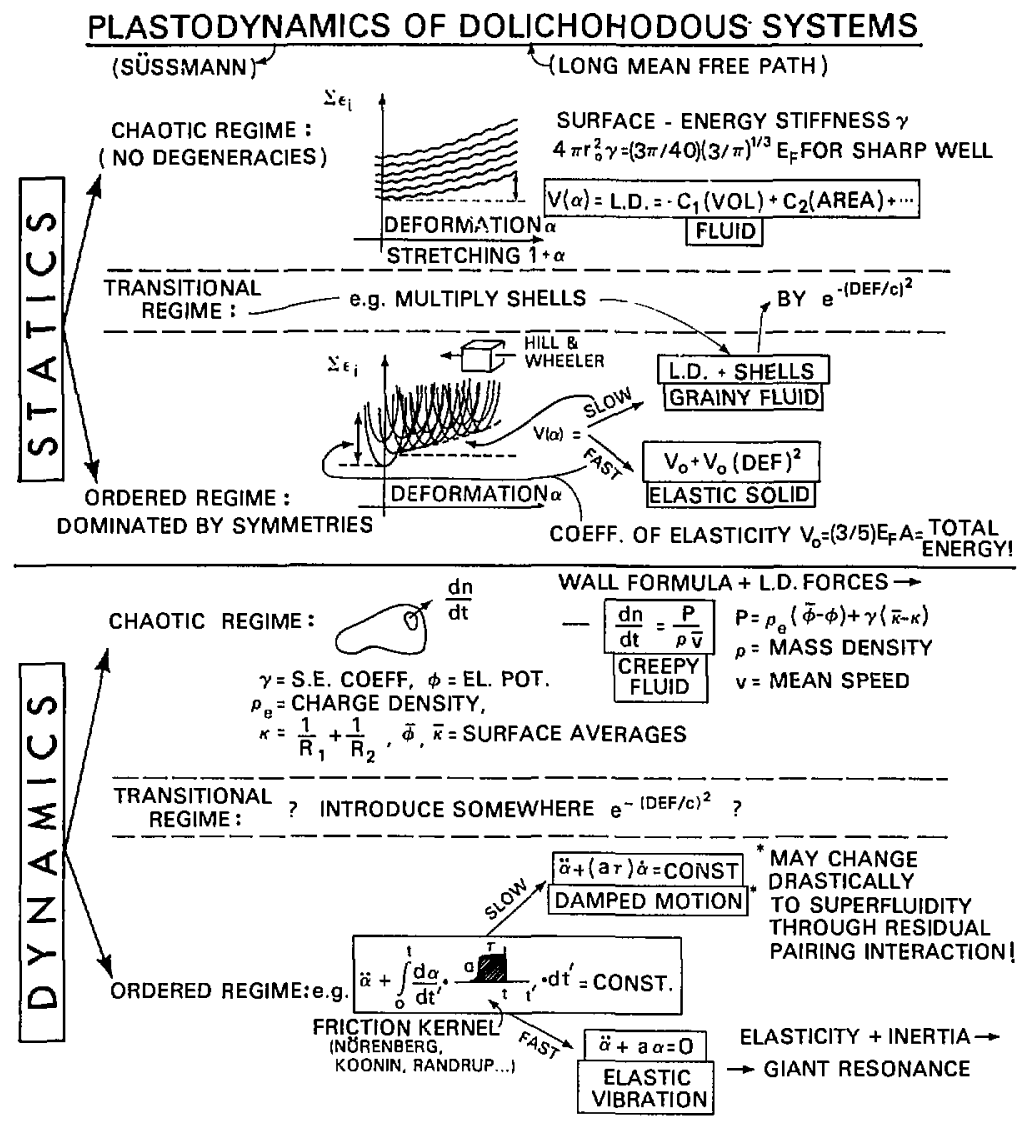

Fig. 1. An attempt to sketch the relation to one another of various limiting theories of nuclear statics and nuclear dynamics. The principal message is that the description of macroscopic nuclear dynamics may be expected to call for a rich mathematical structure, including the theory of fluids (ordinary, superfluid, and superviscid) of elastic solids and of plastic flow, depending on the presence or absence of symmetries and regularities in the configurations and deformations in question.

XBL 823-186

\section{Footnote}

*Condensed from introductory remarks given at the Winter Workshop on Nuclear Dynamics, Granlibakken, Tahoe City, CA, February 21-26, 1982, LBL-14073, LBL-14138. 


\title{
Dynamical Aspects of Nucleus-Nucleus Collisions*
}

\author{
S. Bjiknholm ${ }^{\dagger}$ and W.J. Swiarecki
}

The fusion or reseparation of two colliding nuclei is treated according to a schematic model based on the "Chaotic Regime Dynamics" (liquid-drop potential energy plus one-body dissipation). Attention is focused on three hurdles or "milestone configurations" that a colliding system may be faced with: the touching configuration, the conditional saddle-point configuration at frozen mass asymmetry, and the unconditional saddle-point configuration. Semi-empirical formulae are derived for the "Extra Push" (the extra energy needed in some situations to carry the system from the first to the second hurdle) and for the "Extra-extra Push" (the energy needed to carry the system from the first to the third hurdle). The theoretical formulae are confronted with measurements of fusion and evaporation-residue cross sections (Figs. 1 and 2). A discussion of the implications for superheavy-element reactions is given, using the production of element 107 in the bombardment of ${ }^{200} \mathrm{Bi}$ with ${ }^{54} \mathrm{Cr}$ as a calibrating reaction.

\section{Footnotes}

*Condensed from LBL-14074, and Nucl. Phys. A391(1982) 471.

†Niels Bohr Institute, Blegdamsvej 17, DK-2100, Copenhagen, Denmark

Fig. 1. The excitation functions for various types of reactions. Figure 1 (a) illustrates the qualitative distinction between binary (elastic and quasi-elastic) reactions, dinucleus (deep-inelastic) reactions, mononucleus (fast-fission) reactions, and compound nucleus reactions. The latter divide into (delayed) fission reactions and evaporation residue reactions. Some characteristic lifetimes (in seconds) are indicated on the right. The dotted curve illustrates qualitatively the additional entrance-channel limitation on compound-nucleus formation resulting from the requirement of an extra-extra push to reach the vicinity of the spherical shape. Figures I(b),(c),(d) show a comparison of theory and experiment for three cases.
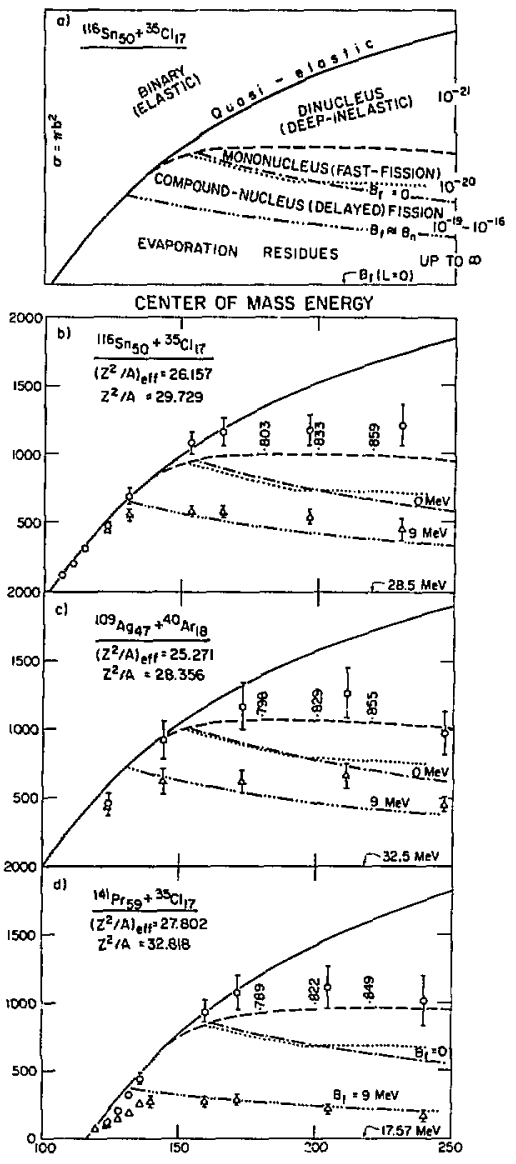

XBL 823-190 


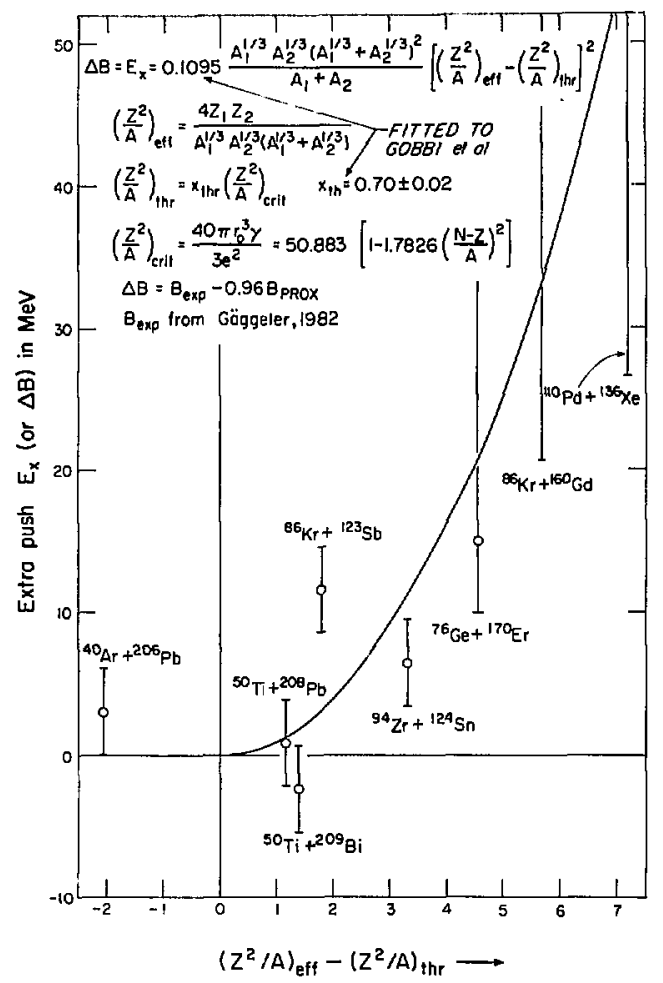

Fig. 2. A comparison of theory and experiment concerning the need for an extra push to form compound nuclei. The experimental points and lower limits are obtained by taking the difference between effective barriers (that have to be inserted in a standard theory to reproduce $x$ n excitation functionj) and baseline barriers estimated from smooth systematics representing average behavior for not too heavy systems (where no extra push is expected). The curve shows the trend of the theoretical extra-push prediction. There are considerable uncertainties both in the experimental points and in the baseline barriers that affect the comparison.

XBL 823-187 


\title{
Nuclear Deformation Energies*
}

\author{
J. Block't and W.J. Swiatecki
}

An atlas of nuclear deformation-energy maps and of other properties of interest, whose contents were described in the Nuclear Science Division Annual Report 1980-1981, LBL-13366, has been completed and printed as a Lawrence Berkeley Lahoratory document, LBL-12811.
Footnotes

"Condensed from LBL-12811

†Institute for Nuclear Research, 05400 Swierk, Poland

\section{Discussions with Jens*}

\author{
W.J. Swiatecki
}

This is the text of a talk given at the Symposium Honouring Jens Lindhard's 60th Birthday, Aarhus, Denmark, February 25-26, 1982. It consists of recollections of discussions with Lindhard, dating back to 1950 and ranging over topics in nuclear and atomic physies, relativity and statistical mechanics.

\section{Footnote}

"Condensed from LBL-14072; to be published in Physica Scripta (1983)

\section{Nonlinear Mean Field Theory for Nuclear Matter and Surface*}

\author{
J. Bogute and S.A. Moszkowskit
}

In this paper nuclear matter properties are studied in a nonlinear relativistic mean field theory. By determining the parameters of the model from bulk properties of symmetric nuclear matter, it is possible to successfully account for other key properties of nuclei, such as the compression modulus, spin-orbit coupling, surface energy, and diffuseness of the nuclear surface. The energy dependence of the nucleon-nucleon optical model is also predicted reasonably well. The nonrelativistic limit of the theory is stressed, and it is shown that most of the results can be obtained, to a good approximation, analytically. The strength of the required nonlinear term is shown to be consistent with that derived using a new version of the chiral mean field theory in which the vector mass as well as the nucleon mass is generated by the sigma field.

Footnotes and References

"Condensed from LBL-14685

†University of California, Los Angeles, CA 90024 


\title{
2. Strongly Damped Collisions
}

\section{Fragment Spin Correlations in Damped Nuclear Reactions*}

\author{
J. Randrup
}

Damped nuclear reactions provide a powerful tool for exploring nuclear dynamical properties. In recent years a number of reaction models have been developed, but the relative importance of the various reaction mechanisms advocated is still not settled. With several different modeis baving comparable success in accounting for the available data, there is a need to consider more exclusive quantities, which are better suited as specific probes. In a recent development of the theory for angular-momentum transport caused by nucleon exchange, it was suggested that the spins of the reaction products might exhibit correlations reflecting the basic mechanism by which the spins have been accumulated. ' This idea has now been pursued quantitatively. This undertaking was stimulated by a suggestion from Lazzarini $^{2}$ that the determination of spin correlations is experimentally feasible by coincidental measurement of fission fragments from both reaction products.

The spin of a nuclear fragment may be probed via the angular distribution of its decay products. We have concentrated on the fission process, but the formulation applies equally well to other decay processes, such as the emission of alpha particles, nucleons, and gamma rays. The fission process is a particularly suitable probe because of the large anisotropy of fission products from nuclei with high spins. The fission direction is determined by $P(K)$ - $\exp \left(-K^{2} / 2 K_{0}^{2}\right)$ where $K$ is the component of the nuclear spin along the emission direction. The quantity $K_{0}$ is characteristic of the nucleus ( $A$ or B) and depends on its excitation.

The theorctical spin distribution function $f_{A B}\left(\vec{S}_{A}, \vec{S}_{B}\right)$ depends on the particular reaction model employed. It is useful to characterize $f_{A B}$ by the mean values $\left\langle\overrightarrow{\mathrm{S}}_{\mathrm{A}}\right\rangle$ and $\left\langle\overrightarrow{\mathrm{S}}_{B}\right\rangle$ together with the covariance tensor $\sigma$, which has the elements $\boldsymbol{\sigma}_{i j}^{F G}=\left\langle S_{i}^{F} S_{j}^{G}\right\rangle-\left\langle S_{i}^{F}\right\rangle\left\langle S_{i}^{G}\right\rangle$, where the superscripts $F, G$ denote the fragment labels $A, B$ and the subscripts $i, j$ denote the spatial coordinates $x, y, z$. The main goal of the present study is to explore the consequences of statistical nucleon exchange on the fragment spin correlations. We therefore employ the model developed in ref. 1. In that theory, the statistical transfer of individual nucleons between the two reactants produces a dissipative exchange of mass, charge, energy, and angular momentum governed by a Fokker-Planck transport equation. The theory relates the various transport coefficients to the elementary kinematics of quasi-free transfers. The temporal evolution of the associated form factor is estimated by idealizing the reaction complex as two spherical nuclides joined by a small cylindrical neck. The computational problem is solved numerically by integrating the moments of the distribution function $f$ along the average dynamical trajectory. Having thus obtained $f_{A B}$, the joint emission probability $P_{A B}(\dot{\alpha}, \hat{\beta})$ is calculated by statistical (Monte-Carlo) integration over the six-dimensional spin-spin space.

In addition to the four angular variables $\dot{\alpha}=\left(\theta_{\alpha} \phi_{\alpha}\right)$ and $\hat{\beta}=\left(\theta_{\beta}, \phi_{\beta}\right), \mathrm{P}_{\mathrm{AB}}$ depends on the various auxiliary observables, such as the kinetic en-rgy loss, the deflection angle, and the mass partition. in the present study, we shall calculate $P_{A B}$ for the average outcome of a collision with a specified value of the total angular momentum $J$. This is expected to be sufficient for a first orientation and a preliminary confrontation with experiment; a more refined treatment can be made subsequently, if called for.

As an instructive case, we considered the reaction $1768 \mathrm{MeV}{ }^{208} \mathrm{~Pb}+{ }^{236} \mathrm{U}$, which has been suggested as suitable for actual experimentation. ${ }^{2}$ In the present context the partially damped reactions are of most interest since they produce fragments with high excitation and high spins and hence optimize the fission probability; the simple dinuclear parametrization employed in the dynamical calculations is also expected to be best in this region.

In order to judge the significance of the calculated results, we also consider the statistical model developed by Moretto. ${ }^{3}$ A striking feature of the statistical results is the approximate symmetry with respect to reflections in the reaction plare (i.e., $\left.\phi_{\alpha} \rightarrow-\phi_{\alpha}\right)$. This simplicity can be understood on the basis of the following discussion: The six normal rotational modes of the disphere can be characterized as either positive or negative according to whether the two spheres turn in the same or in the opposite sense, respectively. In the statistical model, each of these modes carries an average excitation energy equal to half the temperature. By inverting the representation of the normal modes in terms of the individual fragment spins, one obtains the covariance tensor characterizing the statistical spin distribution. This elementary 
manipulation will demonstrate how the equally excited positive and negative normal modes counteract each other in the coupling tensor $\sigma^{A B}$ characterizing the correlations between the two fragment spins. As a consequence, $\sigma^{\mathrm{N}}$ emerges as considerably smaller than $\sigma^{\mathrm{M}}$ and $\sigma^{\mathrm{BB}}$, and the fragment spins are largely uncorrelated. Indeed, in the case under study, the elements of $\sigma^{A B}$ are typically an order of magnitude smaller than those of $\sigma^{M}$ and $\sigma^{B B}$.

In sharp contrast with this scenario, the dynamical nucleon exchange model yields a significant asymmetry with respect to $\phi_{\alpha} \rightarrow-\phi_{\alpha}$. This feature can be understood as follows. When an individual nucleon is transferred, the recoil spins deposited in the two nuclides are nearly parallel. ${ }^{1}$ Therefore, the nucleon exehange mechanism preferentially excites the positive rotational modes in the dinucleus. This tendency is further enhanced for nearly symmetric systems (as are relevant in a double-fission experiment). As a consequence, no cancellation occurs in the coupling tensor $\sigma^{A B}$, which therefore emerges with elements of similar magnitudes as those of $\sigma^{\mathrm{M}}$ and $\sigma^{\mathrm{BB}}$ and thus produces an optimal correlation between the two spins. In this situation, the bias introduced by detecting one of the

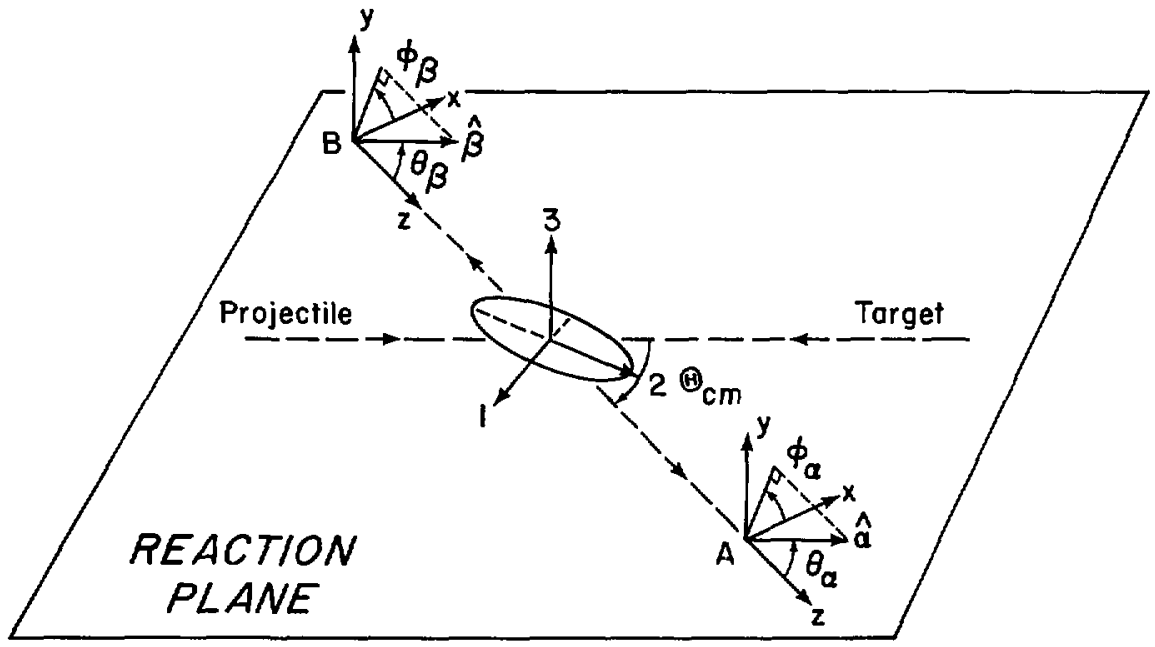

Fig. 1. Ilustration of the geometry of the reaction. The two reaction products $A$. ${ }^{A} B$ emerge in the reaction plane. The angular directions of ejectiles from $A$ are referred to a coordinate system wh is coiniving wib $A$, has its $z$-axis in the direction of motion (as seen in the overall $\mathrm{CM}$ system), and its y-axis paraflel to the reaction normal. The directions of ejectiles from $B$ are referred to a coordinate system with axes parallel to the above one, but comoving with $B$. The contours of the spin distribution are indicated in the reaction plane; the 3-axis points along the reaction normal, while the major in-plane axis (the 2-axis) points approximately in between the directions of the beam and $\mathbf{A}$. 
emission processes significantly modifies the angular distribution of ejectiles from the other emitter. The effect can be stated in the following simplistic way: When the two fragment spins are closely correlated the emission of an ejectile in a given direction enhances the probability that the ejectile from the other fragment also appears in that same direction.

Calculations for several values of $J$, as well as several angular settings, yield the same general features as discussed above. Furthesmore, crode attempts to include the blurring effects associated with the finite angular resolution in practicable experiments indicate that a measurable difference between the two models should remain. It would therefore be valuable to actually carry out such an experiment. If successful, the measurement would establish novel information on the dynamics of damped nuclear reactions and contribute to our quantitative understanding of the specific reaction mechanisms acling.

\section{Footnote and References}

*Condensed from LBL-13403; Phys. Lett. 110B (1982) 25

1. J. Randrup, Nucl. Phys. A383 (1982) 468

2. A. Lazzarini, private communication (December 1980)

3. L.G. Moretto and R.P. Schmitt, Phys. Rev. C21 (1990) 204

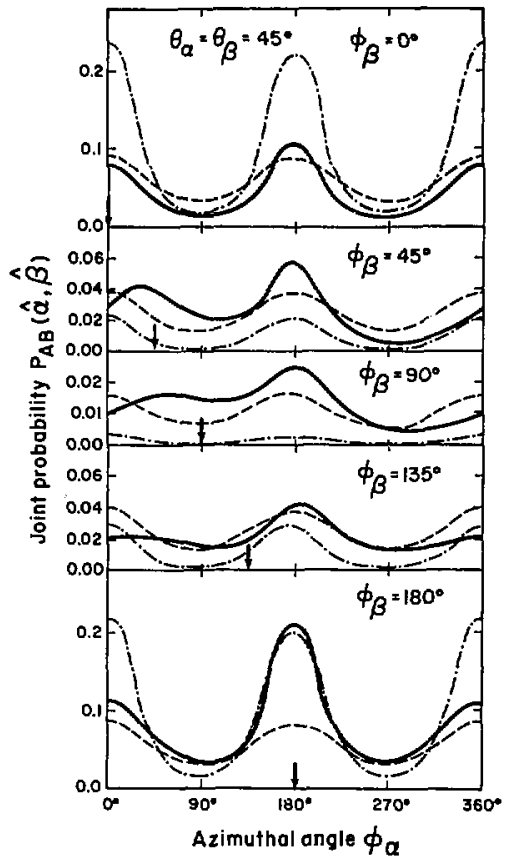

Fig. 2. The joint emission probability $\mathbf{P}_{\mathrm{AB}}(\hat{\alpha}, \dot{\beta})$ for two settings of the polar angles: $\theta_{\alpha}=\theta_{f}=45^{\circ}$ (a) or $\theta_{\alpha}=\theta_{\beta}=90^{\circ}$ (b), as a function of the azimuthal angle $\phi_{a}$ for fixed values of $\phi_{\beta}$. The solid curves indicate the result of the dynamical nucleon-exchange model. while the others are the results of the statistical model with the separation $\mathbf{R}$ taken as either the minimum separation encountered (dot-dasb) or the scission separation (dash). The results are calculated for $1768 \mathrm{MeV}{ }^{201} \mathrm{~Pb}+$ ${ }^{238} \mathrm{U}$ with $\mathrm{J}=500 \mathrm{n}$.

XBI, 8110-1412 


\title{
Equilibrium Treatment of Spin-Depolarizing Modes in
}

\section{Mass Asymmetric Heavy-Ion Systems*}

\author{
Richard P. Schmitt and Alberto J. Pacheco ${ }^{\ddagger}$
}

In a recent work, a macroscopic model has been developed that describes the thermal excitation of collective modes for a symmetric system consisting of two touching, rigid, liquid-drop spheres. ${ }^{1}$ This model has proved useful in the interpretation of gamma-ray anisotropies. In the current work this model is extended to include asymmetric systems. This extension allows a much broader basis for comparison with experimental data.

As an example we shall consider the effect of the various collective modes on the spin alignment in deep. inelastic reactions. Since the problem of the angular distributions of light particles, fission fragments, and gamma rays has recently been dealt with quite extensively, we shall confine ourselves to a simple and schematic example that illustrates the application of the current model. In particular, let us consider the inplane and out-of-plane angular distribntions of sequential fission fragments from the ${ }^{197} \mathrm{Au}+{ }^{86} \mathrm{Kr}$ reaction for $\ell=190 h$.

Using our model we find that $\sigma_{\mathrm{n}}=\sigma_{\mathrm{z}}=13 \hbar$ and $\sigma_{y}=18 \hbar$ for the entrance channel asymmetry ( $U=$ 0.7 ). Assuming $K_{0}=10 \hbar$ and $I_{2}=54 \hbar$, one obtains the in-plane and out-of-plare distributions shown in Fig. I (see solid curves). The out-of-plane distributions are strongly anisotropic as observed experimentally. Interestingly enough, there is also a weak in-plane anisotropy; a shallow minimum occurs along the line of centers. The angular distributions are rather sensitive to the exit-channel asymmetry. As an illustration we have performed a similar calculation for $U=0.9$ (see dashed curves in Fig. 1). For this larger asymmetry, the out-of-plane distribution broadens because of the increased tilting of the decay axis of the projectile and target nuclei. At the same time, the predominant spin fiuctuations about the decay axis give rise to an appreciable in-plane anisotropy.

At the current time, the experimental situation regarding the in-plane angular distributions is unclear. Measurenients on the systems ${ }^{5 B} \mathrm{Ni}$ and ${ }^{90} \mathrm{Zr}+{ }^{203} \mathrm{~Pb}$ and ${ }^{236} \mathrm{U}$ indicate essentially isotropic distributions. On the other hand, studies of the reaction ${ }^{209} \mathrm{Bi}+{ }^{86} \mathrm{Kr}$, ${ }^{197} \mathrm{Au}+{ }^{20} \mathrm{Ne}$, and ${ }^{238} \mathrm{U}+{ }^{20} \mathrm{Ne}$ do show in-plane anisotropies with a maximum along the expected recoil direction of the target-like fragment. In these cases, the anisotropies become mote pronounced as the exitchannel asymmetry increases in qualitative agreement with the present model. However, the quantitative com. parison of the experimental data with the model predictions is not straightforward. In the model, the relevant direction is that of the line of centers at the time of scission when the interaction between the fragments ceases and the instantaneous partition of the total angular momentum is fixed in the system. Because this direction generally does not coiscige with the recoil

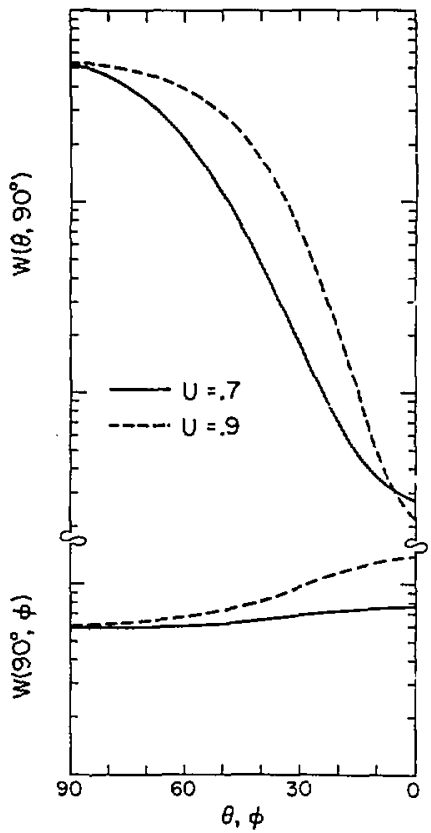

Fig. 1. Calculated out-of-plane and in-plane angular distributions of sequential fission fragments for the ${ }^{197} \mathrm{Au}+600 \mathrm{MeV}{ }^{86} \mathrm{Kr}$ reaction for $\mathrm{U}=0.7$ (the entrance channel) and $U=0.9$. Note that the line of centers is situated at $\theta=90^{\circ}$ and $\Phi=90^{\circ}$.

XBL 833-8961 
direction or any other experimentally determined direction, it is difficult to pass judgement on the statistical model at this time.

\section{Foothotes and References}

*Condensed from Nucl. Phys. A379 (1982) 313
†Permanent address: Department of Chemistry and Cyclotron Institute, Texas A\&M University, College Station, Texas 77843

¥Permanent address: Comisión Nacional de Energía Atómica, Buenos Aires, Argentina

1. L.G. Moretto and R.P. Schmitt, Pbys. Rev. C21 (1980) 204

\title{
The "Soggy Saddle" Theory of Fission
}

\author{
L.G. Morelto and G. Guarino
}

The standard Bohr Wheeler (BW) theory of fission decay ${ }^{1}$, identical with the transition state theory for chemical reactions, calculates the flux of the density distribution in phase space across a suitably chosen hypersurface normal to the reaction coordinate. This flux is then identified with the reaction rate. This is both the beauty and the trap of the theory. The flux and the reaction rate can be identified if and only if no phase-space trajectory, after crossing the hypersurface, comes back and crosses it again returning to the reactant's region.

In a more general approach, we assume high viscosity in the general saddle point neighborhood. As a result, the flux from the compound nucleus is trapped in the saddle region, and the associated randomization leads to a backflow towards the compound nucleus. Furthermore, it is interesting to consider the possibility that while tbe system is trapped in the neighborhood of the saddle point it may undergo particle decay, in particular, neutron emission.

Let us consider a compound nucleus $A$, a saddle point region $B$, a region $C$ far down the scission valley, and a nucleus $D$ after one neutron emission. The transition probabilities are $\lambda_{1}$ (from $A$ to $B$ ), $\lambda_{2}$ (from $B$ to $A$ ), $\lambda_{3}$ (from $B$ to $C$ ), $\lambda_{1}$ (from $A$ to $D$ ), $\lambda_{n^{\prime}}$ (from $B$ to D).

The master equations can be solved in a straightforward way, and from the populations at time infinity one can obtain the following expression:

$$
\Gamma_{\mathrm{N}} / \Gamma_{\mathrm{F}}=\frac{\phi_{D}(\infty)}{\phi_{C}(\infty)}=\frac{\lambda_{\mathrm{B}}}{\lambda_{\mathrm{I}}}+\frac{\lambda_{1} \lambda_{\mathrm{I}^{\prime}}+\lambda_{2} \lambda_{\mathrm{M}}+\lambda_{\mathrm{n}} \lambda_{\mathrm{n}^{\prime}}}{\lambda_{1} \lambda_{3}} .
$$

The new expression (1) favors neutron decay in two ways: a) by allowing neutron decay from the saddle; b) more importantly, by redirecting part of the flux from the saddle region back to the compound nucleus.
An intermediate and more general situation can be envisaged as follows. For a given viscosity at the saddle, there will be a critical velocity along the fission coordinate, above which the system escapes altogether towards fission and below which the system gets trapped in the saddle region. The treatment can be modified by splitting $\lambda_{1}$ as follows:

$$
\begin{aligned}
& \lambda_{1}=\frac{1}{2 \pi \rho(E)}\left[\int_{0}^{0} \rho\left(E-B_{F-t}\right) d t\right. \\
& \left.+\int_{8}^{\infty} \rho\left(E-B_{F}-t\right) d t\right]=\lambda_{1 S}+\lambda_{1 F} .
\end{aligned}
$$

Using (2), expression (1) assumes the form

$$
\Gamma_{F} / \Gamma_{N}=\frac{\lambda_{1 F}}{\lambda_{k}}+\frac{1}{2} \frac{\lambda_{1 S}}{\lambda_{n}}
$$

where the contribution of the neutron decay from the saddle region has been disregarded, and where for the systems trapped in the saddle region we have assumed $\lambda_{2}=\lambda_{3}$. Using the level density in the equidistant model approximation for the transition probabilities, expression (3) yields

$$
\begin{aligned}
& \Gamma_{F} / \Gamma_{N}=\frac{a_{n}}{2 a_{r}\left[2 a_{a}^{1 / 2}\left(E-B_{n}\right)^{1 / 2}-1\right]}\left\{\left[2 a^{1 / 2}\left(E-B_{F-E_{0}}\right)^{1 / 2}-1\right]\right. \\
& \exp \left[2 a_{F}^{1 / 2}\left(E-B_{r}-C_{0}\right)^{1 / 2}-2 a_{n}^{1 / 2}\left(E-B_{a}\right)^{1 / 2}\right] \\
& +\left[2 a_{\mathrm{f}}^{1 / 2}\left(E-B_{F}\right)^{1 / 2}-1\right] \exp \left[2 a_{j} / 2\left(E-B_{F}\right)^{1 / 2}\right. \\
& \left.\left.-2 a_{a}^{1 / 2}\left(E-B_{a}\right)^{1 / 2}\right]\right\} \text {. }
\end{aligned}
$$

Disregarding the effect of the collective motion on the viscosity parameter, we obtain from qualitative microscopic considerations the following dependence of $t_{0}$ on 
the temperature:

$$
\epsilon_{0} \propto\left(1 / \tau_{n}\right)^{2} \approx\left[\frac{1}{\tau_{0}\left(\frac{\varepsilon_{F}}{T}\right)^{2}}\right]^{2} \propto T^{4} .
$$

The effect of the backfiow from saddle to compound nucleus, responsible for the increasing number of neutrons emitted through the inhibition of the fission channel, can be clearly seen in Fig. 1 where the quantity

$$
<u_{n}>-\sum s P_{F, n+1} \prod_{K=1}^{\prime} P_{n, K}
$$

where

$$
P_{\mathrm{L}, \mathrm{K}}=\frac{1}{1+\left(\Gamma_{\mathrm{F}} / \Gamma_{\mathrm{N}}\right)_{\mathrm{K}}} \quad \mathrm{P}_{\mathrm{F}, \mathrm{s}}=\frac{1}{1+\left(\Gamma_{\mathrm{N}} / \Gamma_{\mathrm{F}}\right)_{1+1}}
$$

is plotted versus excitation energy $E^{*}$ for the nuclei ${ }^{170} \mathrm{Yb},{ }^{180} \mathrm{~W},{ }^{186} \mathrm{Os}$.

This general trend seems consistent with the results of recent accurate measurements of prefission neutron emission, ${ }^{1}$ which for systems with high fission barriers show substantially larger values of $\left\langle y_{n}\right\rangle$ than predicted by the standard model.

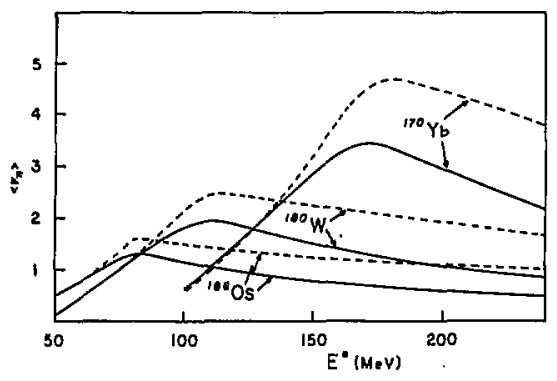

Fig. 1. Average prefission neutron emission $\left\langle y_{n}\right\rangle$ yersus excitation energy for three compound nuclei $\left({ }^{170} \mathrm{Yb},{ }^{180} \mathrm{~W}_{1}{ }^{186} \mathrm{Os}\right)$. The full lines are the result of the calculation with $\epsilon_{0}=0$, the dashed lines with $t_{0}=0.08 \mathrm{~T}^{4}$.

XBL 829-9664

Footnote and Reference

*Condensed from LBL-14404

1. A. Gavron, et al., Phys. Rev. Lett. 47 (1981) 1255

\title{
Energy Equilibration and Anomalous Mass Drift in Heavy Ion Collisions*
}

\author{
Luciano G. Moretto
}

Two striking, apparently unrelated open problems in our understanding of deep inelastic processes are the anomalous drift in mass asymmetry on the one hand and the unexpected thermal partition of the energy between fragments even at small $Q$-values on the other.

The former effect can be observed in the reluctance of the $\mathbf{K r}$-like fragment in reactions such as $\mathbf{K r}+$ $\mathrm{Er}, \mathrm{Kr}+\mathrm{All}$, etc., to drift toward symmetry as suggested by the potential energy surface, except at the largest energy losses. Such experimental facts seem to suggest that the evolution along the mass asymmetry coordinate is controlled by something beyond the relevant static potentials, most likely by dynamical effects. The latter effect is the surprising interfragment thermal equilibration, which is observed even at small Q-values, i.e., at short interaction times. This surprise arises from the short interaction time on the one hand and the straightforward prediction on the other that just about any mechanism responsible for the energy dissipation tends to deposit approximately equal energy on both fragments, thus leading to a nonthermal distribution.

While these two features seem to be unrelated at first glance, they may well be connected at a deeper level. One could make a qualitative argument as follows: Let us assume that particle exchange is the primary means of energy dissipation. It follows that initially the nearly equal amounts of energy given to both 
fragments will create a temperature imbalance in favor of the light fragment, which in turn will grow hotter. Let us further assume that the one-sided particle flux depends on the temperature of the sending fragment. In this way, an imbalance is created in the two flows, the light fragment sending more particles, and of higher energy, than it receives. This response of the system has two effects. On the one hand, it tends to contain the temperature gradient by forcing higher energy particles into the heavy fragment and, much more important, by forcing a greater energy deposition on the heavy fragment through the flux imbalance. On the other, it inevitably forces the light fragment to become progressively lighter. In other words, the light fragment fights the temperature gradient at the expense of its own mass.

To illustrate this effect, a simple model can be set up to capture the flavor of the physics. Let us consider two containers, A, very large, and B, very small, to simulate an asymmetric system. They both contain the same ideal classical gas at the initial temperature $T_{0}$. The containers are connected through a small hole and $B$ moves with initial velocity $V_{0}$ with respect to $A$. Let us calculate for $B$ the particle number $N$, the velocity $V$, and the temperature $T$ as a function of the time $t$. Assuming unity for the particle masses and neglecting the form factor associated with the size of the hole, one obtains for $\mathbf{N}$

$$
\frac{\mathrm{dN}}{\mathrm{dt}}=\sqrt{\frac{\mathrm{T}_{0}}{2 \pi}}-\sqrt{\frac{\mathrm{T}}{2 \pi}}
$$

Similarly, for the momentum loss one has

$$
\begin{aligned}
\frac{d p}{d t} & =\frac{d(N V)}{d t}=-V \sqrt{\frac{T}{2 \pi}} \\
& =V \frac{d N}{d t}+N \frac{d V}{d t}
\end{aligned}
$$

or using (1)

$$
\frac{d \ln V}{d t}=-\frac{1}{N} \sqrt{\frac{T_{0}}{2 \pi}} .
$$

For the energy, one obtains

$$
\begin{aligned}
\frac{\mathrm{dE}}{\mathrm{dt}}= & 2\left(\sqrt{\frac{\mathrm{T}_{0}}{2 \pi}} \mathrm{T}_{0}-\sqrt{\frac{\mathrm{T}}{2 \pi} \mathrm{T}}\right) \\
& +\frac{1}{2} \sqrt{\frac{\mathrm{T}_{0}}{2 \pi}} \mathrm{V}^{2} .
\end{aligned}
$$

For the temperature one obtains

$$
\begin{gathered}
\frac{d T}{d t}=\frac{2}{3 N}\left[2 \sqrt{\frac{T_{0}}{2 \pi}} T_{0}-\left(\frac{3}{2} \sqrt{\frac{T_{0}}{2 \pi}}\right.\right. \\
\left.\left.+\frac{1}{2} \sqrt{\frac{T}{2 \pi}}\right) T+\frac{1}{2} \sqrt{\frac{T_{0}^{-}}{2 \pi}} V^{2}\right] .
\end{gathered}
$$

We can now define the natural units of the problem

$$
n=\frac{N}{N_{0}} ; \tau=t \sqrt{\frac{F_{0}}{2 \pi}} ; v=\frac{V}{\sqrt{T_{0}}} ; T^{*}=\frac{T}{T_{0}} .
$$

In these units, the three differential equations read

$$
\begin{gathered}
\frac{d n}{d \tau}=\left(1-\sqrt{T^{*}}\right) \\
\frac{d \ln v}{d \tau}=-\frac{1}{n} \\
\frac{d T^{*}}{d \tau}=\frac{2}{3 n}\left[2-T^{*}\left(\frac{3}{2}+\frac{1}{2} \sqrt{T^{*}}\right)+\frac{1}{2} v^{2}\right] .
\end{gathered}
$$

The full solutions of equations (6), (7), and (8) are shown in Figs. 1, 2, and 3 for various values of the initial velocity. Fig. 1 shows that the fragment B experiences a temperature pulse, which is of course larger for larger values of initial velocity, which decays almost completely within one relaxation time for the velocity. Conversely, Fig. 2 shows a dramatic drop in particle number as a function of time. Fig. 3 shows the time dependence of the velocity, which is not rigorously exponential.

In conclusion, the model shows that 1) the small fragment becomes hotter than the heavy fragment (in this case the heavy fragment, being infinitely massive, does not warm up); 2) the temperature increase occurs early in time and is quickly checked and reduced by a diffusion imbalance; and 3) the temperature containment is achieved at the expense of the particle number of the small fragment.

\section{Footnote}

"Condensed from Z Phys A 310 (1983) 61. 


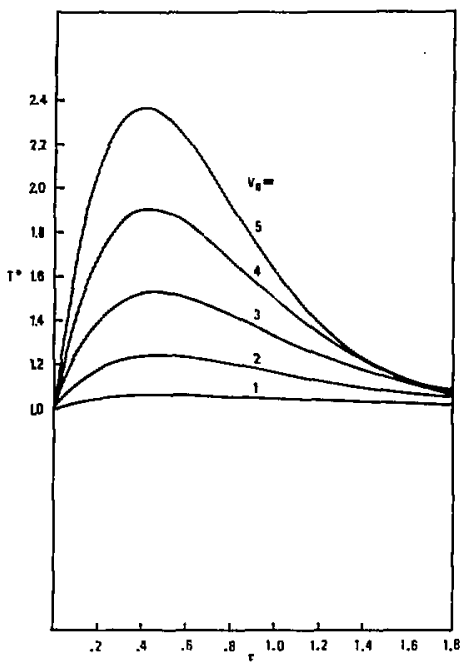

Fig. 1. Dependence of temperature on time for various initial velocities.

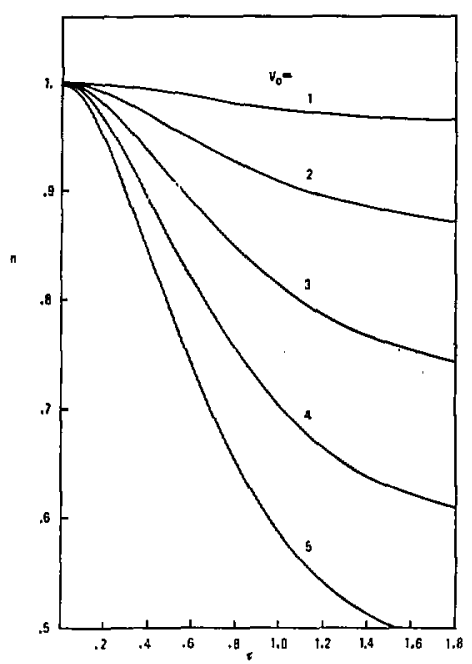

Fig. 2. Dependence of particle number on time for various initial velocities.

XBL $8211-3365$

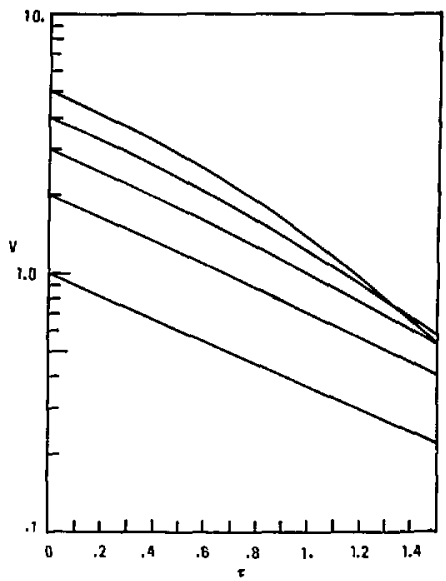

Fig. 3. Dependence of velocity on time for various initial velocities.

XBL $82[1-3366$ 


\title{
Deep Inelastic Reactions:
}

\section{A Probe of the Collective Properties of Nuclear Matter*}

\author{
L.G. Moretto and R.P. Schmint
}

The general features of deep inelastic heavy-ion reactions are reviewed. The most prominent collective degrees of freedom excited in these reactions are discussed within the framework provided by the natural hierarchy of their characteristic relaxation times. Both the quantal and classical aspects of these modes are described. The limitations of the Lagrangian treatment of heavy-ion reactions are pointed out, and a more general approach using transport theory is outlined. This latter approach is illustrated by the Langevin, Master, and Fokker-Planck equations. The four most widely studied collective modes are then described in detail. The damping of the relative motion is dealt with first. The general features of the energy loss spectra are described along with the energy dissipation mechanisms that have been suggested. Evidence for the thermalization of the dissipated energy is considered. Next the discussion focuses on the mass asymmetry degree of freedom. It is shown that the complex experimental features associated with the charge distributions of the fragments can be interpreted as evidence for a diffusion process. Transport theory is applied to the charge transfer process. The coupling between the charge transfer process and the energy damping is also described and empirical prescriptions for deducing transport coefficients are discussed. Simultaneous measurements of the charge and mass of deep inelastic fragments are then considered. The roles of isospin fluctuations and giant isovector modes are analyzed. Last, the various rotational degrees of freedom excited in heavyion reactions are described in connection with measurements of gamma-ray multiplicities and anisotropies and with the angular distributions of light particles and fission fragments. Both the magnitude and the alignment of the transferred angular momentum are explored.

\section{Footnotes}

"Condensed from Rep. Prog. Phys, 44 (1981)

†Department of Chemistry and Cyclotron Institute, Texas A\&M University, College Station, Texas 77843

\section{Multifragmentation and the Partition of Angular Momentum. A General Statistical Theory}

\author{
L.G. Moretto
}

In deep inelastic scattering it has been found that the spin of each fragment is well described by a component aligned with the entrance channel angular momentum arising from rigid rotation and by a random component along the three cartesian axes. These experimental results are well reproduced by a statistical model introduced by Moretto and Schmitt. ${ }^{1}$ The success of the statistical model applied to deep inelastic processes suggests that a similar approach may be useful in describing the fate of angular momentum in collisions leading to the formation of more than two large fragments.
In a collision leading to the formation of $n$ fragments, let us assume statistical equilibrium is maintained in the "expansion" phase until a critical shape is reached. Beyond the critical shape the fragments decouple and the equilibrium at the critical shape remains frozen in. Let us further assume that the critical shape of the system is spherical and that the shape of the fragments is also spherical. We can write the Hamiltonian of such a system as follows: 


$$
\begin{gathered}
H=\Sigma H i=\sum\left\{\frac{l_{x}^{2}+l_{y}^{2}+I_{z}^{2}}{2 f}+\frac{\varepsilon_{x}^{2}}{2 m r^{2}}\right. \\
\left.+\frac{1}{2 m}\left(p_{t}^{2}+p_{z}^{2}\right)\right\} .
\end{gathered}
$$

The sum is over the fragments; $I_{x}, I_{y}$, and $I_{z}$ are the components of the fragment intrinsic angular momentum; $\boldsymbol{J}$ is the fragment moment of inertia; $\ell_{2}$ is the Zprojection of the fragment orbital angular momentum; $P_{z}$ and $P_{z}$ are the other two generalized momenta in cylindrical coordinates. The partition function is

$$
\begin{gathered}
Z=\int e^{\left.-\frac{\sum \pi}{T}-\pi L_{2}+\ell_{2}\right)} d^{3} I d \ell_{2} d p_{1} d p_{2} \\
=\pi(\sqrt{2 \pi T})^{2}(\sqrt{2 m T})^{2} \\
\sqrt{2 \pi \pi} e^{\frac{r^{2} \pi}{2}} \sqrt{2 \pi m r^{2} T} e^{\frac{\mu^{2} r^{2} T}{2}} .
\end{gathered}
$$

The total angular momentum $I_{T}=\Sigma\left(I_{z}+\ell_{z}\right)$ is conserved on the average through the Lagrange muliplier $\mu$. From the partition function, by suitable differentiation one obtains:

$$
\mu=\frac{\mathrm{l}_{\mathrm{T}}}{\mathrm{T} \Sigma\left(\boldsymbol{S}+\mathrm{mr}^{2}\right)}
$$

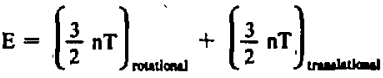

$$
\begin{aligned}
& +\left(\frac{l_{T}^{2}}{2 \Sigma\left(s+m r^{2}\right)}\right)_{\text {ried racelion }} \\
& \tilde{I}^{2}=3 \pi_{T}+\left(\frac{S}{\sum\left(S+m^{2}\right)} I_{T}\right)^{2} .
\end{aligned}
$$

From this one notices that each fragment has two sources of angular momentum, a thermal source whose presence and magnitude is independent of $\mathrm{I}_{T}$, and a rigid rotation source, which gives the fragment a share of the total angular momentum. It is easy to show that $\sigma_{x}^{2}=\sigma_{y}^{2}=\sigma_{x}^{2}$. Consequently, the components of the polarization tensor for each fragment are

$$
\mathrm{P}_{z x}=\frac{\mathrm{P}_{z y} \propto \sigma_{x}^{2}-\sigma_{y}^{2}=0}{1+3 \pi\left(\frac{\sigma\left(\frac{\left.1+m^{2}\right)}{\Lambda_{T}}\right)^{2}}{1} .\right.}
$$

The quantity that needs to be determined is $\Sigma(\boldsymbol{s}+$ $\mathrm{mr}^{2}$ ). As we have no a priori knowledge of it, we can hope to turn the problem around and, after having found satisfactory signs of thermalization, we can infer it from the measurement of the frarnent spins.

\section{Reference}

I. L.G. Moretto, and R.P. Schmitt, Phys. Rev. C21 (1980) 204.

\title{
Statistics at Work in Heavy Ion Reactions*
}

\author{
Luciana G. Moretto
}

In the first part special aspects of the compound nucleus decay are considered. The evaporation of particles intermediate between nucleons and fission fragments is explored both theoretically and experimentally. The limitations of the fission decay width expression obtained with the transition state method are discussed, and a more general approach is proposed. In the second part the process of angular momentum transfer in deep inelastic reactions is considered. The limit of statistical equilibrium is studied and specifically applied to the estimation of the degree of alignment of the fragment spins. The magnitude and alignment of the transferred angular momentum is experimentally determined from sequentially emitted alpha, gamma, and fission fragments.

"Condensed from LBL-14563. Presented at the Enrico Fermi International School of Physics on Nuclear Structure and Heavy Ion Dynamics, Varenna, Italy, July 27-August 6, 1982 


\title{
The Effect of Statistical Fluctuations on the Measurement \\ of the Total Energy and the Multiplicity of $\boldsymbol{\gamma}$-Rays \\ Following Deep-Inelastic Reactions*
}

\author{
A.J. Pachecot and L.G. Moretto
}

The simple relation between the spin and the energy is a well known property of the rotational states in nuclei. This fact provides the rationale for the use of toral $\gamma$-ray energy detectors ("sum spectrometers") to investigate high-spin states of evaporation residues following compound-nucleus and deep-inelastic reactions. ${ }^{1,2}$

Whereas sum spectrometers can be used effectively as spin-selecting devices, the extraction of more quantitative information based on the measured total energy is hindered by several uncertainties. In the case of deep-inelastic reactions a fundamental source of uncertainties is associated with fluctuations, statistical or otherwise, introduced by the reaction mechanism. The most significant problem in this regard is the distribution in both magnitude and orientation of the spins of the emitting population.

The purpose of the present paper is to evaluate the contribution of spin fluctuations to the distributions of the $\gamma$-ray sum energy and $\gamma$-ray multiplicity. Our analysis will be restricted to the case of binary reactions between heavy ions in which the detection of one of the two fragments determines a reaction plane with the orbital angular momentum perpendicular to it. The calculations have been performed in the framework of an equilibrium statistical model that considers the thermal excitation of angular-momentum-bearing modes of the dinuclear complex, assumed to be formed by two touching rigid spheres., 3

If one considers a system with temperature $T=$ 0 , the average intrinsic rotational energy is given by the simple expression

$$
<E_{H}+E_{L}>=\frac{\left|r_{H}\right|^{2}}{2 S_{H}}+\frac{\left|r_{L}\right|^{2}}{2 S_{L}}=\frac{\mathscr{S}_{H}}{2 \mathscr{S}_{\perp}^{2}} I^{2}
$$

where I is the total angular momentum in the system, I is the fragment spin arising from rigid rotation, $\boldsymbol{f}$ is the fragment moment of inertia, and the subscripts $H$ and $\mathbf{L}$ label the heavy and light fragment respectively. In addition, we define

$$
\mathscr{A}=\boldsymbol{x}_{\mathrm{H}}+\boldsymbol{x}
$$

and $\boldsymbol{S}_{1}=\boldsymbol{S}_{1}+\mu \mathrm{d}^{2}$

where $\mu$ is the reduced mass and $d$ is the distance between centers. evaluated:

If $T \neq 0$, the following integral must be

$$
<E_{H}+E_{L}>=\int\left[E_{H}(\{\xi\})+E_{L}(\{\xi\})\right] P(\{\xi\}) d^{6} \xi .
$$

Here, $\{\xi\}$ represents the set of angular momenta associated with a rotational configuration, which is thermally excited with a probability given by $\mathrm{P}(\{\xi\})$.

It is convenient to express the analytical result of the integral (eq. 2) in units of the intrinsic rotational energy at $T=0$ (eq. 1 ):

$$
\left(\frac{A_{1}|!|^{2}}{2 A}\right)^{-1}<E_{H}+E_{L}>=1+\alpha Z^{2} .
$$

The variable $\mathbf{Z}$ desends on the total mass, temperature, and total angular momentum of the system

$$
Z=\frac{\sqrt{\mathcal{S}_{\mathrm{zym}}}}{I}
$$

The constant $\alpha$ is a function of the mass asymmetry only.

The expression in eq. (3) is plotted as a function of $Z$ in Fig. 1 (dark solid line). As an example, let us consider the system ${ }^{165} \mathrm{Ho}+{ }^{165} \mathrm{Ho}$ at $8.5 \mathrm{MeV} / \mathrm{u}$. We estimate a total angular momentum $|!|=350 \mathrm{~h}$ and assume a temperature $\mathrm{T}=2.5 \mathrm{MeV}$; therefore, $\mathrm{Z} \simeq$ 0.038 . From the figure we see that the statistical fluctuations introduce a $20 \%$ increase of the average internal rotational energy over the rigid-rotation prediction. Figure 1 also illustrates the individual contributions from the normal modes (dash-dot, long-dash, and 
shert-dash curves). The dependence of the average reduced rotational energy on mass asymmetry, at constant $Z$, is shown in Fig. 2. As larger asymmetries are considered, the effect of the fluctuations decreases due to the progressive suppression of some of the normal modes. The rotational energy (extracted from the sum energy) and the sum of the spin magnitudes (extracted from the multiplicity) are not related by eq. (3) as might at first appear. This is so because the average spins are also affected by the fluctuations, as shown by the following relation:

$$
\left(\frac{s_{1}}{s_{\perp}} \mid \underline{1}\right)^{-1}<\left|s_{-H}\right|+\left|s_{1}\right|>-1+\beta z^{2} .
$$

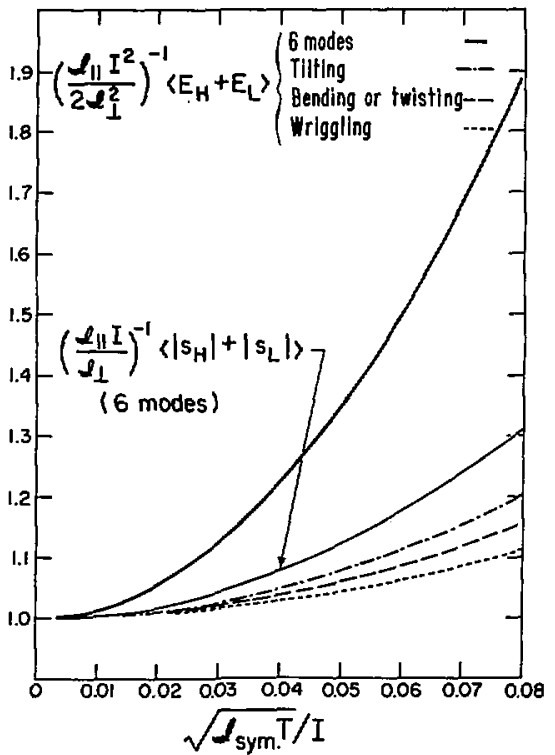

Fig. 1. Reduced average intrinsic rotational energy (dark solid curve) and average sum of the spin magnitudes (light solid curve) as a function of the parameter $Z$, for a mass-symmetric reaction. The three lowest curves illustrate the contribution to the rotational energy from the individual modes.

XBL 8112-12073
In complete analogy with eq. (3), $Z$ is defined by eq. (4), and $\beta$ depends only on the mass asymmetry. The expression in eq. (5) is also plotted in Fig. 1 (light solid curve).

In summary, fluctuations in the spins imparted to the nuclei in a deep-inelastic reaction represent a substantial contribution to the observed average values of physical quantities having an angular momentum dependence. Under the assumption of thermal equilibrium of the normal modes of the dinuclear complex, it has been shown that the average rotational energy may be, typically, $20 \%$ or $30 \%$ higher than the rigid-rotation prediction for a system with zero temperature. The magnitude of the effect decreases with increasing mass asymmetry as a consequence of the increasing stiffness of some of the rotational modes. A similar, although not so strong, effect is predicted for the average sums of the magnitudes of the spins. Second moments of the distributions have also been calculated, and the results indicate that the thermal excitation of the rotational modes of the complex may contribute significantly to the observed widths.

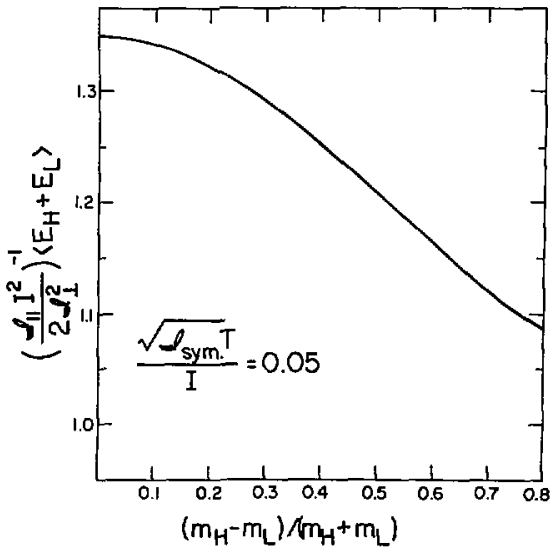

Fig. 2. Reduced average intrinsic rotational energy as a function of mass asymmetry for a fixed value of the parameter $\mathbf{Z}$.

XBL 8112-12075 


\section{Footnotes and References}

'Condensed from Z. Phys. A306 (1982) 259.

†Permanent address: Comisión Nacional de Energía Atómica, Buenos Aires, Argentina

1. P.O. Tjom, F.S. Stephens, R.M. Diamond, J. de Boer and W.E.Meyerhof, Phys. Rev. Lett. 33 (1974) 593.
2. H.J. Kömer, D.L Hills, C.P. Roulet, P. Aguer, C. Ellegaard, D.B. Fossan, D. Habs, M. Neiman, F.S. Stephens, and R.M. Diamond, Phys. Rev. Lett. 43 (1979) 490.

3. LG. Moretto and R.P. Schmitt, Phys, Rev. C21 (1980) 204.

4. R.P. Schmitt and A.J. Pacheco, Nucl. Phys. 1379 (1982) 313. 


\title{
3. High Energy Collisions
}

\section{Calculation of Complete Multi-Fragment Events in a Statistical Model for Nuclear Disassembly*}

\author{
Jorgen Randrup and George Fait
}

We have developed a practical method for genarating samples of multi-fragment distributions within the framework of a statistic model for nuclear disassembly. While the method is quite general and may have wide applicability, the realm of physics we have in mind is that of medium-energy nuclear collisions. In such collisions a highly excited transient system may be formed and subsequently disassemble into many nuclear fragments. This problem has been addressed in refs. [1-2], which study the one-fragment inclusive distributions within a statistical model. Recent developments in detection technique demand that the exclusive distributions be considered, and it is of interest to calculate complete events on the basis of a statistical model. This amounts to assuming that all multi-fragment states compatible with the overall conservation laws are equally probable. The practical task is then to generate a sample of multi-fragment events distributed statistically in accordance with the appropriate exclusive microcanonical distribution function. This undertaking is rather cumbersome, particularly when many different fragment species are included. It is therefore desirable to develop a suitable approximate method. Below we formulate the mathematical task, describe our approximation, and discuss its validity.

In the statistical model, the disassembly depends only on the conserved quantities of the system, which in the present case are the total baryon number $A_{0}$, the total charge number $Z_{0}$, and the total four-momentum $P_{0}=\left(\vec{P}_{0}, E_{0}\right)$. The disassembling system (which shall often be referred to as the source) is thus characterized by the quantities $A_{0} Z_{0} P_{0}$ denoted collectively by $j_{0}$ for notational convenience, and we seek the probability distribution $p(\mathrm{id} f)$ for arriving at various final states $f$. The statistical assumption is that all final states compatible with the conservation laws are equally probable. Hence

$$
p\left(i_{0} \mid f\right)=\boldsymbol{\Lambda}\left(i_{0}\right)^{-1} \delta\left(i_{0}-i[f]\right)
$$

where $\mathrm{i}[\mathrm{f}]=\mathrm{A}_{1} \mathrm{ZP}_{\mathrm{f}}$ are the values of the conserved quantities for the specified final state $f$. The normalization constant is determined from the condition that $p$ be normalized,

$$
\boldsymbol{\sim}\left(i_{0}\right)=\underset{i}{\not \gamma\left(i_{\sigma}-i[f]\right)}
$$

The exclusive distribution $p$ pertains to the situation where the specification of the firal state is complete, corresponding to an exclusive measurement. When only partial specification of the final state is made, as is most often the case, the relevant quantity is the corresponding inclusive distribution $P$. This quantity can be obtai sed from the exclusive distribution by integrating over the unobserved quantities. In particular, when the partial measurement is such that complite information is obtained for some of the fragments, the inclusive distribution is given by

$$
P\left(i_{0} \mid f\right)=\sum_{m} p\left(i_{0} \mid f\right)
$$

Here the observed partial event is denoted by $l$ and the summation is over all final states $f$ that encompass the specified observation $\mathrm{f}$. It is simple to express the inclusive distribution in terms of the phase space integrals (2):

$$
P\left(i_{0} \mid \hat{f}\right)=\sum_{\hat{f}} p\left(i_{0} \mid \hat{f}+\bar{f}\right)=\left\{\left(i_{0}\right)^{-i} \wedge\left(i_{0}-i[\hat{f}]\right)\right.
$$

i.e., the inclusive probability for the partial event 7 is equal to the corresponding complementary phase space integral $\left.\boldsymbol{S}_{i_{0}}-\mathrm{i}[\mathrm{f}]\right)$ divided by the total phase space integral $\boldsymbol{\gamma}\left(\mathrm{i}_{0}\right)$.

By combination of (2) and (4) it is possible to factorize $\mathrm{p}\left(\mathrm{i}_{0} \mid \mathrm{f}\right)$ into simpler quantities:

$$
\begin{aligned}
& p\left(i_{0} \mid n\right)=\left\langle\left(i_{0}\right)^{-1} \delta\left(i_{0}-i[\hat{l}]-i[i]\right)\right. \\
& =P\left(i_{0} \mid \bar{h}\right) p\left(i_{\sigma}-i[\bar{n} \mid \bar{f})\right.
\end{aligned}
$$

where $\hat{f}+\vec{f}=\mathfrak{f}$. This relation expresses the fact that the exclusive probability for obtaining the event $f$ is equal to the inclusive probability for obtaining a part of the event, $\mathbf{f} \leq \mathrm{f}$, times the exclusive probability for obtaining the complementary event $\bar{f}=f-\bar{f}$, given that $f$ has already been obtained. 
By repeated use of the above relation (5), it is possible to factorize the exclusive probability $p$ into one-fragment inclusive distributions:

$$
p\left(i_{0} \mid f=\sum_{k=1}^{i} f_{k}\right)=\prod_{k=1}^{i} P\left(i_{k-1} \mid f_{k}\right) \cdot p\left(i_{k} \mid 0\right)
$$

Here we have defined $i_{k} \equiv i_{k-1}-i\left[f_{k}\right]$ for $k \in\left(1, n_{f}\right)$. The exclusive factor $p\left(i_{n} \mid 0\right)$ vanishes unless the quantities

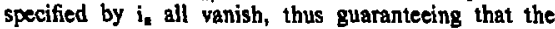
event $f$ is in fact accessible by the disassembling system characterized by $i_{0}$.

The factorization (6) of the exclusive multifragment distribution $p$ into inclusive one-fragment distributions is particularly convenient when one seeks to generate a statistical representation of $p$, i.e., a sample $f$ of multi-fragment events which are statistically distributed in event space according to the probability density $p(i d f)$. However, to accomplish this task, some degree of approximation is therefore necessary. Fortunately, one-fragment distributions, which are the only ones required in the procedure, are much easier to approximate than more exclusive quantities. It is therefore possible to turn the mathematical procedure into a practical method. The key lies in employing the grand canonical approximation separately for each of the inclusive factors in (6). The grand canonical approximation is accurate for one-fragment distributions as long as the fragment considered is only a small part of the system. This condition is reasonably well fulfilled for most of the factors in the product (6), although it is substantially violated for the last few factors. This idea has been implemented and its quantitative validity of the approximation has been demonstrated.

\section{Footnotes and References}

*Condensed from LBL-14179; Phys. Lett. 115B (1982) 281

†Institute of Theoretical Physics, Eötvös University, Budapest, Hungary

1. J. Randrup and S.E. Koonin, Nucl. Phys. A356 (1981) 223

2. G. Fái and J. Randrup, Nucl. Phys. A (in press) .

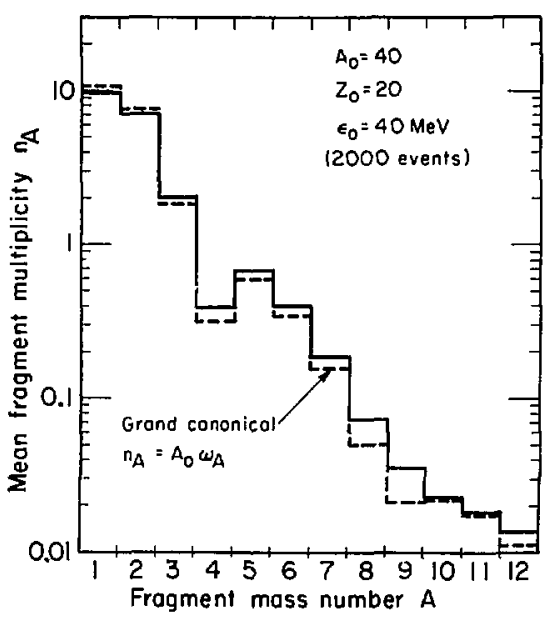

Fig. 1 The mean multiplicity of nuclear fragments with a specified baryon number $A$, for the disassembly of a system characterized by $A_{0}=40$, $Z_{0}=20, \epsilon_{0}=40 \mathrm{MeV}$. The solid histogram is the result of the present model while the dashed histogram displays the corresponding grand canonical approximation. The calculations include the nuclear levels described in ref. 2.

XBL 823-3701 


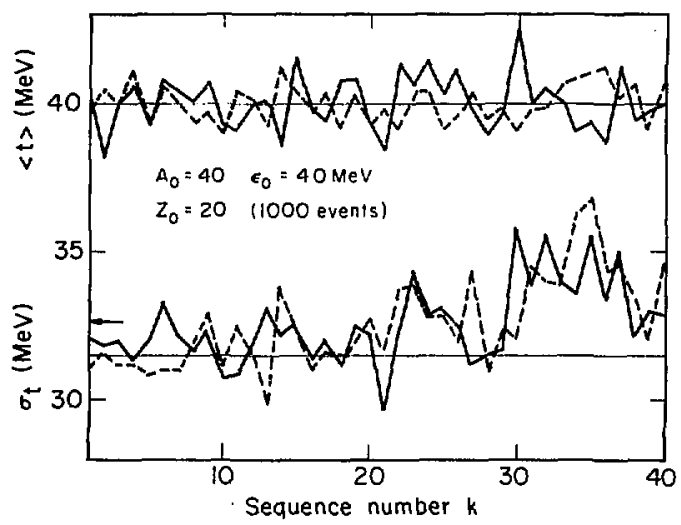

Fig. 2 Total disassembly of a system characterized by $A_{0}=40, Z_{0}=20$, and $\epsilon_{0}=40 \mathrm{MeV}$ into nucleons. The mean binetic energy $\langle\mathrm{t}\rangle=\left\langle\mathrm{E}-\mathrm{mc}^{2}\right\rangle$ and the associated dispersion $\sigma_{1}$ are plotted as functions of the sequence number $k$ (see text). The results for two samples with 1000 events each are shown. The exact overall values of $\langle t\rangle$ and $\sigma_{t}$ (as given by the grand canonical approximation) are indicated bu the horizontal lines while the arrow indicates the actual calculated overall $\sigma_{t}$.

XBL 823-3702

\title{
Statistical Simulation of Complete Events in Energetic Nuclear Collisions*
}

\author{
George Fát and Jprgen Randrup
}

In recent years the theoretical and experimental activity in medium and high energy nuclear collisions has increased rapidly. Previous theoretical studies (as well as earlier experiments) mostly focused on inclusive observables. In addition to statistical calculations based on the grand canonical approximation, several dynamical models have been put forward; they enjoy considerable (and comparable) success in reproducing certain features of inclusive data in the relativistic regime. However, it is now possible to detect electronically practically all charged fragments emerging from a collision, and thus good quality, nearly exclusive data on multifragmentation processes can be obtained. ${ }^{\prime}$ This fact calls on theory to address exclusive quantities. Therefore, we have undertaken to develop a model for calculating complete multifragment events in mediumenergy nuclear collisions. (We have in mind an energy range from a few tens of $\mathrm{MeV}$ to energies where particle creation becomes important.) The goal is to establish a reference model that invokes as few assumptions as possible about the specific dynamics of the collision process. Such a model may find several applications: i) it can provide the theoretical background against which subsequent, more refined and specific calculations can be judged, ii) it can be of help in analyzing the data, in particular in the search for peculiar structures in the individual event patterns, and iii) it can be of value in attempts to assess the bias introduced in the data by the acceptance criteria associated with a particular detection system.

The model developed can be briefly described as follows. The collision system is divided into a few subsystems, sources, each of which is assumed to disassemble in a statistical manner. For the subdivision we use the participant-spectator geometry supplemented with a prescription to share energy and momentum between subsystems. In this way we define one participant source and up to two spectator sources, each characterized by its number of nucleons, charge, and total four- 
momentum. Those sources that have an excitation energy sufficient for exmplete disassembly into free nucleons are said to be above the disassembly threshold. They are assumed to "explode" quickly into a number of pions, rucleons, and composite nuclei that are generally excited and particle unstable. Sources below the disassembly threshold are assumed to deexcite by sequential light particle evaporation; the same is assumed fo: the particle unstable explosion products.

We introduce two parameters governing the sharing of energy and momentum among the participants and spectators. By varying these parameters many different physical scenarios can be encompassed in the model. The explosion of a sufficiently excited source is assumed to $p$ pulate the accessible multif ragment channels accordir. to their statistical weight, as obtained by summing over the available phase space. This picture has been used to study the one-fragment inclusive quantities in medi im-energy nuclear collisions by employing the grand c.nonical approximation. ${ }^{2,3}$ However, the focus of the present study is on exclusive quantities, and the conservation laws must be obeyed event by event. We bave therefore developed an approximate microcanonic! treatment of the disassembly process (see other cintribution by the same authors in this report). The evaporation process is treated in a way rather simila: to that of ref. 3 .

As illus 'rations of the use of the model, we show in Fig. 1 the fragment multiplicity as a function of impact parameter and in Fig. 2 a sphericity-coplanarity contour plot for the global event shape in velocity space.

We ant:cipate extended use of the model in the time ahead, fartly for systematic theoretical studies and partly for aid.ng in the analysis of data.

\section{Footnotes and References}

*condensed f.om LBL-15044, submitted to Nuclear Phy$\operatorname{sics} \mathrm{A}$

tOn leave from the Roland Eötvös University, Budapest, Hungary

1. A. Bad n, et al., GSI-82-5 report (1982)

2. J. Rar trup and S.E. Koonin, Nucl. Phys. A356 (1981) 223

3. G. Fái and J. Randrup, Nucl. Phys. A381 (1982) 557

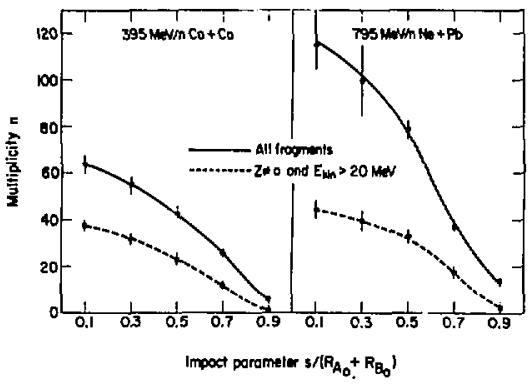

Fig. 1. Multiplicity of fragments as a function of impact parameter in reactions of experimental interest. The dashed curve corresponds to the schematic acceptance criterion that only charged fragments with kinetic energies above $20 \mathrm{MeV}$ be considered.

XBL $829-4613$

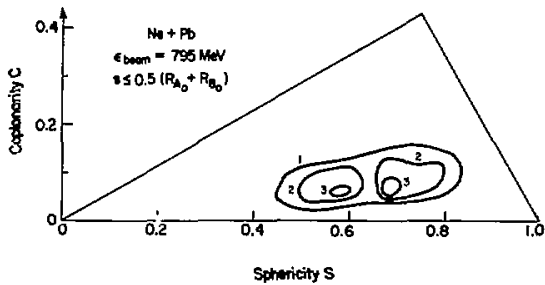

Fig. 2. Contour plot of the global sphericity and coplanarity in the case of $\mathrm{Ne}+\mathrm{Pb}$. The same schematic acceptance criterion has been applied as in Fig. 1.

XBL $829-4612$ 


\title{
Formulation of the Coulomb Effects of Spectator Fragments on Pions from Heavy Ion Collisions*
}

\author{
Hafez M.A. Radi, J.O. Rasmussen, J.P. Sullivan, \\ K.A. Frankel, and O. Hashimoro
}

Coulomb effects on heavy-ion pion production cross sections are formulated in terms of weighted averaging over various projestile fragments. Satisfactory fits to zero-degree pion data are found for $\mathrm{Ar}+\mathrm{C}$ and $\mathrm{Ne}+\mathrm{C}$ systems. The fragment distributions of excited compound nuclei before nusleon evaporation must be used. Average charge Z $Z_{\text {c }}$ values are determined and compared with those from an experimental paper. In the heavy-ion energy range of the $0^{\circ}$ pion studies $(300 \mathrm{MeV}<\mathrm{E} / \mathrm{A}<600 \mathrm{MeV}$ ) it is clear that targetprojectile factorization for fragmentation cross sections does not hold; that is, the relative yields of projectile fragments are not target-independent.

The strong $\pi^{-}$peak and $\pi^{+}$depression near beam velocity for heavy-ion-produced pions ${ }^{1}$ is qualitatively understood as a Coulomb effect of projectile spectator charges. The theoretical studies of refs. $2-4$ all show this effect. Lihbrecht and Koonin ${ }^{3}$ approximated the Coulomb field after the collision by spreading part of the proton charge along a line between nuclear centers with the remainder continuing with unaltered velocities in the original Gaussian distributions. In ref. 4 various approximate analytical expressions were derived for thermally expanding charge distributious. In particular, the $\pi^{-} / \pi^{+}$ratio at its peak is very sensitive to the temperature assumed for the spectator source, a temperature of $-4 \mathrm{MeV}$ appearing reasonable for the data of ref. 1.

The collaborators of ref. I meanwhile upgraded their zero-degree pion spectrometer with wire chambers to measure double differential cross sections at higher resolution than before. The resulting new data ${ }^{5}$ with more closely spaced points in the $\pi^{-}$peak region are no longer consistent with an expanding spectator iemperature of $4 \mathrm{MeV}$.

In Sullivan, et al., ${ }^{5}$ pion data fits gave temperatures of the order of $1 \mathrm{MeV}$ when the spectator temperature was used as a free parameter. The theoretical expressions of ref. 4 are based on unbound, free-gas expansion of the spectator source with the characteristic temperature. Here there is an inconsistency, for if the projectile spectator protons began to evaporate with characteristic temperature of $1 \mathrm{MeV}$ the nucleus would soon cool to a bound fragment containing most of the original charge.
Since production of bound projectile fragments at near-beam velocity is known to be appreciable, ${ }^{6}$ these bound fragments ought to be taken explicitly into account in pion-production Coulomb effects. We shall see that the velocity dispersion of projectile fragments plays the role of the mean expansion velocity of the free-gas source.

The Coulomb correction to the pion production cross section depends on the regular Coulomb function $F_{l}\left(\eta_{ \pm}, \rho_{u_{ \pm}}\right)$, where $\eta$ is the Sommerfeld parameter, $\eta_{ \pm}= \pm Z e^{2} / h|\vec{v}|, \vec{v}$ is the velocity of the produced pion relative to the spectator, and $Z$ is the charge of the spectator. For the cases of interest, ${ }^{7}$ the s-wave term is predominant in the expansion, and it is a good approximation to write the cross section as

$$
\sigma_{ \pm \pm} \propto\left|\frac{F_{\alpha}\left(\eta_{ \pm}, \rho_{s_{ \pm}}\right)^{2}}{\rho_{s_{ \pm}}}\right| \simeq G\left(\eta_{ \pm}\right)\left(1+\rho_{s_{ \pm} \eta_{ \pm}}+\ldots\right)^{2}
$$

where

$$
\begin{gathered}
\rho_{S_{ \pm}}=k_{ \pm} r_{s}=r_{0} \frac{m_{1} c}{h} \beta_{ \pm \pm} A_{F}^{1 / 3} \quad \text { with } r_{s}=r_{Q} A_{F}^{1 / 3}, \\
G(\eta)=\frac{2 \pi \eta}{\exp (2 \pi \eta)-1},
\end{gathered}
$$

and the + , signs stand for $\pi^{+}$and $\pi^{-}$, respectively.

For comparison with inclusive pion data we should calculate an average value of $\mathrm{Fq}$. (1) over all products and relative velocity of pion and fragment rather than for one set oi $A, Z$, v. The velucity dispersion formula obtained by Greiner, et al., ${ }^{6}$ is used and it has the form

$$
P \propto \exp \left[-\frac{\theta_{f}^{2}}{2 \alpha_{f}^{2}}\right],
$$

where $\beta_{E}=v_{F} / c$ is the fragment velosity in units of $\mathrm{c}$, and the velocity dispersion width $\alpha_{\mathrm{F}}$ is given as

$$
\alpha_{F}=\left[\sigma_{0} /\left(m_{F}\right)\right]\left[\left(A-A_{F}\right) A_{F} /\left(A_{0}-1\right)\right] .
$$

In eq. (4) the constant $\sigma_{0}$ has been found to be about 86 $\mathrm{MeV} / \mathrm{c}$ by experiment, $\mathrm{m}_{\mathrm{N}} \mathrm{c}^{2}$ is the nucleon rest mass energy constant, $A_{0}$ is the projectile mass, and $A_{f}$ is the mass of fragment under consideration. 
Furthermore, the averaging technique requires weighting with respect to the cross section of forming a fragment of mass $A_{F}$ and charge $Z_{F}, \sigma\left(A_{F}, Z_{F}\right)$ before evaporation of protons and neutrons, and also with respect to the differential cross section (before Coulomb correction) of forming a pion simultaneously with a fragment of mass $A_{F}$ near beam velocity, which is assumed to be a function of the mass loss, $F\left(A_{0}-A_{F}\right)$.

It is now straightforward to integrate over $\beta_{\mathrm{F}}$ and have the analytical formula for the average value of $\eta_{\text {s }}$ in terms of an error function as

$$
\left\langle\eta_{ \pm}\right\rangle_{\beta_{F}}= \pm\left[\frac{Z_{F} e^{2}}{\hbar c}\right] \frac{\operatorname{erf}\left[\beta_{r:} /\left(\sqrt{2} \alpha_{F}\right)\right]}{\beta_{T \pm}} .
$$

It is a good approximati n to replace the average over $\beta_{\mathrm{F}}$ in the pion cross sectio. by the average over the Sommerfeld parameter and get the grand average of the pion cross section as

$$
\begin{aligned}
& <_{\pi \pm}>=N_{\pi} \frac{\left.\sum_{F} Z_{F} N_{A_{F}} \sigma\left(A_{F}, Z_{F}\right) F\left(A_{\sigma}-A_{F}\right) G\left(<\eta_{ \pm}\right\rangle_{A_{F}}\right)}{\sum_{A_{F} Z_{F}} N_{A_{F}} \sigma\left(A_{F}, Z_{F}\right) F\left(A_{\sigma}-A_{F}\right)} \\
& \left\{1+\frac{m_{r} r_{0} C}{\hbar} \beta_{\pi^{ \pm}}<\eta=>_{\sigma_{F}} A_{t}^{1 / 3}\right\}^{2}
\end{aligned}
$$

where $N_{\Lambda^{T}}=\sqrt{2 \pi} \alpha_{F}^{3}$.

To employ our code for averaging pion Coulomb effects over projectile fragments, we must have cross sections for fragments as input.

Thus, we used the theoretical firestreak code ${ }^{8}$ for fragmentation cross sections.

Since the pions should move out many nuclear diameters during a mean life of compound nuclei, it scems more correct to use the primary fragment distribution as $\sigma\left(A_{F}, Z_{F}\right)$ rather than the final distribution in Eq. (6). Figure 1 shows the results of our theoretical code compared to ${ }^{40} \mathrm{Ar}+\mathrm{C}$ experimental pion data of Sullivan, et al. ${ }^{s}$ The firestreak code fragment yields at $533 \mathrm{MeV} / \mathrm{N}$ were used as input. Here we made the simplest assumption for the pion production factor, namely, purely linear dependence on fragment mass loss $\left(\mathrm{A}-\mathrm{A}_{F}\right)$. A momentum dispersion constant of $\sigma_{0}$ $=60 \mathrm{MeV} / \mathrm{c}$ and a radius constant $\mathrm{r}_{0}=1.5 \mathrm{fm}$ were used in eq. (6). The theoretical curve was corrected by folding in the experimental resolution. The agreement seems quite satisfactory. In ref. 5 this $\mathrm{Ar}+\mathrm{C}$ case could not be fit well using Coulomb factors for a single effective $Z_{F}$ fragment charge. Since the momentum dispersion of primary fragments before particle evaporation is unknown, we did not constrain the calculations to the experimental constant for inclusive fragmentation measurements, ${ }^{6} \sigma_{0}=86 \mathrm{MeV} / \mathrm{c}$ but left $\sigma_{0}$ as a free paranieter. The somewhat smaller value of $60 \mathrm{MeV} / \mathrm{c}$ seems favored for fragmentation associated with pion product.

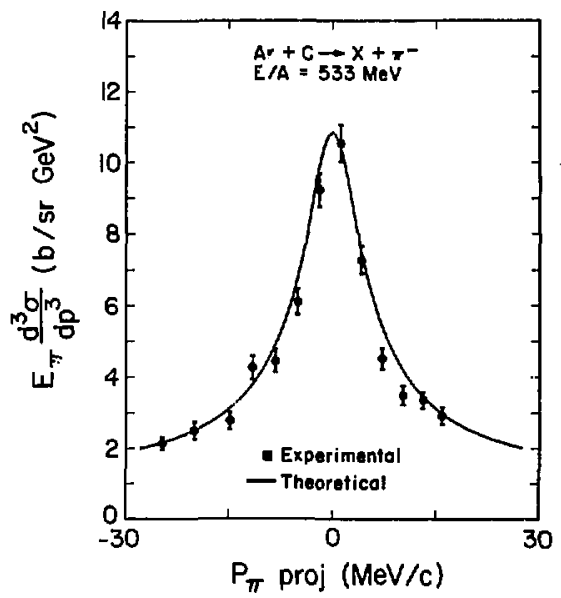

Fig. 1. The $\pi^{-}$spectrum at $0^{\circ}$ calculated by eq. (6) and compared to data of Sullivan, et al. ${ }^{5}$ The solid curve is the theory after folding with experimental resolution. The abscissa is pion momentum in the projectile frame.

XBL 816-10170

\section{Foothotes and References}

"Condensed from Physical Review C25 (1982) 1518

†Physics Department, Kuwait University, Kuwait

1. W. Benenson, G. Bertsch, G.M Crawley, E. Kashy, J.A. Nolen, J̃r., H. Bowman, J.G. Ingersoll, J.O. Rasmussen, J. Sullivan, M. Koike, M. Sasao, J. Peter, and T.E. Ward, Phys. Rev. Lett. 43 (1979) 683

2. G. Bertsch, Pbys. Rev. C15 (1977) 713

3. K.G. Libbrecht and S.E. Koonin, Phys. Rev. Lett. 43 (1979) 1581; J. Cugnon and S.E. Koonin, Nucl. Phys. A355 (1981) 488

4. M. Gyulassy and S.K. Kauffmann, Nucl. Phys. A362 (1981) 503

5. J. Sullivan, et al., Phys. Rev. C25 (1982) 1499 
6. D.E. Greiner, P.J. Lindstrom, H.H. Heckman, Bruce Cork, and F.S. Bieser, Phys. Rev. Lett. 35 (1975) 152

7. J.O. Rasmussen, H.M.A. Radi, J. Sullivan,
K. Frankel, and O. Hashimoto, LBL-12623, also in Proceedings of 5th High Energy Heavy Ion Study (May, 1981), LBL-1 2652

8. P. McGaughey, Ph.D. Thesis, U.C. Berkeley, LBL-15325 (Nov. 1982) unpublished

\title{
Calculation of Muon Final Probabilities After Mron-Induced Fission in Four-State Basis*
}

\author{
Zhong-yu Ma, ${ }^{\dagger}$ Xi-zhen $\mathrm{Wu}_{*}^{\dagger}$ Jing-shang Zhang, \\ Yi-zhong Zhuo, ${ }^{\dagger}$ and J. O. Rasmussen
}

Our earlier theoretical work ${ }^{1}$ on the relative muon capture between heavy and light fission fragments is extended by including $2 \mathrm{po}$ states as well as $1 \mathrm{s \sigma}$. We calculate about $0.8 \%$ population of the $2 p$ state in the heavy fragment with negligible change from our earlier two-state basis regarding the 1s population of light and heavy fragments.

We first solve the stationary problem of a muon in the Coulomb field of two centers by the LCAO method. We have chosen four nonorthogonal basis functions, i.e., the wave functions from equations of $1 \mathrm{~s}$ and $2 p$ states for light and heavy fragments. The Hamiltonian matrix in this basis set can be obtained analytically. Then the generalized eigenvalue problem is solved numerically. The eigenvalue as a function of the separation distance $\mathbf{R}$ between the two charge centers for the most probable asymmetric case is shown in Fig. 1. When $\mathbf{R} \rightarrow \infty$, the four energy levels and wave functions correspond to the one-center asymmetric solutions for heavy and light fragments. The dashed line in the figure is for the case of two is basis states. The energy levels for the two cases almost overlap except for the region of $\mathbf{R}<20 \mathrm{fm}$.

We then solve he time-dependent Schroedinger equation numerically. We assumed that the two parts of the fissioning nucleus move with uniform speed ( $3 \mathrm{x}$ $10^{8} \mathrm{~cm} / \mathrm{sec}$ ) from saddle point to scission point. Our starting point is chosen as $11 \mathrm{fm}$, at which point the moon is assumed to be lying entirely in the ground state. The two parts $o_{i}$ the fissioning nucleus are accelerated in a pure Coulomb field from scission at 18 fm. This distance was chosen in order to match the final kinetic energy to the experimental $170 \mathrm{MeV}$.

The results for the most probable asymmetric case are shown in Fig. 2. The solid line represents the final occupation probability of the muon on the light

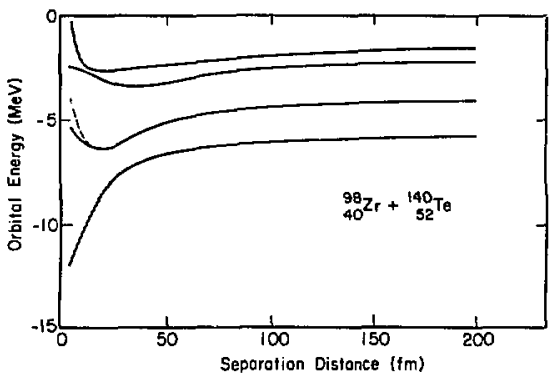

Fig. 1. Calculated four lowest energy (1s $\sigma \mathrm{h}, 1 \mathrm{~s} \sigma \ell$, $2 \mathrm{poh}$, and $2 \mathrm{po} \ell$ in increasing order of energy) levels of the muon for most probable fission asymmetry. The dashed line shows the earlier result ${ }^{1}$ for the two-state basis. An exponential nuclear charge distribution matching experimental ims charge radii was used.

XBL 8012-13581

fragment; the dash-dot line is for the case of the 2-state basis. The lowest line sbows the probability of the muon lying in the $2 p$ state of the heavy fragment. It shows that the final occupation probability of the muon on the light fragment is about 3.5 and the probability for the $2 \mathrm{p}$ state of the heavy fragment is about $0.8 \%$. The population of the $2 p$ level in the light fragment is negligibly small, and the dominant $1 s_{h}$ population can be determined by difference. 
The resuits show that the inclusion of the two $2 p$ orbitals along with the 1s did not make much change for final is populations of fission fragments, compared with the case of two 1s states as basis.

We should comment about the oscillation on the $2 p$ probability of large separation distance. This oscillation is not physical and represents a small numerical instability in the calculations giving transitions between Is and $2 p$ states in the separated fragment.

Our result of $0.8 \%$ for the heavy fragment $2 p$ final state probability is a bit lower than that of Maruhn, et al. ${ }^{2}$ who found $1.7-2 \%$ probability. Given the many differences in assumptions and methods between our calculations and theirs, the agreement seems satisfactory. The percentage of $\mathrm{p}$ state unfortunately seems so low as to make experimental detection unlikely.

\section{Footnotes and References}

*Condensed from Physics Letters 106B (1981)

†Institute of Atomic Encrgy, Academia Sinica, Beijing, Peoples Republic of China

1. J.O. Rasmussen, et al., Chinese Journal of Nucl. Phys. 2 (1980)(in Chinese); Z.Y. Ma, et al., Nucl. Phys. A348 (1980) 446

2. J.A. Maruhn, V.E. Oberacker, and V. Maruhn-Rezwani, Phys. Rev. Lett. 44 (1980) 1576

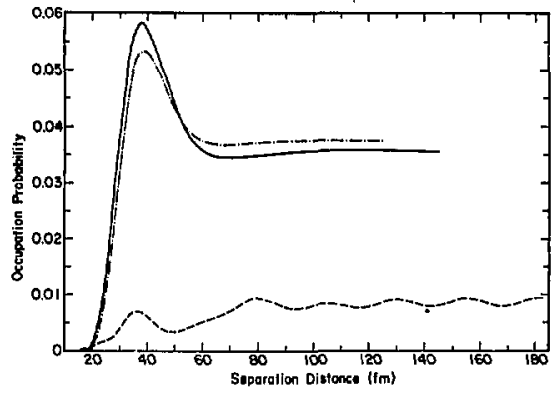

Fig. 2. Calculated muon orbital occupation probabilities as a function of fission fragment separation energies. The upper curves refer to the ls level of the light fragment, the solid curve being present results for the 4state basis and the dash-dot line for the 2-state basis. The lowest (dashed) line is the probability for being in the state that correlates with the $2 p$ level of the heavy fragment. The system shown is ${ }^{98} \mathrm{Zr}+{ }^{140} \mathrm{Te}$.

XBL 8012-13579 


\title{
Deuteron Formation in Nuclear Collisions*
}

\author{
M. Gyulossy, K. Frankel, and E.A. Remlert
}

We have completed calculations begun last year. The Cugnon cascade code was supplemented by Remler's theory to obtain results shown in Fig. 1. The basis for these calculations is the formula

$$
" \sigma_{d} "=\frac{3}{4} \sum\left\langle\delta\left(\mathrm{P}-\mathrm{P}_{\mathrm{m}} \mathrm{P}_{\mathrm{p}}\right) \mathrm{D}\left(r_{n}-\tau_{R_{1}}\left(\mathrm{P}_{n}-\mathrm{P}_{\mathrm{p}}\right) / 2\right)>\right.
$$

where $D$ is the deuteron Wigner density,

$$
D(r, q)=8 \exp \left(-r^{2} / d^{2}-q^{2} d^{2}\right), d \approx 1.7 f m .
$$

The momenta $p_{n}, P_{p}$ and coordinates $r_{n}, r_{n}$ appearing in eq. (1) are determined from the dynamical caleulation and correspond to the coordinates of the pair immediately after the last collision of either nucleon with a third one. There is thus mo ad hoc freezeout time or density in eq. (1). There are no free parameters in the calculation. Nevertheless, the exact theory includes a correction term $\sigma_{\sigma_{b}}$ which is related to the sensitivity of " $\sigma_{\mathrm{D}}$ " to the deuteron wave function. In fact, we showed that

$$
\frac{\delta \sigma_{D}}{" \sigma_{D} "}-\left\{\frac{\partial \ln " \sigma_{D} "}{\partial \ln d^{2}}\right\}^{H}
$$

In our calculation we found $\delta \sigma_{\mathrm{D}} /$ " $\sigma_{\mathrm{D}}$ " $<0.5$ over several decades of inclusive cross sections. This, by the way, justifies the generalized coalescence formula given by eq. (1).

\section{Footnotes}

- College of William and Mary preprint submitted to Nuclear Physics A

†Permanent address: Physics Department, College of William and Mary, Williamsburg, VA 23185

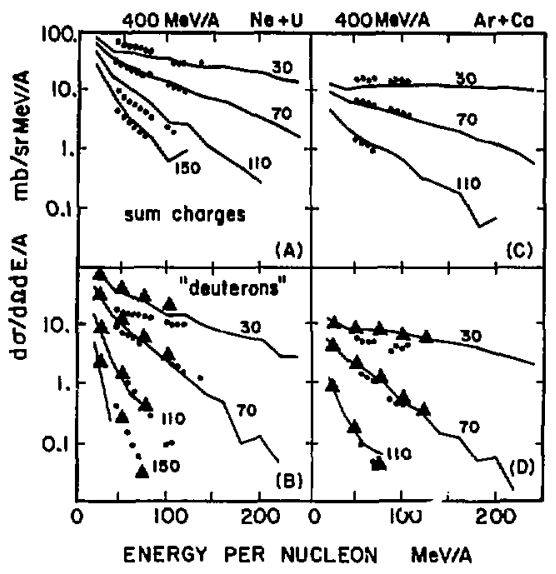

Fig. 1. Sum charge inclusive data compared with Cugnon cascade results. The primordial deuteron yields are predicted in parts $B$ and $D$. The data are represented by triangles; the dots refer to deuteron spectra alone.

XBL 822-7955 


\title{
Global Flow Analysis of Nuclear Collisions*
}

\author{
M. Gyulassy, K.A. Frankel, and H. Stockert
}

We proposed the kinetic flow tensor

$$
F_{i j}=\sum_{\nu} p_{i}(\nu) p_{j}(\nu) / 2 m_{N}
$$

to study the flow characteristics of nuclear collisions in the 100-1000 AMeV domain. Extensive cascade calculations using the Cugnon cascade code were made for systems up to ${ }^{230} \mathrm{U}+{ }^{238} \mathrm{U}$. In addition, results were compared with fluid dynamic calculations. The flow plot for $U+U$ collisions at $\mathbf{4 0 0} \mathrm{AMeV}$ is shown in Fig. 1.

From Fig. 1 we conclude that there are considerable differences between the flow characteristics of hydrodynamic and cascade models. For light $(A<$ $100)$ systems we found that finite number fluctuation effects wash out this difference. Global analysis such as this will be vital in searching for hydrodynamical effects.

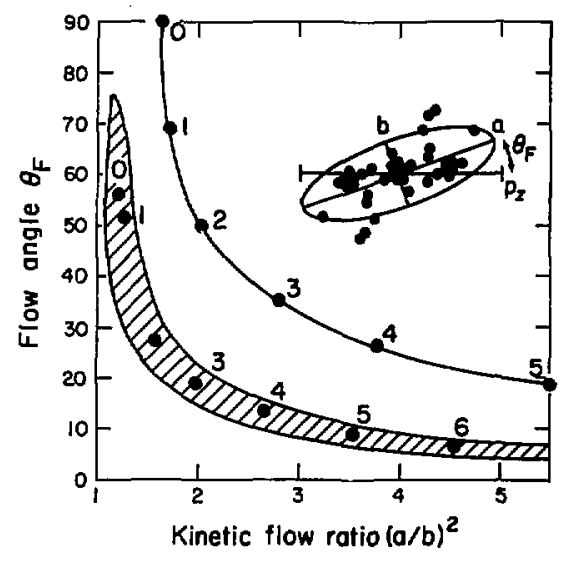

\section{Fcotnotes}

* Condensed from Phys. Lett. 103B (1981) 269

†Permanent address: Physics Department, Michigan State University, East Lansing, Michigan

Fig. 1. Flow diagram based cascade (shaded region) and hydrodynamies (solid curve). Numbers indicate relative intensities. In this plot $\theta_{F}$ is the polar angle of the principal axis of the ellipse with aspect ratio $\mathrm{a} / \mathrm{b}$ describing the shape of the event in momentum space. Insert jllustrates how an ellipse characterizes an event.

XBL. 822-4485 


\title{
Spacial Gradients in the Fragmentation Regions at ATeV Energies*
}

\author{
M. Gyulassy
}

Using the inside-outside cascade scenario, the spacial gradients of the proper energy density deposited in the fragmentation regions were estimated. The basic formula derived is that the energy density deposited at depth $\mathbf{z}$ into the target nucleus is given by

$$
E(z)=\rho_{0}<m_{\perp}>\int_{0}^{x(1)} \cosh y \frac{d N}{d y} d y .
$$

where $y(z)=\sinh ^{-1}\left(m_{\perp} z / 2\right)$. In addition, there is a recoil energy per nucleon $E_{7}=m_{N} \cosh \Delta y_{r}$ where the recoil rapidity shift $\Delta y_{1} \approx-\ln (1-\eta)$ with $\eta \approx 1 / 2$ being the inclasticity. Including the energy deposited because of secondary particle cascading, eq. (1), and nuclear recoil, we obtain the results of Fig. 1. Note that only on the far side of uranium is the proper energy density $>2 \mathrm{GeV} / \mathrm{fm}^{3}$. Therefore, any signature of the quark-gluon plasma in the fragmentation regions will be contaminated by a background because of ordinary hadronic processes occurring in the front half of the nucleus, where $\mathrm{c}<1 \mathrm{GeV} / \mathrm{fm}^{3}$. Figure 1 also demonstrates that not much is gained from increasing the nuclear mass from $\mathbf{A} \sim 100$ to $A \sim 200$. For this discussion only curves 3 and 4 are relevant.

\section{Footnote}

"Condensed from LBL-14512 (1982), to be published in Proceedings of Bielefeld Workshop, May 1982 (ed. H. Satz).

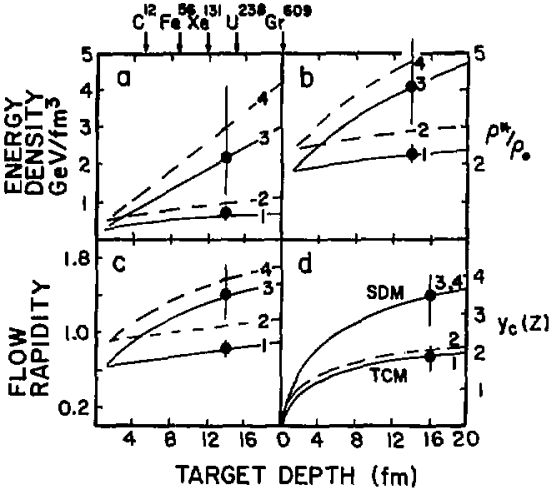

Fig. 1. The maximum comoving frame energy density (a) and compression (b) are shown vs the depth into the target. The flow rapidity $Y_{(}(z)$ and the stopping rapidity $y_{c}(z)$ are shown in (c) and (d). Curves 3, 4 correspond to the inside-outside cascade predictions for $\sqrt{8}=30$ and $100 \mathrm{GeV}$, respectively.

XBL 825-1029?

\section{Maximum Baryon Densities at $10 \mathrm{GeV} /$ nucleon*}

\author{
M. Gyulassy
}

One of the fascinating questions is how far up in energy can we pursue the goal of extracting the equation of state of nuclear matter, $P(\rho, T)$. Clearly, an upper bound is given by the energy at which nuclei can no longer stop nucleons in the midrapidity frame. That energy, $E_{\text {mon }}$ has been estimated ${ }^{1}$ to be $E_{\text {ilop }} \sim 50 \mathrm{GeV}$ for uranium. For $E_{\text {lab }}<E_{\text {suep }}$ heavy nuciei have enough stopping power so thermal equilibrium could be established at least in parts of the nuclear volume. A natural question is how high a baryon compression $\rho$ could be achieved in this energy domain.

Three simple estimates of $\rho$ for $\mathrm{E}<\mathrm{E}_{200 \mathrm{~g}}$ can be made: (1) Jower bound, (2) upper bound, and (3) relativistic quantum bound.

(1) Consider two colliding nuclei, each with thickness $R / \gamma_{\text {end }}$, touching at $t_{0}=0$ in the $\mathrm{cm}$ frame. If $R$ is thick enough to stop a nucleon of rapidity $y_{\mathrm{om}}=\cosh ^{-1} \gamma_{\mathrm{cm}}$ in the $\mathrm{cm}$ frame, then the nucleons stop within $R / 2 \gamma_{\mathrm{cm}}$ of the origin. Therefore 


$$
\rho>\frac{2 A}{\left(v_{0} / \gamma_{c a n}\right)}=2 \gamma_{c o m} D_{0} .
$$

This is the Goldhaber lower bound on the average baryon density achieved assuming instantaneous recoil of nucleons.

(2) The upper bound consistent with baryon and four-momentum conservations follows from the Rankine-Hugoniot equation. This equation gives the density of shocked matter and is valid as long as the shock front thickness $d_{w}$ is less than the nuclear dimensions $\left(R / 2 \gamma_{\mathrm{cm}}\right.$ in the $\left.\mathrm{cm}\right)$. For an equation of state $P=$ $\alpha \epsilon$, we get $^{2}$

$$
\rho / \rho_{0}<\alpha^{-1}+\left(1+\alpha^{-1}\right) \gamma_{a n} .
$$

For the softest equation of state possible $\alpha=1 / 3$.

(3) Relativistic quantum considerations lead to a finite recoil time $r_{0}-2 / \mathrm{m}_{\perp}-1 \mathrm{fm} / \mathrm{c}$ in the nucleon rest frame. Therefore, in the $\mathrm{cm}$ frame it takes at least $T_{0} \gamma_{\mathrm{em}}$ to stop a nucleon. Hence, $T_{0} \gamma_{\mathrm{cm}}$ must also correspond to the minimum thickness of any shock part. Since eq. (2) is valid only as long as $d_{t h}<R / 2 \gamma_{c m}$, we are led to the conclusion that shock formation can no longer be maintained for energies

$$
E_{1 s b}>E_{i b b}^{*}=\frac{R}{T_{0}} m_{\mathrm{N}}-10 \mathrm{GeV} .
$$

At $E^{*}, \rho$ reaches its maximum value and hence,

$$
\rho / \rho_{0}<3+4 \sqrt{\frac{\mathrm{K}}{2 \tau_{0}}}<14 .
$$

The actual maximum density may be considerably less because of a stiffer equation of state or viscosity effects. Nevertheless, these considerations show that $E_{k b} \sim 10$ $\mathrm{GeV} /$ nucleon marks a turning point beyond which the proper baryon density does not increase.

\section{Footnote and References}

*Condensed from LBL-15175

1. M. Gyulassy, LBL-15175 (1982), to be published; A.S. Goldhaber, Nature 275 (1978) 114

2. H. Stocker, M. Gyulassy, and J. Boguta, Phys. Lett. 103B (1981) 269

\title{
Energy Densities in TeV/nucleon Nuclear Collisions*
}

\author{
M. Gyulassy
}

To produce a quark-gluon plasma, an energy density $\varepsilon \sim 2 \mathrm{GeV} / \mathrm{fm}^{3}$ is required. To see whether such high values of $\mathrm{c}$ can be generated in nuclear collisions, I have analyzed cosmic-ray data using the inside-outside cascade model. The basis of this model is the observation that the uncertainty principle together with relativistic kinematics leads to longitudinal growth of the reaction volume at high energies. Specifically, particles are produced along a trajectory

$$
\left.\begin{array}{l}
t(y) \\
z(y)
\end{array}\right\}=\frac{2}{m_{\perp}}\left\{\begin{array}{l}
\cosh y \\
\sinh y
\end{array},\right.
$$

where $y$ is the rapidity and $m_{\perp}=\left(m^{2}+p_{\perp}^{2}\right)^{y_{1}}-0.3 \mathrm{GeV}$. Since eq. (1) provides a one-to-one connection between rapidity and distance, we can compute the energy loss per unit length $d E / d z$, knowing the rapidity density $\mathrm{dN} / \mathrm{dy}$ of produced particles. Thus,

$$
\frac{d E}{d z}=m_{\perp} \operatorname{coshy} \frac{d N}{d y} \frac{d y}{d z}=\frac{m_{\perp}^{2}}{2} \frac{d N}{d y} .
$$

The energy density follows by dividing by the effective area of the beam. Tuking into account that the maximum energy density in a simple geometrical model is $3 / 2$ times the average and using $\left\langle m_{\perp}\right\rangle^{-} 0.3 \mathrm{GeV}$, we obtain

$$
E_{\operatorname{men}} \approx\left(0.1 \frac{\mathrm{GeV}}{\mathrm{fm}^{3}}\right) \mathrm{A}^{-2 / 3} \frac{\mathrm{dN}}{\mathrm{dy}},
$$

where $\mathrm{A}$ is the mass number of the lighter nucleus. With eq. (3) we can convert measured $d N / d y$ in cosmic-ray events to an estimated energy density at proper time $r=1$ in the midrapidity frame. Figure 1 shows this analysis based on 16 events I could find. The theoretical curves are based on the color neutralization model of Brodsky, which predicts $\mathrm{dN} /$ dy using eq. (3).

The obvious conclusion from Fig. 1 is that above $100 \mathrm{GeV} /$ nucleon, energy densities $\epsilon>2 \mathrm{GeV} / \mathrm{fm}^{3}$ can indeed be reached with nuclear collisions. Furthermore, the events studied are consistent with conservative theoretical expectations. Thus, the prospects of 
studying the quark-gluon phase of hadronic matter using nuclear collisions in the $\mathrm{TeV} /$ nucleon region seem very promising and I have suggested a novel signature of the plasma transition.

\section{Footnote}

•Condensed from LBL-15I75 (1982), to be published

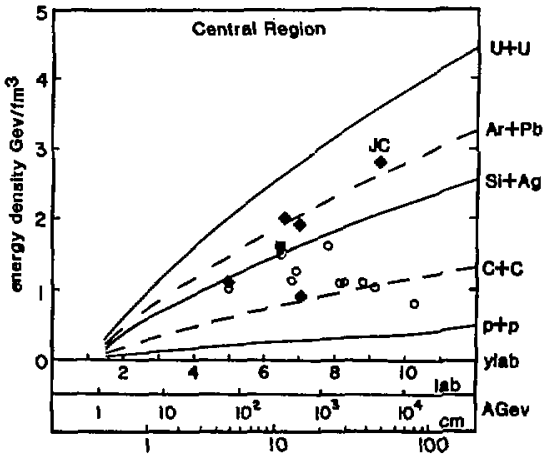

Fig. 1. Maximum energy density achieved in low baryon density regions (midrapidity). Equation 3 was used to convert measured multiplicities into proper energy densities. Diamonds correspond to $\mathrm{Si}+\mathrm{Ag}$, square to $\mathrm{Ar}+\mathrm{Pb}$, open circles to "light" ( $\alpha, B, C, N)+A g$ collisions. Theoretical estimates for various systems are also shown.

XBL 8211-3252 


\title{
Anomalons as Pineuts Bound to Nuclear Fragments:
}

\section{A Possible Explanation*}

\author{
Wm.C. McHarrist and J.O. Rasmussen
}

\begin{abstract}
"Aromalons" is the name given to certain relativistic projectile fragments from high-energy heavy-ion collisions, those fragments that have anomalously short reaction mean free paths (mfp's) immediately following vieir formation.

We propose here a possible explanation of anomalons. It falls within the framework of "conventional" nuclear physics and requires no exotic or esoteric additions.
\end{abstract}

Anomalons could well result from a nuclear halo of "pineuts", 1 hadronically bound states of a $\pi$ " and a few neutrons surrounding a nucleus. Thus, the possibility exists that neutron-rich nuclei (or nuclei with locally neutron-rich domains, such as the neck in a f.ssioning system) might have a sufficiently attractive velocity-dependent potential to allow $\pi^{-}-x n$ hadronically bound "polyneutron" systems. This possibility was first considered by Ericson and Myhrer, ${ }^{2}$ who noted that a finite piece of nuclear matter might bind a $\pi^{-}$at lower than normal nuclear density; however, their deeply bound inner solutions based on a parameterization of the optical potential showed extremely short l. . - imes. We believe their solutions bear reinvestigation bie for: the case of especially neutron-rich projectile fragmin: The true absorption process is not allowed in neuu on matter, since a $\pi^{-}$must transform a proton into a neutron in an orbital not already occupied. It thus has an energy threshold equal to the $\beta$-decay energy, which can easily exceed $10 \mathrm{MeV}$ for neutron-rich light nuclei. Also, for $a \pi^{-}$bound by greater than $m_{2} c^{2}-\left|Q_{j}\right|$, neither true absorption on a pair nor charge exchange on a proton can occur. For $\pi^{-3} s$ bound somewhat less strongly, the final states available for absorption will still be severely limited.

Following the prescriptions of Mandelzweig, Gal, and Friedman, ${ }^{3}$ we have made illustrative calculations for neutron-rich $\mathrm{Na}$ of inner pionic states with the values of Carr, McManus, and Stricker-Bauer (CMS) ${ }^{4}$ for the pion optical potential, considering the pion to build up the local neutron density. The lowest $\mathrm{L}=4$ and 5 solutions are bound by more than $100 \mathrm{MeV}$. Our proposal then is that anomalons are deep $\pi^{-}$inner bound states.

However, just as it is appropriate in light nuclei to consider $\alpha$-particle clusters in the surface region, so also do our model caiculations make appropriate the picture of a pineut orbiting in the nuclear surface region, since the $\pi^{-}$wave function could extend the outside and convect-neutron density.

Note added in proof: Garcillazo $^{5}$ has recently published theoretical estimates of stability of clusters of neutrons and negative pions.

\section{Foomotes and References}

*Condensed from Phys. Lett. 120B (1983) 49

†Permanent address: National Superconducting Cyclotron Laboratory and Departments of Chemistry and Physics, Michigan State University, East Lansing, Michigan 48824

1. R. vanDantzig and J.M. van der Velden (NIKHEF, Amsterdam), preprint (1982)

2. T.E.O. Ericson and F. Myhrer, Phys. Lett. $7 \$ B$ (1978) 163

3. V.B. Mandelzweig, A Gal, and E. Friedman, Ann. Phys. (NY) 124 (1980) 124

4. J.A. Carr, H. McManus, and K. Stricker-Bauer, Phys. Rev. C25 (1982) 952

5. H. Garcillazo, Phys. Rev. Lett. 50 (1983) 1567 


\title{
Centauro as a Superfluorescence Phenomenon*
}

\author{
G.N. Fowler, E.M. Friedlander, and R.M. Weinert
}

One of the most recent challenges of cosmic-ray high-energy physics is the observation of Centauro events. These events were observed by means of a twolayer detector and appear to originate in the air above the detector, the photons from $\pi^{0}$ decay close to the primary interaction should be detected by the upper layer (visual threshold $\mathbf{E}_{1} \simeq 1-2 \mathrm{TeV}$ ) while the longer-lived ("quasji-stable") hadrons are efficiently recorded by the lower layer. The Centaurca are distinguished by the fact that practically no photons tracenble to the primary interaction are observed in the upper detector layer. In the assumption of isotopic spin conservation this is equivalent to the abence of pions since the most important source of photons are $\pi^{\circ}$ s. In "normal" events, on the other hand, pions dominate. Furthermore, the average transverse mordentum ( $p_{T}$ ) of hadrons in Centauros is of the order of $\left\langle\mathrm{p}_{\tau}\right\rangle \simeq 1-1.5 \mathrm{GeV}$, which is a factor of $\geq 3$ larger than the value observed in "normal" events.

We suggest that present physics knowledge might be sufficient to explain Centauro events provided this knowledge is not restrained to the narrow domain of what is called usually "theoretical high-energy physics" and includes also some recent advances in atomic physics. In particular, we connect the appearance of Centauro events with the phenomenon of superfluorescence and with the energy threshold for photons of the measuring device used for the detection of these events. There are important consequences that follow from this interpretation for present and future experi-

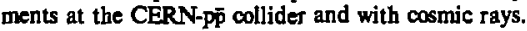

We are faced with a system in preequilibrium, i.e., a succession of equilibrium states characterized by a time-dependent temperature $T(t)$. To each value of $T(t)$ corresponds an equilibrium configuration of different particle species governed by the statistical function

$$
f^{-} p_{3}^{2} g_{i} / \exp \left[\left(m_{i}^{2}+p_{i}^{2}\right)^{1 / 2} / T(t) \pm 1\right] .
$$

where $g_{i}$ is the statistical weight factor corresponding to species $i$, and $m_{i}$ the respective mass. From the form of this function it follows that the higher $T(t)$ the more important the contribution of large masses to the spectrum of produced particles at a moment $t$. The contribution of higher masses will eventually be cut down because of energy conservation. It is useful to distinguish now "primary" pions from "secondary" pions.
The former arise directly from the fireball while the latter are decay products of baryonic and mesonic resonances, At a sufficiently high energy, if particles could be produced from the outset when the temperature is maximum, most primary particles would be not pions but beavier particles.

We make the hypothesis that Centauros are events in which, as a consequence of superfluorescence, the primary fireball decays "immediately", i.e., before cooling down to $T \simeq \mathrm{m}_{\mathrm{r}}$. From the argument given above it follows that the higher the temperature, the less energy will be left for primary pions. The energy carried by secondary pions will always be much smaller.

It follows that if one could in a few events inhibit the expansion and let the system decay completely at an early stage of its history, at the very high energies at which Centauro events are found, no pions (and, a fortiori, photons) with energies exceeding the experimental threshold of $3000 \mathrm{GeV}$ would be seen. Furthermore, because of the higher decay temperature the average $p_{T}$ of particles produced would be much higher than in "normal" Pomeranchuk-like events.

Such a precocious decay can be understood in analogy with the optical superfluorescence phenomenon. It consists in the cooperative emission of particles from a coherently excited system. Coherence in the quantum optical sense has become recently a subject of current interest for high-energy physics. It may manifest itself in identical particle correlations and may explain the absence of cascading in nuclear matter at high energies in terms of self-induced transparency, which is a nonlinear effect. Another nonlinear effect is superfluorescence, and its relevance to Centauro consists in the fact that in this effect fluciuations play a major role, inducing the decay of an unstable equilibrium state. The results of our calculation for a certain range of values of interest show that, in the range of multiplicities $\mathrm{N}_{\mathbf{h}}$ seen in Centauro events, i.e., $60-70$ quasi-stable hadrons, out of $\sim 20$ emitted $\pi^{\circ} s$ only $1-2$ ${ }^{2} \mathrm{~s} s$ exceed the threshold $2 \mathrm{E}_{1}$ of the upper detector (taken as $3 \mathrm{TeV}$ ).

The mean transverse momentum of the quasistable hadrons lies then in the vicinity of $\left\langle\mathrm{p}_{\mathrm{T}}\right\rangle \simeq 1.7$ $\mathrm{GeV} / \mathrm{c}$, a value reported by the discoverers of the Centauro events. Energy conservation requires the mass spectrum to be cut off at $\mathrm{M}=1.4 \mathrm{GeV}$. 


\title{
Particle Multiplicities at the $\bar{p}-\mathrm{p}$ Collider and the $\mathrm{s}^{1 / 4} \mathrm{Law}^{*}$
}

\author{
E.M. Friedlander and R.M. Weinert
}

In two recent pepers ${ }^{1,2}$ the authors of the CERN UAS collaboration report on the first results from the CERN $\bar{p}-p$ coliider concerning the mean multiplicity of produced particles as well as their (pseudo) rapidity distribution.

In Ref. 1 the mean charged multiplicity $\left\langle\mathrm{n}_{\mathrm{eh}}\right\rangle$ is found to have reached a value of $\sim 27$, which "excludes an $s^{1 / 4}$ behavior of $\left\langle n_{\mathrm{eh}}\right\rangle$ in going from ISR $10 \mathrm{col}$ lider energies".

Since the oniy theoretical models predicting an $s^{1 / 4}$ behavior of $\left\langle n_{c h}\right\rangle$ are of the thermohydrodynamical family, the collider result would seem to contradict the predictions of such models.

However, in Ref. 2 the same authors find that the spread of the central rapidity plateau is smaller than would be allowed by kinematics; they discuss this fact in the framework of a cylindrical phase-space MonteCarlo calculation and suggest that the data require an increase of the mean transverse momentum $\left\langle\mathrm{pT}_{\mathrm{T}}\right\rangle$ to a value of $\sim 500 \mathrm{MeV} / \mathrm{c}$.

No correlation between these two new and important results has been proposed so far.

In this note we suggest that the two results, considered together, confirm, rather than contradict, the hydrodynamical model.

Within the context of this model, an essential ingredient in the calculation of the mean particle multiplicity $\langle\Omega\rangle$ is the inelasticity $\mathbf{K}$ of the collision

$$
K \equiv \text { (Total energy of created particles) } / \sqrt{s}
$$

The hydrodynamical model predicts that

$$
<n>^{-} K^{3 / 4} s^{1 / 4}
$$

It is this $\mathrm{s}^{1 / 4}$ law that follows from hydrodynamics and any energy dependence of $K$ will reflect in the energy dependence of $\left\langle n_{\mathrm{eh}}\right\rangle$.
The findings of Ref. 2 concerning the width of the rapidity distribution are equivalent to the observation that the mean inelasticity $\mathrm{K}$ has decreased by $\sim 40 \%$ between $\sqrt{\mathrm{s}}=53$ and $\sqrt{\mathrm{s}}=540 \mathrm{GeV} / \mathrm{c}$. This follows from the integration of the rapidity distributions presented in Fig. 16 of Ref. 2, assuming factorizable rapidity and $\mathrm{p}_{\mathrm{T}}$ distributions, as well as an increase of $\left\langle\mathrm{p}_{\mathrm{T}}\right\rangle$ to $500 \mathrm{MeV} / \mathrm{c}$ at the higher energy (these assumptions are consistent with the particle density observed in Ref, 1 and the transverse energy flow seen by the UAl collaboration.

Using eq. (2) at $\sqrt{\mathrm{s}}=540 \mathrm{GeV}$ we predict a mean multiplicity of 25.4 , which lies within $\sim 1$ standard deviation of the experimental findings.

Thus, there is no contradiction with the $s^{1 / 4}$ law, as long as the complete physical picture, which includes inelasticity, is taken into account. Furthermore, the substantial increase in $\langle\mathrm{pr}\rangle$ is also consistent with the predictions of the hydrodynamical model ${ }^{3}$ since this model allows for "leakage" of "high- $\mathrm{p}_{\mathrm{T}}$ " secondaries from the early stages of evolution of the system, and this leakage effect increases with energy.

\section{Footnotes and References}

*Submitted to Physical Review C (Rapid Communications)

†Physics Department, University of Marburg, Marburg, Germany

1. UA5-collaboration-CERN, Phys. Lett. 107B (1981) 315

2. UA5-collaboration-CERN, Phys. Lett. 107B (1981) 310

3. E. Friedlander and R. Weiner, Phys. Rev. Lett. 43 (1979) 15 


\title{
"Mass Scaling" Phenomena in Heavy Fragment \\ Production in Relativistic Heavy Ion Collisions*
}

\author{
Fumiyo Uchiyamat
}

Scaling phenomena in inclusive reactions in which a single particle in the final state is measured for hadron-hadron collisions ( $\mathrm{A}+\mathrm{B} \rightarrow \mathrm{C}+$ anything) have been known and studied for a long time. The scaling is derived by assuming the existence of a momentum distribution function for the free and structureless constituents of the hadron in the high energy limit for the impulse type of interaction. The scaling variable that is used to describe the distribution depends on both the specific reactions and the kinematical regions of interest. One kind of scaling tested widely in hadron inclusive reactions is that the longitudinal momentum distribution of the observed hadron is independent of the beam momentum.

Since the nucleus consists of nucleons that play the role of constituents, a similar situation may occur for the inclusive nucleus-nucleus reaction in which a nuclear fragment in the final state is measured. Indeed, that was observed in the first high energy heavy ion reaction done by the Greiner-Heckman group ${ }^{2}$ in 1974. They observed that both the inclusive cross sections and the momentum distributions of heavy nuclear fragments of charge $Z$ and neutron number $N$ in the forward direction are independent of incoming heavy ion entergies of 1.05 or $2 \mathrm{GeV} /$ nucleon (of ${ }^{12} \mathrm{C}$ and ${ }^{12} \mathrm{O}$ beams). Considering the fact that the nucleons are loosely ( $10 \mathrm{MeV} /$ nucleon) bound inside of the nucleus and can be treated as structureless in the kinensatical region of peripheral interactions, 1 and 2 $\mathrm{GeV} /$ nucleon beam momentum is bigh enough to satisfy the assumptions for the scalings mentioned above. Furthermore, since the observed secondary nucleus fragment is nothing but a bunch of constituent nucleons, there may exist a different type of scaling unique to the nucleus fragmentation.

In this paper, we point out that there is a new scaling called "mass scaling", which connects the different inclusive channels. We find that the momentum distribution of the secondary nucleus $\mathrm{C}$ in the forward direction can be described by a universal function of the scaling variable that is the momentum $\mathrm{K}$ where
$K$ is defined to be equal in the "beam rest" system to

$$
K=\left(\frac{m_{C}\left(m_{B}-m_{C}\right)}{m_{B}^{2}}\right)^{1 / 2} P_{C}
$$

where $m_{c}$ and $P_{c}$ are the mass and the momentum of the observed fragment $C$ respectively and $m_{B}$ is the mass of the beam nucleus $B$. We proceed with the argument as follows: First, we briefly review the twostep kinematical model ${ }^{3,4}$ to seq where the mass scaling and the factor $\left(\frac{m_{C}\left(m_{B}-m_{C}\right)}{m_{B}^{2}}\right)^{1 / 2}$ originate. Then we investigate whether the model of composite hadrons ${ }^{5}$ can accommodate the mass scaling under some assumptions unique to nuclei in the kinematical region of our interest. We find that it does and that the observation of heavy fragments in the peripheral regions of both projectile jons and the target may be related directly to the major cluster structure inside the nucleus.

\section{Footnotes and References}

*Condensed from LBL-13681

†Permanent address: Institute of Applied Physics, University of Tsukuba, Sakura, Ibarakj 305, Japan

1. J. Benecke, T.T. Chan, C.N. Yang, and E. Yen, Phys. Rev. 188 (1969) 2159; R.P. Feynman, Phys. Rev, Lett. 23 (1969) 1415

2. D.E. Greiner, P.J. Lindstrom, H.H. Heckman, B. Cork, and F.S. Bieser, Phys. Rev. Lett. 35 (1975) 152

3. F. Uchiyama, Phys. Rev. C23, 935 (1981); N. Masuda and F. Uchiyama, Phys. Rev. C15 (1977) 972

4. N. Masuda and F. Uchiyama, Phys. Rev. C15 (1977) 1598

5. I.A. Schmidt and R. Blankenbecler, Phys. Rev. D15 (1977) 3321 


\title{
Subthreshold K--Production by Coherently Produced $\phi$-Mesons in Heavy Lon Collisions* ${ }^{\dagger}$
}

\author{
K.-H. Müllert
}

The enhanced subthreshold $\mathrm{K}^{\prime}$-production found recently in the reaction ${ }^{28} \mathrm{Si}^{28} \mathrm{Si}$ at $2.1 \mathrm{GeV}^{1}$ is assumed to be due to the decay of coherently produced $\phi-$ mesons. We calculate the differential $\mathrm{K}$-production cross section by treating the source term of the $\phi$-meson field, which is the nucleon current, as an external cnumber source. This current is parameterized by assuming a diving-, a compression-, and an expansionstage during the nucleus-nucleus collision where the results of the intranuclear cascade caiculations serve as a reference. Assuming a reasonable time ior building up three times nuclear matter density, agreement with the experimental data is obtained.

In a relativistic quantum field theory the strongly interacting nucleon system is described by a Lagrangian where the interaction Lagrangians are assumed to be due to $\pi, \eta, \sigma, \rho, \omega, \phi \ldots$ meson exchange. The equations describing the space and time behavior of the corresponding meson fields are inhomogeneous KleinGordon-Poca-equations, respectively. In the case of a relativistic heavy ion collision where the nucleons are decelerated these equations not only describe the virtual mesons inside of the colliding systems but also the production and emission of real mesons. While emitted $\eta, \sigma, \rho$, and $\theta$ mesons decay mainly into photons or pions, which might be difficult to detect in the background of photons produced by decaying $\pi^{\circ} \mathrm{s}$ and pions produced via decaying $\Delta$ 's, the $\phi$-meson has the advantage to decay into a $\mathrm{K}^{+} \mathbf{K}^{-}$pair. At bombarding energies far enough below the $\mathrm{NN}^{\rightarrow} \mathrm{NNK}^{+} \mathrm{K}^{*}$ threshold, the $\mathrm{K}^{-}$ meson produces a background-free signal from coherently created $\phi$-mesons assuming that the $\mathrm{K}$ production due to the processes $\pi \Lambda^{\rightarrow} \mathrm{NK}^{*}$ and $\pi \boldsymbol{\Sigma} \rightarrow \mathrm{NK}$ are already sufficiently small.

In the measurements of ref. 1 the $\mathrm{K}$ production threshold is $210 \mathrm{MeV}$ above the available nucleonnucleon centre of mass energy. If an attempt is made to explain these data solely in terms of individual nucleon-nucleon $\mathrm{K}$-production taking Fermi motion in form of double Gaussian momentum distributions for projectile and target into account, the $\mathrm{K}^{\circ}$-yjeld is underestimated by about a factor of 20 .

The aim of this paper is to show that the surprisingly large number of subthreshold $\mathrm{K}$-mesons observed might be due to coherently produced $\phi$-mesons during the nucleus-nucleus collision where the participant nucleons contribute cooperatively ${ }^{3}$. The picture proposed here is that the nuclear matter distribution of the participant nucleons is surrounded by (virtual and real) I-mesons in a similar way as a moving single nucleon. In analogy to photon-bremsstrahlung where a deceleration of a charged current produces real photons, in this model, the deceleration of the baryonic current during the nuclear collision leads to the creation of real $\mathbf{\Phi}$ mesons. A similar picture for the pion cloud surrounding the projectile nucleus was recently proposed by B. Hiller and H.J. Pirner ${ }^{4}$ to describe coherent pion production.

\section{Footrntes and References}

"Condensed from LBL-14715

${ }^{\dagger}$ This work was supported in part by the Deutsche Forschungsgemeinschaft, 5300 Bonn-Bad Godesberg, West Germany.

‡Current address: Institut für Theoretische Physik, Philosopheniveg 19, 6900 Heidelberg, West Germany

1 A. Shor, K Ganezer, S. Abachi, J. Carnoll, J. Geaga, G. Igo, P. Lindstrom, T. Mulera, V. Perez-Mendez, 1 ale, D. Woodard, and F. Zarbakhsh, Phys. ? भ. Lett. 48 (1982) 1592

2. K-H. Müller and '3. Bohrmann, Lawrence Berkeley Laboratory Report, LBL-12663 (1381)

3. K-H. Müller, Proceedings of Workshop on Nuclear Dynamics, Granlibakken, Tahoc City, California (1982) 139

4. B. Hiller and H.J. Pirner, Phys. Lett. 109B (1982) 338 


\title{
A Saturating Chiral Field Theory of Nuclear Matter*
}

\author{
J. Boguta
}

Relativistic quantum field theory appraach to the study of nuclear matter and finite nucie. : structure can account for a large number of data in terms of a few phenomenologically determined parameters.' These parameters can be adjusted so that relevant nuclear matter properties, such as nuclear matter saturation, its compressibility, and the effective nucleon mass in the medin have reasonable values. In the mean field approximation, the model is constructed to describe these essential properties reasonably well. Clearly, such an approech must be phenomenological in its very nature, since quantum corrections, pion contribution, two body correlations are known to be important.

The pienomenological approech yields a Lagrangian that in fact does not have a lower bound for the energy. The best fits are obtained when the scalar meson quartic self interactions are attractive and not repulsive, as they should be. A related problem is that pions, in the linear realization of chiral symmetry, cannot be introduced into the theory without spoiling nuclear matter saturation in an essential way. It was shown by A.K. Kerman and L.D. Miller ${ }^{2}$ and independently by the author that the usual chiral theory of pions leads to a theory of nuclear matter that does not have a saturating normal matter ground state if the theory is solved in the mean field approximation. The reason for this is that the spontaneous breaking of chiral symmetry requires the potential functional to have a double minimum as a function of the fields. This leads to the well-known cusp catastrophe. ${ }^{3}$ The field solutions bifurcate. The aim of this work is to show a simple and elegant solution to this problem. If an isoscalar vector field is introduced into the theory via the Higgs mechanism, these bifurcations disappear and a saturating equation of state can be obtained for a potential functional having a touble minimum. Physi. cally, the Higgs mechanism in this case amounts to the statement that the mass of the vector field is not static, but dynamically determined. Thus a chiral theory of pions can be constructed, in which not only the mass of the nucleon and the scalar meson are spontaneously produced, but also the mass of the vector field.

Footnotes and References

"Condensed from LBL-13874

I. J. Boguta, Phys. Lett. 106B (1981) 250; J. Boguta, Finite Nuclei in Non-Linear Relativistic Quantum Field Theory of Nuclear Matter, LBL13161

2. A.K. Kerman and L.D. Miller, Field Theory Methods for Finite Nuclear Systems and the Possibility of Density Isomerism, MIT-CTP PIJB 449 (unpublished)

3. R. Thom, Structural Stability and Morphogenesis, W.A. Benjamin, Inc., Reading, Massachusetts (1975)

4. P.W. Higgs, Phys. Rev. Lett. 12 (1964) 132 


\section{PART V: INSTRUMENTATION}




\title{
1. Detectors and Techniques
}

\section{Ion Sources for Cyclotrons*}

\author{
David J. Clark
}

This paper describes cyclotron ion sources for light and heavy ions. Sources of polarized ions are not included, since there are recent good reviews of this subject.

The cyclotron ion source has the responsibility for producing well collimeted intense beams of various charge states of many atomic species, polarized and unpolarized. The source should have bigh reliability, long lifetime and quick change time. The first sources were "internal sources", designed to fit within the few centimeters of space inside the first orbit of acceleration in the central region. The approximately uniform magnetic field in this region was a great asset in producing intense ion source ares.

With the advent of more advanced sources of heavy and polarized ions, a space of $1 \mathrm{~m}^{3}$ or more was required for the source, so it had to be placed outside

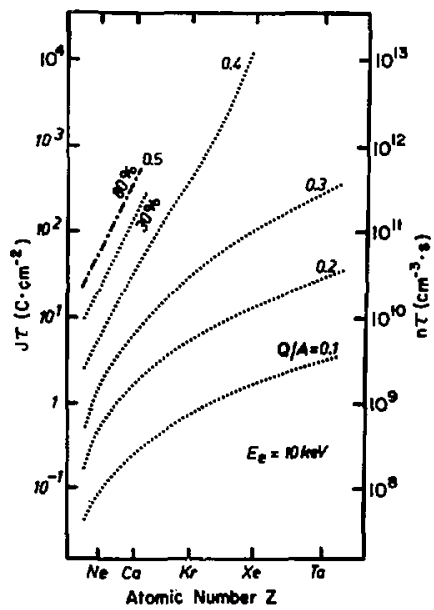

Fig. 1. Charge to mass ratio, $Q / A$, obtained when atom of number $Z$ is bombarded with $10 \mathrm{keV}$ electrons with ionization factor $\mathbf{J} \boldsymbol{\tau}$. the cyclotron as an "external source". Injection systems were developed to transport the beam to the cyclotron center region, either axially or radially.

To create singly or multiply charged positive ions we must remove one or more electrons from atoms and ions. This ionization process is accomplished by electron bombardment, in which the maximum electron energy is 2-3 time the ionization potential.

For the production of high charge state ions we need bombardment with electrons of sufficient energy, $E_{e}$ and density $n$ (electrons $/ \mathrm{cm}^{3}$ ) or current density $J$ (amps $/ \mathrm{cm}^{2}$ ) for a sufficient time $\tau$ (sec) to reach the high charge state. Fig. I shows the charge/mass ratios produced for values of electron bombardment parameters $J_{\tau}$ and $n \tau$, neglecting charge exchange and recombination.

Fig. 2 shows the operating regions of several high charge state ion sources for a range of $E_{e}$ and $n \tau$ values.

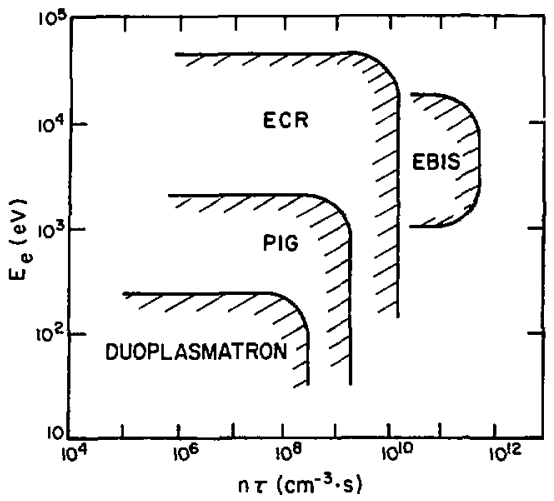

Fig. 2. Operating regions of ion sources used in present accelerators.

XBL $8211-3261$ 
The PIG source is the standard cyclotron heavy ion source. External ECR (electron cyclotron resonance) sources are being installed on several cyclotrons to increase their energies. Although the EBIS (electron beam ion source) produces the highest charge states, it is a pulsed source with lower average beam intensity, and is not as well matched to the cyclotron. The principles and performance of these sources are described in more detail in this paper.

\section{Footnote}

* Condensed from Proc. Ninth Intl. Conf, on Cyclotrons and their Applications, Caen, France, September 7-10, 1981, pg. 231

\title{
The Plastic Ball Spectrometer: an Electronic 4 $\pi$ Detector with Particle Identification*
}

\author{
A. Baden, H.H. Gutbrad, ${ }^{\dagger} H$. Löhner, ${ }^{\ddagger}$ M.R. Maier, ${ }^{\ddagger}$ A.M. Poskanzer, T. Renner. \\ H. Riedesel, H.G. Ritter, ${ }^{\dagger}$ H. Spieler, ${ }^{\dagger}$ A. Warwick, ${ }^{\dagger}$ F. Weik, ${ }^{\dagger} H$. Wieman ${ }^{\dagger}$ \\ GSI-LBL Collaboration
}

A concept of a $4 \pi$ detector consisting of many individual $\triangle \mathrm{E}-\mathrm{E}$ and time-of-flight telescopes was chosen that promises fast data analysis. For the coverage of most of $4 \pi$ the Plastic Ball was built, completely surrounding the target except for the very backward angles, where the beam enters the system, and the extreme forward angles. These forward angles of $0^{\circ}-9^{\circ}$ are covered with a multielement time-of-flight system (called the Plastic Wall) taking into account the large fragment velocities at forward angles. The following gives a detailed description of the whole system, as it is schematically shown in Fig. 1.

The SLAC Crystal Ball design was adopted, which is based on the geometry of an icosahedron: a 20-faced solid figure in which each face is an equilateral triangle of the same dimensions. Each face is divided into 36 triangles, resulting in the division of the surface into 720 triangles, with only 11 different twodimensional shapes. For the Plastic Ball modifications were made in details of the entrance and exit ports. Modules in the backward cone between $160^{\circ}$ and $180^{\circ}$ and in the fr-trard cone between $0^{\circ}$ and $90^{\circ}$ had to be omitted to allow room for the beam pipe and prevent the beam halo from hitting the detector modules.

A further modification was necessary for the region between $10^{\circ}$ and $30^{\circ}$ to guarantee good particle identification despite the high multiplicities expected at forward angles. This region (known as the Mall) is subdivided into 160 modules instead of the 40 in the original design and positioned at a larger radius from the target position. The central spherical cavity of the ball has a radius of $25.4 \mathrm{~cm}$; the outer radius is 61.4 $\mathrm{cm}$. The aforementioned surface division scheme is applied to both the outer and inner surfaces. For assembly and access to the target, the ball is divided into two half-spheres along the $\theta=90^{\circ}$ plane. A thin sheet of $1.2 \mathrm{~mm}$ steel with a hole $50 \mathrm{~cm}$ in diameter forms the baseplate of each hemisphere.

\section{Individual Detector Modules}

Each of the 815 detector modules represents a particle identifying telescope with a $\Delta \mathrm{E}$ and $\mathrm{E}$ detector using a slow and a fast scintillator read out via one photomultipler. The $\triangle \mathrm{E}$ counter is a $\mathrm{CaF}_{2}(\mathrm{Eu})$ crystal with a characteristic decay time of $1 \mu \mathrm{s}$ for the emission of the scintillation light. Unlike $\mathrm{NaI}$, the $\mathrm{CaF}_{2}(\mathrm{Eu})$ is not bygroscopic and therefore needs no bulky canning, which would have jeopardized the system goal of a total coverage of $4 \pi$ of solid angle. Each module is wrapped with only a double layer of aluminized mylar foil as an optical separator between adjacent modules.

The thickness of the $\triangle \mathrm{E} \mathrm{CaF}(\mathrm{Eu})$ crystal was. chosen to be $4 \mathrm{~mm}$ with $35.6 \mathrm{~cm}$ as the length of the $E$ plastic scintillator. This allowed good $\Delta \mathbf{E}$ signals even for minimum ionizing particles and also a minimum low energy cutoff for particles stopping in the $\Delta \mathrm{E}$ counter. To obtain clean proton spectra up to $200 \mathrm{MeV}$, the detector length was chosen to stop $240 \mathrm{MeV}$ protons. This length represents a physical upper limit for particle identification for stopped particles due to the onset of reaction losses.

The readout of the $\triangle \mathrm{E}-\mathrm{E}$ module is done via a conically shaped lightguide (lucite), which couples to a 2-inch, 10-stage photomultiplier. The phototube was 
selected for good gain and high linearity up to the high currents that are necessary for the subsequent pulse shape analysis. The gain of the phototube is monitored by a light pulse fed into the light guide via an optical fiber from a pulsed $X_{e}$-light flasher.

\section{Pion Identification}

In designing the Plastic Ball, special care was taken to detect the positive pions. Since the yield of the $\pi^{+}$is only about $10 \%$ of the proton yield at beam energies well above the pion production threshold, in a pure $\triangle E-E$ identification scheme the pions would be overshadowed by the background produced by heavier particles. Therefore, the $\pi^{+}$are additionally identified by their delayed decay. Stopped $\pi^{+}$decay into $a \mu^{+}$ and a neutrino with a mean life of $26 \mathrm{~ns}$ and $a$ Qvalue of $4.12 \mathrm{MeV}$. The $\mu^{+}$subsequently decays into a positron and two neutrino with mean life of $2.2 \mu \mathrm{s}$. In this decay the $e^{+}$is emitted with an energy of up to 53 $\mathrm{MeV}$ and therefore produces signal that is easily detectable in the plastic scintillators. A discriminator in the electronies detects the occurrence of this second spike and IDC messures the delay time. The TDC covers the range between 250 as and 10ys so about $90 \%$ of all $\pi^{+} \rightarrow \mu^{+} \rightarrow e^{+}$decays can be recorded, provided the $e^{+}$deposits an energy greater than 1 to $2 \mathrm{MeV}$ in the scintillator. This method can not be used to detect negative pions because the $\pi^{*}$ are promptly absorbed by a nucleus in the detector and the $140 \mathrm{MeV}$ rest mass of the pion is released as kinetic energy.

\section{The Plastic Wall}

The Plastic Wall (Pilot F) covers an ares of 192 $\mathrm{cm} \times 192 \mathrm{~cm}$ and provides fine position resolution coverage of the angular region between $\theta=0^{\circ}$ and the forwardmost sections of the Plastic Ball $\left(\theta \sim 10^{\circ}\right)$. It comprises two sections of scintillators, the outer Wall and the inner Wall (Fig. 1).

The outer Wall serves to extend the acceptance of the Ball-Wall system from $\theta=10^{\circ}$ to $\theta=2.5^{\circ}$, detecting particles in the same range of mas and cherge as the Ball itself, but measuring velocity and $Z$ instead of enersy, and $Z$ and $A$ as in the Ball. The inner Wall extends the angular measurement to $0^{\circ}$. In addition, the center region of the inner Wall makes up the event trigger.

The outer Wall region consists of 60 pairs of counters, each $72 \times 8 \times 3.8 \mathrm{~cm}$, providing coincidence units sensitive to charged particles passing through both bars of a pair. Neutron and gamma background is rejected by this coincidence requirement. A time

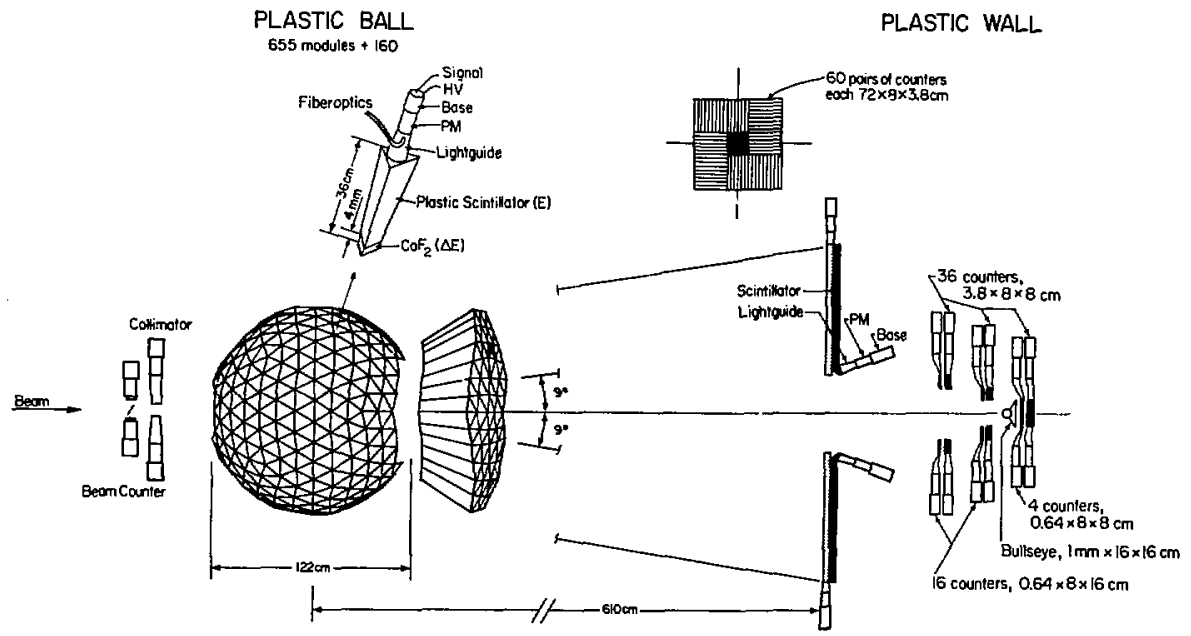

Fig. 1. Schematic view of the Plastic Ball and the Plastic Wall.

XBL 792-329A 
resolution of 350 psec (FWHM) was achieved for minimum ionizing protons. The mean time of the pair gives the flight time from the target and hence the particle velocity.

Each scintillator signal is fed to an $A D C$, and $Z$ identification is achieved (as suggested hy the Bethe Block equation) by generating a function $v^{2} \Delta E$, where $v$ is the measured particle velocity and $\Delta \mathrm{E}$ is one of the two $\Delta \mathrm{E}$ signals.

The inner Wall covers the region within $2^{\circ}$ of the beam and is finely divided because of the high multiplicity of fragments near the beam. Its main purpose is to form the fast trigger with the upstream beam counter and collimator to decide which events are to be recorded. Or course, it also gives nuclear charge and velocity information for the particles it detects and can be used for centering the beam on the Wall.

The inner Wall consists of a $6 \times 6$ array of 36 thick scintillators $8 \times 8 \mathrm{~cm}^{2}$ each, $3.8 \mathrm{~cm}$ thick. In front of each is a $0.64 \mathrm{~cm}$ thin scintillator. For the center four counters the thin and thick scintillators are of the same area and in one to one correspondence, but for the remaining 32 thick scintillators, only 16 thin scintillators are used, each $8 \times 16 \mathrm{~cm}^{2}$ in area and covcring a pair of thick detectors. The 56 scintillators have light guides and phototubes coupled on one edge. In addition, in front of the center four pairs of scintillators is placed one $16 \times 16 \mathrm{~cm}^{2}$ "Bull's Eye" scintillator, only $1 \mathrm{~mm}$ thick. The purpose of the bull's eye is to reject beam particles, and it was made thin to minimize nuclear interactions in the detector itself. The Bull's Eye has light gujdes and phototubes coupled onto two opposite edges.

\section{Upstream Beam Counter}

The upstream counter is a thin plastic film scintillator with a projected diameter of $2.5 \mathrm{~cm}$ and together with an active collimator $3 \mathrm{~m}$ upstream defines the beam acceptance of the ball detector system. This counter establishes the timing reference for the fast trigger and also the start signa' for the TOF measurement in the Plastic Wall. It was designed to have very low mass and also for optimal light collection in order to achieve good time resolution while minimizing the background from material in the beam $A$ film with a thickness of roughly $3.6 \mathrm{mg} / \mathrm{cm}^{2}$ is supportcd in the beam at $45^{\circ}$ on a stretched film of $0.275 \mathrm{mg} / \mathrm{cm}^{2}(2.5$ $\mu \mathrm{m})$ hostaphan. The frame for this is a cylinder of aluminized mylar $(125 \mu \mathrm{m})$, which doubles as a reflector. Two Phototubes (RCA 8575) view the scintillator from each end of the cylinder. A coincidence requirement between the two tubes eliminates a small background caused by beam halo striking a photocathode. The timing reference is extracted from the analogue sum of the two phototube signals with a constant fraction discriminator. The time resolution achieved between this detector and the inner Plastic Wall is $350 \mathrm{ps}$. A large area $(30 \mathrm{~cm} \times 30 \mathrm{~cm}$ ) active collimator, which is sensitive to minimum ionizing particles, largely rejects nuclear interactions in the upstream thin film beam counter, which would otherwise be confused with target interactions. Although the rejection of beam in the active collimator is done online with hardware, the rejection of minimum ionizing particles is done using its TDC off-line so as not to reject particles coming backward from the target.

\section{Signal Processing}

The event trigger is derived from the beam start counter and from the inner Plastic Wall detectors. Presently, the Plastic Ball is not involved in the trigger decision. Therefore, the signals from all 815 Ball modules are delayed via $70 \mathrm{~m}$ cables (RG 58) to allow time for the trigger decision, whereas the other counters have the shortest possible cables (RG 58) with a length of only $20 \mathrm{~m}$.

A passive split generates three input signals for the $\mathrm{E}_{3} \Delta \mathrm{E}_{\mathrm{s}}$ (short $\triangle \mathrm{E}$ ), and $\triangle \mathrm{E} A D C$ s and one for an amplifier. "The first three signals are fed via 25 fold 50-ohm ribbon cables to LRS 2282B ADCs. The fourth amplified signal is given to a discriminator and via twisted pair cables to LRS 4291 TDCs with a $10 \mu \mathrm{s}$ range. The Wall signals are split in the octal constant fraction discriminators, and both analogue and logical outputs are given via $50 \mathrm{~m}$ long 8 fold $50 \mathrm{ohm}$ ribbon cables to ADCs and 100 ns full-scale TDCs. The lnner Wall discriminator outputs are also used in the trigger logic described below. The Wall discriminator signals to the TDCs are regenerated just before the TDCs because of attenuation in the long cables.

\section{Trigger}

The simplest trigger for high energy heavy ion studies is to require that the beam particle undergo a reaction between the beam counter and the Wall counters. This so-called "minimum bias trigger" is of great importance since all more restrictive trigger selections are normalized to it. After detecting a beam particle in the start counter, one demands that the projectile lose at least one charge. The "central trigger" used requires that no beam velocity particles of proton pulse height or higher were recorded in the inner Wall.

\section{On-Line Data Handling}

The torrent of information coming from the Plastic Ball and the Plastic Wall requires sophisticated early inspection and selection in order to restrict the data flow to the significant data, "Smart" readout 
processors are applied in the Camac branch and a microprocessor is used for other Camac modules. One PDP-11/50 processor is dedicated for data collection and writing on tape, while a second PDP-11/44 is used for on-line analysis of the data. All data produced by an event are converted and read out by Camac modules. There are a total of 2631 ADC channels, 817 long-range TDC channels, and 188 high resolution TDCs.

The quality of the particle identification for the 655 modules between $30^{\circ}$ and $160^{\circ}$ is shown in Fig. 2. The dashed curve represents the raw data after gainmatching, while the solid line shows the particle separation after all particles scattered out have been reconstructed by taking into account up to 12 neighboring modules that surround a center module and that contain only $E$ but no $\triangle E$ information. Windows were selected along the valleys of those distributions, allowing one to assign a mass and charge value with high confidence.

The performance figures allow one to draw a figure for the response as a function of fragment momentum and rapidity. Figure 3 shows the response of the Ball and the Wall for protons.

\section{Footnotes}

"condensed from Nucl. Instr. and Meth. 203 (1982) 189

†Gesellschaft für Schwerionenforschung, 6100 Darmstadt, Postfach 110541, West Germany
łUniversität Marburg, 355 Marburg, Renthof 5, West Germany

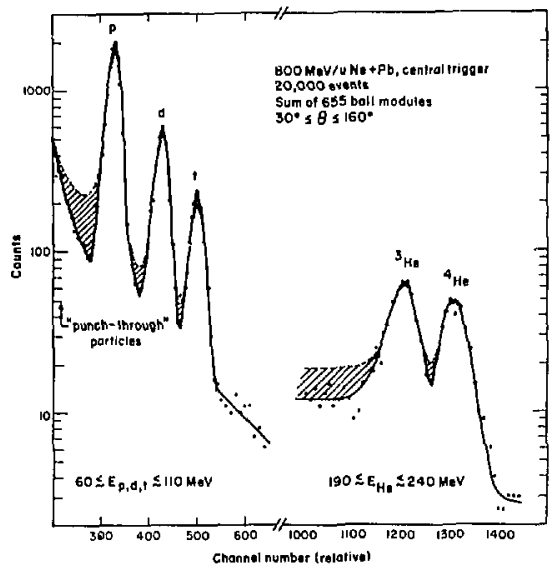

Fig. 2. Particle identification spectrum for 655 modules after gain-matching and with and without scattering out reconstruction.

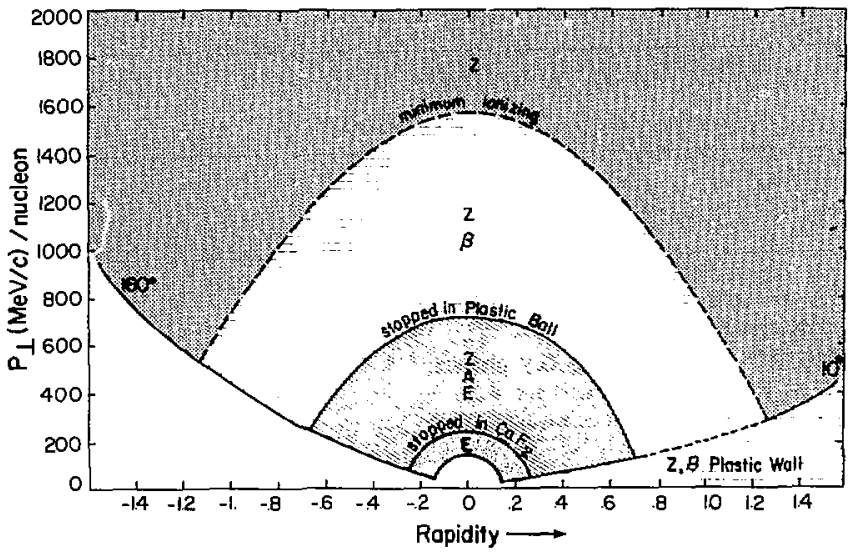

Fig. 3. Plastic Ball and Plastic Wall response in the transverse momentum versus rapidity plane. The limits labeled "stopped in $\mathrm{CaF}_{2}$ " and "stopped .n Plastic Ball" refer to protons and "He fragments.

XBL 8110-1526 


\title{
A Method for Precise Charge Measurements of Relativistic
}

\author{
Nuclei $Z \leq 3$ in Nuclear Emulsion*
}

\author{
M.A. Bloomer, H.H. Heckman, and Y.J. Karant
}

We have developed a technique for the precise charge determination of particle tracks in nuclear emulsion based on me:surements of the lacunarity, i.e., fractional transparency; and/or the fractional opacity in the linear track structure. The method yields estimates of charge for relativistic $Z=1$ and $Z=2$ nuclei to a precision of $\sim \pm 0.05$ charge units, in agreement with Barkas' theoretical model of track structure. ${ }^{1}$ This method of charge measurement complements a variety of techniques that extract similar information from an ionization track. The method is not applicable to tracks of particles whose rates of ionization are so high as to produce completely saturated, i.e., opaque, tracks. In this case, methods of charge analysis commonly used are those that measure $\delta$-ray densities and track widths.

Beginning with the experimentally observed fact that the gap-length distribution for a track of an ionizing particle in emulsion is exponential, we show that the charge of a particle is related to the lacunarity $L$ of its track by the relation

$$
Z=k_{0} \sqrt{-\ln L}
$$

where $k_{0}$ is a proportionality constant determined empirically.

The precision $\delta Z$ with which the charge $Z$ can be determined is (see Ref. 1, Sec. 9.7):

$$
\delta \mathrm{z}=\frac{\mathrm{k}_{0}^{2}}{2 \mathrm{Z}}\left[\frac{2 \alpha}{\Lambda}\left[-\frac{1-\mathrm{L}}{\mathrm{L} \ln \mathrm{L}}-\mathrm{l}\right)\right]^{/ 2} \text {. }
$$

where $\alpha$ is the mean diameter in microns of the developed grains and $\Lambda$ is the path length (microns) over which $\mathbf{L}$ is measured.

Given that $k_{0} \sim 3.3$ for Ilford G-5 electronsensitive emulsion, Eq. (2) predicts that for $Z \leq 4$, charge measurements can be made to an accuracy $\delta Z \sim$ 0.05 units in $\Lambda \lesssim 1 \mathrm{~mm}$.

In our measurements, an observer determined the lacunarity $L$ for a minimum of 16 cells (cell length $=$ $62 \mu \mathrm{m}$ ), terminating the measurement of $L$ when the observed rass deviation of mean charge $Z, S_{Z} \leq 0.045$ was satisfied. For $Z=2$ nuclei, this condition was met by the time 16 cells were measured in about $70 \%$ of the measurements.
Figure I shows the charge distribution of $\mathrm{He}$ fragments emitted from $1.9 \mathrm{AGeV} F e$ interactions in emulsion. The charge distribution is a composite of measurements of three different observers, the data of each observer being normalized to $Z=2.00$ after corrections for depth gradients in the emulsions and time variations of the scanner-dependent value of $k_{0}$. Also shown in Fig. 1 are values of $\bar{Z}$ obtained by each observer assuming $\bar{k}_{0}=3.1$, the average value of $k_{0}$ for all observers.

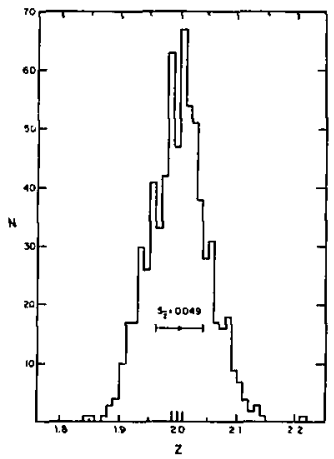

Fig. 1. Measured charge distribution of $\mathrm{He}(Z=$ 2) projectile fragments of ${ }^{56} \mathrm{Fe}$ at $1.9 \mathrm{AGeV}$.

XBL 8349469

Footnote and Reference

"Based on Senior Thesis, Undergraduate Honors Program, Department of Physics, University of California (MAB)

1. W.H. Barkas, Nuclear Research Emu/sions, Vol. 1, New York: Academic Press, 1963, 518 pp 


\title{
Observation of Large Deviations from the Bethe-Bloch Formula for \\ Relativistic Uranium Ions*
}

\author{
S.P. Ahlen' and G. Tarle't
}

By measuring the range deficit of $955 \mathrm{MeV} / \mathrm{amu}$ ${ }^{238} \mathrm{U}$ ions in copper, we have identified the largest deviation ever observed of the stopping power of fast $(\beta$ $>>\alpha$ ) heavy ions from the Bethe-Bloch formula. We show that the most likely explanation of this discrepancy is the existence of higher order terms in the Mott cross section. The significance of this result with regard to several high-energy astrophysics experiments is discussed.
Footnotes and Reference

*Condensed from Phys. Rev, Lett. 50 (1983) 1110 †Also at Space Sciences Laboratory

1. W.H. Barkas, W. Birnbaum, and F.M. Smith, Phys. Rev. 101 (1956) 778

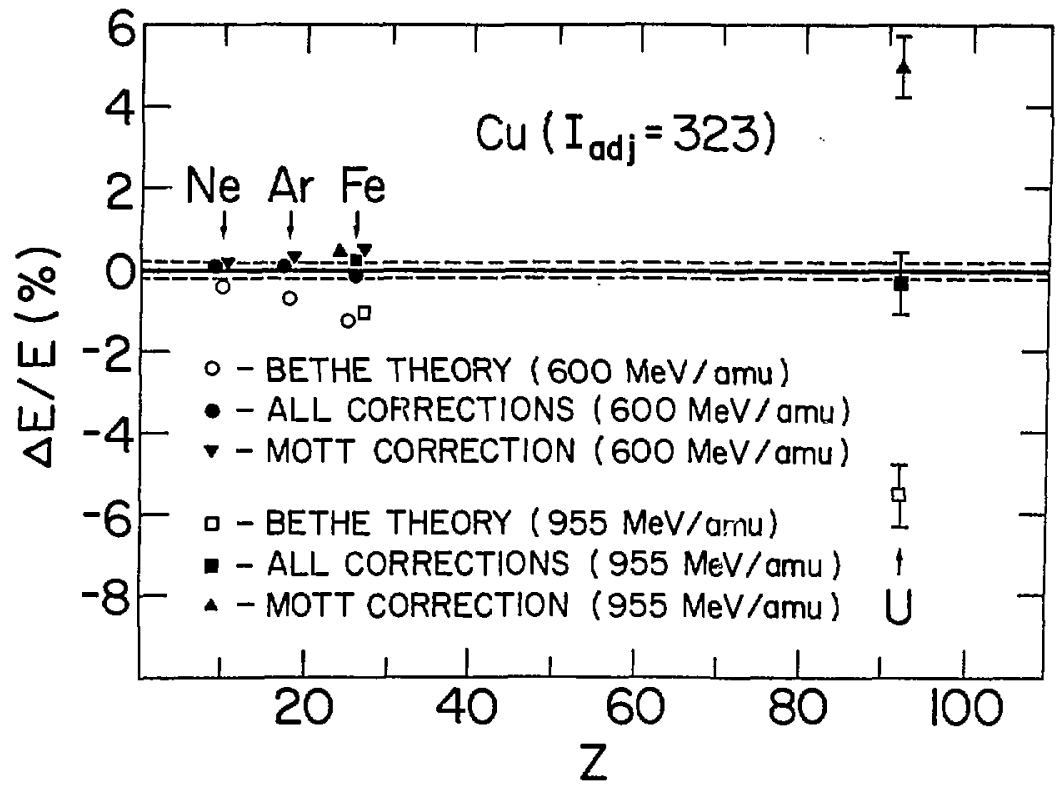

Fig. 1. Deviation of energies calculated from measured ranges, from those determined using time-of-flight methods. Data for $600 \mathrm{MeV} / \mathrm{amu} \mathrm{Ne}, \mathrm{Ar}$, and Fe were obtained from ref. 1 while the $955 \mathrm{MeV} / \mathrm{amu} \mathrm{Fe}$ and U data are from this work. In all cases most of the range was in Cu. Large deviations from the Bethe theory are evident for $U$.

XBL 834-9036A 


\title{
Calculation of the Stopping Power of Very Low Velocity Magnetic Monopoles*
}

\author{
S.P. Ahlent and K. Kinoshitat.t
}

One cannot interpret dynamic searches for very slowly moving $\left(v<10^{-2} \mathrm{c}\right)$ grand unification magnetic monopoles without knowing both their stopping power and the response of the detector as a function of stopping power. In this paper we consider the first question. We calculate the electronic stopping power of slow magnetic monopoles in condensed materials and find that, for $\beta \leqslant 0.01, \mathrm{dE} / \mathrm{dx}$ is proportional to the monopole velocity. For monopoles with $8= \pm 137 \mathrm{e}$ it is found that the monopole stopping power is at least as large as that for a proton with the same velocity. Figure 1 shows the results of our calculations for a Dirac monopole in silicon. The results presented here are not directly relevant to the evaluation of the response of excitation and ionization sensitive particle detectors to slow, super massive monopoles. However, it is hoped that the techniques presented will be a useful guide in thinking about such problems.

\section{Footnotes}

*Condensed from Phys. Rev. D27 (1983) 688

†Also at Space Sciences Laboratory

\$Now at Department of Physics, Harvard University, Cambridge, Massachusetts

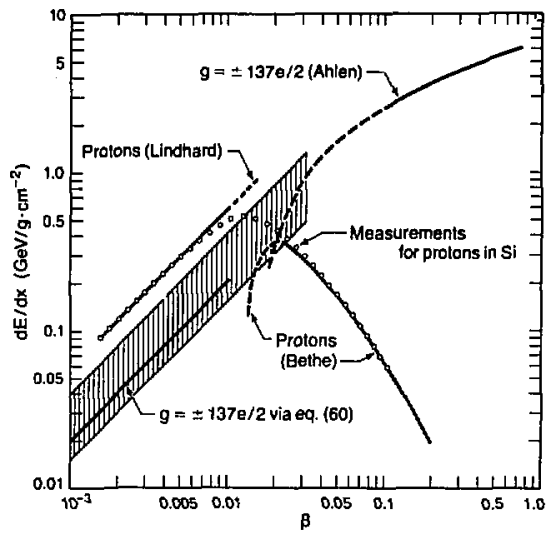

Fig. 1. Stopping powers in silicon for protons and for monopoles with $\mathrm{g}= \pm 137 \mathrm{e} / 2$

XBL 833-8963 


\title{
Can Grand Unification Monopoles be Detected with Plastic Scintillators*
}

\author{
S.P. Ahlent and G. Tarlet
}

Numerous groups throughout the world, including our own group, are preparing experiments to search for grand unification monopoles using large arrays of plastic scintillators. Such experiments require for their interpretation an understanding of the light output as a function of monopole velocity, in the region of very low velocities. By drawing upon results from work dealing with the interactions of slow protons with matter, we have estimated the light yield of GUT monopoles from organic scintillators. Figure I shows the results. We conclude that monopoles having velocities as low as $6 \mathrm{x}$ $10^{-4} \mathrm{c}$ can be detected.

\section{Footnotes}

*Condensed from a paper submitted to Physical Review Letters

†Also at Space Sciences Laboratory

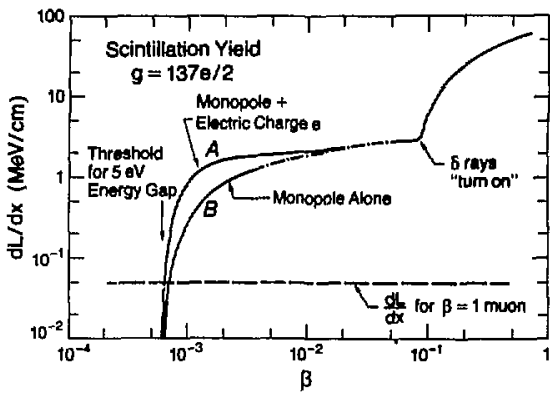

Fig. 1. Estimates of scintillation yield for magnetic monopoles.

XBL $833-8962$

\section{The Maximum Likelihood Method Applied to Decay During and After Accumulation of a Sample of Radioactive Nuclei}

\section{L.P. Somerville*}

The maximum likelihood method is a very old method $^{1}$ that has been used to determine the half life from a list of decay times for a sample of radioactive nuclei. In a previous publication the method was extended to multi-component decay. ${ }^{2}$ In this report the method is applied to systems in which the decay rate first increases with time, as nuclei are accumulated in the sample, for example, by production from an accelerater bombardment, and later decreases with time after the accumulation has stopped. Experiments of the "flipper" type described by J.M. Nitschke, et al. in this report initialed the development of this half life analysis code. One approximation to the flipper situation is used, namely that possible decays after the total observation time $T$ are not observed during the next accumulation period. Figure 2 of the report by J.M.
Nitschke, et al., is an example of how one of the decay curves is analyzed with this method.

During the time 0 to to of accumulation or production at rate $R$ the number of nuclei $N(t)$ in the sample must obey the differential equation

$$
\mathbf{N}=\mathbf{R}-\lambda \mathbf{N}, \quad 0 \leq \mathrm{t} \leq \mathrm{t}_{0}
$$

$\left(\begin{array}{l}\text { where } \lambda \\ \lambda=\frac{\ln (2)}{T_{1 / 2}}\end{array}\right)$. Since after the accumulation ceases at time $t_{0}$ the accumulation rate $R$ is zero, solution of this equation for al! times yields 


$$
N(t)=\left\{\begin{array}{c}
\frac{R}{\lambda}\left(1-e^{-\lambda(t)}, t \leq t_{0}\right. \\
\frac{R}{\lambda}\left(1-e^{-\lambda t}\right) e^{-\lambda\left(1-t_{0}\right)}, t \geq t_{0}
\end{array} .\right.
$$

The decay rate for the entire sample of nuclei is given by $\lambda N(t)$, or, alternatively, $\lambda N(t) / R$ is the decay probability at time $t$ for each nucleus in the sample. Since some nuclei decay during accumulation and others decay after the accumulation ceases, the likelihood function for a set of decay times consists of a product of the two likelihood functions in the brackets of the following equation.

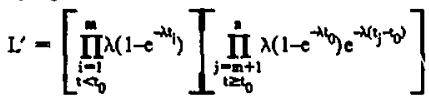

Each of these likelihood functions is a product of the decay probabilities for each of the individual nuclei from equation (2): first for the set of decay times $t_{1}, t_{2}, \ldots t_{m}$ during accumulation and second for the set of decay tumes $t_{m+1}, t_{m+2}, \ldots t_{n}$ after accumulation. This total likelihood function must now be normalized so the total decay probability for any observed decay is unity over the observation time from time 0 to time $T$. If the normalized likelihood function $\mathrm{L}$ is defired as $\mathrm{L}$ $\equiv A^{n} L^{\prime}$, the normalization constant $A$ is required to satisfy the following equation:

$$
A\left[\int_{0}^{0} \lambda\left(1-e^{-\lambda}\right) d t+\int_{0}^{T} \lambda\left(1-e^{-\lambda t} y\right) e^{-\lambda(1-t)} d t\right]=1 .
$$

The simplified normalized likelihood function $\mathrm{L}$ then is

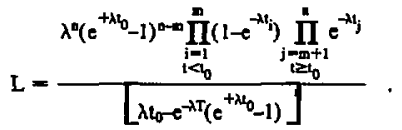

This likelihood function is now to be maximized with respect to $\lambda$ to determine the most probable $\lambda=\bar{\lambda}$ or half life $\bar{T}_{1 / 2}=\frac{\ln (2)}{\bar{\lambda}}$ for all the observed decay times.

To calculate the standard deviation $\sigma_{\tau_{1 / 2}}$ of $\bar{T}_{1 / 2}$ from the standard deviation $\sigma_{\bar{\lambda}}$ of $\bar{\lambda}$ the likelihood funcion can be approximated by a Gaussian, ${ }^{3}$ which is valid for a large number of events $n$, or

$$
L=L_{0} \exp \left[-\left(\lambda-\bar{\lambda} / 2 e_{2}^{2}\right)\right] \text {. }
$$

The standard deviation $\sigma_{\mathbf{t}_{1 / 2}}$ in $\overline{\mathbf{T}}_{1 / 2}$ is then given by

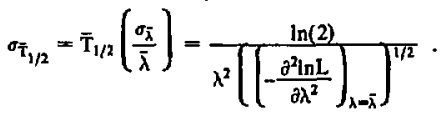

The rate of accumulation or production $\mathbf{R}$ is given by requiring that the integral of the decay rate $\lambda N(t)$ over the observation time 0 to $T$ equal the total number of decays $n$ observed. From equations 2 and 4

$$
R=\frac{n \bar{\lambda}}{\bar{\lambda} t_{0}-e^{-\bar{\lambda} T}\left(e^{+\bar{\lambda}_{0}}-1\right)} \text {. }
$$

The standard deviation $\sigma(R)$ of $R$ is given by

$$
\begin{aligned}
& \sigma(R)=\left[\sigma_{n}^{2}\left(\frac{\partial R}{\partial n}\right)_{\lambda-\bar{\lambda}}^{2}+\sigma_{\lambda}^{2}\left(\frac{\partial R}{\partial \lambda}\right)_{\lambda-\bar{\lambda}}^{2}\right)^{1 / 2}
\end{aligned}
$$

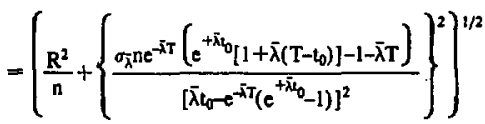

A computer code has been written to determine the most probable half life $\bar{T}_{1 / 2}$, the accumulation rate $R$, and the standard deviations of these quantities. The code was tested by using an artificially generated data set with a 5-s half life. A half life of $5.03 \mathrm{~s}$ was determined by the code, in close agreement with the 5-s half life used in generating the data. The code bins the events with a constant number of counts per $\mathrm{bin}^{2}$ for plotting decay curves and for calculating the chi square per degree of freedom of the fit.

Future developments of the maximum likelihood method will include the effect of a constant background, the determination of asymmetric half life error bars, and application to parent-daughter growth and decay.

\section{Footnotes and References}

*Present address: Physics Division, Argonne National Laboratory, Argonne, IL 60439

1. R.A. Fisher, Messenger Math. 41 (1912) 155

2. R.C. Eggers and L.P. Somerville, Nucl. Instr. Meth. 190 (1981) 535

3. E. Segre, Nuclei and Particles, W.A. Benjamin Press (1965) 176; 2nd edition (1977) 


\title{
Time of Flight Detector for HISS
}

\author{
Ryoichi Wada
}

A time-of-flight detector for HISS is designed to measure velocity ard charge of scattered particles that are produced in relativistic heavy-ion collisions. To cover the large solid angle of the HISS magnet, this detector is just like a big wall.

The time-of-flight well detector of phase I ( $2 \mathrm{~m} \times$ $3 \mathrm{~m})$ and phase II ( $3 \mathrm{~m} \times 4 \mathrm{~m})$ was completed. The new wall for, thase II consists of 40 pieces of $3 \mathrm{~m} \times 10 \mathrm{~cm}$ pilot F plastic scintillators. All slats have XP2230 phototubes at both ends. Actual attenuation length of the scintillator is $2.4 \mathrm{~m}$ and actual light propagation time for $3 \mathrm{~m}$ is about $20 \mathrm{~ns}(15 \mathrm{~cm} / \mathrm{ns})$.

All H.Y.s are supplied by a LeCroy 32-channel power supply (model 4032A) and controlled by a PDP

TDC Resolution for Carbon Beam

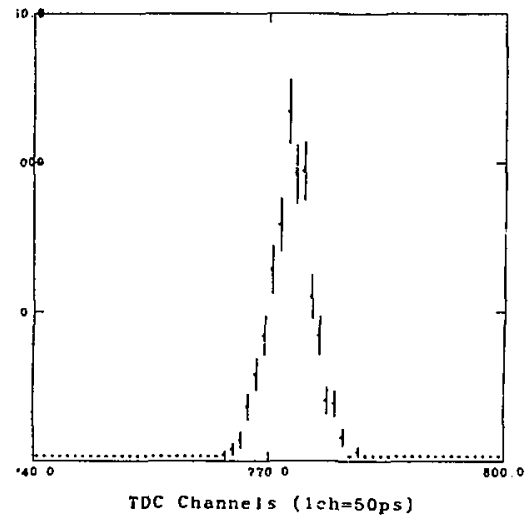

Fig. 1. Time resolution of $1 \mathrm{GeV} / \mathrm{n}{ }^{12} \mathrm{C}$ beam. Start signal was taken by $0.5 \mathrm{~mm} \times 5 \mathrm{~mm} \times 5 \mathrm{~mm}$ scintillator. True flight time was obtained by averaging two flight times from both ends of a $3 \mathrm{~m}$ scintillator. All corrections for the dependences of amplitude and charge were done by sof tware.

XBL 8349096
11/45 through a CAMAC module. Bases are specially designed to be very stable for a high current load. Two anode outputs are used for a TDC and an ADC.

Timing is determined by a LeCroy 620D leading edge discriminator located in the cave (experimental area) and a 12-channel TDC module (2226A) in the shack (control area). The ADC signal is directly connected to a 48-channel ADC module (2282).

Time and charge resolution are measured by a ${ }^{12} \mathrm{C}$ beam $(1 \mathrm{GeV} / \mathrm{n})$ and its fragments. Time resolution DT(FWHM) is $<250 \mathrm{ps}$ for $Z=$; and $<450 \mathrm{ps}$ for $Z$ - 1, using $0.5 \mathrm{~mm} \times 5 \mathrm{~cm} \times 5 \mathrm{~cm} \mathrm{~s}$ intillator for the start signal (Fig. 1). Charge resolution DZ(FWHM) is 0.4 for $Z=6$ and 0.2 for $Z=1$ ( $F: g$. 2).

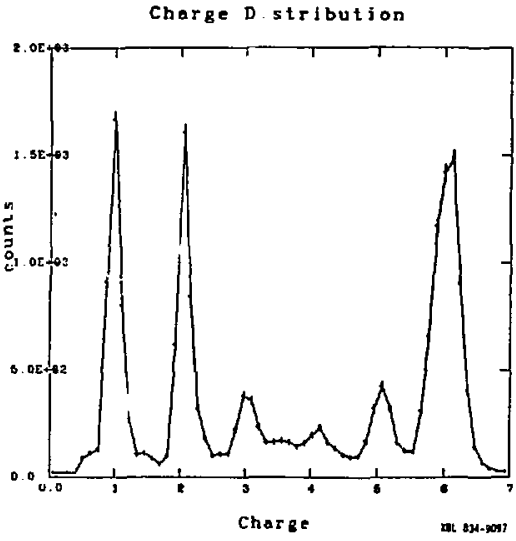

Fig. 2 Charge resolution of projectile fragments from $2 \mathrm{GeV} / \mathrm{n}{ }^{12} \mathrm{C}$ beam. An empirical formula was ised to calculate the charge of each particle.

XBL 834-9097 


\section{MUSIC Ionization Chamber}

\section{H. Sann}

To be able to determine experimentally the charges $(Z>6)$ of the projectile fragments produced in relativistic heavy-ion reactions, we built a large Multiple Sampling Ionization Chamber (MUSIC).

The detector measures the energy loss of a relativistic heavy ion 64 times in a gas ionization chamber and will enable us to determine the charge of the particle in the final state to a very high accuracy ( $<10 \%$ misidentification). In the last year we finished the detector and took the first data.
To cover the total area in which the HISS magnet deflects the charged particles with $Z>6$ at $4 \mathrm{~m}$ distance, the active area of the detector has to be $1 \mathrm{~m} \times 2$ m.

The detector (Fig. 1) consists of a stainless steel barrel with a diameter of $3.2 \mathrm{~m}$ and a length of $2 \mathrm{~m}$. The vessel can be evacuated and then refilled with the detector gas, so the required gas purities (<10ppm $\mathrm{O}_{2}, \mathrm{H}_{2} \mathrm{O}$ ) can be reached. After removal of the vessil heads the detector is operational. The entrance and

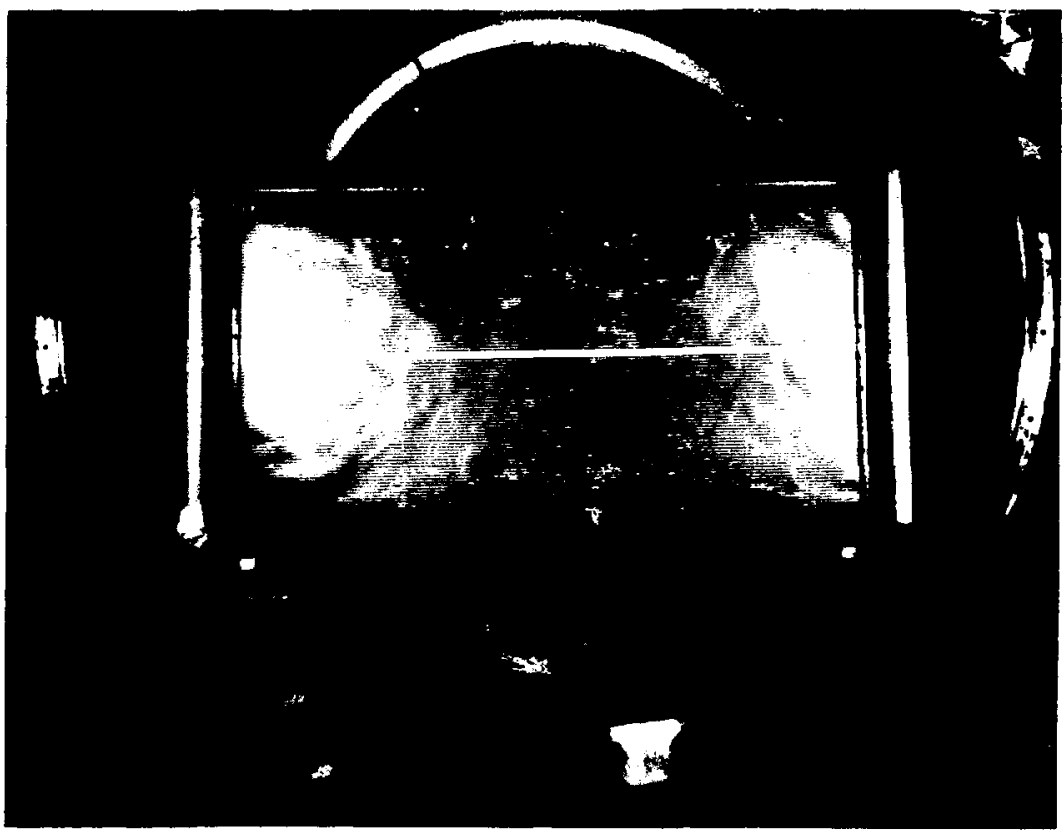

Fig. 1. The picture shows the detector just before mounting the endplates with the entrance window. In the 3.3 $\mathrm{m}$ diameter stainless steel vessel, the field cage with an active area of $1 \mathrm{~m} \times 2 \mathrm{~m}$ and the high-voltage cathode on top is shown. Below the field cage one can see a frame that contains the Frisch grid and the anode structure.

BBC 82111-0073 
exit window for the particles consists of two canpounded polypropylene-aluminum-mylar foils that are separated by a $1.5 \mathrm{~cm}$ gas gap. A clean gas (Ar) is purged through this gap to maintain the high gas purity in the detector volume.

The sensitive volume of the detector consists of a field cage with a sensitive area of $1 \mathrm{~m} \times 2 \mathrm{~m}$ and a depth of $1.6 \mathrm{~m}$. The field cage consists of a $1 \mathrm{mil}$ mylar foil onto which horizontal copper st $-j$ pes are evaporated. These stripes are connected by a resistor chain (20 $\mathrm{M} \Omega / \mathrm{cm}$ ), which divides the negative cathode voltage $(<80 \mathrm{kv}$ ) down to the grounded Frisch grid.

The anode structure is mounted $2 \mathrm{~cm}$ below the Frisch grid and is operated at positive voltage (750 volts). It consists of a right and a left part, each of which contains $60 \mathrm{~cm} \times 2 \mathrm{~cm}$ and $4 \mathrm{~cm} \times 6 \mathrm{~cm}$ divisions. The 128 preamplifiers are mounted outside the tank.

The detector is positioned under very small angles and normally has to digest the bigh fiux of elast dilly scattered particles; therefore the readout system itas to be capable of multiple track recognition. Th-t is why we copied the complete TPC system (preamplifier, main amplifier, CCD's, and digitizers).

The multiple (64) energy loss signals shown in Fig. 2 allow us to determine the energy loss distribution for each detected charge. As expected from the energy deposited in the detector, the distribution of the energy luss signals resemble an asymmetric Gauss function. For $F_{e}$ the energy loss distribution shows a width of about $13 \%$, whereas Tanata, et al., give a value of $8 \%$. The difference can easily be attributed to the difficulties in the data extraction because of the electronics used. The present TPC readout system is designed for a very spcciai purpose with a much smaller dynamic signal range and is thus not adequate in all details for our use. We need a better calibration, readout of the momentary $C C D$ baseline, and adjustment to a dynamic range of $(1 / 150)$.

In the far future it seems necessary to avoid all the problems connected with the analog signal storage in the CCD part of the TPC electronics. Each amplifier channel should have its own digitizer and the data should be stored in a digitized form. So we could build an $A D C$ system with a quadratic response function, the best way to handle the big dynamic range of $>1,150$ (carbon, uranium).

We believe that the MUSIC detector is in a state where it has shown that it is possible to build a large surface detector for the determination of the charge of the fragments in relativistic heavy ion reactions. Together with the proposed gas $\left(\mathrm{CF}_{4}\right)$ it will be possible to determine the complete charge range from carbon to uranium with a misidentification level below $10 \%$.

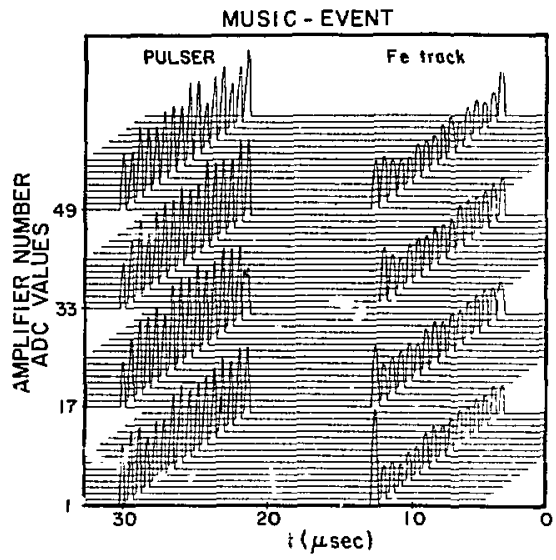

Fig. 2. The time dependence of the signals observed by the 64 anode amplifiers is shown for an Fe event. $12 \mu \mathrm{sec}$ after the trigger the signal from an Fe particle arrives at each amplifier. This corresponds to an eleciron drift distance of $0.5 \mathrm{~m}$. At $30 \mu \mathrm{sec}$ a pulser connected to all amplifiers is seen. The energy loss distribution must be extracted from the area under the signals.

XBL 834-9025 


\title{
Investigation of the Operation of Wire Chambers in the Self Quenching Streamer Mode*
}

\author{
T. Mulera, V. Perez-Mendez, A. Del Guerra, ${ }^{\dagger}$ and G. Schwartz
}

The production of anomalously large pulses in wire counters with thick anodes has been reported since the very early work with multiwire proportional chambers'. These signals have comparatively fast $(\sim 10 \mathrm{nsec})$ risetimes and short $(\sim 100 \mathrm{nsec})$ durations and are not followed by the large dead times typical of the Geiger-Muller mode of operation. Recent work 2.3 reveals that these pulses do not arise from some "limited Geiger" mechanism but from another phenomenon in which narrow (150-200 $\mu \mathrm{m}$ thick) streamers propagate orthogonally away from the anod: for several millimeters and then quench themselves in a well-controlled way. This phenomenon has thus come to be referred to as "self quenching streamer" (SQS) behavior. The large (typically $50 \mathrm{mV}$ into $50 \Omega$ ) pulses would allow the building of very inexpensive wire chambers as low noise, high gain amplifiers would not have to be provided to read out the wires.

Our investigations reveal that $S Q S$ behavior takes place in many noble gas-complex molecule gas mixtures. To achieve good timing characteristics fairly high concentrations of the quenching gas are required. Figure I shows the signals produced in various mixtures of argon and carbon dioxide. The shape of the pulse is strongly affected by the quencher concentration. Besides argon-carbon dioxide, we observed $\mathrm{SQS}$ behavior in $\mathrm{AR}-\mathrm{C}_{2} \mathrm{H}_{6}, \mathrm{Ar}-\mathrm{CH}_{4}, \mathrm{Ar}-\mathrm{CF}_{4}$ and $\mathrm{Ne}-\mathrm{CO}_{2}$.
The signals varied in amplitude but were all large (>40mV-50R) and were more or less saturated (independent of the initial amount of charge deposited).

The effects of gas pressure, anode wire size, and anode cathode geometry were also investigated in hopes of providing input to the design of detectors employing this mode of operation.

\section{Footnotes and References}

"Condensed from LBL 14003 and Nucl. Instr. and Meth. 203 (1982) 609, and LBL 14412 and IEEE Trans. Nucl. Sci NS-30 (1983) 355

†Permanent address: Istituto di Física, Pisa, I-56100, Italy

1. R. Bouclier, et al., Nucl. Instr, and Meth. 88 (1970) 149

S. Brehin, et al., Nucl. Instr. and Meth. 123 (1975) 225

2. G.D. Alekseev, et al, Nucl. Instr, and Meth. 177 (1980) 385

3. M. Atac, A. Tollestrup and D. Potter, IEEE Trans. Nucl. Sci. NS-29 (1982) 388

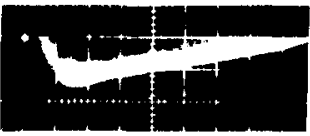

a

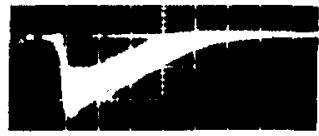

b

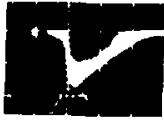

6

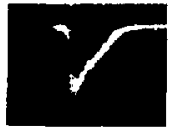

d

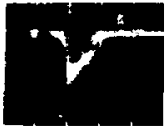

e

Fig. 1. Effect of carbon dioxide concentration on pulse shape: (a) $10 \%$, (b) $33 \%$, (c) $50 \%$, (d) $66 \%$, (e) $90 \%$, balance argon. Operating voltages were chosen to give comparable pulse heights. 20 tnv/div. into $50 \Omega, 50 \mathrm{nsec} / \mathrm{div}$.

XBB $824-3120$ 


\title{
HISS Phase II Drift Chamber Electronics
}

\author{
Fred Bieser
}

The HISS Phase II drift chamber system includes 3000 channels of time digitizers (LeCroy 4291B), 1000 channels of pulse height digitizers (LeCroy 2282) and 3000 channels of front-end ampiifer/discriminators. All the TDC's and ADC's have been purchased and 1500 channels of second generation front-end circuits have been built during 1982 .

The new front-end design offers the following improvements over the older version:

(1) Improved delta-ray rejicition due to greatly increased dynamic range;
(2) Increased sensitivity to ensure high efficiency for charge one particle detection;

(3) A delayed analog output for pulse height analysis;

(4) On-board steering of test pulses for computer controlled system testing.

The hasic concept of using a peak detector and delay line to pick off thi: largest pulse within a drift cell is still employed; the enhanced performance comies from using discrete compunent amplifiers rather than the MVL-100 chips used before. The minimum threshold can now be set as low as $5 \%$ of the average proton pulse height.

\section{Fast Trigger Processor for HISS Experiments}

\author{
J. Carroll, 'J. Geaga, 'S. Abachi, ${ }^{\dagger}$ T. Mulera and V. Perez-Mendez
}

To obtain a statistically adequate data sample for our program of studying particle correlations in the mid-rapidity region we have found it necessary to have some means of rapidly selecting events which have the proper number of reconstructable tracks. Otherwise the dead time incurred in digitizing and recording the data associated with false triggers is too large. To solve this problem we are constructing a modular, CAMAC-based system designed by Nash, Barsotti and others at Fermilab.

For our application the processor will receive, for each event, the hit patterns (in bit-parallel) from the $\mathrm{x}$-measuring planes of 3 drift chambers mounted downstream of the HISS magnet. The processor will search the hit patterns for three-points-in-a-line tracks, check that the tracks that are found have a slope/intercept combination that corresponds to a particle coming from the target with an acceptable momentum, and then count the number of such tracks in the event. In parajIel with this process, the full information concerning the event is being digitized in standard CAMAC modules (ADC's, TDC's). If the event has an appropriate number of good tracks, the signal is given to $\mathrm{read} / \mathrm{record}$ this event, otherwise a "fast clear" signal is issued to the CAMAC modules and the system is readied for the next event. This whole process occurs within about 10 microseconds for a rejected event, permitting us to handle about $10^{5}$ triggers $/ \mathrm{sec}$. The processor modules have been assembled and are being iested in their CAMAC operating environment. Interfaces to connect the processor to our specific signal sources are being constructed.

Two of the drift chambers used as input are the existing HISS drift chambers. For the third chamber we have constructed an inexpensive, coarse resolution wire chamber which operates in the "self quenching streamer"' mode of operation.

Although we have described a particular application, the modularity and CAMAC based control of system function permit the apparatus to be re-configured and expanded to meet a variety of trigger processing needs.

Footnote and Reference

†University of California Los Angeles, Los Angeles, CA 90024

1. Described elsewhere in this report 


\title{
HISS Cryogenic Control System
}

\author{
C. McParland*
}

The cryogenic HISS magnet requires a large physical plant to compress, cool, and recirculate liquid helium and nitrogen within two solenoid coils. Instrumentation of this large physical plant was found necessary to achieve both better control of operating parameters and greater efficiency in use of liquid nitrogen coolant.

Monitored parameters include temperatures at key locations of the support cylinder and liquid helium supply system, settings of critical flow control valves, and compressor speed controls. In addition, status and vacuum levels of the magnet's four vacuum systems and their associated gate valves are monitored. In all, 96 analog and 48 digital parameters are encoded and monitored.

The above monitoring is accomplished with an LSI 11/23 microcomputer system interfaced to a single CAMAC instrumentation crate. The CAMAC standard was chosen for the wide variety and availability of measurement and interface modules. The LSI $11 / 23$ is connected to the HISS VAX computer via DECNET network software and in turn appears as the node "COLDBX" on the LBL-wide network. This has allowed program development and debugging on the larger and better equipped host (VAX) with few changes required for program execution on the target system (LSI 11/23). The monitoring system was designed to be quite independent of the VAX. Once running, the LSI $11 / 23$ should provide monitoring, display, control, and feedback functions without reference to the host. It was found necessary, however, to curtail some display functions when the HISS VAX was not available.

One novel feature of this monitoring system is the presence of a touch-sensitive screen on the system's display CRT. This screen replaces the normal keyboard for all program input. This touch screen is used for the usual display selection and menu-selection functions. In addition, the program responsible for closed-loop feedback control of several parameters can be "tuned" (i.e., time constants and response functions adjusted) by use of this display and screen. Future plans include the use of this touch screen along with its $512 \times 512$ bitmapped display CRT to edit various feedback and response curves on-line.

All monitored data and a representative sample of computer-gererated feedback control commands are sent to the HISS VAX via DECNET. They are then available for a facility status display program. Future plans will have these data included in the HISS engineering data base on a daily, weekly, and monthly basis.

\section{Footnote}

*Space Sciences Laboratory and U.C. Berkeley

\section{Design of a Drift Collection Calorimeter with a Combined Radiator and Field Shaping Structure of Lead Glass Tubing*}

\author{
V. Perez-Mendez, A. Del Guerra, ${ }^{\dagger}$ H. Hirayama, ${ }^{\ddagger}$ \\ T. Mulera and W. Nelson ${ }^{\ddagger}$
}

The list of attractive features of calorimeters as particle detectors includes their sensitivity to neutral as well as charged particles; the fact that the longitudinal size of the detector scales as $\log E$ rather than $\sqrt{p}$, as is the case for magnetic spectrometers of equal resolution; and the capability of exploiting their differences in response to electrons, muons, and hadrons as a means of particle identification. The advantages of gas sampling calorimeters over devices based on sampling with scintillators or Cerenkov radiators are the easily achieved higher degree of transverse segmentation, allowing precision measurement of the angles of incidence and positions of the detected particles, and the avoidence of the problems associated with photomultiplier tubes in awkward geometries and high magnetic fields. Collecting ionization electrons over long drift paths on a relatively 
small number of sampling wires (ala ISIS, TPC, or many other "image readout" schemes) alleviates the disadvantageous need for finer longitudinal sampling to overcome the inherently poor resolution of gas filled devices.

We are investigating an economical and convenient scheme, adopted from some of our past medical instrumentation work ${ }^{1}$, for drifting electrons over long distances. This scheme combines radiator and drift field shaping structures in the form of lead glass tubes having a resistive metal coating obtained by surface reduction of the lead oxide. This allows construction of a self-supporting structure having high density, very fine sampling geometry, and smooth drift field shaping. Various lead glass configurations are being studied using the SLAC Monte Carlo routine, EGS ${ }^{2}$ and prototype calorimeters are being planned.
Footnotes and References

*Condensed from LBL-15149, to be published in Nucl. Instr. and Meth.

†Permanent address: Istituto di Física, Pisa, I-56100, Italy

¥Stanford Linear Accelerator Center, Stanford, CA 94305

§Permanent address: National Lab. for High Energy Physics (KEK), Tskuba, Japan

1. G.K. Lum, et al., IEEE Trans. Nucl. Sci. NS-27 (1980) 157

2. R.L. Ford and W.R. Nelson, SLAC-210 (1978).

\title{
A Threshold Cerenkov Counter for Isotopic Identification of High-Energy heavy Ions*
}

\author{
J. Stevenson, ${ }^{\dagger}$ J. Musser, and P.B. Pricet
}

We have developed an apparatus for isotope identification of heavy-ion projectile fragments with energies from $\sim 330$ to $600 \mathrm{MeV} /$ rucleon. The "detector" uses a Cerenkov intensity measurement in conjunction with a measurement of magnetic rigidity to determine mass. An advantage of this techniqua is that it allows isotope identification of projectile fragments with higher energies than are possible for "stopping type" detectors. Higher fragment energies allow the use of much thicker targets and, therefore, provide higher sensitivity to rare isotopes. In a recent test of the apparatus using a $670 \mathrm{MeV} /$ nucleon ${ }^{20} \mathrm{Ne}$ beam and a $30 \mathrm{~g} / \mathrm{cm}^{2}$ aluminum target a mass resolution of $\sigma_{\mathrm{A}}=$ 0.2 amu was obtained for projectile fragments with charges $5 \leq \mathrm{Z} \leq 9$.

Figure 1 shows a schematic of the detector. The detector elements each have $a 6^{\prime \prime} \times 12^{\prime \prime}$ active area with the $12^{\prime \prime}$ dimension being in the focal plane of the spectrometer. The detector elements, labeled $\mathrm{SCl}$ and $\mathrm{SC} 2$, are $3 / 8^{*}$ thick plastic scintillators made of NEl10. Two Cerenkov counters, labeled CK1 and CK2, are located between the scintillators $\mathrm{SCl}$ and $\mathrm{SC2}$. The Cerenkov counters are $1 / 2^{\prime \prime}$ thick Nuclear Enterprises Pilot 425. Following the second scintillator is a pair of wire chambers, each containing a set of orthogonal wire planes. The final element in the detector is a $1 / 4^{\prime \prime}$ thick NE110 scintillator, which is used as a trigger.
Figure 2 shows mass histograms for carbon and nitrogen isotopes from the test run. The mass resolution is typically $\sigma \sim 0.2$ mass units. The mass resolution is dominated by Cerenkov photon statistics and may be improved by making more efficient or thicker radiators. For example, a $2^{\prime \prime}$ radiator with the same collection efficiency would have a resolution of $\sim 0.15$ mass units for the same data. The mass resolution of the detector is expected to degrade to 0.3 mass units at mass 50, due entirely to the limitations of the focusing of the spectrometer.

\section{Footnotes}

* Condensed from a paper submitted to Nuclear Instruments and Methods

$\dagger$ Also at Space Sciences Laboratory 


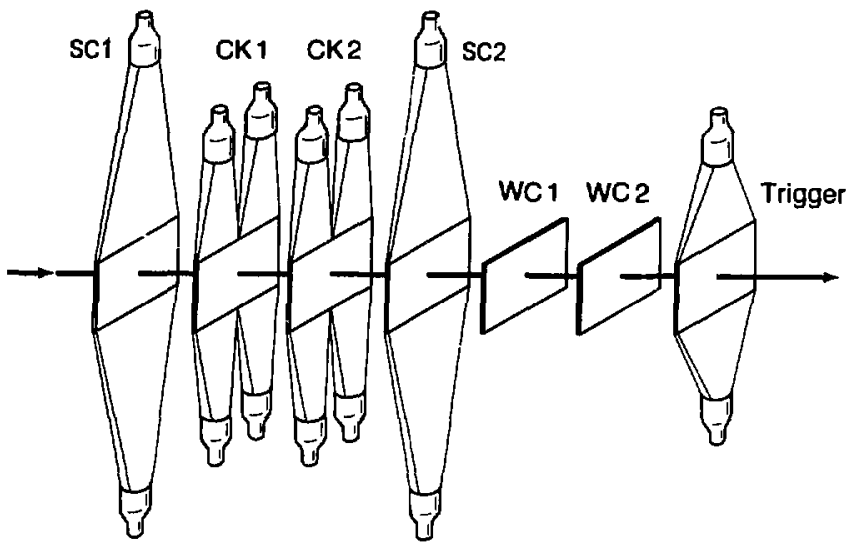

Fig. 1. Exploded view of detector: elements $\mathrm{SCl}$ and $\mathrm{SC2}$ are 3/8" thick NE110 scintillators; CK1 and CK2 are 1/2" thick Pilot 425 Cerenkov counters. WC1 and WC2 are wire chambers, each consisting of a pair of orthogonal planes. The trigger is $1 / 4^{\prime \prime} \mathrm{NE} 110$ scintillator. The active area of each detector is $6^{\prime \prime} \times 12^{\prime \prime}$.

XBL 835-9613A

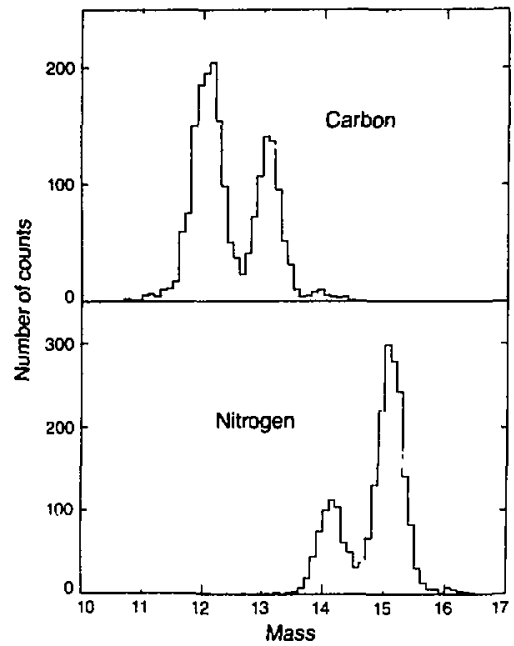

Fig. 2. Isotope histograms for carbon and nitrogen.

XBL 835-9614 


\title{
2. Computers
}

\section{Performance of the MIDAS Prototype}

\author{
Creve Maples, Daniel Weaver, William Rathbun, and Douglas Logan
}

The goal of the MIDAS project is the development of a computer system specifically targeted to deal with the problems associated with the reduction and analysis of scientific data. The system was designed to provide a highly interactive, graphies oriented, mutliuser environment capable of handling relatively large data bases for each user. The architecture was organized in such a fashion that multiple users could operate independently and simultaneously, without any significant effect on the syeed of individual data processing.

For those classes of problems involving sequenti : $^{-}$ data analysis, it is anticipated that the proposed paralle. processor approach should provide each real-time user with a processing power an order of magnitude greater than is currently availabic on our best uppc- mid-range computers (DEC VAX or ModComp Classic). This should roughly be equivalent to one $\mathrm{CDC} 7600$. The design is capable of supporting at least five such distributed processing subsystems, if required. The total processing power of a full system should therefore be equivalent to approximately 50 of the best of our current mid-range computers. It would be possible to dynamically allocate to either many users or fewer users with more demanding analyses. If necessary, the total resources of the facility could be used on a single problem.

An important design goal of this facility is the creation of a highly modular hardware and software environment. In such an environment the high technology devices could be isolated in a manner to facilitate their relatively low-cost replacement as need and future technological advances dictate. In this way it would appear possible to create a system, in the rapidly changing areas of computer and peripheral development, which is capable of planned growth, both with technology and with the needs of the user community.

\section{Architecture}

As described previously, ${ }^{1 \rightarrow}$ the MIDAS design is based on a hierarchy of multiple, high-level processors, which permits the high-speed parallel analysis of experimental data. The basic structure of data, discrete events packets, makes its analysis ideally suited to parallel processing. In this parallel framework events are analyzed simultaneously and asynchronously by multiple CPU's, as opposed to the serial, one-event-ata-time approach of conventional computers. Such a design is now practical because of the commercial availability of high-level CPU's at relatively modest cost. The system would combine interactive graphics capability, high-speed computation, and large mass storage capacity to guarantee a rapid response time to each user. For a currently typical data-analysis problem, a turnaround time of under I minute for every magnetic tape equivalent of data processed would be a desirable goal to reach. This requires that each user have avai]able, on demand, a processing power approaching that of the CDC7600. The system design is intended to provide a highly effective interface between the computer and the scientist, thereby minimizing the real time necessary to complete a given analysis problem. The key features this data analysis facility is designed to provide are as follows: 1) highly interactive performance, 2) high-speed processing; 3) optimized L/O operations; 4) modular construction, and 5) ease of use.

The basic philosophy of the architecture involves three independent levels of computer processing in a general tree structure, integrated with an independent intelligent mass storage system. The first processing level has a single multi-tasking computer refer :d to as the "primary computer". The main function of this level is to handle all user interaction and to manage all resources of the system. The problem of interactive analysis is conceptually divided at this level into interactive requirements, handled by the primary computer, and a batch analysis phase handled by level two. the "secondary computer" system.

This secondary system may consist of up to five computers, which, although dynamically allocable, are independently devoted to the solution of only a single analysis problem at a time. The function of the secondary computer is to receive a specific batch analysis problem from the primary, to break the problem into components (e.g., input, output, arithmetic transformation, spectrum generation, etc.), and to load the problem into the elements of a third processing level, known as the "multiprocessor array". Having set up the problem and started the processing, the secondary computer will constantly monitor and, if necessary, control the 
operation of the multiprocessor array as well as maintain contact with the primary computer.

A multiprocessor array (which exists for each secondary computer) consists of at least eleven somewhat specialized, high-speed processors, a multi-bussed memory area for data, and a local storage area for results. The assumption is that once an analysis problem is specified data events are all analyzed in a parallel fashion, rather than serially as would be done in a conventional computer. At least eight of the eleven processors would be powerful, pipelined CPU's whose function is to perform mathematical transformations of the data base. Each of these CPU's would have a processing capability equivalent to the most powerful computers currently used by the Nuclear Science Division. Thus, a general system containing five distributed or secondary systems would have at least 40 processors of this type in multiprocessor arrays.

Separate from these three processing levels, the design also incorporates an intelligent, multi-bussed, parallel processing storage environment. At least five intelligent controliers would oversee the storage and retrieval of user data and results from various mass storage devices. Initially these devices would consict of magnetic tapes, large disks, and bulk histogramming memory.

\section{Development}

The development of the MIDAS Project was planned in three stages: Phase 1, the development of a working prototype; Phase 2, the creation of a fully operational model; and Phase 3, an expansion of the architecture into a complete multi-user system. Construction of the Phase 1 MIDAS prototype was begun in May 1980 and, after passing all tests, became operational in January 1982. The hardware cost of this initial system was approximately $\$ 110 \mathrm{~K}$. This prototype used three commercial mid-range CPU's, as shown in Fig. 1, to demonstr: the feasibility of the basic parallel-processor approach. The central processing units of commercial mid-range computers, which includes floating-point and pipeline hardware, can be obtained at about one-tenth the cost of a complete computer system using the same CPU. A major advantage of using powerful commercial CPU's is the availability of software support, particularly in the form of support for high-level languages such as FORTRAN.

In the protocype system, shown in Fig. 1, user programs are compiled and assembled in a standard manner by the secondary CPU. Complete binary copies of this program are then downloaded by the secondary into each parallel CPU (Programmable Arithmetic Processor) for execution. During execution, a special processor, the input formatter, independently directs the incoming information into separate blocks of external memory (the data area). A hardware switching unit, the conductor, supplies new information to each CPU, on demand, by switching a new block into its memory map. Calculational results are collected from the "completed" memory blocks by the output formatter and stored in a common bulk mernory unit, the storage memory. All these processors operate simultaneously and asynchronously. Results of the analysis are available to the user from the secondary computer by means of a second access port on the storage memory.

In January 1982, benchmark tests were conducted on the prototype system to compare its performance both with our current romputers (ModComp IV/25) and with performance predicted by our software models. Based on results of software simulations, the performance of the system was expected to be dependent on the relative amounts of I/O versus CPU use of each problem. For this reason, testing was conducted using several (very different) existing codes under different calculational conditions. This involved converting several existing large-scale programs to run in a MIDAS environmen:. These included EVA, an event analysis language originally developed in Copenhagen; OKDER, the analysis portion of the MINUS3 system running at the SuperHILAC and 88-inch Cyclotron; SATURN, a Monte Carlo fission simulation code; and an analysis program used to reduce data taken by the Bevatron's Plastic Ball apparatus. To assist in using MIDAS, sophisticated program translation and debugging tools have been created to facilitate program conversion and development. These include a generalized translator program that converts higher level, freeform FORTRAN or RATFOR-type codes into ModComp-standard FORTRAN, which can then be compiled and run on MIDAS. Substantial program simplification occurred in the course of converting these programs because of the architecture of MIDAS, which permits some operations (particularly those involving $1 / O$, such as event unpacking) to be performed by specialized hardware devices rather than by software.

Ferformance was, therefore, tested under three classes of conditions. The first class of problems involved programs with heavy $1 / 0$ demands; the second set of problems included calculations with relatively balanced I/O and CPU requirements; and the third class of problems invclved sompletely CPU-bound calculations, with minimal $\mathrm{I} / \mathrm{O}$ operations. Table I summarizes the results of the benchmark tests. These tests were first run on the prototype consisting of three paralIel CPU's (Programmable Arithmetic Modules). A fourth CPU, in orporating new memory that enables this CPU to perform fetches from local memory $20 \%$ faster than the three original CPU's, was iater added, and the benchmark tests were repeated. The EVA program was used as representative of I/O-intensive 
operations; the ORDER program was run with two different analysis conditions - one case incorporating basic histogram building (moderately I/O intensive) and another case in " "ding extensive gated-spectra and pseudo-parameter creution (mostly CPU intensive). The SATURN code was included as a completely CPUlimited program Table $I$ indicates that in a CPUlimited environment MIDAS performance shows approximately linear increase in speed with number of CPUS, as would be expected. In I/O-intensive problems, however, the increase is far more dramatic, showing a pro. cessing throughput more than $50 \%$ greater than would be predicted by a strictly linear increase with the number of CPU's. The comparison tests were performed by analyzing real experimental data on both the prototype facility and existing computers.

Work on the Phase 2 model began in February 1982 and is expected to be completed in early FY83. To accomplish this, the existing prototype is being expanded to incorporate ten active processors. To this end a fourth CPU was added to the prototype in March 1982 and a fifth in May. Projections of the performance of a Phase 2 system running an "average", or class 2, program are shown in Table it. Thise projections are based on an emulation of the system using the measured benchmark performances. A projection based on a CPU-bound problem run on bot:- the CDC7600 and the MIDAS prototype indicates that the processing power of a single Phase 2 subsystem should be the equivalent of about $85 \%$ of a 7600 . Total estimated capital equipment cost for the completion of Phase 2 is $\$ 390 \mathrm{~K}$ (inclusive of Phase 1 ). This working system will support one or two interactive users performing CPU. intensive operations. The Phase 2 model will ultimately constitute a single distributed subsystem within the Phase 3 facility. By using additional distributed subsystems, the Phase 3 system is capable of considerable expansion, both in terms of processing power and the number of simultaneous users supported.
Summary

Computers continue to assume an increasingly important role in scientific research. The MIDAS Project represents state-of-the-art research into the forefront of computer architecture and its specific application in a scientific environment. In the decade of the 60 's computing speeds increased by a factor of about 100. During the 70's, however, only a factor of 10 increase in speed was realized. Traditional serial computers are approaching their theoretical limit - the transmission time of signals are being limited by the speed of light. Current estimates are that serial computers are limited to $10^{9}$ operations per second, or only a factor of 10 better than is currently available.

To achieve significant improvements in future computer processing, it is necessary to develop a parallel architecture. This can be achieved in two ways: executing instructions in parallel, as in a vector machine (e.g., Cyber 205 or CRAY-1), or using parallel processors. For certain classes of problems, vectororiented computers provide assistance. The main difficulty here lies in efficiently using these computers ("Converting problems for efficieat execution on the new class of highly parallel machines requires an alinost complete reanalysis bind rewrite of the programs." - J.E. Wirsching and T. Kishi, LLLi "Many current large-scale programs cannot run efficiently on any known bigh nerformance machine..." - Hogan, Jensen, and Cornish, Tex is Instruments Inc.).

The use of multiple processors operating in parallel, such as employed in the MIDAS architecture, provides an alternative to achieve necessary high-speed computation in the future. A recent Livermore study on high-performance computers states: "Computers incorporating arrays of processors are beginning to emerge...The optimum arithmetic performance of thesc devices is potentially much greater than that of current supercomputers." Work at SLAC is currently being completed on utilizing multiple specially designed processors (emulating an IBM 370/168) to analyze experimental data.

\begin{tabular}{|l|c|cc|c|}
\hline \multicolumn{4}{|c|}{ Table I: Results of Benchmark Tests on MIDAS Prototype System } \\
\hline & \multicolumn{4}{|c|}{ Running Time in Seconds (Ratio) } \\
\cline { 2 - 5 } & 1/O Limited & \multicolumn{2}{c|}{ Average Mix } & CPU Limited \\
\hline Single Computer & $272(6.3)$ & $612(5.5)$ & $2314(4.9)$ & $(\sim 4)$ \\
MIDAS(3) & $49(1.1)$ & $159(1.4)$ & $673(1.4)$ & $(1.4)$ \\
MIDAS(4) & $<43>(1)$ & $112(1)$ & $472(1)$ & $(1)$ \\
\hline
\end{tabular}

a)Indicates number of parallel CPU's $4 / 16 / 82$ 


\begin{tabular}{|l|c|c|c|}
\hline \multicolumn{3}{|c|}{ Table II: MIDAS Relative Performance } \\
\hline & Problem: Average CPU and I/O Mix \\
\hline & Time & Relative & I/O Rate \\
\hline ( & (sec.) & Speed & (KB/sec) \\
2 PAMs & 480 & 2.0 & 26 \\
3 PAMs & 241 & 3.0 & 52 \\
4 PAMs & 159 & 4.3 & 83 \\
(8) & 112 & - & 115 \\
(8AMs) & - & $(9.0)$ & $(243)$ \\
\hline
\end{tabular}

b) Lmproved model $4 / 16 / 82$

The MIDAS Project is not designed as a general purpose coinputer but rather to fill a specific need within the nuclear science community. Experience at other research institutions and examination of current computer performance suggest that this need cannot reasonably be filled by currently available computers in any cost-effective manner. Further, given current computing trends, research and development into the application of parallel processing could have broad applications in otber areas besides nuclear science.

References

1. C.C. Maples, IEEE Transactions on Nuclear Science, NS-26 (1979) 4580

2. C. Maples, W. Rathbun, D. Weaver, and J. Meng, IEEE Transactions on Nuclear Science, NS-28 (1981) 3746

3. C. Maples, D. Weaver, W. Rathbun, and J. Meng, IEEE Transactions on Nuclear Science, NS-28 (1981) 3880

4. W. Rathbun, C. Maples, J. Meng, and D. Weaver, IEEE Transactions on Nuclear Science, NS28 (1981) 3875

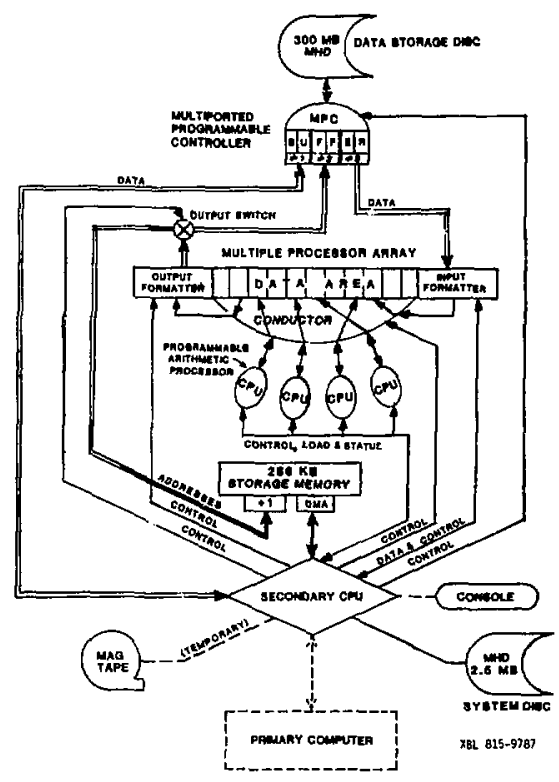

Fig. 1. The layout of the MIDAS Phase 1 prototype system, currently in operation.

XBL 815-9787 


\title{
Dynamic Expression Evaluator for Real Time Applications
}

\author{
Daniel Weaver, William Rathbun, and Creve Maples
}

\begin{abstract}
A dynamic expression evaluator ${ }^{1}$ developed for on-line data analysis gives experimenters at the 88-Inch Cyclotron and SuperHILAC both flexibility and high throughput. It uses standard FORTRAN rules for expression, including operator precedence, and allows built-in functions such as sine and cosine. Evaluation techniques using a combination of FORTRAN and Assembly and XPL (a dialect of PL/1) have been compared. An XPL evaluator was selected because of development time and evaluator speed. It can evaluate expressions at approximately $50 \mu \mathrm{sec}$ per operand on a ModComp Classic 7870 with $20 \mu s e c$ overhead per expression.
\end{abstract}

\section{Introduction}

The quadrupole expression evaluator was designed to enhance on-line data analysis with the ModComp computers at the SuperHILAC and 88-Inch Cydotson. Data acquisition at these accelerators is handled by the package, MINUS3 ${ }^{2}$ to which the expression eviluator was added. Expressions may be evaluated with integer or floating point arithmetic.

A dynamic expression evaluator consists of two parts. In the first phase (which is executed unly once for each new expression) the translator takes the text of the expression and converts it to an interpretive language. Then the runtime evaluator uses the interpretive code to evaluate the expression. This operation occurs once for each new piece of data for each expression. Since data are produced in large quanities at research accelerators, the second phase is used many more times than the first. If the evaluator is to be fast, the intermediate code must be designed to sinimize the evaluation time needed for each use. Definition of the code is therefore of key importance. In addition, the runtime evaluator should be written in tie most efficient language available. The language in which the translator is written, however, need not affect the efficiency of the evaluator since identical interpretive code can be produced by translators written in different languages. A significant factor in selecting a language for a translator is the amount of time needed to write the program.
History

The MINUS3 data acquisition package provides on-line display and analysis of real time events. The analysis capability was designed primarily for speed in calculation and uses a set of nine general equations built into the code. An experimenter may select an expression to use, the parameters that ase fed into the expression, and values for constants. Two primary techniques are used to increase speed. First, the limited precision of the data ( 12 bits) permits, in many cases, the calculations to be performed using 32-bit integer aritbmetic without loss of accuracy. The second method also capitalizes on this limited precision by recognizing that an expression with one input variable bas a fixed number of results (4096) and all possible results may be stored in a table. Table look-up of results is significantly faster than actual calculation for each use. Although the nine equations were selected to give a wide variety of computation, any limitation in the number of expressions reduces the scope of calculation available to an experimenter. It became clear that a more flexible method was needed, one that each experimenter could tailor to his or her needs. The method decided on was the addition of an evaluator that would permit user selection of expressions and dynamic change of expressions during experimentation.

As a first requirement, the evaluator had to be as fast as possible within system constraints. Its second requirement was to increase fiexibility of computation. Third, it had to be relatively easy for new users to learn to ise and to use at 3 am when they have been running for two days straight. Finally, it had to interface with MINUS3, to receive input and display results.

Two different translators using different parsing techniques and three different runtime evaluators were implemented. The first translator was written in FORTRAN with a runtime evaluator also written in FORTRAN. After preliminary timing tests, the runtime evaluator was rewritten in Assembly, producing an increase in throughput of about $30 \%$. The original FORTRAN version of the evaluator did not fully meet the design criteria. While useful, it suffered because operator precedence had to be explicitly stated through

the use of parentheses. For example, with no parentheses, $1+2 * 3$ would be calculated as $(1+2)^{*}$, 
since all expressions were evaluated from left to right. Standard operator precedence would have zerformed the multiplication first. Two solutions presented themselves to solve the problem of operator precedence. The first was to redesign the existing evaluator to include operator hierarchy. The second was to redevelop the evaluator using the XPL language and its automatic compiler writing system. This second approach resulted in a second translator written in XPL with a runtime evaluator also written in XPL.

\section{XPL Expression Evaluator Design}

The runtime evaluator is stack oriented. Each operand is placed on the stack, then an operation is performed on the two elements on top of the stack (one if unary minus or functions) and the result is returned to the stack.

\section{Example:}

Consider the expression P1 + P2 3 - 2 The first operation that must be performed is multiply, followed by add, and then subtract. In reversePolish the expression would be P1 P2 $3^{*}+2$

This is, in fact, how the ixpression appears to the runtime evaluator. It loads $P 1, P 2$, nnd 3 into the stack, then does the multiplication on the top two stack siements (P2 and 3). The result is placed on the second position on the stack (where P2 used to be). The top two elements then are added with the result being returned to the stack as its only element. Finaliy the constant 2 is pushed onto the stack and the subtraction is performed. After an expression is evaluated, the result is ajways the last element on the stack. This value is then returned to the data acquisition package, MINUS3.

\section{Expression Syntax}

The learning curve for physicists using the evaluator was minimized by making mathematical syntax basically the same as that foind in FORTRAN and most other bigh-level languages. This permits nesting by use of parentheses, with multiplication and division being performed before addition and subtraction within one nesting level. Input expressions may be up to 80 characters in length and may contain the following standard operator symbols:

$$
+-* /() \text { and ** (exponentiation). }
$$

Parameters, pseudo-parameters, constants, and built-in functions may be used within each expression. A parametet is the data from a given detector. Par?meters are specified as $\mathrm{Pl}$ thru $\mathrm{Pn}$ where $\mathrm{n}$ is the number of detectors in a given experiment. A pseudo-parameter is the output of some other hard-coded expression or dynamic expression. Pseudo-parameters are specified as $\mathrm{Pn}+1$ thru P32 where $\mathrm{n}$ is the number of detectors in the experiment. If desired, the results of a pseudoparameter may replace the value of a given detector input. Constants may be imbedded as part of an expression (e.g., PI + 6.3) or given identifier names of $\mathrm{Cl}$ through C16. Identifier names for constants permit change of values for "constants" within a run. The built-in functions SINe, COSine, TANgent, SQRT (square root), EXPonent, LOG (base 10), LN (natural $\log$ ), and ABSolute value are supported. Expressions may be evaluated as integer or real, specified by the structure of the equation.

Recent improvements to the evaluator permit the use of logical operators and conditional statements. The logical operators $A N D, O R$, and NOT permit experimenters to process digital and non-ADC type data. Conditional statements provide the capability to set limits for acceptable data and vary the kind of analysis done for each piece of data. In addition, the original limitation of 80 -character expressions has been removed by recognizing a sontinuation character that permits multiple lines of code.

\section{Editing Expressions}

An editing command was included in MINUS3 to permit alteration of expressions without retyping. This command can be invoked while an experiment is in progress or during subsequent analysis. In either case, the experimenter can retrieve the input source expressions, change text (by typing alterations beneath it), delete code (by typing an underscore beneath character(s) to be deleted), or make additions (by typing an up-arrow at the point of insertion). The changed expression is processed back through the translator, and the internal runtime table is altered. 
Below is an example of defining and editing a

dynamic expression (boldface denotes user response):

\# 1 ... FORTRAN EXPRESSION EVALUATOR

(USE FORTRAN ALGEBRAIC NOTATION WITH

SYMBOL PN DENOTING PARAMETER N AND CN

TO DENOTE A DYNAMIC VARIABLE N)

* * ENTER EXPRESSION (80 CHAR, MAX)

1.0+C1*P2-C2*(2.7E-4*P2**2 + P2**3)+C2*P1**4

***EDIT EXPRESSION ( $Y$ / $)$ ?

$Y$

**ENTER CHANGE(S) UNDER ORIGINAL EXPRESSION

(USE \& FOR A SPACE; " TO INSERT; AND - TO DELETE)

$1.0+C 1 * P 2-C 2 *(2.7 E-4 * P 2 * * 2+P 2 * * 3)+C 2 * P 1 * * 4$

$P$ C $\mathrm{CB}^{*}-42$

$1.0+C 1 * P 2-C 2 * P 2 * P 2+C 3 * P 2 * * 3+C 4 * P 2 * * 4$

Results

Development time for the FORTRAN evaluator was approximately two man months and for the XPL expression evaluator about aalf that. The difference in the amount of effort is directly attributable to the use of the XPL compiler writing system.

The XPL evaluator can evaluate expressions at approximately 50 microseconds per operand on a ModComp Classic 7870 with 20 microseconds overhead per expression. Table I shows the times in microseconds for the various evaluators. Hard-coded FORTRAN times are included for comparison.

Timings for the XPL runtime evaluator as it currently exists would be improved by rewriting the code in Assembly, but this has not been done to date. The Assembly language evaluator written for the FORTRAN translator cannot be used with the XPL translator because the two packages use different intermediate corie.

After initial timing tests showed the XPL expression evaluator to be faster, the XPL system was integrated into the data anlysis package of MINUS3. The FORTRAN expression evaluator is currently being used to provide graphies display and modeling.

The major advantage of a FORTRAN expression evaluat or is portability. The XPL system is limited to machines with an XPL compiler. This is not a major cisadvantage at LBL because the MINUS3 data acquisition system is only used on ModComp equipment.
Table I

\begin{tabular}{|c|c|c|c|}
\hline EXPRESSION & $\begin{array}{c}\text { Compiled } \\
\text { FORTRAN }\end{array}$ & XPL & $\begin{array}{r}\text { FORTRAN } \\
\text { Amerbbly }\end{array}$ \\
\hline$X / A+Y / B+Z$ & 24 & 305 & 500 \\
\hline$(X / A+Y) \cdots P . Y * P$ & 232 & 605 & 804 \\
\hline$A+B X+C X * 2+D X * 3+E X * 4$ & 79 & 780 & 1270 \\
\hline SQRT $(X)$ & 34 & 145 & 280 \\
\hline
\end{tabular}

All variables and constants are 32-bit floating point numbers.

Times for the FORTRAN runtime evaluator were $50 \%$ slower than the Assembly timings.

\section{References}

1. D. Weaver, W. Rathbun, and C. Maples, IEEE Transactions in Nuclear Science, NS-28 (1981) 3895

2. C. Maples and J. Sivak, IEEE Transactions in Nuclear Science, NS-26 (1979) 4409 


\title{
Status of Bevalac Software
}

\author{
C. McParland*
}

Several software and hardware items of Bevalacwide interest are worthy of note.

Distribution tapes for the DATACQ' data acquisition system have been produced. These contain both the sources and command procedures for generating the DATACQ system. Preliminary documentation on the generation procedure and the design philosophy behind DATACQ are included. New in this distribution is a procedure for building the MBD driver and all its associated utilities. Copies have been installed on all three HISS front-end machines, the GSI Plastic Ball 11/44, and the Streamer Chamber 11/45.

A CAMAC-DATACQ test module (called a CATS module) has been designed and produced. This module is a response to the intolerable levels of unreliability experienced on CAMAC branch highways by several groups at the HISS facility. The CATS module contains a 24bit read/write register used to check the integrity of the branch highway data lines. An additional register latches the function $(F)$ and subaddress (A) lines on each CAMAC cycle. This allows a verification of the previous CAMAC cycle. These features are used by a new CAMAC test program (current distribution), which locates all CATS modules present in any on-line CAMAC crate and proceeds to exercise them all simultaneously. Errors are reported and the offending module is dropped from the test. Since this program can be run during any pause in experimental data- taking, CAMAC branch highway and crate controller problems should be diagnosed simply and quickly.

Non-CAMAC features include a random frequency pulse generator and a Bevatron spill simulator. When used in conjunction with the DATACQ program, a complete data-taking sequence with randomly spaced events of random length can be simulated. Wher performed at the end of a DATACQ system generation, this facility provides a complete systen! checkout.

Planning has proceeded for the purchase of the Bevalac-NSD VAX in FY 83. This machine will be a dedicated experimental acquisition and on-line analysis tool for beam areas not currently connected to either the HISS or GSI VAX. The logical connection will be the same as that used by both the HISS and GSI machines - a large shared disk between front-end data acquisition computer and the VAX. It will be housed along with the GSI VAX in a building near the Bevatron A door. Order is anticipated in early 1983 with delivery in May-June 1983.

\section{Footnote and Reference}

-Space Sciences Laboratory and U.C. Berkeley

1. F. Weik, "DATACQ. a New Data Acquisition Program" in Nuclear Science Annual Report 1980-81, LBL-13366. 
PART VI: APPENDICES

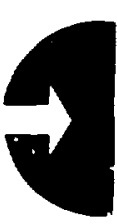


Aystö, J. (See Wouters, J.M., LBL-13990)

Abachi, S. (See Shor, A., LBL-14229)

Alekett, K. (See Loveland, W., LBL-13201)

Aleklett, K. (See Groening, H., LBL-13483)

Alekłett, K. (See Loveland, W., LBL-14477)

Andersen, O. (See Ellegaard, C., LBL-11913)

Anderson, L., W. Brüchner, E. Moeller, S. Nagamiya, S. Nissen-Meyer, L.Schroeder, G. Shapiro, and H. Steiner Inclusive Particle Production at Forward Angles From Collisions of Light Relativistic Nuclei, Part I: Nuclear Fragments, LBL-14328, May 1982, Phys. Rev. C

Anderson, L., E. Moeller, S. Nagamiya, S. Nissen-Meyer, L. Schroeder, G. Shapiro, and H. Steiner Inclusive Particle Production at Forward Angles from Collisions of Light Relativistic Nuclei, Part IIl: Data Tables, LBL-14330, May 1982

Anderson, L. (See Moeller, E., LBL-14329)

Baden, A. (Sec Gutbrod, H.H., LBL-14218)

Baden, A. (See Warwick, A.I., LBL-13831)

Banerjee, B. (See Glendenning, N.K., LBL-12704)

Bendjaballah, N. (See Deleplanque, M.A., LBL-14056)

Benenson, W. (See Frankel, K.A., LBL-12585)

Benenson, (See Frankel, K.A., LBL-12641)

Benenson, W. (See Sullivan, J.P., LBL-11971)

Berman, B.L. (See Olson, D. LBL-12292)

Betlach, M.R. (See Firestone, R.B., LBL-10919)

Bice, A.N., A.C. Shotter, and Joseph Cerny

${ }^{12} \mathrm{C}^{*}$ and ${ }^{8} \mathrm{Be}$ Production in ${ }^{12} \mathrm{C}+{ }^{208} \mathrm{~Pb}$ Collisions, LBL-14100, May 1982, Nucl. Phys. A390, 161 (1982).

Bice, Alden Newell

Nuclear Reaction Studies Via the Observation of Unbound Ontgoing Systems, LBL-13859, January 1982, Ph.D thesis

Bice, A.N. (See Shotter, A.C., LBL-11376)

Bice, A.N. (See Shyam, R., LBL-14740)

Bistirlich, J.A. (See Frankel, K.A., LBL-12585)

Bistirlich, J.A. (See Frankel, K.A., LBL-12641) 
Bistirlich, J.A. (See Sullivan, J.P., LBL-1 1971)

Bjornholm, S. and W.J. Swiatecki

Dynamical Aspects of Nucleus-Nucleus Collisions, LBL-14074, May 1982, Nucl. Phys. A391, 471 (1982).

Blocki, J. and W.J. Swiatecki

Nuclear Deformation Energies, LBL-12811, Presented at the IEEE Meeting on Nuclear Science Washington, DC, October 20-22, 1982., May 1982

Bock, R. (See Stock, R., LBL-14583)

Boguta, J.

Nucleon-Nucleus Optical Potential in a Relativistic Theory of Nuclear Matter, LBL-11466, August 1980, Phys. Lett. 106B, 245 (1981).

Boguta, J.

Relativistic Quantum Field Theory of Finite Nuclei, LBL-1 1467, August 1980, Phys. Lett. 106B, 241 (19i 1).

Boguta, J.

A Saturating Chiral Field Theory of Nuclear Matter, LBL-13874, May 1982, Phys. Lett. 120B, 34 (1983).

Boguta, J.

Baryonic Degrees of Freedom Leading to Density Isomers LBL-12333, April 1981, Phys. Lett. 109B, 251 (1982).

\section{Boguta, J.}

Beta Stable Neutron Stars in Non-Linear Relativistic Mean Field Models, LBL-12584, November 1981, Phys. Lett.

\section{Bogute, J.}

Ground State Properties of ${ }^{16} \mathrm{O},{ }^{40} \mathrm{Ca}$ and ${ }^{48} \mathrm{Ca}$ in a Relativistic Hartree Theory of Nuclear Matter, LBL-11894, December 1980, Nucl. Phys. A372, 386 (1981).

Boguta, J. and H. Stocker

Systematics of Nuclear Matter Properties in Non-Linear Relativistic Field Theory, LBL-13004, Aug. 1981, Phys. Lett. 120B, 289 (1983).

Boguta, J. and S.A. Moszkowski

Nonlinear Mean Field Theory for Nuclear Matter and Surface, LBL-14685, July 1982, Nucl. Phys. A

Boguta, J. (See Stocker, H., LBL-12095)

Bohrmann, S. (See Mïller, K.-H. LBL-12663)

Bohrmann, S. (See Nagamiya, S., LBL-14033)

Bolotin, H.H. (See McDonald, R.J., LBL-11608)

Bonner, B.E. (See Sugle, A.L., LBL-13232)

Bossingham, R. (See Frankel, K.A., LBL-12585)

Bossingham, R. (See Frankel, K.A, LBL-12641)

Bossingham, R. (See Sullivan, J.P., LBL-11971) 
Bowman, D.J., R.E. Eppley, EK. Hyde

Alpha Spectroscopy of Nuclides Produced in the Interaction of $5 \mathrm{GeV}$ Protons with Heavy Element Targets, LBL8895, August 1981, Phys. Rev. C25, 941 (1982).

Bowman, H. (See Hicks, K.H, LBL-15405)

Bowman, H.R. (See Frankel, K.A., LBL-12585)

Bowman, H.R. (See Frankel, K.A, LBL-12641)

Bowman, H.R. (See Sullivan, J.P., LBL-11971)

Brady, F.P. (See Sagle, A.L., LBL-13232)

Brandan, ME. (See Cole, A.J., LBL-13011)

Brandan, ME. (See Cole, A.J., LBL-14550)

Brockmann, R. (See Stock, R., LBL-14583)

Brüchner, W. (See Anderson, L., LBL-14328)

Brüchner, W. (See Moeller, E., LBL-14329)

Buchwald, G. (See Stocker, H., IBL-12634)

Burde, J., E.L. Dines, S. Shih, R.M. Diamond, J.E Draper, K.H. Lindenberger, C. Schuck, and F.S. Stephens A Third Discontinuity in the Yrast Levels of ${ }^{158} \mathrm{Er}$, LBL-13610, December 1981, Phys. Rev. Lett. 48, 530 (1982).

Buctke, T. (See Sullivan, J.P., LBL-11971)

Cable, M.D., J. Honkanen, R.F. Parry, H.M. Thierens, J.M.Wouters, Z.Y. Zhou, and Joseph Cerny Beta-Delayed Proton Decay of an Odci-Odd $T_{z}=-2$ Isotope, ${ }^{2}$ Al, LBL-14419, May 1982, Phys. Rev. C26, 1778 (1982).

Cable, M.D. (See Wotsters, J.M, LBL-13990)

Canto. L.F. (See Radi, H.M.A., LBL-13901)

Carroll, J. (See Shor, A., LBL-14229)

Cauvin, B. (See Mathews, G.J., LBL-13226)

Cerny, J. (See Wouters, J.M., LBL-13990)

Cerny, J. (See Bice, A.N., LBL-14100)

Cerny, J. (See Cable, MLD., LBL-14419)

Cerny, J. (See Shotter, A.C., LBL-11376)

Cerny, J. (See Shyam, R., LBL-14740)

Cheng Luo (See Liu, Y.-f., LBL-12909) 


\section{Appendix I}

\section{Publications}

\section{Cheng Lwo (See Loveland, W., LBL-11658)}

\section{Clark, D.J.}

Ion Sources for Cyclotrons, LBL-13267, Presented at the Ninth International Conference on Cyclotrons and their Applications, Caen, France, Sept.7-10, 1981, September 1981

\section{Clawson, Carl William}

Zero Field Spin Relaxation of the Positive Muon in Copper, LBL-14489, July 1982, Ph.D. thesis

Cole, A.J., W.D.M. Rae, M.E. Brandan, A. Dacal, B.G. Harvey, R. Legrain, M.J. Murphy and R.G. Stokstad Elastic Scattering of ${ }^{12} \mathrm{C}+{ }^{12} \mathrm{C}$ Between 121 and $290 \mathrm{MeV}, \mathrm{LBL}-14550$, June 1982, Phys. Rev. C

Cole, A.J., W.D.M. Rae, M.E. Brandan, A. Dacal, B.G. Harvey, R. Legrain, M.J. Murphy, and R.G. Stokstad

${ }^{12} \mathrm{C}+{ }^{12} \mathrm{C}$ Reaction Cross Section Between 70 and $290 \mathrm{MeV}$ Obtained from Elastic Seattering, LBL-13011, July 1981, Phys. Rev. Lett. 47, 1705 (1981).

Cole, A.J. (See Rae, W.D., LBL-12568)

\section{Conzett, H.E.}

Concerning Tests of Time-Reversal Invariance Via the Polarization-Analyzing Power Equality, LBL-13992, invited paper presented at the First International Conference on Non-potential Interactions and Their Lie-Admissible Treatment, Orleans, France, January 5-9, 1982 and to be published in the proceedings

Conzett, H.E. (See Roy, R., LBL-14343)

Conzett, H.E. (See Sagle, A.L., LBL-13232)

Conzett, H.E. (See Slobodrian, R.J. LBL-13892)

Conzett, H.E. (See Slobodrian, R.J., LBL-13411)

Cork, B. (See Hoang, T.F., LBL-12362)

Crawford, HJ. (See Murphy, MJ., LBL-14272)

Crawford, H.J. (See Hoang, T.F., LBL-12362)

Crawford, H.J. (See Olson, D. LBL-12292)

Crawley, G.M. (See Frankel, K.A., LBL-12585)

Crawley, G.M. (See Frankel, K.A., LBL-12641)

Crawley, G.M. (See Sullivan, J.P., LBL-11971)

Crowe, K.M. (See Frankel, K.A., LBL-12585)

Crowe, K.M. (See Frankel, K.A., LBL-1264)

Crowe, K.M. (See Sullivan, J.P., LBL-11971)

Csernai, L.P., Walter Greiner, Horst Stocker, Isao Tanihata, Shoji Nagamiya, and Jörn Knoll Macroscopic Nucleon-Nucleon Correlations Caused by the Bounce-Off Process in the Energetic Collisions of Heavy 
Nuclei, LBL-13944, November 1981, Phys. Rev. C

Csernai, L.P., and H. Stocker

Central Heavy Ion Collisions by Viscous Nuclear Fluid Dynamics, LBL-12788, May 1981, Phys. Rev. C

Csernai, L.P. (See Stocker, H., LBL-12634)

Dacal, A. (See Cole, A.J., LBL-13011)

Dacal, A. (See Cole, A.J., LBL-14550)

Dacal, A. (See Harris, J.W., LBL-12334)

Dacal, A. (See Rae, W.D., LBL-12568)

Ducal, A. (See Stock, R., LBL-14583)

Dairiki, J.M (See Firestone, R.B., LBL-14070)

Deleplanque, i.A.

Nuclear Moments of Inertia at High Spins, LBL-15162, presented at the Conference on High Angular Momentum Properties of Nuclei, Oak Ridge. TN Novemher 2-4, 1982, October 1982

Deleplanque, M.A., H.J. Korner, H. Kluge, A.O. Macchiavelli, N. Bendjaballah, R.M. Diamond, and F.S. Stephens

The Rotational Inertia of Nuclei, LBL-14056, July 1982, Phys. Rev. Lett.

Deleplanque, M.A. (See Ellegaard, C., LBL-11913)

Diamond, R.M.

Nucicar Coilectivity in Highly Excited States, LBL-13723, Presented at the Symposium on Nuclear Collectivity, Dogashima, Japan, October 14-16, 1981, October 1981

Diamond, R.ME (See Burde, J., LBL-13610)

Diamond, R.M. (See Deleplanque, M.A. LBL-14056)

Diamond, R.M. (See Draper, J.E., LBL-14274)

Diamond, R.M. (See Ellegaard, C., LBL-11913)

Diamond, R.M. (See McDonald, R.J., LBL-11608)

Diamond, R.M. (See Pacheco, A.J., LBL-14091)

Diamond, R.M. (See Wozniak, G.J., LBL-946I)

Dines, E.L. (See Burde, J., LBL-13610)

Donangelo, R. (See Radi, H.M.A., LBL-13901)

Draper, J.E., J.O. Newton, L.G. Sobotka, H. Lindenberger, G.J. Wozniak, L.G. Moretto, F.S. Stephens, R.M. Diamond, and R.J. McDonald 
Dependence of the Giant Dipole Strength Function on Excitation Encrgy, LBL-14274, June 1982, Phys. Rev. Lett. 49. 434 (1982).

Draper, J.E. (See Burde, J., LBL-13610)

Draper, J.E (Sec Ellegaard, C, LBL-11913)

Eggers, R.C. and L.P. Somerville

The Maximum Likelihood Method Applied to the Problem of the Multi-Component Decay, LBL-12060, January 1981, Nuclear Instruments and Methods 190, 535 (1981).

Ellegard, C., M.A. Deleplanque, O. Andersen, B. Herskind, F.S. Stephens, R.M. Diamond, H. Kluge, C. Schuck, S. Shih, and J.E Draper

Alignment Effects in Correlation Spectra, LBL-11913, Novemuer 1981, Phys. Rev. Lett.

Eppley, R.E. (See Bowman, D.J., LBL-8895)

Fäi, G. and J. Randrup

Explosion-Evaporation Model for Fragment Production Medium-Energy Nuclear Collisions, LBL-13357, October 1981, Nucl. Phys. A

Fái, G.

The Clutching of Two Colliding Nuclei, LBL-14413, May 1982, Nucl. Phys.

Fäi, G. (See Randrup, J., LBL-14179)

Firestone, M.K. (See Firestone, R.B., LBL-10919)

Firestone, R.B.

Complex Beta-Decay Schemis: Pandemonium Lost and Paradise Regained, LBL-14136, March 1982, Phys, Lett. 113B, 129 (1982).

Firestone, R.B., M.K. Firestone, M.R. Betlach and J.M. Tiedje

The Feasibility of Producing ${ }^{11} \mathrm{C},{ }^{13} \mathrm{~N},{ }^{15} 0$, and ${ }^{18} \mathrm{~F}$ with Heavy-Ion Beams, LBL-10919, May 1980, Advances in Chemistry Series, 197, 251 (1981).

Firestone, R.B., R.C. Pardo, R.A. Warner, Wm. C. McHarris, and W.H. Kelly

The $\epsilon^{+}$Decay of ${ }^{145} \mathrm{Gd}$ : Resolution of $\epsilon^{+}$Decay Branching Ratio Anomalies and Evidence for Pronounced Structures in the $\beta$-Decay Strength, LBL-12424, March 1981, Phys. Rev. C25, 527 (1982).

Firestone, R.B., V.S. Shirley, J.M. Dairiki, Editors:

Proceecings of the First Conference on Nuclear Structure Data Evaluation; LBL-14070, APS Division of Nuclear Physics Fall Meeting, Asilomar Conf. Grounds, Pacific Grove, CA, October 27-30, 1981, April 1982

Fowler, G.N., E.M. Friedlander, and R.M. Weiner

Coherence and Self-Induced Transparency in High Energy Hadronic Collisions, LBL-12279, May 1981, Phys. Lett.

Frankel, K.A., J.A. Bistirlich, R. Bossingham, H.R Bowman, KM. Crowe, C.J. IMartoff, D. Murphy, J.O. Rasmussen, J.P. Sullivan, W.A. Zajc, J.P. Miller, O. Hashimoto, M. Koike, J. Peter, W. Benenson, G.M. Crawley, E. Kashy, J.A Nolen, Jr., and J. Quebert

Pions Produced Near the Center-of-Mass Velocity in Heavy fon Collisions, LBL-12585, August 1981, Phys. Rev. C25, 1102 (1982). 
Frankel, K.A., J.A. Bistirlich, R. Bossingham, H.R. Bowman, K.M. Crowe, C.J. Martof, J. Miller, D.L. Murphy, J.O. Rasmussen, J.P. Sullivan, W.A Zajc, O. Hashimoto, M. Koike, J. Peter, W. Benenson, G.M. Crawley, E. Kashy, J.A. Nolen, Jr., and J. Quebert

Mid-Rapidity Pion Cross Sections: New Data Confront Theories, LBL-1264I, Presented at the Fifth High Energy Heavy Ion Studies, Lawrence Berkeley Laboratory, University of California, Berkeley, CA May 18-22, 1981. July 1981

Frankel, K. (See Stocker, H., LBL-12634;

Frankel, K.A. (See Gyulassy, M., LBL-13379)

Frankel, K.A. (See Radi, H.M.A., LBL-12591)

Frankel, K.A. (See Rasmussen, J.O., LBL-12623)

Frankel, K.A. (See Sullivan, J.P., LBL-11971)

Freedman, M. (See Warwick, A.I., LBL-13831)

Friedlander, E.M. and H.H. Heckman

Relativistic Heavy Ion Collisions: Experiment, LBL-13864, to be published as a chapter in Heavy fon Science, D. Allan Bromley, Ed., Plenum Press, 1982, April 1982

Friedlander, E.M. H.H. Heckman and Y.J. Karant Nuclear Collisions of Uranium Nuclei up to $\sim 1 \mathrm{GeV} /$ Nucleon, LBL-15444, December 1982, Phys. Rev. C

Friedlander, E.M., R.W. Gimpel, H.H. Heckman, Y.J. Karant, B. Judek, and E. Ganssauge Anomalous Reaction Mein Free Paths of Nuclear Projectile Fragments from Heavy Ion Collisions at 2 AGeV LBL10573 ; August 1982, Phys. Rev. C

Friedlander, E.M. (See Fowler, G.N., LBL-12279)

Friedlander, E.M. (See Heckman, H.H. LBL-14629)

Ganezer, K. (See Shor, A., LBL-14229)

Ganssauge, E. (See Friedlander, E.M., LBL-10573)

Geaga, J. (See Shor, A., LBL-14229)

Geaga, J.V. (See Harris, J.W., LBL-12334)

Ghiorso, A. (See Leino, M.E., LBL-12816)

Gimpel, R.W. (See Friedlander, E.M., LBL-10573)

Glendenning, N.K.

The Hyperon Composition of Neutron Stars, LBL-14062, April 1982, Phys. Lett. 114B, 392 (1982).

Glendenning, N.K., B. Benerjee, and M. Gyulassy

Normal and Pion Condensed States in Neutron Stat Matter in a Relativistic Field Theory Constrained by Bulk Nuclear Properties, LBL-12704, January 1982, Nucl. Phys. 
Glendenning, N.K., P. Hecking

On "Pion-Condensation in A Relativistic Field Theory and $\pi-\rho$ Interaction", LBL-14241, April 1982, Phys. Lett. 116B, 1 (1982).

Gosset, J. (See Wolf, K.L., LBL-14345)

Graebner, G. (See Stocker, H, LBL-12634)

Gregorich, K.E., K.J. Moody and G.T. Seaborg

The Half-Life and Absolute Gamma Ray Intensities of ${ }^{151} \mathrm{Gd}$, LBL-15046, September 1982, Radiochemica Acta

Greiner, D.E. (See Olson, D. LBL-12292)

Greiner, W. (See Csernai, L.P., LBL-13944)

Greiner, W. (See Stocker, H., LBL-12634)

Greiner, W. (See Stocker, H., LBL-12971)

Griffin, J.J. (See Myers, W.D., LBL-14138)

Groening, H., K Aleklett, K.J. Moody, P.L. McGaughey, W. Loveland and G.T. Seaborg

Isomer Ratio Measurements for the Reaction ${ }^{29} \mathrm{Si}\left({ }^{18} 0, \mathrm{p} 2 \mathrm{n}\right){ }^{44 \mathrm{~m}}{ }^{44} \mathrm{Sc}$, LBL-13483, December 1981, Nucl. Phys. A38s, 80 (1982).

Groening, H., K.J. Moody, and G.T. Seaborg

On the Use of Isomer Ratios in ${ }^{44} \mathrm{Sc}$ for Predicting Spin Populations in High Energy Heavy-Ion Nuclear Reactions, LBL-13484, September 1982, Nucl. Phys. A

Groening, H. (See Nguyen, HD., LBL-13485)

Guarino, G. (See Moretto, L.G. LBL-13850)

Guarino, G. (See Moretto, LG. LBL-14404)

Gutbrod, H.H., A. Baden, H. Löhner, M.R. Maier, AM. Poskanzer, T. Renner, H. Riedesel, H.G. Ritter, H. Spieler, A.I. Warwick, F. Weik, and H. Wieman

First Experiments with the Plastic Ball, LBL-14218, presented at the MARIA Heavy lon Workshop, Banff, Alberta, Canada, February 22-26, 1982, March 1982

Gutbrod, H.H, A.l. Warwick, and H. Wieman

Nuciear Disintegration in Relativistic Heavy Ion Collisions, LBL-14371, presented at the International Conference on Selected Aspects of Heavy Ion Reactions, Saclay, France, May3-7, 1982, April 1982, Nucl. Phys. A387, 177 (1982).

Gutbrod, H.H, A.l. Warwick, and H. Wieman

The Target Nucleus in Relativistic Nuclear Collisions, LBL-14059, presented at the MARIA Heavy Ion Workshop, Banff, Alberta, Canada, February 22-26, 1982, March 1982

Gutbrod, HH. (See Warwick, A.I., LBL-1383L)

Gutbrod, H.H. (See Wolf, K.L., LBL-]4345)

Gyulassy, $M$. 


\section{Appendix I}

\section{Publications}

Dynamics Versus Symmetrization in Hadron Interferometry, LBL-12389, August 1981, Phys. Rev. Lett. 48, 454 (1982).

\section{Gyulassy, M.}

Formation of a Quark-Gluon Plasma in Nuclear Collisions, LBL-14512, Proceedings of the Conference on Quark Matter Formation and Heavy Ion Collisions, Bielefeld, West Germany, May 8-16, 1982, World Scientific Publishing Co., pp. 81-93, Eds. M. Jacob and H. Satz., June 1982

\section{Gyulassy, M.}

Non-Linear Vacuum Polarization in Strong Fields, LBL-12972, Presented at the North Atlantic Treaty Organization Advanced Summer Institute Summer School on GED of Strong Fields, Lanhstein, West Germany, June 1426, 1981, July 1981

Gyulassy, M., K.A. Frankel, and H. Stocker

Do Nuclei Flow at High Energies, LBL-13379, September 1981, Phys. Rev. Lett. 110B, 185 (1982).

Gyulassy, M. (See Glendenning, N.K., LBL-12704)

Gyulassy, M. (See Nagamiya, S., LBL-14035)

Gyulassy, M. (See Stocker, H., LBL-12095)

Gyulassy, M. (See Stocker, H., LBL-12634)

Hamagaki, H. (See Nagamiya, S., LBL-14033)

Harris, J.W., A Sandoval, R. Stock, H. Stroebele, R.E. Renfordt, J.V. Geaga, H.G. Pugh, L.S. Schroeder, K.L. Wolf, and A Dacal

Production Near Threshold in Central Nucleus-Nucleus Collisions, LBL-12334, Phys. Rev. Lett. 47, 229 (1981).

Harris, J.W. (See Stock, R., LBL-14583)

Haryey, B.G.

Small Momentum Widths in Heavy-Ion Fragmentation at $20 \mathrm{MeV} / \mathrm{amu}$ and Below, LBL-12787, Phys. Rev. Lett. 47, 454 (1981).

Harvey, B.G. (See Cole, A.J., LBL-13011)

Harvey, B.G. (See Cole, A.J., LBL-14550)

Harvey, B.G. (See Murphy, M.J., LBL-14226)

Harvey, B.G. (See Rae, W.D., LBL-12568)

Harvey, B.G. (See Van Bibber, K., LBL-15105)

Hashimoto. O. (See Frankel, K.A., LBL-12585)

Heshimoto, O. (See Frankel, K.A., LBL-12641)

Hashimoto, O. (See Radi, H.M.A., LBL-125\%)

Hashimoto, O. (See Rasmussen, J.O., LBL-12623) 
Hashimoto, O. (See Sullivan. J.P., LBL-11971)

Haustein, P.E. (See Wouters, J.M., LBL-13990)

Hecking, P.

Pion-Cendensation Threshold in Nuclear Matter and Thermal $\Delta$-Isobars LBL-12254, February 1981, Phys. Rev. C24, 1691 (1981).

Hecking, $P$.

Abnormal Nuclear Matter and Pion Condensation, LBL-13043, August 1981, Nucl. Phys. A

Hecking, $\mathbf{P}$.

Optical Model Solutions for Pions in Nuclear Matter, LBL-12911, July 1981, Nucl. Phys.

Hecking, P. (See Glendenning, N.K., LBL-14241)

Hecking, P. (See Nagamiya, S., LBL-14033)

Heckmen, H.H.

Anomalons, LBL-14562, to be published as a book chapter in the Yearbook of Science and Technology, McGraw-Hill, Inc., New York, NY, June 1982

Heckmen, H.H., Y.J. Kar:sit, and E.M. Friedlander

Characteristics of the Ionization Tracks and Interactions of $\sim 100 \mathrm{~A} \mathrm{MeV}{ }^{238} \mathrm{U}$ Nuclei in Emulsion, LBL-14629, July 1982, Science Magazine 217, 1137 (1982).

Heckman, H.H. (See Friedlander, E.M., LBL-10573)

Heckman, H.H. (See Friedlander, E.M., LBL-13864)

Heckman, H.H. (See Friedlander, E.M., LBL-15444)

Heckman, H.H. (See Olson, D. LBL-12292)

Henderson, D.J. (See Warwick, A.I., LBL-13831)

Hendrie, D.I. (See Murphy, M.J., LBL-14226)

Hendrie, D.L. (See Van Bibber, K., LBL-15105)

Herskind, B. (See Ellegaard, C., LBL-11913)

Hicks, K.H., T.E. Ward, H. Bowman, J.G. Ingersoll, J.O.Rasmussen, J.P. Sullivan, M. Koike, J. Peter Interaction of 4.22 GeV and 7.54 GeV ${ }^{20}$ Ne with Cu, LBL-15405, Phys. Rev. C26, 2016 (1982).

Hinterberger, F. (See Slobodrian, R.J., LBL-13411)

Hoang, T.F., Bruce Cork, and H.J. Crawford

Space Structure of a Fireball and the Pion Radius, LBL-12362, July 1981, Zeitschrift für Physik C - Particles and Fields

Honkanen, J. (See Cable, M.D., LRL-14419) 
Hsu, C.C., D.J. Morrissey, L.W. Richardson, G.J. Wozniak, and L.G. Moretto

Linear Momentum Transfer from Gamma-Ray Multiplicities in Intermediate Energy Heavy-Ion Reactions, LBL12519. August 1981, Nucl. Phys. A

Hsu, C.C. (See Morrissey, D.J., LBL-12181)

Hsu, C.C. (See Morrissey, D.J., LBL 13055)

Hsu, C.C. (See Pacheco, A.J., LBL-14091)

Hsu, C.C. (See Sobotka, LG., LBL-11840)

Hsu, C.C. (See Wozniak, G.J., LBL-1353)

Hsu, C.C. (See Wozniak, G.J., LBL-13637)

Hî̉bel, H. (See Wozniak, G.J., LBL-9461)

Huizenga, J.R. (See Myers, W.D., LBL-14138)

Hyde, E.K. (See Bowman, D.J., LBL-8895)

Igo, G. (See Shor, A., LBL-14229)

Ingersoll, J.G. (See Hicks, K.H., LBL-15405)

Jourdain, J.-C. (See Wolf, K.L., LBL-14345)

Judek, B. (See Friedlander, E.M, LBL-10573)

Kadota, S. (See Nagamiya, S., LBL-14033)

Karant, Yasha J.

Observation of Anomalous Reaction Mean Free Paths of Nuciear Projectile Fragments in Research Emulsion from 2 A GeV Heavy lon Collisions, LBL-13103, March 1981, Ph.D thesis

Karant, Y.J. (See Friedlander, E.M, LBL-10573)

Karant, Y.J. (See Friedlander, E.M, LBL-15444)

Karant, Y.J. (See Heckman, H.H., LBL-14629)

Kashy, E. (See Frankel, K.A., LBL-12585)

Kashy, E. (See Frankel, K.A., LBL-12641)

Kashy, E. (See Sullivan, J.P., LBL-11971)

Kaufman, S.B. (See Warwick, A.I., LBL-13831)

Kelly, W.H. (See Firestone, R.B., LBL-12424)

King, C.H. (See Wolf, K.L., LBL-14345) 


\section{Appendix I}

\section{Publications}

King, G. (See Wolf, K.L., LBL-14345)

King, N.S.P. (See Sagle, A.L., LBL-13232)

Kluge, H. (See Deleplanque, M.A., LBL-14056)

Kluge, H. (See Ellegaard, C., LBL-11913)

Knoll, J., J. Randrup

Proton-Proton Correlations in High Energy Nuclear Collisions, LBL-11418,Phys. Lett. 103B, 264 (1981).

Knoll, J. (See Csernai, L.P., LBL-13944)

Knoll, J. (Sec Nagamiya, S., LBL-14033)

Koike, M. (See Frankel, K.A., LBL-12585)

Koike, M. (See Frankel, K.A., LBL-12641)

Koike, M. (See Hicks, K.H., LBL-15405)

Koike, M. (See Sullivan, J.P., LBL-11971)

Korner, H.J. (See Deleplanque, M.A., LBL-14056)

Kraus, R.H., Jr. (See Loveland, W., LBL-13201)

Le, H.D. (See Nguyen, H.D., LBL-13485)

Lederer, M.C.

E1 Transition Probabilities from $K^{x}=0$ and $K^{x}=1^{-}$States of ${ }^{238} \mathrm{Pu}$, LBL-12075, January 1981, Phys. Rev. C24, 1175 (1982).

Legrain, R. (See Cole, A.J., LBL-13011)

Legrain, R. (See Cole, A.J., LBL-14550)

Legrain, R. (See Murphy, M.J., LBL-14226)

Legrain, R. (See Rae, W.D., LBL-12568)

Leino, M.E., S. Yashita, and A. Ghiorso

Alpha Decay of Neutron Deficient Polonjum and Bismuth Isotopes, LBL-12816, May 1981, Phys. Rev. C24, 2370 (1981).

Lemaire, M-C. (See Nagamiya, S., LBL-12123)

Lemaire, M.-C. (See Schnetzer, S., LBL-14523)

Lindenberger, K.H. (See Burde, J., LBL-13610)

Lindenberger, H. (See Draper, J.E., LBL-14274) 


\section{Appendix I}

\section{Publications}

Lindstrom, P.J. (See Olson, D. LBL-12292)

Lindstrom, P. (See Sher, A, LBL-14229)

Liu, Y.-f., Cheng Luo, Hans R. Von Guten, and Glenn T. Seaborg

A Procedure for a Fast Separation of Berkelium and Cerium, LBL-12909, April 1981, Inoig. Nucl. Chem. Lett. 17, 257 (1981).

Lōhner, H. (See Gutbrod, H.H., LBL-14218)

Lombard, R. (See Nagamiya, S., LBL-14033)

Lombard, R. (See Schnetzer, S., LBL-14523)

Loveland, W., Cheng Luo, P.L. McGaughey, D.J. Morrissey and G.T. Seaborg

Target Fragment Energies and Momenta in the Reaction of $4.8 \mathrm{GeV}{ }^{12} \mathrm{C}$ and $5.0 \mathrm{GeV}{ }^{20 \mathrm{Ne}}$ with ${ }^{238} \mathrm{U}, \mathrm{LBL}-11653$, October 1980, Phys. Rev. C24, 464 (1981).

Loveland, W., K. Aleklett, P.L. McGaughey, K.J. Moody, R.M. McFarland, and G.T. Seaborg

Target Fragmentation at Intermediate Energies, LBL-14477, May 1982, Phys. Lett.

Loveland, W., P.L. McGaughey, K Alekett, K.J. Moody, R.H. Kraus, Jr., R.M. McFarland, and G.T. Seaborg

Target Fragmentation from 10-2100 MeV. LBL-13201, August 1981, Phys. Rev. Lett.

Loveland, W. (See Groẹning, H., LBL-13483)

Loveland, W. (See Morita, Y., LBL-13425 Rev.)

Loveland, W.D. (See Seaborg, G.T., LBL-13994)

Macchiavelli, A.O. (See Deleplanque, MA., LBL-14056)

Mahoney, J. (See Rae, W.D., LBL-12568)

Mahoney, J. (See Van Bibber, K., LBL-15105)

Maier, M. (See Stock, R., LBL-14583)

Maier, M.R. (See Gutbrod, H.H., LBL-14218)

Maier, MR. (See Watwick, A.I., LBL-13831)

Manko, V.I. and S. Nagamiya

Kinematical Analysis of the Experimental Data on Nucleus Nucleus Collisions at $800 \mathrm{MeV} / \mathrm{nucleon}$, LBL-14032,

February 1982, Nucl. Phys. A384, 475 (1982).

Maples, C., D. Weaver, W. Rathbun and J. Meng

The Utilization of Parallel Processors in a Data Analysis Environment, LBL-12505, May 1981, IEEE Transactions on Nuclear Science

Maples, C., W. Rathbun, D. Weaver, and J. Meng

The Design of Midas - A Modular Interactive Data Analysis System, LBL-12504, May 1981, IEEE Transactions on Nuclear Science 
Maples, C. (See Rathbun, W., LBL-12498)

Maples, C. (See Weaver, D., LBL-12503)

Martoff, C.J. (See, Frankel, K.A., LBL-12585)

Martoff, C.J. (See Frankel, K.A., LBL-12641)

Martoff, C.J. (See Sullivan, J.P., LBL-1 1971)

Maruhn, J.A. (See Stocker, H., LBL-12634)

Mathews, G.J., J.B. Moulton, G.J. Wozniak, B. Cauvin, R.P. Schmitt, J.S. Sventek, and L.G. Moretto A study of ${ }^{20} \mathrm{Ne}$ Induced Reactions with ${ }^{\text {mit }} \mathrm{Cu}$ and ${ }^{197} \mathrm{Au}$ at 8.6 and $12.6 \mathrm{MeV} /$ nucleon, LBL-13226, August 1981, Phys. Rev. C

Mathews, G.J. (See Wozniak, G.J., LBL-9461)

McDonald, R.J., A.J. Pacheco, G.J. Wozniak, H.H. Bolotin, L.G.Moretto, C. Schuck, S. Shih, R.M. Diamond, and F.S. Stephens

Evidence for Spin Fluctuations in the Deep Inelastic Reaction ${ }^{163} \mathrm{Ho}+{ }^{165} \mathrm{Ho}$ at $8.5 \mathrm{MeV} / \mathrm{amu}$, LBL-11608, May 1981, Nucl. Phys. A373, 54 (1982).

McDonald, R.J. (See Draper, J.E., LBL-14274)

McDonald, R.J. (See Morrissey, D.J., LBL-12181)

McDonald, R.J. (See Morrissey, D.J., LBL-13055)

McDonald, R.J. (See Pacheco, A.J., LBL-14091)

McDonald, R.J. (See Sobotka, L.G., LBL-13319)

McFarland, Rose Marie

Recoil Range Studies of Heavy Products of Multinucleon Transfer from ${ }^{18} \mathrm{O}$ to ${ }^{245} \mathrm{Cm}$ and ${ }^{249} \mathrm{C}$, LBL-15027, September 1982, Ph.D. thesis

McFarland, R.M. (See Loveland, W., LBL-13201)

McFarland, R.M. (See Loveland, W., LBL-14477)

McGaughey, P.L. (See Groening, H., LBL-13483)

McGaughey, P.L. (See Loveland, W., LBL-11658)

McGaughey, P.I. (See Loveland, W., LBL-13201)

McGaughey, P.L. (See Loveland, W., LBL-14477)

McGaughey, P.I. (See Morita, Y., LBL-13425 Rev.)

McHarris, Wm.C. and J.O. Rasmussen

Anomalons as Pineuts Bound to Nuclear Fragments: A Possible Explanation, LBL-14075, March 1982, Phys. Lett. 
McHarris, W.C. (See Firestone, R.B., LBL-12424)

McNaughton, M.W. (See Sagle, A.L., LBL-13232)

Meng, J. (See Maples, C., LBL-12504)

Meng, J. (See Maples, C., LBL-12505)

Meng, J. (See Rathbun, W., LBL-12498)

Meyer, W.G. (See Wolf, K.L, LBL-14345)

Miake, Y. (See Nagamiya, S., LBL-14033)

Miller, J. (See Frankel, K.A., LBL-12641)

Miller, J. (See Sullivan, J.P., LBL11971)

Miller, J.P. (See Frankel, K.A, LBL-12585)

Moeller, E., L. Anderson, W. Brüchner, S. Nagamiya, S. Nissen-Meyer, L. Schroeder, G. Shapiro, and H. Steiner Inclusive Particle Production at Forward Angles From Collisions of Light Relativistic Nuclei, Part It: Negative Pions, LBL-14329, May 1982, Phys. Rev. C

Moeller, E. (See Andersm, L, LBL-14328)

Moeller, E. (See Anderson, L, LBL-14330)

Moeller, E. (See Nagamiya, S., LBL-12123)

Moeller, E. (See Nagamiya, S., LBL-14033)

Moeller, E. (See Schnetzer, S., LBL-14523)

Molitoris, J.D., J.M. Nitschke

Evaluation of Thin Metal Foils as Targets and Windows for Heavy-Ion Experiments, LBL-9725, January 1981, Nuclear Instruments and Methods 186, 659 (1981).

Moody, K.J. (See Groening, H., LBL-13483)

Moody, K.J. (See Groening, H., LBL-13484)

Moody, KJ. (See Gregorich, K.E., LBL-15046)

Moody, K.J. (See Loveland, W., LBL-13201)

Moody, K.J. (See Loveland, W., LBL-14477)

Moody, KJ. (See Nguyen, H.D., LBL-13485)

Moretto, L.G.

Particle Angular Distributions and Gamms-Ray Multiplicities as Experimental Probes for Angular Momentum Fluctuations, LBL-14101, presented at the 20th International Meeting on Nuclear Physics, Bormio, Italy, January 25-29, 


\section{Appendix I}

1982, March 1982

Moretto, L.G.

Statistical Fluctuations in Heavy Ion Collisions, LBL-14137, presented at the International Conference on Fission and Related Phenomena, Baden Honnef, W. Germany, October26-30, 1981, February 1982

Moretto, L.G.

Statistics at Work in Heavy Ion Reactions, LBL-14563, presented at the Enrico Fermi International School of Physics on Nuclear Structure and Heavy Ion Dynamics, Varenna, Italy, July 27-August 6, 1982, July 1982

Moretto, L.G.

The Role of Rotational Degrees of Freedom in Heavy Ion Collisions, LBL-14283, presented at the International Conference on Selected Aspects of Heavy Ion Reactions, Saclay, France, May3-7,1982, April 1982, Nucl. Phys. A387, 331 (1982).

Moretto, L.G. and G. Guarino

Fission Probability for High Viscosity at the Saddle Point, LBL-14404, August 1982, Zeitschrift für Physik A

Moretto, L.G. and G. Guarino

The Soggy Saddle Theory of Fission, LBL-13880, presented at the Gross Properties of Nuclei and Nuclear Excitation Conference, Hirschegg, Austria, January 18-22,1982, January 1982

Moretto, LG. and L.G. Sobotka

The Influence of Fluctuation on the Correlation Between Exit-Channel Kinetic Energy and Entrance-Channel Angular Momentum for Heavy Ion Collisions, LBL-13007, July 1981, Z. Phys. A - Atoms and Nuclei 303, 229 (1981).

Moretto, LG. and R.P. Schmitt

Deep Inelastic Reactions: A Probe of the Collective Properties of Nuclear Matter, LBL-12859, November 1981, Reports on Progress in Physics 44, 53381).

Moretto, LG. (See Draper, J.E., LBL-14274)

Moretto, L.G. (See Hsu, C.C., LBL-12519)

Moretto, LG. (See Mathews, G.J., LBL-13226)

Moretto, LG. (See McDonald, R.J., LBL-11608)

Moretto, LG. (See Morrissey, D.J., LBL-12181)

Moretto, LG. (See Morrissey, D.J., LBL-13055)

Moretto, LG. (See Pacheco, A.J. LbL-13703)

Moretto, L.G. (See Pacheco, A.J., LBL-14091)

Moretto, LG. (See Sobotka, LG., LBL-11840)

Moretto, LG. (See Sobotka, LG., LBL-13319)

Moretto, L.G. (See Wozniak, G.J., LBL-1353)

Moretto, L.G. (See Wozniak, G.J., LBL-13637) 
Maretto, L.G. (See Wozniak. G.J., LBL-9461)

Morita, Y., W. Loveland, P.L. McGaughey, and G.T. Seaborg

Target Fragment Angular Distributions from the Interaction of $3.0 \mathrm{GeV}$ and $12.0 \mathrm{GeV}{ }^{12} \mathrm{C}$ with ${ }^{197} \mathrm{Au}$ and ${ }^{238} \mathrm{U}$, LPL-13425 Rev., November 1981, Phys. Rev. C26, 511 (1982).

Morrissey, D.J., G.J. Wozniak, L.G. Sobotka, A.J. Pacheco, C.C. Hsu, R.J. McDonald and L.G. Moretto Angular Momentum, Statistical Equillibrium and Sequential Fission in Very Asymmetric Systems, LBL-12181, March 1981, Z. Phys. A - Atoms and Nuclei 305, 131 (1982).

Morrissey, D.J., G.J. Wozniak, LG. Sobotka, A.J. Pacheco, R.J., McDonald, C.C. Hsu, and LG. Moretto Intrinsic Fragment Spins Generated in the Reactions of ${ }^{20} \mathrm{Ne}$ with ${ }^{197} \mathrm{Au}$ and ${ }^{238} \mathrm{U}$ at $12.6 \mathrm{MeV} / \mathrm{Nucleon}$, LBL-13055, March 1982, Nucl. Phys. A389, 120 (1982).

Morrissey, D.J. (See Hsu, C.C., LBL-12519)

Morrissey, D.J. (See Loveland, W., LBL-11658)

Morrissey, D.J. (See Pacheco, A.J., LBL-14091)

Morrissey, D.J. (See Sobotka, LG., LBL-11840)

Morrissey, D.J. (See Sobotka, LG., LBL-13319)

Morrissey, D.J. (See Wozniak, G.J., LBL-1353)

Morrissey, D.J. (See Wozniak, G.J., LBL-13637)

Moszkowski, S.A. (See Boguta, J. LBL-14685)

Moulton, J.B. (See Mathews, G.J., LBL-13226)

Müller, K.-H.

A Relativistic Quantum Field Theoretical Model for Nuclear Slab Collisions Dynamics, LBL-12667, May 1981, Nucl. Phys.

M(üler, K.-H.

Nuclear Slab Collision in a Relativistic Quantum Field Theory, LBL-12893, Presented at the Fifth High Energy Heavy Ion Study, Lawrence Berkeley Laboratory, University of California, Berkeley, CA May 18-22, 1981, June 1981

\section{Müller, K.-H.}

The Energy Dependent Single Nucleon Potential in a Relativistic Field Theory of Nuclear Matter, LBL-12684, Junc 1981, Phys. Lett. 107B, 253 (1981).

Müller, K.-H. and S. Bohrmann

Interaeting Streaming Nuclear Matter Systems in a Relativistic Quantum Field Theory, LBL-12663, May 1981, Phys. Lett. B

\section{Müller, Karl-Heinz}

Subthreshold K-Production by Coherently Produced II-Mesons in Heavy Ion Collisions, LBL-14715, August 1982, Nucl. Phys. A 
Mulera, T. (See Shor, A., LBL-14229)

Murphy, D. (See Frankel, K.A., LBL-12585)

Murphy, D.J. (See Sullivan, J.P., LBL-11971)

Murphy, D.L. (See Frankel, K.A., LBL-12641)

Murphy, M.J., B.G. Harvey, D.L. Hendrie, W.W. Pang, K. Van Bibber, and R. Legrain

Trensfer and Fregmentation Reactions in ${ }^{16} 0+\operatorname{CsI}$ at $16.4 \mathrm{MeV} / \mathrm{n}, \mathrm{LBL}-14226$, March 1982, Phys. Rey. Lett. 120B, 75 (1983).

Murphy, M.J., T.J.M. Symons, G.D. Westfall, and H.J. Crawford

Beta-Decay Half-Lives of Isotopes Produced in Projectile Fragmentation, LBL-14272, March 1982, Phys. Rev. Lett. 49, 455 (1982).

Murphy, M.J. (See Cole, AnJ., LBL-13011)

Murphy, M.J. (See Cole, A.J., LBL-14550)

Murphy, MJ. (See Rae, W.D., LBL-12568)

Murphy, MJ. (See Van Bibber, K., LBL-15105)

Myers, W.D.

Droplet Model Predictions of Charge Moments, LBL-14310, presented at the Conference on Lasers in Nuclear Physics, Oak Ridge, TN, April 21-23, 1982, April 1982

Myers, W.D., J.J. Griffin, J.R. Huizenga, J.R Nix, F. Plasil and Y.E. Viola, Organizers:

Workshop on Nuclear Dynamics 22-26 February 1982, Granlibakken, Tahoe City, California April 1982, LBL-14138, April 1982

Myers, W.D., and W.J. Swiatecki

The Macroscopic Approach to Nuclear Masses and Deformations, LBL-13929, March 1982, Ann. Rev. Nucl. Part. Sci. 32, 309 (1982).

Nagamiya, S.

1. Mechanism of Light Particle Emission II. Current Puzzles and Future Possibilities, LBL-14031, presented at the Relativistic Heavy-Ion Winter School, Banf, Alberta, Canada, February 22-26, 1982, February 1982

Nagamiya, $S$.

Particle Correlations In Proton-Nucleus and Nucleus-Nucleus Collisions, LBL-14034, February 1982, Soviet Journal of Particles and Nuclei

Nagamiya, $\mathrm{S}$.

Possibilities for Experiments with Deuteron and Light-Ion Bears at $4<\mathrm{E}_{\text {Beam }} / \mathrm{A}<10 \mathrm{GeV}$, LBL-14030, presented at the Los Alamos Meson Physics Fecility II Workshop, Los Alamos, NM, February 1-4, 1982, February 1982 
Nagamiya, $S$.

What can we learn from Inclusive Spectra, LBL-12950. Presented at the Fifth High Energy Heavy Ion Study, Lawrence Berkeley Laboratory, University of California, Berkeley, CA May 18-22, 1981., May 1981

Nagamiya, S. and M. Gyulassy

High-Energy Nuclear Collisions, LBL-14035 To be Published as a chapter in Advances in Nuclear Physics Plenum Pub. Corp. New York, NY, February 1982

Nagamiya, S., H. Hamagaki, P. Hecking, S. Kadota, R. Lombard, Y. Miake, E. Moeller, S. Schnetzer, H. Steiner, 1. Tanihata, S. Bohrmann, and J. Knoll

Production of Negative Pions With $183 \mathrm{MeV} /$ nucleon Ne Beams, LBL-14033, February 1982, Phys. Rev. Lett.

Nagamiya, S., M.-C. Lemaire, E. Moeller, S. Schnetzer, G. Shapiro, H. Steiner, and I. Tanihata

Production of Pions and Light Fragments at Large Angles in High Energy Nuclear Collisions, LBL-12123, March 1981, Phys. Rev. C24, 971 (1981).

Nagamiya, S. (See Anderson, L., LBL-14328)

Nagamiya, S. (See Anderson, L., :-BL-14330)

Nagamiya, S. (See Csernaj, L.P., LBL-13944)

Nagamiya, S. (See Manko, V.I. LBL-14032)

Nagamiya, S. (See Moeller, E., LBL-14329)

Naganiya, S. (See Schnetzer, S., LBL-14523)

Nagamiya, S. (See Tanihata, I., LBL-11488)

Nagarajan, M.A. (See Shyam, R., LBL-14740)

Newton, J.O. (See Draper, J.E., LBL-14274)

Nguyen Van Sen (See Wolf, K.L., LBL-14345)

Nguyen, H.D., H.N. Trinh, H.D. Le, K.J. Moody, H. Groening, and G.T. Seaborg

Excitation Functions and Isomer Ratio Measurements for the Reaction ${ }^{11} \mathrm{~K}\left({ }^{6} \mathrm{Li}, \mathrm{p} 2 \mathrm{n}\right){ }^{4} \mathrm{Sc}^{\mathrm{m}},{ }^{44} \mathrm{Sc}^{4}, \mathrm{LBL}-13485$, October, 1981

Nissen-Meyer, S. (See Anderson, L., LBL-14328)

Nissen-Meyer, S. (See Anderson, L., LBL-14330)

Nissen-Meyer, S. (See Moeller, E., LBL-14329)

Nitschke, J.M.

A Digital Calorimeter, LBL-13900, January 1982, Nuclear Instruments and Methods

Nitschke, J.M.

OASIS: The SuperHILAC on-line Isotope Separator, LBL-14041, February 1982, Nuclear Instruments and Methods

Nitschke, J.M. (See Molitoris, J.D., LBL-9725) 
Nix, J.R. (See Myers, W.D., LBL-14138)

Six, J.R. (See Stocker, H., LBL-12634)

Nolen, J.A, Jr. (See Sullivan, J.P., LBL-11971)

Nolen, J.A, Jr. (See Frankel, K.A, LBL-12585)

Nolen, J.A., Jr. (See Frankel, K.A., LBL-12641)

Ogloblin, A.A. (See Stocker, H, LBL-12971)

Oliveria, L.F. (See Radi, H.M.A., LBL-13901)

Olson, D. L., B. L. Berman, D. E. Greiner, H. H. Heckman, P. J. Lindstrom, G. D. Westfall, and H. J. Crawford Electromagnetic Dissociation of Relativistic "'O Nuclei LBL-12292, February 1981, Phys. Rev. C24, 1529 (1981).

Ortiz, M.E. (See Stock, R., LBL-14583)

Pacherô, A.J. and L.G. Moretto

The Eifect of Statistical Fluctuations on the Measurement of the Total Energy and the Multiplicity of $\gamma$ Rays Following Deep-Inelastic Reactions, LBL-13703, January 1982, Z. Phys. A - Atoms and Nuclei 306, 259 (1982).

Pacheco, A.J., G.J. Wozniak, R.J. McDonald, R.M. Diamond, C.C.Hsu, L.G. Moretto, D.J. Morrissey, L.G. Sobotka, and F.S.Stephens

Angular-Momentum Transfer and Alignment in Deep-Inelastic Reactions for Nearly Symmetric Heavy-Ion Systems, LBL-14091, July 1982, Nucl. Phys.

Pacheco, A.J. (See McDunald, R.J., LBL-11608)

Pacheco, A.J. (See Morrissey, D.J., LBL-12181)

Pacheco, A.J. (See Morrissey, D.J., LBL-13055)

Pacheco, AJ. (See Sobotka, L.G., LBL-13319)

Pang, W.W. (See Murphy, M.J., LBL-14226)

Pang, W.W. (See Van Bibber, K., LBL-15105)

Pardo, R.C. (See Firestone, R.B., LBL-12424)

Parry, R.F. (See Cable, M.D., LBL-14419)

Parry, R.F. (See Wouters, J.M., LBL-13990)

Perez-Mendez, V. (See Shor, A., LbL-14229)

Peter, J. (See Frankel, K.A, LBL-12585)

Peter, J. (See Frankel, K.A., LBL-12641)

Peter, J. (See Hicks, K.H., LBL-15405) 
Peter, J. (See Sullivan, J.P., LBL-11971)

Peter, J. (See Warwick, A.I., LBL-13831)

Plasil, F. (See Myers, W.D., LBL-14138)

Poskanzer, A.M. (See Gutbrod, H.H, LBL-14218)

Poskanzer, A.M. (See Wolf, K.L, LBL-14345)

Pugh, HG.

Accelerator Prospects for High Energy Heavy Ion Collisions, LBL-14561, Proveedings of the Conference on Quark Matter Formation and Heavy Ion Colljsions, Bielefeld, West Germany, May 8-16, 1982, World Scientific Publishing Co., pp.319-328, Eds. M. Jacob and H. Satz., June 1982

Pugh, H.G.

Overview of Very High Energy Heavy lons, LBL-12835, Presented at the Inter-Regional Program for Very High Energy Heavy Ions, Niels Bohr Institute, Copenhagen, Denmark, June 10, 1981, June 1981

\section{Pugh, H.G.}

Recent Results from The Bevalac, LBL-14513, Proceedings of the Conference on Quark Matter Formation and Heavy Ion Collisions, Bielefeld, West Germany, May 8-16, 1982, World Scientific Publishing Co., pp. 185-212, Eds. M. Jacob and H. Satz., June 1982

\section{Pugh, H.G.}

Relativistic Heavy lon Experiments, LBL-13029, Invited paper presented at the XII International Symposium on Multiparticle Dynamics, Notre Dame, South Bend, IN, June 21-26, 1981, August 1981

Pugh, H.G. (See Harris, J.W., LBL-12334)

Pugh, H.G. (See Stock, R., LBL-14583)

Quebert, J. (See Frankel, K.A., LBL-12641)

Quebert, J. (See Frankel, K.A., LBL-12585)

Rad, F.N. (See Roy, R., LBL-14343)

Radi, H.M.A., J.O. Rasmussen, J.P. Sullivan, K.A. Frankel, and O. Hashimoto

Formulation of the Coulomb Effects of Spectator Fragments on Pions from Heavy Ion Collisions, LBL-12591, April 1981, Phys. Rev. C25, 1518 (1982).

Radi, H.M.A., John O. Rasmussen, Raul Donangelo, Luiz F. Canto, and Luiz F. Oliveria Monte Carlo Studies of Alpha-Accompanied Fission, LBL-13901, June 1982, Phys. Rev. CC26, 2049 (1982).

Radi, H.M.A. (See Rasmussen, J.O., LBL-12623)

Rae, W.D., A.J. Cole, A. Dacal, R. Legrain, B.G. Harvey, J. Mahoney, M.J. Murphy, R.G. Stokstad, and I. Tserruya

Coherent and Incoherent Processes in Projectile Breakup, LBL-12568, April 1981, Phys. Lett. 105B, 417 (1981).

Rae, W.D.M. (See Cole, A.J., LBL-13011) 
Rae, W.D.M. (See Cole, A.J., LBL-14550)

Randrup, J.

Dynamics of Damped Nuclear Reactions, LBL-14786, invited talk presented at the Nordic Meeting on Nuclear Physics, Fugls $\phi$, Denmark, August 16-20, 1982, September 1982

Randrup, J.

Fragment Spin Correlations in Damped Nuclear Reactions, LBL-13403, Phys. Lett. 110B, 25 (1982).

Kandrup, J.

Transport of Angular Momentum in Damped Nuclear Reactions, LBL-12676, July 1981, Nucl. Phys. A383, 468 (1982).

Randrup, J. and G. Fai

Calculation of Complete Multi-Fragment Events in A Statistical Model for Nuclear Disassembly, LBL-14170 " 1982, Phys. Lett. 115B, 281 (1982).

Randrup, J. (See Fả, G. LBL-13357)

Randrup, J. (See Knoll, J., LBL-11418)

Rasmussen, J.0.

Funny Hills in Pion Spectra from Heavy-ion Collisions, LBL-14174, presented at the Relativistic Heavy Ion Winter School, Banff, Alberta, Canada, February 22-26, 1982, March 1982

\section{Rasmussen, J.O.}

Heavy Ion Pion Production: Spectral Irregularities, LBL-14956, presented at the International Conferefice on Nucleus-Nucleus Collisions, Michigan State L'niversity, East Lansing, MI, September 26 - October 1, 1982, September 1982

\section{Rasmussen, J.O.}

Pions From and About Heavy Ions, LBL-14852, invited paper to appear in the Proceedings of the Summer School on Nuclear Dynamics, Pearson College, Victoria, BC Canada, August 23-September 3, 1982, September 1982

Rasmussen, J.O., Hafez M.A. Radi, J.P. Sullivan, K.A. Frankel, and O. Hashimoto

Theory of Coulomb Distortion of Pion Spectra by Heavy-Ion Spectator Fragments, LBL-12623, Presented at the Fifth High Energy Heavy Ion Study, Lawrence Berkeley Laboratory, University of California, Berkeley, CA May 18-22, 1981. July 1981

Rasmussen, J.O. (See Frankel, K.A., LBL-12585)

Rasmussen, J.O. (See Frankel, K.A., LBL-12641)

Rasmussen, J.O. (See Hicks, K.H., LBL-15405)

Rasmussen, J.O. (See McHarris, Wm.C., LBL-14075)

Rasmussen, J.O. (See Radi, H.M.A., LBL-12591)

Rasmussen, J.O. (See Radi, H.M.A., LBL-13901)

Rasmussen, J.O. (See Sullivan, J.P., LBL-11971) 


\section{Publications}

Rathbun, W., C. Maples, J. Meng, and D. Weaver

A Fast, Time-Sliced, Multiple Data Bus Structure for Overlapping I/0 and CPU Operations, LBL-12498, May 1981, IEEE Transactions on Nuclear Science

Rathbun, W. (See Maples, C., LBL-12504)

Rathbun, W. (Sec Maples, C., LBL-12505)

Rathbun, W. (See Weaver, D., LBL-12503)

Regimbart, R. (See Wozniak, G.J., LBL-9461)

Renfordt, R.E. (See Harris, J.W., LBL-12334)

Renfordt, R.E. (See Stock, R., LBL-14583)

Renner, T. (See Gutbrod, H.H., LBL-14218)

Richardson, L.W. (See Hsu, C.C., LBL-12519)

Richardson, L.W. (See Wozniak, G.J., LBL-1353)

Richardson, L.W. (See Wozniak, G.J., LBL-13637)

Riedel, C. (See Stocker, H, LBL-12634)

Riedesel, H. (See Gutbrod, H.H., LBL-14218)

Rioux, C. (See Slobodrian, R.J., LBL-13411)

Ritter, H.G.

Plastic Ball and Streamer Chamber Experiments at the Bevalac, LBL-14541, presented at the Varenna Conference on the Nuclear Reaction Mechanism, Varenna, Italy, June 1419, 1982, July 1982

Ritter, H.G. (See Gutbrod, H.H., LBL-14218)

Ritter, H.G. (See Warwick, A.I., LBL-13831)

Romero, J.L. (See Sagle, AL., LBL-13232)

Roy, R., F. Seiler, H.E. Conzett, and F.N. Rad Cross

Section and Vector Analyzing Power iT $\mathrm{T}_{11}$ of the Processes ${ }^{3} \mathrm{He}(\overrightarrow{\mathrm{d}}, \mathrm{d}){ }^{3} \mathrm{He}(\overrightarrow{\mathrm{d}}, \mathrm{p}){ }^{4} \mathrm{He}$ Between 15 and $40 \mathrm{MeV}$, LBL14343, December 1981, Phys. Rev. C, 24, 2421 (1981).

Roy, R. (See Slobodrian, R.J., LBL-13411)

\section{Ruck, H.M.}

Classical Field Theory with Z(3) Symmetry, LBL-12852, Presented at the Center for Nonlinear Studies Conference "Nonlinear Problems: Present and Future", Los Alamos National Laboratory, Los Alamos, NM, March 2-6, 1981., Nonlinear Problems: Present and Future, A.R. Bishop, D.K Campbell, B.Nicolaenko (eds.) North-Holland Publishing Co. pp 237-244 (1982), June 1981 


\section{Ruck, H.M.}

Inseparability of Fermions in a Model of Cyclic Symmetric Field Theory, LBL-13378, October 1981, Phys. Rev. D

\section{Ruck, HM.}

Polynomial Chromodynamic, LBL-12872, Presented at the Fifth High Energy Heavy lon Study, Lawrence Berkeley Laboratory, University of California, Berkeley, CA May 18-22, 1981, June 1981

Sagle, A.L., F.P. Brady, J.L. Romero, B.E. Bonner, N.S.P. King, M.W. McNaughton, and H.E. Conzett Time Reversal and Charge Symmetry Studies in Single Nucleon Transfer Reactions in the A $=5$ System, LBL-13232, August 1981

Sagle, A. (See Shor, A., LBL-14229)

Sendoval, A. (Sec Harris, J.W., LBL-12334)

Sundoval, A. (See Stock, R., LBL-14583)

Sandoval, A. (See Wolf, K.L., LBL-14345)

Sandoval, A. (See van Bibber, K. LBL-12883)

Schmitt, R.P. (See Mathews, G.J., LBL-13226)

Schmitt, R.P. (See Moretto, L.G. LBL-12859)

Schmitt, R.P. (See Wozniak, G.J., LBL-9461)

Schnetzer, S., M.-C. Lemaire, R. Lombard, E. Moeller, S. Nagamiya, G. Shapiro, H. Steiner, and I. Tanihata Production of $\mathrm{K}^{+}$Mesons in $2.1 \mathrm{GeV} /$ nucleon Nuclear Collisions, LBL-14523, April 1982, Phys. Rev. Lett. 49, 989 (1982).

\section{Schnetzer, Stephen $\mathbf{R}$.}

Meson Production in Relativistic Heavy Ion Collısions, LBL-13727, August 1981, Ph.D thesis

Schnetzer, S. (See Nagamiya, S., LBL-12123)

Schnetzer, S. (See Nagamiya, S., LBL-14033)

Schnetzer, S. (See Tanihata, I., LBL-11488)

Schroeder, L. (See Anderson, L, LBL-14328)

Schroeder, L. (See Anderson, L, LBL-14330)

Schroeder, L. (See Moeller, E., LBL-14329)

Schroeder, L.S. (See Harris, J.W., LBL-12334)

Schroeder, L.S. (See Stock, R., LBL-14583)

Schuck, C. (See Burde, J., LBL-13610)

Schuck, C. (See Ellegaard, C., LBL-11913) 


\section{Appendix I}

\section{Publications}

Schuck, C. (See McDonald, R.J., LBL-11608)

Schurmann, B. (See Stocker, H., LBL 12634 )

Seaborg, G.T.

Forty Years of Plutonium Chemistry: The Beginnings, LBL-15098, presented at the 184th National Meeting of the American Chemical Society, Kansas City, MO, September 12-17, 1982, October 1982

\section{Seaborg, G.T.}

The Plutonium Story, LBL-13492, Presented at the Lawrence Berkeley Laboratory, Lawrence Livermore National Laboratory Conference, Actinides 1981, Asilomar Conference Center Pacific Groue, CA September 10-15 1981

\section{Seaborg, G.T.}

The Research Style of Gilbert N. Lewis: Acids and Bases, LBL-14785, presented at the 183rd National Meeting of the American Chemical Society, Las Vegas, NV March 30, 1982, October 1982

Seaborg, G.T. and W.D. Loveland

Transuranium Nuclei, LBL-13994, submitted as a chapter in Heavy Ion Nuclear Science (Vols.I and II - Nuclear Science), D. Allan Bromley, Ed., Plenum Publishing Corp, New York NY, February 1982

Seaborg, G.T. (See frcening, H., LBL-13483)

Seaborg, G.T. (See Groening, H, LBL-13484)

Seaborg, G. I. (See Gregorich, K.E., LBL-15046)

Seaborg, G.T. (See Liu, Y.-f., LBL-12909)

Seaborg, G.T. (See Loveland, W., LBL-11658)

Seaborg, G.T. (See Loveland; W., LBL-1320I)

Seaborg, G.T. (See Loveland, W., LBL 14477)

Seaborg, G.T. (See Morita, Y., LBL-13425 Rev.)

Seaborg, G.T. (See Nguyen, H.D., LBL-13485)

Seiler, F. (See Roy, R., LBL-14343)

Shapiro, G. (See Anderson, L., LBL-14328)

Shapiro, G. (See Anderson, Lo, LBL-14330)

Shapiro, G. (See Moeller, E., LBL-14329)

Shapiro, G. (See Nagamiya, S., LBL-12123)

Shapiro, G. (See Schnetzer, S., LBL-14523)

Shih, S. (See Burde, J., LBL-13610)

Shih, S. (See Ellegaard, C., LBL-11913) 
Shih, S. (See McDonald, R.J., LBL-11608)

Shirley, V.S. (See Firestone, R.B., LBL-14070)

Shor, A., K. Ganezer, S. Abachi, J. Carroll, J. Geaga, G. Igo, P.Lindstrom, T. Mulera, V. Perez-Mendez, A. Sagle, D. Woodard, and F. Zarbakhsh

A Measurement of "Subthreshold" Production of $\mathrm{K}$ " and Antiprotons in Relativistic Nuclear Collisions, LBL-14229, March 1982, Phys. Rev. Lett.

Shotter, A.C., A. N. Bice, D. P. Stahel and Joseph Cerny

The Breakur of $187 \mathrm{MeV}{ }^{12} \mathrm{C}$ lons Into the $3 \alpha$ Channel, LBL-11376, July 1980, J. Phys. G. Nucl. Phys. 8, 355 (1982).

Shotter, A.C. (See Bice, A.N., LBL-14100)

Shotter, A.C. (See Shyam, R., LBL-14740)

Shyam, R., M.A. Nagarajan, A.C. Shotter, A.N. Bice, and J. Cerny

Inelastic Scattering of ${ }^{12} \mathrm{C}$ from ${ }^{208} \mathrm{~Pb}$ to the Second $0^{+}$State of ${ }^{12} \mathrm{C}$, LBL-14740, June 1982, Phys. Lett. 116B, 99 (1982).

Slobodrian, R.J. and H.E. Conzett

The ${ }^{4} \mathrm{He}(\alpha, \mathrm{p}){ }^{7} \mathrm{~L},{ }^{7} \mathrm{Li}^{* 478}$ Reaction Near Threshold, LBL-13892, January 1982, Z. Phys. A - Atoms and Nuclei 308 , 15 (1982).

Slobodrian, R.J., C. Rjoux, R. Roy, H.E. Conzett, P. von Rossen, F. Hinterberger

Evidence of Time Symmetry Violation in the Interaction of Nuclear Particles, LBL-13411, October 1981, Phys. Rev. Lett. 47, 1903 (1981).

Sobotka, L.G., C.C. Hsu, G.J. Wozniak, D.J. Morrissey,and L.G. Moretto Angular Momentum Transfe, and Partition in the Deep-Inelastic Reaction: $664 \mathrm{MeV}^{84} \mathrm{Kr}+{ }^{\mathrm{nat}} \mathrm{Ag}, \mathrm{LBL}-11840$, April 1981, Nucl. Phys. A371, 510 (1981).

Sobotka, L.G., R.J. McDonald, G.J. Woznizt, D.J. Morrissey, A.J. Pacheco, and L.G. Moretto Alpha-Particle Emission from the Deep-Inelastic Reaction $1354 \mathrm{MeV}{ }^{165} \mathrm{Ho}+{ }^{181} \mathrm{Ta}$, LBL-13319, October 1981, Phys. Rev. C25, 1693 (1982).

Sobotka, Lee G.

Light Particle Emission as A Probe of the Rotational Degrees of Freedom in Deep-lnelastic Reactions, LBL-13863, May 1982, Ph.D. thesis

Sobotka, L.G. (See Jraper, J.E., LBL-14274)

Sobotka, L.G. (See Moretto, L.G. LBL-13007)

Sobotka, L.G. (See Morrissey, D.J., LBL-12181)

Sobotka, L.G. (See Morrissey, D.J., LBL-13055)

Sobotka, L.C. (See Pacheco, A.J., LBL-14091)

Somerville, Lawrence Patrick

Observation of New Spontaneous Fission Activities from Elements 100 to 105, LBL-14050, March 1982, ?.1.D thesis 
Somerville, L.P. (See Eggers, R.C. LBL-12060)

Syieler, H. (See Gutbrod, H.H., LBL-14218)

Stahel, D.P. (See Shotter, A.C., LBL-11376)

Steinberg, E.P. (See Warwick, A.I., LBL-13831)

Steiner, H. (See Anderson, L, LBL-14328)

Steiner, H. (See Anderson, L, LBL-14330)

Steiner, H. (See Moeller, E., LBL-14329)

Steiner, H. (See Nagamiya, S., LBL-12123)

Steiner, H. (See Nogumiya, S., LBL-14033)

Steiner, H. (See Schnetzer, S., LBL-14523)

Steiner, H. (See Tanihath, I., LBL-11488)

Stelzer, H. (See Warwick, A.I., LDL-13831)

Stephens, F.S.

Behavior of Nuclei at High Angular Momentum, LBL-14641, presented at the 1982 INS International Symposium on Dynamics of Nuclear Collective Motion, Mt.Fuji,Japan, July 6-10, 1982, July 1982

\section{Stephens, F.S.}

Collective Bands in Some Rotational and Transitional Nuclei, LBL-14610, to be published as a chapter in Collective Bands in Nuclei, Ed. D. Wilkinson, Pergamon Press, Ltd. 1982, August 1982

\section{Stephens, F.S.}

High Spin States, LBL-14824, to be published in the Proceedings of the International Conference on Nuclear Structure, Free University of Amsterdam, Amsterdam, Netherlands, August 30 - September 3, 1982, August 1982

\section{Stephens, F.S.}

Properties of Nuclei at High Spins, LBL-15070, presented at the Nordic Meeting on Nuclear Physics, Fugls $\phi$, Denmark, August 16-20, 1982, October 1982

Stephens, F.S. (See Burde, J., LBL-13610)

Stephens, F.S. (See Deleplanque, M.A., LBL-14056)

Stephens, F.S. (See Draper, J.E., LBL-14274)

Stephens, F.S. (See Ellegaard, C, LBL-11913)

Stephens, F.S. (See McDonald, R.J., LBL-1 1608)

Stephens, F.S. (See Pacheco, A.J., LBL-14091)

Stevenson, J.D. (See Stocker, H., LBL-12634) 
Stock, R.

Multi-Particle Correlation Observables in High Energy Nucleus-Nucleus Collisions, LBL-12884, Presented at the Fifth High Energy Heavy Ion Study, Lawrence Berkeley Laboratory, University of California, Berkeley, CA May 18-22, 1981, July 1981

Stock, R., R. Bock, R. Brockmann, A Dacal, J.W. Harris, M. Maier, M.E. Ortiz, H.G. Pugh, R.E. Renfordt, A Sandoval, LS. Schroeder, H. Stroebele, and KL. Wolf

Compression Effects in Relativistic Nucleus-Nucleus Collisions, LBL-14583, June 1982, Phys. Rev. Lett. 49, 1236 (1982).

Stock, R. (See Harris, J.W., LBL-12334)

Stock, R. (Sec Wolf, K.L., LBL-14345)

Stocker, H., A.A. Ogloblin, and W. Greiner

Significance of Temperature Measurements in Relativistic Nuclear Collisions, LBL-12971, May 1981, Zeitschrift für Physik

Stocker, H, C. Riedel, Y. Yariv, L.P. Csernai, G. Buchwald, G. Graebner, J.A. Maruhn, W. Greiner, K. Frankel, M. Gyulassy, B. Schurmann, G. Westfall, J.D. Stevenson, J.R Nix, and D. Strottman

Nuclear Fiuid Dynamics versus Intranuclear Cascade - Possible Evidence for Collective Flow in Central High Energy Nuclear Collisions, LBL-12634, May 1981, Phys. Rev. Lett.

Stocker, H, M. Gyulassy and J. Boguta

Probing Dense Nuclear Matter Via Nuclear Collisions, LBL-12095, January, 1981, Phys. Lett. 103B, 269 (1981).

Stocker, H. (See Boguta, J. LBL-13004)

Stocker, H. (See Csernai, L.P., LBL-127\$8)

Stocker, H. (See Csernai, L.P., LBL-13944)

Stocker, H. (See Gyulassy, M., LBL-13379)

Stokstad, R.G.

The Use of the Statistical Model in Heavy-Ion Reaction Studies, LBL-12636, To be published as a Chapter in Heavy-Ion Science D. Allan Bromley, Ed., Plenum Publishing New York, 1982., April 1981

Stokstad, R.G. (See Cole, A.J., LBL-14550)

Stokstad, R.G. (See Cole, A.J., LBL-13011)

Stokstad, R.G. (See Rae, W.D., LBL-12568)

Stroebele, H. (See Harris, J.W., LBL-12334)

Stroebele, H. (See Stock, R., LBL-14583)

Strottman, D. (See Stocker, H., LBL-12634)

Sullivan, J.P., J.A. Bistirlich, H.R. Bowman, R. Bossingham, T. Buttke, K.M. Crowe, K.A. Frankel, C.J. Martoff, J. Miller, D.J. Murphy, J.O. Rasmussen, W.A Zajc, O. Hashimoto, M. Koike, J. Peter, W. Benenson, G.M. Crawley, E. Kashy, and J.A. Nolen, Jr. 
Strong Coulomb Effects on Pions Produced in Heavy Ion Collisions, LBL-11971, June 1981, Phys. Rev. C25, 1499 (1982).

\section{Sullivan, John Peter}

Coulomb Effects on Pions Produced in Heavy Ion Reactions, LBL-12546, Ph.D thesis, November 1981

Sullivan, J.P. (See Frankel, K.A., IBL-12585)

Sullivan, J.P. (See Frankel, K.A., LBL-1264l)

Sullivan, J.P. (See Hicks, K.H., LBL-15405)

Sullivan, J.P. (See Radi, H.M.A., LBL-12591)

Sullivan, J.P. (See Rasmussen, J.O., LBL-12623)

Sventek, J.S. (Sce Mathews, G.J., LBL-13226)

\section{Swiatecki, W.J.}

Discussions with Jens, LBL-14072, presented at the Symposium Honouring Jens Lindhard's 60th Birthday, Aarhus, Denmark, February 25-26, 1982 and to be published in Physica Scripta, Apri] 1982

\section{Swiatecki, W.J.}

The Dynamics of the Fusion of Two Nuclei, LBL-12642, Nucl. Phys, A376, 275 (1982).

\section{Swiatecki, W.J.}

The Nature of Nuclear Dynamics, LBL-14073: Introductory Remarks given at the Winter Workshop on Nuclear Dynamics, Granlibakken, Tahoe City, CA, February21-26, 1982 and to be published in the Proceedings, April 1982

Swiatecki, W.J. (See Bjornholm, S., LBL-14074)

Swiatecki, W.J. (See Blocki, J. LBL-12811)

Swiatecki, W.J. (See Myers, W.D., LBL-13929)

\section{Symons, T.J.M.}

$\alpha-\alpha$ Colljsions at the CERN ISR, LBL-14902, presented at the MARIA Heavy Ion Workshop, Banf, Alberta, Canada, February 18-22, 1982, September 1982

Symons, T.J.M. (See Murphy, M.J., LBL-14272)

Tanihata, I., S. Nagamiya, S. Schnetzer, and H. Steiner

One- and Two-Proton Inclusive Spectra in $800 \mathrm{MeV}$ Proton-Nucleus Collisions and the Mean Free Path of Protons in Nuclei, LBL-11488, October 1980, Phys. Lett. 100B, 121 (1981).

Tanihata, I. (See Csernai, L.P., LBL-13944)

Tanihata, I. (See Nagamiya, S., LBL-12123)

Tanihata, I. (See Nagamiya, S., LBL-14033)

Tanihata, I. (See Schnetzer, S., LBL-14523) 


\section{Appendix I}

\section{Publications}

Thierens, H.M. (See Cable, M.D., LBL-14419)

Thierens, H.M. (See Wouters, J.M., LBL-13990)

Tiedje, J.M. (See Firestone, R.B., LBL-10919)

Treuhaft, Robert Neil

A (p,2p) Study of High Momentum Components at 2.1 GeV, LBL-14677 Ph.D, thesis

Trinh, H.N. (See Nguyen, H.D., LBL-13485)

Tserruya, I. (See Rae, W.D., LBL-12568)

Uchiyama, F.

"Mass Scaling" Phenomena in Heavy Fragments Production in Relativistic Heavy Ion Collisions, LBL-13681, December 1981, Phys. Rev. Latt.

Van Bibber, K. and A. Sandoval

Streamer Chambers for Heavy lons, LBL-12883, To be published as a book chapter in Heavy Ion Science, D. Allan Bromley, Ed., Plenum Press, New York, 1982., June 1981

Van Bibber, K., B.G. Harvey, D.L. Hendrie, J. Mahoney, M.J. Murphy, and W.W. Pang A Hybrid Streamer Chamber for Intermediate Energy Heavy Ion Physics, LBL-15105, November 1981, Nucl. Instr. and Methods 198, 253 (1982).

Van Bibber, K. (See Murphy, M.J., LBL-14226)

Viola, V.E. (See Myers, W.D., LBL-14138)

Von Guten, H.R. (See Liu, Y.-f., LBL-12909)

Ward, T.E. (See Hicks, K.H., LBL-15405)

Warner, R.A. (See Firestone, R.B., LBL-12424)

Warwick, A.I., A. Baden, H.H. Gutbrod, M.R. Maier, J. Peter, H.G. Ritter, H. Stelzer, H.H. Wieman, F. Weik, M. Freedman, D.J. Henderson, S.B. Kaufman, E.P. Steinberg, and B.D. Wilkins

Fission and Deep Spallation Characteristics in Relativistic Nuclear Collisions, LBL-13831, March 1982, Phys. Rev. Lett. 48, 1719 (1982).

Warwick, A.I. (See Gutbrod, H.H., LBL-14059)

Warwick, A.I. (See Gutbrod, H.H, LBL-14218)

Warwick, A.I. (See Gutbrod, H.H., L.BL-14371)

Weaver, D., W. Rathbur, and C. Maples

Dynamic Expression Evaluator for Real Time Applications, LBL-12503; May 1981, IEEE Transactions on Nuclear Science

Weaver, D. (See Maples, C., LBL-12504)

Weaver, D. (See Maples, C., LBL-12505) 
Weaver, D. (See Rathbun, W., LBL-12498)

Weik, F. (See Gutbrod, H.H, LBL-14218)

Weik, F. (See Warwick, A.I., LBL-13831)

Weiner, R.M. (See Fowler, G.N., LBL-12279)

Westfall, G. (See Stocker, H., LBL-12634)

Westfall, G.D. (See Murphy, M.J., LBL-14272)

Westfall, G.D. (See Oison, D. LBL-12292)

Westfall, G.D. (See Wolf, K.L., LBL-14345)

Wieman, H. (See Gutbrod, II.H., LBL-14059)

Wieman, H. (See Gutbrod, H.H., LBL-14218)

Wieman, H. (See Gutbrod, H.H, LBL-14371)

Wieman, H.H. (See Warwick, A.I., LBL-13831)

Wilkins, B.D. (See Warwick, A.I., LBL-13831)

Wolf, K.L., H.H. Gutbrod, W.G. Meyer, A.M. Poskanzer, A. Sandoval, R. Stock, J. Gosset, J.-C. Jourdain, C.H. King, G. King, Nguyen Van Sen, and G.D. Westfall

Pion Production and Charged-Particle Multiplicity Selection in Relativistic Nuclear Collisions, LBL-14345, April 1982, Phys. Rev. C26, 2572 (1982).

Wolf, K.L. (See Harris, J.W., LBL-12334)

Wolf, K.L. (See Stock, R., LBL-14583)

Woodard, D. (See Shor, A., LBL-14229)

Wouters, Jan Marc

Decay Studies of the Highly Neutron-Deficient Indium Isotopes, LBL-13860, February 1982, Ph.D. thesis

Wouters, J.M., H.M. Thierens, J. Aystö, M.D. Cable, P.E. Haustein, R.F. Parry and Joseph Cerny

Beta Decay Energies and Masses of ${ }^{103-105}$ In, LBL-13990, February 1982, Phys. Rev. C

Wouters, J.M. (See Cable, M.D., LBL-14419)

Wozniak, G.J., G.J. Mathews, R.P. Schmitt, R. Regimbart, H. Hübel, R.M. Diamond, and L.G. Moretto A Study of Charge, Energy and Angular Momentum Transfer in the ${ }^{56} \mathrm{Fe}+{ }^{197} \mathrm{Au}$ and ${ }^{56} \mathrm{Fe}+{ }^{107,109} \mathrm{Ag}$ Reactions at 7.2 and 8.3 MeV/Nucleon, LBL-9461, September 1982, Nucl. Phys.

Wozniak, G.J., C.C. Hsu, D.J. Morrissey, L.W. Richardson and L.G. Moretto Angular Momentum and Linear Momentum Transfer in Intermediate Energy Heavy-Ion Reactions, LBL-1353, November 1981 
Wozniak, G.J., C.C. Hsu, D.J. Morrissey, L.W. Richardson, and L.G. Moretto

Angular Momentum and Linear Momentum Transfer in Intermediate Energy Heavy-Ion Reactions, LBL-13637, Presented at the XIV Masurian School of Nuclear Physies, Mikolajki, Poland, August 31-September 12, 1981

Wozniak, G.J. (See Draper, J.E., LBL-14274)

Wozniak, G.J. (See Hsu, C.C., LBL-12519)

Wozniak, G.J. (See Mathews, G.J., LBL-13226)

Wozniak, G.J. (See McDonald, R.J., LBL-11608)

Wozniak, G.J. (See Morrissey, D.J., LBL-1218I)

Wozniak, G.J. (See Morrissey, D.J., LBL-13055)

Wozniak, G.J. (Sree Pacheco, A.J., LBL-14091)

Wozniak, G.J. (See Sobotka, L.G., LBL-11840)

Wozniak, G.J. (See Sobotka, L.G., LBL-13319)

Yariv, Y. (See Stocker, H., LBL-12634)

Yashita, S. (See Leino, M.E., LBL-12816)

\section{Zajc, William Allen}

Two-Pion Correlations in Heavy Ion Collisions, LBL-14864 Ph.D. thesis, August 1982

Zajc, W.A. (See Frankel, K.A., LBL-12585)

Zajc, W.A. (See Frankel, K.A., LBL-12641)

Zajc, W.A. (See Sullivan, J.P., LBL-11971)

Zarbakhsh, F. (See Shor, A., LBL-14229)

Zhou, Z.Y. (See Cable, M.D., LBL-14419)

Zisman, M.S.

National Uses and Needs for Separated Stable Isotopes in Physics, Chemistry, and Geoscience Research, LBL-14068, presented at the Workshop on Stable Isotopes and Derived Radioisotopes, National Academy of Sciences, Washing.ton, DC, February 3-4, 1982, January 1982

von Rossen, P. (See Slobodrian, R.J., LBL-13411) 


\title{
Nuclear Reaction Studies Via the Observation of Unbound Outgoing Systems
}

\author{
Alden Newell Bice
}

The mechanisms involved in the production of fast $\alpha$-particles in ${ }^{12} \mathrm{C}$ induced reactions have been investigated for the ${ }^{12} \mathrm{C}+{ }^{208} \mathrm{~Pb}$ system at bombarding energies of $E\left({ }^{12} \mathrm{C}\right)=132,187$ and $230 \mathrm{MeV}$. Absolute cross sections have been determined for the reactions ${ }^{208} \mathrm{~Pb}\left({ }^{12} \mathrm{C},{ }^{8} \mathrm{Be}\right.$ (g.s.)), ${ }^{208} \mathrm{~Pb}\left({ }^{12} \mathrm{C},{ }^{8} \mathrm{Be}(2.94 \mathrm{MeV})\right)$ and ${ }^{200} \mathrm{~Pb}\left({ }^{12} \mathrm{C},{ }^{12} \mathrm{C} \rightarrow \alpha+{ }^{8} \mathrm{Be}\right)$ by double and triple coincidence measurements of the sequential decay $\alpha$ particles. Inclusive $\alpha$-particle production cross sections were also measured at $\mathrm{E}\left({ }^{12} \mathrm{C}\right)=187 \mathrm{MeV}$ for comparison. It is found that the simple inelastic scattering process $\left({ }^{12} \mathrm{C},{ }^{12} \mathrm{C} * \rightarrow \alpha+{ }^{8} \mathrm{Be}\right)$ does not contribute significantly to the production of fast $\alpha$-particles but that the production of ${ }^{8} \mathrm{Be}$ nuclei by projectile fragmentation is an important source of $\alpha$-particles. At the highest bombarding energy investigated ( $19 \mathrm{MeV} / \mathrm{A})$ it appears that the ${ }^{12} \mathrm{C} \rightarrow 3 \alpha$ fragmentation reaction becomes more prominent at the expense of the ${ }^{12} \mathrm{C} \rightarrow \alpha$
$+{ }^{8} \mathrm{Be}$ fragmentation channel. Although qualitatively these results are not in disagreement with the incomplete fusion model predictions of Wilczynski it is concluded that projectile spectroscopic properties and/or final state interactions are important in fragmentation reactions for these bombarding energies.

In a kinematically complete experiment the direct and the sequential breakup channels of $10 \mathrm{MeV} / \mathrm{A}^{7} \mathrm{Li}$ projectiles have been investigated with ${ }^{12} \mathrm{C}$ and ${ }^{208} \mathrm{~Pb}$ targets. By appropriate arrangement of detector telescopes it was possible to define a kinematical window which allowed for the unambiguous observation of both the direct (to the $\alpha-t$ continuum) and the sequential components of a heavy-ion projectile breakup reaction. A semiclassical Monte Carlo type projectile breakup calculation was constructed which qualitatively reproduced the measured $\alpha-t$ coincidence cross section as a function of the laboratory angle.

\section{Zero Field Spin Relaxation of the Positive Muon in Copper}

\author{
Carl William Clawson
}

The spin relaxation of the $\mu^{+}$in high purity single crystal and polycrystalline copper has been measured at temperatures between $0.5 \mathrm{~K}$ and $5.2 \mathrm{~K}$ by the zero-field $\mu^{+}$SR technique. In both types of sample the experiments show a temperature independent dipolar width $\Delta_{z}=0.389 \pm 0.003 \mu \mathrm{s}^{-1}$ and a hopping rate decreasing from $\sim 0.05 \mu \mathrm{s}^{-1}$ at $0.5 \mathrm{~K}$ to $\sim 0.05 \mu \mathrm{s}^{-1}$ above $5 \mathrm{~K}$. This is the first direct proof of a dynamic effect in the low temperature $\mu^{+}$spin relaxation in copper.

The relationship between the zero-field and transverse-field dipolar widths is discussed, and the measured zero-field width is found to be $-10 \%$ larger than expected based on the known transverse-field widths. A new $\mu^{+}$SR spectrometer has been constructed and used in this work. The spectrometer and the associated beam lines and data acquisition facilities are discussed. 


\title{
Observation of Anomalous Reaction Mean Free Paths of Nuclear Projectile Fragments in Research Emulsion from $2 \mathrm{AGeV}$ Heavy Ion Collisions
}

\author{
Yasha J. Karant
}

From an analysis of 1460 projectile fragment collisions in nuclear research emulsion exposed to 2.1 $\mathrm{AGeV}{ }^{16} \mathrm{O}$ and $1.9 \mathrm{AGeV}{ }^{36} \mathrm{Fe}$ at the Bevalac, evidence is presented for the existence of an anomalously short interaction mean free path of projectile fragments for the first several $\mathrm{cm}$ after emission. The result is significant to beyond the 3 standard deviation confidence level.

\section{Recoil Range Studies of Heary Products of Multinucleon Transfer from ${ }^{18} \mathrm{O}$ to ${ }^{245} \mathrm{Cm}$ and ${ }^{249} \mathrm{CF}$}

\author{
Rose Marie McFarland
}

\begin{abstract}
Recoil range distributions were measured for alpha and spontaneous fission activities made in the bombardment of ${ }^{245} \mathrm{Cm}$ and ${ }^{249} \mathrm{Cf}$ with ${ }^{18} \mathrm{O}$ from 6.20 $\mathrm{MeV} /$ nucleon down to the interaction barrier. The shape of the distributions indicates that transfers of up to four protons take place via a combination of quasielastic (QET) and deep inelastic (DIT) mechanisms, rather than complete fusion-de-excitation (CF) or massive transfer (MT). Angular distributions constructed from recoil range distributions, assuming QET/DIT,
\end{abstract}

indicate that the QET component contributes more significantly to the heavy product residue cross section than the DIT, even though primary cross sections are expected to be higher for DIT than for QET. This may be explained qualitatively as a result of the high excitation energies associated wih DIT; the very negative $Q$. of projectile stripping for these systems combined with the lower expected optimal $Q_{r}$ of QET compared to DIT can give QET products comparatively low excitation.

\section{Meson Production in Relativistic Heavy Ion Collisions}

\author{
Stephen R. Schnetzer
}

We have measured the inclusive $\mathrm{K}^{+}$production cross sections at angles from $15^{\circ}$ to $80^{\circ}$ in collisions of protons $(2.1 \mathrm{GeV})$ and deuterons $(2.1 \mathrm{GeV} / \mathrm{amu})$ on $\mathrm{NaF}$ and $\mathrm{Pb}$, and $\mathrm{Ne}(2.1 \mathrm{GeV} / \mathrm{amu}$ ) on $\mathrm{C}, \mathrm{NaF}, \mathrm{KCl}$, $\mathrm{Cu}$ and $\mathrm{Pb}$. The kaons were identified by measuring the time of flight and the momentum in a magnetic spectrometer, and by detecting the particles from the kaon decays in a lead-glass Cerenkov counter. The momentum range of the detected kaons extended from $350 \mathrm{MeV} / \mathrm{c}$ to $750 \mathrm{MeV} / \mathrm{c}$. The multiplicity of each event was measured bu a set of scintillation counter telescopes which were situated around the target.

The differential cross section of the kaons falls off exponentially with center of mass energy in the nucleon-nucleon center of mass frame. In addition, the angular distribution of the kaons is nearly isotropic in this frame even for $\mathrm{p}-\mathrm{NaF}$ and $\mathrm{Ne}-\mathrm{Pb}$ collisions. The data are compared with a row-on-row model and a thermal model. Neither are able to explain all features of the data. The row-on-row model does not reproduce the near-isotropy in the nucleon-nucleon frame, and the 


\title{
APPENDIX II Thesis Abstracts
}

thermal model overpredicts the kaon yield by a factor of approximately twenty.

Analysis of the A-dependence shows that the increase in the cross section for kaon production between $\mathrm{Ne}-\mathrm{NaF}$ and $\mathrm{Ne}-\mathrm{Pb}$ collisions is greater than that between $\mathrm{d}-\mathrm{NaF}$ and $\mathrm{d}-\mathrm{Pb}$. This may be an indica- tion of a collective effect.

In $\mathrm{Ne}-\mathrm{Pb}$ collisions the associated multiplicity is approximately $10 \%$ higher when a kaon is detected in the spectsometer than when a proton or pion is detected. This indicates that the kaons may come from more central collisions.

\section{Light Particle Emission as A Probe of the Rotational}

\section{Degrees of Freedom in Deep-Inelastic Reactions}

\author{
Lee G. Sobotka
}

The emission of alpha particles in coincidence with the most deeply inelastic heavy-ion reactions has been studied for ${ }^{18} \mathrm{Ta}+{ }^{165} \mathrm{Ho}$ at $1354 \mathrm{MeV}$ laboratory energy and ${ }^{\text {mit }} \mathrm{Ag}+{ }^{84} \mathrm{Kr}$ at $664 \mathrm{MeV}$. Alpha particle energy spectra and angular distributions, in coincidence with a projectile-like fragment, were acquired both in the reaction plane and out of the reaction plane at a fixed in-plane angle.

The in-plane data for both systems are employed to show that the bulk of the alpha particles in coincidencc with the deep-inelastic exit channel can be explained by evaporation from the fully accelerated fragments. Average velocity diagrams, $\alpha$-particle energy spectra as a function of angle in several rest frames, and $\alpha$-particle angular distributions are presented.

The out-of-plane alpha particle angular distribu- tions and the gamma-ray multiplicities are used to study the transfer and partitioning of angular momentum between the two fragments. For the ${ }^{n 21} \mathrm{Ag}+{ }^{84} \mathrm{Kr}$ system individual fragment spins are extracted from the alpha particle angular distributions as a function of mass asymmetry while the sum of the fragment spins is derived from the gamma-ray multiplicities. These data, together with the fragment kinetic energies, are consistent with rigid rotation of an intermediate complex consisting of two substantially deformed spheroids in near proximity. These data also indicate that some angular momentum fractionation exists at the largest asymmetries examined. Out-of-plane alpha particle distributions, gamma-ray multiplicities, fragment spins as well as the formalism for the spin evaluation at various levels of sophistication are presented.

\section{Observation of New Spontaneous Fission Activities from Elements 100 to 105}

\section{Lawrence Patrick Somerville}

Several new Spontaneous Fission (SF) activities have been found. Their half-lives and production cross sections in several reactions bave been measured by collecting and transporting recoils at known speed past mica track detectors. No definite identification could be made for any of the new SF activities; however, half-lives and possible assignments to element-104 isotopes consistent with several cross bombardments include ${ }^{257} \mathbf{R}(3.8 \mathrm{~s}, 14 \% \mathrm{SF}),{ }^{259} \mathrm{Rf}(13 \mathrm{~ms}),{ }^{259} \mathbf{R}(\sim 3$ s, $8 \%$ SF $),{ }^{260} \mathrm{Rf}(\sim 20 \mathrm{~ms})$, and ${ }^{262} \mathrm{Rf}(\sim 50 \mathrm{~ms})$. The 80-ms SF activity claimed by the Dubna group for the discovery of element $104\left({ }^{200} 104\right)$ was not observed. A difficulty exists in the interpretation that ${ }^{260} \mathrm{Rf}$ is a 20-ms activity: in order to be correct, for example, the SF activities with half-lives between 14 and $24 \mathrm{~ms}$ produced in the reactions 109 - to $119 \mathrm{MeV}{ }^{18} \mathrm{O}+$ ${ }^{248} \mathrm{Cm}$, 88- to $100-\mathrm{MeV}{ }^{15} \mathrm{~N}+{ }^{249} \mathrm{Bk}$, and $96-\mathrm{MeV}{ }^{18} \mathrm{O}$ $+{ }^{249} \mathrm{Cf}$ must be other nuclides due to ther large production cross sections, or the cross sections for production of ${ }^{260} \mathrm{Rf}$ must be enhanced by unknown mechan- 


\title{
APPENDIX II · Thesis Abstracts
}

isms. Based on calculated total production cross sections a possible $\sim 1 \%$ electron-capture branch in ${ }^{258} \mathrm{Lr}(4.5 \mathrm{~s})$ to the SF emitter ${ }^{258} \mathrm{No}(1.2 \mathrm{~ms})$ and an upper limit of $0.05 \%$ for SF branching in ${ }^{254} \mathrm{No}(55 \mathrm{~s}$ ) were determined. Other measured half-lives from unknown nuclides produced in respective reactions include $\sim 1.6 \mathrm{~s}\left({ }^{16} \mathrm{O}+{ }^{248} \mathrm{Cm}\right)$, indications of a $\sim 47 . \mathrm{s} \mathrm{SF}$ activity (75-MeV ${ }^{12} \mathrm{C}+{ }^{249} \mathrm{CF}$ ), and two or more $\mathrm{SF}$ activities with $3 \mathrm{~s} \leq \mathrm{T}_{1 / 2} \leq 60 \mathrm{~s}\left({ }^{18} \mathrm{O}+{ }^{249} \mathrm{Bk}\right.$ ). The most exciting conclusion of this work is that if the tentative assignments to even-even element-104 isotopes are correct, there would be a sudden change in the SF half-life systematics at element 104 which has been predicted theoretically by Randrup et al, and Baran et al. and attributed to the disappearance of the second hump of the double-humped fission barrier. This disappearance of the second barrier also explains the tentative low hindrance factors compared to lighter elements for SF of the odd-mass isotopes ${ }^{257} \mathrm{Rf}\left(\sim 4 \times 10^{3}\right)$ and ${ }^{259} \mathrm{Rf}\left(\sim 2 \times 10^{3}\right)$. On the basis of recent odd-mass alpha-decay energy data, the 152-neutron sub-shell effect is probably weaker for element 104 than for element 102, confirming predictions of Randrup et al., and $n^{n t}$ strong enough to significanatly alter the SF half-life predictions. This weakening sub-shell effect is in contrast to the continuing strong effect assumed in the Ghiorso half-life systematics. The possibilities of enhanced stability against SF with 157 neutrons for ${ }^{261} \mathrm{RP}(65 \mathrm{~s})$ and theoretical arguments concerning the SF-mass distribution for element-104 nuclei are discussed.

\section{Coulomb Effects on Pions Produced in Heavy Ion Reactions}

John Peter Sullivan

Double differential cross sections for the production of $\pi^{+}$and $\pi^{-}$near the velocity of the incident beam for pion lab angles less than 40 degrees are presented. The experimental apparatus and the techniques are discussed. Beams of $20 \mathrm{Ne}$ with $\mathrm{E} / \mathrm{A}$ from 80 to $655 \mathrm{MeV}$ and ${ }^{40} \mathrm{Ar}$ with $\mathrm{E} / \mathrm{A}=535 \mathrm{MeV}$ incident on $\mathrm{Be}, \mathrm{C}$, $\mathrm{NaF}, \mathrm{KCl}, \mathrm{Cu}$, and $\mathrm{U}$ targets were used. A sharp peak in the $\pi^{-}$spectrum and a depression in the $\pi^{+}$spectrum were observed at zero degrees near the incident beam velocity. The effect is explained in terms of Coulomb interactions between the pions and fragments of the incident beam. Least squares fits to the data using the Coulomb correction formulas of Gyulassy and Kauffmann and an effective projectile fragment charge are made. The relationship between these data and previously measured pion production and projectile fragmentation data is discussed. The data are also compared to some theoretical models. A simple expression is given for the differential cross section as a function of the projectile mass, target mass, and beam energy.

\section{A $(p, 2 p)$ Study of High Momentum Components at 2.1 GeV}

\author{
Robert Neil Treuhaft
}

A ( $p, 2 p)$ experiment designed to isolate interactions with small numbers of fast nuclear constituents is described. Special attention is paid to the experimental manifestation and description of a correlated pair of nucleons in the nucleus. Phase space calculations are presented for the proton-pair three-body final state and for final states with larger numbers of particles. The Two Armed Spectrometer System (TASS) is described in detail. The data suggest the possibility of isolating an interaction with one or two nucleons in the nucleus which may have momenta far in excess of those described in a Fermi gas model. 


\title{
Decay Studies of the Highly Neutron-Deficient Indium Isotopes
}

\author{
Jan Marc Wouters
}

An extension of the experimentally known nuclidic mass surface to nuclei far from the region of beta-stabiliy is of fundamental interest in providing a better determination of the input parameters from the various nuclear mass formulae, allowing a more accurate prediction of the ultimate limits of nuclear stability. In addition, a study of the shape of the mass surface in the vicinity of the doubly-closed nuclide ${ }^{100} \mathrm{Sn}$ provides initial information on the behavior of the shell closure to be expected when $Z=N=50$. Experiments measuring the decay energies of ${ }^{103-105}$ In by $\beta$-endpoint measurements are described with special attention focused on the development of a plastic scintillator $\beta$ telescope coupled to the on-line mass separator RAMA (Recoil Atom Mass Analyzer). Attempts to measure the $\beta$-endpoint energy of ${ }^{102}$ In is also briefly described.

The experimentally determined decay energies and derived masses for ${ }^{103-105}$ In are compared with the predictions of different mass models to identify which models are more successful in this region. Furthermore, the inclusion in these comparisons of the avail- able data on the neutron-rich indium nuclei permits a systematic study of their ground state mass behavior as a function of the neutron number between the shell closures at $\mathrm{N}=50$ and $\mathrm{N}=82$. These analyses indicate that the binding energy of ${ }^{103}$ In is $1 \mathrm{MeV}$ larger than predicted by the majority of the mass models.

An examination of the $Q_{E C}$ surface and the singleand two-neutron separation energies in the vicinity of ${ }^{103-105}$ In is also performed to investigate further the above deviation and other possible systematic variations in the mass surface in a model-independent way. The ${ }^{103}$ In $Q_{E C}$ and the ${ }^{105}$ In $s_{2 n}$ are shown to diverge seriously from systematics. Ascribing these effects to the mutual support of shell closures, which would cause a strengthening of the proton binding energy with decreasing neutron number, requires additional investigation. Interestingly, the neighboring even cadmium $2^{+}$and $4^{+}$excited state systematics indicate that these nuclei are becoming more spherical with decreasing neutron number as expected near a double shell closure.

\section{Two-Pion Correlations in Heavy Ion Collisions}

\author{
William Allen Zajc
}

An application of intensity interferometry to relativistic heavy ion collisions is reported. Specifically, the correlation between two like-charged pions is used to study the reactions $\mathrm{Ar}+\mathrm{KCl} \rightarrow 2 \pi^{ \pm}+\mathrm{X}$ and $\mathrm{Ne}+\mathrm{NaF} \rightarrow 2 \pi^{-}+\mathrm{X}$. Source sizes are obtained that are consistent with a simple geometric interpretation. Lifetimes are less well determined but are indicative of a faster pion production process than predicted by Monte Carlo cascade calculations. There appears to be a substantial coherent component of the pion source, although measurement is complicated by the presence of final state interactions. Additionally, the generation of spectra of uncorrellated events is discussed. In particular, the influence of the correlation function on the background spectrum is analyzed, and a prescription for removal of this influence is given. A formulation to describe the statistical errors in the background is also presented.

Finally, drawing from the available literature, a self-contained introduction to Bose-Einstein correlations and the Hanbury-Brown-Twiss effect is provided, with an emphasis on points of contact between classical and quantum mechanical descriptions. 
August 17, 1981

R. Scharenberg

Purdue U.

August 31, 1981

J.L. Egido

TUM, Garching

September 14, 1981

Julius Kuti Inst.Theoretical Phys. Santa Barbara

October S, 1981

October 12, 1981

October 19, 1981

October 26, 1981

November 2, 1981

November 3, 1981

November 9, 1981

November 10, 1981

November 16, 1981

November 23, 1981

November 30, 1981

December 7, 1981
A. Gavron LASL

Y. Morita, J. Sullivan, LBL

Joseph Comfort Ariz. State Univ.

Friedrich Riess

U. of Murich and GSI

H. Robertson LASL/MSU

Arto Passoja U. of Jyväskylä

A. Bice, L. Sobotka LBL

Dr. Madhu Mehta Bhabha Inst., India

\section{A. Budzanowski}

U. of Cracow

M. Gyulassy

LBL

Itshak Tserruya Weizmann Inst. and LBL

P. McGaughey, D. Murphy LBL
Possible Evidence for a Phase Transition in Hadronic Matter from Mass Yields of High Energy pNucleus Collisions (Gas-jet Target Experiment at Fermilab)

Description of Excited Rotational Bands in Deformed Nuclei

Toward a Numerical Solution of QCD

Target Fragment Angular Distribution from ${ }^{12} \mathrm{C}+{ }^{238} \mathrm{U}$ and ${ }^{197} \mathrm{Au}$ at Relativistic Energies

Inelastic Scattering at Intermediate Energies and Precritical Phenomena

Band Structure of Neutron Deficient $\mathrm{Yb}$ and $\mathrm{Ra}$ Isotopes

Observation of the ${ }^{2} \mathrm{H}(\alpha, \gamma){ }^{6} \mathrm{Li}$ Reaction and its Astrophysical Implications

A New Technique for High-Resolution Internal-Pair Spectroscopy and Recent Results on the Structure of Excited $0^{+}$States in $\mathrm{f}$-p Shell Nuclei

Onset of Non-equilibrium Neutron Emission in Heavy Ion Reactions

${ }^{12} \mathrm{C}^{*}$ and ${ }^{8} \mathrm{Be}$ Production in ${ }^{12} \mathrm{C}+{ }^{208} \mathrm{~Pb}$ Collisions and Alpha-Particle Emission from the Deep Inelastic Reaction: $1354 \mathrm{MeV}{ }^{165} \mathrm{Ho}+{ }^{181} \mathrm{Ta}$

Study of pn Reactions at Sub-Coulomb Energies on Medium Mass Nuclei

Evidence for Local Heating of the Nucleus Using the $\left(\alpha, \alpha^{\prime} p\right)$ Reaction

Do Nuclei Flow at High Energi

Preequilibrium Neutron Emission in ${ }^{86} \mathrm{Kr}$ on ${ }^{166} \mathrm{Er}$ at $1.02 \mathrm{GeV}$

Heavy Target Fragmentation at Intermediate Energies and Subthreshold Charged Pion Production in Nuclear Collisions 
Januery 11, 1982

Jenuary 18, 1982

January 29, 1982

February I, 1982

March 1, 1982

March 8, 1982

March 29, 1982

April 5, 1982

April 5, 1982

April 12, 1982

April 19, 1982

May 3, 1982

May 10, 1982

May '7, 1982

May 24, 1982
S.Chessin, R.B. Welch, LBL
T. Tombrello W.K. Kelloge Radiation Lab and Caltech

Walter Greiner U. of Frankfurt

K. Moody, R Parry LBL

M. Cable and E,L. Dines LBL

M.J. Murphy LBL

W. McHarris MSU and LBL

Goran Nyman Chalmers U. of Technology Göteborg

Prof, M. Moshindky

U. of Mexico

R. McFarland, A.J. Pacheco LBL

C. Maples

LBL

Dr. Fujia Yang

Fudan U., Shanghai

Karl van Bibber Stanford U.

Isoo Tanihata INS - U. of Tokyo

Dr. R. P. Schmitt Texas A\&M U.
Pion Production at $180^{\circ}$ in Nucleus-Nucleus Collisions and Production of Neutron- Deficient Actinide Nuclides from ${ }^{16} \mathrm{O}+{ }^{237} \mathrm{~Np}$ and ${ }^{233} \mathrm{U}$

Nuclear Physics for Fun and Profit

Superheavy Nuclear Systems with $Z \geq 184$ and Their Detection Through Positron Emission

The Decay of ${ }^{231} \mathrm{Bk}$ and Beta Decay Energies from Ground and Isomeric States of ${ }^{122} \mathrm{Cs}$

Observation of the Beta Delayed Proton Decay of a New Isotop: ${ }^{22} \mathrm{~A}$ and High-Spin States in ${ }^{158} \mathrm{Er}$

Transfer and Fragmentation Reactions at 15 $\mathrm{MeV} /$ nucleon

Rotational Bands in Odd-Odd ${ }^{182}$ Re

Beta Delayed Emission of Two and Three Neutrons and the Influence of Beta Recoil and Level Widths on Delayed Particle Spectra

Unified Approach to Nuclear Collective Models

Recoil Range Studies of ${ }^{18} \mathrm{O}+{ }^{249} \mathrm{Cf}$ and AngularMomentum Transfer in the Deep-Inelastic Reactions $1400 \mathrm{MeV}{ }^{165} \mathrm{Ho}+{ }^{176} \mathrm{Yb},{ }^{148} \mathrm{Sm}$ and ${ }^{\text {ant }} \mathrm{Ag}$

MIDAS Overview

Decay Law of Complex Cascade Processes and its Application to Lifetime Measurements and a Review of the Shanghai Restarch Program

Coincidence Electron Scattering and Giant Multipole Resomances in ${ }^{238} \mathrm{U}$

Twc-Particle Correlations in High-Energy Nuclear Collisions

Excitation of Angular Momentum Bearing Collective Modes in Heavy Ion Reactions 
June 7, 1982

June 14, 1982

June 17,1982

June 21, 1982

June 28, 1982

July 12,1982

July 19,1982

July 26, 1982

August 2, 1982

August 4, 1982

August 27, 1982

August 31, 1982

September 2, 1982

September 20, 1982

September 21, 1982
Marie-Agnes Deleplanque LBL

Dr. Peter Armbruster GSI

Dr. Peter Kleinheinz IKP Julich \& LLNL

Dieter Fick

U. of Marburg

A.O. Macchiavelii, R. Treuhaft LBL

\section{J. Rafelski}

U. of Frankfurt

Keh-Fei Liu

U. of Kentucky

G.M. Crawley MSU

\section{A. I. Warwick} GSI

E.C. Schloemer Yale

H. Bertschat Hahn-Meitner Inst.

J.L. Egido TUM, Garching

B. Goldring Weizmann Inst.

Renee Lucas CEN, Saclay

J. Wilson LLNL
Nuclear Moments of Inertia at High Energy Spin

The Soft Way to Element 107 and Maybe Beyond

The Pauli Principle in Octupole Excitation around ${ }^{146} \mathrm{Gd}$

Polarized Heavy Ions

One- and Two-Neutron Transfer Reactions in 690 $\mathrm{MeV}{ }^{132} \mathrm{Xe}+{ }^{154} \mathrm{Sm}$ and $\mathrm{A}(\mathrm{p}, 2 \mathrm{p})$ Study of High Momentum Components at $2.1 \mathrm{Gev}$

Formation of a Quark-Gluon Plasma

Multi-Quark Hadrons and the Color Van der Waals Force Revisited

Ml Giant Resonances Observed in High Energy (p,p) Reactions

The Break-up of Highly Excited Nuclear Residues

Resonance Characterization of the ${ }^{12} \mathrm{C}+{ }^{16} \mathrm{O}$ System

Valence Determination in Gamma-Cerium

Description of Excited Rotational Bands in Deformed Nuclei

Fencing with Tillted Foils

Isospin Transfer in Deep Inelastic Reactions

Stellar Collapse and the Supernova Problem 
July 22,1981

July 27, 1981

July 29, 1982

August 3, 1981

August 5, 1981

September 9, 1981

September 14, 1981

September 16, 1981

November 4, 1981

Dec. 12,1981

January 6, 1982

January 20, 1982

February 3, 1982

March 4, 1982

March 17, 1982

April 19, 1982

July ?, 1982
Peter Möller

Lund University, Sweden

J.P. Blaizot CEN, Saclay, France

Xin-Hua Yang Beijing Univ.

P. Hecking LBL

\section{Jörg Hüfner} Heidelberg

Paul Bonche CEN, Saclay, France

G. Chew

Phys. Div., LBL

Kai Neergaard Giessen

M. Dworzecka University of Maryland

Jacob Bondorf Niels Bohr Inst.

T. Matsui Stanford

N.K Glenderning LBL

Pawel Danielewicz I.BL

Hans Feldmeier Darmstadt

Gordon Baym U. of Illinois

C. Maples LBL

Sven Bjørnholm Niels Bohr Institute
Nuclear Shapes

Time Dependent Variational Principles and Semiclassical Bound States

Many Body Theory of Confined Quarks and Gluons and the Calculation or the Exchange and Self-energy

Pion-Production in Heavy-Ion Collisions at $90-400$ $\mathrm{MeV} / \mathrm{N}$

Nuclear Fragmentation and Spallation Reactions

Supernova Matter

Topological Bootstrap

Deformed Independent-Particle Model of Yrast Spectra

Large Amplitude Collective Dynamies Using Periodic Solutions to TDHF

Spectator Fragmentation

Fermi Liquid Properties of Nuclear Matter

The Strangeness of Neutron Stars

-Introduction to Nonequilibrium Green's Functions, Part 1

Stochastic Behavior of a Deterministic Quantal System

Pion Condensation at Finite Temperature

MIDAS Overview

The Fusion of Heavy Nuclei 


\section{Appendix III}

\section{Theory Seminars}

July 14, 1982

August 3, 1982

September 8, 1982

September 21, 1982

B. Giraud

Saclay

September 22, 1982 Wolfgang Cassing,
Science and Art in Heavy Ion Scattering

Fission at Finite Excitation

Parameters for Quark Matter Formation from Lattice QCD

Variational Estimates of Exclusive Heavy-Ion Cross Sections are not Impossible

Aspects of Diabatic Single Particle Motion in Heavy-Ion Collisions 
Abachi, $\mathbf{S}$.

Abegg, $R$.

Aguer, $P$.

Ahlen, S.P.

Al-Ghazi, M.S.A.L

Aleklett, $K$.

Alexander, J.M.

Anderson, $\mathrm{L}$.

Baden, A.

Bendjaballah, N.

Benenson, W.

Bethune, G.R.

Bice, A.N.

Bieser, F.

Birchall, J.

Bistirlich, J.A.

Bjornholm, $S$.

Blockj, J.

Bloomer, M.A.

Bock, R.

Bogucki, P.

Boguta, J.

Bohrmann, S.

Bonner, W.A.

Bossingham, $\mathbf{R}$.

Bowman, H.R.

Brockmann, R.

Bronson, J.D.

Browne, $\mathrm{E}$.

Budzanowski, A.

Burde, J.

Butler, P.A

Buttke, T.

Cable, M.D.

Cairns, E.B.

Carroll, J.B.

Cauvin, B.

Cerny, J.

Chan, Y.D.

Clark, D.J.

Colombari, P.

Conzett, H.E.

Coombes, $\mathrm{H}$.

Crawford, H.J.

Crawley, G.M.

Crowe, KM.

Dacal, A.

Dairiki, J.M.

Danielewicz, $\mathbf{P}$.

\author{
$180,195,257$ \\ $165,166,250,249,251$ \\ 100 \\ $152,154,155,156$ \\ 124 \\ 169 \\ 172,244 \\ 84 \\ 186 \\ 69 \\ 55,137 \\ 257 \\ 100,101 \\ 186 \\ 207 \\ 209 \\ 248 \\ 177 \\ 55 \\ 209,241 \\ 194 \\ 108 \\ 186 \\ 186 \\ $174,177,184$ \\ 55 \\ 97,99 \\ 130 \\ 90 \\ 88 \\ 186 \\ $58,61,62,65,68$ \\ $180,195,257$ \\ 128 \\ $55,58,61,68,137$ \\ 121 \\ 243 \\ 88 \\ $100,101,103,105,107,108$ \\ 101 \\ 169 \\ 186 \\ 186 \\ 177 \\ 95 \\ 201 \\ Davis, C.A. \\ Davison, N.E. \\ Del Guerra, A. \\ Deleplanque, M.A. \\ Diamond, R.M. \\ Dines, E.L. \\ Dougan, R.J. \\ Draper, J.E. \\ Duck, E. \\ Ellegaard, C. \\ Fái, $\mathbf{G}$. \\ Firestone, R.B. \\ Fossan, D.B. \\ Fowler, G.N. \\ Frankel, K.A. \\ Friedlander, E.M. \\ Ganezer, K. \\ Ganssauge, E. \\ Garg, U. \\ Geaga, J, \\ Ghiorso, A. \\ Gimpel, R.W. \\ Glendenning, N.K. \\ Grabmayr, P. \\ Green, P. \\ Greeniaus, L.G. \\ Gregorich, KE. \\ Grotowski, K. \\ Guarino, G. \\ Gubler, H.P. \\ Guerreau, D. \\ Gutbrod, H.H. \\ Gyulassy, M. \\ Hats, D. \\ Hamagaki, $\mathrm{H}$. \\ Harilton, J.H. \\ Hanis, J.W. \\ Harvey, B.G. \\ Hashimoto, $\mathrm{O}$. \\ Hecking, P. \\ Heckman, H.H. \\ Hendrie, D.L. \\ Hinterberger, F. \\ Hirayama, $\mathrm{H}$. \\ Hoffman, $D$. \\ Honkanen, J. \\ Hoover, A.D. \\ Hsu, C.C.
}

101
101

256,258

84

$77,84,87,88,90,92$,

$111,113,114,117$

90,113

69

$87,90,113$

124

92

223,225

$95,97,98,99$

92

$159,160,162,163,237,238$

180,195

159

55

195,257

$69,72,74,75,142$

159

$197,198,203$

55

101

101

146,147

135

$119,121,135,214$

101

124

$148,172,173,182,244$

$231,232,233,234$

. 88

181,194

88

$174,177,184$

139

186,227

194,197

$159,162,163,248$

139

105

258

144

$58,61,68$

69

$111,117,126$
$165,186,227,231,232$ 
Hübel, $\mathbf{H}$.

Hulet, E.K.

Igo, G.

Jacak, B.V.

Johnson, $\mathbf{P}$.

Judek, $B$.

Kadota, S.

Karant, Y.J.

Kashy, E.

Kaufman, S.B.

Kinoshita, K.

Kluge, $\mathbf{H}$.

Knöple, K.T.

Knoll, J.

Koike, M.

Kolb, B.

Korner, H.J.

Kowalski, L.

Kraus, R.H., Ju.

Landrum, J.H.

Lapointe, $C$.

Larimer, R.M.

Lee, $D$.

Lee, I.Y.

Lee, W.P.

Legrain, $R$.

Lemmon, R.M.

Lindenberger, $\mathbf{H}$.

Lindstrom, $P$.

Liss, T.M.

Liu, Y.-W.

Löbner, $\mathrm{H}$.

Logan, D.

Lombard, $\mathbf{R}$.

Lougheed, R.W.

Loveland, W.

Ludewigt, B.

Ma, Zhong-yu

Mac Gregor, M.H.

Macchiavelli, AO.

Maguire, C.F.

Maier, M.R.

Majka, Z.

Maples, C.

Martinis, J.

Martoff, C.J.

Mathews, G.J.
92,114 McDonald, R.J.

69,72

180,195

149

154

159

194

$159,161,162,163,248$

186

148

250

84,92

55

194

186

172,182

84

124

156

69,72

100

100

144,146

88

101

139

108

87,90

195

166

55

$172,173,182,244$

124,261

194

69,72

$149,151,152$,

$154,155,156,157$

172,182

229

161

84

88

$172,174,177,182,184,244$

135

261,265

168

186

114,128
McDonald, W.J.

McFarland, R.M.

MoGaughey, P.L.

McHarris, Wm.C.

McKee, C.J.S.

McParland, C.

Miake, Y.

Miller, C.A.

Miller, J.P.

Moeller, E

Moody, K.J.

Moretto, L.G.

Morita, Y.

Morrissey, D.J.

Moss, G.A.

Moszkowski, S.A.

Moulton, J.B.

Müller, K-H.

Mulera, T.A.

Murphy, D.L.

Murphy, M.J.

Musser, J.

Myers, W.D.

Nagamiya, $\mathrm{S}$.

Nagarajan, M.A.

Nelson, $W$.

Newton, J.O.

Nissen-Meyer, $\mathbf{S}$.

Nitschke, J.M.

Nolen, J.A., Jr.

Nurmia, M.J.

Oertel, C.

Okumusoglu, N.T.

Ortiz, M.E.

Pacheco, A.J.

Padgett, M.L.

Pang, W.W.

Parry, R.F.

Perez-Mendez, V.

Perlmutter, S.

Perry, M.D.

Peter, J.

Plattner, G.R.

Poskanzer, A.M.
$87,111,113,117$

$119,120,123,126$

101

142

$149,152,154,155,151,156$

236

100

258,268

181,194

101

186

169,194

$69,144,146,147$

$87,111,113,114,117,119$,

$120,121,123,126,128,135$,

$214,215,218,219,220$

156,157

$117,119,120,123$,

$126,135,149,151$

101

209

128

240

$180,195,256,257,258$

186

$130,132,139,140$

259

203,204

$169,181,194$

55

258

87

169

$65,72,74,75,62$

186

$69,72,74,75,144$

151

100

177

$111,113,117,119$.

$120,121,123,126,213,220$

121

139

$58,61,68$

$180,195,256,257,258$

165

147

186

101

$172,173,182,244$ 
Price, P.B.

Pugh, H.G.

Quebert, J.

Radi, H.M.A.

Ramayya, AV.

Randrup, J.

Rasmussen, J.O.

Rathbun, W.

Regimbart, R.

Remler, E.A.

Renfordt, RE.

Renner, T.

Rjedesel, $\boldsymbol{H}$.

Riess, F.

Rioux, C.

Ritter, H.G.

Rivet, M.F.

Ronningen, RM.

Roy, G.

Roy, R

Ruck, V.

Sagle, AL.

Sandoval, A

Sann, $\mathbf{H}$.

Sayer, RO.

Schädel, M.

Schmitt, R.P.

Schnetzer, S.

Schroeder, L.S.

Schuck, C.

Schwartz, G.

Seaborg, G.T.

Shapiro, G.

Shida, Y.

Shih, S.

Shirley, V.S.

Shor, A.

Shotter, A.C.

Shyam, R.

Slobodrian, RJ.

Smilanski, U.

Sobotka, L.G.

Somerville, L.P.

Soukup, J.

Spieler, $\mathrm{H}$.

Steinberg, E.P.

Steiner, $\mathrm{H}$.

Stelzer, $\mathrm{H}$.
$165,166,168,259$

$174,177,184$

186

227

88

$210,223,225$

$186,189,192,227,229,236$

261,265

114

231

$174,177,184$

$172,173,182,244$

$55,172,173,182,244$

174

105

$148,172,173,182,244$

124

88

101

105

197

180,195

$174,177,184$

.254

88

69,72

$114,128,213,218$

181,194

$169,174,177,184$

90,92

256

$69,142,144,146,147,149$,

$151,152,154,155,156,157$

169

181

90,92

95

180,195

55,137

55

105,107

92

$87,111,113,117,119$,

$120,121,123,126,135$ $69,72,74,75,251$

101

$172,182,244$

148

$169,181,194$

148
Stephens, F.S.

Stevenson, J.D.

Stinson, G.M.

Stock, R.

Stocker, $H$.

Stokstad, R.G.

Stroebele, H.

Sullivan, J.P.

Svenne, J.P.

Sventek, S.

Swiatecki, W.J.

Tanihata, I.

Tarlé, G.

Thierens, H.M.

Tincknell, M.L.

Tittel, K.

Tserruya, I.

Uchiyama, F.

Van Bibber, $K$

yan Oers, H.W.T

Vaz, L.C.

von Gunten, $H$.

von Rossen, P.

Wada, Ryoichi

Wagner, G.J.

Wald, $S$.

Ward, D.

Warwick, A.J.

Weaver, D.

Weik, F.

Weiner, R.M.

Welch, R.B.

Wieman, $\mathrm{H}$.

Wild, J.F.

Wilkins, B.D.

Wilmarth, P.

Wolf, KL.

Woodard, D.

Wouters, J.M.

Wozniak, G.J.

Wu, Xi-zhen

Yamada, $\mathrm{H}$.

Youngblood, D.H.

$77,84,87,88,90$,

$92,111,113,117$

168,259

101

$174,177,184$

232

$121,130,132,135$

$174,177,184$

186,227

101

128

$204,205,207,209$

181,194

$165,166,249,251$

58

165

174

$121,130,135$

239

139

101.

124

144

100,105

253

55

$121,130,135$

$148,172,173,182,244$

261,265

$148,172,173,182,244$

237,238

146,147

$172,173,182,148,244$

69

148

146,147

$174,177,184$

195

58,68

$87,111,113,1 ! 4,117,119$,

$120,121,123,126,128,135$

229

Zajc, W.A.

Zarbakhsh, F. 


\section{Appendix IV}

\section{Author Index}

Zeitz, W.-D.

Zhang, Jing-shang

62,65

Zhou, S.H.

Zhou, Z.Y.

Zhuo, Yi-zhong

Zisman, M.S.

229

61,68

$58,61,68$

229

124 
This report was done with support from the Department of Energy. Any conclusions or opinions expressed in this report represent solely those of the author(s) and not necessarily those of The Regents of the University of California, the Lawrence Berkeley Laboratory or the Department of Energy.

Reference to a company or product name does not imply epproval or recommendation of the product by the University of California or the U.S. Department of Energy to the exclusion of others that may be suitable. 